\title{
Investigating the role of the apelinergic system in glioblastoma
}

\author{
Varun Subramaniam Venkatesh
}

\author{
A thesis \\ submitted to Victoria University of Wellington \\ in fulfilment of the requirements for the degree of \\ Doctor of Philosophy in Biomedical Science
}

Victoria University of Wellington

2018 
This thesis was conducted under the supervision of

Dr Darren J. Day (primary supervisor)

Victoria University of Wellington

Wellington, New Zealand

\author{
And \\ Professor John H. Miller (secondary supervisor) \\ Victoria University of Wellington \\ Wellington, New Zealand
}


"It is possible to make no mistakes, and still lose.

That is not a weakness. That is life."

- Jean Luc Picard 


\section{Abstract}

Elucidating the molecular signalling circuitry that underpins the pathogenesis of cancers is critical to understanding and developing effective treatment paradigms for cancer. The apelinergic system is a signalling pathway primarily consisting of the apelin peptide $(A P L N)$ and the apelin receptor $(A P L N R)$. The apelinergic system has been implicated in the pathophysiology of several cancers. However, there have been very few reports regarding the role of the apelinergic system in the brain cancer glioblastoma. Glioblastoma is a highly recalcitrant malignancy with a poor prognosis which makes understanding the underlying molecular pathogenesis critical for developing new treatments. The goal of this thesis was to investigate the role of the apelinergic system in glioblastoma. This primary goal was divided into three specific aims that comprised of the following.

i) Determine the expression of $A P L N$ and $A P L N R$ mRNA in glioblastoma and glioblastoma-derived cell lines as well as use public data to investigate the expression of $A P L N$ and $A P L N R$ in other astrocytic and oligodendroglial tumours.

ii) Develop a cellular model for the apelinergic system in glioblastoma.

iii) Determine the response of the apelinergic system to common glioblastoma stressors.

$A P L N$, but not $A P L N R$, mRNA expression was elevated in glioblastoma compared to normal tissue. Analysis of mRNA expression from several public data sources through the GLIOVIS portal demonstrated that both $A P L N$ and $A P L N R$ were upregulated in glioblastoma relative to lower grade tumours. Significantly, it was also noted that $A P L N$ expression was strongly associated with regions of hypoxia whilst APLNR expression was elevated in astrocytes, tumour cells and vascular cells.

The mRNA expression of $A P L N R$ and $A P L N$ was high in resected glioblastoma tissue but 100 - 1000 fold lower in glioblastoma-derived cell lines. The low expression of APLNR in glioblastoma-derived cells suggested that these cell lines would not be a suitable model for testing the apelinergic system in glioblastoma. To overcome this limitation, the established 
glioblastoma-derived cell line U87MG was stably transfected with a plasmid expressing an APLNR-GFP fusion protein to recapitulate the expression of APLNR seen in resected tissue, and this cell line was used as a model of the apelinergic system in glioblastoma. This U87.APLNR cell line demonstrated that exogenous $\left[\mathrm{Pyr}^{1}\right]$ apelin-13 could induce migration in the transwell migration assays, indicating a possible role in glioblastoma tumour cell migration.

Glioblastomas like other solid tumours are exposed to significant stresses from sources such as hypoxia, nutrient deprivation and chemotherapeutic treatment. The apelinergic system has been reported to protect against cell death caused by several stressors, including hypoxia and glucose deprivation, but this has not been investigated in glioblastoma cells. The effects of hypoxia and glucose deprivation and chemotherapeutic treatments were investigated in regard to mRNA expression of $A P L N$ and APLNR in glioblastoma-derived cell lines. APLN mRNA expression was upregulated in hypoxic (1\% oxygen) conditions. However, the addition of exogenous $\left[\mathrm{Pyr}^{1}\right.$ ]apelin-13 did not affect U87.APLNR cell number when cultured under hypoxic, glucose deprived or combined oxygen-glucose deprived conditions. This may suggest hypoxiamediated APLN expression has a role outside of protection. No significant changes in mRNA expression of $A P L N$ or $A P L N R$ were detected in response to the chemotherapeutic agents doxorubicin and temozolomide. Treatment of U87.APLNR cells with exogenous [Pyr ${ }^{1}$ ]apelin13 failed to protect against chemotherapeutic-induced cell death. It was concluded that the treatment of U87.APLNR cells with $\left[\mathrm{Pyr}^{1}\right]$ apelin-13 did not protect against hypoxic, hypoglycaemic, combination or chemotherapeutic induced stress.

Collectively, this thesis presents an exploration of $A P L N$ and $A P L N R$ expression in glioblastoma. It provides the first evidence of hypoxia-induced $A P L N$ expression in glioblastoma-derived cell lines. The results of this research suggest, however, that it is unlikely that the apelinergic system has a significant role in protecting against cell stress in glioblastoma but suggests that hypoxia-regulated $A P L N$ may play a critical role in glioblastoma, possibly as a signalling molecule, and this warrants further research. 


\section{Declaration}

During this thesis, some of the work was carried out in conjunction with Dr Ryan Steel (RS). Specifically, RS carried out some of the PCRs on the expression of APLNR and APLN in glioblastoma tissue samples presented in Chapter 3 as well as initial optimization of primer sets. In Chapter 3, immunohistochemistry (IHC) was performed on a sample of infantile haemangioma to verify APLNR localisation with CD34 and $\alpha S M A$, this was performed by RS and interpreted by myself. APLNR IHC expression was also performed on xenograft glioblastoma tissue by RS. The qPCR expression studies in Chapter 5 were performed together by RS and myself, however, all data were analysed by myself. I performed all other experimental work in this thesis.

All data analysis, interpretation and subsequent writing was done by myself with assistance from my supervisors Dr Darren Day, Professor John Miller and Dr Janet Pitman.

With these disclosures, I hereby declare that this thesis is my work and that all sources quoted, paraphrased or otherwise referred to have been appropriately acknowledged in the references at the end of the thesis.

Varun Subramaniam Venkatesh

2018 


\section{Acknowledgements}

I believe a man's wealth is not measured in his possessions or achievements, but in the friends he surrounds himself with. The resubmission of this $\mathrm{PhD}$ thesis showed me that, irrespective of the results, I am truly a wealthy man because I was surrounded and supported by the most amazing people. I wish my acknowledgements could be longer, but I simply can't list you all; so to all of you who helped get me to where I am, thank you.

To Dr Darren Day, you were my supervisor from day one and have advised me in all aspects of my scientific life enabling me to develop into the scientist I am today, and for that, I am so very grateful. In addition, thank you for taking a chance on me six years ago, I couldn't have got here without you, and I can't thank you enough for that.

To my secondary supervisor Professor John Miller, I thank you for your kindness, friendship, help with improving my writing technique and most crucially, your belief in me. I still have a long way to go, but thanks to you, I am getting there.

Additionally, I would like to thank Dr Janet Pitman for encouraging me at my lowest point to try again; I'm not sure if I can express what this meant to me. Moreover, thank you for helping me with proofreading my thesis when you never had to.

I would also like to thank Dr Shalen Kumar for his help with writing and your scientific mentorship over the years. I would also like to specifically thank Dinindu Senanayake and Dr Remy Schneider - there's not enough space here to say it, but your help meant everything to me.

Many people have given me good advice, proofread my thesis, helped my scientific development or just listened to me rant over the years and to name just a few - Professor Paul Teesdale-Spittle, Dr Clint Oakley, Dr Kate Oakley, Dr Meghan Cooling, Dr Fabian Westermann and Dr Katarina Robichon, thank you for all your help over the years. Additionally, thank you, Dr Diane Ormsby, for the teaching, mentoring and support.

To the members of the Day lab past and present, I thank you for all your help and support both emotional and scientific. Especially Drs Ryan Steel and Sandi Dempsey for being my first teachers and to Jennifer Soundy, Wouter Aarts and Euan Russell, thank you for being the little siblings I never knew I needed, your help and friendships are much appreciated.

To the SBS staff, especially, Derek, Sushila, Pisana, Chris and Ange, thank you for all your help in tissue culture on microscopes and every other odd scientific need I had. Neville, Shaun, Craig and Paul, thanks for keeping everything running, the laughs and the friendship! To the 
office staff, thanks for all the help over the years. A special thank you to Mary, Sandra and Lesley for just being so helpful and kind to me in every way and Paul Marsden for the laughs and friendship.

To my friends who helped me during my thesis, I can't list you all, but please know your friendships meant everything to me. Especially my Kirk office mates Balam Jiménez and Antoine Felden and all my TTR friends. Antoine, mon frère, merci pour ton amitié, ça n'aurait pas été possible sans toi.

To the Ackerley/Owen lab, you were all so friendly, helpful and great fun to drink with. I appreciated it very much. Specifically, thank you to Luke Stevenson, Mitch Ganley and Alistair Brown for the friendship and support over the years. You guys were my rocks.

To the Kivell lab, especially Dr Amy Ewald and Dr Peter Bosch your friendship and advice were much appreciated. To Kelly Paton, thanks for being there till the end, it was a gift to have your support as we both went crazy. Additionally, thanks to Dr Bronwyn Kivell for a number of good conversations and the donation of antibodies.

To the La Flamme lab, thank you, especially Pirooz for your flow cytometry support, belays and friendship. Sven Sondhauss for all your help on the MALDI and finally Nikki, thank you for the coffees, friendship, histology support and everything else you've done for me.

To my rock climbing friends, Ben and Taikata the key components of the Oreo trio, Brook, Lyall, Jasmine, Sean, Jacob, Bea, Rose, Nora, Sam Lee, Sam M, Oli Dickie, Fred and everyone else, thanks for asking me how I was, being there for me and all the advice, encouragement and feeding me. Especially, thank you to Michael Houghton for your friendship, encouragement and teaching me to trad climb, which gave me a great outlet from my $\mathrm{PhD}$ and Rosie Percival for the support, treats and teaching me to be kinder to myself.

To Dr Sarrabeth Stone, I don't think I can express just what you did for me during this time, but your love, strength and kindness have helped me immeasurably through this challenge. I'm looking forward to spending a lot more time with you.

To my parents, Vinny and Vanaja, there is not enough space to say thank you for the support you have given me. You have been there for me for this entire journey and, without you, my thesis would not have got done. I am eternally grateful for this opportunity, and I dedicate this thesis to you two.

Finally, I would like to thank Dr Melanie McConnell and Professor Ian Hermans for providing me with the cell lines and tissue samples from which I built this thesis and the Wellington Medical Research Foundation and the Cancer Society of New Zealand for the funding to complete this study. 


\section{Contents}

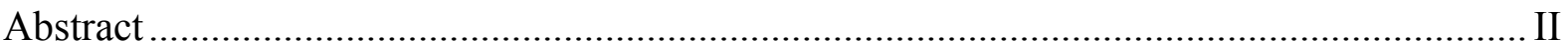

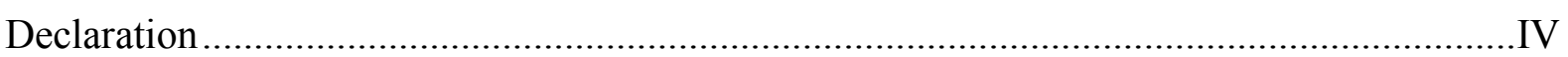

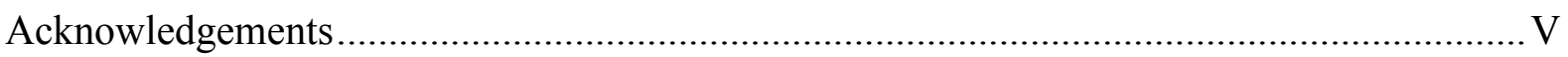

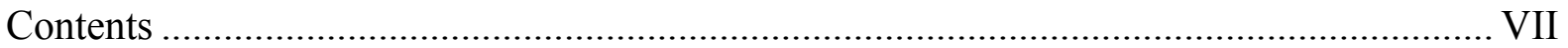

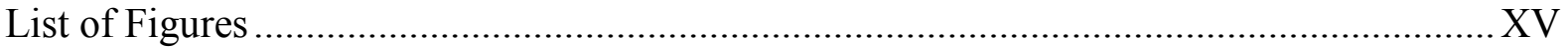

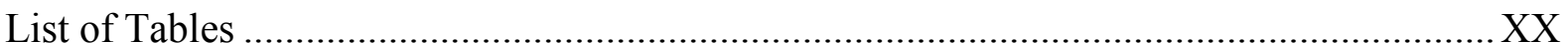

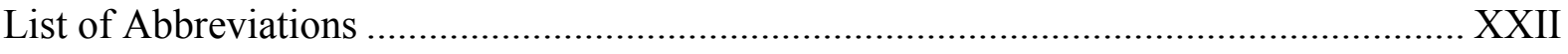

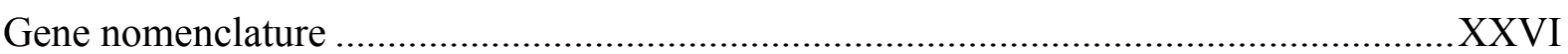

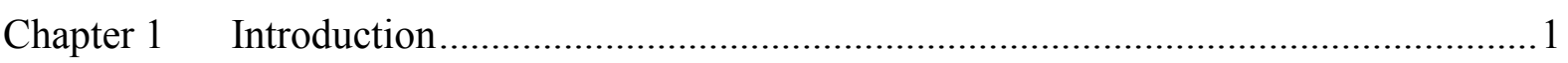

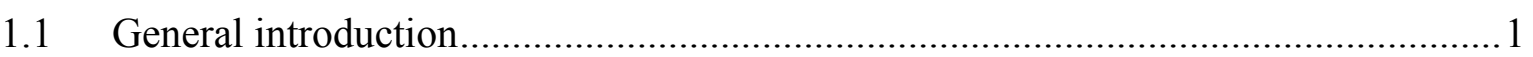

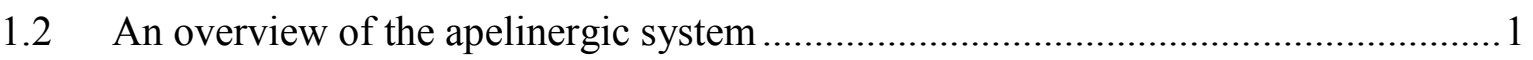

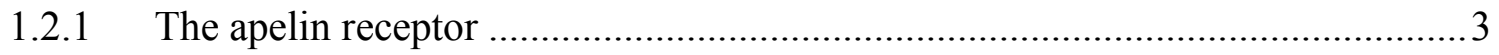

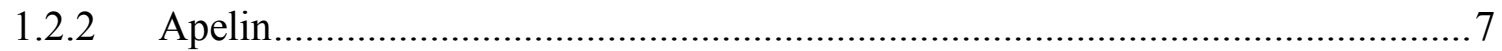

1.2.3 Apelin Receptor Early Endogenous Ligand ...............................................

1.2.4 The role of the apelinergic system in tumour pathogenesis .............................. 10

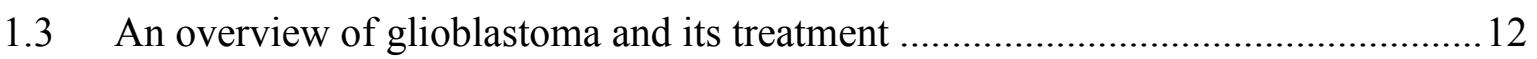

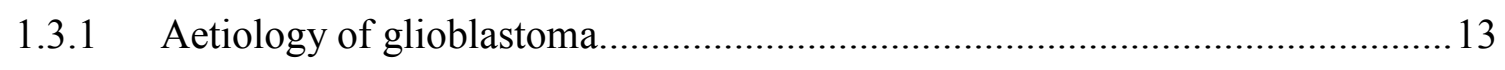

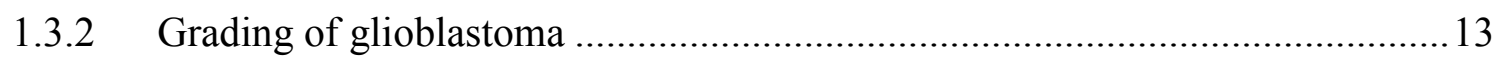

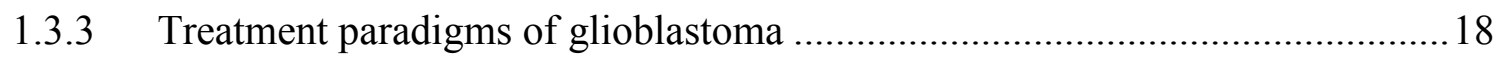

1.4 The hypothetical roles of the apelinergic system in glioblastoma. ..........................21

1.4.1 The protective role of the apelinergic system against cellular stress .................22

1.4.2 Hypoxia, the apelinergic system and glioblastoma.........................................24

1.4.3 The role of glucose in glioblastoma and the potential role of the apelinergic

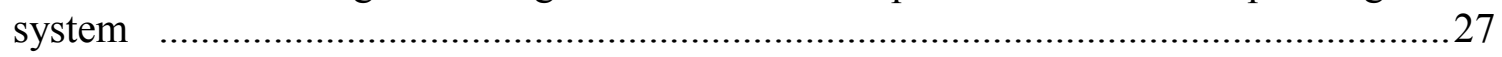

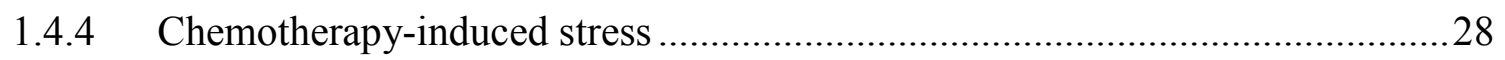

1.4.5 The role of the apelinergic system in cell migration.......................................2. 29

1.4.6 Recent insights regarding the role of the apelinergic system in glioblastoma... 30

1.5 Detecting serum APLN using aptamer technologies ............................................. 31

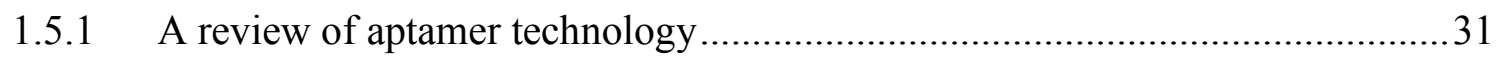

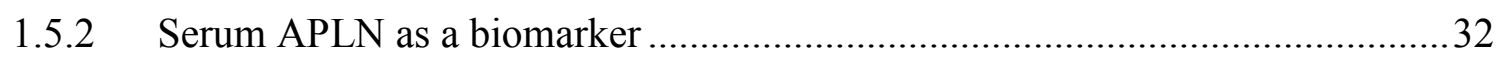

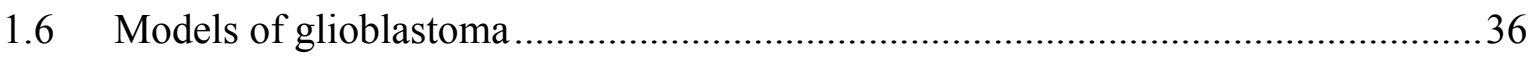

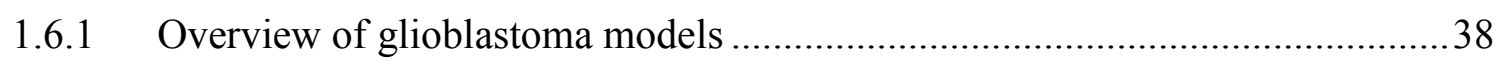

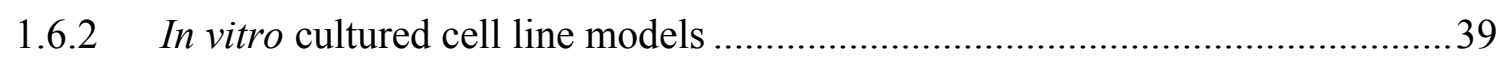


1.7 Goals of this thesis

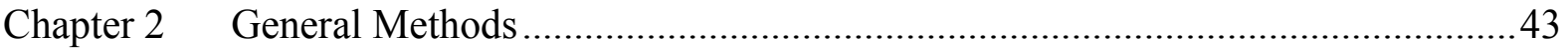

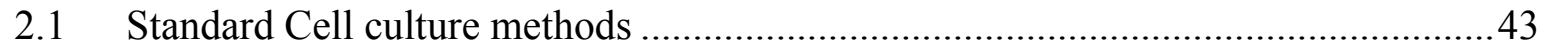

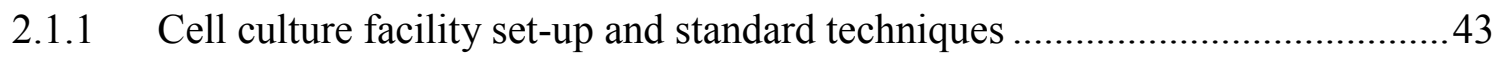

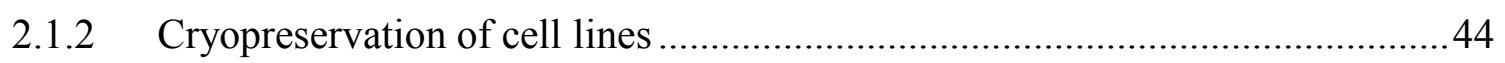

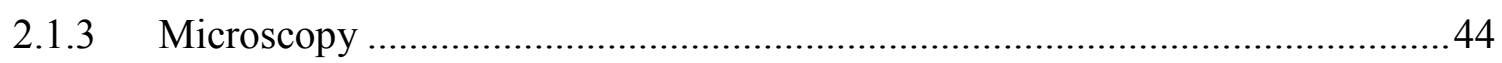

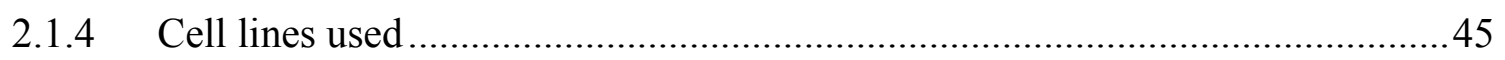

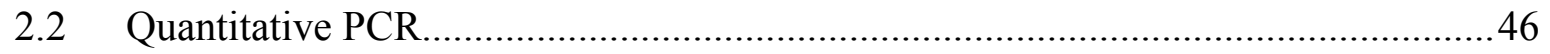

2.2.1 Cell sample preparation and RNA extraction ............................................... 46

2.2.2 Quantification of RNA via Nanodrop spectrophotometer ................................46

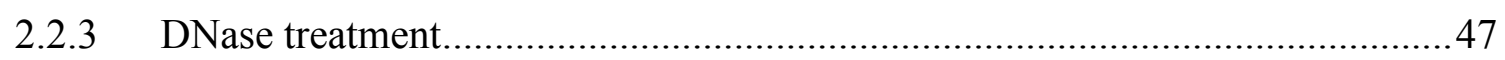

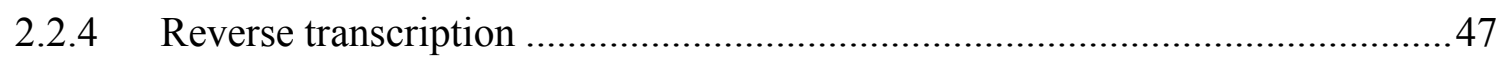

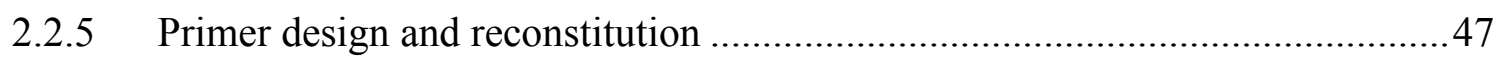

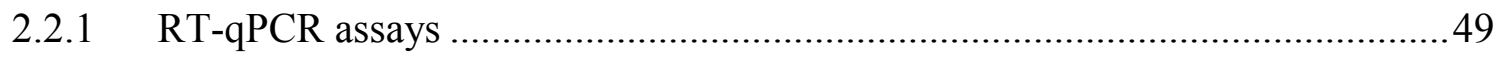

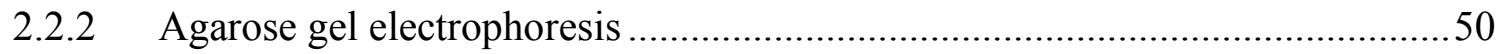

2.2.3 Small molecule and peptide reconstitution and usage .....................................50

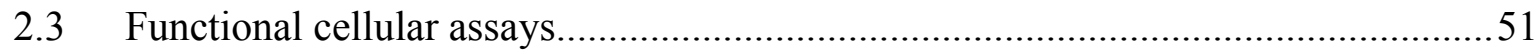

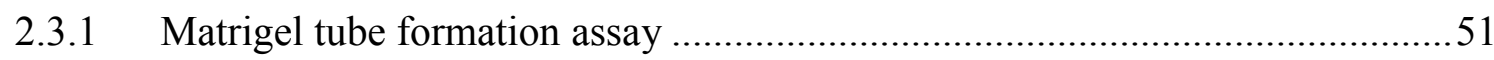

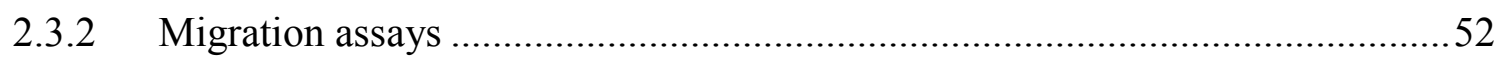

2.4 Immunohistochemistry and Immunocytochemistry ..........................................57

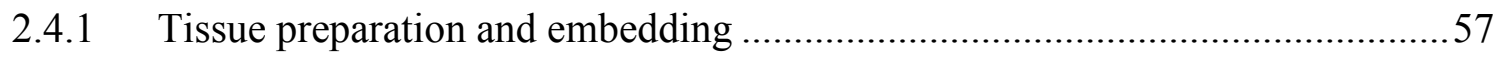

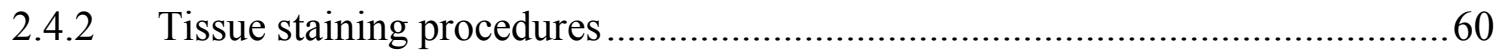

2.5 Chemically competent DH5a E. Coli cultivation and preparation............................ 63

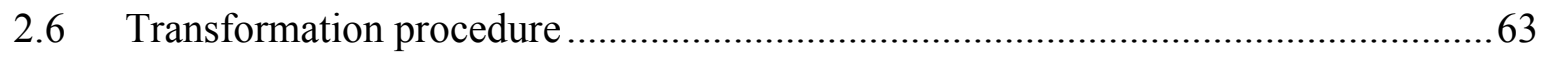

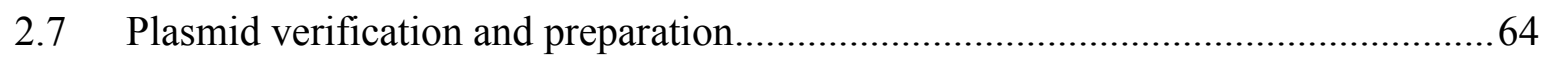

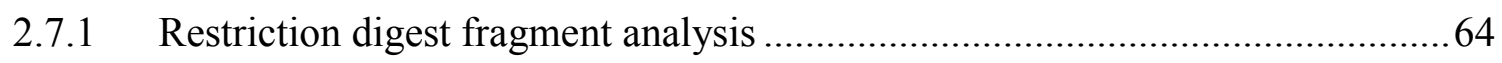

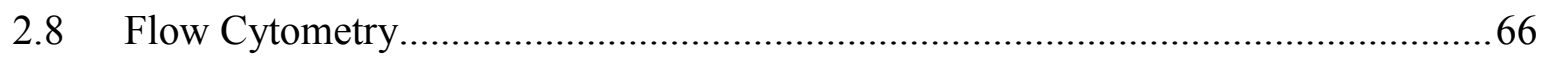

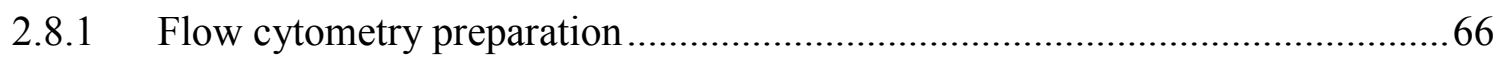

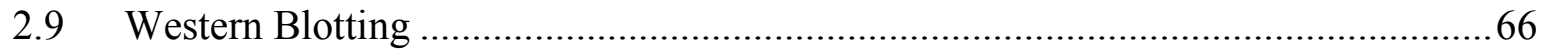

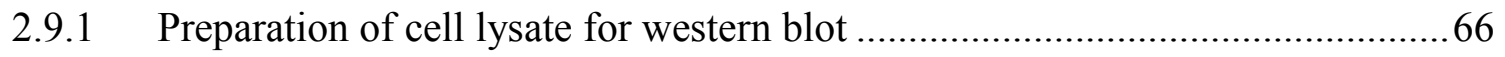

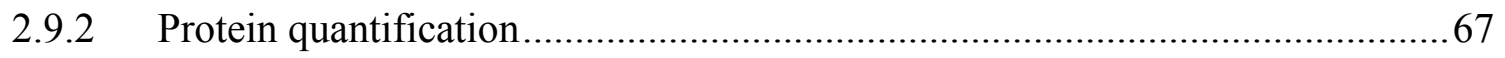

2.9.3 Sodium dodecyl sulphate-polyacrylamide gel electrophoresis (SDS PAGE) ...67

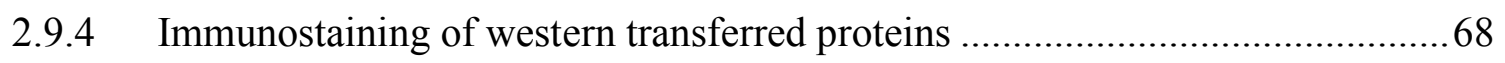

Chapter 3 The expression of the apelinergic system in glioblastoma ..............................69 


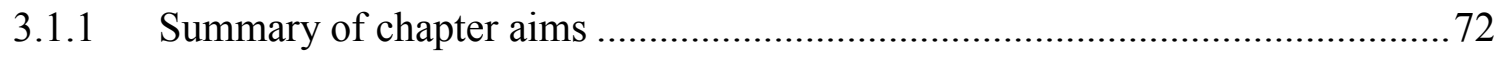

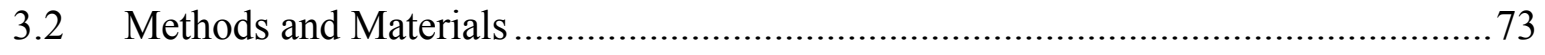

3.2.1 Reverse transcription Quantitative Polymerase Chain Reaction ....................... 73

3.2.2 Verification of APLNR antibodies binding specificity by western blotting...... 74

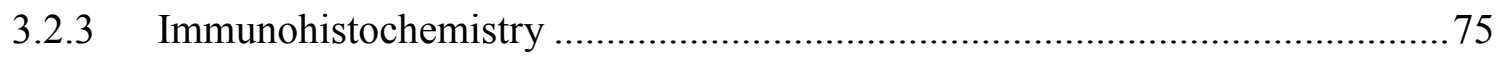

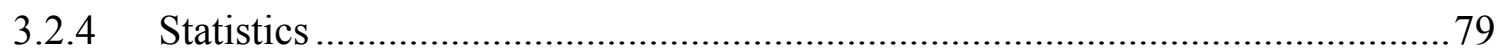

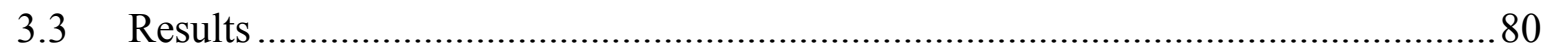

3.3.1 Expression of APLN and APLNR mRNA in human glioblastoma tissue and cell

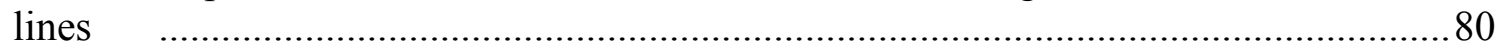

3.3.2 The mRNA expression of APLN and APLNR in glioblastoma subtypes .........84

3.3.3 Expression of APLN and APLNR mRNA in diffuse astrocytic and

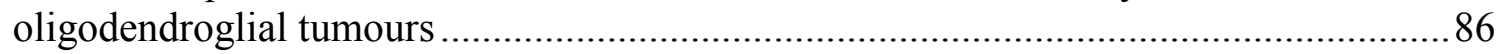

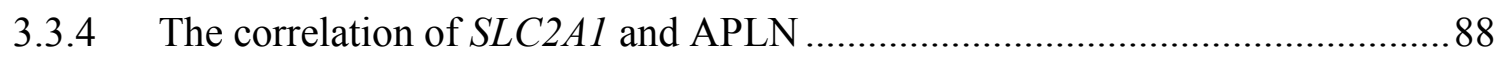

3.3.5 Investigating the potential cause of the correlation between SLC2A1 and APLN mRNA

3.3.6 Intratumoural analysis of APLN and APNLR expression in glioblastoma tissue.

3.3.7 The analysis of single-cell RNA sequenced public datasets to determine

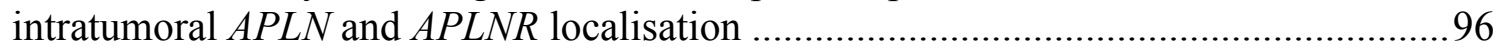

3.3.8 Immunohistochemical and immunocytochemical expression of APLNR in

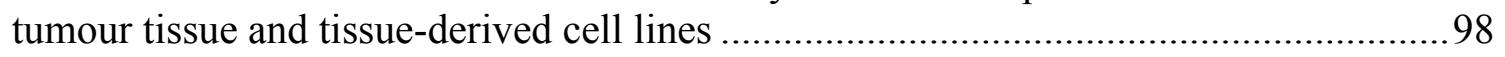

3.3.9 Analysis of APLNR expression in archival GL261 orthotopic xenograft tissue

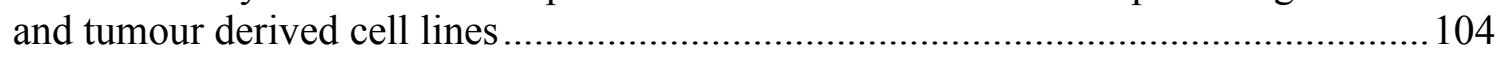

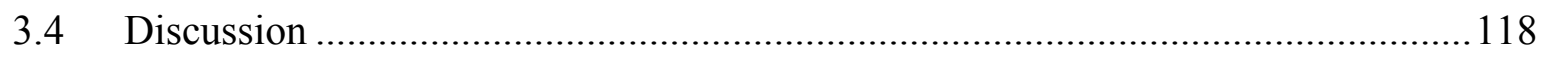

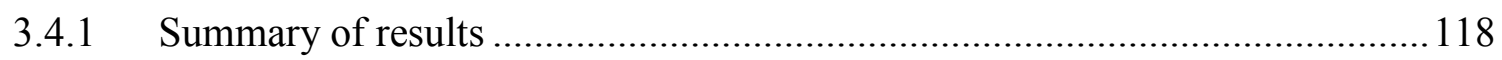

3.4.2 Analysis of APLN and APLNR mRNA expression in glioblastoma tissue and

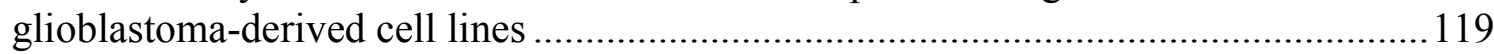

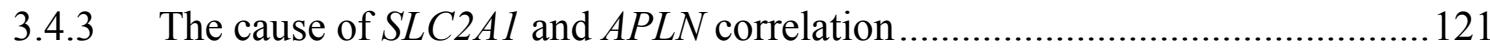

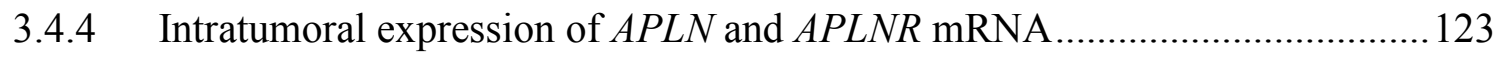

3.4.5 Single cell mRNA expression of $A P L N$ and $A P L N R$.................................... 125

3.4.6 The mRNA expression of the APLNR receptor in tumour-derived cell lines and

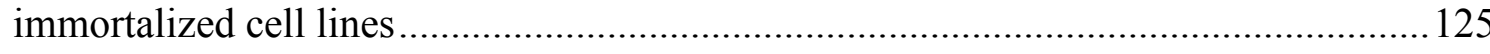

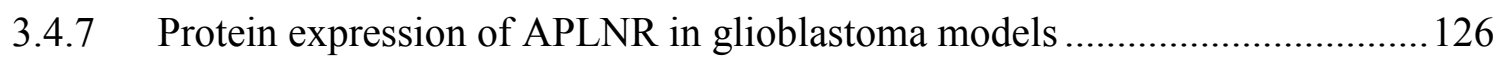

3.4.8 APLNR expression in orthotopic xenograft glioblastoma tissue and tumour-

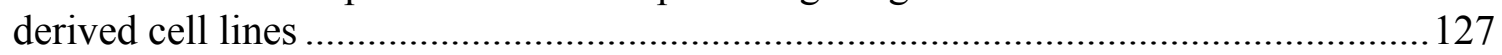

3.4.9 The expression of APLNR in the GL261 model of glioblastoma ................... 128 
3.4.10 Future directions

3.4.11 Choosing a cell model for investigation of the apelinergic system in glioblastoma

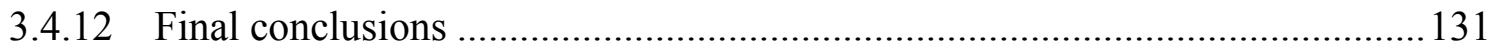

Chapter 4 Creation of an apelinergic system model in glioblastoma .............................. 133

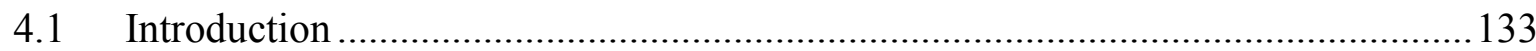

4.1.1 The selection of an in vitro model of the apelinergic system glioblastoma..... 133

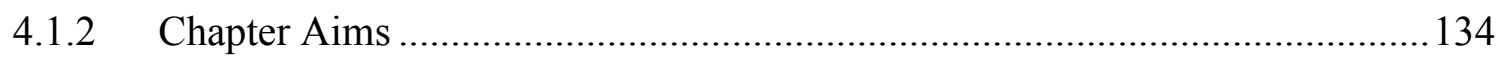

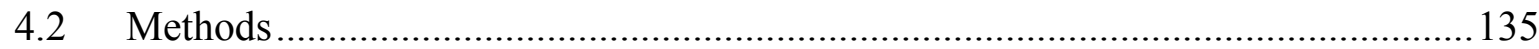

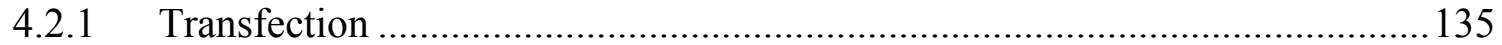

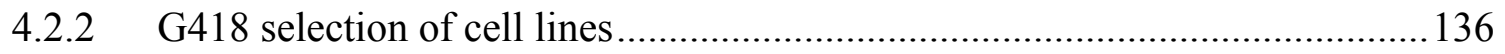

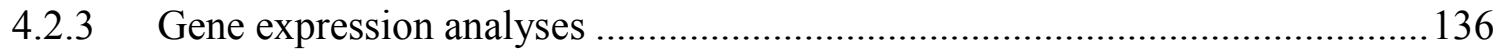

4.2.4 Testing literary reported apelinergic system signalling pathways ................... 137

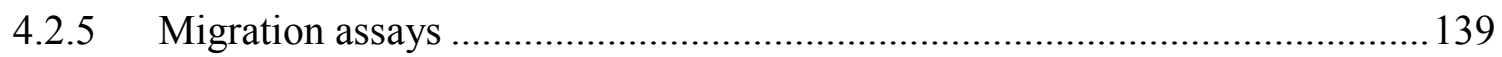

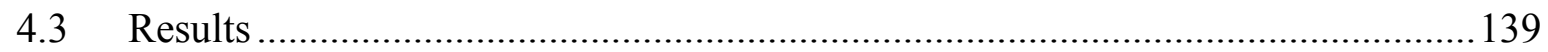

4.3.1 The creation of cell lines stably expressing APLNR-GFP .............................. 139

4.3.2 Confocal analysis of stable cell lines ........................................................... 150

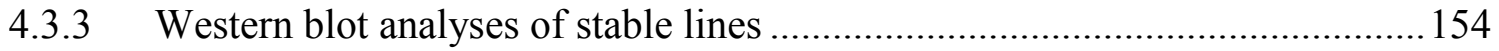

4.3.4 Stable Expression of APLNR in U87.APLNR and HEK.APLNR Cell Lines .. 156

4.3.5 Analyses of APLN-Mediated Cellular Processes ........................................... 157

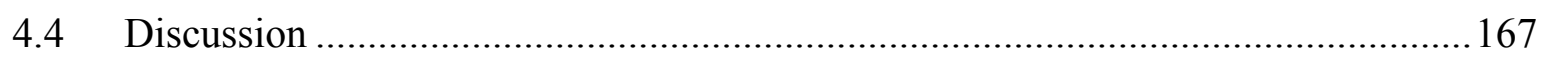

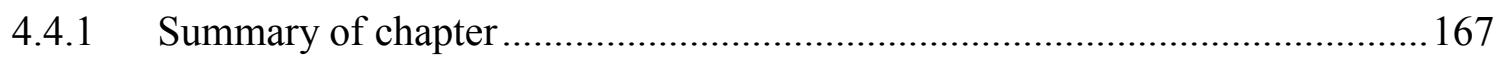

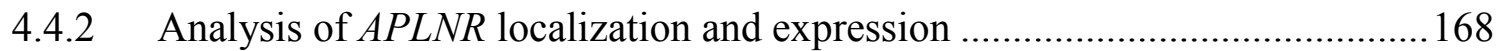

4.4.3 Analysis of commonly activated APLNR pathways. ..................................... 168

4.4.4 Migratory role of the apelinergic system in glioblastoma ............................. 169

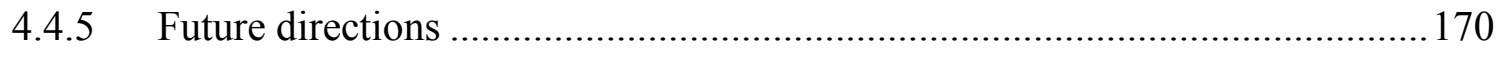

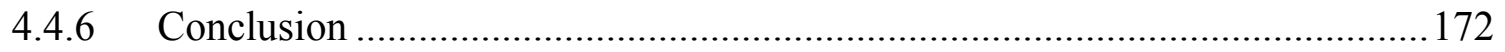

Chapter 5 The role of the apelinergic system in cell stress ........................................... 174

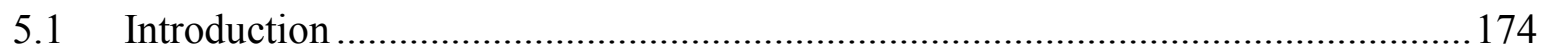

5.1.1 Role of the apelinergic system in stress response in glioblastoma ................... 174

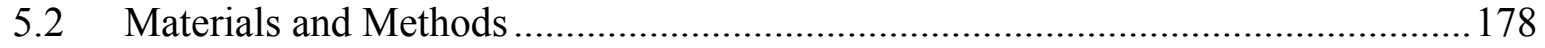

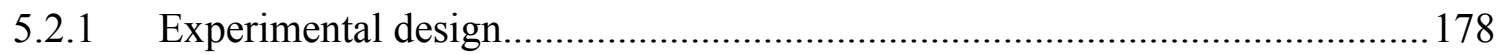

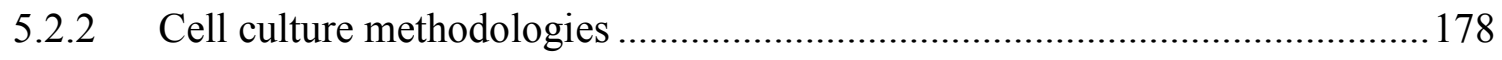

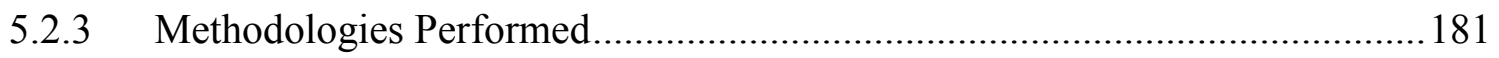




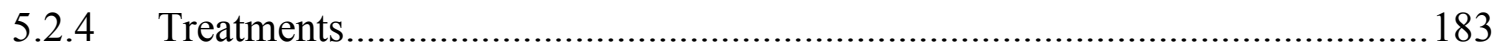

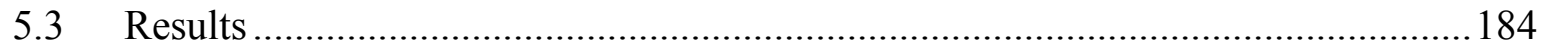

5.3.1 Effects of hypoxia on the apelinergic system ................................................... 184

5.3.2 Changes in gene expression during glucose deprivation and combined oxygenglucose deprivation in glioblastoma-derived tumour cells ............................................ 189

5.3.3 The effects of chemotherapy drugs on APLN and APLNR mRNA .................196

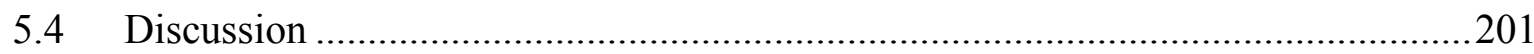

5.4.1 The role of the apelinergic system in glioblastoma response to hypoxia ........201

5.4.2 The role of the apelinergic system on glucose deprivation and combined

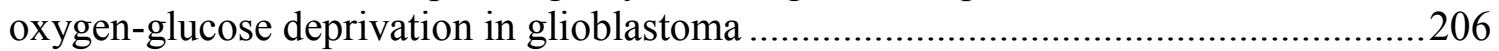

5.4.3 Role of apelin protecting against GD and OGD cell death..............................207

5.4.4 The effects of anti-cancer agents and the apelinergic system...........................210

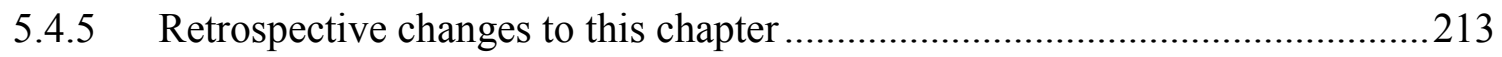

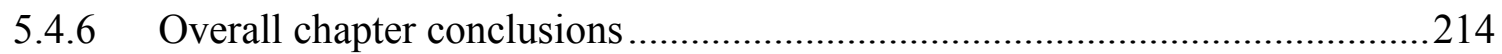

Chapter 6 Generation of an aptamer to apelin-13.......................................................2217

6.1 General introduction: The generation of an aptamer against apelin 13 .................217

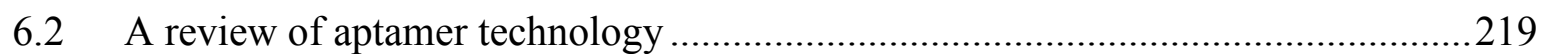

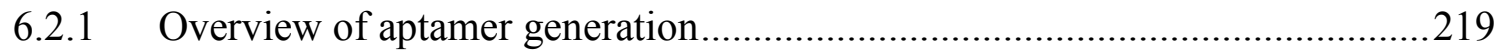

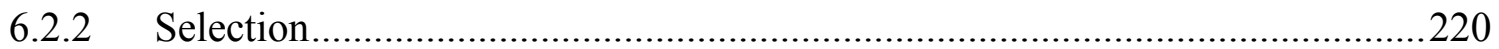

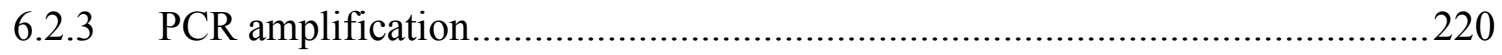

6.2.4 Generation of single-stranded oligos ........................................................... 221

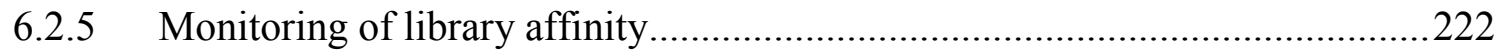

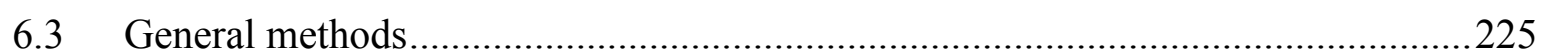

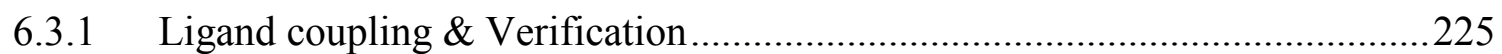

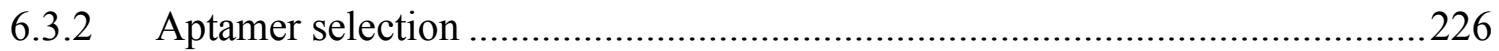

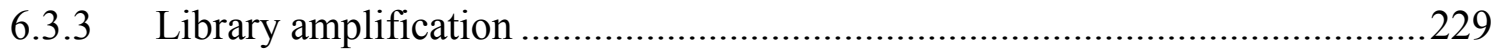

6.3.4 Generating ssDNA library using asymmetric PCR ……………………......230

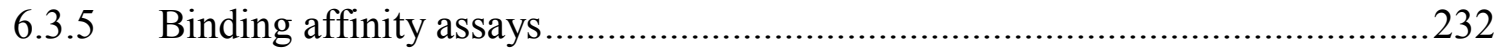

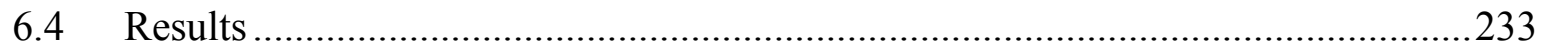

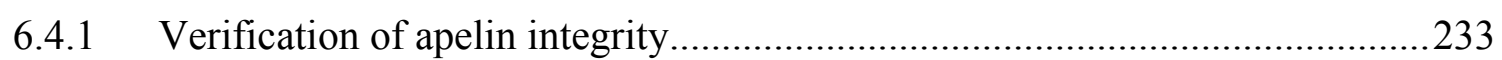

6.4.2 PCR amplification of candidates and regeneration of single-stranded library 234

6.4.3 Measurements of library binding affinity with different methods....................236

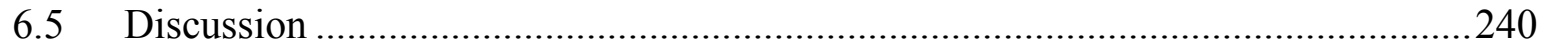

6.5.1 Coupling of apelin-13 to paramagnetic beads ................................................240

6.5.2 PCR amplification of libraries and the use of evolutionary methods ...............241 


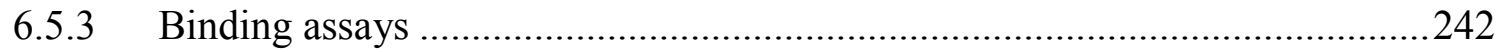

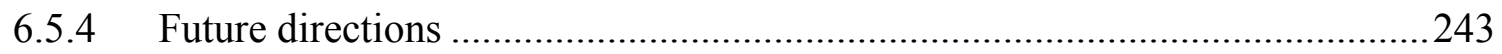

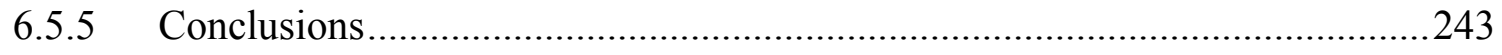

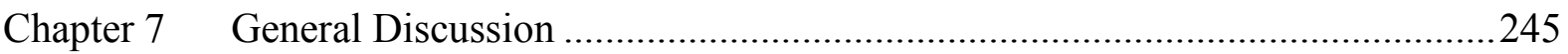

7.1 The potential role of the apelinergic system in glioblastoma................................245

7.2 Characterisation of the mRNA expression of APLN and APLNR in glioblastoma. 247

7.2.1 APLN and APLNR mRNA levels in glioblastoma ......................................247

7.2.2 APLNR and APLN mRNA levels in glioblastoma-derived cell lines ..............248

7.2.3 APLN and APLNR mRNA levels in different intratumoral regions and cell types

7.2.4 Correlation between SLC2A1 and APLN mRNA levels ...............................252

7.2.5 Future directions and limitations of Chapter 3 ...........................................253

7.3 Selection and development of an in vitro glioblastoma cell model .......................254

7.3.1 Alternative models for studying the apelinergic system in glioblastoma ........256

7.4 Investigating the role of the apelinergic system in glioblastoma ..........................259

7.4.1 Role of apelinergic system in migration in glioblastoma cells ........................259

7.4.2 Role of the apelinergic system in cell stress response. ...................................261

7.4.3 Role of the apelinergic system under conditions of glucose deprivation and

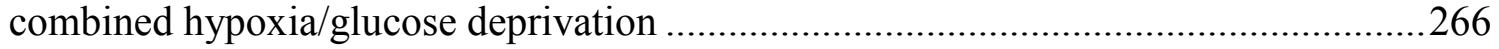

7.4.4 Role of the apelinergic system in response to chemotherapeutic agents .........269

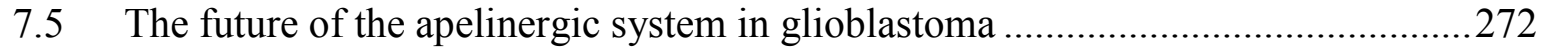

7.5.1 The apelinergic system in modulating glioblastoma pathological angiogenesis ...

7.5.2 The potential intercellular signalling role of the apelinergic system ...............272

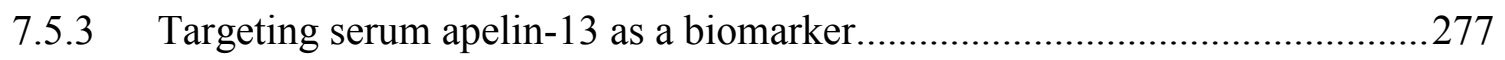

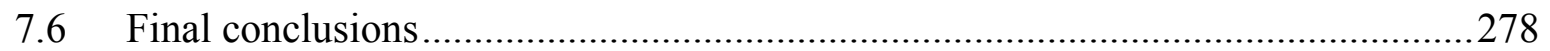

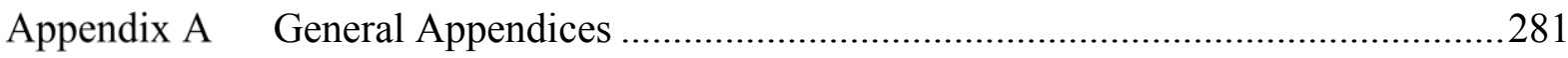

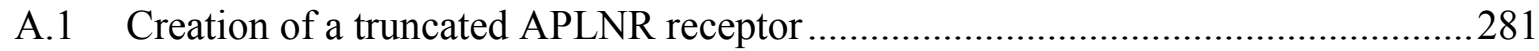

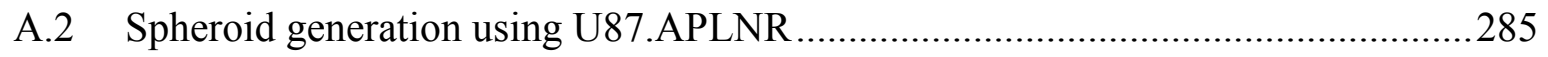

A.3 Summary of methods trialled for the generation of a clonal stable cell line...........288

A.4 Preliminary investigations into the APELA peptide ...........................................28

A.5 Optimization of concentrations of doxorubicin and TMZ on glioblastoma cell lines ..

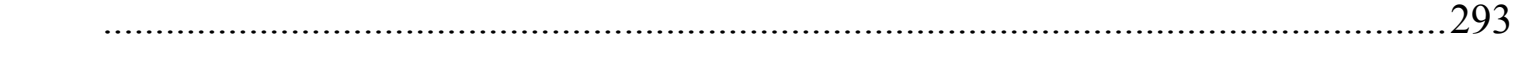

A.6 Optimization of hypoxia and glucose deprivation conditions...............................298

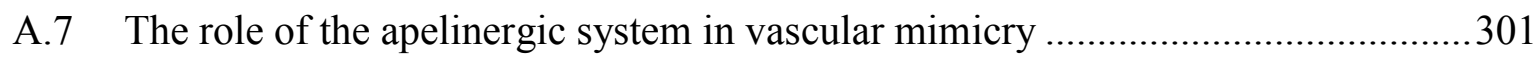




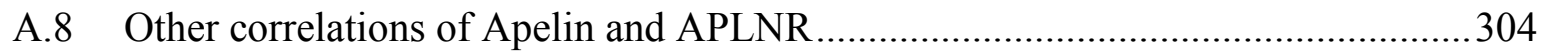

A.9 Delta cycle threshold $(\triangle \mathrm{CT})$ statistical analysis of qPCR data ..............................305

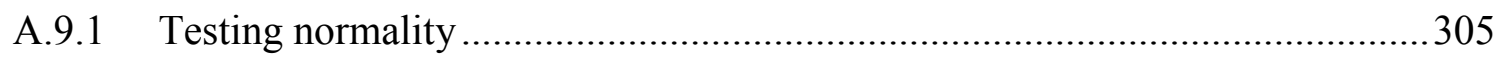

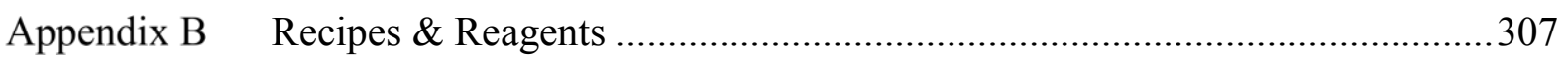

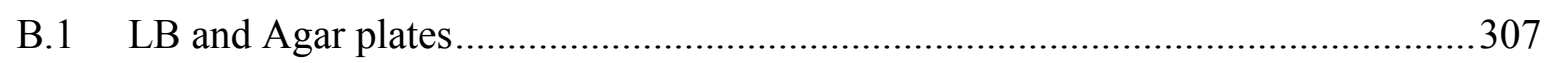

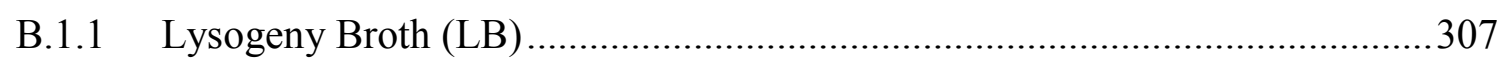

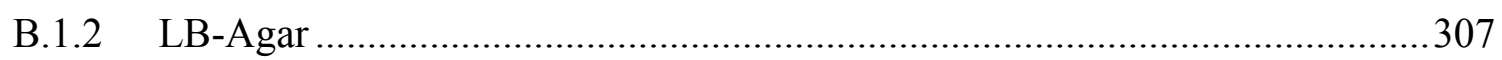

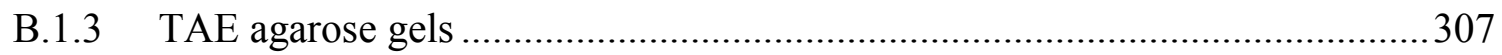

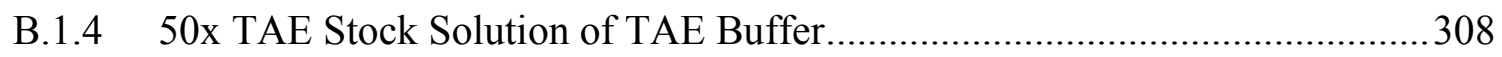

B.1.5 Super optimal broth with Catabolite repression (SOC) medium .......................308

B.1.6 Preparation and transformation of electrocompetent cells...............................309

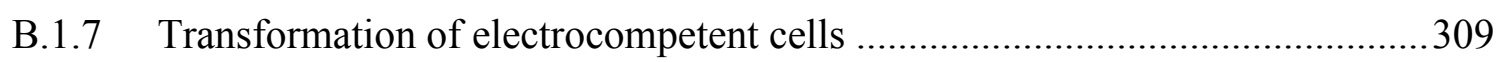

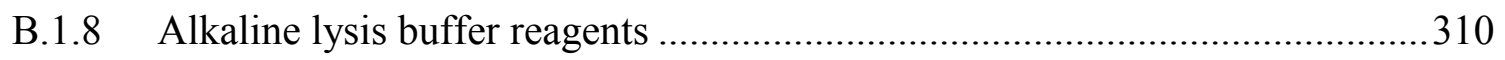

B.1.8.1 Alkaline Lysis Buffer I (Aka GTE buffer) .............................................. 310

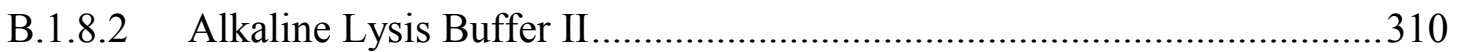

B.1.8.3 Alkaline Lysis buffer III (5 M potassium acetate) .................................310

B.1.9 Alkaline Lysis Miniprep protocol (from Sambrook et al. 3rd edition) .............310

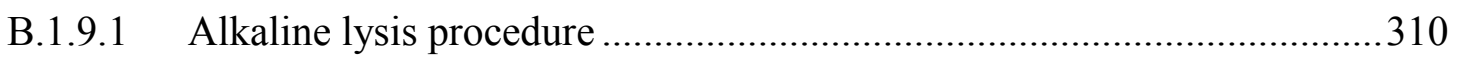

B.1.9.2 RNAse treatment and phenol-chloroform-isoamyl alcohol (PCIA)

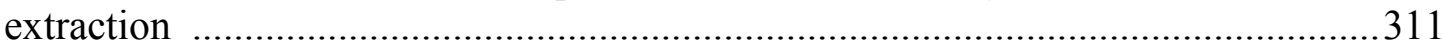

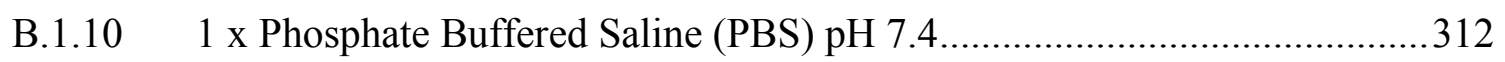

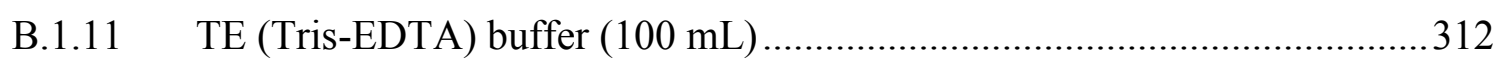

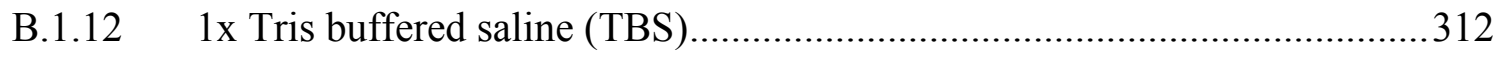

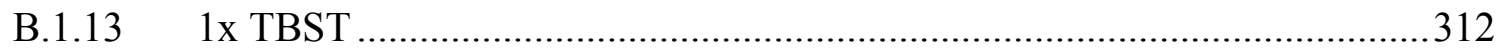

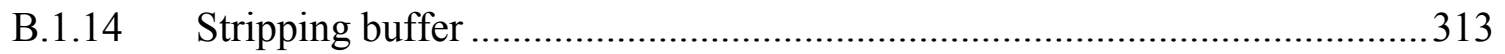

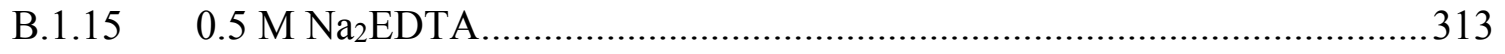

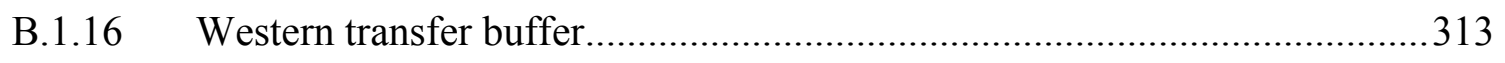

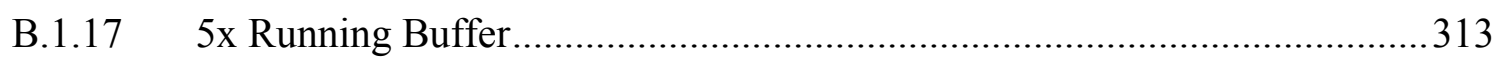

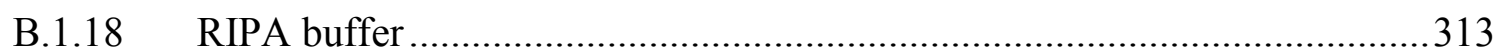

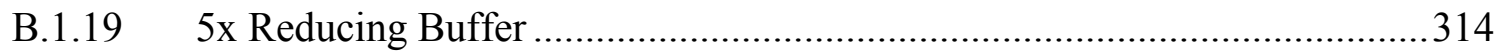

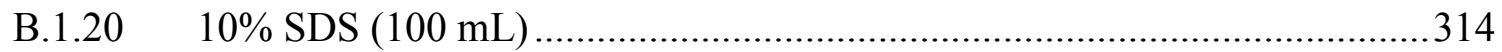

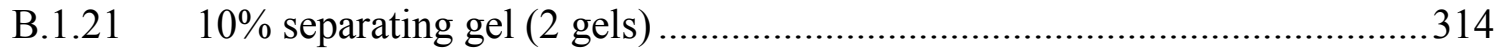

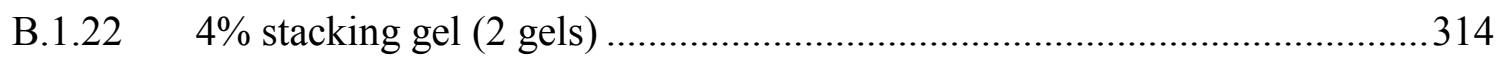

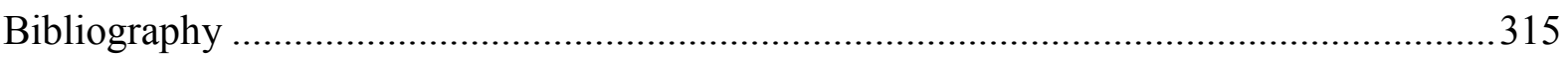




\section{List of Figures}

Figure 1.1. A review of the apelinergic system signalling pathways reproduced from the Kyoto Encyclopaedia of Genes and Genomes (KEGG) atlas.............................................. 6

Figure 1.2. A summary of the processing of APLN and key peptides that bind to APLNR ... 10

Figure 1.3. A representation of the classification algorithm of central nervous system (CNS) tumours from the 2016 WHO classification of tumours of the CNS................................... 17

Figure 1.4. MRI images demonstrating the highly invasive nature of glioblastoma............... 18

Figure 1.5. The in situ hybridisation expression of endothelial-associated markers PECAM1 (CD31), VEGF, APLNR and APLN in glioblastoma blood vessels. ....................................24

Figure 1.6. The difference in tumour recapitulation from two glioblastoma cell lines, U87MG

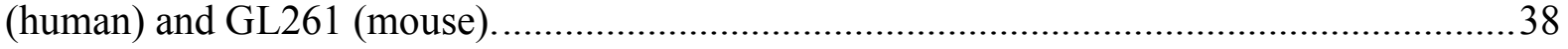

Figure 2.1. A representative image of a Matrigel-based tube formation assay ......................52

Figure 2.2. Images of the proprietary wound healing assay generating device which was

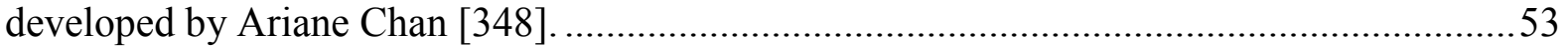

Figure 2.3. The inter and intra wound variation using the proprietary scratch generation

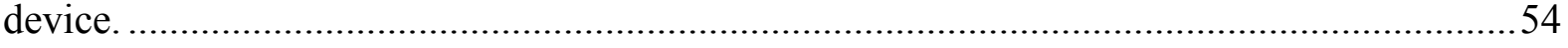

Figure 2.4. Representative images of the transwell assay. ...............................................56

Figure 2.5. Macroscopic images of GL261 burdened C57BL/6 brains...................................58

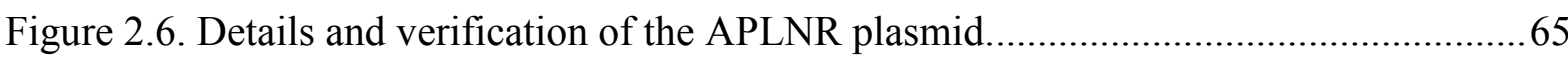

Figure 3.1. Expression levels of $A P L N, S L C 2 A 1$ and $A P L N R$ mRNA in human glioblastoma and healthy brain tissue samples.

Figure 3.2. A comparison of glioblastoma tissue, glioblastoma-derived primary and long-term immortalized glioblastoma-derived cell lines expression of APLN and APLNR.

Figure 3.3.The expression of $A P L N, A P L N R$ and SLC2A1 in glioblastoma and glioblastoma subtypes

Figure 3.4. A comparison of the mRNA expression of $A P L N, A P L N R$ and SLC2A1 in the different grades of diffuse astrocytic and oligodendroglial tumours from public TCGA datasets.

Figure 3.5. An analysis of the correlation between $S L C 2 A 1$ and $A P L N$ in glioblastoma and glioblastoma subtypes.

Figure 3.6. The Spearman's correlation of $S L C 2 A 1$ and $A P L N$ in different diffuse astrocytic and oligodendroglial tumours.

Figure 3.7. Multiple individual Spearman's correlation analyses of hypoxia and blood vessel markers with the $A P L N$ mRNA in glioblastoma. 
Figure 3.8. The expression of $A P L N, A P L N R, C A 9$ and PECAM1 (CD31) in intratumoral locations of glioblastoma tumours.

Figure 3.9. Single cell RNAseq analysis using public data.

Figure 3.10. A representative western blot of APLNR protein expression in cell line and mouse heart lysates

Figure 3.11. IHC analysis of infantile haemangioma (IH) samples for APLNR and blood vessel associated markers CD34 (endothelial cells) and alpha smooth muscle actin ( $\alpha$-SMA).

Figure 3.12. Examples of APLNR receptor expression in glioblastoma tissue samples....... 107

Figure 3.13. Expression of APLNR in the corpus callosum (Panel A) and piriform cortex

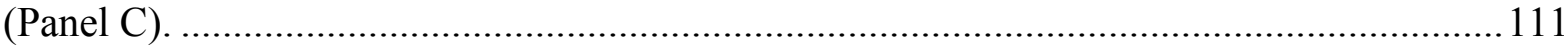

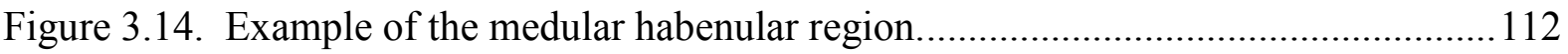

Figure 3.15. Medial habenular region APLNR expression in C57/BL6 tissue. .................... 115

Figure 3.16. Expression of APLNR at the tumour margin of the orthotopically implanted

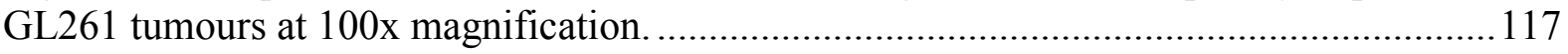

Figure 3.17. Summary of the roles of the apelinergic system in glioblastoma..................... 119

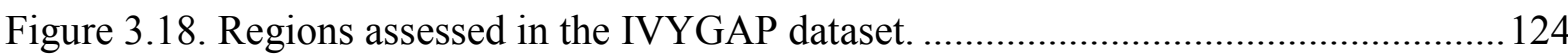

Figure 4.1. Schematic showing the areas of binding of the three pairs of APLNR-GFP directed primers.

Figure 4.2. Effect of transfection time on transfection efficiency and morphology on U87MG cells.

Figure 4.3. Representative image of U87MG cells transfected with APLNR (green cells) after iterative rounds of G418 selections with large populations of non-GFP, G418 resistant cells.

Figure 4.4. Flowchart of the single clonal cell line selection procedure.

Figure 4.5. Representative figure of overlay histogram of 20,000 HEK293 (colourless peak)

and 20,000 HEK293.APLNR (green peak) cells assessed by flow cytometry.

Figure 4.6. Qualitative and quantitative assessment of the purity of the U87.APLNR and HEK293.APLNR cell lines

Figure 4.7. Localisation of GFP-labelled APLNR in the stably transfected U87.APLNR cell

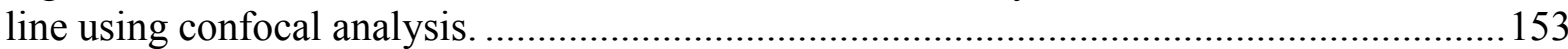

Figure 4.8. Representative western blots (WB) for endogenous APLNR and transfected APLNR-GFP.

Figure 4.9. End-point-PCR bands representing the expression of APLNR (APLNR CDS), GFP and APLNR-GFP mRNA in human brain tissue.

Figure 4.10. Quantitative analyses of APLNR mRNA expression levels in U87.APLNR cells and non-transfected U87MG cells.

Figure 4.11. Geometric mean \pm SEM GFP expression in HEK293.APLNR (A) and U87.APLNR (B) cell lines in the absence (vehicle) or presence of [Pyr1]apelin-13. 
Figure 4.12. Representative images of GFP expression as a measure of APLNR localization in

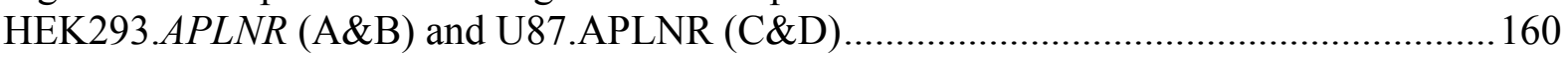

Figure 4.13. A western blot showing the effect of [ $\left.\mathrm{Pyr}^{1}\right]$ apelin-13 over time on ERK phosphorylation (pERK) in HEK293.APLNR cells.

Figure 4.14 The effect of apelin-13 on pERK levels in U87.APLNR and U87MG cells. .... 162

Figure 4.15. Change in phosphorylated ERK (pERK) after $\left[\mathrm{Pyr}^{1}\right]$ apelin-13 treatment in U87MG and U87.APLNR cells.

Figure 4.16. The effect of $\left[\mathrm{Pyr}^{1}\right]$ apelin-13 on HEK293.APLNR migration using the wound

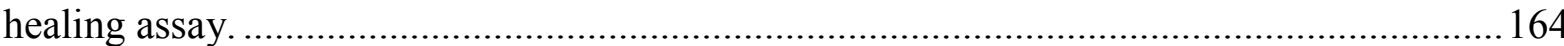

Figure 4.17. The effects of $\left[\mathrm{Pyr}^{1}\right]$ apelin-13 and ML221 on migration ................................ 165

Figure 4.18: The effects of [Pyr ${ }^{1}$ ]apelin-13 on the chemotactic migration of U87.APLNR and U87MG assessed using the transwell assay.

Figure 5.1. The effects of hypoxia on expression levels of APLN, APLNR, SLC2A1 and PROM1 mRNA.

Figure 5.2. The effect of [Pyr1] apelin-13 on the survival of U87MG and U87.APLNR cell lines cultured in hypoxic and normoxic conditions.

Figure 5.3. The effects of apelinergic inhibitor (ML221) on the migration of 0906 cells in hypoxic and normoxic conditions.

Figure 5.4. The effects of glucose deprivation on APLN, APLNR, SLC2A1 and PROM1 mRNA expression in four glioblastoma cell lines.

Figure 5.5. The effects of combined hypoxia and glucose deprivation on mRNA expression levels of $A P L N, A P L N R, S L C 2 A 1$ and PROM1 in four glioblastoma cell lines.

Figure 5.6. The effects of low glucose concentrations and the APLNR inhibitor (ML221) on U87MG cells incubated under normoxic and hypoxic conditions.

Figure 5.7. The effects of [Pyr1] apelin-13 on cell death induced by glucose deprivation (GD) in U87.APLNR and U87MG cells (A) and the correlation between glucose concentrations and total cell number (B).

Figure 5.8. The effects of doxorubicin treatment on expression levels of PROM1, APLN and APLNR mRNA in U87MG and LN18 cells.

Figure 5.9. The effects of doxorubicin and TMZ treatment on expression levels of PROMI (2n), APLN (3n) and APLNR (3n) in 0906 cells.

Figure 5.10. The effects [Pyr1]apelin-13 on doxorubicin-mediated death in U87.APLNR cells.

Figure 5.11. The effects of [Pyr1] apelin-13 on the cell number of temozolomide (TMZ)treated U87MG and U87.APLNR cells.

Figure 5.12. A possible role for hypoxia-induced APLN.

Figure 6.1. A summary of the process of SELEX.

Figure 6.2. An example of a fluorescence intensity shift due to aptamer binding, reproduced from Sefah et al. [529]. 
Figure 6.3. Amplification of eluted aptamer candidates using qPCR

Figure 6.4. Representative figure showing an example of asymmetric PCR products and FAM

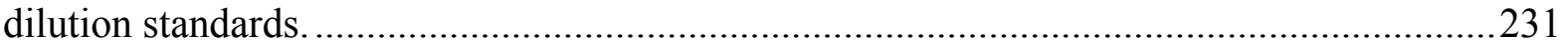

Figure 6.5. Representative image of apelin-13 MALDI-TOF spectrum. …........................2234

Figure 6.6. An example of a polydispersed emulsion generated for emulsion PCR ............235

Figure 6.7. Representative image of apelin-13 conjugated PMBs bound with the round 0, 3 and 4 library run on a flow cytometer to test binding.

Figure 6.8. Optimization of dot blot detection levels through serial dilution of FAM primer scanned at $600 \mathrm{~V}$.

Figure 6.9. The binding affinity of the round 10 library to apelin and glycine beads.

Figure 7.1. Summary of expression data from Chapter 3 regarding the intratumoral expression of $A P L N$ and $A P L N R$.

Figure 7.2. The potential roles of the apelinergic system in glioblastoma.

Figure A.1.1. The generation of the truncated APLNR plasmid and sequence confirmation.

Figure A.2.1. Preliminary analysis of the morphology of U87MG and U87.APLNR spheroids.

Figure A.4.1. The mRNA expression of APELA in glioblastoma tissue samples.

Figure A.4.2. A preliminary experiment examining the effects of oxygen and glucose deprivation on the primary cell line 0906 (Panel A) and 0814 (Panel B).

Figure A.4.3. The effect of APELA-11 (Panel A) $(n=3)$ and APELA-33 (Panel B) $(n=1)$ on

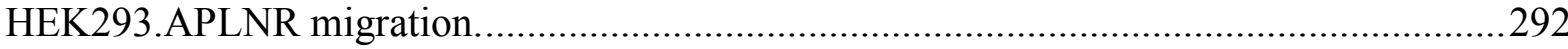

Figure A.5.1. Titration of the optimal doxorubicin concentration.....................................294

Figure A.5.2. Effect of doxorubicin on cell morphology. .................................................295

Figure A.5.3. The effect of endogenous doxorubicin fluorescence on the PI channel.........296

Figure A.5.4. The effect of APLNR inhibition on cell viability of U87MG cells treated with

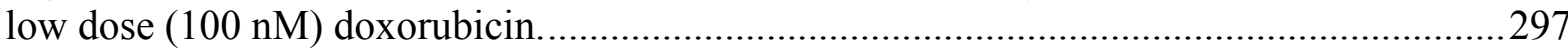

Figure A.6.1. The effect of hypoxia $\left(1 \% \mathrm{O}_{2}\right)$ on HEK293.APLNR cell viability.................298

Figure A.6.2.The effect of GD on HEK293.APLNR cells. ................................................299

Figure A.6.3. Effect of oxygen and glucose deprivation on viability of glioblastoma cell lines

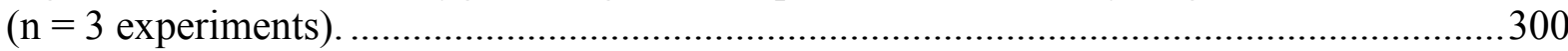

Figure A.7.1. An Initial investigation into the role of the apelinergic system on vasculogenic mimicry.

Figure A.7.2. Comparison of two separate experimental replicates of un-transfected U87MG cultures under hypoxic conditions during the Matrigel tube formation assay. 303

Figure A.8.1. A representative example of a Kaplan-Meier survival plots derived from GlioVis using the TCGA_GBM dataset. 304

Figure A.9.1. Comparing the spread of gene expression to assess normality. 306 XVIII 


\section{List of Tables}

Table 1.1. A broad overview of the physiological roles of the apelinergic system. 2

Table 1.2. A summary of the reported expression of the APLNR in brain tissue. 4

Table 1.3. Review of the literature regarding reports of the apelinergic system in cancers other than glioblastoma.

Table 1.4. The changes in WHO classification of CNS tumours. ......................................... 15

Table 1.5. Summary of current FDA approved treatment options for glioblastoma...............20

Table 1.6. Review of the different apoptotic roles of the apelinergic system. Adapted from [185] reproduced with permission from Oxford University Press.......................................23

Table 1.7. Summary of cell line models treated with hypoxia and subsequent responses. .....26

Table 1.8. Review of papers that utilise immunological based techniques to quantify serum APLN levels and correlated to physiological outcomes

Table 1.9. Review of papers that used non-immunological based methods to quantify APLN

in human serum.

Table 1.10. An overall summary of available human and murine models of glioma and their strengths and weaknesses.

Table 2.1. A summary of the cell lines and the corresponding medias used during the study.

Table 2.2 Summary of primers used during thesis.

Table 2.3 A summary of the peptides using during this study, including their origin and the solvent used to assist their aqueous suspension....

Table 2.4. Leica Tissue Processor TP1020 embedding procedure.

Table 2.5. Primary antibodies used in this study, their dilutions and sources. IHC $=$ Immunohistochemistry, ICC $=$ Immunocytochemistry and $\mathrm{WB}=$ Western blotting

Table 2.6. Secondary antibodies used in this study, their dilutions and sources....................62

Table 3.1. Summary of antibodies used in this study to detect APLNR ............................... 75

Table 3.2. Description of the analysed areas of glioblastoma tissue used from the Ivy Gap

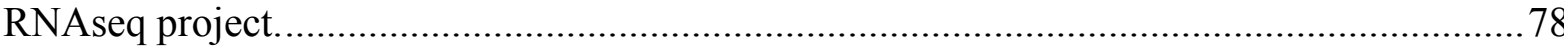

Table 3.3. Descriptive terms used for Spearman's correlation coefficients. .......................... 80

Table 3.4. A summary of the correlations of APLN and SLC2A1 between hypoxic and endothelial markers.

Table 5.1. The average delta cycle threshold $(\Delta \mathrm{CT})$ values for all genes under hypoxic and normoxic conditions. 
Table 6.1. Details of aptamer library and primer sequences. $\mathrm{N}$ refers to a random nucleotide.

Table 6.2. Summary of selection stringencies applied during SELEX ...............................228

Table 7.1. A summary of the physiological roles of the apelinergic system that could contribute to glioblastoma pathophysiology.

Table 7.2. Summary of key papers that use stable transfection to modulate endogenous APLN or APLNR expression to study the apelinergic system in tumour biology. .258

Table 7.3. Summary of effects of hypoxia on APLN, APLNR, SLC2A1 and PROM1 mRNA expression in four glioblastoma cell lines. 263

Table 7.4. Summary of mRNA expression changes due to glucose deprivation and combined hypoxia/glucose deprivation. 268

Table A.3.5. A summary of the potential methods able to create a clonal stable cell line....288

Table A.9.1. A table summarising the normality of each gene for each treatment group. ....305 


\section{List of Abbreviations}

\begin{tabular}{ll}
\hline Contraction & Definition \\
\hline A260 & Absorbance at 260 nm \\
aa & Amino acid \\
ab & Antibody \\
AC & Adenocarcinoma \\
ACE & Angiotensin-converting enzyme \\
ACE2 & Angiotensin-converting enzyme 2 \\
Akt & Otherwise known as PKB (protein kinase B) \\
AngII & Angiotensin type II \\
APLNR & Apelin receptor \\
ATCC & American Type Culture Collection \\
bFGF & Basic fibroblast growth factor \\
bp & Base pairs \\
BSA & Bovine serum albumin \\
C57BL/6 & C57 black 6 mouse strain \\
CA9 & Carbonic anhydrase 9 \\
cAMP & Cyclic adenosine monophosphate \\
CC & Colon cancer \\
CC-RCC & Clear cell renal carcinoma \\
CD133 & Cluster of differentiation 133 \\
CD34 & Cluster of differentiation 34 \\
CD31 & Cluster of differentiation 31 (PECAM1) \\
cDNA & Complementary deoxyribonucleic acid \\
CNS & Central nervous system \\
CRC & Colorectal Cancer \\
CSCs & Cancer Stem Cells \\
CT & Cycle Threshold \\
DAPI & $4 ', 6$-diamidino-2-phenylindole \\
ddH2O & Double distilled water \\
DMEM & Dulbecco's modified Eagle medium \\
DMSO & Dimethyl sulfoxide \\
DNase & Deoxyribonuclease \\
EGF & Epidermal growth factor \\
EGFR & Endothelial growth factor receptor \\
APELA & Apelin receptor early endogenous ligand \\
ELISA & Enzyme-linked immunosorbent assays \\
Erk & Extracellular regulated kinase \\
& XXII \\
& \\
&
\end{tabular}


ENU

FACS

FBS

FDA

FSC

GD

GEC

GFAP

GLUT1

GPCR

GCSC

$\mathrm{HCC}$

HEK

HIF $1 \alpha$

HPRT

HRE

hrs, min, sec

HUVECS

ICC

IDH

IH

IHC

IR

$\mathrm{kDa}$

Da

KEGG

KOR

LB

MAPK

mRNA

NSCLC

NTC

NTSR1

OGD

OGG1

oligo

oligo(dT)

OSCC
N-ethyl-N-nitrosourea

Fluorescence-assisted cell sorting

Foetal Bovine Serum

Federal drug administration

Forward (light) scatter

Glucose deprivation

Gastroesophageal cancer

Glial fibrillary acidic protein

Glucose transporter 1

$\mathrm{G}$ protein-coupled receptor

Glioblastoma Cancer Stem cells

Hepatocellular carcinoma

human embryonic kidney

Hypoxia Inhibitory Factor 1 alpha

Hypoxanthine-guanine

phosphoribosyltransferase

Hypoxia response element

Hours, Minutes, Seconds

Human Umbilical Vein Endothelial Cells

Immunocytochemistry

Isocitrate dehydrogenase

Infantile haemangioma

Immunohistochemistry

Immunoreactivity

Kilo Dalton

Dalton

Kyoto Encyclopaedia of Genes and

Genomes

Kapa Opioid receptor

Luria Broth

Mitogen-activated protein kinase

Messenger ribonucleic acid

Non-small cell lung cancers

No template control

Neurotensin 1 receptor

Oxygen and glucose deprivation

8-Oxoguanine glycosylase

Oligonucleotide, a DNA or RNA

polynucleotide molecule

A short sequence of single-stranded deoxythymine

Oral Squamous cell carcinoma 
PAGE

PBS

PCR

PCSK3

PECAM1

PEG

PENSTREP

pERK

PET

$\mathrm{pH}$

PI

PI3K

PVDF

PVN

PDX

PROM1

qPCR

RCF

RNA

RNase

RPM

RPMI

rs

RT

RT

RT-qPCR

SCID/NOD

SCNH2

SD

SDS

SELEX

SLC2A1

SON

Sox-2

SSC

ssDNA

TAE

TBP

TBS
Polyacrylamide gel electrophoresis

Phosphate buffered saline

Polymerase chain reaction

Proprotein convertase subtilisin/kexin 3

Platelet and endothelial cell adhesion

molecule 1

Polyethylene glycol

Penicillin \& Streptomycin

Phosphorylated Extracellular regulated

kinase

Positron emission tomography

Potential of hydrogen

Propidium iodide

Phosphoinositide 3-kinase

Polyvinylidene difluoride

Paraventricular nuclei

Patient Derived Xenograft

Prominin 1

Quantitative PCR

Relative centrifugal force

Ribonucleic acid

Ribonuclease

Revolutions per minute

Roswell Park Memorial Institute medium

Spearman's correlation coefficient

Room Temperature

Radiation therapy

Reverse transcription quantitative PCR

Severe combined

immunodeficiency/Nonobese diabetic

Selective apelin-36 cutting and amidation

Standard deviation

Sodium dodecyl sulphate

Systematic evolution of ligands by

exponential enrichment

Solute carrier family 2 A 1

supraoptic nuclei

(sex determining region Y)-box 2

Side (light) scatter

Single-stranded DNA

Tris-acetate EDTA

TATA-box-binding project

Tris Buffered Saline 
TBS-T

TCA

TCGA

TE

TMZ

Tris

TSS

TTF

UV

$\mathrm{v} / \mathrm{v}$

VEGF

WHO

$\alpha$-SMA
Tris Buffer Saline with Tween

Tricarboxylic acid

The cancer genome atlas

Tris-EDTA

Temozolomide

Tris(hydroxymethyl)aminomethane

Transformation Storage Solution

Tumour treating fields

Ultraviolet

Volume per volume

Vascular Endothelial Growth Factor

World Health Organisation

Alpha smooth muscle actin 


\section{Gene nomenclature}

The HUGO Gene Nomenclature Committee (HGNC) is the worldwide authority with regards to gene nomenclature. During this thesis every effort was made to adhere to HGNC nomenclature rules; however, this may lead to some confusion as certain genes used during this thesis are referred to as their HGNC name and not their more ubiquitously used synonym. To prevent confusion, a table of the key genes and their appropriate nomenclature and any variants in the nomenclature used during this thesis is provided below. This thesis follows HGNC recommendations with regard to italicizing the gene symbol when referring to the gene or mRNA but non-italicised when referring to protein. Moreover, when referring to the murine version of the gene, the first letter of the symbol is capitalised, and the remaining are lower case. To avoid confusion with the apelin protein isoforms used during this thesis for experimentation and referring to the apelin isoforms in a hypothetical biological setting, exogenously added apelin are written out in full, i.e. apelin-13 refers to the 13 amino acid form of apelin and $\left[\mathrm{Pyr}^{1}\right]$ apelin-13 refers to the pyroglutamated form.

\begin{tabular}{lll}
\hline HGNC approved name & HGNC approved gene symbols & Synonyms \\
\hline Apelin & $A P L N$ & apelin, AGTRL1 ligand \\
Apelin receptor & $A P L N R$ & APJ, AGTRL1 receptor \\
Solute carrier family 2 member 1 & $S L C 2 A 1$ & GLUT1, GLUT \\
Hypoxanthine phosphoribosyltransferase 1 & HPRT1 & HPRT, HGPRT \\
Apelin receptor early endogenous ligand & $A P E L A$ & Toddler, ELABELA, ELA \\
Prominin 1 & PROM1 & CD133 \\
\hline
\end{tabular}




\section{Chapter 1 Introduction}

\subsection{General introduction}

The apelinergic system is a peptide signalling system that is thought to contribute to the growth of cancer via pathophysiological processes including angiogenesis [1-3] and cell migration [47]. Moreover, it is stimulated under hypoxic conditions [8-10]. These pathophysiological processes, together with the presence of hypoxia, are central to solid tumour pathogenesis including the highly recalcitrant brain tumour glioblastoma. The overall objective of this thesis was to investigate the role of the apelinergic system in glioblastoma using an in vitro cell model.

\subsection{An overview of the apelinergic system}

The apelinergic system primarily consists of the apelin receptor (APLNR) also known as APJ and its cognate peptide ligand apelin $(A P L N)$ [11]. Since the discovery of the APLN peptide, several isoforms of APLN have been discovered as well as other ligands that can bind to APLNR. The structure and function of the apelinergic system are well reviewed elsewhere [12] but is also discussed briefly herein. The apelinergic system has regulatory roles in several homeostatic functions (see Table 1.1) including glucose and lipid metabolism [13], fluid homeostasis [14] and cardiovascular control [15]. Additionally, it is also known for its pathological roles in conditions such as diabetes [16] and cardiovascular diseases [17]. 
Table 1.1. A broad overview of the physiological roles of the apelinergic system.

\begin{tabular}{|c|c|c|}
\hline $\begin{array}{l}\text { Physiological } \\
\text { system }\end{array}$ & Apelinergic system physiological role & Reference \\
\hline $\begin{array}{l}\text { Cardiovascular } \\
\text { system }\end{array}$ & $\begin{array}{l}\text { Affects vascular tone and blood pressure as well as cardiac } \\
\text { contractility }\end{array}$ & {$[18]$} \\
\hline $\begin{array}{l}\text { Blood vessel } \\
\text { formation }\end{array}$ & Regulates angiogenesis; regulates blood vessel diameter & {$[19]$} \\
\hline $\begin{array}{l}\text { Diabetes, obesity } \\
\text { and energy } \\
\text { regulation }\end{array}$ & $\begin{array}{l}A P L N \text { is up-regulated in human and murine obesity and } \\
\text { can inhibit diet-induced obesity in mice. } A P L N \text { is required } \\
\text { for the maintenance of insulin sensitivity and can stimulate } \\
\text { mitochondrial function. } A P L N \text { regulates food satiety } \\
\text { through the hypothalamus and can also increase glucose } \\
\text { uptake. }\end{array}$ & {$[20],[21],[22],[23]$} \\
\hline $\begin{array}{l}\text { Metabolism } \\
\text { (Glucose and } \\
\text { Lipid) }\end{array}$ & $\begin{array}{l}A P L N \text { stimulates uptake and utilization of glucose. } A P L N \\
\text { modulates lipid metabolism. }\end{array}$ & {$[13,24-27]$} \\
\hline $\begin{array}{l}\text { Gastrointestinal } \\
\text { system }\end{array}$ & $\begin{array}{l}\text { Exogenous APLN stimulates gastrointestinal cells to } \\
\text { proliferate. }\end{array}$ & {$[28]$} \\
\hline Fluid homeostasis & $\begin{array}{l}A P L N \text { is associated with arginine vasopressin (AVP) } \\
\text { synthesis and release and co-localizes with AVP. APLNR } \\
\text { knockout mice drink less water and have a decreased } \\
\text { ability to concentrate urine. }\end{array}$ & {$[29]$} \\
\hline Lung development & $\begin{array}{l}A P L N \text { is highly expressed during foetal lung development, } \\
\text { and its effects are mediated through the p } 38 \text { and JNK } \\
\text { pathways. }\end{array}$ & {$[30]$} \\
\hline Atherosclerosis & $\begin{array}{l}A P L N \text { abrogates the effects of angiotensin II-induced } \\
\text { atherosclerosis. }\end{array}$ & {$[31]$} \\
\hline Early development & $\begin{array}{l}A P L N \text { promotes haematopoiesis of human embryonic stem } \\
\text { cells and endothelial cells. The second } A P L N R \text { ligand, } \\
\text { APELA, is a global mitogen and essential for heart } \\
\text { development }\end{array}$ & {$[32],[33] .[34,35]$} \\
\hline $\begin{array}{l}\text { Reproductive } \\
\text { physiology }\end{array}$ & $\begin{array}{l}\text { APLN/APLNR are expressed in hypothalamic-pituitary } \\
\text { gonad axis tissue. APLN modulates gonadotropin } \\
\text { secretion. APLN/APLNR axis is implicated in granulosa } \\
\text { cell steroidogenesis and is hypothesised to play a role in } \\
\text { follicular development. }\end{array}$ & {$[36,37]$} \\
\hline
\end{tabular}




\subsubsection{The apelin receptor}

The apelin receptor (APLNR) was discovered in 1993 but remained an orphan receptor until the discovery of the putative ligand [38]. APLNR is a G protein-coupled receptor (GPCR) that shares homology with the angiotensin type I receptor but is not bound by angiotensin II [38]. APLNR encodes a 380 amino acid receptor in humans [38]. APLNR has been sequenced in several species and appears to be well-conserved with rat Aplnr sharing 96 and $89.7 \%$ homology to mouse Aplnr and human APLNR, respectively. APLNR has also been identified in non-mammalian species such as the African clawed toad [39] and zebrafish [40].

$A P L N R$ is expressed extensively in humans, rats and mice [41, 42]. Localized tissue expression of $A P L N R$ has been examined at both a mRNA and protein level. APLNR mRNA was initially detected in the brain of rats [38] and humans [43, 44]. It has since also been detected in the lung, heart, skeletal muscle, kidney and liver, indicating widespread expression [45]. As this current study investigates the role of the apelinergic system in glioblastoma, the expression of $A P L N R$ in the brain is further discussed below.

APLNR mRNA transcripts have been detected in numerous brain regions [38, 44]; however, these findings have been contradicted by others who reported the more localised expression of APLNR to only certain regions of the brain [45]. The regions of the brain in which APLNR protein have been detected in rats, mice and humans are summarised in Table 1.2. In summary, expression of APLNR has been reported in both neurons and glial cells within the hypothalamus, hippocampus and cortex $[42,46]$. Specifically, the localisation of APLNR was often observed in neurons within the supraoptic nucleus (SPN) and paraventricular nucleus (PVN) of the hypothalamus. In these regions, APLNR expression may be associated with arginine vasopressin neurons; which alludes to the role of the apelinergic system in fluid homeostasis $[14,15]$. 
Table 1.2. A summary of the reported expression of the APLNR in brain tissue. Abbreviations: APLNR, apelin receptor; PVN, paraventricular nuclei; SON, supraoptic nuclei

Method of detection and species Summary of expression

APLN-13 autoradiography in C57BL/6 mice

APLN-13 autoradiography in Sprague Dawley rats

Immunohistochemistry and western blotting using antiAPLNR antibody (Santa Cruz Biotechnology) in primary cultured mouse cortical neurons

Immunocytochemical detection using MAB856 from R\&D systems in hippocampal cultures from Sprague Dawley rats

Immunohistochemistry of fixed samples from human brains, using the R \& D systems monoclonal antibody MAB856 in human tissue samples

Immunohistochemistry with an in-house rabbit-raised polyclonal antibody against rat APLNR in unreported rat models

An in-house monoclonal antibody made in conjunction with $R \& D$ systems (MAB856) in human tissue samples

Immunocytochemistry and western blotting using in-house generated APLNR specific rabbitraised polyclonal antibody in human foetal and adult-derived cell lines and SON. including U87MG. or microglia.
Protein expression in the hypothalamus, specifically the PVN and SON and the pituitary gland.

Binding to hypothalamic diencephalon, specifically in the PVN

Expression of APLNR in cortical neuronal cultures and localised with neuronal marker NeuN. Also, expression was reported on glial cells, but data was not shown.

APLNR expression in hippocampal neurons.

Abundant expression of APLNR in the PVN of the hypothalamus, specifically the pyramidal cells. Expression was also in the cerebellum; specifically, the neuronal cell nuclei in the granular and molecular layer, and Purkinje cells.

APLNR was widely expressed in the brain; specific regions included: Cerebral cortex, specifically the frontal and piriform cortices; colocalization of APLNR with GFAP positive cells in the cortex was observed. White matter tracts including the corpus callosum. The hippocampal region including the pyramidal cell layer, granule cell layer, the CA2 and CA3 regions and dentate gyrus and several subcortical nuclei including the hypothalamus, thalamus, midbrain and reticular formation.

Abundant expression of APLNR in the CNS including pyramidal neurons located in the hippocampus as well as cells in the cerebellum; APLNR was not detected in several cell lines of note

Abundant expression in foetal astrocytes and neurons as well as mature oligodendrocytes but not monocyte-derived macrophages 


\subsubsection{Signalling mechanisms of APLNR}

APLNR has multiple signalling mechanisms that range from typical GPCR signalling pathways that occur through the $\mathrm{G} \alpha$ subunit to more intricate signalling processes, such as heterodimerization with other GPCRs [52]. The canonical signalling pathway for APLNR is initiated with G-proteins [53]. APLNR was initially thought to be coupled to the $G \alpha_{i}$ subunit and thus inhibit the adenylate cyclase production of cAMP [54] and activate secondary signalling molecules such as extracellular signal-regulated kinases (ERK's;) [55]. It was then found that APLN can signal through other $\mathrm{G} \alpha$ subunits such as the pertussis insensitive $\mathrm{G} \alpha_{\mathrm{q} / 11}$ [56] or $\mathrm{G} \alpha_{13}$ [57]. APLNR is also internalised by the $\beta$-arrestin-mediated signalling [58] which has been reported as isoform-dependent, i.e. different isoforms of APLN will yield different fates for the internalised receptor [59]. The complete signalling pathways of APLNR are beyond the scope of this thesis; however, the activation of mitogen-activated protein kinases such as ERK 1/2 [60] and internalisation of receptors provide measures of apelinergic system signalling used later in this study. A summary of the known pathways modulated by APLNR activation is shown in Figure 1.1. 


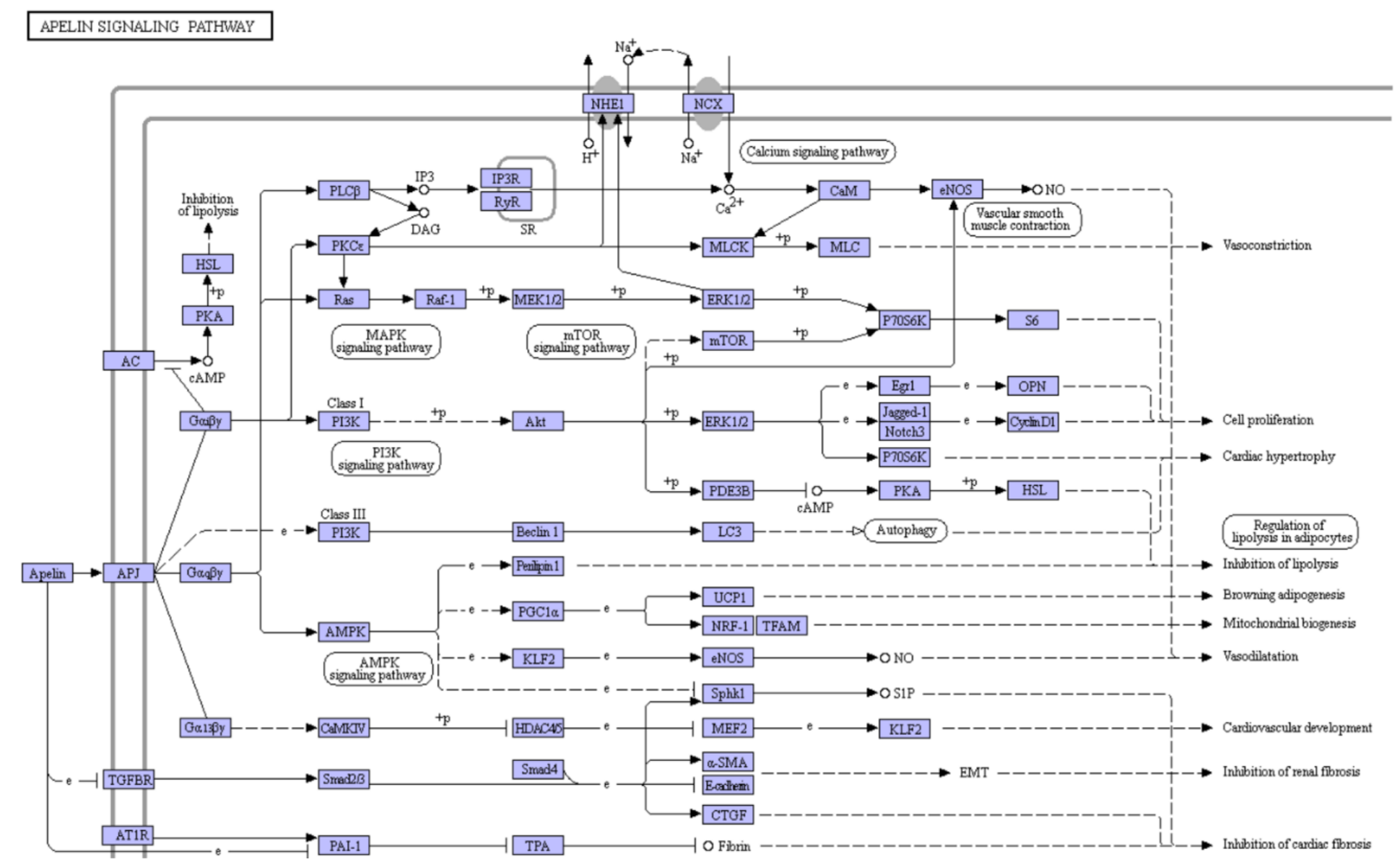

Figure 1.1. A review of the apelinergic system signalling pathways reproduced from the Kyoto Encyclopaedia of Genes and Genomes (KEGG) atlas. The figure provides an overview of putative apelinergic system signalling taken from the KEGG atlas. Reproduced with permission from Miwako Matsumoto, Kanehisa Laboratories. 


\subsubsection{APLNR oligomerisation and synergy}

APLNR was initially reported to share homology with the angiotensin II type 1 receptor (AngII type 1) but was thought to not interact with angiotensin or its receptor [38]. However, APLNR was later found to dimerise with the AngII type 1 receptor which modified angiotensin II affinity for its receptor [61-63]. APLNR also dimerises with the kappa opioid receptor (KOR) [64] and the neurotensin 1 receptor (NTSR1) [52] leading to increased signalling via the ERK pathway.

The apelinergic system can also work synergistically with other systems to exert its effects. APLN stimulation of APLNR positively modulates angiotensin-converting enzyme 2 (ACE2) activity [65], thus modulating the renin-angiotensin system. The apelinergic system also regulates the development of blood vessels synergistically with vascular endothelial growth factor (VEGF). In combination, the two signalling peptides produce longer, thicker blood vessels than either one of the peptides alone [66].

\subsubsection{Apelin}

The endogenous ligand of the apelinergic system was discovered in 1998 and was subsequently named APJ endogenous binding ligand or apelin (APLN) [67]. APLN is synthesised as a 77 amino acid prepropeptide that is subsequently cleaved into a 55-amino acid propeptide containing the $\mathrm{C}$ terminus; this form is called pro-APLN [68-70]. Pro-APLN is further cleaved to form several biologically active isoforms [11]. APLN isoforms have been reported to be differentially expressed in several regions of the body [68] and can have numerous effects upon binding of APLNR [59].

APLN was initially identified in rats but is highly conserved in vertebrates, with rat APLN sharing 82 and 77\% homologies with human and bovine APLN, respectively [71]. There is a high level of conservation at the C-terminus end of the prepropeptide. Subsequent papers have reported the C-terminus to be the primary source of the bioactive isoforms that have high 
affinity to APLNR $[11,71]$. The C-terminus of the APLN prepropeptide also has many basic amino acid residues that suggest variable peptidase cleavage sites which can result in a number of different isoforms with different bioactivities [70]. In contrast, the $\mathrm{N}$-terminus region is rich in hydrophobic amino acids suggesting the N-terminus has a role in trafficking [11].

The apelinergic signalling system is complex, and the mechanisms by which the prepropeptide is cleaved into shorter isoforms are not fully understood [72]. Cleavage of pro-APLN is catalysed by several different enzymes, including angiotensin-converting enzyme 2 (ACE2) $[18,65,73]$ and proprotein convertase subtilisin/kexin 3 (PCSK3 or furin; ) [68]. ACE2 has also recently been implicated in the regulation of APLN peptide activity through further cleavage and inactivation of the shorter APLN peptide isoforms [74]. Each isoform may vary in regards to APLNR binding kinetics and receptor internalisation, i.e., stimulation of APLNR with APLN-13 leads to receptor internalisation and recycling back to the cell membrane while APLN-36 stimulation leads to receptor internalisation and intracellular sequestering [59].

The potencies of the different isoforms of APLN are outside the scope of this PhD study, but a general pattern exists whereby potency appears to be directly correlated with isoform size, i.e. APLN -13 > APLN -17 > APLN -36 > APLN -55 [75, 76]. As APLN-13 is the most potent isoform, it was selected for use during this study. However, APLN-13 also spontaneously cyclizes its N-terminal glutamine into a pyroglutamated form, namely [ $\mathrm{Pyr}^{1}$ ]apelin-13 (or pGlu-Apelin 13) $[11,68]$. [ $\mathrm{Pyr}^{1}$ ]apelin-13 has been reported as one of the most potent isoforms [77], but this conclusion is assay-dependent with some reports indicating equal potency between apelin-13 and $\left[\mathrm{Pyr}^{1}\right.$ ]apelin-13 [78]. It also has been reported that the interconversion of APLN-13 to [ $\mathrm{Pyr}^{1}$ ]apelin-13 to is a source of potential variability in assays [76]. As such, for functional assays in this current study, the $\left[\mathrm{Pyr}^{1}\right]$ apelin-13 isoform was used.

There are numerous regulatory pathways that can regulate APLN secretion and allude to the role of the apelinergic system in various pathological and physiological processes. Its role in metabolism is well known [13]; one component of this is APLN secretion by adipocytes [79] which is regulated by angiotensin type 1 and 2 receptors. The apelinergic system's role in fluid homeostasis is also well known, and APLN secretion can be regulated by hormones such as vasopressin [29] and aldosterone [80]. Hypoxia has also been reported to stimulate APLN secretion in several cell lines including cardiomyocytes [81], adipocytes [82, 83] and 
endothelial cells [10]. The relationship between $A P L N$ and hypoxia is of great interest to this study due to the importance of hypoxia in glioblastoma and is further discussed in Section 1.4.2.

\subsubsection{Apelin Receptor Early Endogenous Ligand}

A new ligand that is not formed from APLN prepropeptide was recently identified and named Apelin Receptor Early Endogenous Ligand (APELA). Prior to its official naming, several other names were given to the peptide including Epiboly LAte Because of Endoderm LAte (ELABELA) and Toddler [33, 40]. This discovery was significant as APELA was found not to be derived from the original APLN prepropeptide but from a newly-identified 54 amino acid propeptide that is conserved in vertebrate species [33]. The 54 amino acid peptide is subsequently cleaved into active forms much like APLN [84]. APELA is expressed in the primitive mesoderm $[33,85,86]$ and has a role in embryogenesis and cardiogenesis $[33,40]$. APELA was briefly assessed during this study (Appendix, Section A.4) but was eschewed for focus on the APLN peptide. 


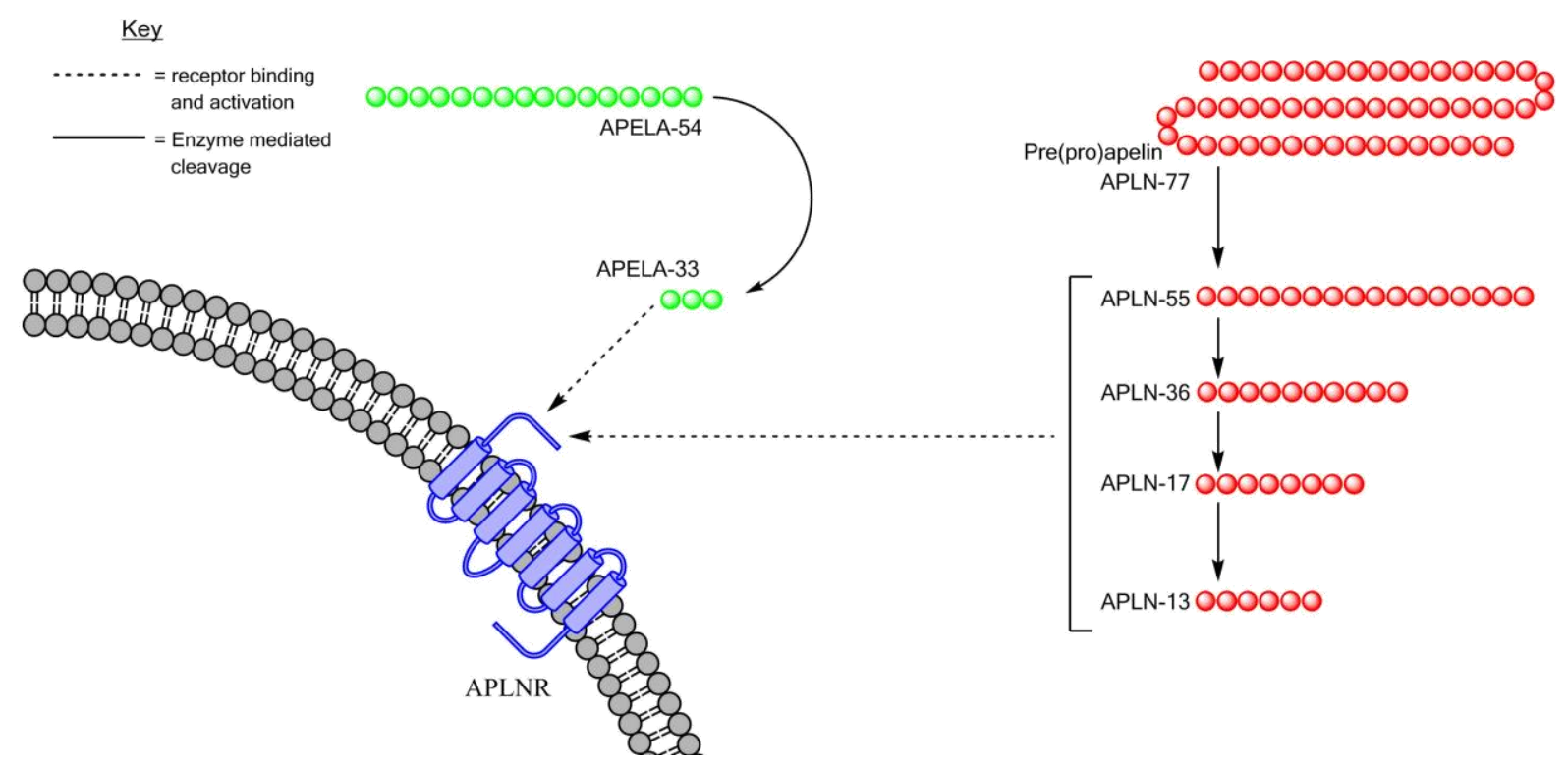

Figure 1.2. A summary of the processing of APLN and key peptides that bind to APLNR. The cleavage of the APLN prepropeptide (77 aa) into is subsequent bioactive peptides is shown (in red) along with the other principal APLNR binding ligand ELABELA (in green) that is cleaved from a 54 aa preproprotein and subsequently cleaved further into more bioactive form. Note that this pathway doesn't have all post-translation modifications of isoforms and all pathways of enzymatic cleavage that generate these isoforms.

\subsubsection{The role of the apelinergic system in tumour pathogenesis}

The apelinergic system has been reported to be overexpressed in a number of different cancers including in half of all colon adenocarcinomas [87] and in human non-small cell lung cancers (NSCLC) [1]. The apelinergic system has potential as a prognostic indicator in several cancers, having been associated with poor patient outcome in oral squamous cell carcinoma [6], prostate cancer [88] and gastric cancer [7]. APLNR expression has also been associated with higher rates of tumour invasion, local lymph node and distant metastasis in gastric cancer patients [89]. All these data suggest that there may be a role for the apelinergic system in cancer pathophysiology. The roles of the apelinergic system in cancers other than glioblastoma are reviewed in Table 1.3. The first report of the apelinergic system in glioblastoma describes the up-regulation of $A P L N$ and $A P L N R$ in glioblastoma-associated blood vessels and in tumour cells surrounding necrotic regions, compared to control tissue [2]. 
Table 1.3. Review of the literature regarding reports of the apelinergic system in cancers other than glioblastoma.

\begin{tabular}{|c|c|c|c|}
\hline Cancer & Models used & Synopsis of literature & References \\
\hline $\begin{array}{l}\text { Lung cancers } \\
\text { Adenocarcinoma (AC), } \\
\text { non-small cell lung } \\
\text { cancer (NSCLC) }\end{array}$ & $\begin{array}{l}\text { Lung AC tissue, plasma from } \\
\text { lung AC patients, A549 lung } \\
\text { AC line, NSCLC tumour } \\
\text { tissue, NSCLC cell lines }\end{array}$ & $\begin{array}{l}\text { APLNR levels are elevated in } \\
\text { AC; } A P L N \text { mRNA is elevated } \\
\text { in NSCLC; APLN has several } \\
\text { effects on in vitro lung cancer } \\
\text { cell lines. APLN is reported as } \\
\text { a biomarker for diagnosis of } \\
\text { lung cancer }\end{array}$ & $\begin{array}{l}{[90],[1]} \\
{[91]}\end{array}$ \\
\hline $\begin{array}{l}\text { Gastrointestinal } \\
\text { cancers } \\
\text { Colon adenocarcinoma } \\
\text { (AC), Colon Adenoma } \\
\text { (CA), Gastroesophageal } \\
\text { cancer (GEC), Gastric } \\
\text { cancer (GC), colorectal } \\
\text { cancer (CRC) }\end{array}$ & $\begin{array}{l}\text { Human colon adenoma and } \\
\text { AC, LoVo cells, blood } \\
\text { samples of GEC patients, }\end{array}$ & $\begin{array}{l}A P L N \text { and } A P L N R \text { levels are } \\
\text { overexpressed in AC, and CA } \\
\text { APLN has a } \\
\text { pathophysiological role in } \\
\text { colon AC, serum APLN is } \\
\text { elevated in GEC. In GC } \\
\text { APLN has been proposed as a } \\
\text { marker of clinical and } \\
\text { prognostic outcomes in GC. } \\
\text { APLN and APLNR are } \\
\text { upregulated in CRC. APLN } \\
\text { has also been reported as a } \\
\text { predictive biomarker for } \\
\text { bevacizumab response in } \\
\text { CRC }\end{array}$ & $\begin{array}{l}{[87],[92]} \\
{[7]} \\
{[93][94]}\end{array}$ \\
\hline $\begin{array}{l}\text { Hepatocellular } \\
\text { carcinoma (HCC) }\end{array}$ & $\begin{array}{l}\text { Tissue from HCC patients, in } \\
\text { vivo model of HCC. }\end{array}$ & $\begin{array}{l}A P L N \text { and APLNR are } \\
\text { overexpressed in HCC and } \\
\text { may have a role in } \\
\text { arteriogenesis }\end{array}$ & {$[95]$} \\
\hline Prostate & $\begin{array}{l}\text { Human prostate cancer lines } \\
\text { LNCaP and Du145, patient } \\
\text { prostate cancer tissue. }\end{array}$ & $\begin{array}{l}\text { Dysregulation of miR- } \\
224 / \text { APLN axis may } \\
\text { contribute to the progression } \\
\text { of prostate carcinoma and } \\
\text { may be a predictor of poor } \\
\text { prognosis }\end{array}$ & {$[88]$} \\
\hline Endometrial & $\begin{array}{l}\text { Serum of endometrial cancer } \\
\text { patients }\end{array}$ & $\begin{array}{l}\text { Circulating APLN may be a } \\
\text { predicting factor for } \\
\text { endometrial cancer }\end{array}$ & [96] \\
\hline $\begin{array}{l}\text { Oral Squamous cell } \\
\text { carcinoma (OSCC) }\end{array}$ & $\begin{array}{l}\text { Human OSCC tissue, human } \\
\text { tongue OSCC cell lines HSC- } \\
2 \text {, HSC-3 and Ca9.22 }\end{array}$ & $\begin{array}{l}\text { APLN presents as a potential } \\
\text { prognostic and therapeutic } \\
\text { target of oral squamous cell } \\
\text { carcinoma }\end{array}$ & {$[6]$} \\
\hline Breast & $\begin{array}{l}\text { MCF-7 and Hs } 578 \mathrm{~T} \text { breast } \\
\text { cancer cells, breast cancer } \\
\text { tissue, TS/A mammary } \\
\text { carcinoma line }\end{array}$ & $\begin{array}{l}\text { APLN is present in breast } \\
\text { carcinoma tissue; breast } \\
\text { cancer derived cell line data } \\
\text { are suggestive of a potential } \\
\text { APLN role in breast cancer. }\end{array}$ & {$[97],[98],[3]$} \\
\hline Skin cancer & B16 murine melanoma & $\begin{array}{l}\text { APLN confers growth } \\
\text { advantages to tumour grafts } \\
\text { and promotes }\end{array}$ & {$[4]$} \\
\hline
\end{tabular}




\begin{tabular}{|c|c|c|c|}
\hline Cancer & Models used & Synopsis of literature & References \\
\hline & & $\begin{array}{l}\text { lymphangiogenesis and } \\
\text { lymph node metastasis }\end{array}$ & \\
\hline $\begin{array}{l}\text { Clear cell renal } \\
\text { carcinoma (CC-RCC) }\end{array}$ & CC-RCC tissue samples & $\begin{array}{l}\text { APLN is upregulated in CC- } \\
\text { RCC }\end{array}$ & [99] \\
\hline $\begin{array}{l}\text { Multiple Myeloma } \\
\text { (MM) }\end{array}$ & Patient samples (plasma) & $\begin{array}{l}\text { Plasma APLN levels were } \\
\text { upregulated in MM patients } \\
\text { over both control and patients } \\
\text { suffering Non-Hodgkin } \\
\text { lymphoma }\end{array}$ & {$[100]$} \\
\hline $\begin{array}{l}\text { Cholangiocarcinoma } \\
\text { (CCA) }\end{array}$ & Patient tissue samples & $\begin{array}{l}\text { APLN and APLNR } \\
\text { expression is increased in } \\
\text { human CCA tissues }\end{array}$ & [101] \\
\hline Metastatic Melanoma & $\begin{array}{l}\text { Whole exome sequencing of } \\
\text { patient tissue }\end{array}$ & $\begin{array}{l}\text { Revealed potential mutations } \\
\text { loss-of-function of APLNR in } \\
\text { immunotherapy resistant } \\
\text { patients. }\end{array}$ & {$[102]$} \\
\hline
\end{tabular}

\subsection{An overview of glioblastoma and its treatment}

The term glioblastoma multiforme (GBM), was initially coined in 1926 [103, 104] using a histogenetic approach and was termed "multiforme" due to the number of different presentations of the disease. This term is no longer recommended since it is apparent the multiple presentations of glioblastoma are, more accurately, different subtypes; thus the term GBM or glioblastoma multiforme is eschewed during this thesis for the more appropriate term, glioblastoma.

Glioblastoma is the most common primary malignancy of the central nervous system (CNS) [105-107]. Patients diagnosed with glioblastoma have a median survival time of approximately 16 months with treatment $[108,109]$. Currently, there are limited treatment options, as such, increasing our understanding of the underlying pathophysiological mechanisms of glioblastoma is extremely important, including any part played by the apelinergic system. 


\subsubsection{Aetiology of glioblastoma}

Tumours of the CNS can arise from the two major cell types of the brain, namely the working neuronal cells and the supporting glial cells. Originally, the cellular origin of glioblastoma was thought to be astrocytes due to the expression of the astrocyte marker glial fibrillary acidic protein (GFAP) [110]; however, this cellular origin of glioblastoma is more contentious with multiple cells being reported to be able to form the disease [111-114]. Glioblastoma presents more frequently in men, and like many cancers incidence increases with age [115], although cases in children have been reported [116]. Patients with glioblastoma present with a variety of symptoms including neurological issues such as seizures and headaches, as well as neurological deficits and personality changes [117]. There are only a few proven risk factors for developing glioblastoma, including exposure to ionising radiation [118] and rare hereditary diseases such as Turcot syndrome or Li-Fraumeni syndrome [119].

Neuroimaging is used to confirm the presence of a tumour. The most common method is magnetic resonance imaging (MRI), but other imaging tools such as computed tomography or positron emission tomography (PET) can also be utilised [120]. The patient is then assessed for neurosurgical treatment which varies depending on the location and structure of the tumour [121]. This is followed by either biopsy or resection, and then the tumour is graded by the grading system discussed below (1.3.2) [122].

\subsubsection{Grading of glioblastoma}

The characterisation of tumours by phenotypic and genotypic markers is imperative as it enables accurate grading of the tumour and treatment. During the conception of this thesis, the question arose whether components of the apelinergic system may be exclusively expressed in certain CNS tumours or particular subtypes of glioblastoma. This was reported in gastric cancer where elevated APLN expression was associated with tumour stage, lymph node and distance metastases [7]. In Chapter 3, several CNS tumours and subtypes of glioblastoma are referred to in detail; here, for reference, is a brief review of the history and current methodology of classification and grading of tumours of the CNS. 
Since its recognition by Bailey and Cushing, the classification of glioblastoma has been an area of constant development [123][123] and subsequent changes to the classification of tumours are published by the World Health Organisation (WHO) in the WHO classification of tumours of the CNS. Recently the WHO classification of CNS tumours 2016 was released and differs significantly from previous guidelines in that it utilizes a combination of histopathological and molecular characteristics to further diagnose and classify tumours; which has resulted in several new categories [124]. The new grouping category "diffuse astrocytic and oligodendroglial tumours" encompasses over 20 different tumours including, critically, glioblastoma [107]. The changes from 2007 to 2016 are summarised in Table 1.4, critically, astrocytic (which contains glioblastoma), oligoastrocytic and oligodendroglial tumours are all concatenated into one category.

Both the 2016 and previous WHO classification of tumours of the CNS utilizes the numerical grading scheme (WHO grade I to IV) as seen in Table 1.4. The numbering system is based, in part, on morphological and histopathological features such as cellular atypia, necrosis, the formation of blood vessels and the presence of certain genetic markers [125]. The grading scheme also alludes to the malignancy of the disease. Grade I and II tumours are characterised by a slow and less aggressive phenotype while grade III and IV are far more malignant and aggressive. The grade IV tumour is referred to as glioblastoma [107]. 
Table 1.4. The changes in WHO classification of CNS tumours. Deleted classifications are in orange, and new classifications are in blue, provisional classifications are in italics indicating their potential to be redefined. This table is reproduced from [124] with permission from Wolters Kluwer Health Inc.

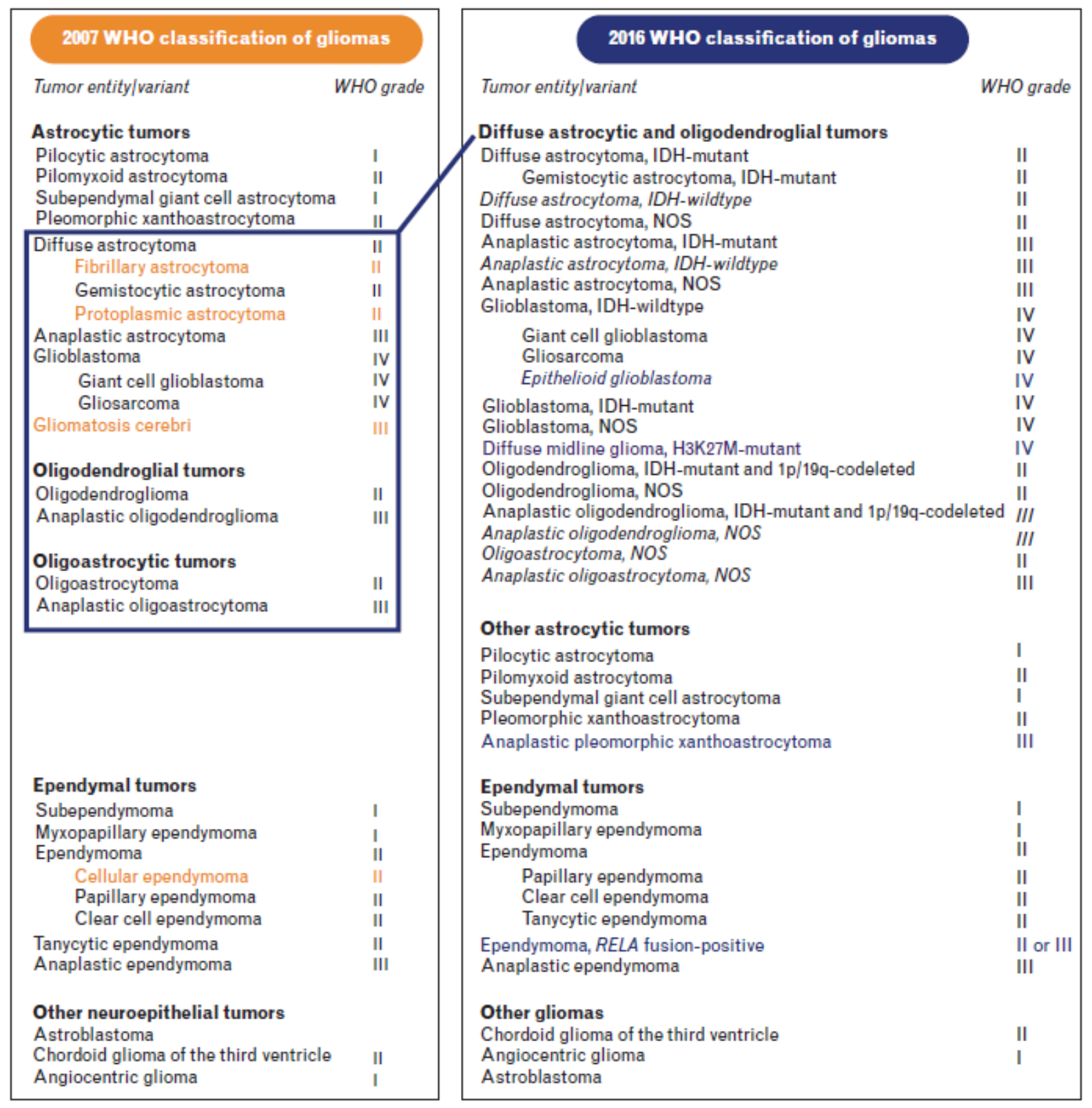

The new WHO guidelines describe a three-stage approach (summarised in Figure 1.3) to classify glioblastoma. The morphological and histopathological features are utilised in conjunction with several critical molecular markers described below, to allow better grading of the tumour and thus, better treatment. 
The first stage of investigation uses traditional histopathological methods [126] such as haematoxylin, and eosin staining [124] are utilised to investigate common pathological characteristic features (Figure 1.3). In the case of glioblastoma, these features include necrotic regions in the tumour surrounded by tumour cells in a palisading pattern (termed "pseudopalisading necrosis") and significant pathological blood vessel formation [127].

The second stage of investigation includes detection of immunohistochemical markers such as GFAP [128, 129]. The 2016 guidelines also recommend the assessment of the important genetic markers such as isocitrate dehydrogenase $(I D H)$.

$I D H$ is an enzyme from the tricarboxylic acid (TCA) cycle that exists in three isoforms, namely IDH1, 2 and 3 [130]. IDH1 has been established as a significant prognostic marker of outcome in glioblastoma patients [131] following a seminal paper that identified an IDH1 mutation in a significant proportion of glioblastoma samples [132]. The $I D H 1$ gene is mutated in several grade II and III tumours, as well as $70-80 \%$ of secondary glioblastomas [131], but not primary glioblastoma [107]. Thus, determining the status of the $I D H 1$ gene as being either "wildtype" or "mutant", is one of the key genes recommended for identifying the subtype in the new WHO CNS tumour guidelines [107]. This is especially important as there is a distinction between "primary" glioblastoma which develops de novo and secondary glioblastoma which is less prevalent and progresses from lower grade tumours [122]. The terms primary and secondary are classified as synonyms in the new WHO guidelines and are superseded by $I D H$ status.

Finally, several other genetic parameters are assessed to refine the classification of the tumour further. For example, testing for co-deletion of the short arm of chromosome 1 and the long arm of chromosome 19 (co-deletion of $1 \mathrm{p} / 19 \mathrm{q}$ ). Other markers that do not correlate to classification such as the methylguanine methyltransferase gene (MGMT) can be used to determine optimal treatment strategies. $M G M T$ is linked to an enhanced sensitivity of the tumour to DNA alkylating agents such as temozolomide (TMZ) [119].

Beyond the WHO CNS tumour classification guidelines, there has been other research into determining the subtypes of glioblastoma through understanding the unique molecular signatures of resected tumour samples. In 2008 the cancer genome atlas (TCGA) published a landmark paper that attempted to identify all the genetic changes on a large scale in 
glioblastoma [133]. Subsequently, Verhaak et al. [134] identified four subtypes of glioblastoma based on gene expression: classical, mesenchymal, proneural and neural. These subtypes each express different genes signatures and are clinically relevant as each subtype shows a different prevalence and response to treatment [134]. Moreover, the TCGA has also gone on to analyse lower-grade glioma (Grade II and III) [135]. The 4 glioblastoma subtypes are also used in Chapter 3 alongside the 2016 WHO CNS tumour classifications to stratify public data of APLN and $A P L N R$ mRNA expression from the TCGA.

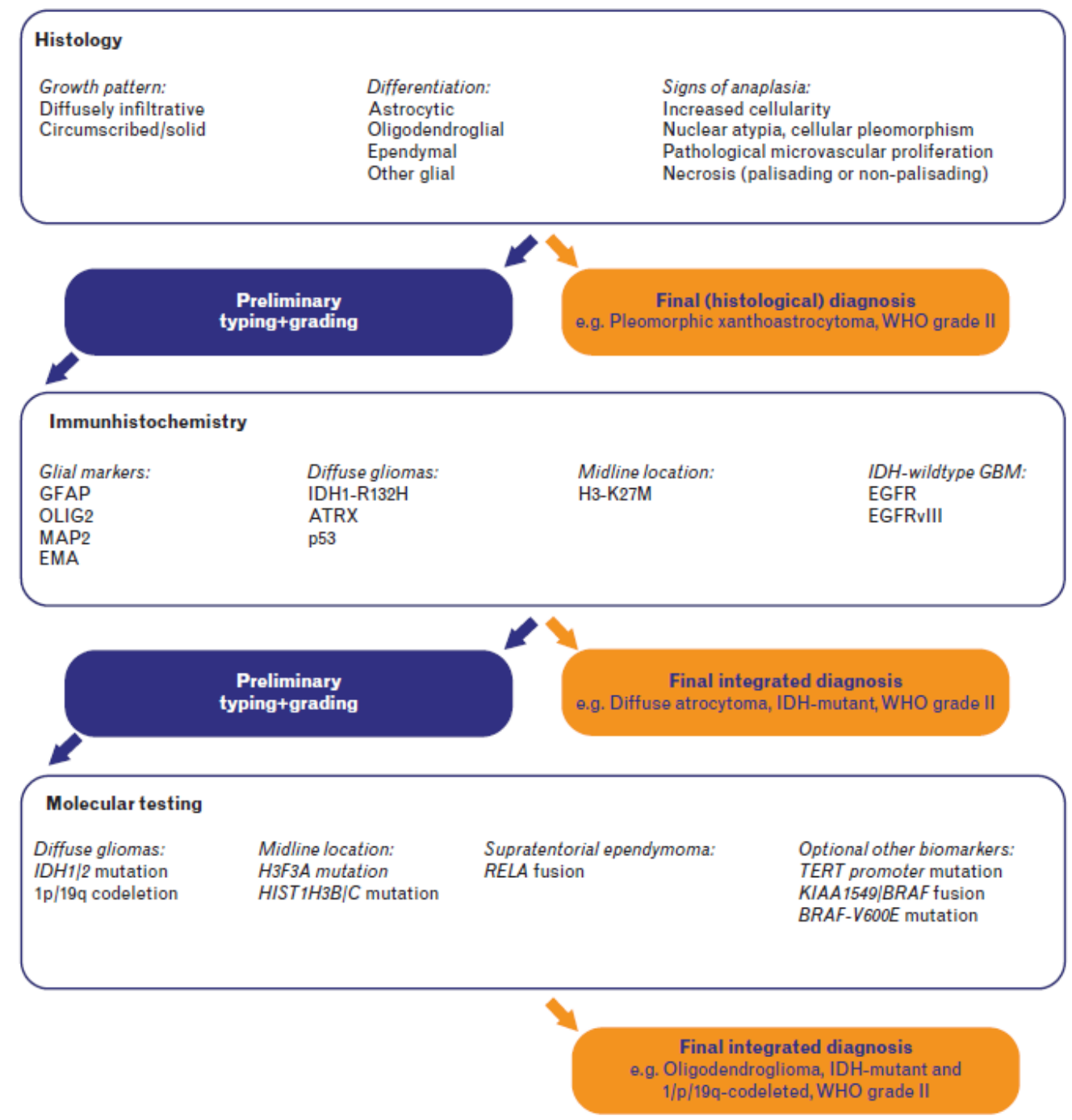

Figure 1.3. A representation of the classification algorithm of central nervous system (CNS) tumours from the 2016 WHO classification of tumours of the CNS. Reproduced from [124]. Reproduced with permission from Wolters Kluwer Health, Inc. 


\subsubsection{Treatment paradigms of glioblastoma}

\subsubsection{Neurosurgical treatment}

Neurosurgical intervention is the first step in glioblastoma treatment and involves the partial or total removal of the tumour, which aids in patient survival [136]. Despite surgical removal, glioblastoma recurrence is usually inevitable [137] due to not only it's highly aggressive, invasive and diffuse growth pattern which makes resection challenging but also due to other factors such as residual cancer stem cells $[121,138]$. For example, Figure 1.4 illustrates an MRI image of a glioblastoma before resection (Panel A, white arrow), immediately following resection (Panel B) and six months following resection (Panel C). This indicates reoccurrence not only at the resection margin but also across the Sylvian fissure in the frontal lobe.
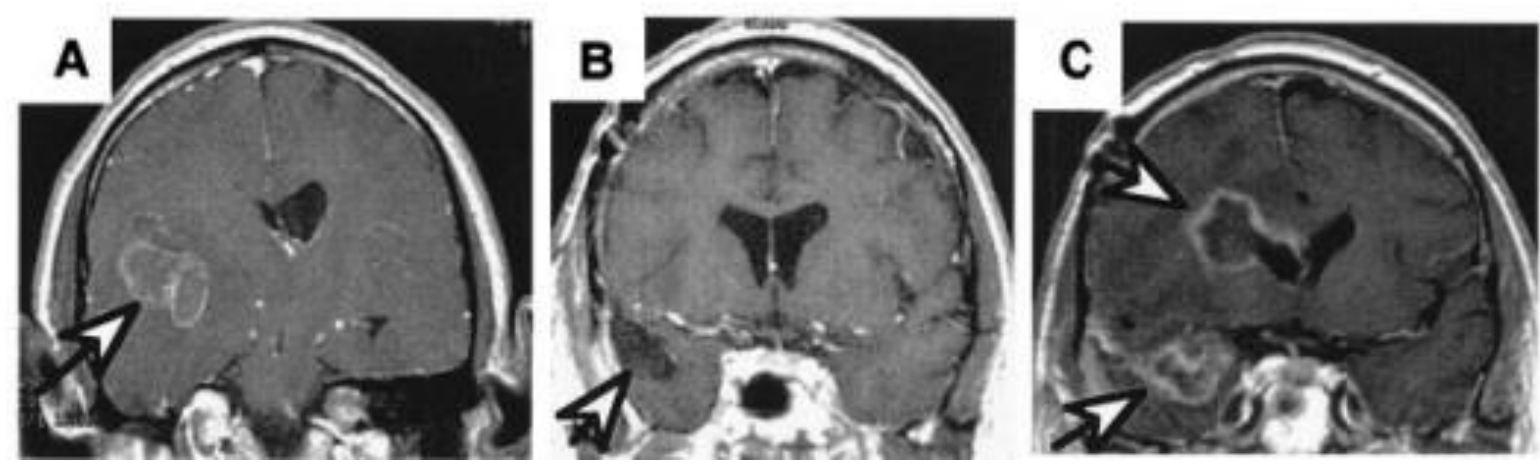

Figure 1.4. MRI images demonstrating the highly invasive nature of glioblastoma. Panel A shows a glioblastoma (arrow) before surgery. Panel B is the same area after a gross total resection, showing a clear cavity. Panel C demonstrates reoccurrence of the glioblastoma at the same site and another site across the Sylvian fissure (arrows). Adapted from [139] with permission from the Proceedings of the National Academy of Sciences. Copyright (2000) National Academy of Sciences, U.S.A.

\subsubsection{Treating recurrent glioblastoma}

The high rate of glioblastoma tumour reoccurrence after resection means that follow-up radiation and chemotherapy is required [140]. Courses of radiation therapy significantly improve survival [141] however, glioblastoma is often associated with radiotherapy resistance due to efficient DNA repair mechanisms being present $[142,143]$. Thus a combination of treatments is required, such as the current gold standard treatment paradigm established by Stupp et al. [109] whereby surgical resection is followed by both radiotherapy and 
simultaneous treatment with TMZ [109]. TMZ is the recommended leading treatment for glioblastoma. It may be administered alone or in combination with other anticancer agents [144] and is utilised in this thesis. Briefly, the TMZ mechanism of action is preferential methylation of purine bases which leads to double-strand breaks in DNA [145], resulting in replication fork collapse [146]. Despite this treatment, reoccurrence and ultimately progression of the disease is inevitable [147]. Chemotherapy and radiation therapy often leads to apoptosis, which is of interest as the apelinergic system has been implicated in a variety of anti-apoptotic roles (as discussed below; Section 1.4.1).

\subsubsection{Additional therapies for glioblastoma}

There is a handful of current federal drug administration (FDA) approved treatments for glioblastoma outside of the gold standard TMZ and radiation treatment; these are reviewed in Table 1.5 below. Beyond this, there are a number of targeted therapies and other chemotherapeutic compounds in various stages of development for the treatment of glioblastoma that are not discussed in this study with the exception of one class which are the topoisomerase inhibitors. Topoisomerase inhibitors target the DNA unwinding enzymes topoisomerase 1 and 2. Inhibition of these enzymes leads to double-strand breaks causing DNA damage. Irinotecan is a topoisomerase I inhibitor that can readily cross the blood-brain barrier (BBB) and demonstrates cytotoxicity against glioblastoma [148]. In combination with bevacizumab, a humanized at VEGF monoclonal antibody that inhibits the growth of new

blood vessels, it is suggested to be an effective treatment of glioblastoma [149-152]. The topoisomerase II inhibitor doxorubicin also shows promise in treating glioblastoma if modified to enable it to cross the BBB more readily $[153,154]$. This can be done by methods such enveloping doxorubicin in a liposome which also increases its half-life $[155,156]$ but is yet to be translated into a clinical setting. Doxorubicin is utilised in this thesis as an inexpensive and readily available model of a topoisomerase. 
Table 1.5. Summary of current FDA approved treatment options for glioblastoma.

\begin{tabular}{|c|c|c|c|c|}
\hline \multicolumn{2}{|l|}{ Treatment } & Description & Mechanism of action & References \\
\hline \multicolumn{2}{|c|}{ Radiation therapy (RT) } & $\begin{array}{l}\text { Standard ionizing radiation therapy in } \\
\text { the form of a 3D conformal beam or } \\
\text { intensity-modulated RT } 60 \mathrm{GY} \text { in } 1.8 \\
-2 \text { fractions. }\end{array}$ & $\begin{array}{l}\text { Ionization of DNA } \\
\text { that results in DNA } \\
\text { damage and cell death }\end{array}$ & {$[140,141]$} \\
\hline \multicolumn{2}{|c|}{ Temozolomide (TMZ) } & $\begin{array}{l}\text { The current gold standard treatment } \\
\text { choice in conjunction with RT; } \\
\text { dubbed the "Stupp" protocol after the } \\
\text { seminal } 2005 \text { paper demonstrating } \\
\text { combined TMZ and RT increases . }\end{array}$ & $\begin{array}{l}\text { A DNA alkylation } \\
\text { agent which leads to } \\
\text { double-stranded DNA } \\
\text { breaks and thus cell } \\
\text { death. }\end{array}$ & {$[109,157]$} \\
\hline \multicolumn{2}{|l|}{ Bevacizumab } & $\begin{array}{l}\text { Commonly used to treat recurrent } \\
\text { glioblastoma or as a last line therapy } \\
\text { alone or as a combined treatment with } \\
\text { other compounds such as irinotecan or } \\
\text { lomustine. Some evidence for } \\
\text { treatment of newly diagnosed } \\
\text { glioblastoma exists but is contentious. }\end{array}$ & $\begin{array}{l}\text { Monoclonal antibody } \\
\text { targeted to VEGF-A } \\
\text { thus inhibiting } \\
\text { angiogenesis }\end{array}$ & [158-162] \\
\hline Nitrosoureas & $\begin{array}{l}\text { Lomustine } \\
\text { (CCNU) }\end{array}$ & $\begin{array}{l}\text { Early glioblastoma treatment, now } \\
\text { suggested for recurrent glioblastoma } \\
\text { treatment in conjunction with } \\
\text { bevacizumab with some controversy } \\
\text { about the efficacy. }\end{array}$ & $\begin{array}{l}\text { The primary } \\
\text { mechanism of action } \\
\text { is through DNA } \\
\text { alkylation which } \\
\text { causes DNA-DNA } \\
\text { and DNA-protein } \\
\text { crosslinking. In } \\
\text { addition, inhibition of } \\
\text { RNA synthesis and } \\
\text { DNA repair occurs. }\end{array}$ & $\begin{array}{l}{[158,163-} \\
168]\end{array}$ \\
\hline \multicolumn{2}{|c|}{$\begin{array}{l}\text { Electric field therapy } \\
\text { (Tumour treating fields } \\
\text { TTF) (NovoTTF-110A } \\
\text { system) }\end{array}$} & $\begin{array}{l}\text { Novel treatment that emits electrical } \\
\text { fields at } 200 \mathrm{kHz} \text {. Worn continuously } \\
\text { for } 4 \text {-week blocks of treatment with } \\
\text { short breaks ( } 1 \mathrm{hrs}) \text { daily. }\end{array}$ & $\begin{array}{l}\text { Disrupts mitosis and } \\
\text { induces cycle arrest } \\
\text { and apoptosis. }\end{array}$ & [169-174] \\
\hline
\end{tabular}




\subsection{The hypothetical roles of the apelinergic system in glioblastoma.}

The role of the apelinergic system in glioblastoma is hitherto unknown. However, the apelinergic system has known roles in several biological processes that are important to glioblastoma pathogenesis. These processes could be considered avenues for further research into what the role of the apelinergic system is in glioblastoma.

The expression of $A P L N$ and $A P L N R$ was first reported in glioblastoma in 2007 by Kälin et al. [2] A decade later, the same authors reviewed the physiological and pathophysiological roles of the apelinergic system and underlined some of the possible roles of the apelinergic system in glioblastoma; such as blood vessel formation and glycolysis [8]. In addition, the authors suggested that pathological features which influence the apelinergic system like hypoxia may play a role in glioblastoma progression. To investigate this hypothesis further, Kälin et al. [2] examined gene clusters that were co-expressed with up-regulated $A P L N$ expression in a provisional dataset of glioblastoma samples from TCGA [8]. The identified gene clusters that were associated with angiogenesis, glycolysis and cell migration; common processes associated with glioblastoma which is a highly glycolytic, invasive tumour with significant pathological angiogenesis [175-177]. These findings supported the hypothesis that the apelinergic system may play a role in glioblastoma pathophysiology. This section further describes some of the established physiological roles of the apelinergic system and links them to mechanisms of glioblastoma pathophysiology, thus, suggesting areas for further research. 


\subsubsection{The protective role of the apelinergic system against cellular stress}

Cancer cells are under a number of stresses in the tumour microenvironment [178-181]. Cell stress in the tumour microenvironment can arise from many different sources, examples of this include endogenous stresses such as nutrient deprivation and hypoxia which are in part due to the rapid growth of tumour cells and aberrant vasculature found in glioblastoma $[182,183]$. Stresses can also derive from exogenous sources such as chemotherapeutic treatments [181, 184]

The apelinergic system is reported to have an anti-apoptotic role under several types of cell stress, as reviewed in Table 1.6. An extensive review of the literature suggests that the antiapoptotic actions of the apelinergic system are likely to be mediated through the phosphoinositide 3-kinase (PI3K) and map kinase (MAPK) pathways irrespective of the method through which cell stress is induced [185]. The PI3K and MAPK pathways are commonly implicated in signalling of DNA damage repair and cell stress response pathways in glioblastoma [186-189]. It is therefore plausible that the apelinergic system may have a protective role in glioblastoma in response to stress induced by glucose deprivation, hypoxia or chemotherapeutics; potentially through the PI3K and MAPK pathways. The general protective effects of the apelinergic system were investigated during this study. 
Table 1.6. Review of the different apoptotic roles of the apelinergic system. Adapted from [185] reproduced with permission from Oxford University Press.

\begin{tabular}{|c|c|c|c|c|}
\hline System & Disease/model & Cell or tissue & Effect & Reference \\
\hline \multirow[t]{8}{*}{ Cardiovascular system } & Stress-related heart disease & Wistar rat heart tissue & Anti-apoptotic & [190] \\
\hline & \multirow[t]{2}{*}{ Post-myocardial infarction } & Myocardial progenitor cells & Anti-apoptotic & [191] \\
\hline & & Bone marrow cells & Anti-apoptotic & [192] \\
\hline & \multirow{3}{*}{$\begin{array}{l}\text { Ischemia-reperfusion (I/R) } \\
\text { injury }\end{array}$} & Rat $\mathrm{I} / \mathrm{R}$ model & Anti-apoptotic & [193] \\
\hline & & $\mathrm{H} 9 \mathrm{C} 2$ cells & Anti-apoptotic & {$[190]$} \\
\hline & & Rat cardiomyocytes & Anti-apoptotic & [194] \\
\hline & $\begin{array}{l}\text { Pulmonary artery } \\
\text { hypertension }\end{array}$ & $\begin{array}{l}\text { Primary pulmonary artery smooth muscle } \\
\text { cells }\end{array}$ & Pro-apoptotic & {$[195]$} \\
\hline & Diabetes mellitus & Mesenchymal stem cells & Anti-apoptotic & {$[196]$} \\
\hline \multirow[t]{4}{*}{ Nervous system } & \multirow[t]{2}{*}{ Brain I/R injury } & PC12 cells & Anti-apoptotic & [197] \\
\hline & & $\begin{array}{l}\text { Middle cerebral artery occlusion (MCAO) } \\
\text { model }\end{array}$ & Anti-apoptotic & [198] \\
\hline & \multirow[t]{2}{*}{ Retinal cells } & Muller cells & Anti-apoptotic & [199] \\
\hline & & Pericytes & Anti-apoptotic & [200] \\
\hline \multirow[t]{2}{*}{ Skeletal homeostasis } & \multirow[t]{2}{*}{ Skeletal homeostasis } & MC3T3-E1 cells & Anti-apoptotic & {$[201]$} \\
\hline & & Osteoblasts & Anti-apoptotic & {$[201,202]$} \\
\hline \multirow[t]{3}{*}{ Gastrointestinal system } & \multirow{3}{*}{$\begin{array}{l}\text { Colon adenomas and } \\
\text { adenocarcinomas }\end{array}$} & LoVo cells & Anti-apoptotic & [87] \\
\hline & & Rat crypt (IEC-6) & Anti-apoptotic & [203] \\
\hline & & Human enterocyte model (Caco-2) & Pro-apoptotic & [203] \\
\hline
\end{tabular}




\subsubsection{Hypoxia, the apelinergic system and glioblastoma}

There have been numerous reports that hypoxia upregulates $A P L N$ mRNA expression in multiple cell lines summarised below (Table 1.7) which lead to the hypothesis that hypoxia would induce $A P L N$ mRNA expression in glioblastoma-derived tumour cells. It was hypothesised, based on the number of reports regarding the effects of hypoxia on the apelinergic system that the relationship between hypoxia and the apelinergic system is important; however, it is unclear what this role may be in the highly hypoxic glioblastoma tumour [204].

The presence of $A P L N$ and $A P L N R$ was reported in the cells surrounding necrotic regions in the glioblastoma (as seen in Figure 1.5 denoted with *), these cells surrounding necrosis are termed pseudopalisading necrosis $[2,175]$. The cells surrounding pseudopalisading necrosis are often hypoxic [205] which lead the authors to hypothesise that hypoxia may modulate the apelinergic system with glioblastoma [2].

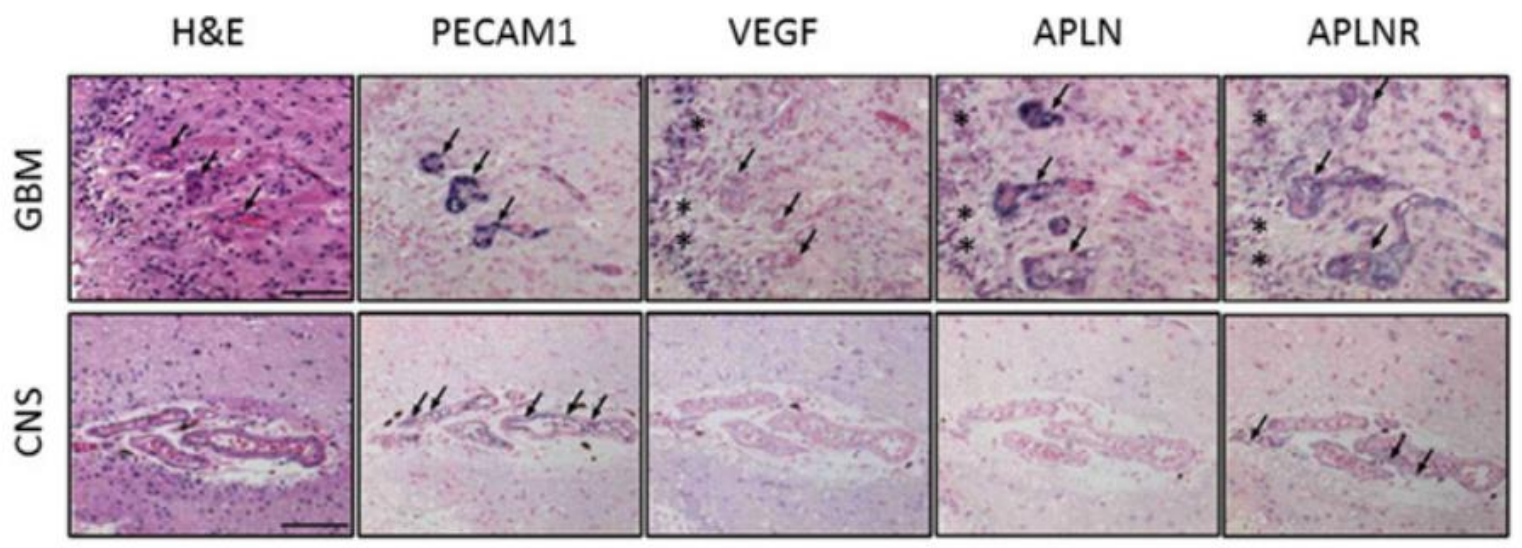

Figure 1.5. The in situ hybridisation expression of endothelial-associated markers PECAM1 (CD31), VEGF, $A P L N R$ and APLN in glioblastoma blood vessels. The arrows denote the positive expression of the marker; asterisks show expression of markers in neoplastic cells that surround necrotic regions. Image reprinted with permission from Springer [8].

Hypoxia is detrimental to non-cancerous cells but essential to the pathophysiology of many solid tumours [177] including glioblastoma [206-209]. Hypoxia is implicated in the regulation of a number of different pathophysiological mechanisms essential to glioblastoma pathogenesis 
[207, 210] such as migration and invasion [210-213], angiogenesis [214-216], glucose uptake [217], and maintenance of the glioblastoma stem cell niche [218, 219]. The apelinergic system can also modulate some of these processes $[6,10,25]$, and this further suggests potential roles for the hypoxia-apelinergic system relationship in glioblastoma.

One of the key transcription factors of hypoxia is hypoxia-inducible factor 1 alpha (HIF $1 \alpha$ ) which is upregulated under hypoxic conditions. HIF $1 \alpha$ is critical in the mediation of hypoxiarelated physiological functions. An example of this is hypoxia-mediated drug resistance in glioblastoma. HIF $1 \alpha$ is upregulated in glioblastoma in response to hypoxia and modulates methylguanine methyltransferase (MGMT), a critical gene in glioblastoma resistance to TMZ treatment [220]. MGMT is just one example of over 40 possible genes that HIF1 $\alpha$ regulates that promote glioblastoma progression and resistance $[220,221]$. The link between the apelinergic system and hypoxia was first established in 2007 [81] and first noted in breast cancer tumour cells where it was demonstrated that hypoxia could upregulate $A P L N$ expression [3]. It was later reported that HIF1 $\alpha$ bound to a hypoxia response element (HRE) on the $A P L N$ gene, stimulating expression of $A P L N$ [10]. HIF2 $\alpha$ has also been reported to regulate the apelinergic system [222, 223].

Since these initial studies, the role of the apelinergic system under hypoxic conditions has been investigated in several diseases [224] but only sparingly in cancers. In oral squamous cell carcinoma, it was found that $A P L N$ up-regulation was a predictor of poor prognosis and this was hypothesised to be due, in part, to hypoxia [6]. The well-established link between hypoxia and endothelial cell biology may provide one mechanism by which the hypoxia-APLN link contributes to glioblastoma biology [3, 93, 95, 225]. This is particularly pertinent as aberrant blood vessel formation is a hallmark of glioblastoma that is less prevalent in lower grade astrocytic and oligodendroglial tumours [226-228]. However, while the interaction between the apelinergic system, hypoxia and blood vessel formation has been researched [10], the other mechanisms by which the possible hypoxia- $A P L N$ interaction affects glioblastoma pathogenesis has not yet been determined. 
Table 1.7. Summary of cell line models treated with hypoxia and subsequent responses. Adapted and reproduced with permission from Elsevier APLN/APLNR signalling in hypoxia-related diseases [224] Upregulation $=\uparrow$, Downregulation $=\downarrow$.

\begin{tabular}{|c|c|c|c|}
\hline Cell or tissue & Type of hypoxia & Effect (protein/ mRNA) & Reference \\
\hline $\begin{array}{l}\text { Human pulmonary endothelial, smooth } \\
\text { muscle }\end{array}$ & Hypoxia $\left(1 \% \mathrm{O}_{2}\right)$ & $A P L N$ mRNA $\uparrow$ & {$[10]$} \\
\hline $\begin{array}{l}\text { Human ventricular myocytes and human } \\
\text { cardiomyocyte cell line AC16 }\end{array}$ & $\begin{array}{l}\text { Hypoxia }\left(5 \% \mathrm{O}_{2}\right) \\
\text { and } \mathrm{CoCl}_{2}\end{array}$ & $A P L N$ protein and mRNA $\uparrow$ & [229] \\
\hline Rat cardiomyocytes & Hypoxia $\left(2 \% \mathrm{O}_{2}\right)$ & APLN mRNA $\uparrow$ & {$[81]$} \\
\hline Neonatal cardiomyocytes & $\mathrm{CoCl}_{2}$ & $\begin{array}{l}A P L N \text { and APLNR protein } \\
\text { and mRNA } \uparrow\end{array}$ & {$[230]$} \\
\hline Cardiac myocyte cells & $\mathrm{CoCl}_{2}$ & $A P L N$ mRNA $\uparrow$ & {$[231]$} \\
\hline Human endothelial cells & Hypoxia $\left(1 \% \mathrm{O}_{2}\right)$ & $A P L N$ mRNA $\uparrow$ & {$[222]$} \\
\hline Endothelial progenitor cells & Hypoxia $\left(1 \% \mathrm{O}_{2}\right)$ & $\begin{array}{l}A P L N \text { and APLNR protein } \\
\text { and mRNA } \uparrow\end{array}$ & {$[232]$} \\
\hline 3T3-L1 Adipocytes & $\begin{array}{l}\text { Hypoxia }\left(1 \% \mathrm{O}_{2}\right) \\
\mathrm{CoCl}_{2}\end{array}$ & $A P L N$ protein $\uparrow$ & {$[82]$} \\
\hline $\begin{array}{l}\text { Human Simpson-Golabi-Behmel syndrome } \\
\text { adipocytes }\end{array}$ & $\begin{array}{l}\text { Hypoxia }\left(1 \% \mathrm{O}_{2}\right) \\
\mathrm{CoCl}_{2}\end{array}$ & $A P L N$ protein and mRNA $\uparrow$ & {$[83]$} \\
\hline $\begin{array}{l}\text { Endothelial cells, mouse preadipose cell line } \\
\text { 3T3F442A }\end{array}$ & Hypoxia $\left(1 \% \mathrm{O}_{2}\right)$ & $A P L N$ mRNA $\uparrow$ & [233] \\
\hline $\mathrm{TS} / \mathrm{A}$ breast cancer cells & Hypoxia $\left(1 \% \mathrm{O}_{2}\right)$ & $A P L N \mathrm{mRNA} \uparrow$ & {$[3]$} \\
\hline $\begin{array}{l}\text { Human oral tongue squamous cell } \\
\text { carcinoma cell lines HSC-2, HSC-3 and } \\
\text { Ca9.22 }\end{array}$ & Hypoxia & $A P L N$ protein and mRNA $\uparrow$ & {$[6]$} \\
\hline $\begin{array}{l}\text { Liver (HepG2), enteroendocrine (STC-1), rat } \\
\text { primary enteric cells stomach, ileal and } \\
\text { colon cells were measured for APLNR } \\
\text { mRNA }\end{array}$ & $\begin{array}{l}\text { Hypoxia }(0.5 \% \\
\left.\mathrm{O}_{2}\right)\end{array}$ & $\begin{array}{l}A P L N \text { mRNA } \uparrow, \text { APLNR } \\
\text { mRNA } \uparrow\end{array}$ & {$[28]$} \\
\hline Retinal glial cell (Müller) & $\mathrm{CoCl}_{2}$ & $A P L N$ mRNA $\uparrow$ & {$[199]$} \\
\hline Retinal glial cell (Müller) & $\mathrm{CoCl}_{2}$ & $A P L N$ mRNA $\uparrow$ & \\
\hline Rat retinal pericytes & $\mathrm{CoCl}_{2}$ & $\begin{array}{l}\text { APLN and APLNR mRNA } \\
\uparrow,\end{array}$ & {$[200]$} \\
\hline RAW264.7 cells & Hypoxia $\left(1 \% \mathrm{O}_{2}\right)$ & $\begin{array}{l}\text { APLNR mRNA and protein } \\
\uparrow\end{array}$ & {$[234]$} \\
\hline HEPG2 and LX-2 cells & Hypoxia (5\% O2) & APLNR mRNA $\uparrow$ & {$[235]$} \\
\hline $\begin{array}{l}\text { Bone marrow-derived mesenchymal stem } \\
\text { cells (BMSCs) }\end{array}$ & Hypoxia $\left(1 \% \mathrm{O}_{2}\right)$ & APLN and APLNR protein $\uparrow$ & {$[236]$} \\
\hline
\end{tabular}




\subsubsection{The role of glucose in glioblastoma and the potential role of the apelinergic system}

Glioblastoma tumour cells experience not only hypoxic conditions in vivo but also low glucose (hypoglycaemic) conditions [237, 238]; particularly in oxygen-depleted regions of the tumour $[239,240]$. Like hypoxia, hypoglycaemic conditions can be attributed to rapid tumour growth [241], poor vascularization, as well as high glucose demand [242]. Glioblastoma also exhibits high glucose demand [243, 244]. This is known as the Warburg effect, and it is a central feature of a number of cancers [245] including glioblastoma cells [243]. To survive these low glucose environments, glioblastoma has a number of adaptive processes [183, 240, 242, 246-249].

Glioblastoma's reliance on glucose metabolism together with the ability of glioblastoma cells to adapt to low nutrient conditions is intriguing as the apelinergic system both modulates glucose metabolism [13] and protects against glucose deprivation-induced cell death in retinal glial cells [250] as well as other cell types [194, 251, 252]. Finally, glucose metabolism is also modulated by hypoxia through the HIF $1 \alpha$ transcription factor inducing glycolysis-associated genes [253]. This relationship between hypoxia, low glucose conditions, and the apelinergic system in glioblastoma is hitherto un-investigated despite evidence of the apelinergic system being modulated by hypoxia and modulating glucose metabolism. Thus, the relationship between the apelinergic system, glucose metabolism and hypoxia led to the hypothesis that the apelinergic system may play a role in protecting glioblastoma cells against glucose deprivation or combined glucose and oxygen deprivation. This was assessed during this study by measuring changes in $A P L N$ and $A P L N R$ expression as well as assessing if exogenously added $\left[\mathrm{Pyr}^{1}\right]$ apelin-13 modulated cell number under the aforementioned conditions. 


\subsubsection{Chemotherapy-induced stress}

Chemotherapy induces stress responses in cancer cells that can lead to cell death. There is a wealth of evidence linking the apelinergic system to anti-apoptotic pathways in several cell lines [185] as summarised prior (Table 1.6). The apelinergic system has also been shown in one study to respond to DNA damage by regulating the DNA damage repair enzyme 8oxoguanine glycosylase (OGG1) [203]; however, no other evidence has been reported of a direct link between the apelinergic system and DNA-damaging agents.

The alkylating agent TMZ, which is the first-line treatment for glioblastoma [254, 255], works by inducing DNA single and double strand breaks which result in cell death. Indirectly related to the main mechanism of action of TMZ, it also induces endoplasmic reticulum stress [256] and oxidative stress through the generation of reactive oxygen species (ROS) [257]. The apelinergic system has been reported to play a role in mitigating these stress responses. APLN is transcriptionally upregulated by ER stress [258] and can attenuate ROS production [259, 260]. While not demonstrative of an apelinergic system role in response to chemotherapeutic induced stress, it is suggestive of a potential role.

During this thesis, the chemotherapeutic drugs TMZ and doxorubicin were used as models of DNA damaging inducing chemotherapeutics. While there is no concrete evidence of the apelinergic system protecting against stress induced by chemotherapeutics the numerous antiapoptotic reports and evidence of APLN involvement in response to oxidative and ER stress responses suggest it is beneficial to investigate the response of the apelinergic system under stress induced from chemotherapeutics. This was done in the current study by measuring the changes of APLN and APLNR mRNA expression in response to treatment with TMZ and doxorubicin along with assessing if exogenously added $\left[\mathrm{Pyr}^{1}\right]$ apelin-13 modulated cell number. 


\subsubsection{The role of the apelinergic system in cell migration}

A hallmark of glioblastoma is a diffuse invasive phenotype $[261,262]$ that makes glioblastoma difficult to resect and allows tumour reoccurrence to occur far from its site of origin [263]. This contributes to poor patient outcomes [264]. The apelinergic system has been reported to have a pro-migratory role in several cell lines [199, 265-267] including a number of cancer cell lines $[5,6,88]$ such as MCF-7 breast [97] and LNCaP prostate [88] cancer cells. Recently, the apelinergic system was also linked to promoting metastases in B16 melanoma mice [4] and in lung adenocarcinoma cell lines, where it abrogated the anti-metastatic effects of doxorubicin $[5]$.

The migratory and invasive characteristics of glioblastoma cells can provide information about invasion in the tumour [268]. Understanding migration and invasion with an aim to target these processes as therapies for glioblastoma has been considered [269] but never in the context of the apelinergic system. In this study, it was hypothesised that the apelinergic system has a promigratory role in glioblastoma-derived cell lines. 


\subsubsection{Recent insights regarding the role of the apelinergic system in glioblastoma}

During the final submission of this thesis, two critical papers were published regarding the role of the apelinergic system in cancer biology. These papers, though important, ultimately could not shape the experimental design of this thesis due to their recent publication. These are summarised briefly below and are discussed later in this thesis.

The first paper by Harford-Wright et al described the role of the apelinergic system in glioblastoma [270]. Specifically, the authors wished to investigate the cues secreted by brain endothelial cells (BEC) that support the glioblastoma stem cells (GSCs) in the perivascular niche $[270,271]$. The authors utilized a mass spectrometry approach to characterize the secretome of BECs in comparison to epithelial cells and noted apelin was strongly enriched. Apelin-13 was found to potently promote tumoursphere formation in patient isolated GSCs through the APLNR receptor in vitro. Genetic depletion of APLNR reduced the size of xenograft tumours derived from GSCs, inferring the apelinergic system has a role in glioblastoma pathogenesis. Finally, the authors demonstrated that pharmacological inhibition of the APLNR receptor in both ectopic and orthotopic GSC derived tumours reduced tumour size. This indicates that the premise of this thesis, that the apelinergic system has a salient role in glioblastoma pathogenesis, is valid.

The second paper by Patel et al describes the importance of the APLNR receptor in the mediation of immunotherapy in vitro [102]. Patel et al developed a " 2 cell type" CRIPSR screen to determine which genes were important in immune therapies. The screen consisted of a chimeric antigen receptor T-cell (CAR-T) as an effector cell and melanoma cells as a target. The authors then utilized a genome scale CRISPR-CAS9 approach to edit out numerous genes putatively believed to be involved in the melanoma evasion of CAR-T mediated cell death. As certain melanoma cells with essential immunotherapy genes evaded T-cell mediated tumour killing, the sgRNAs that knocked out the genes were enriched. These were investigated using a bioinformatics approach. Loss of APLNR was found to exhibit $>50 \%$ resistance to CAR-T mediated lysis suggesting APLNR has a critical role in immunotherapy mediated killing. This 
also indicates the apelinergic system may hold a role in the immune system. This is discussed in more depth in the final discussion.

\subsection{Detecting serum APLN using aptamer technologies}

\subsubsection{A review of aptamer technology}

Aptamers are nucleic acid or protein molecules that are selected by a process called SELEX (systematic evolution of ligands by exponential enrichment) from a large random library of candidates to bind to targets with very high specificity. The use of aptamers is a rapidly growing new biotechnology. Aptamers have a variety of functions, the most basic of which can be to bind to target proteins but can also act as agonists and even exhibit biased agonism [289]. Aptamers are rapidly chemically synthesised with much less batch to batch variation than polyclonal antibodies [290] which is highly advantageous as the human APLN ELISA Kit from Phoenix Pharmaceuticals relies on the use of a polyclonal antibody which is subject to batch to batch variation. Aptamers are more structurally stable than antibodies at a broader range of pHs, temperatures, and ionic conditions [291] enabling detection in a number of solutions. Aptamers are readily excreted or biodegraded in serum due to their small size and nucleic acid properties; however, aptamers are also able to be stabilised relatively simply through chemical modification [292-294]. Aptamer-based biosensors have been developed to femtomolar sensitivity [295]; thus, the development of an aptamer against APLN-13 could open up possibilities for detecting APLN-13 at more sensitive levels than current ELISA based alternatives. This would better-allow the correlation of APLN levels to diseases such as the ones described in Table 1.8. It is apparent that the generation of an aptamer to APLN-13 would be invaluable. The process of aptamer generation is described in more detail in Chapter 7. 


\subsubsection{Serum APLN as a biomarker}

The primary goal of this $\mathrm{PhD}$ study was to investigate the role of the apelinergic system in glioblastoma. The previous sections have primarily explored the potential functional roles of the apelinergic system in glioblastoma. However, several researchers have also suggested that the serum APLN levels may be a biomarker in a number of pathologies, including major adverse cardiovascular events [272], diabetic retinopathy [273], hepatitis C [274], obesity [275], diabetes [276, 277], chronic obstructive pulmonary disease [278] and coronary disease [279]. More recently, a correlation between serum APLN concentrations and cancer presence was reported [280]. The numerous examples of serum APLN showing value as a biomarker, this suggests there is value in developing a methodology to accurately detect serum APLN.

However, current studies detecting serum levels of APLN and correlating these to disease states (summarised in Table 1.8) has shown limitations. Firstly, the reviewed studies (Table 1.8) largely utilised immunological assays, such as enzyme-linked immunosorbent assays (ELISA) in which antibodies are used for detection purposes. Some of the antibodies used in Table 1.8 are highly cross-reactive with the different isoforms of APLN [58, 281]. Discrimination of these isoforms is critical as the various isoforms exert different biological effects $[59,78]$. This indicates a need to have a highly isoform-specific detection method.

Secondly, there have also been significant discrepancies in serum APLN levels between studies using immunoassays and those utilising more sensitive techniques such as mass spectrometry (MS) [281-283]. To better understand these discrepancies in serum APLN concentration, a review of all studies quantifying serum APLN using immunological assays (Table 1.8) and more sensitive methodology such as mass spectrometry (MS) (Table 1.9) was performed. 
Most reported literature that attempts to quantify APLN in serum utilises the ELISA assay from Phoenix Pharmaceuticals that detects APLN in the linear range of $0.07-0.79 \mathrm{ng} / \mathrm{mL}$ [284]. All the methods in Table 1.8 detect within this range, however, none of the results corroborated measurements by MS (Table 1.9), of which one group could not detect APLN at all by MS in human serum [283], and one detected APLN at $0.007-0.023 \mathrm{ng} / \mathrm{mL}$ [281]; less than described using the ELISA (Table 1.8). It seems unlikely that MS could not detect what antibodies could and casts doubt on the data presented in Table 1.8. This also indicates that there is a need for a more sensitive detection method for serum APLN. While an MS-based method to detect serum APLN, such as described by Zhen et al. [281], could be a sensitive enough technique; given Mesmin et al. [283] could not detect APLN in serum using MS, this suggests there is some difficulty in establishing an MS-based detection method. Moreover, a more accessible detection method that does not require costly MS equipment with specialised knowledge to optimise methods would increase the utility of detecting serum APLN.

The biological relevance of serum APLN is demonstrated by the data presented in Table 1.8. However, the discrepancy in reported APLN concentrations between ELISA based methods (Table 1.8) and MS approaches (Table 1.9) suggests a more sensitive and consistent detection method is required for APLN-13. Furthermore, a more cost-effective approach to serum APLN detection would enable widespread use. Finally, while serum APLN's utility as a biomarker marker is evident, there is yet to be any method that discriminates the different APLN isoforms. As such a method of detecting the most abundant isoform of APLN, APLN-13 [281] would be beneficial. A DNA aptamer would efficiently cover these requirements more so than antibody alternatives; DNA aptamers are briefly reviewed in Section 1.5.1. 
Table 1.8. Review of papers that utilise immunological based techniques to quantify serum APLN levels and correlated to physiological outcomes

\begin{tabular}{|c|c|c|c|}
\hline Biological question asked & Detection mechanism & $\begin{array}{l}\text { What was observed and } \\
\text { concluded }\end{array}$ & $\begin{array}{l}\text { Reference/detected } \\
\text { range of APLN }\end{array}$ \\
\hline $\begin{array}{l}\text { Is APLN a predictor of major } \\
\text { adverse cardiac events? }\end{array}$ & $\begin{array}{l}\text { APLN }-12 \text { detecting } \\
\text { ELISA assay kit from } \\
\text { Phoenix Pharmaceuticals }\end{array}$ & $\begin{array}{l}\text { Stipulated that detecting APLN } \\
\text { levels in patients' serum as a } \\
\text { prognostic marker for major } \\
\text { cardiac events }\end{array}$ & $\begin{array}{l}{[272]} \\
\text { Detected range: } \\
0.34-2.15 \mathrm{ng} / \mathrm{mL}\end{array}$ \\
\hline $\begin{array}{l}\text { Is APLN a predictor of patients } \\
\text { with advanced heart failure? }\end{array}$ & $\begin{array}{l}\text { APLN -12 detecting } \\
\text { ELISA assay kit Phoenix } \\
\text { pharmaceuticals }\end{array}$ & $\begin{array}{l}\text { Believed serum APLN did not } \\
\text { serve as a prognostic marker for } \\
\text { patients with advanced heart } \\
\text { failure secondary to left } \\
\text { ventricular systolic dysfunction }\end{array}$ & $\begin{array}{l}{[285]} \\
\text { Detected range: } \\
0.52-2.02 \mathrm{ng} / \mathrm{mL}\end{array}$ \\
\hline $\begin{array}{l}\text { Is APLN a predictor of poor } \\
\text { outcome in oral squamous cell } \\
\text { carcinoma? }\end{array}$ & $\begin{array}{l}\text { IHC staining of APLN in } \\
\text { patient-derived tissues } \\
\text { using an antibody against } \\
\text { APLN-36 from Phoenix } \\
\text { Pharmaceuticals }\end{array}$ & $\begin{array}{l}\text { APLN expression was correlated } \\
\text { with recurrence but not } \\
\text { associated with tumour size, } \\
\text { metastases, age, gender or } \\
\text { smoking status. }\end{array}$ & {$[6]$} \\
\hline $\begin{array}{l}\text { Do plasma APLN levels predict } \\
\text { arrhythmia reoccurrence in } \\
\text { patients with atrial fibrillation? }\end{array}$ & $\begin{array}{l}\text { APLN -12 detecting } \\
\text { ELISA assay kit from } \\
\text { Phoenix Pharmaceuticals }\end{array}$ & $\begin{array}{l}\text { Low APLN levels correlated with } \\
\text { arrhythmia }\end{array}$ & $\begin{array}{l}{[286]} \\
\text { Detected concentration } \\
730 \pm 150 \mathrm{pg} / \mathrm{mL}\end{array}$ \\
\hline $\begin{array}{l}\text { Do plasma APLN levels act as a } \\
\text { prognostic marker for chronic } \\
\text { liver disease (CLD)? }\end{array}$ & $\begin{array}{l}\text { Human total APLN ELISA } \\
\text { kit from MyBioSource }\end{array}$ & $\begin{array}{l}\text { APLN was deemed to be a weak } \\
\text { prognostic marker and marker of } \\
\text { disease severity based on } \\
\text { histological grades of CLD. }\end{array}$ & $\begin{array}{l}{[287]} \\
\text { Detected concentration: } \\
863.6 \pm 219.7 \mathrm{pg} / \mathrm{mL}\end{array}$ \\
\hline $\begin{array}{l}\text { Are plasma concentrations of } \\
\text { APLN are altered in chronic heart } \\
\text { failure patients? }\end{array}$ & $\begin{array}{l}\text { APLN }-12 \text { detecting } \\
\text { ELISA assay kit from } \\
\text { Phoenix Pharmaceuticals }\end{array}$ & $\begin{array}{l}\text { APLN concentrations were lower } \\
\text { in patients with chronic heart } \\
\text { failure. }\end{array}$ & $\begin{array}{l}{[288]} \\
\text { Detected range: } \\
0.85-5.13 \mathrm{ng} / \mathrm{mL} \text { (healthy } \\
\text { patients) }\end{array}$ \\
\hline $\begin{array}{l}\text { Are serum APLN levels altered } \\
\text { in obese patients with } \\
\text { endometrial cancer? }\end{array}$ & $\begin{array}{l}\text { APLN-36 ELISA (USCN } \\
\text { Life Sciences) Range: } \\
37.04-3000 \mathrm{pg} / \mathrm{mL}\end{array}$ & $\begin{array}{l}\text { Women with endometrial cancer } \\
\text { exhibited higher concentrations } \\
\text { of serum APLN-36 than controls }\end{array}$ & $\begin{array}{l}{[96]} \\
\text { APLN in endometrial } \\
\text { cancer patient: } 215.1 \pm \\
59.8 \mathrm{pg} / \mathrm{mL} \text {; control } \\
\text { patients: } 177.3 \pm 55.2 \\
\text { pg/mL }\end{array}$ \\
\hline $\begin{array}{l}\text { What are the changes in APLN } \\
\text { levels in patients with cancers } \\
\text { compared to healthy subjects? }\end{array}$ & $\begin{array}{l}\text { APLN detecting ELISA } \\
\text { assay kit from Phoenix } \\
\text { Pharmaceuticals }\end{array}$ & $\begin{array}{l}\text { Assaying } 95 \text { patients with lung, } \\
\text { gastrointestinal breast, prostate } \\
\text { and gynaecological cancers found } \\
\text { there to be higher serum APLN } \\
\text { levels than } 35 \text { control patients }\end{array}$ & $\begin{array}{l}{[280]} \\
\text { Serum APLN in cancer } \\
\text { patients: } 532.5 \pm 223.4 \\
\text { pg/mL; in healthy subjects: } \\
231.0 \pm 47.1 \mathrm{pg} / \mathrm{mL}\end{array}$ \\
\hline $\begin{array}{l}\text { What is the expression of APLN } \\
\text { serum concentrations in } \\
\text { gastroesophageal cancer (GEC) } \\
\text { patients }\end{array}$ & $\begin{array}{l}\text { APLN detecting ELISA } \\
\text { assay kit from Phoenix } \\
\text { Pharmaceuticals }\end{array}$ & $\begin{array}{l}85 \text { GEC patients were compared } \\
\text { with } 60 \text { control patients, and } \\
\text { APLN serum concentrations were } \\
\text { found to be elevated in cachectic } \\
\text { GEC patients }\end{array}$ & $\begin{array}{l}{[92]} \\
\text { Serum APLN in GEC } \\
\text { patients was } 855.0 \pm 195 \\
\text { pg/mL vs in healthy } \\
\text { subjects } 635 \pm 365 \mathrm{pg} / \mathrm{mL}\end{array}$ \\
\hline
\end{tabular}


Table 1.9. Review of papers that used non-immunological based methods to quantify APLN in human serum

\begin{tabular}{|c|c|c|c|}
\hline $\begin{array}{l}\text { Biological question } \\
\text { asked }\end{array}$ & Detection mechanism & $\begin{array}{l}\text { What was observed and } \\
\text { concluded }\end{array}$ & $\begin{array}{l}\text { Reference/APLN } \\
\text { concentration noted }\end{array}$ \\
\hline $\begin{array}{l}\text { What is the effect of APLN } \\
\text { on the regulation of osmotic } \\
\text { stimuli? }\end{array}$ & $\begin{array}{l}\text { Utilized HPLC followed by } \\
\text { radioimmunoassay with an } \\
\text { antibody developed by the } \\
\text { authors }\end{array}$ & $\begin{array}{l}\text { Detected APLN in human } \\
\text { serum, not for diagnosis }\end{array}$ & $\begin{array}{l}{[29]} \\
739 \mathrm{pg} / \mathrm{mL} \text { in healthy } \\
\text { volunteers }\end{array}$ \\
\hline $\begin{array}{l}\text { What is the concentration of } \\
\text { APLN peptides in plasma? }\end{array}$ & $\begin{array}{l}\text { Utilised a novel liquid } \\
\text { chromatography-tandem } \\
\text { mass spectrometry ( LC- } \\
\text { MS/MS) approach and } \\
\text { compared to the APLN-12 } \\
\text { immunoassay Phoenix } \\
\text { Pharmaceuticals }\end{array}$ & $\begin{array}{l}\text { The LC-MS/MS } \\
\text { methodology could not } \\
\text { detect any of the five } \\
\text { expected circulating forms } \\
\text { of APLN in human serum; } \\
\text { however, using the same } \\
\text { methodology, numerous } \\
\text { APLN isoforms in bovine } \\
\text { colostrum and milk were } \\
\text { detected }\end{array}$ & $\begin{array}{l}{[282,283]} \\
\text { Immunoassay detected: } 208 \\
\text { - } 466 \mathrm{pg} / \mathrm{mL} \text { in healthy } \\
\text { donors }\end{array}$ \\
\hline $\begin{array}{l}\text { What is the concentration of } \\
\text { plasma APLN-13 } \\
\text { concentration }\end{array}$ & $\begin{array}{l}\text { Utilised a cation exchange } \\
\text { purification alongside LC- } \\
\text { MS/MS methodology to } \\
\text { quantify serum APLN. }\end{array}$ & $\begin{array}{l}\text { The authors reported the } \\
\text { detection of APLN-13 using } \\
\text { LC-MS/MS at a lower } \\
\text { concentration than detected } \\
\text { with the ELISA assay. } \\
\text { Moreover, the authors } \\
\text { commented on the Mesmin } \\
\text { et al. [281] study and were } \\
\text { unsure why the authors } \\
\text { could not detect APLN in } \\
\text { serum. }\end{array}$ & $\begin{array}{l}{[281]} \\
\text { ELISA detection of APLN } \\
49 \mathrm{pg} / \mathrm{mL}-273 \mathrm{pg} / \mathrm{mL} \\
\\
\text { LCMS detection of APLN } \\
7.7-23.3 \mathrm{pg} / \mathrm{mL}\end{array}$ \\
\hline
\end{tabular}




\subsection{Models of glioblastoma}

The selection of an appropriate model to study the apelinergic system in glioblastoma for this thesis was challenging. Current literature researching the apelinergic system in cancer biology have utilised primary tissue in conjunction with stably expressing cell lines [4] or in vivo xenograft models [30], but none of these models have been verified to accurately replicate the apelinergic system in glioblastoma. The numerous models of glioblastoma have been proficiently reviewed elsewhere [296-298], but a summary table of the potential models are provided below (Table 1.10). During this study in vivo models were eschewed due to cost, model complexity, and time-consuming nature [219] in favour of in vitro models with a view to initially establish potential roles of the apelinergic system in glioblastoma which could then be later examined within in vivo models. This section briefly addresses the potential models that could be used and describes the rationale behind the models selected for this study. 
Table 1.10. An overall summary of available human and murine models of glioma and their strengths and weaknesses. Adapted and reproduced under Creative Commons Attribution 4.0 International License (http://creativecommons.org/licenses/by/4.0/), from [296]. Abbreviations: PDX - Patient derived xenograft, ENU - N-ethyl-N-

nitrosourea.

\begin{tabular}{|c|c|c|c|c|c|}
\hline Model type & Heterogeneity & $\begin{array}{l}\text { Immuno- } \\
\text { competent }\end{array}$ & Brain micro-environment & $\begin{array}{l}\text { Blood- } \\
\text { brain } \\
\text { barrier }\end{array}$ & $\begin{array}{l}\text { Stable/ } \\
\text { reproducible }\end{array}$ \\
\hline $\begin{array}{l}\text { ENU-induced murine } \\
\text { tumours }\end{array}$ & $\begin{array}{l}\text { Genetically heterogeneous, different neural cells may } \\
\text { be initiator cells }\end{array}$ & Yes & Relevant & Yes & No \\
\hline $\begin{array}{l}\text { GEMMs (conditional } \\
\text { expression of } \\
\text { oncogenes/loss of tumour } \\
\text { suppressor genes) }\end{array}$ & $\begin{array}{l}\text { Genetically homogeneous, initiator cell type dependent } \\
\text { on promoter driving Cre expression } \\
\text { (CMV/nestin/GFAP) }\end{array}$ & Yes & $\begin{array}{l}\text { Relevant when Cre expression is induced in the CNS } \\
\text { (intracerebral injection of Cre-lentivirus, crossing mice with } \\
\text { developmental CNS- expression of Cre }\end{array}$ & Yes & Yes \\
\hline PDX-subcutaneous & $\begin{array}{l}\text { Genetically homogeneous, but intratumoural } \\
\text { heterogeneity due to lack of pre-existent vasculature, } \\
\text { development of hypoxia and angiogenesis dependence }\end{array}$ & No & Non-relevant & No & Yes \\
\hline PDX—orthotopic & $\begin{array}{l}\text { Partly, it is not known to which extent PDX models } \\
\text { represent most aggressive parts of the originating } \\
\text { tumour }\end{array}$ & No & $\begin{array}{l}\text { Partly, only relevant in case PDXs have retained the capacity } \\
\text { to grow via diffuse infiltration }\end{array}$ & Yes & Yes \\
\hline Cell lines — adherent & No & No & Non-relevant & No & Yes \\
\hline Cell lines_-spheroids & $\begin{array}{l}\text { No *Can be made more heterogeneous by using } \\
\text { through the generation of multicellular spheroids [299] }\end{array}$ & No & Non-relevant & No & Yes \\
\hline
\end{tabular}




\subsubsection{Overview of glioblastoma models}

Established in vivo patient-derived xenograft (PDX) models such as the U87MG [300] and LN18 [301] are long-standing cell lines isolated from patients and have provided a valuable understanding of glioblastoma [302]. These cell lines can be studied in vitro and can also be used as an orthotopic or heterotropic in vivo models. In vivo, these cell lines can provide a representation of the glioblastoma; however, some in vivo models may not exhibit specific molecular features that are salient to glioblastoma pathogenesis; such as, a diffuse invasion front or an extensive microvasculature [303]. Mutagen induced cell line models such as the GL261 [304], which was used during this study, provide an alternative to traditional established PDX models but can also be limited in that they do not express all the salient features of glioblastoma. For example, the murine glioblastoma line GL261 exhibited a more diffuse invasion front akin to native glioblastoma while the human glioblastoma line U87MG exhibits a more defined border as seen below (Figure 1.6) (Newcomb \& Zagzag, 2009).
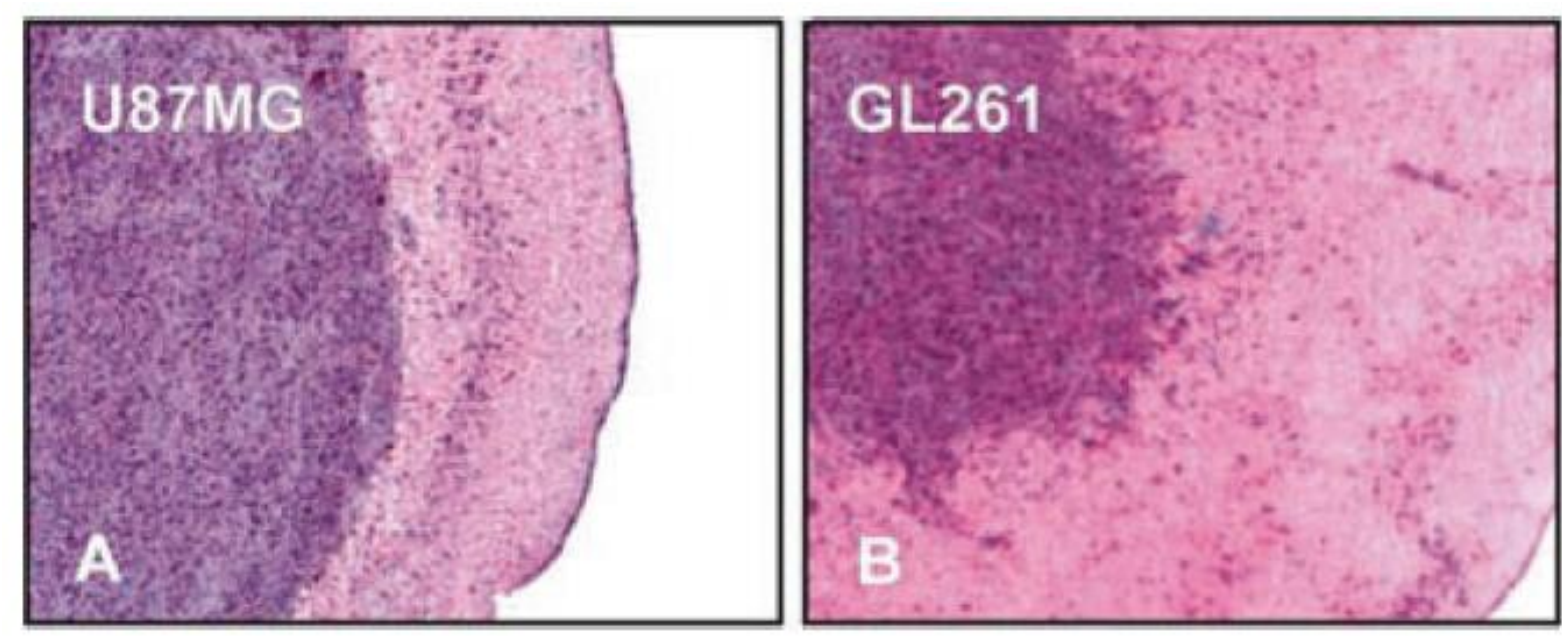

Figure 1.6. The difference in tumour recapitulation from two glioblastoma cell lines, U87MG (human) and GL261 (mouse). Mice were injected with U87MG or GL261 cells. Brains were harvested 15 days after implantation and stained with haematoxylin and eosin. Images are reproduced with permission from Springer [305] 
More representative in vivo models of glioblastoma are available that use fresh glioblastoma tissue from patients and generate primary cell lines from these samples; however, fresh tissue was unavailable during this study. As such, it was concluded that use of established glioblastoma cell lines as an in vitro cell culture model would be more appropriate to initially investigate the role of the apelinergic system in glioblastoma with the potential to be extended to in vivo models. Especially, given that at the time of inception of this study there was no real evidence as to what the role of the apelinergic system may be.

\subsubsection{In vitro cultured cell line models}

In vitro models provide a reductionist system to initially establish the potential roles of the apelinergic system for further investigation at a later time. To overcome some of the limitations of using established in vitro cultured glioblastoma-derived cell lines in this study, studies were performed on primary tissue-derived cell line and the established immortalised cell line [297]. The in vitro cultured cell lines used in this study are reviewed below.

The established immortalised human cell line models U87MG and LN18 were the two immortalised cell lines used during this thesis alongside the mutagen induced immortalised murine GL261 cell line. Archival tissue from the orthotopic recapitulated GL261 cell line was also used in this study to investigate in situ localisation of APLNR expression. The cell lines U87MG, LN18 and GL261, were selected because they are highly reproducible and consistent [306] despite not necessarily providing total clinical relevance due to differences in their in vivo features to in situ glioblastoma tumours in patients [307, 308]. These cell lines are discussed briefly in the next paragraph but are reviewed in depth elsewhere [301, 309].

U87MG and LN18 are human cell lines established from human glioblastoma samples. U87MG was established approximately 50 years ago [300], and since this time it has become one of the most widely studied glioblastoma cell lines with over 1700 publications [310]. LN18 is more recently established and was characterised in 1981 [301]. Recently the relevance and veracity of the U87MG cell line were investigated, and it was found that while it was different from the original isolated cell line, it was still of glioblastoma origin indicating some validity to its use [311]. The U87MG cell line is useful in that it has been extensively studied in several aspects of glioblastoma pathophysiology such as cancer stem cell markers [312] and tumour 
vasculature development [309], but it does not wholly represent original glioblastoma tumours found in patients $[311,313]$. Despite this, it still provides a well-studied initial model to investigate the apelinergic system in glioblastoma.

GL261 cells are murine astrocytic cells, chemically mutated to resemble glioblastoma and can be directly injected into the brain of immunocompetent mice [314] which allows for immunological aspects of glioblastoma to be studied. The GL261 cell line also displays a number of important pathological features of glioblastoma [305, 315] including PROM1 ${ }^{+}$cell subpopulations [316] and has glioblastoma-relevant point mutations in its genome [304]. Researchers consider GL261 a valid model in pre-clinical research [314, 315] however, it lacks the characteristic vascularisation of glioblastoma and displays irregular growth patterns [317]. Despite these limitations, the GL261 model is a useful, well-used model for initial investigation and translational research in glioblastoma [305, 314].

During this project, to account for the limitations of established immortalised in vitro cell lines, i.e. loss of salient genetic features, primary cell lines were also used [318, 319]. Primary tissuederived cell lines are tumour cells that have been dissociated from the resected tissue of patients and propagated in vitro [320]. Primary cell lines differ from immortalised cell lines because they have been more recently isolated from the original tumour tissue and can represent the original tumour characteristics more accurately [313, 319, 321]. Three primary glioblastoma cell lines were used during this thesis. These lines, 0906, 1003 and 0814 were derived from patients undergoing tumour debulking surgery as described elsewhere [322] and have been published prior [323].

During this study foetal bovine serum (FBS) was used to maintain cultures. The use of FBS in tissue cultures can drastically decrease the salient molecular features thus altering the phenotype of cancer cell lines from the original tumour making them less representative models $[313,324-326]$. An example is the expression of endothelial growth factor receptor (EGFR), which is characteristic of glioblastoma but down-regulated in serum culture [327]. It is possible to maintain relevant molecular expression by isolating cells fresh from tumours and culturing using neurobasal medium supplemented with basic fibroblast growth factor (bFGF) and epidermal growth factor (EGF) $[313,328]$ or complex cell culture methods, such as the 
Cambridge protocol [329]. Unfortunately, these options were not available for this study due to a lack of fresh tumour tissue and specialised media.

Two dimensional (2D) in vitro cell culture is the most widely used culturing system whereby cells are cultured in a flat layer on plastic dishes [330]. 2D in vitro cell culture models are commonly used to elucidate fundamental molecular interactions due to their ease of culture, reproducibility, and predictability; however, are limited in that their gene expression profiles differ from glioblastoma tumours [302]. Additionally, 2D in vitro models lack several features observed in situ or within in vivo models; such as hypoxic cores, reduced penetration of drugs, and multicellular interactions common in stem cell and perivascular niches. 2D models can further be extended into three dimensional (3D) models in which in vitro cells are cultured in 3D structures such as spheroids or using unique scaffolds [331]. 3D models can be effective solutions to the limitations of $2 \mathrm{D}$ culture by replicating features such as hypoxic cores and reduced penetration of drugs, but do not account for genetic differences between tumours and in vitro cell lines. Furthermore, 3D models can be costly especially when using freshly isolated glioblastoma tissue samples which require special culturing conditions to maintain genetic profiles as seen in the original tumour [324, 329, 332]. 3D models were trialled during this thesis (Appendix A.2) but ultimately eschewed for 2D models due to the availability, costeffective and reproducible nature. 


\subsection{Goals of this thesis}

The overall goal of this $\mathrm{PhD}$ study was to investigate the role of the apelinergic system in glioblastoma to address three aims.

The first aim was to characterise and compare the mRNA expression of APLN and APLNR in resected glioblastoma tissues; primary tissue-derived cell lines and the immortalised glioblastoma cell lines. This included determining the expression of APLNR protein in archival paraffin-embedded GL261 recapitulated tumour specimens, and glioblastoma tumour derived cell lines.

The second aim was to develop an appropriate in vitro cell model for investigating the role of the apelinergic system in glioblastoma and to characterise this model by examining known APLN activated pathways and physiological responses.

The third aim was to utilise an appropriate cell model to determine the role of the apelinergic system in response to cellular stress, specifically stress induced by hypoxia, low glucose conditions and chemotherapeutic compounds.

The secondary goal of this thesis was to generate an aptamer to apelin-13. This could have been used in future studies to develop an assay capable of detecting apelin-13 in serum. 


\section{Chapter 2 General Methods}

\subsection{Standard Cell culture methods}

\subsubsection{Cell culture facility set-up and standard techniques}

All cell culture plasticware was obtained sterile from Thermofisher Scientific (Nunc, Thermofisher Scientific, Auckland, New Zealand). The handling of all tissues and liquids were conducted under aseptic conditions in a sterile Email Air Handling Class II Biological safety cabinet (AES Environmental Pty LTD, Auburn Australia).

Cultures were incubated in a Heracell incubator (Kendro Laboratory Products GmbH, Hanau, Germany) at $37^{\circ} \mathrm{C}$ in humidified air with $5 \% \mathrm{CO}_{2}$. Multiple cell lines were used with varying culture conditions as listed in Table 2.1. Cell culture reagents: heat-inactivated foetal bovine serum (FBS), Penicillin and Streptomycin 5000 units/mL (hereafter referred to as penstrep), Roswell Park Memorial Institute 1640 cell culture media (RPMI), Optimem reduced serum media and Dulbecco's Modified Eagle Medium (DMEM) were all purchased from Gibco (Thermofisher Scientific, Auckland, NZ).

To defrost cells, a vial was removed from liquid nitrogen and placed in a water bath at $37^{\circ} \mathrm{C}$. Cells were then transferred to a $15 \mathrm{~mL}$ centrifuge tube with $10 \mathrm{~mL}$ of $37^{\circ} \mathrm{C}$ culture media, and pelleted at $300 \mathrm{RCF}$ for $5 \mathrm{~min}$ at $25^{\circ} \mathrm{C}$. Cells were resuspended in fresh culture medium and transferred to a $25 \mathrm{~cm}^{2}$ sterile culture flask for expansion.

Trypsinisation was carried out by removing the cell culture medium and the cells rinsed with sterile 1 x phosphate buffered saline (PBS) (Gibco, Thermofisher Scientific, Auckland, NZ) 
followed by incubation with $1-2 \mathrm{~mL}$ of TrypLE (Gibco, Thermofisher Scientific, Auckland, NZ) preheated to $37^{\circ} \mathrm{C}$ for $1-3 \mathrm{~min}$. Once cells were detached the TrypLE was diluted with three times the initial volume of TrypLE in culture media, transferred to a $15 \mathrm{~mL}$ centrifuge tube and pelleted as previously described. When required, the percentage of viable cells was determined using the trypan blue exclusion method.

\subsubsection{Cryopreservation of cell lines}

Cryopreservation of cell cultures involved the use of a cryopreservation chamber known as a "Mr Frosty" (Nalgene, Sigma Aldrich, Auckland, NZ). Cells were placed into the Mr Frosty device and then incubated at $-80^{\circ} \mathrm{C}$ overnight to allow for the slow freezing of cells. The cells were then transferred to a liquid nitrogen dewar.

\subsubsection{Freezing methodology}

Cultures were always frozen at a logarithmic growth phase which is approximately $70 \%-80 \%$ confluency. Cultures were checked before freezing to confirm the absence of bacterial and fungal contaminants as well as general cell health by using an Olympus IX51 inverted microscope (Olympus, Hamburg, Germany).

Cells were trypsinised as described prior and re-suspended in $1 \mathrm{~mL}$ culture medium without serum. The cells were briefly vortexed, and $50 \mu \mathrm{l}$ was withdrawn to be used for trypan counting. The remaining culture was centrifuged at $300 \mathrm{RCF}$ for $5 \mathrm{~min}$ at $25^{\circ} \mathrm{C}$. Cells were resuspended at a concentration of $1 \times 10^{6}$ cells per $\mathrm{mL}$ in freeze medium $(69 \%$ base media, $20 \% \mathrm{FBS}, 10 \%$ DMSO and $1 \%$ penstrep) and aliquots of $1 \mathrm{~mL}$ were made in cryogenic vials (Grenier bio-one, Frickhausen Germany). Vials were placed in a "Mr Frosty" unit and frozen at $-80^{\circ} \mathrm{C}$ for 12 to $16 \mathrm{hrs}$. The cryogenic vials were stored long-term in liquid nitrogen.

\subsubsection{Microscopy}

Cells in culture were viewed using an Olympus IX51 inverted microscope (Olympus, Hamburg, Germany) fitted with phase contrast and fluorescence objectives and a DP70 digital camera. Live cell and immunostained slides were also viewed on an Olympus Fluoview 
FV1000 confocal laser scanning biological microscope (inverted model IX81) fitted with a heating chamber (INU-21LCS-F1 model, Tokai Hit Co., Ltd, Japan)

\subsubsection{Cell lines used}

The U87MG and LN18 cell lines were kindly provided by Dr Melanie McConnell at Victoria University School of Biological Sciences, and the GL261 cell line was kindly provided Professor Ian Hermans at Malaghan Institute of Medical Research. Primary glioblastoma tumour-derived cell lines were also utilised in this study (kindly donated by Dr Melanie McConnell). Three primary lines, namely 0906, 0814 and 1003, were used in this thesis and are previously described [322, 333-335]. All primary cell lines were analysed with 25 passages of isolation

Table 2.1. A summary of the cell lines and the corresponding medias used during the study.

\begin{tabular}{lll}
\hline Cell line name & Media composition & Cell line origin \\
\hline 0906 & $10 \%$ FBS 1\% PS RPMI 1640 & Primary glioblastoma derived line \\
1003 & $10 \%$ FBS 1\% PS RPMI 1640 & Primary glioblastoma derived line \\
0814 & $10 \%$ FBS 1\% PS RPMI 1640 & Primary glioblastoma derived line \\
U87MG & $10 \%$ FBS 1\% PS DMEM & Immortalised glioblastoma cell line \\
LN18 & $10 \%$ FBS 1\% PS DMEM & Immortalised glioblastoma cell line \\
HEK293 & $10 \%$ FBS 1\% PS DMEM & Immortalised Human Embryonic Kidney line \\
GL261 & $10 \%$ FBS 1\% PS DMEM & $\begin{array}{l}\text { Immortalised glioblastoma cell line } \\
\text { Stable cell line created during this study. }\end{array}$ \\
U87.APLNR & $10 \%$ FBS 1\% PS DMEM & $\begin{array}{l}\text { Requires maintenance concentration of } \\
\text { geneticin } 400 \mu \text { g/mL G418 (Geneticin) }\end{array}$ \\
& & $\begin{array}{l}\text { Stable cell line created during this study, no } \\
\text { maintenance G418 required }\end{array}$ \\
\hline
\end{tabular}




\subsection{Quantitative PCR}

\subsubsection{Cell sample preparation and RNA extraction}

Cells were plated in a $35 \mathrm{~mm}$ dish at the appropriate density determined for the cell line and allowed to adhere and grow to approximately $60 \%$ confluence in standard cell media. Cells were then treated for $24 \mathrm{hrs}$ at low serum (1\%) conditions to prevent serum interference with the drug. Following treatment, the medium was removed by aspiration and $1 \mathrm{~mL}$ of TRIzol (Ambion, Thermofisher Scientific, Auckland, NZ) was added to the $35 \mathrm{~mm}$ dish and left for 30 - 60 sec. The TRIzol triturated to dislodge cells and then the cell suspension was transferred into a microcentrifuge tube.

The homogenised sample was left at $25^{\circ} \mathrm{C}$ for $5 \mathrm{~min}$, following which $0.2 \mathrm{~mL}$ of chloroform was added per $1 \mathrm{~mL}$ of TRIzol and shaken vigorously for $15 \mathrm{sec}$. The tube was left at room temperature for $5 \mathrm{~min}$, then centrifuged at $14,000 \mathrm{RCF}$ for $15 \mathrm{~min}$ at $4^{\circ} \mathrm{C}$. Approximately $80 \%$ of the aqueous phase was removed and placed in a fresh microcentrifuge tube. Further purification of the RNA was done using a GeneJET column (Thermofisher Scientific, Auckland, NZ); $0.5 \mathrm{~mL}$ volumes of $100 \%$ absolute ethanol (Thermofisher Scientific, Auckland, NZ) was added to the aqueous phase and triturated to mix. This was added directly to the GeneJET column and centrifuged at 14,000 RCF for $1 \mathrm{~min}$. Following this, the standard GeneJET manufacturer's protocol was followed; briefly, three subsequent wash steps were performed and a final clearance spin. Finally, the RNA was eluted in $12 \mu \mathrm{L}$ of nuclease-free water.

\subsubsection{Quantification of RNA via Nanodrop spectrophotometer}

RNA was quantified by measuring the absorbance at $260 \mathrm{~nm}$ (A260) using a Nanodrop ND1000 spectrophotometer (Thermofisher Scientific, Auckland, NZ). RNA purity was assessed by examining the A260/A280 and A260/A230 ration. Ratios of 2.0 indicate pure RNA. 


\subsubsection{DNase treatment}

Contaminating gDNA was removed using DNase I (Cat\# EN0521, Thermofisher Scientific, Auckland, NZ) treatment as per manufacturer's protocol. In brief, 1 unit of DNase was added per $1 \mu \mathrm{g}$ of total RNA and incubated at $37^{\circ} \mathrm{C}$ for $30 \mathrm{~min}$. The DNase was then inactivated with $2 \mathrm{mM}$ EDTA and heated to $75^{\circ} \mathrm{C}$ for $10 \mathrm{~min}$. DNase treatment efficacy was tested using separate no reaction template (NRT) PCRs due to the sample being scarce for PCRs.

\subsubsection{Reverse transcription}

RNA was reverse-transcribed using Superscript III reverse transcriptase (Thermofisher Scientific, Auckland) using gene-specific primers (2 pmol each) or in the case of OligoDT (50 pmol). The reaction was performed as per manufacturer's protocol with the maximal amount of RNA available up to $1 \mu \mathrm{g}$ of total RNA. RNAse Out ribonuclease inhibitor was also included (Thermofisher Scientific, Auckland, NZ).

\subsubsection{Primer design and reconstitution}

PCR primers for this study were selected from published literature having been verified prior by the authors of the publication. On the occasion that primers needed to be designed, they were generated using Primer3Plus software [336]. When possible, intron-spanning primers and intron-exon boundary primers were used. All primers were synthesised by Integrated DNA Technologies (IDT, Singapore) and were reconstituted in TE buffer (10mM Tris, $0.1 \mathrm{mM}$ EDTA, $\mathrm{pH} 8.0$ ) to a final concentration of $250 \mu \mathrm{M}$. Efficiencies were validated by Dr Ryan Steel. 
Table 2.2 Summary of primers used during thesis. The (+) refers to the forward primer and (-) refers to the reverse primer. Target size refers to the target amplicon size in base pairs (bp).

\begin{tabular}{|c|c|c|c|}
\hline Primer Name & Sequence $5^{\prime}$-> 3' & $\begin{array}{l}\text { Target size } \\
\text { (bp) }\end{array}$ & Reference \\
\hline $\operatorname{APLN}(+)$ & GATGCCGCTTCCCGATG & \multirow{2}{*}{76} & \multirow{2}{*}{ [87] } \\
\hline $\operatorname{APLN}(-)$ & ATTCCTTGACCCTCTGGGCT & & \\
\hline $\operatorname{APLNR}(+)$ & GCCCTTGCTTTCTGAAAATCA & \multirow{2}{*}{66} & \multirow{2}{*}[337]{} \\
\hline $\operatorname{APLNR}(-)$ & GGACAGTTAAAGGATGTGCAT & & \\
\hline SCL2A1 $(+)$ & TCATCGTGGCTGAACTCTTC & \multirow{2}{*}{140} & \multirow{2}{*}[338]{} \\
\hline SCL2A1 (-) & GATGAAGACGTAGGGACCAC & & \\
\hline HPRT1 (+) & CCCTGGCGTCGTGATTAG & \multirow{2}{*}{179} & \multirow{2}{*}{ [339] } \\
\hline HPRT1 (-) & GGGCTACAATGTGATGGCCT & & \\
\hline Toddler $(+)$ & ATCAGCGGACAGAGACCAGT & \multirow{2}{*}{108} & \multirow{2}{*}{ [33] } \\
\hline Toddler $(-)$ & TCAGGGAAAGGGTACTCGTG & & \\
\hline PROM1 (+) & GCATTGGCATCTTCTATGGTT & \multirow{2}{*}{170} & \multirow{2}{*}[340]{} \\
\hline PROM1 (-) & CGCCTTGTCCTTGGTAGTGT & & \\
\hline APLNR.Human.CDS $(+)$ & СССТСАТСССТGCСАТСТАС & \multirow{2}{*}{111} & \multirow{2}{*}{ Self-designed } \\
\hline APLNR.Human.CDS (-) & TATCAGCTGAGCGCCTCTTC & & \\
\hline tGFP $(+)$ & GATGGGCTACGGCTTCTACC & \multirow{2}{*}{109} & \multirow{2}{*}{ Self-designed } \\
\hline tGFP $(-)$ & TACAGCCAGGAGACCCTTGT & & \\
\hline APLNR.GFP $(+)$ & TACAGCCAGGAGACCCTTGT & \multirow{2}{*}{183} & \multirow{2}{*}{ Self-designed } \\
\hline APLNR.GFP (-) & CATCTTGTTGGTCATGCGGC & & \\
\hline Apln (murine) $(+)$ & GCTGCTCTGGCTCTCCTTGA & \multirow{2}{*}{60} & \multirow{2}{*}{ [341] } \\
\hline Apln (murine) $(-)$ & CCATCTGGAGGCAACATCA & & \\
\hline Aplnr (murine) $(+)$ & CCACCTGGTGAAGACTCTCTACA & \multirow{2}{*}{110} & \multirow{2}{*}{ [54] } \\
\hline Aplnr (murine) (-) & TGACATAACTGATGCAGGTGC & & \\
\hline PROM1 (murine) $(+)$ & AGGCTACTTTGAACATTATCTGCA & \multirow{2}{*}{344} & \multirow{2}{*}{ [342] } \\
\hline PROM1 (murine) $(+)$ & GGCTTGTCATAACAGGATTGT & & \\
\hline SCL2A1 (murine) $(+)$ & ATGGATCCCAGCAGCAAG & \multirow{2}{*}{92} & \multirow{2}{*}{ [343] } \\
\hline SCL2A1 (murine) (-) & CCAGTGTTATAGCCGAACTGC & & \\
\hline
\end{tabular}




\subsubsection{RT-qPCR assays}

The relative cDNA amount was measured using Bioline Sensimix (Quantace, Total Lab Systems, Auckland, NZ) in $25 \mu \mathrm{L}$ reactions in duplicate as per manufacturer's protocols. Sensimix contains DNA polymerase, buffer constituents, dNTPs, $\mathrm{MgCl}$, fluorescein and SYBR Green in a $2 X$ reaction mix. Primers were added to a final concentration of $200-250$ $\mathrm{nM}$. Template cDNA was added to a final amount of 70-100 ng. All reactions included a no template control (NTC). The primer sequences used in this study are provided in Table 2.2. Cycling conditions were an initial denaturation of $10 \mathrm{~min}$ at $95^{\circ} \mathrm{C}$ followed by $35-40$ cycles of three-step amplification from $95^{\circ} \mathrm{C}$ for $10 \mathrm{sec}, 55^{\circ} \mathrm{C}$ or $60^{\circ} \mathrm{C}$ degrees for $15 \mathrm{sec}$ (assay dependent) and $72^{\circ} \mathrm{C}$ for $15 \mathrm{sec}$ extension time. High-resolution melt analysis was performed to determine the specificity of the PCR. This was done using a temperature gradient from $65^{\circ} \mathrm{C}$ to $95^{\circ} \mathrm{C}$ in $0.5^{\circ} \mathrm{C}$ increments. All RT-qPCR was performed in a Biorad CFX 96 (Biorad, Auckland, NZ). The relative fold change in mRNA expression was determined using the deltadelta CT method [344].

\subsubsection{Calculation of relative gene expression}

Relative gene expression was analysed using the $2^{-\Delta \Delta \mathrm{Ct}}$ method as described elsewhere [344, 345]. Briefly, to compare between two treatment groups, the cycle threshold (CT) values for each transcript were compared against an internal housekeeping gene HPRT which has been reported in the literature to be appropriate for glioblastoma cells [346, 347]. This yields the $\Delta \mathrm{CT}$ value $(\Delta \mathrm{CT}=\mathrm{CT}$ target $-\mathrm{CT}$ reference gene). The $\Delta \Delta \mathrm{CT}$ is calculated by subtracting the control $\Delta \mathrm{CT}$ from the treatment $\Delta \mathrm{CT}(\Delta \Delta \mathrm{CT}=\Delta \mathrm{CT}$ (treatment) - $\Delta \mathrm{CT}$ (housekeeping)). The fold mRNA expression of the gene of interest was then calculated using $2^{-\Delta \Delta C T}$. In the event that the $2^{-\Delta \Delta \mathrm{CT}}$ is less than 1 , the reciprocal of the $2^{-\Delta \Delta \mathrm{CT}}$ is taken $\left(\frac{-1}{2^{-\Delta \Delta \mathrm{CT}}}\right)$ to proportionally show downregulation of the gene. 


\subsubsection{Agarose gel electrophoresis}

Agarose gel electrophoresis was performed to determine correct product size. Agarose gels ranging from $1-3 \%$ were used. Gels were run at 100 volts in 1 x TAE buffer for $30-60$ min, dependent on the length of gel and size of the product. All gels were run with an Invitrogen 50 base pair ladder (Invitrogen, Thermofisher Scientific, Auckland, and NZ) or a KAPA universal ladder (Custom Science, Auckland, NZ).

\subsubsection{Small molecule and peptide reconstitution and usage}

Table 2.3 A summary of the peptides using during this study, including their origin and the solvent used to assist their aqueous suspension.

\begin{tabular}{lll}
\hline Peptide & Manufacturer & Solvent \\
\hline Toddler 11 (Synthesised) & Peptides \& elephants GmbH (Hennigsdorf, Germany) & DMSO \\
Toddler 33 (Synthesised) & Chempeptide (Shanghai, China) & High Purity $\mathrm{H}_{2} \mathrm{O}$ \\
ML221 Cat \# 4748 & Tocris Bioscience (Bristol, UK) & DMSO \\
Apelin-13 Cat \# 13523 & Cayman Pharmaceuticals (Michigan, USA) & DMSO \\
{$[$ Pyr1]-Apelin-13 ab141012 } & Abcam (Melbourne, Australia) & High Purity $\mathrm{H}_{2} \mathrm{O}$ \\
\hline
\end{tabular}

\subsubsection{Apelin}

During this study, the $\left[\mathrm{Pyr}^{1}\right]$ apelin-13 isoform was used for all functional studies while apelin13 was used for aptamer generation. In all instances of exogenous peptide the full name is written out for clarity. This was purchased from Abcam (Melbourne, Australia) and prepared in Milli-Q water to a stock concentration of $100 \mu \mathrm{M}$. Apelin-13 has a binding affinity $\mathrm{IC}_{50}$ of $5.7 \pm 0.3 \mathrm{nM}$ in the competitive binding assay and an $\mathrm{EC}_{50}$ of $1.89 \pm 1 \mathrm{nM}$ in the forskolininduced cAMP assay [348]. The concentrations of [ $\mathrm{Pyr}^{1}$ ]apelin-13 used for functional assays during this study $(1-2 \mu \mathrm{M})$ far exceed the reported $\mathrm{EC}_{50}$ of both apelin-13 isoforms in several assays [11, 70, 78]. During aptamer generation, the apelin-13 purchased from Cayman Pharmaceuticals was used. This was made up to a $10 \mathrm{mM}$ stock in DMSO and used as described extensively in Chapter 6. 


\subsubsection{ML221}

An inhibitor of the APLNR, namely ML221, was purchased from Tocris Bioscience (Bristol, UK). ML221 was prepared in DMSO at a stock concentration of 10mM. To control for vehicle effects on cells, DMSO was added to all untreated control cultures, and the final concentration kept below $0.1 \%(\mathrm{v} / \mathrm{v})$. ML221 has an $\mathrm{IC}_{50}$ of approximately $1.75 \mu \mathrm{M}$ in the beta-recruitment assay and $0.70 \mu \mathrm{M}$ in the cyclic adenosine monophosphate (cAMP) inhibition assay [349]. As such, ML221 was used at a final concentration of $5 \mu \mathrm{M}$ as this was approximately two-fold higher than the reported $50 \%$ inhibitory concentration $\left(\mathrm{IC}_{50}\right)$ values.

\subsection{Functional cellular assays}

\subsubsection{Matrigel tube formation assay}

A Matrigel (BD Bioscience, Auckland, NZ) based tube formation assay as described by Francescone et al. [350] was used to determine vascular mimicry potential. Matrigel was thawed on ice, $50 \mu \mathrm{L}$ of Matrigel per well was added to 96-well plate, and incubated for 60 $\min$ at $37^{\circ} \mathrm{C}$. Cells were then trypsinised as previously described, diluted to $2 \times 10^{5}$ cells $/ \mathrm{mL}$ and $50 \mu \mathrm{L}$ of cell suspension added to each well. Complete cell culture medium $(50 \mu \mathrm{L})$ was then added and cells incubated for $12-16$ hrs. The cells were micrographed using an Olympus IX51 inverted light microscope using a 4x objective. Then Cell-A software (Olympus) was used to make composite images of each well, and the tubule formations counted. An example of a composite image is shown in Figure 2.1. 


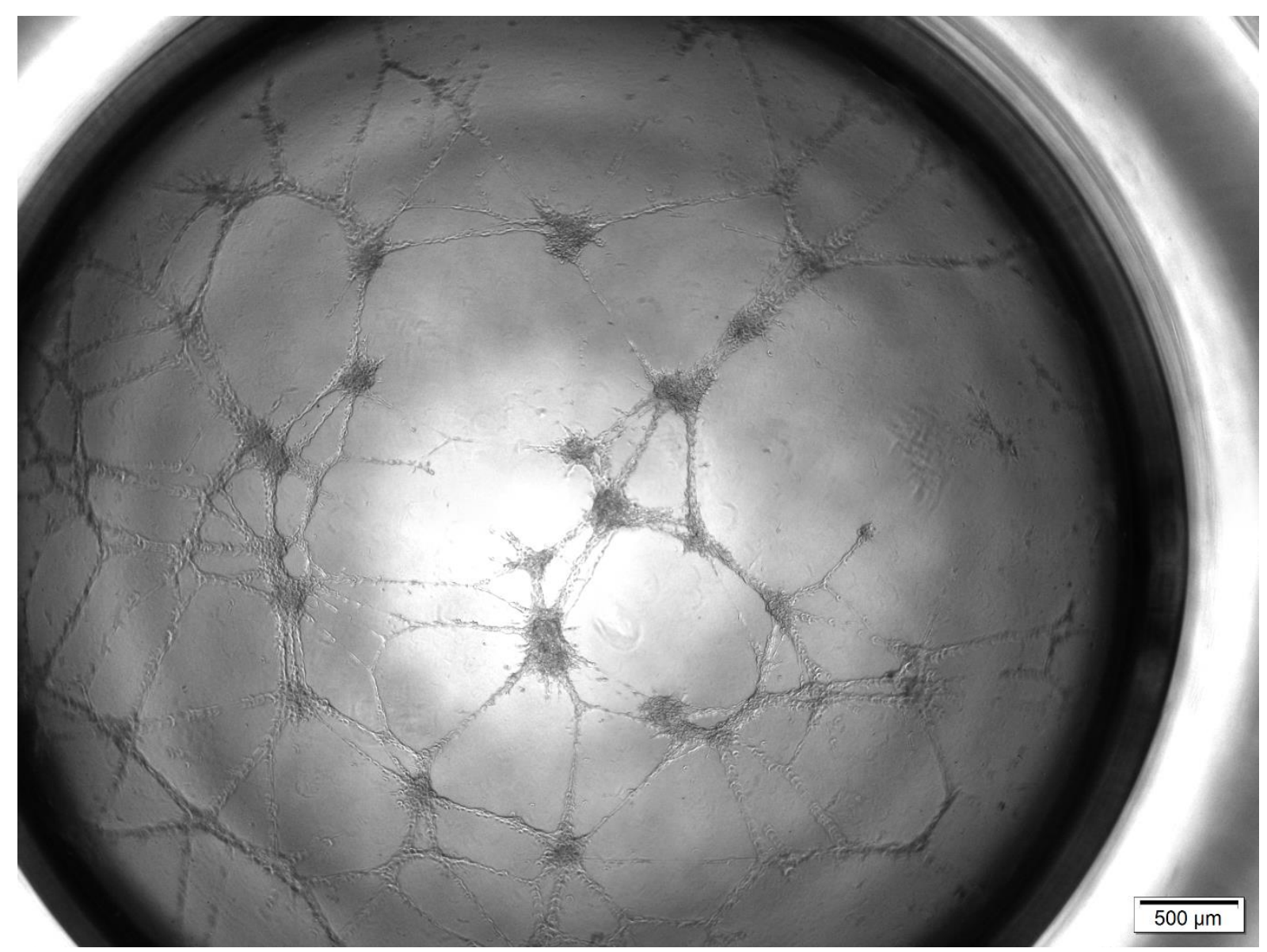

Figure 2.1. A representative image of a Matrigel-based tube formation assay as described by [350] used to assess the vasculogenic activity of tumour cells. U87MG cells were seeded on a Matrigel surface overnight and micrographed the following morning.

\subsubsection{Migration assays}

\subsubsection{Wound healing assay}

The wound healing assay is a migration assay that tests the kinetic movement of cells into a denuded area. A layer of confluent cells was grown on a dish, and a wound made with a pipette tip down the centre of the well. Photos were taken before and after a selected period to determine the migration of cells. The methodology used is a modification of that described by Chan et al. [351] and makes use of a Perspex guide shown below in Figure 2.2. 
A

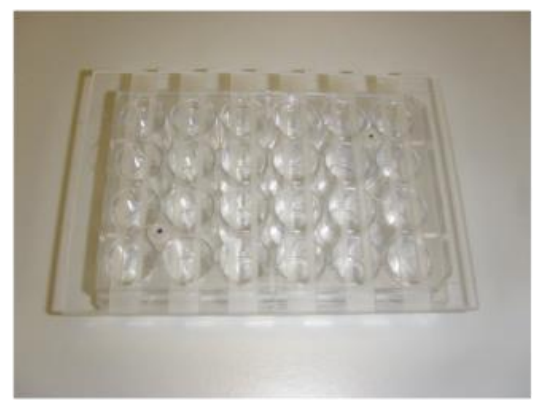

B

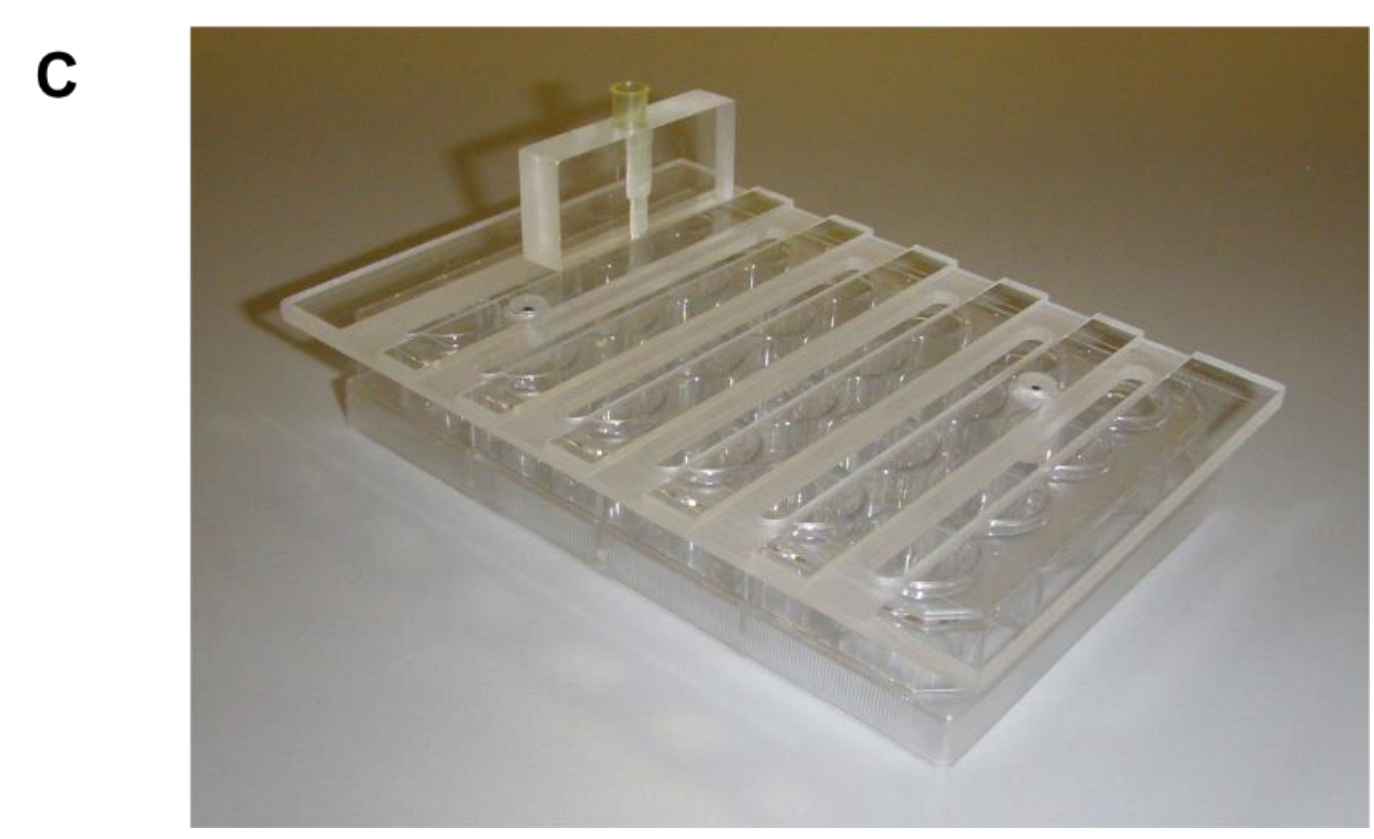

Figure 2.2. Images of the proprietary wound healing assay generating device which was developed by Ariane Chan [352]. A $200 \mu \mathrm{L}$ pipette tip is inserted into the guide pictured in panel B and inserted in the device as seen in panel $\mathrm{C}$; this is then moved up and down to facilitate accurate, consistent wounds. Reproduced under creatives commons attribution (CC BY-NC-ND 3.0 NZ) from the Thesis of Dr Ariane Chan [352].

Before seeding of cells, all equipment was sterilised with 70\% ethanol and treated with UV for $45 \mathrm{~min}$. Four horizontal lines were drawn on the underside of each well at approximate equidistance to each other as markers for micrographs.

Cells were trypsinised as described previously and seeded to approximately $70 \%$ confluency in a 24-well plate. Cells were then grown to a very high confluence of approximately 90 $100 \%$. A wound was placed vertically in the centre of the dish using a $200 \mu \mathrm{L}$ pipette tip placed in the device described above. 
To assess the consistency and accuracy of wounds, four wounds were made and the width measured at three different points on the wound (Figure 2.3). Figure 2.3 shows low intra-wound variation but a higher inter wound variation.
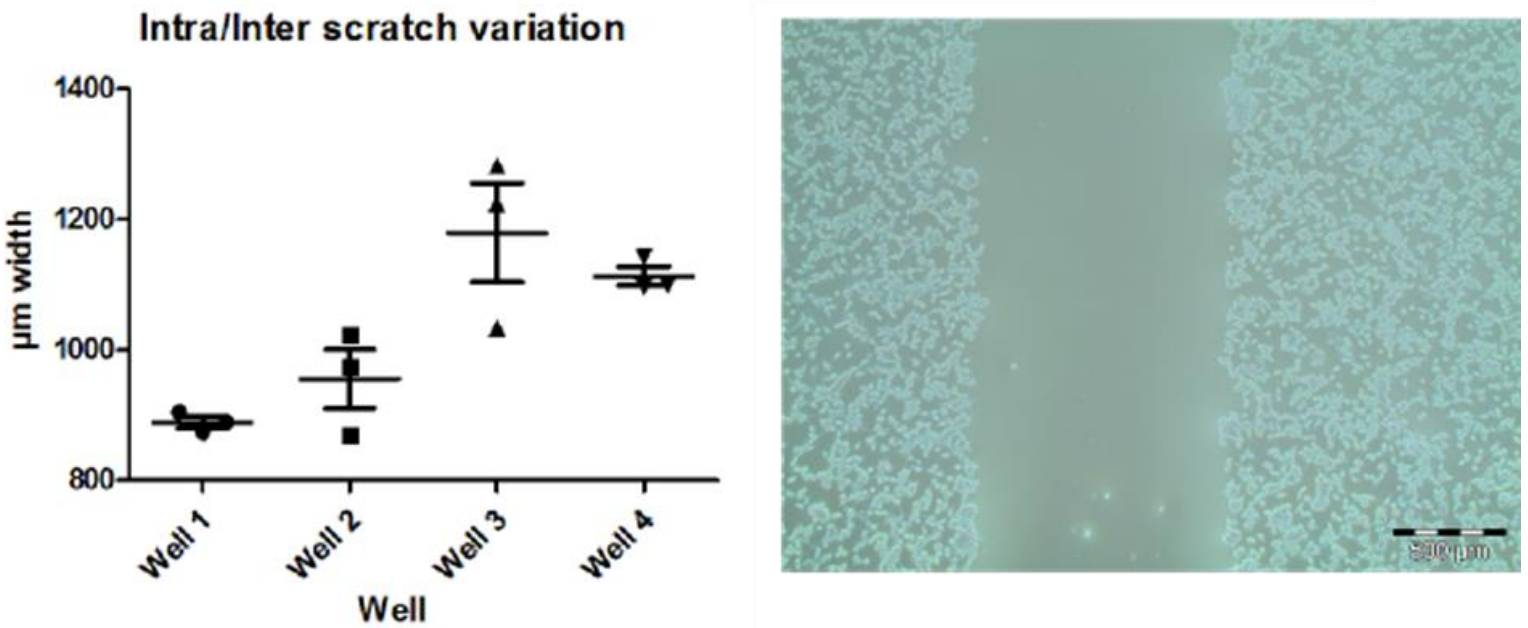

Figure 2.3. The inter and intra wound variation using the proprietary scratch generation device. This figure shows the intra-wound variation and inter scratch variation between 4 separate wounds (left) and a representative image of a cell layer post-scratch (right)

After placement of the wound, cell media was removed and the well washed with $300 \mu \mathrm{L}$ of basal medium. This was then replaced with cell culture medium containing $1 \%$ FBS, $1 \%$ penstrep and the appropriate treatments. Cells were incubated for $16 \mathrm{hrs}$ with photos taken at $0 \mathrm{hrs}$ and $16 \mathrm{hrs}$. To ensure repeatability, photos were taken at the junction where the horizontal lines intersected with the perpendicular wound, and at $16 \mathrm{hrs}$ the photo was aligned with the initial photo.

Photos were analysed using the T Scratch analysis software (Tobias Gebäck and Martin Schulz, ETH Zürich, Switzerland). T Scratch uses an edge detection algorithm that determines the open space between cells automatically, once defined by the programme; a paintbrush style tool was utilised to correct errors due to contrast or presence of debris. The photos were blinded to ensure bias-free measurement, and the $\mathrm{T}$ Scratch programme was allowed to determine the migration front. Following this, the free selection tool was utilised to select the most consistent migration front (single cells were not counted as the migration front). The $\mathrm{T}$ Scratch 
programme was compared against standard measuring methods in the original paper [353] and showed that it was just as accurate and more rapid.

\subsubsection{Transwell migration assay}

The transwell migration assay [354] is a chemotactic migration assay that determines the ability of a cell to pass through a semi-permeable membrane. While more expensive than a wound healing assay, it is more robust and more accurately depicts the chemotactic migratory behaviour of the cells than the chemokinetic movement of the wound healing assay.

BD Falcon 24-well plate transwell inserts (In Vitro Technologies, Auckland, NZ) with $8 \mu \mathrm{m}$ pores were used. Cells were trypsinised and counted in a haemocytometer as previously described. Cells were resuspended to $500,000 / \mathrm{mL}$ in cell culture medium supplemented with $1 \%$ FBS and 1\% penstrep and recounted to ensure accuracy. An aliquot of cells in medium $(100 \mu \mathrm{L})$ was added to each insert, and a further $200 \mu \mathrm{L}$ of cell medium ( $1 \% \mathrm{FBS}, 1 \%$ penstrep) was added. After approximately $5 \mathrm{hrs}, 1 \mathrm{~mL}$ of cell culture medium supplemented with $1 \%$ FBS and $1 \%$ penstrep with the chemoattractant added was placed into the lower chamber. The cultures were incubated for $18 \mathrm{hrs}$. A representative image is shown below along with a schematic of the assay Figure 2.4. 

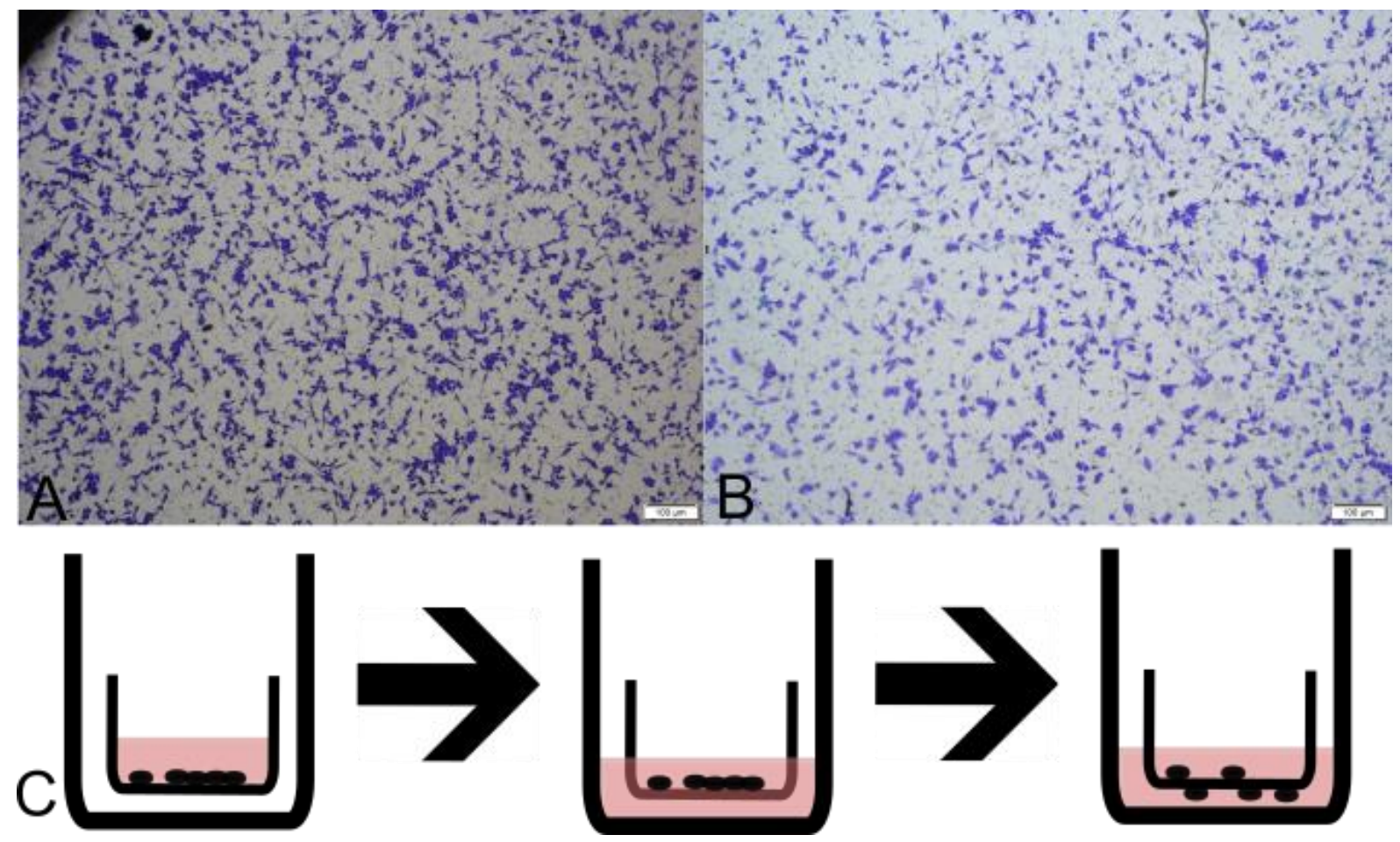

Figure 2.4. Representative images of the transwell assay. Panel A and B are representative images from a transwell assay; images were taken at 4x magnification, the scale bar represents $100 \mu \mathrm{m}$. Panel A is [Pyr ${ }^{1}$ ]apelin-13 treated U87.APLNR cells and panel B is vehicle treated U87.APLNR cells. Panel C is a schematic of the transwell assay, as shown in the schematic a smaller insert is placed inside a large well with cells seeded inside, after this a chemoattractant is added to the outside well stimulating the movement of cells inside to the outside, after which the un-migrated cells are wiped off, and the cells on the underside are visualised with crystal violet and counted.

After the incubation period, un-migrated cells were removed, and the remaining cells were stained with crystal violet and micrographed as follows. Transwell inserts were removed, and excess medium blotted off the membrane using a Kimwipe. The remaining medium and unmigrated cells were removed from the top of the membrane using a cotton bud. Cells were fixed in $70 \%$ ethanol for $10 \mathrm{~min}$ at room temperature. Excess ethanol and any remaining unmigrated cells were removed as prior. The insert was allowed to dry for $10 \mathrm{~min}$, then placed in $0.2 \%$ crystal violet (Sigma Aldrich, Auckland, NZ) for 5 min. Excess stain was removed as above. The insert was repeatedly dipped very gently into a beaker of distilled water to remove the remainder of the excess crystal violet. Following this, the excess water was removed and the insert again allowed to dry. Micrographs were taken of only the migrated side of the transwell insert on an inverted light microscope with the insert in position in the 24-well plate. 


\subsection{Immunohistochemistry and Immunocytochemistry}

\subsubsection{Tissue preparation and embedding}

\subsubsection{Paraffin-embedded tissue sections}

Brains for use in paraffin-embedded tissue experiments were provided from the lab of Dr Ian Hermans (Malaghan Institute of Medical Research, Wellington, New Zealand) from the PhD work of Dr Cameron Field. Brain tissue was from C57BL/6 mice injected with GL261 cells as described fully by Field [355]. Briefly, mice were tumour challenged intracranially with 10,000 to 50,000 GL261 cells and monitored for weight loss of greater than $10 \%$ or significant discomfort, after which mice were sacrificed. Mice were sacrificed and transcardially perfused via the left ventricle with $10 \mathrm{~mL}$ ice-cold $1 \mathrm{x}$ PBS. The brains were fixed in $4 \%$ paraformaldehyde (PFA) (Sigma-Aldrich, Auckland, NZ) for $72 \mathrm{hrs}$ and were transferred to $70 \%$ ethanol for storage prior to use. Macroscopic and microscopic examples of the tissue can be seen below (Figure 2.5) from the thesis of Dr Field. 

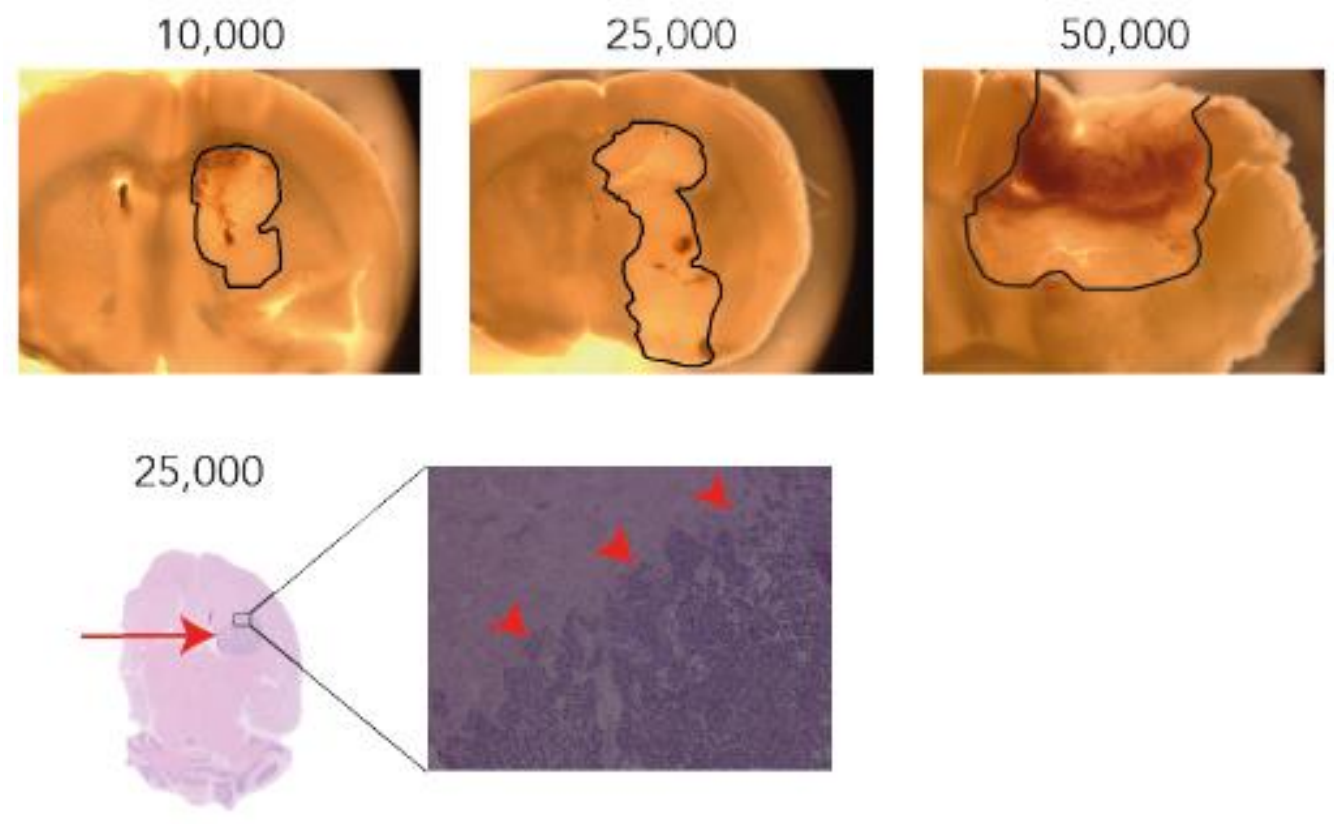

Figure 2.5. Macroscopic images of GL261 burdened C57BL/6 brains. Upper panels show coronal sections of mice brains which were injected with 10,000, 25,000 and 50,000 GL261 cells with tumour mass outlined. Lower images show H\&E sections from brains challenged with 25,000 cells with the tumour mass indicated by red arrows. Reproduced under creatives commons attribution (CC BY-NC-ND 3.0 NZ) from the Thesis of Dr Cameron Field [355].

Whole brains were received from Dr Hermans and before embedding, brains were gross sectioned coronally and transferred to a tissue processing cassette (Shandon, Thermofisher Scientific, Auckland, NZ). Cassettes containing tissue were processed using a Leica TP1020 processor (Leica Biosystems, Wetzlar, Germany) using the tissue processing protocol below (Table 2.4). Tissue was embedded into paraffin wax using a Leica EG1160 Tissue embedding system (Leica Biosystems, Wetzlar, Germany). 5 - $7 \mu \mathrm{M}$ sections were made using an RM2235 Manual Rotary Microtome (Leica Biosystems, Wetzlar, Germany) and dried overnight before use. 
Table 2.4. Leica Tissue Processor TP1020 embedding procedure. The tissue processing protocol for paraffinembedded brain tissue.

\begin{tabular}{|c|c|c|}
\hline Step & Reagent & Incubation time \\
\hline 1 & $70 \%$ ethanol & $60 \mathrm{~min}$ \\
\hline 2 & $70 \%$ ethanol & $60 \mathrm{~min}$ \\
\hline 3 & $95 \%$ ethanol & $60 \mathrm{~min}$ \\
\hline 4 & $95 \%$ ethanol & $60 \mathrm{~min}$ \\
\hline 5 & $100 \%$ ethanol & $60 \mathrm{~min}$ \\
\hline 6 & $100 \%$ ethanol & $60 \mathrm{~min}$ \\
\hline 7 & $100 \%$ ethanol & $60 \mathrm{~min}$ \\
\hline 8 & 50:50 xylene:ethanol & $80 \mathrm{~min}$ \\
\hline 9 & Xylene under vacuum & $45 \mathrm{~min}$ \\
\hline 10 & Xylene under vacuum & $45 \mathrm{~min}$ \\
\hline 11 & Paraffin vacuum & $80 \mathrm{~min}$ \\
\hline 12 & Paraffin vacuum & $80 \mathrm{~min}$ \\
\hline
\end{tabular}

\subsubsection{Fresh frozen embedded tissue sections}

Fresh frozen brain tissue was also used. Healthy brains from C57/BL6 mice were obtained from the lab of Professor Anne La Flamme (Victoria University of Wellington, New Zealand). These were fixed in 4\% PFA in $1 \times$ PBS (pH 7.0) overnight and stored in $70 \%$ ethanol until ready for use. Brains were cryoprotected in 30\% sucrose made up in $1 \times$ PBS with one change at $5 \mathrm{hrs}$ and ended when tissue sank in the tube. Embedding of brains was as follows, $30 \%$ sucrose was blotted off using paper towels, and the brain was incubated for 2 min in TissueTek optimum cutting temperature compound (OCT) (Global science, Auckland) and then transferred to moulds and immersed in fresh OCT. Moulds were placed onto the powdered dry ice until frozen solid and then transfer to a $-80^{\circ} \mathrm{C}$ freezer for storage until sectioning. $10 \mu \mathrm{M}$ sections were made using a Leica CM3050 S Research Cryostat (Leica Biosystems, Wetzlar, Germany).

\subsubsection{Cell growth for immunocytochemistry}

Cells were grown in 24-well plates on $13 \mathrm{~mm}$ diameter glass coverslips. Coverslips were sterilised by immersion in 70\% ethanol overnight followed by exposure to UV light for $1 \mathrm{hrs}$. Cell medium $(100 \mu \mathrm{L})$ containing approximately 5000 cells was pipetted directly onto the 
coverslip and allowed to adhere for 2 hrs. Coverslips were covered in cell line specific culture medium and left $12-16 \mathrm{hrs}$ for fixing the next day.

\subsubsection{Tissue staining procedures}

\subsubsection{Fluorescence immunohistochemistry and $H \& E$ staining of paraffin-embedded tissue sections}

Tissue sections were deparaffinised in xylene ( 2 changes, 3 min each) followed by a stage in xylene-ethanol mix 1:1 (1 change $3 \mathrm{~min}$ ). Slides were rehydrated through a graded ethanol series $(100 \%, 95 \%, 70 \%$ and $50 \%$ each for $3 \mathrm{~min})$ and rinsed in $\mathrm{ddH}_{2} \mathrm{O}$.Following this sections were stained with Mayer's haematoxylin and eosin (H\&E) (Sigma Aldrich, Auckland, NZ) to examine tissue architecture. Slides were treated with haematoxylin for $20 \mathrm{sec}$ and rinsed with $\mathrm{ddH}_{2} \mathrm{O}$ followed by eosin for $5-10 \mathrm{sec}$ and rinsed once more with $\mathrm{ddH}_{2} \mathrm{O}$. The slides were then dehydrated using an ethanol gradient $(70 \%, 90 \%, 1$ change of $5 \mathrm{~min})$ and $100 \%$ ethanol ( 2 changes, $5 \mathrm{~min}$ each) and finally cleared in xylene (1 change, $5 \mathrm{~min}$ ). Slides were mounted in D.P.X (BDS Chemicals Ltd, Poole, United Kingdom).

All other slides underwent fluorescent immunohistochemistry. Antigen retrieval was performed by immersing slides in coplin jars containing boiling $10 \mathrm{mM}$ sodium citrate ( $\mathrm{pH}$ 6.0) for 10 min equilibrated in a pressure cooker with the lid left on loosely. The coplin jar was then removed from the pressure cooker and allowed to cool at room temperature for $40 \mathrm{~min}$. Slides were rinsed with $1 \mathrm{x}$ Tris-buffered saline (TBS) (2 changes, 5 min each). Autofluorescence was quenched using $0.5 \%(\mathrm{w} / \mathrm{v})$ sodium borohydride, made up fresh in $1 \mathrm{x}$ TBS (3 changes, $15 \mathrm{~min}$ each). Slides were rinsed with TBST ( 2 changes, 5 min each). Slides were blocked using 5\% (w/v) bovine serum albumin (BSA) (Sigma Aldrich, Auckland, NZ) dissolved in 1x TBST for $60 \mathrm{~min}$ at room temperature. Cells were washed three times with TBST for 5 min each and incubated in primary antibody diluted in 1\% BSA prepared in 1x TBST, overnight in a darkened, humidified chamber at $4^{\circ} \mathrm{C}$ with constant agitation from a rocker device. Negative controls were used in which the primary antibody was omitted. The following day the coverslips were washed $3 \mathrm{x}$ with TBST for $5 \mathrm{~min}$ each and incubated in the appropriate dilution of secondary antibody conjugated to a fluorophore. The secondary antibody was diluted in $1 \%$ 
BSA in TBST for $120 \mathrm{~min}$ at room temperature in a darkened chamber. The coverslips were washed in TBST $3 \mathrm{x}$ and mounted in ProLong Gold Antifade (Invitrogen, Thermofisher Scientific, Auckland, NZ), containing cell nuclei counter stain 4', 6-diamidino-2-phenylindole (DAPI) (Invitrogen, Thermofisher Scientific, Auckland, NZ).

\subsubsection{Fluorescence immunohistochemistry fresh frozen embedded tissue sections}

Slides were left to warm to room temperature before use. Slides were fixed with acetone (Thermofisher Scientific, Auckland) for $15 \mathrm{~min}$ and rinsed with 1 x TBS ( 3 changes, $5 \mathrm{~min}$ ). Slides were blocked using 5\% (w/v) BSA dissolved in $1 \times$ TBST with an addition 0.3 M glycine to reduce background fluorescence, for $120 \mathrm{~min}$ at room temperature. Fresh frozen tissue was stained with antibodies as per section 2.4.2.1.

\subsubsection{Fluorescence immunocytochemistry staining procedures}

Coverslips with adhered cells were fixed with ice-cold methanol: acetone 1:1 (v/v) for $1 \mathrm{~min}$ and washed $2 \mathrm{x}$ in TBST (TBS with $0.1 \%$ Tween-20) for 5 min each. If endogenous GFP expression was required cells were fixed in 4\% PFA made up in 1 x PBS (pH 7.0). If methanol-acetone fixation was performed, permeabilisation was not performed otherwise, cells were permeabilised with $0.5 \%(\mathrm{w} / \mathrm{v})$ saponin (Sigma Aldrich, Auckland, NZ) made up in $1 \mathrm{x}$ TBS. Saponin interacts with membrane cholesterol, selectively removing it and permeabilising the membrane without stripping it totally, as is preferable for examining membrane-bound proteins. Non-specific antibody binding sites were blocked with 5\% BSA prepared in 1x TBST for $60 \mathrm{~min}$ at room temperature. Cells were stained with antibodies as per section 2.4.2.1 above. The concentrations of primary and secondary antibodies are presented in Table 2.5 and Table 2.6 respectively. 
Table 2.5. Primary antibodies used in this study, their dilutions and sources. IHC $=$ Immunohistochemistry, ICC $=$ Immunocytochemistry and $\mathrm{WB}=$ Western blotting

\begin{tabular}{ccccc}
\hline Marker & Ab raised in & Antibody Dilution & Source of Antibody & Usage \\
\hline pERK & Rabbit & $1: 500$ & $\begin{array}{c}\text { Cell Signaling } \\
\# 9101\end{array}$ & WB \\
APLN & Rabbit & $1: 200$ & Abcam (ab59469) & ICC \\
APLNR & Rabbit & $1: 500(\mathrm{ICC} / \mathrm{IHC})$, & Abcam (ab84296) & ICC, IHC and WB \\
APLNR & Rabbit & $1: 1000(\mathrm{WB})$ & WB \\
ERK & Rabbit & $1: 200(\mathrm{WB})$ & Abcam (ab214369) & WB \\
$\begin{array}{c}\alpha \text {-tubulin } \\
\text { R-smooth muscle }\end{array}$ & Rabbit & Cell Signaling & (\#9102) \\
actin & Mouse & $1: 5000$ & Abcam (ab18251) & WB \\
CD34 & Rabbit & $1: 200$ & Abcam & (ab7817) \\
\end{tabular}

Table 2.6. Secondary antibodies used in this study, their dilutions and sources.

\begin{tabular}{cccc}
\hline Secondary Antibody & Antibody Dilution & $\begin{array}{c}\text { Source of } \\
\text { Antibody }\end{array}$ & Usage \\
\hline Alexa Fluor 488 anti-rabbit IgG & $1: 500$ (ICC), 1:1000 & $\begin{array}{c}\text { Life Technologies } \\
\text { (AB) }\end{array}$ & $\begin{array}{c}\text { IHC/ICC and WB } \\
\text { Life Technologies } \\
\text { (AHC (IH samples) }\end{array}$ \\
Alexa Fluor 555 anti-rabbit IgG & $1: 1000$ (IHC) & $\begin{array}{c}\text { Life Technologies } \\
\text { (A21245) }\end{array}$ & IHC \\
Alexa Fluor 647 anti-rabbit IgG & $1: 1000(\mathrm{IHC})$ & $\begin{array}{c}\text { Abcam } \\
\text { (ab150113) }\end{array}$ & IHC (IH samples) \\
\hline
\end{tabular}




\subsection{Chemically competent DH5a E. Coli cultivation and preparation}

Chemically competent $E$. coli were made using the TSS protocol (see below) described by Chung et al. [356]. DH5 $\alpha$ cells were plated onto Luria Broth (LB) agar plates and cultured overnight at $37^{\circ} \mathrm{C}$. A single colony was selected and inoculated into $10 \mathrm{~mL}$ of LB medium and left overnight at $37^{\circ} \mathrm{C}$ in a shaking incubator at $200 \mathrm{rpm}$. The next morning, $50 \mathrm{~mL}$ of LB was inoculated with $500 \mu \mathrm{L}$ of overnight culture and grown to an $\mathrm{OD}_{600}$ of approximately 0.4 . The culture was then split into two $50 \mathrm{~mL}$ falcon tubes and chilled on ice for $10 \mathrm{~min}$ to stop growth. Cells were then centrifuged at $1000 \mathrm{RCF}$ for $10 \mathrm{~min}$ at $4^{\circ} \mathrm{C}$. The medium was gently and wholly decanted, and the pellet resuspended in 1/10th the volume of TSS buffer (LB broth supplemented with 10\% (w/v) PEG 8000, 5\% (v/v) DMSO, $50 \mathrm{mM} \mathrm{MgCl}_{2}$, pH 6.5) and gently triturated to resuspended cells. Cells in TSS were directly pipetted into microcentrifuge tubes chilled at $-80^{\circ} \mathrm{C}$ rapidly in a steel tube block.

\subsection{Transformation procedure}

The transformation protocol used was a modification of the protocol of Chung et al. [356]. Up to $10 \mathrm{ng}$ of DNA was added to $100 \mu \mathrm{L}$ of competent DH5 $\alpha$ cells and the DNA mixed with gentle tapping. The mixture was incubated on ice for $30 \mathrm{~min}$ to $1 \mathrm{hr}$. The cells were briefly heat shocked in a water bath at $42^{\circ} \mathrm{C}$ for $50 \mathrm{sec}$, then chilled on ice for $2 \mathrm{~min} .0 .9 \mathrm{~mL}$ of Super Optimal broth with catabolite repression (SOC) medium (Recipe in Appendix-B.1.5) was added, and the cells were incubated at $37^{\circ} \mathrm{C}$ with shaking at $200 \mathrm{rpm}$ for $1 \mathrm{hrs}$ before plating onto LB plates with the appropriate plasmid antibiotic overnight at $37^{\circ} \mathrm{C}$. Transformation efficiency (TE) was checked by transforming a control plasmid and calculated from the following equation.

$$
\frac{\text { Colonies }}{\mu \mathrm{g}}=\frac{\# \text { colonies } * 1000 \mathrm{ng}}{\text { concentration of DNA }\left(\frac{n g}{\mu \mathrm{L}}\right) \text { in tranformation } * \text { volume plated in } \mu \mathrm{L}}
$$




\subsection{Plasmid verification and preparation}

A single colony was isolated and cultured overnight in $10 \mathrm{~mL}$ of LB Broth. The next day a further $1 \mathrm{~mL}$ of this was added to $100 \mathrm{~mL}$ of $\mathrm{LB}$ for another overnight culture, and the remainder was used for verification using restriction digest as described below. The overnight culture was used the following day for miniprepping as per the method described in appendix (Appendix B.1.9).

\subsubsection{Restriction digest fragment analysis}

Plasmid identity was checked using restriction digest fragment analysis. Restriction enzymes were purchased from NEB (Thermofisher Scientific, Auckland, NZ) and used as per the manufacturer's instructions. DNA fragments were then analysed on a $1 \%$ agarose gel as described above.

\subsubsection{Restriction digest fragment analysis of APLNR plasmid}

APLNR plasmid was purchased from Origene (Australia) product number RG207576 and was prepared and purified by a commercial alkaline lysis plasmid purification kit (Pure link, Life Technologies) with a resin column to remove endotoxins before transfection. Full sequences were available on the Origene website. The basic structure is described below (Figure 2.6) and shows an uncut plasmid, a single cut Xho1 (New England Biolabs, MA, USA) plasmid and a double cut XhoI and EcoRI (New England Biolabs, MA, USA) digest yielding the appropriate size insert. 


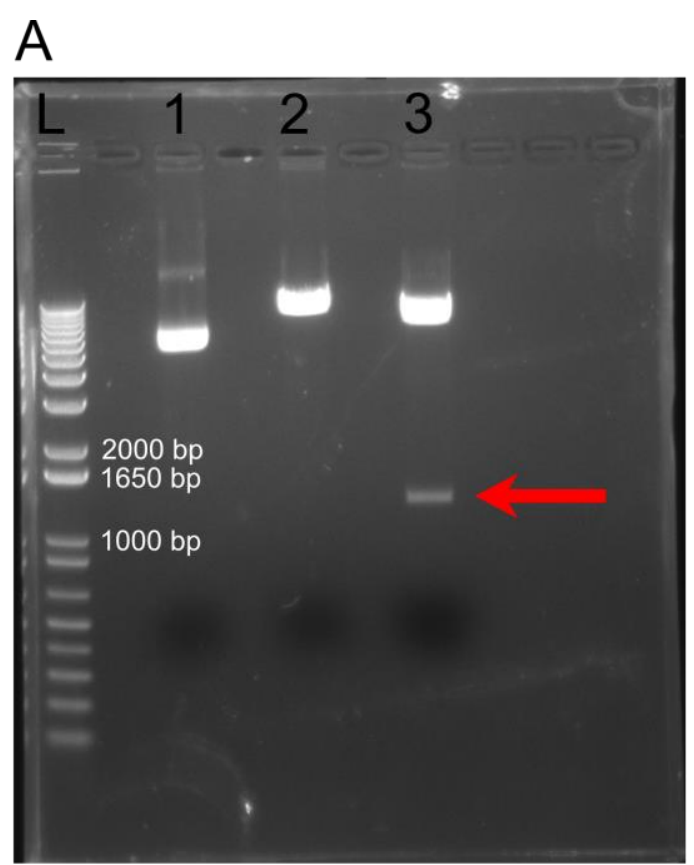

B

C schematic of the multiple cloning sites:

pCMV6-AC-GFP

ECORI BamHIKpnI RBS

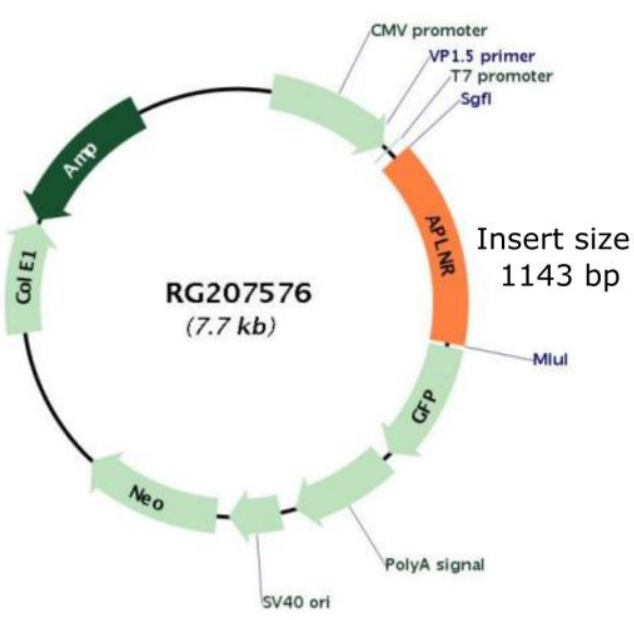

Cloning scheme for RG207576:

$\begin{array}{lll}\text { ECORI BamHIKpnI RBS } & \begin{array}{c}\text { Kozac } \\ \text { Consensus }\end{array} \\ \text { Ascl } & \text { Sgfl }\end{array}$

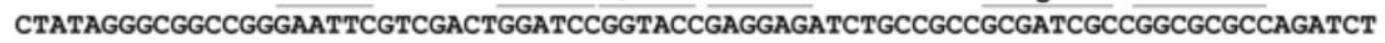

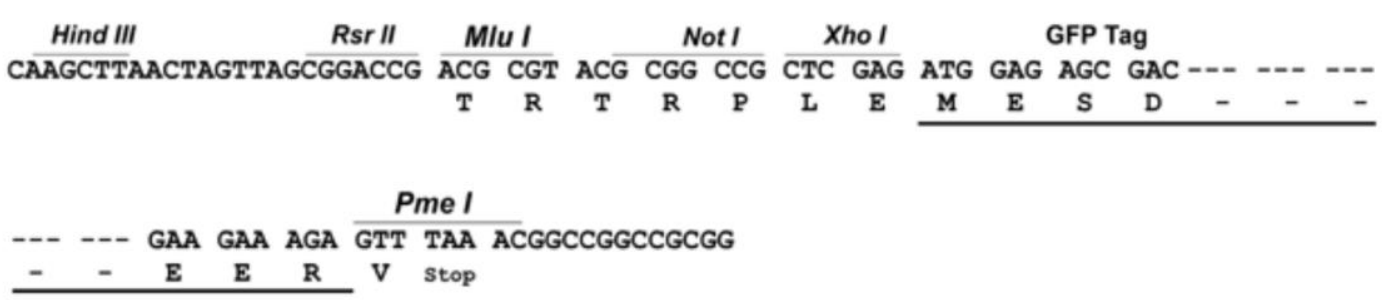

Figure 2.6. Details and verification of the APLNR plasmid. Panel A shows a restriction digest of the APLNR plasmid. L refers to the $1 \mathrm{~kb}$ plus ladder (Invitrogen, Life technologies); lane 1 shows uncut plasmid; lane 2 is a single cut of APLNR with EcoR1; lane 3 shows APLNR plasmid double digested with Xho1 and EcoR1 with the appropriate excised insert size of $1205 \mathrm{bp}$ indicated with a red arrow. Panel B shows a plasmid map of APLNR plasmid. Panel C shows the multiple cloning site of the empty pCMV6-AC-GFP vector. The APLNR region (1143 bp) is cloned in between the Sgf 1 and Mlu 1 sites and the restriction digest cut sites EcoR1, and Xho 1 can be seen. Panels B and C were reproduced with permission from OriGene Technologies, Inc. (Origene.com, Cat\# RG207576). 


\subsection{Flow Cytometry}

\subsubsection{Flow cytometry preparation}

For flow cytometry, cell medium was removed and the cells detached by trypsin as previously described in Section 2.1. The cells were collected by centrifugation at $300 \mathrm{RCF}$ for $5 \mathrm{~min}$. The medium was gently decanted and the cells resuspended in $300 \mu \mathrm{L}$ of fresh FACS buffer $(2 \%$ FBS, $1 \times$ PBS). The tubes were centrifuged at 300 RCF for 5 min and the buffer decanted off. The tubes were allowed to drain on a paper towel in a laminar flow hood. Finally, $300 \mu \mathrm{L}$ of FACS buffer was added, and the cells kept on ice for analysis. If required, $20 \mu \mathrm{L}$ of $20 \mu \mathrm{g} / \mathrm{mL}$ propidium iodide (PI) was added. Cells were analysed on a FACS Canto II flow cytometer (BD Biosciences, USA) and analysed using FLOWJO 7.6.1 software (Tree Star, Ashland, OR, USA) or Flowing software 2.5.1 (Terho Turku Centre from Biotechnology, Turkey)

\subsection{Western Blotting}

\subsubsection{Preparation of cell lysate for western blot}

Cells were seeded at approximately $60 \%$ confluence and allowed to grow until $80 \%$ confluence was achieved to ensure healthy, log-phase cells. After treatment, the medium was removed, and the cells were rinsed with $0.3-0.5 \mathrm{~mL}$ of sterile $1 \mathrm{x}$ PBS. The cells were then treated with $0.08 \mathrm{~mL}$ to $0.3 \mathrm{~mL}$ radio-immunoprecipitation assay (RIPA) buffer (Appendix B.1.18) supplemented with $10 \mu \mathrm{L} / \mathrm{mL}$ protease inhibitor (P8340, Sigma) and $10 \mu \mathrm{L} / \mathrm{mL}$ phosphatase inhibitor (P5726, Sigma Aldrich, Auckland). The cells were then incubated on a shaker table for $30 \mathrm{~min}$ at $4^{\circ} \mathrm{C}$. Cells were further homogenised by trituration of the cell suspension and scraping using the pipette tip, and the cell suspension was then transferred to a microcentrifuge tube and centrifuged for $30 \mathrm{~min}$ at $14,000 \mathrm{rpm}$ at $4^{\circ} \mathrm{C}$. The DNA pellet was removed and discarded, or the supernatant was removed in small $50 \mu \mathrm{L}$ aliquots and placed into a fresh microcentrifuge tube. 


\subsubsection{Protein quantification}

Protein was quantified using a Pierce bicinchoninic acid (BCA) assay kit (Thermofisher Scientific, Auckland) Performed as per manufacturers protocol and scanned at $570 \mathrm{~nm}$ on a plate reader (Versamax, Molecular Devices, Sunnyvale, CA).

\subsubsection{Sodium dodecyl sulphate-polyacrylamide gel electrophoresis (SDS PAGE)}

SDS-PAGE was performed using a BioRad Miniprotean system, (BioRad, CA, USA). A $10 \%$ resolving gel (B.1.21) with a 4\% stacking gel (B.1.22) was used to separate proteins. Protein sample $(20 \mu \mathrm{g})$ was added to a microcentrifuge tube, and the total volume made up to $20 \mu \mathrm{L}$ with RIPA buffer. To each microcentrifuge tube, $5 \mu \mathrm{L}$ of $5 \mathrm{x}$ reducing buffer (Appendix 8.1.15) containing freshly added 2-mercaptoethanol (Sigma Aldrich, Auckland, NZ) at a 1:10 ratio). The 10-20 $\mu \mathrm{g}$ of cell protein lysate was loaded into each well and run alongside $1.0 \mu \mathrm{L}$ Precision Plus Protein TM Standards Dual colour molecular weight marker (BioRad, CA, USA) for molecular weight comparison. Gel electrophoresis was carried out at $120 \mathrm{~V}$ for approximately $1-1.5 \mathrm{hrs}$ at room temperature in $1 \mathrm{x}$ running buffer (Appendix B.1.17)

Proteins were then transferred to a polyvinylidene difluoride (PVDF) (Immobilon-FL, 0.22 $\mu \mathrm{m}$ pore size, Millipore, USA) membrane for immunological probing using a BioRad tetracell set. The transfer of proteins from gel to membrane was carried out in a $4^{\circ} \mathrm{C}$ cold room at 20 volts for $17 \mathrm{hrs}$ in Western transfer buffer (Appendix B.1.16). After transfer, the original SDS-PAGE gel was stained with Coomassie brilliant blue (Sigma Aldrich, Auckland, NZ) to estimate the amount of non-transferred protein. 


\subsubsection{Immunostaining of western transferred proteins}

Following transfer, the PVDF membrane was placed in 5\% (w/v) BSA (Sigma) made up in 1 $\mathrm{x}$ TBST to block non-specific binding sites for $1-2 \mathrm{hrs}$ at $4^{\circ} \mathrm{C}$ on a rocking platform. Primary antibody diluted in $1 \%$ BSA in TBST was added and the membrane incubated overnight with shaking of $150 \mathrm{rpm}$ at $4^{\circ} \mathrm{C}$.

The primary antibody was then washed from the membrane with 1 x TBST with three washes for 5 min on a shaking platform. The membrane was then incubated with secondary antibody at the appropriate dilution in $1 \times$ TBST in a light-proof bag for $2 \mathrm{hrs}$ at room temperature on a rocking platform. After incubation with the secondary antibody, the membrane was washed three times for $5 \mathrm{~min}$ on a rocking platform with $1 \mathrm{x}$ TBST and the immunostained bands visualised using a membrane scanner (FUJIFILM FLA 5100; Tokyo, Japan). Densitometry analyses were performed using ImageJ (Rasband, W.S., ImageJ, U. S. National Institutes of Health, Bethesda, Maryland, USA, (http://imagej.nih.gov/ij/, 19972015.). All antibodies used for western blots are described in Table 2.5 and Table 2.6. 


\section{Chapter 3 The expression of the apelinergic system in glioblastoma}

\subsection{Introduction}

Since its discovery, the number of studies reporting the role of the apelinergic system in cancer pathophysiology have increased, but there have been few reports of its role in glioblastoma. This is surprising considering the apelinergic system has been reported to be overexpressed in glioblastoma compared to normal brain tissue [2]. In addition, it has also been implicated in physiological processes that contribute to glioblastoma pathogenesis like angiogenesis [357, 358], glucose metabolism [13] and it is regulated by hypoxia [3] which is a critical modulator of glioblastoma [206, 221].

Identifying the expression of genes at a mRNA or protein level is a critical step in understanding the contribution of the gene to the cancer pathophysiology. The components of the apelinergic system have been reported to be upregulated in several cancers. This includes APLN mRNA and APLNR protein in colon adenocarcinomas [87], APLN mRNA and protein in non-small cell lung cancers [1] and APLN protein in hepatocellular carcinomas [95]. The apelinergic system has also been reported as a biomarker in several cancers. For example, increased $A P L N$ mRNA expression has been associated with poor prognosis in prostate cancer [88] and APLN protein levels correlated with poor outcome in both oral squamous cell carcinoma [6] and gastric cancer [7]. The current body of literature seems to suggest that the apelinergic system has a role in cancers, but its expression and role in glioblastoma is unclear. 
Elucidating the expression and localisation of $A P L N$ and $A P L N R$ mRNA in glioblastoma is essential. Furthermore, the expression and role of the apelinergic system in lower grade CNS tumours is also unknown. APLN and APLNR mRNA expression were first detected using fluorescence in situ hybridisation (FISH) in human glioblastoma in 2007 [2]. The authors reported that $A P L N$ and $A P L N R$ expression was elevated in microvasculature proliferations (MVP) and pseudopalisading cells around necrosis (PAN) but not in normal brain tissue [2]. Since this study, there have been few papers investigating the expression or the role of the apelinergic system in glioblastoma. As such, it was deemed necessary in this to investigate the mRNA expression of $A P L N$ and $A P L N R$ in human glioblastoma compared to normal brain tissue using reverse transcription quantitative PCR (RT-qPCR).

The mRNA expression $A P L N R$ and $A P L N$ using RT-qPCR has not been investigated in glioblastoma tissue before. As such, solute carrier family 2 member 1 (SLC2A1) was used as a comparative gene because of its similarity in function and regulation with $A P L N$ expression, and it is expressed in glioblastoma $[359,360]$. SLC2A1 has been found to localise to endothelial cells of the brain and tumour cells [361-363]. SLC2A1 is also involved in glucose uptake [364] and modulated by hypoxia [365]. These functions overlap with APLN which has also been reported to modulate glucose homeostasis [15], is modulated by hypoxia [10] and also expressed by endothelial cells [3]. It has also been suggested that $S L C 2 A 1$ can be used as a measure of hypoxia in glioma due to $S L C 2 A 1$ modulation by HIF1 $\alpha$ [366]. This suggests $S L C 2 A 1$ is an appropriate gene for comparison to the APLN and APLNR.

During the inception of this study, it was recognised that the pathological features (MVP and PAN) where Kalin et al. [2] reported APLN and APLNR expression are far less prevalent in low-grade tumours. This lead to the question, what is the expression of APLN and APLNR in glioblastoma and compared to other tumours of the same category as glioblastoma. The WHO CNS tumour classification categorises glioblastoma as part of the "diffuse astrocytic and oligodendroglial" category of CNS tumours. In addition to the categorisation of tumours by name, a numerical grading scheme is also applied which indicates the degree of malignancy of the tumour. Grade I and II tumours are characterised by a slow and less aggressive phenotype while Grade III and IV were far more malignant and aggressive. The grade IV tumour is referred to as glioblastoma [107]. Due to the rarity of human CNS tumour tissue, it was decided 
public RNAseq data would be used to investigate the mRNA expression of APLNR and APLN in several astrocytic and oligodendroglial tumours to ascertain if there is any correlation between $A P L N$ or $A P L N R$ mRNA expression and tumour classification. A better understanding of $A P L N$ and APLNR mRNA expression in a range of CNS tumours including different glioblastoma subtypes may lead to a better understanding of the disease and potentially direct future research which may result in better treatment paradigms [367].

Cancer-derived in vitro cell lines are often used as models but have been reported not to replicate mRNA expression of salient genes seen in the tumour [368, 369]. Due to the prohibitive cost of in vivo models and lack of primary tissue, in vitro cell lines were intended to be used during this study. Equivalent expression of $A P L N R$ in cell lines compared to glioblastoma tissue is critical for modelling the role of the apelinergic system in glioblastoma. If $A P L N R$ expression is low, this could lead to no response when modulating the apelinergic system with exogenous APLN peptide or APLNR antagonists. As such, the expression of $A P L N$ and $A P L N R$ mRNA were also assessed in several primary glioblastoma-derived cell lines and established immortalized cell lines. Finally, APLNR levels were also assessed using immunochemistry in glioblastoma-derived cell lines and in recapitulated glioblastoma tumour tissue to provide further insight into which cell lines express APLNR for use as a model. 


\subsubsection{Summary of chapter aims}

In summary, the apelinergic system has been implicated in the pathogenesis of several cancers and has been reported to be overexpressed in glioblastoma. Thus, it is plausible it may have a role in glioblastoma pathophysiology. The overall aim of Chapter 3 is to determine the potential role of the apelinergic system in glioblastoma through assessing expression of APLN and APLNR in glioblastoma.

1. In order to determine if $A P L N$ and $A P L N R$ mRNA expression were upregulated in glioblastoma, the expression of $A P L N$ and $A P L N R$ was measured in glioblastoma tissue samples and compared to normal brain tissue. The results were confirmed using public RNAseq data.

2. Further, RNAseq public datasets were used to assess the expression of $A P L N$ and $A P L N R$ in different types of CNS tumours to assess if the expression was correlated with grade or classification of the tumour.

3. APLN and APLNR expression was also analysed in primary derived cell lines 0906 , 0814 and 1003, as well as the established immortalised glioblastoma, derived cell lines U87MG, LN18 and GL261

4. APLNR expression examined in the primary derived cell line 0906 and the immortalised line U87MG as well as in the recapitulated glioblastoma tumour model GL261. 


\subsection{Methods and Materials}

\subsubsection{Reverse transcription Quantitative Polymerase Chain Reaction}

Messenger RNA (mRNA) expression was assessed in this chapter using reverse transcription Quantitative Polymerase Chain Reaction (RT-qPCR). Isolation of RNA and subsequent reactions were performed as described in Chapter 2 Section 2.2.

\subsubsection{Samples}

The mRNA expression of $A P L N$ and $A P L N R$ was measured in 12 glioblastoma samples derived from patients. Glioblastoma samples were provided as total RNA by Professor Ian Hermans at Malaghan Institute of Medical Research (Wellington, New Zealand). Human brain total RNA was commercially obtained (Cat\#: AM7962, Thermofisher Scientific, Auckland, NZ).

The mRNA expression of $A P L N$ and $A P L N R$ RNA was also measured in mRNA isolated from immortalised glioblastoma lines (U87MG and LN18) provided by Dr Melanie McConnell at Victoria University School of Biological Sciences and Professor Ian Hermans at Malaghan Institute of Medical Research (GL261). Primary glioblastoma tumour-derived cell lines were also utilised in this study (kindly donated by Dr Melanie McConnell). Three primary lines, namely 0906, 0814 and 1003, were used in this thesis and are previously described [322, 333335]. 


\subsubsection{Analysis}

Gene expression was analysed using the $2^{-\Delta \mathrm{Ct}}$ method as described in the literature [345]. Briefly, this method is commonly used for gene expression profiling, i.e. heat maps and is similar to the $2^{-\Delta \Delta \mathrm{Ct}}$ method but allows the presentation of normalized individual data points. Briefly, to compare between different glioblastoma tissues the delta cycle threshold $(\Delta \mathrm{CT})$ was computed by the following, $\Delta \mathrm{CT}=\mathrm{CT}$ target gene - CT housekeeping gene. The fold expression of the mRNA of interest was then calculated using $2^{-\Delta \mathrm{CT}}$ after which the values were expressed on a $\log _{10}$ scale. The housekeeping gene used was Hypoxanthine-Guanine Phosphoribosyltransferase 1 (HPRT1).

\subsubsection{Verification of APLNR antibodies binding specificity by western blotting}

The expression of APLNR was investigated at a protein level during this study using anti APLNR antibodies. To verify antibody specificity to the APLNR receptor, two anti-APLNR antibodies from Abcam were tested using western blotting. The first antibody used was a rabbit polyclonal anti-APLNR (catalogue number: ab84296) raised against the $\mathrm{C}$ terminus of APLNR (hereafter APLNR-Cterm) which has a strong publication history [200, 234, 370]. This antibody was compared against another rabbit polyclonal anti-APLNR (catalogue number: ab214369) raised against the $N$ terminus of APLNR (hereafter APLNR-Nterm) which has been published but not clearly verified $[102,371]$. Anti-APLNR antibodies are predicted to generate a band of $\sim 42 \mathrm{kDa}$, but neither antibodies do. The salient details of each antibody are summarised below (Table 3.1). 
Table 3.1. Summary of antibodies used in this study to detect APLNR. All data was retrieved from respective antibody datasheets and summarised.

\begin{tabular}{llll}
\hline Antibody name & $\begin{array}{l}\text { Manufacturers observed band } \\
\text { size (additional bands) }\end{array}$ & $\begin{array}{l}\text { Manufacturer recommended positive } \\
\text { control tissue }\end{array}$ & Catalogue \# \\
\hline APLNR-Cterm & $58 \mathrm{kDa}(110 \mathrm{kDa})$ & $\begin{array}{l}\text { U87MG (Human glioblastoma } \\
\text { astrocytoma), HeLa (Human epithelial } \\
\text { carcinoma cell line) }\end{array}$ & $\begin{array}{l}\text { Abcam } \\
\text { ab84296 }\end{array}$ \\
APLNR-Nterm & $\sim 50 \mathrm{kDa}$ & Mouse heart lysate & $\begin{array}{l}\text { Abcam } \\
\text { ab214369 }\end{array}$ \\
\hline
\end{tabular}

\subsubsection{Immunohistochemistry}

Immunohistochemistry (IHC) was performed on several tissues described below. All methodology pertaining to IHC can be found in Chapter 2, Section 2.4. Immunocytochemistry was performed as described in Chapter 2, Section 2.4.1.3 using the 0906 and U87MG cell lines described above. All APLNR staining was performed using the APLNR-Cterm antibody previously described.

\subsubsection{Infantile haemangioma positive control tissue}

APLNR is well reported to associate with microvasculature, specifically vascular endothelial and smooth muscle cells [358, 372-375]. Infantile haemangioma (IH) is a benign tumour of the microvasculature [376] that was used as a positive control for APLNR staining. IH samples were used to correlate APLNR staining with established vascular endothelial cell marker CD34 [376-378] and the vascular pericytes marker alpha-smooth muscle actin ( $\alpha$-SMA)[379]. The details of IH tissue resection and isolation is described elsewhere [380]. The CD34 and SLC2A1 antibodies have been verified prior, and both $S L C 2 A 1$ and CD34 were shown to be expressed in the endothelium of microvessels in haemangioma [381]. By looking for co-localisation of APLNR to CD34 and $\alpha$-SMA staining this can verify accurate APLNR antibody binding specificity. This staining was performed in conjunction with Dr Ryan Steel. 


\subsubsection{Orthotopic glioblastoma tissue}

A section of primary human glioblastoma tissue grown as an orthotopic xenograft in a SCID/NOD mouse was provided on a slide by Dr Martin Hunn of the Malaghan Institute for Medical research. The slide was stained using standard immunohistochemical techniques described in Chapter 2 Section 2.4 using the APLNR antibody and counterstained with DAPI.

\subsubsection{GL261 tissue}

Immunohistochemistry (IHC) was performed on C57BL/6 brain tissue with recapitulated GL261 tumours provided by Professor Ian Hermans of the Malaghan Institute for Medical Research. A full description of these GL261 tumour samples is given in Chapter 2, Section 2.4.1.1 Control C57BL/6 brains were provided from the lab of Prof Anne La Flamme of Victoria University of Wellington.

\subsubsection{Expression analysis of the cancer genome atlas datasets}

In silico analysis of public glioblastoma data derived from the cancer genome atlas (TCGA) was performed using the online data tool GlioVis (URL: http://gliovis.bioinfo.cnio.es/) [382]. The GlioVis datasets TCGA_GBM [133, 367] and TCGA_LGG-GBM (a combination of the TCGA_GBM and the TCGA_LGG [135] datasets) were used. The TCGA data sets have all been processed under similar means and compared prior [383].

The TCGA project utilised microarray as well as RNA sequencing (RNAseq) to assess gene expression. During this study, RNAseq generated data was focused on and stratified for adult glioblastoma. While there is an opportunity to interrogate data generated from microarray platforms this was not done because poor agreement has been shown between microarrays and RNAseq datasets [384]. This does not exclude the validity of data generated by microarray methods; merely, microarray data was not used to prevent confounding results which may merely occur due to technical differences. Furthermore, the TCGA_LGG dataset was only examined using RNAseq, so as to ensure comparability with TCGA_GBM data, only RNAseq data could be used. Finally, RNAseq data was selected because these datasets represent the 
most abundant source of information including data on the different glioblastoma subtypes sequenced.

\subsubsection{Expression analysis of $A P L N$ and $A P L N R$ mRNA in intratumoral regions of glioblastoma}

Public data from the Ivy Glioblastoma Atlas Project (Ivy GAP) was used to analyse the intratumoral expression of $A P L N$ and $A P L N R$. The Ivy GAP dataset [385] uses a combination of histochemistry, laser capture microdissection and RNA sequencing to allow interrogation of gene expression across different intratumoral regions of glioblastoma.

Complete documentation of the generation of the Ivy GAP data including specific details regarding the identification and isolation of brain regions set may be found: (URL: http:/help.brain-map.org/display/glioblastoma/Documentation). Briefly, samples from 10 independent human glioblastoma samples were analysed using haematoxylin \& eosin staining to identify five intratumoral regions (Reviewed in Table 3.2). Each intra-tumoural region has differing ratios of tumour to normal cells allowing comparisons about the expression of $A P L N$ in $A P L N R$ in regions of mostly normal cells and tumour cell dense regions. The regions were then excised using laser capture microdissection and the mRNA isolated and sequenced. Samples of glioblastoma encompassed the four previously described Verhaak subtypes (proneural, neural, mesenchymal and classical) [134].

The aforementioned data portal GlioVis (http://gliovis.bioinfo.cnio.es/) [382] was used to download data from the IVY GAP dataset (http://glioblastoma.alleninstitute.org). RNA sequencing data stratified explicitly for $A P L N$ and $A P L N R$ mRNA expression in the predefined regions of glioblastoma and was analysed further using Prism 5.01. 
Table 3.2. Description of the analysed areas of glioblastoma tissue used from the Ivy Gap RNAseq project. This table shows that the different sampled regions of the glioblastoma tumours and the different ratios of tumour to normal cells in each area. The text was reproduced from Ivy GAP technical documentation as per Creative Commons Attribution 3.0 Unported License. URL: (http://help.brainmap.org/display/glioblastoma/Documentation?preview=/8028197/8454231/IvyOverview.pdf).

\begin{tabular}{ll}
\hline Name of area & Description \\
\hline Leading edge (LE) & $\begin{array}{l}\text { Outermost boundary of tumour, a ratio of 1-3: } 100 \text { tumour cells to normal } \\
\text { cells }\end{array}$ \\
Infiltrating Tumour (IT) & $\begin{array}{l}\text { IT is located between the LE and CT, a ratio of 10-20: } 100 \text { tumour cells to } \\
\text { normal cells }\end{array}$ \\
$\begin{array}{l}\text { Cellular Tumour (CT) } \\
\text { cell numbers due to oedema or necrosis, a ratio of 100-500: } 1 \text { tumour } \\
\text { cells to normal cells }\end{array}$ \\
$\begin{array}{l}\text { Microvasculature proliferations } \\
\text { (MVP) }\end{array}$ & $\begin{array}{l}\text { Regions of abundant blood vessels mainly containing endothelial cells } \\
\text { and smooth muscle cells }\end{array}$ \\
$\begin{array}{l}\text { Pseudopalisading cells around } \\
\text { necrosis (PAN) }\end{array}$ & $\begin{array}{l}\text { Largely located in the core, includes a large number of tumour cells that } \\
\text { surround the necrosis. }\end{array}$ \\
\hline
\end{tabular}

\subsubsection{Expression analysis of $A P L N$ and $A P L N R$ mRNA in single cell RNAseq public data}

Single cell RNAseq analysis was performed on public data published previously by [386]. Data were analysed using the online portal GBMseq (www.gbmseq.org) developed by the Gephart lab. Full details of sample generation are found elsewhere [386]. Briefly, the data set contains 3,589 cells from 4 patients with primary glioblastoma. Samples were taken from the tumour core and the peritumoral space adjacent to the tumour core, as determined by contrast magnetic resonance imaging. Samples were dissected, single cell suspensions generated, and the different cell types isolated using fluorescence-activated cell sorting (FACS) with antibodies directed against unique clustal differentiation makers (CDs) for each cell type. Specifically; anti-CD45 for myeloid cells, anti-GalC for oligodendrocytes, anti-BSL-1 for endothelial cells, anti-Thy1 for neurons and anti-HepaCAM for astrocytes. Sequencing was then performed as described in Darmanis et al. [387]. 


\subsubsection{Statistics}

All data with the exception of the single-cell RNAseq data was downloaded from the GlioVis portal and graphed in Prism 5.01 for Windows (GraphPad Software, La Jolla CA, USA) with statistical analysis performed within Prism. Correlation analysis was performed several times on the expression of genes. First, normality was tested using a Shapiro-Wilk and D'AgostinoPearson omnibus normality tests. If these tests show statistical significance, this confirms the null hypothesis can be rejected, and the data is not normally distributed. There were several data sets which did not follow a gaussian distribution, as such Spearman's correlation was used. This differs from the Pearson's correlation analysis in that gaussian distributed data, and a linear relationship is not assumptions. However, in the occasional event that the data was gaussian distributed, Spearman's correlation analysis was used for consistency and comparability because it does not assume normality. Spearman's correlation coefficient denoted "rs", numerically describes the strength of the relationship between two values. This correlates with the descriptive terms as listed in Table 3.3 below. In addition to correlation analyses, comparisons of the mean expression of multiple genes were performed. Initially, data was assessed using the previously described normality tests; despite some datasets presenting gaussian distributed data the differences in differences in expression were tested using KruskalWallis (non-parametric) for consistency. Results of the Kruskal-Wallis test are presented as follows (Kruskal Wallis (number of groups) = Kruskal-Wallis statistic, p value). To compare individual groups the Dunn's multiple comparison test was performed at a significance level alpha $=0.05$. PRISM 5.0 only reports significant values as $<0.05$; PRISM also denotes the smallest significance level at which the comparison is significant as follows: $*=<0.05, * *=$ $<0.01$, or $* * *=<0.001$. In the instance of comparing two groups, a Mann-Whitney (nonparametric) was used. 
Table 3.3. Descriptive terms used for Spearman's correlation coefficients. These descriptive interpretations are derived from Applied Statistics for the Behavioural Sciences 5th edition [388]

\begin{tabular}{lll}
\hline Spearman's value & Description & Interpretation \\
\hline $0.00-0.30$ & "very weak" & Little if any correlation \\
$0.30-0.50$ & "weak" & Low positive or negative correlation \\
$0.50-0.70$ & "moderate" & $\begin{array}{l}\text { Moderate positive or negative } \\
\text { correlation }\end{array}$ \\
$0.70-0.90$ & "strong" & $\begin{array}{l}\text { High positive or negative correlation } \\
0.90-1.0\end{array}$ \\
& "very strong" & $\begin{array}{l}\text { Very high positive or negative } \\
\text { correlation }\end{array}$ \\
\hline
\end{tabular}

\subsection{Results}

\subsubsection{Expression of $A P L N$ and $A P L N R$ mRNA in human glioblastoma tissue and cell lines}

The mRNA expression of $A P L N, A P L N R$ and $S L C 2 A 1$ was measured in glioblastoma samples from twelve different patients (depicted as black points below), and one healthy human brain tissue sample measured independently 3 times (depicted as red points) as seen in Figure 3.1 below. Due to low biological replicates of normal brain tissue, statistical comparison of tumour and normal brain tissue could not be performed.

The expression of each gene was compared between glioblastoma and the normal tissue sample by comparing the black to red points. The mRNA expression of APLNR and SLC2A1 in glioblastoma tissue is similar to that in normal human brain tissue with approximately 6 of 12 glioblastoma samples being higher and 6 of 12 glioblastoma samples being lower than the control brain tissue expression (red points). In contrast, APLN mRNA expression in 11 out of the 12 tumour samples was markedly higher than expressed in the healthy human brain tissue sample. The one sample of glioblastoma that had lower expression of $A P L N$ (represented by a star in Figure 3.1, A) also had lower SLC2A1 and APLNR expression than the other 
glioblastoma samples which suggests this sample, for unknown reasons, had lower expression overall.

Due to several overlapping biological functions of $A P L N$ and $S L C 2 A 1$, the hypothesis was formed that the expression of $A P L N$ may be correlated with SLC2A1 expression. Spearman's correlations were performed between $A P L N, A P L N R$ and SLC2A1. It was found that there was a statistically significant, moderate positive correlation between $A P L N$ and $S L C 2 A 1$ (rs = $0.6506, \mathrm{P}=0.0086$ ) (Figure 3.1, B). There was a very weak correlation between $S L C 2 A 1$ and APLNR (rs $=-0.174, \mathrm{p}=0.536)$ and $A P L N$ and $A P L N R(\mathrm{rs}=0.1769, \mathrm{p}=0.5281)$ (data not shown).
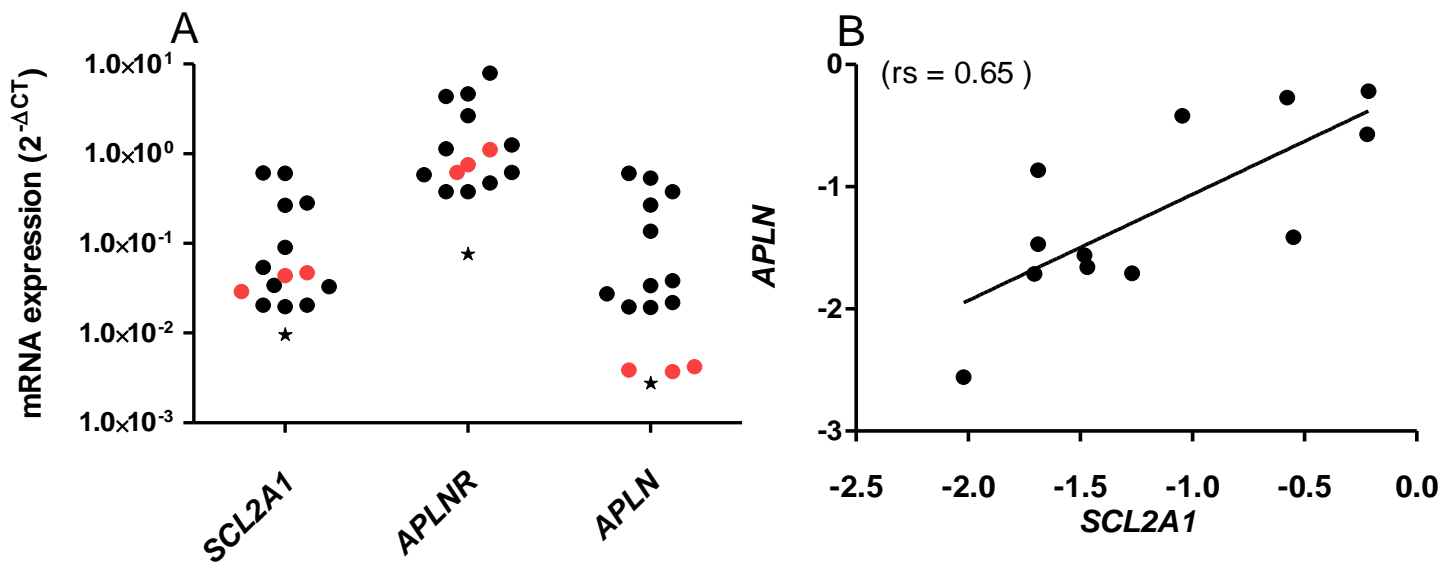

Figure 3.1. Expression levels of APLN, SLC2A1 and APLNR mRNA in human glioblastoma and healthy brain tissue samples. All data were normalized to expression levels of the reference gene HPRT and are expressed as $2^{-}$ $\triangle \mathrm{CT}$ values on either a $\log _{10}(\mathrm{~A})$ or linear (B) scale. (A) The mRNA expression levels of SLC2A1, APLNR and $A P L N$, were measured in 12 human glioblastoma tumour samples (black points), and one healthy human brain RNA sample measured independently 3 times (red points). One tumour sample was represented as a star (*) and expressed lower levels of $A P L N, A P L N R$ and $S L C 2 A 1$. (B) Spearman's correlation between mRNA expression levels of $A P L N$ and $S L C 2 A 1$ ( $\mathrm{rs}=0.6506, \mathrm{P}=0.0086, \mathrm{n}=12$ ). 
The previous data from Figure 3.1 suggests that the mRNA expression of $A P L N$ is upregulated and $A P L N R$ is equivalent to glioblastoma tumour samples. Several glioblastoma-derived cell lines were available for use as a model of glioblastoma during this study; however, it was unknown what the mRNA expression of $A P L N$ and $A P L N R$ was and if it was similar to glioblastoma tumour samples. Thus, mRNA expression of APLN and APLNR was measured in primary derived (0906, 0814 and 1003) and immortalized glioblastoma cell lines (U87MG, GL261 and LN18) (Figure 3.2) and compared to the mRNA expression seen in Figure 3.1.

Figure 3.2 shows $A P L N$ expression in glioblastoma tissue, primary and immortalised glioblastoma cell lines. A Kruskal-Wallis test showed there was a statistically significant difference in the median expression of APLN between each tissue type (Kruskal Wallis (3) = 18.00, $\mathrm{p}=0.0001$ ). Dunn's multiple comparison test (Dunn's MCT) was performed to determine if the differences between groups was significant. The primary and immortalised cell lines had approximately 100-fold lower expression of APLN mRNA compared to that in glioblastoma tissue samples $(\mathrm{p}<0.05)$. However, expression levels of $A P L N$ mRNA were not different between the primary glioblastoma cells and immortalised cell lines.

The median APLNR mRNA expression between groups, like APLN mRNA, was significantly different (Kruskal Wallis $(3)=21.24, \mathrm{p}<0.0001)$. When the mRNA expression of APLNR was compared between glioblastoma tissue and the glioblastoma-derived primary and immortalised cell lines using Dunn's MCT, there was significant difference $(\mathrm{p}<0.05)$. Though the expression of APLNR mRNA appeared 10 fold less in immortalised lines than the expression in primary cell lines, there was no statistically significant difference between primary and immortalised cell lines. This suggests that a loss of APLNR and APLN mRNA expression can occur when cells are cultured for longer periods of time. 

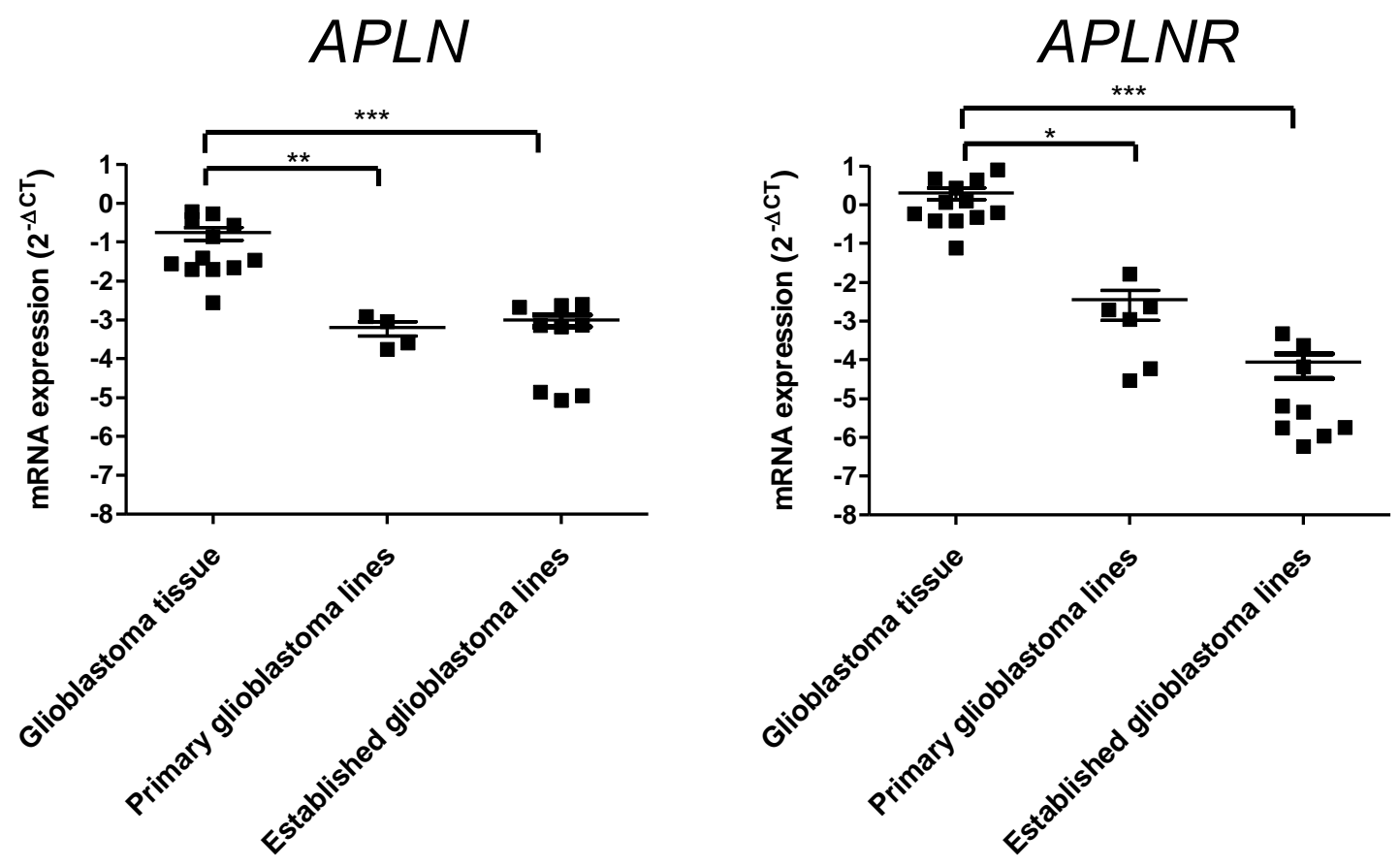

Figure 3.2. A comparison of glioblastoma tissue, glioblastoma-derived primary and long-term immortalized glioblastoma-derived cell lines expression of $A P L N$ and $A P L N R$. Panel A compares the difference in $A P L N$ expression between glioblastoma tissue $(\mathrm{n}=12), 3$ different glioblastoma-derived cell lines (Total $\mathrm{n}=4 ; 0906 \mathrm{n}$ $=2,0814 \mathrm{n}=1,1003 \mathrm{n}=1$ ) and 3 different immortalized glioblastoma cell lines (Total $\mathrm{n}=9$, U87MG $\mathrm{n}=3$, LN18 $\mathrm{n}=3$ and GL261 $\mathrm{n}=3)$. Panel B compares the difference in APLNR expression glioblastoma tissue $(\mathrm{n}=$ 12) and 3 different glioblastoma-derived cell lines (Total $n=6 ; 0906 n=3,0814 n=1,1003 n=2$ ) and 3 different immortalised glioblastoma cell lines (Total $n=9$, U87MG $n=3$, LN18 n=3 and GL261 $n=3$ ). All tissue-derived cell lines were derived from primary glioblastoma tissues and were tested within 20 passages of isolation. All data is normalized to HPRT and expressed as $2^{-\Delta \mathrm{CT}}$ on a $\log$ scale expressed as mean $\pm \mathrm{SEM}$. The difference in means was tested using Kruskal - Wallis test with Dunn's multiple comparisons test. 


\subsubsection{The mRNA expression of APLN and APLNR in glioblastoma subtypes}

The data shown in Figure 3.1 suggests $A P L N$ mRNA expression was upregulated in glioblastoma over normal brain tissue, but that mRNA expression of SLC2A1 and APLNR was unchanged. However, the number of available biological replicates was limited; as such, public data from the TCGA was used to confirm expression seen in Figure 3.1 and further examine APLN and APLNR expression in glioblastoma subtypes. The mRNA expression of APLN, $A P L N R$ and SLC2A1 were compared between glioblastoma and normal brain tissue samples from the TCGA_GBM dataset using a Mann-Whitney test (Figure 3.3). Figure 3.3 shows there is a statistically significant difference in $A P L N$ mRNA expression $(\mathrm{U}=35.00, \mathrm{p}=0.0025)$ but no difference in $A P L N R$ and $S L C 2 A 1$. This corroborates data shown in Figure 3.1.

Next, the expression of $A P L N, A P L N R$ and SLC2A1 were examined in the Verhaak subtypes of glioblastoma (neural, proneural, mesenchymal and classical) [134] in Figure 3.3. There was no statistical difference in the expression of $A P L N$ or $A P L N R$ between the different glioblastoma subtypes. 

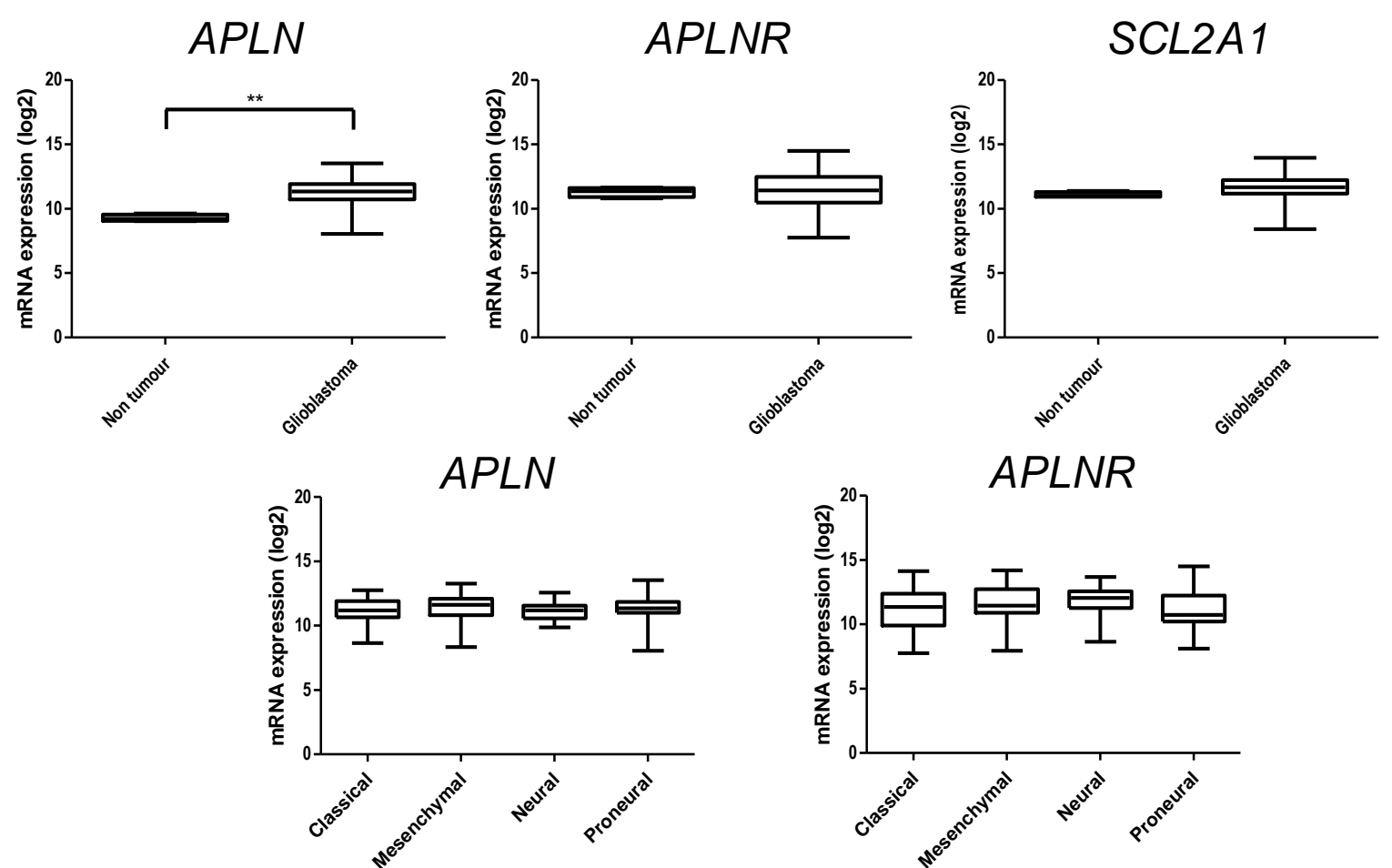

Figure 3.3.The expression of $A P L N, A P L N R$ and $S L C 2 A 1$ in glioblastoma and glioblastoma subtypes. The mRNA expression of $A P L N, A P L N R$ and $S L C 2 A 1$ are measured between non-tumour tissue and glioblastoma. There was a significant increase in the median $A P L N$ expression in glioblastoma tissue when compared to normal tissue (MannWhitney test, $\mathrm{U}=35.00, \mathrm{p}=0.0025, \mathrm{n}=4$ non-tumour tissue, $\mathrm{n}=156$ glioblastoma). There was no difference in expression of $A P L N R$ and $S L C 2 A 1$ between tumour and nontumour tissue. The expression of $A P L N$ and $A P L N R$ is also measured in glioblastoma subtypes as reported by Verhaak et al. [134]: classical, mesenchymal, neural and proneural. No significant difference in $A P L N$ or $A P L N R$ mRNA expression was seen across the 4 subtypes. All values are presented as mean $\pm \mathrm{SEM}$. 


\subsubsection{Expression of $A P L N$ and $A P L N R$ mRNA in diffuse astrocytic and oligodendroglial tumours}

The expression of $A P L N$ and APLNR mRNA in differing grades of diffuse astrocytic and oligodendroglial tumours were examined using the TCGA LGG-GBM dataset (Figure 3.4). Samples were stratified using the numerical grading system described in Chapter 1 Section 1.3.2. The median mRNA expression significantly differed between grades for $A P L N$ (Kruskal Wallis (3) $=141.0, \mathrm{p}<0.0001), A P L N R$ (Kruskal Wallis $(3)=17.42, \mathrm{p}=0.0002)$ and SLC2A1 (Kruskal Wallis $(3)=50.26, \mathrm{p}<0.0001)$. To compare the differences in mRNA expression between tumour grades Dunn's MCT was performed. APLN and APLNR mRNA expression were significantly elevated in Grade IV over grade II and III $(\mathrm{p}<0.05)$. As a point of comparison, SLC2A1 mRNA expression across the differing grades is presented in Figure 3.4. SLC2A1 has been reported to correlate with the grade of glioma [366, 389] and was found to be significantly elevated in grade IV over grade II and III $(\mathrm{p}<0.05)$. While the increase in $A P L N R$ and $A P L N$ expression with grade is statically significance, the biological relevance is unclear. 


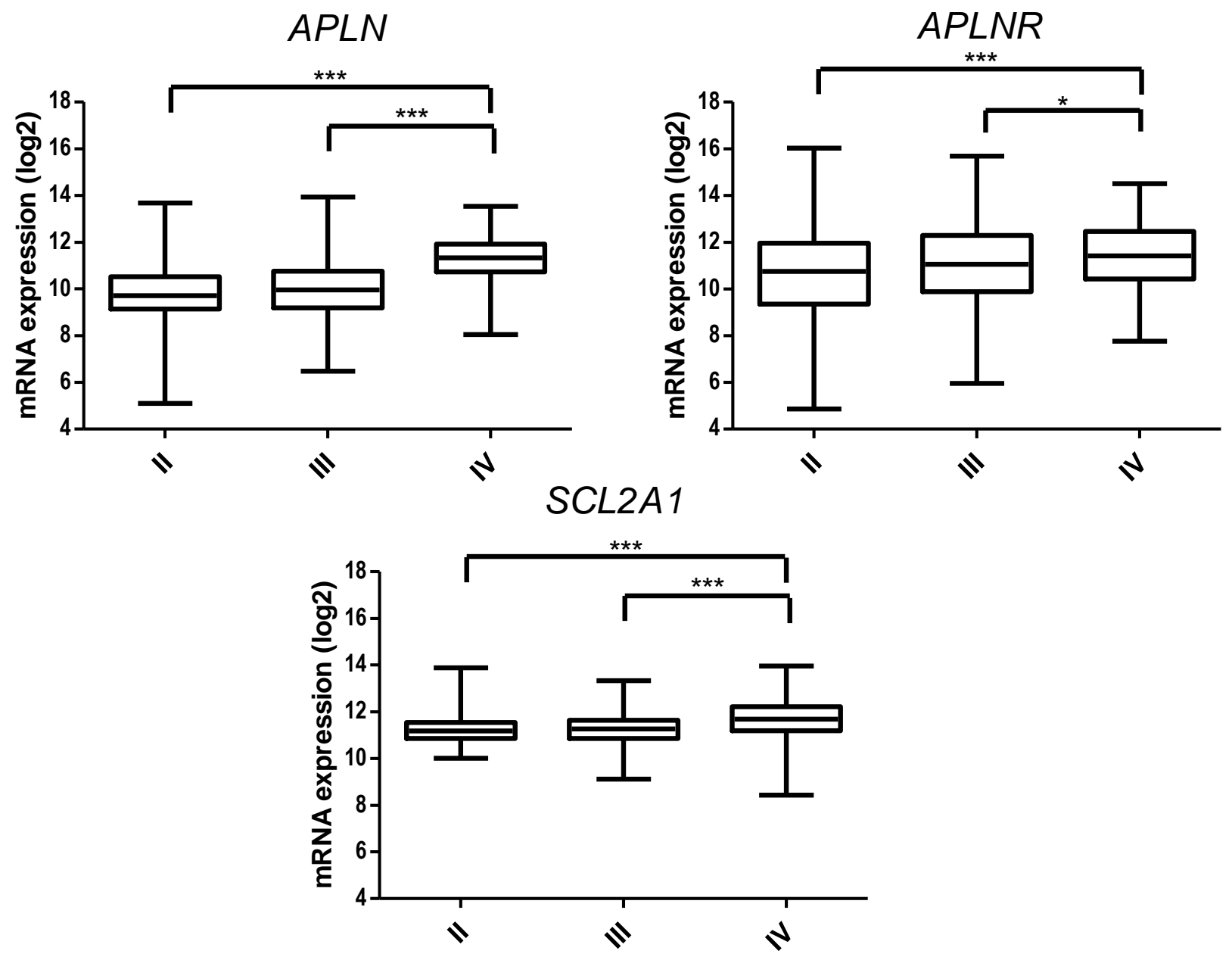

Figure 3.4. A comparison of the mRNA expression of APLN, APLNR and SLC2A1 in the different grades of diffuse astrocytic and oligodendroglial tumours from public TCGA datasets. All values are expressed as mean \pm SEM. Data were analysed from 226 grade II, 244 grade III and 150 grade IV glioblastomas. The difference in means was tested using Kruskal - Wallis and the means compared using Dunn's multiple comparisons test. 


\subsubsection{The correlation of $S L C 2 A 1$ and APLN}

The strong positive correlation between $S L C 2 A 1$ and $A P L N$ seen in Figure 3.1 Panel B suggests that high expression of $S L C 2 A 1$ is associated with a high expression of APLN. However, it is unclear what the cause of this correlation is and whether it is unique to glioblastoma or also present in other lower grade glioma. Thus, to further investigate the correlation, the same public datasets from the TCGA LGG- GBM database described above were analysed for a correlation between $S L C 2 A 1$ and $A P L N$. The correlation between $A P L N$ and $S L C 2 A 1$ was examined in the glioblastoma (Figure 3.5) and there was found to be a weak to moderate correlation ( $\mathrm{rs}=$ $0.4678, \mathrm{p}<0.0001, \mathrm{n}=156)$ rather than Figure 3.1 where the correlation was stronger. This relationship could not be assessed in normal tissue because the low sample number $(n=4)$ makes determining any relationship unlikely. The data from glioblastoma in Figure 3.5, was stratified into three of the different subtypes of glioblastoma described by Verhaak et al. [134] to further examine the correlation between $S L C 2 A 1$ and $A P L N$ [134]. The positive correlation was maintained in the three subtypes: classical, mesenchymal and proneural to differing strengths as shown in Figure 3.5. This indicates the correlation between SLC2A1 and APLN is consistent across subtypes but does not suggest the cause. 


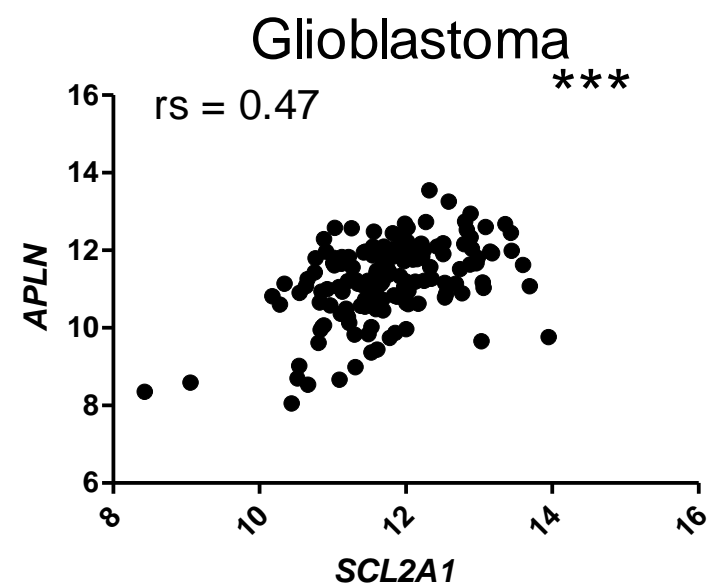

Mesenchymal

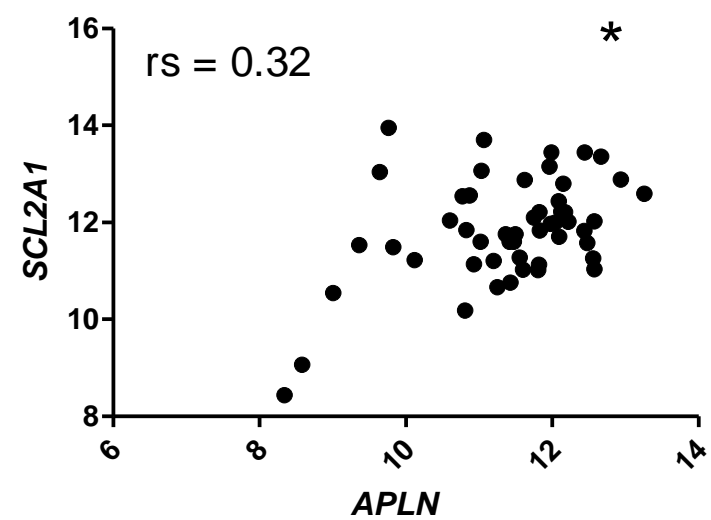

Classical

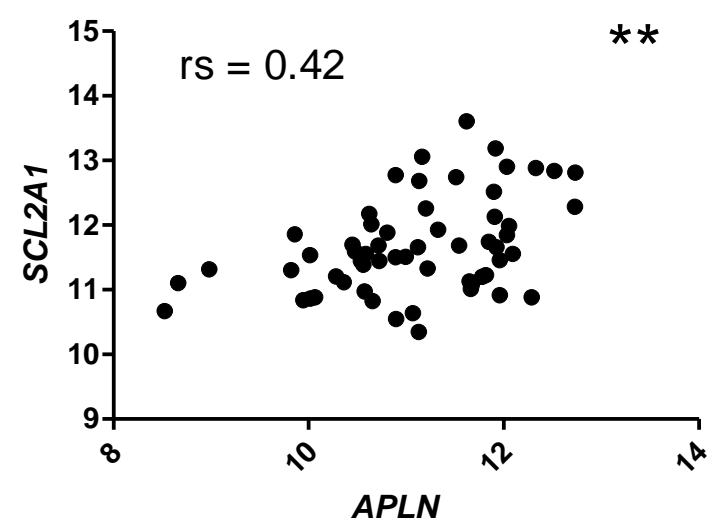

Proneural

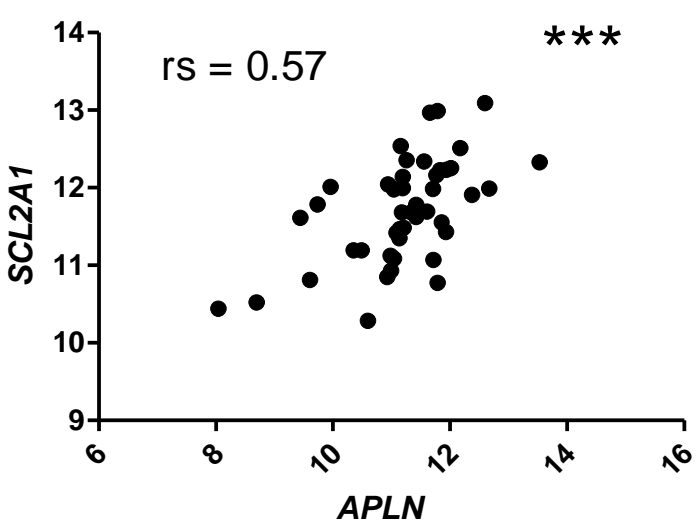

Figure 3.5. An analysis of the correlation between $S L C 2 A 1$ and $A P L N$ in glioblastoma and glioblastoma subtypes. Spearman's correlation was performed between $S L C 2 A 1$ and $A P L N$ in glioblastoma using the TCGA_GBM public dataset. The Glioblastoma data were stratified for three different subtypes: classical, mesenchymal and proneural. Each panel shows the correlation of SLC2A1 and APLN in glioblastoma ( $\mathrm{rs}=0.4678, \mathrm{p}=<0.0001, \mathrm{n}=156$ ) classical glioblastoma $(\mathrm{rs}=0.4159, \mathrm{p}=<0.0011, \mathrm{n}=59)$, mesenchymal glioblastoma $(\mathrm{rs}=0.3178, \mathrm{p}=<0.0230$, $\mathrm{n}=51)$ and proneural glioblastoma $(\mathrm{rs}=0.5677, \mathrm{p}=<0.0001, \mathrm{n}=46)$. 
Glioblastoma presents with unique pathophysiological features such pseudopalisading necrosis (which are areas of necrosis with hypoxic cells surrounding it) and florid microvasculature proliferation [390], which are not seen in lower grade tumours. These same features are likely to express $S L C 2 A 1$ and $A P L N$ mRNA because both genes are modulated by hypoxia and expressed in the microvasculature. These two features are less prevalent in other diffuse astrocytic and oligodendroglial tumours. To confirm that the correlation of APLN and SLC2A1 was due to unique features found in glioblastoma, the correlation of APLN and SLC2A1 was analysed using the TCGA GBM-LGG dataset specifically examining glioblastoma, astrocytoma, oligoastrocytoma and oligodendroglioma (Figure 3.6).

Glioblastoma showed a statistically significant weak positive correlation between $A P L N$ and $S L C 2 A 1$. There was a very weak statistically significant correlation of SLC2A1 and APLN in oligoastrocytoma and astrocytoma (Figure 3.6) and statistically insignificant correlation in oligodendroglioma (Figure 3.6). This suggests the correlation of SLC2A1 and APLN is only present in glioblastoma. 
Oligoastrocytoma

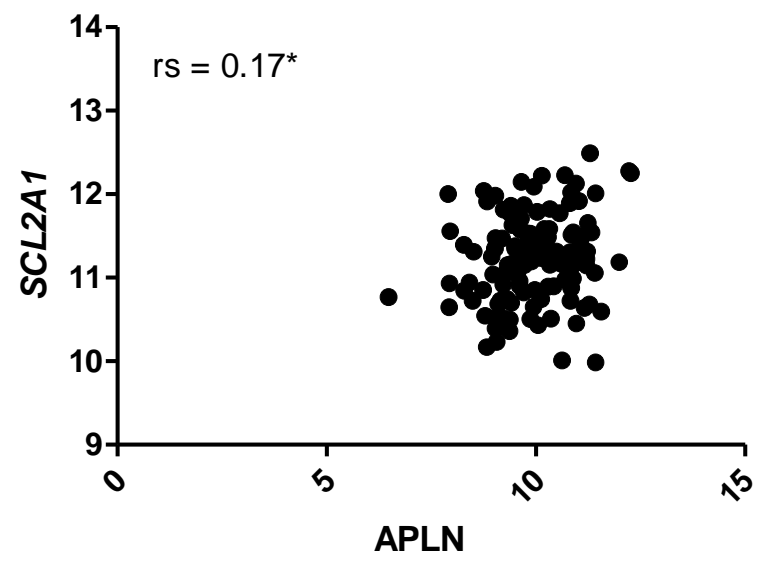

Astrocytoma

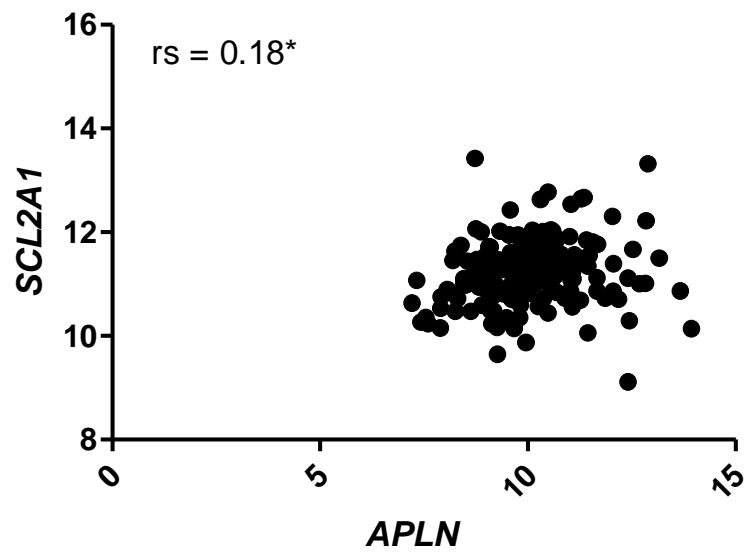

Oligodendroglioma

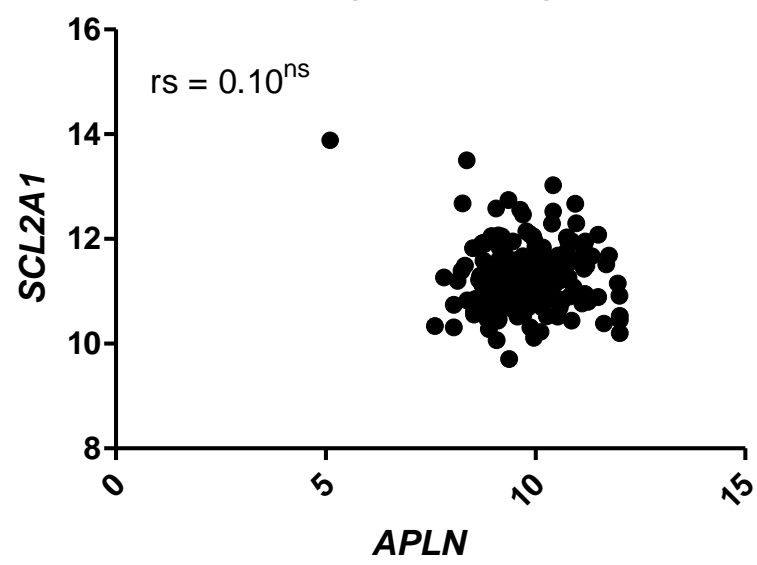

Glioblastoma

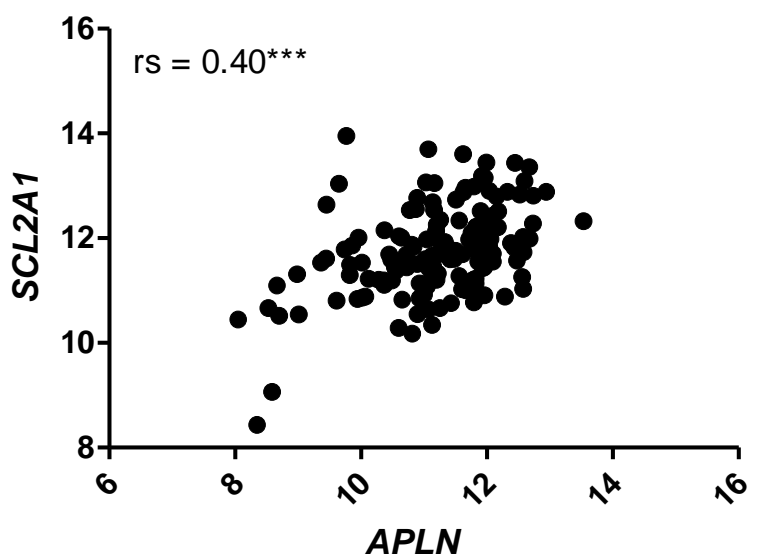

Figure 3.6. The Spearman's correlation of $S L C 2 A 1$ and $A P L N$ in different diffuse astrocytic and oligodendroglial tumours. The correlation between $S L C 2 A 1$ and $A P L N$ was examined in Oligoastrocytoma, Oligodendroglioma, Astrocytoma and Glioblastoma. There is a statistically significant very weak correlation of $S L C 2 A 1$ and $A P L N$ in oligoastrocytoma ( $\mathrm{rs}=0.1746, \mathrm{p}<0.0469, \mathrm{n}=130$ ), no statistically significant correlation between $S L C 2 A 1$ and $A P L N$ in oligodendroglioma ( $\mathrm{rs}=0.1044, \mathrm{p}<0.1486, \mathrm{n}=193$ ), a statistically significant correlation between $S L C 2 A 1$ and $A P L N$ in astrocytoma ( $\mathrm{rs}=0.1840, \mathrm{P}<0.04007, \mathrm{n}=194)$ and a statistically significant correlation between $S L C 2 A 1$ and $A P L N$ in glioblastoma ( $\mathrm{rs}=0.4043, \mathrm{P}<0.0001, \mathrm{n}=152$ ). All data was taken from the TCGA_LGG_GBM dataset and extracted using GlioVis. 


\subsubsection{Investigating the potential cause of the correlation between $S L C 2 A 1$ and APLN mRNA}

It was hypothesised that $A P L N$ and $S L C 2 A 1$ mRNA expression correlation could be due to several factors, two most likely of which were hypoxia-mediated expression of APLN and $S L C 2 A 1$ or endothelial cell expression of APLN and SLC2A1. To ascertain the cause of the $A P L N$ and SLC2A1 correlation, multiple correlation analyses were performed (Figure 3.7) comparing APLN and SLC2A1 to the common endothelial cell marker, platelet and endothelial cell adhesion molecule 1 (PECAM1 aka CD31) and the hypoxia markers carbonic anhydrase 9 (CA9) and Hypoxia-Inducible Factor 1 Alpha (HIFl $\alpha)$. This will help inform what the cause of the $S L C 2 A 1$ and $A P L N$ correlation may be due to.

There was a very weak correlation between the hypoxia markers $C A 9$ and $H I F 1 \alpha$ (rs $=0.20, \mathrm{p}$ $=0.0147)$; in addition, literary reports suggest that alternative hypoxia markers such as CA9 are more beneficial as hypoxia markers [391]. Thus, CA9 was used as the primary hypoxia marker for future comparisons.

The relationship between the endothelial marker PECAM1 to APLN, SLC2A1 and CA9 was examined. There was no correlation between CA9 and PECAM1 (rs =0.02), SLC2A1 and PECAM1 ( $\mathrm{rs}=0.05)$ and a weak correlation between APLN and PECAM1 ( $\mathrm{rs}=0.3542, \mathrm{P}<$ $0.0001)$. However, a moderate correlation was seen between $S L C 2 A 1$ and $C A 9$ (rs $=0.62, \mathrm{p}<$ 0.0001 ) and a weak positive correlation between SLC2A1 and APLN (rs $=0.43,<0.0001)$. There was also a weak positive correlation between $A P L N$ and $C A 9$ ( $\mathrm{rs}=0.39,<0.0001)$. This suggests that the $A P L N$ and $S L C 2 A 1$ correlation may be due to hypoxia. 

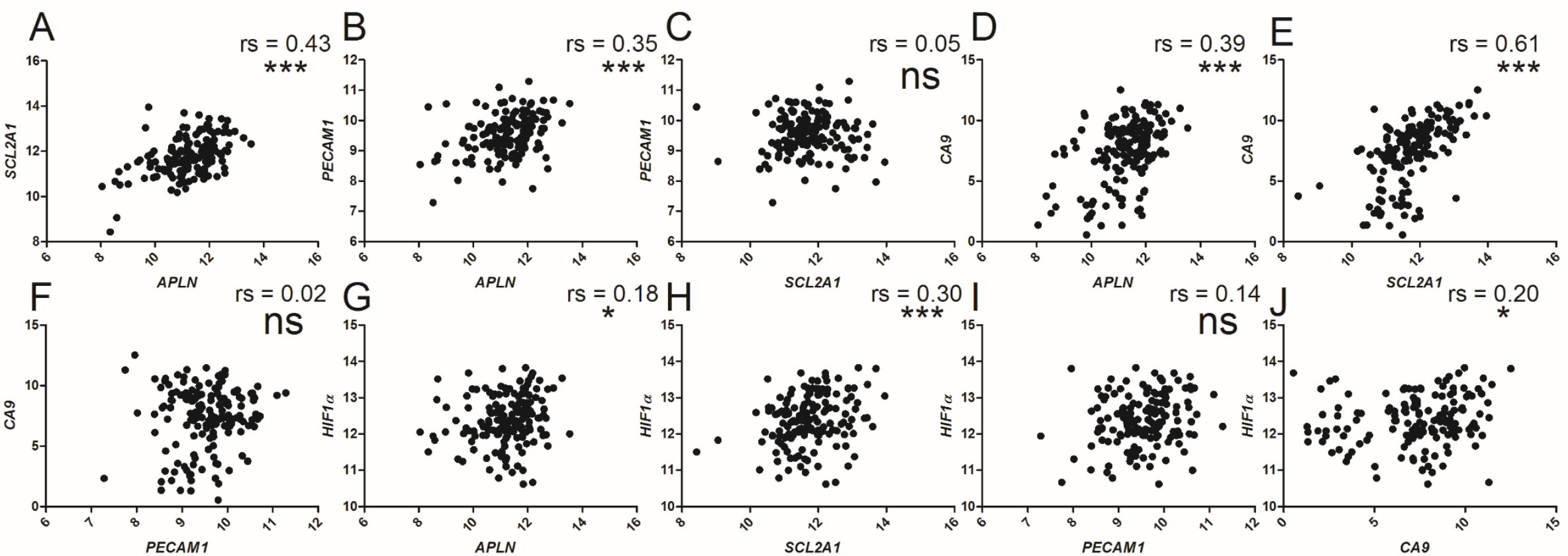

Figure 3.7. Multiple individual Spearman's correlation analyses of hypoxia and blood vessel markers with the $A P L N$ mRNA in glioblastoma. Panel A shows the correlation between $A P L N$ and $S L C 2 A 1$ ( $\mathrm{rs}=0.4344, \mathrm{P}=<0.0001, \mathrm{n}=156$ ). Panel B shows the correlation between $A P L N$ and the endothelial marker $P E C A M 1$ ( $\mathrm{rs}=0.3542, \mathrm{P}<0.0001$, $\mathrm{n}=156)$. Panel $\mathrm{C}$ shows the correlation between SLC2A1 and PECAM1 ( $\mathrm{rs}=0.0 .0485, \mathrm{P}=0.0001, \mathrm{n}=156)$. Panel $\mathrm{D}$ shows the correlation between the hypoxia marker CA9 and APLN ( $\mathrm{rs}=0.3886, \mathrm{P}=<0.0001, \mathrm{n}=156)$. Panel E shows the correlation between $C A 9$ and $S L C 2 A 1$ ( $\mathrm{rs}=0.6158, \mathrm{P}<0.0001, \mathrm{n}=156)$. Panel $\mathrm{F}$ shows the correlation between CA9 and PECAM1 ( $\mathrm{rs}=0.02926, \mathrm{P}=<0.717, \mathrm{n}=156)$. Panel $\mathrm{G}$ shows the correlation between $A P L N$ and $H I F 1 \alpha(\mathrm{rs}=0.1840, \mathrm{P}<0.0215, \mathrm{n}=156)$. Panel $\mathrm{H}$ shows the correlation between $S L C 2 A 1$ and HIFl $\alpha(\mathrm{rs}=0.2976, \mathrm{P}=0.0002, \mathrm{n}=156)$. Panel I shows the correlation between PECAMI and HIF1 $(\mathrm{rs}=0.1427, \mathrm{P}=0.0755, \mathrm{n}=156)$. Panel J shows the correlation between $C A 9$ and $\operatorname{HIFl\alpha }(\mathrm{rs}=0.1950, \mathrm{P}<0.0147, \mathrm{n}=156)$. All data was taken from the TCGA_GBM data set and extracted using GlioVis. 


\subsubsection{Intratumoural analysis of APLN and APNLR expression in glioblastoma tissue}

The Ivy GAP public dataset [385] was investigated through the GlioVis data portal to further understand the mRNA expression of $A P L N$ and $A P L N R$ in different intratumoral regions of glioblastoma reviewed in Table 3.2. Figure 3.8 shows the mRNA expression of $A P L N, A P L N R$, $C A 9$ and PECAM1 in the 5 different regions of glioblastoma. Median expression of the aforementioned genes was statistically compared using a Kruskal-Wallis test and all medians were found to vary significantly $(p<0.0001)$. The difference in gene expression between the different tumour regions and the leading edge (LE) was performed using Dunn's MCT because the LE is the area with the most normal (non-tumorous) cells.

$A P L N R$ expression varied significantly from the LE to features in the tumour core. APLNR expression was higher in the infiltrating tumour (IT) and microvasculature proliferations (MVP) but not significantly different in the cellular tumour (CT) and pseudopalisading cells around necrosis (PAN). However, the wider spread of data in the LE sample group is suggestive of a varied range of expression making statistical comparisons more challenging.

APLN expression (Figure 3.8) was higher in the CT, MVP and PAN but not in the IT. The CT, MVP and PAN are more centrally located in the tumour and could suggest a correlation with hypoxic conditions. To investigate this, the mRNA expression of $C A 9$ (Figure 3.8) was examined within the same dataset found to be slightly higher in the CT. As expected, $C A 9$ was significantly overexpressed in the PAN. The high expression of CA9 and APLN, but not, $A P L N R$ in the PAN is highly suggestive that hypoxia could modulate APLN expression.

The endothelial marker PECAMI was examined to confirm appropriate expression of endothelial markers in the MVP. As expected, PECAM1 expression was higher in the MVP than the leading edge. APLNR and APLN were both upregulated in the MVP which suggests the apelinergic system has a role in the MVP of glioblastoma. 

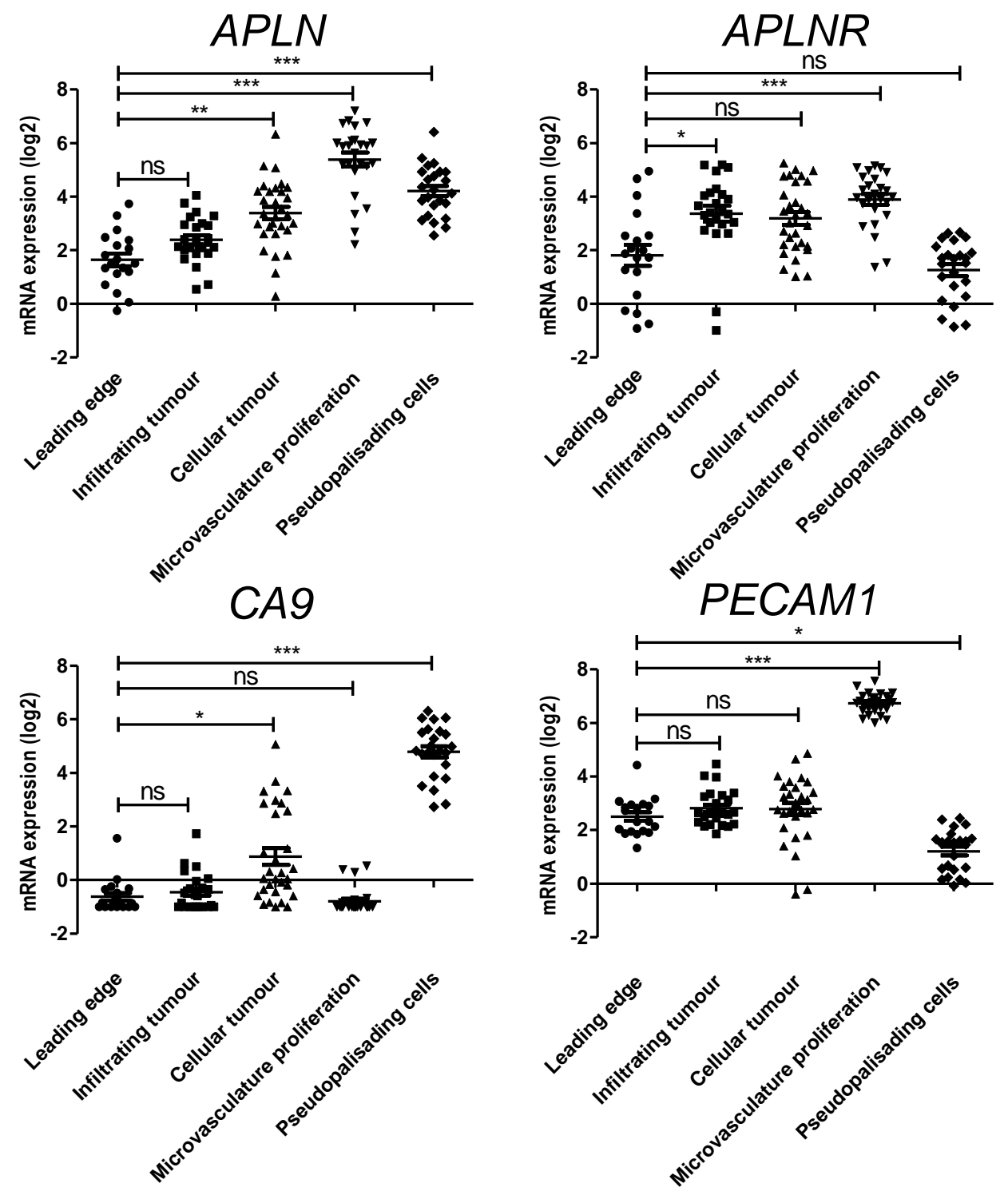

Figure 3.8. The expression of APLN, APLNR, CA9 and PECAM1 (CD31) in intratumoral locations of glioblastoma tumours. Data from the Ivy GAP public dataset was downloaded using the GlioVis data portal and analysed in Prism 5.01; all data were expressed as mean $(\log 2)$ transformed data \pm SEM. The difference in medians was tested using the Kruskal - Wallis test. The medians of APLN (Kruskal Wallis (5) =70.72, p < 0.0001), APLNR (Kruskal Wallis (5) = 47.06, $\mathrm{p}<0.0001$ ), CA9 (Kruskal Wallis $(5)=77.71, \mathrm{p}<0.0001$ ) and PECAM1 (Kruskal Wallis (5) $=82.91, \mathrm{p}<0.0001)$ were all found to vary significantly. The comparison between regions was performed using Dunn's multiple comparison test to compare the gene expression of all groups to the leading edge sample group. (Leading edge $n=29$, Infiltrating tumour $n=24$, Cellular tumour $n=30$, Microvasculature proliferation $n=25$ and pseudopalisading cells $n=24)$. 


\subsubsection{The analysis of single-cell RNA sequenced public datasets to determine intratumoral $A P L N$ and $A P L N R$ localisation}

Public data were analysed to look for expression of $A P L N$ and $A P L N R$ in specific cell types and regions of a glioblastoma tumour. $A P L N$ expression (Figure 3.9, Panel A) was expressed in most cell types apart from myeloid cells and oligodendrocyte precursor cells (OPC's). APLN expression was also higher in the tumour core than in the periphery (Figure 3.9, Panel C) whereas APLNR expression was not different when comparing the same tissues (Figure 3.9, Panel D). It's important to note that the authors of this dataset noted the higher expression of hypoxia markers in the tumour core [386]. This suggests, once more, that APLN expression is associated with hypoxic regions.

Elevated mRNA expression of $A P L N R$ was found in neoplastic cells, astrocytes and vascular cells (Figure 3.9, Panel B). The high expression of APLNR in vascular cells corroborates data seen above (Figure 3.8) and suggests APLNR mRNA expression is elevated on cells of the vasculature. In addition, APLNR expression is noted on neoplastic cells as well as astrocytes but not all glial cells. Taken together the single cell RNAseq data suggests that APLNR has a more localised expression to some cell types whereas $A P L N$ is more widely expressed. 

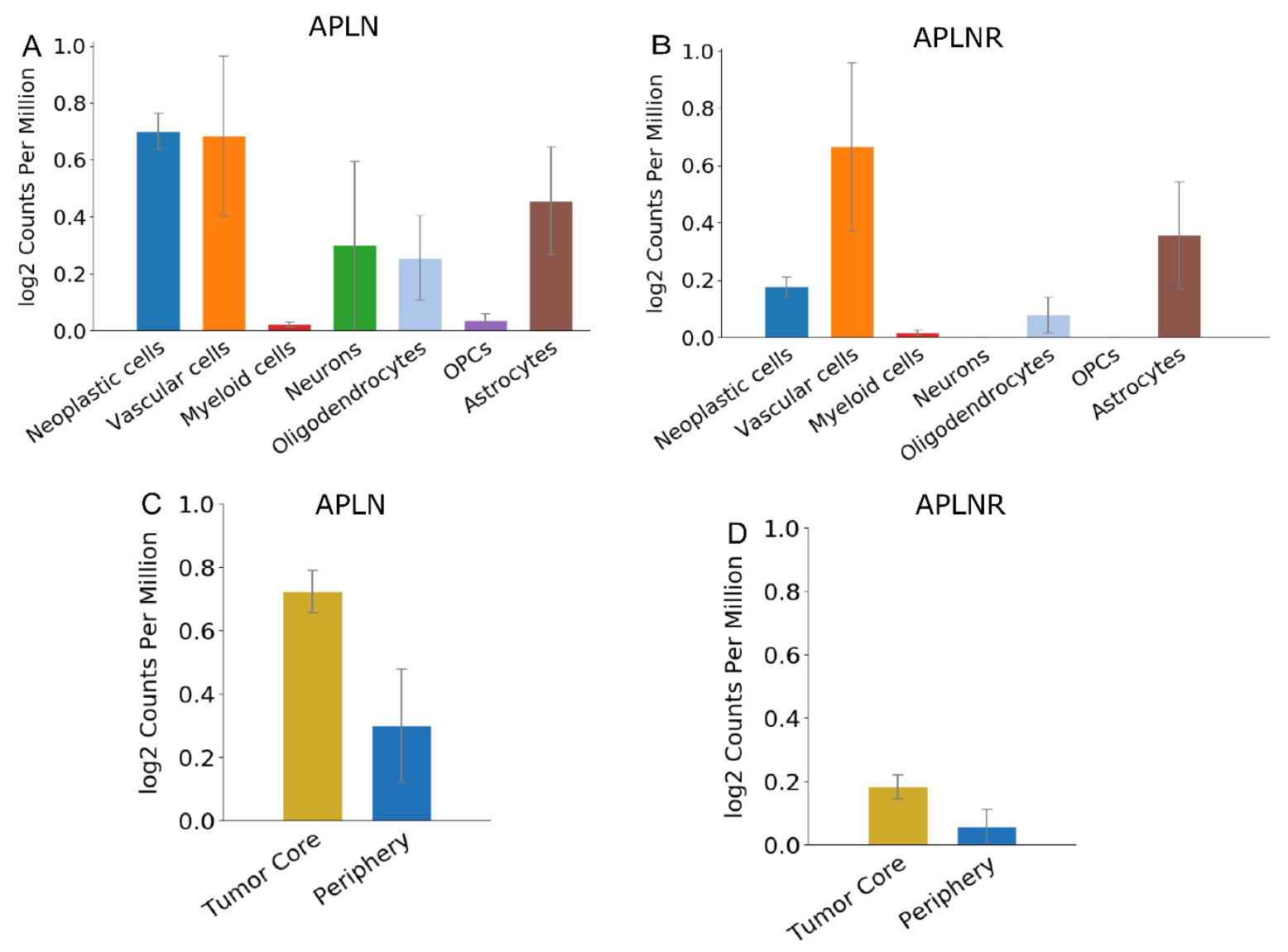

Figure 3.9. Single cell RNAseq analysis using public data. Panel A shows $A P L N$ expression across 7 different cell types from glioblastoma patients, panel B shows $A P L N R$ expression across cell types, panel C shows $A P L N$ expression in the tumour core and periphery and panel D shows the expression of $A P L N R$ in the tumour core and periphery. Data was analysed using the GBMseq.org portal [386]. Values are the mean \pm SEM 


\subsubsection{Immunohistochemical and immunocytochemical expression of $A P L N R$ in tumour tissue and tissue-derived cell lines}

The data in Figure 3.1 showed there was an increase of $A P L N$ and no difference in APLNR mRNA expression when comparing glioblastoma tissue to normal tissue. This was corroborated using public data from the TCGA (Figure 3.3). Interestingly, the expression of APLN and APLNR appears to be lost after resection and subsequent in vitro culture. APLNR expression is critical because it is implicated in response to APLN. As such, APLNR expression was examined in cell lines as well as in the GL261 glioblastoma tumour model using two commercially available APLNR antibodies.

\subsubsection{Western blot verification of antibody target specificity in glioblastoma cell lines}

Figure 3.10 Panel A shows a western blot (WB) using the APLNR-Nterm antibody. The positive control was mouse heart with an expected band of approximately $50 \mathrm{kDa}$ which was seen. Immunoblotting with the APLNR-Nterm antibody for the equivalent amount of protein $(\sim 10 \mu \mathrm{g})$ yielded no immunoreactivity (IR) at $50 \mathrm{kDa}$ in U87MG and HEK293 cells. This suggests that the APLNR-Nterm antibody does not bind human-derived cell lines.

Figure 3.10 panel B shows a WB using the APLNR-Cterm antibody with the positive control (U87MG) and HEK293. Two bands are present in the U87MG control for the APLNR-Cterm antibody. The smaller band indicated by the red arrow in Figure 3.10 panel B denotes an approximately $50 \mathrm{kDa}$ product, and the blue arrow denotes a band larger than $50 \mathrm{kDa}$ that was the manufacturers expected APLNR band. When probing for APLNR in the positive control mouse heart lysate using the APLNR-Cterm antibody, four bands were detected (Figure 3.10, panel B, mouse heart lane). One of the bands (yellow arrow, Figure 3.10, Panel B) corresponds to the appropriate approximate size for APLNR as stated by the manufacturer. There is the presence of a band indicated by the red arrow (Figure 3.10, Panel B) which is hypothesised to 
be the APLNR with the potentially different glycosylation pattern. Two other bands were detected, one at $\sim 37 \mathrm{kDa}$ (indicated by the green arrow) and one band indicated by the black arrow which may correspond to endogenous APLNR targets with different levels of PTM and can be seen consistently in mouse heart lysate but not cell lines.

It was concluded that the APLNR-Nterm antibody could recognise APLNR isoforms in the mouse heart but not in the human cell lysate; whereas, the APLNR-Cterm antibody recognises the appropriate APLNR in human cells but different isoforms in the mouse heart. Thus the APLNR-Cterm antibody was carried forward for further verification.

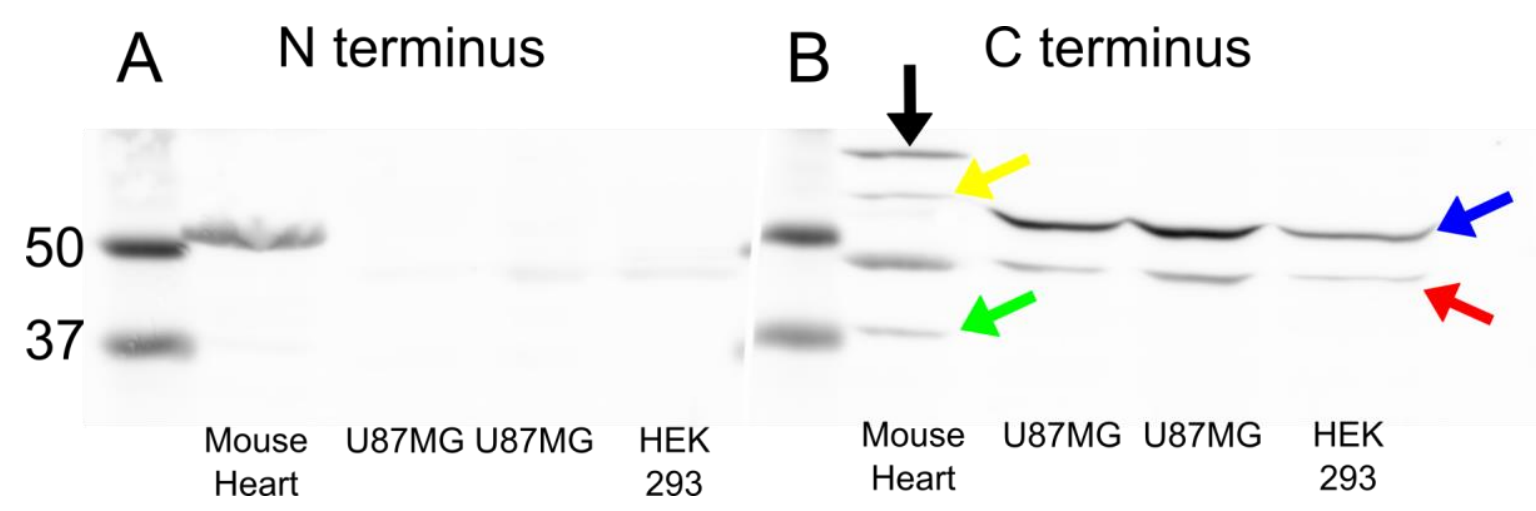

Figure 3.10. A representative western blot of APLNR protein expression in cell line and mouse heart lysates when probed with antibodies raised against the $\mathrm{N}$ and $\mathrm{C}$ terminus of APLNR. APLNR immunoblotting was performed on mouse heart, U87MG and HEK293 lysate. Both panels were run on the same membrane with the same amount of protein $(\sim 10 \mu \mathrm{g})$, divided post transfer and probed with antibodies against the $\mathrm{N}$ terminus (Panel $\mathrm{A})$ and $\mathrm{C}$ terminus (Panel B) of APLNR. Both membranes were then scanned at the same settings. Yellow and blue arrows correspond to the approximate size of APLNR as suggested by the manufacturer. The red arrow indicates bands that are hypothetically APLNR with differing glycosylation modification. The green and black arrows indicate bands of unknown origin. 


\subsubsection{Antibody verification of APLNR via immunohistochemical analysis in infantile haemangioma samples}

APLNR expression is well reported in the microvasculature, thus, to further verify the APLNRCterm antibody binding specificity to APLNR, immunohistochemical (IHC) staining was performed on samples of the highly vascular disease, infantile haemangioma (IH). The expression and colocalisation of APLNR were compared to the standard endothelial cell marker CD34 and the vascular smooth muscle cell marker $\alpha$-smooth muscle actin ( $\alpha$-SMA).

Figure 3.11 Panels A - D show below shows the staining of APLNR (green) with CD34 (red) in early developing "proliferating” IH. Strong APLNR (Figure 3.11 Panel B) and CD34 (Figure 3.11 Panel C) IR can be seen throughout the tissue surrounding blood vessels. CD34 and APLNR expression appear to be co-localised around blood vessel structures as seen in Figure 3.11 Panel D outline with a white dotted line; auto-fluorescent erythrocytes may be seen inside the blood vessel. During "involuting” mid-stage development of IH more $\alpha$-SMA positive pericytes can be seen. Figure 3.11 Panel E - H show an involuting IH stained with APLNR (green) (Figure 3.11, panel F) and $\alpha$-SMA (red) (Figure 3.11, panels G). Colocalisation of $\alpha$ SMA and APLNR to blood vessels outlined with the dotted line can be seen in Figure 3.11, Panel H.

Strong APLNR IR seen in the blood vessels of IH samples indicating positive APLNR staining (Figure 3.11, panels B and F). Furthermore, APLNR colocalisation with both CD34 and $\alpha-$ SMA was seen on blood vessels (Figure 3.11, panels D and H. This data combined with the western blotting data shown above is strongly suggestive that the APLNR-Cterm antibody is binding the appropriate target. 



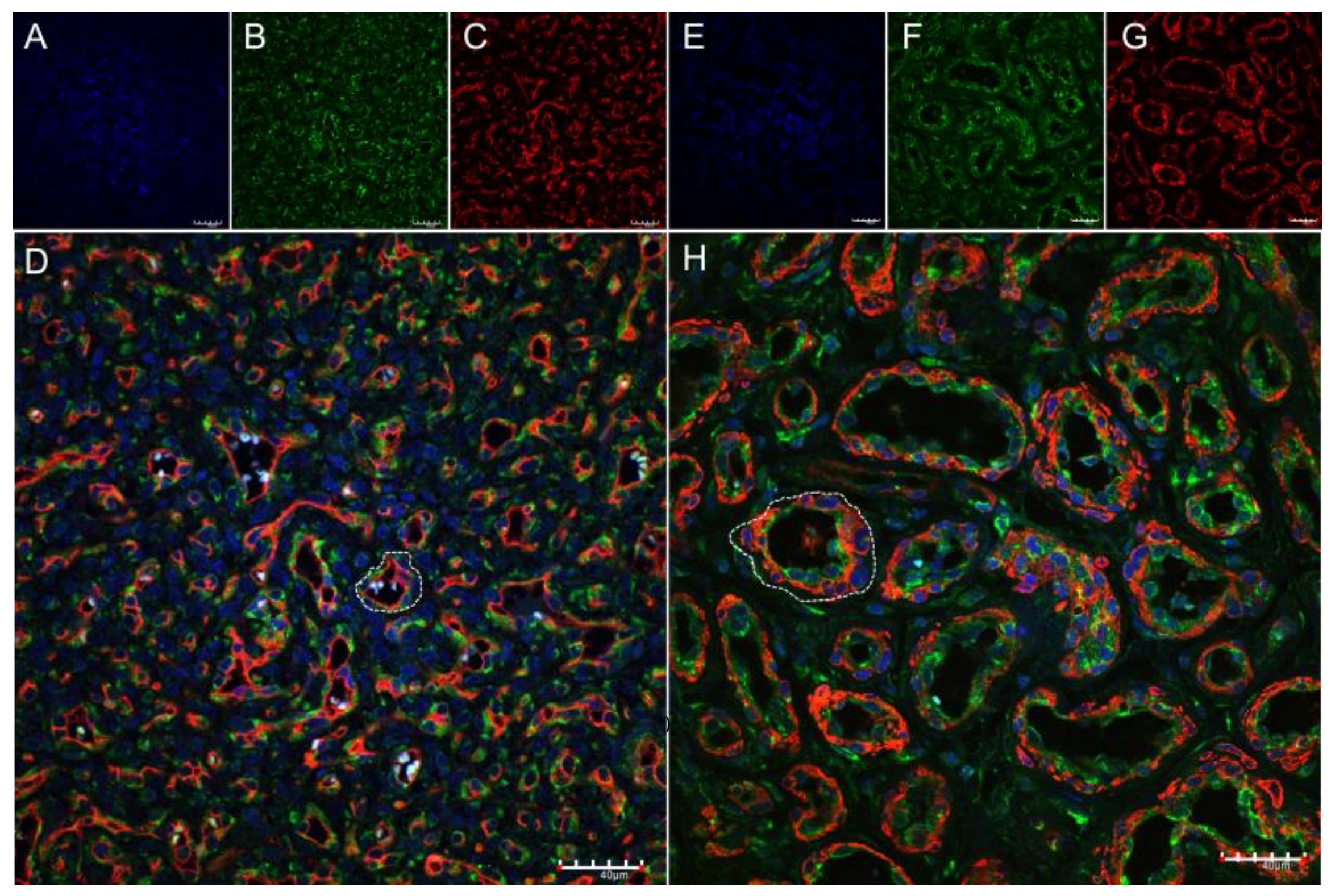


Figure 3.11. IHC analysis of infantile haemangioma (IH) samples for APLNR and blood vessel associated markers CD34 (endothelial cells) and alpha smooth muscle actin ( $\alpha$ -

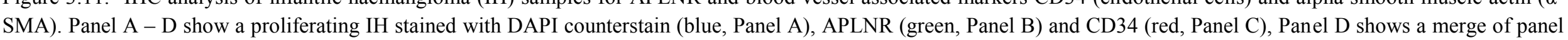

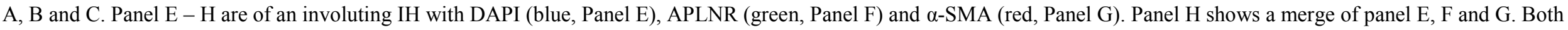
panel $\mathrm{D}$ and $\mathrm{H}$ show examples of blood vessels outlines with dashed white lines. All scale bars are $40 \mu \mathrm{M}$. 


\subsubsection{Analysis of APLNR expression in archival GL261 orthotopic xenograft tissue and tumour derived cell lines}

APLNR expression was assessed in several different glioma tissue types. The expression of APLNR was initially assessed in a sample of recapitulated human glioblastoma tissue. APLNR expression (green staining) can be seen in the recapitulated human glioblastoma tissue (Figure 3.12 panel A) as indicated by the green IR within the tumour tissue. A close-up image of Panel A is presented in Figure 3.12 panel B more clearly showing tumour cells expressing APLNR (tumour cells are identified counterstaining nuclei with DAPI (blue) and examining for nuclei size and the higher density of cell nuclei). It is of note that the APLNR expression is heterogeneous across the tumour.

Due to the unavailability of more xenograft tissue, APLNR expression was examined in the primary glioblastoma cell line 0906 and in the immortalized glioblastoma cell line U87MG. Both 0906 and U87MG showed IR for the APLNR receptor (Figure 3.12 panel C and E). Figure 3.12 panel $\mathrm{C}$ and $\mathrm{E}$ show that APLNR expression is predominantly perinuclear and not membrane localized as expected. Interestingly, the expression of APLNR was noted to be in the membrane protrusions and especially between the cell to cell connections of the U87MG cell line (Figure 3.12, Panel E) as indicated by the white arrows.

To clarify the expression of APLNR in tumour tissue samples, further IHC experimentation was required; however, due to a lack of orthotopic xenograft glioblastoma tissue as seen in Figure 3.12, panel A, expression of APLNR was further studied in the recapitulated GL261 glioblastoma model [304, 392]. 



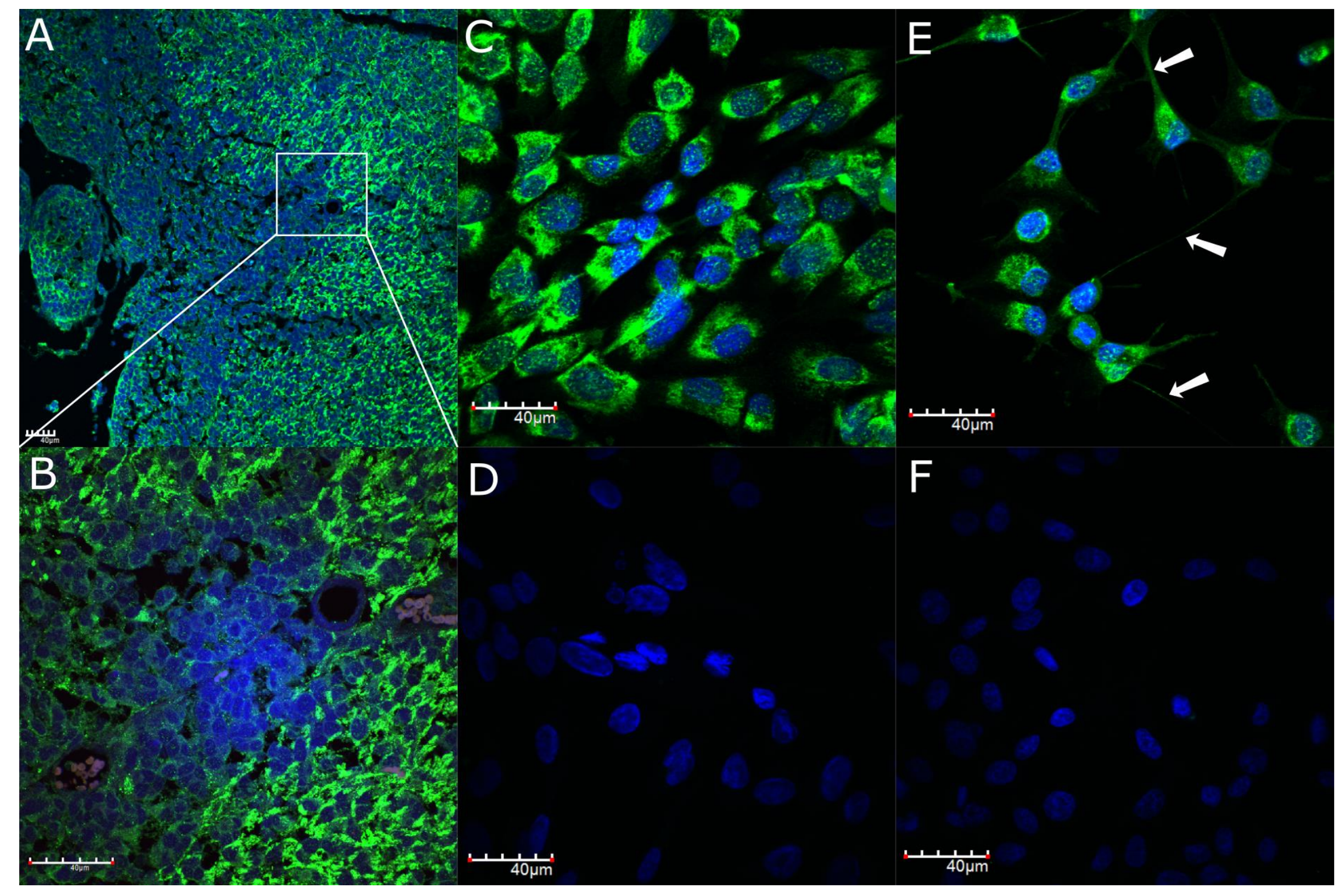


Figure 3.12. Examples of APLNR receptor expression in glioblastoma tissue samples. Panel A is a section of orthotopic xenograft glioblastoma tumour tissue which shows abundant APLNR protein expression (green) on the tumour cells. Panel B is a higher magnification image of the area outlined by the white square in Panel A and shows expression of APLNR (green) in greater detail in tumour cells. Panel C shows APLNR expression (green) in the primary glioblastoma cell line, 0906 and panel D shows the primary omitted control. Panel E shows the U87MG cell line stained with the APLNR-Cterm antibody (green), the white arrows indicate cell to cell contacts the express APLNR. Panel F shows the primary omitted control. In all panels, the nuclei are counterstained with DAPI (blue), and scale bars are $40 \mu \mathrm{M}$. 


\subsubsection{Immunohistochemical analysis of APLNR expression in control C57/BL6 brains}

To further ensure the specificity of APLNR IR in the murine brain, APLNR expression and localization was examined in select regions where there have been previous reports of APLNR expression. It was hypothesised that if localization of APLNR-IR was detected in these previously reported areas, it would confirm the specific APLNR antibody staining and increase confidence in any data generated when examining the expression of APLNR in GL261 tumour tissue samples.

The reports of APLNR expression in the brain are thoroughly reviewed in Chapter 1 Table 1.2. There are several areas where APLNR has been more consistently reported, and these areas were examined below. APLNR expression has been reported several times in the corpus callosum [41-44]. The corpus callosum is located above the pyramidal cell layer (white arrow Figure 3.13, panel A). APLNR expression was found in the corpus callosum Figure 3.13, panel A as indicated by the yellow arrow. The hippocampus has been reported in the past to express APLNR; the pyramidal cell layer forms part of the hippocampus, small amounts of IR (red) can be seen surrounding the pyramidal cell layer suggesting some hippocampal expression.

The piriform cortex has also been reported to have APLNR expression [41,393] which can be seen below (Figure 3.13, panel C), to the left of the fibrous tract indicated by the white arrow which is the end of the corpus callosum. APLNR appears to be localized to the radial glial projections several of which are exemplified by the dashed arrows (Figure 3.13, panel C). 



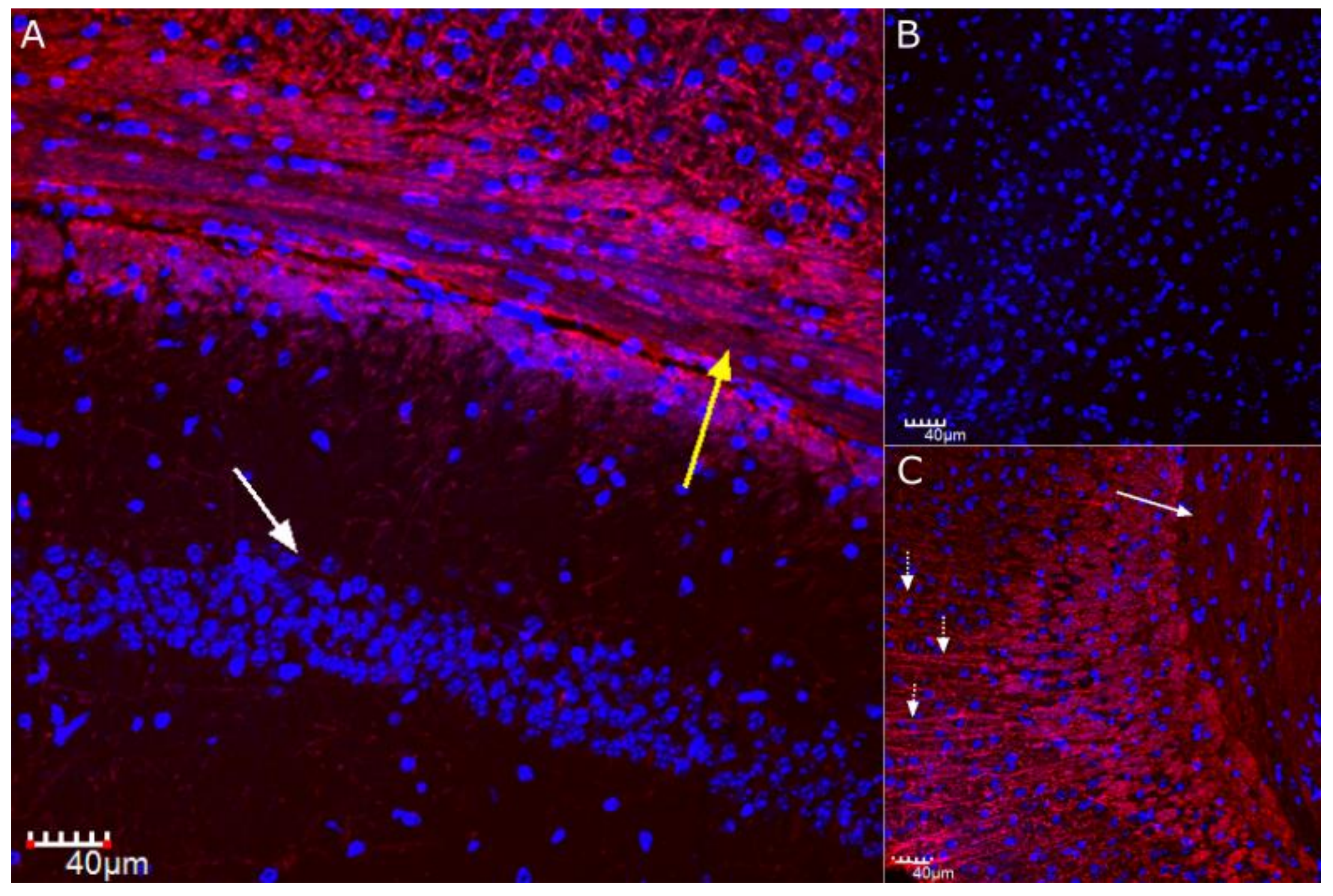


Figure 3.13. Expression of APLNR in the corpus callosum (Panel A) and piriform cortex (Panel C). Panel A shows APLNR IR (red) in the corpus callosum layer (indicated by a yellow arrow) and cortex beyond, but less expression around the pyramidal layer below (indicated by a white arrow). Panel B shows a primary antibody omitted control of the piriform cortex. Panel C shows APLNR expression in the piriform cortex to the left of the fibrous tract indicated by the white arrow. The piriform cortex is seen as striations on the left of the image with projections extending from the fibrous region on the right, into the cortex region on the left. The striations could be radial glia (denoted by the white dashed arrows). In all panels, the nuclei are counterstained with DAPI (blue), and scale bars are $40 \mu \mathrm{M}$. 
APLNR expression has also been reported in the medial habenular $(\mathrm{MH})$ nuclei region of the thalamus $[15,49]$. The $\mathrm{MH}$ is found on the border of the dorsal $3^{\text {rd }}$ ventricle, labelled as $\mathrm{MH}$ in Figure 3.14 and indicated with a black arrow.

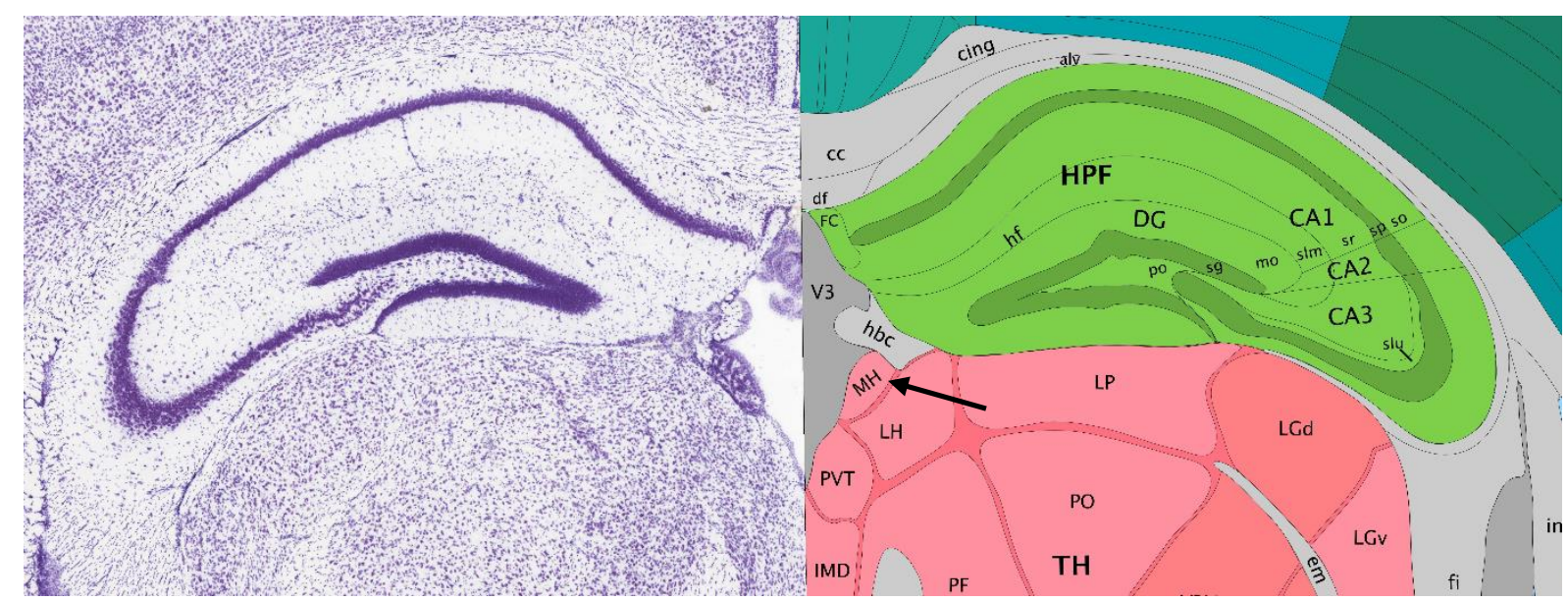

Figure 3.14. Example of the medular habenular region. The medular habenular (Labelled MH) region and indicated with a black arrow. The image was reproduced with permission from Allen Mouse Brain Atlas (2004). Available from: URL: http://mouse.brain-map.org.

The dorsal $3^{\text {rd }}$ ventricle is approximately outlined below (Figure 3.15, panel A) using a dashed white line. The white arrows in Figure 3.15, panel A show the MH region of the murine brain with APLNR expression (red). APLNR expression is lower in surrounding regions. A magnified image of the MH region (Figure 3.15, panel C) shows APLNR expression in detail, with a primary omitted control provided (Figure 3.15 , panel B). 



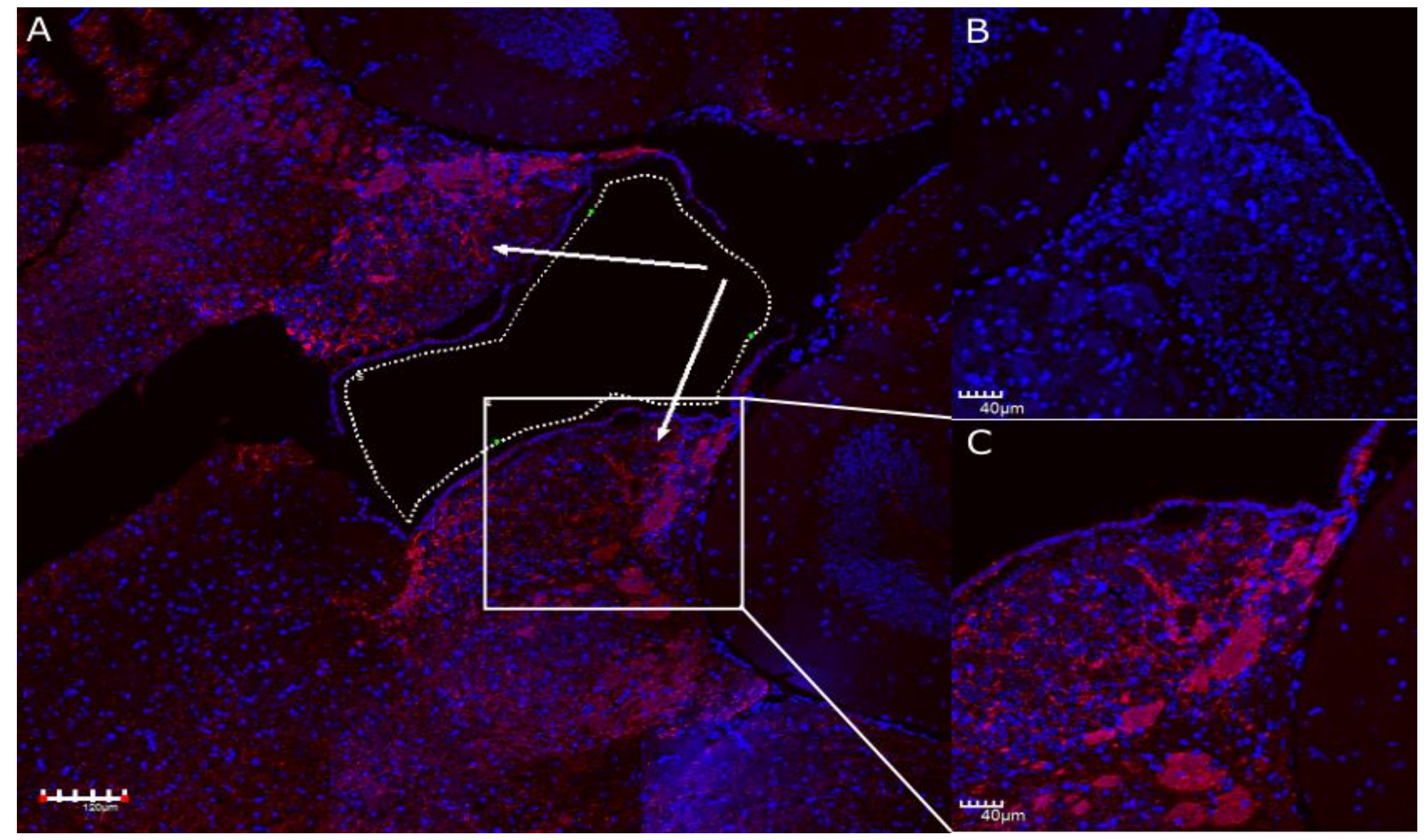


Figure 3.15. Medial habenular region APLNR expression in C57/BL6 tissue. Panel A shows a central brain section with the dorsal $3^{\text {rd }}$ ventricle outline with white dashed line. APLNR expression is shown in red and nuclei are counterstained with DAPI (blue). The medial habenular regions are indicated by the arrows. The scale bar is $120 \mu$ M. Panel $\mathrm{B}$ shows a magnified region of the medial habenular with the primary antibody omitted and nuclei counterstained with DAPI (blue). Panel C shows a magnified region of panel A (outlined with a white square) with APLNR expression (red) and the nuclei are counter stained with DAPI (blue). The scale bars for panel B and C are $40 \mu \mathrm{M}$. 


\subsubsection{GL261 tumour expression of APLNR}

To determine if APLNR expression was present in recapitulated GL261 tumours an IHC study of archival brains from C57/BL6 mice, cranially injected with GL261 cells was performed. Figure 3.13 and Figure 3.15 show APLNR expression in previously reported regions of the brain suggesting APLNR-Cterm antibody was specific and that the expression seen below is valid.

Initially all tumour sections were stained with haematoxylin and eosin to determine the cellular architecture and location of tumours, which were noted by the presence of common tumour features such as increase in the number of cell nuclei in a region, changes in cell nuclei size and alteration of the nuclear to cytoplasm's ration from $1 / 4: 1$ to $1: 1$. Following H\&E analysis, confocal microscopy was utilized to confirm localization of APLNR in the tumour. The GL261 cells were injected in the striatum which has been noted for its expression of APLNR [42, 49, 394] as such; expression would be expected surrounding the tumour.

Figure 3.16 displays tumours from three separate mice each micrographed at the tumour margin with a dashed line to delineate the approximate margin of the tumour which was located by looking for increased number and size of nuclei (stained by DAPI in blue) as an indicator of tumour cells. Figure 3.16 shows that there is no tumour expression of APLNR, but expression is present in the areas surrounding the tumour as indicated by the red IR. This lack of expression in the tumour was noted irrespective of where the image was taken, but APLNR expression can clearly be seen in the striatal fibre bundles outside of the tumour in Figure 3.16, panel B outlined with solid white lines. The tumour margin below the dashed line (Figure 3.16 panel C) exhibits a dull red which could potentially be due to a low level of APLNR expression, but this is unclear. 


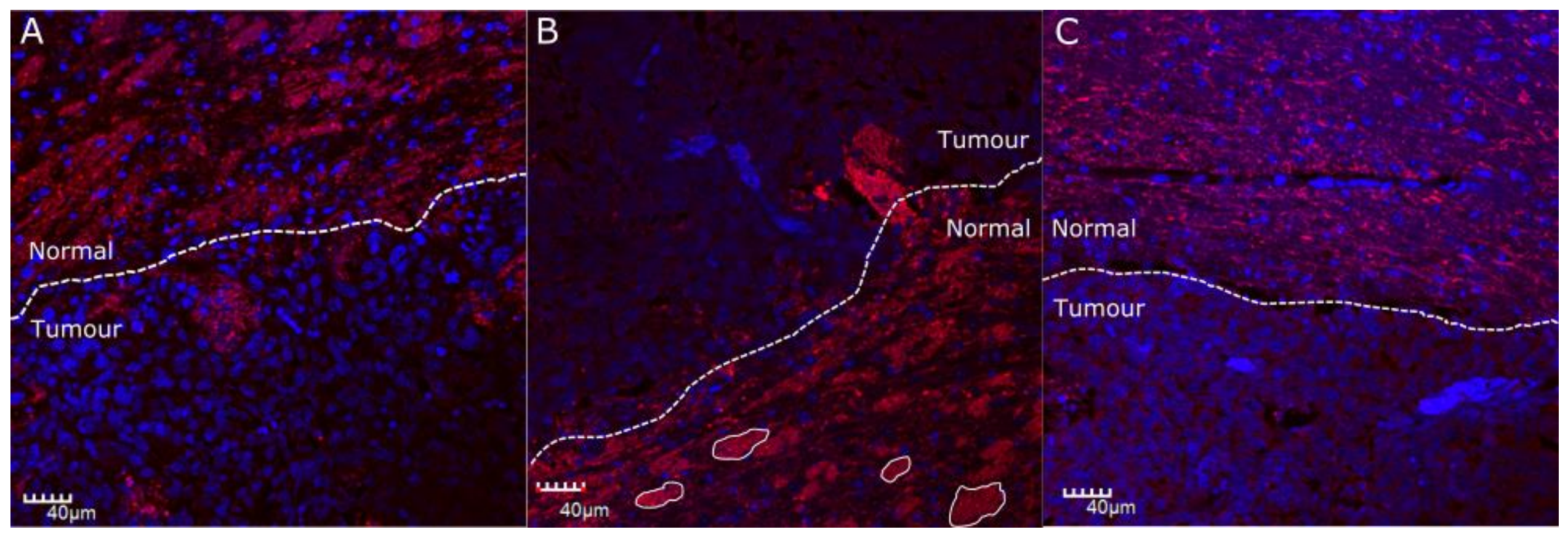

Figure 3.16. Expression of APLNR at the tumour margin of the orthotopically implanted GL261 tumours at 100x magnification. Panel A-C show APLNR (red) localization counterstained with DAPI (blue). The dashed lines indicate the approximate tumour margin which was identified by examining for larger numbers of nuclei with irregular sizing. The tumour size and normal side are labelled for each panel. Panel B also shows striatal axon fibre bundles outline with white lines. The striatum has been reported to express APLNR. All scale bars are $40 \mu \mathrm{M}$. 


\subsection{Discussion}

\subsubsection{Summary of results}

At the inception of this study, there was one report of the apelinergic system in glioblastoma. In addition, it was noted that the apelinergic system had roles in biological processes that could contribute to the pathophysiology of glioblastoma. Examples of these are, glucose uptake [20], blood vessel formation [19, 395], migration/invasiveness $[6,88,396]$ and is modulated by hypoxia [10]. Glucose uptake is essential to glioblastoma [397], aberrant microvasculature proliferation is ubiquitous in glioblastoma [398], the invasive/migratory nature of glioblastoma is well established [262], and hypoxia is a master regulator of glioblastoma [399]. This idea is summarised graphically in Figure 3.17 below. Taken in total, the expression of APLN and APLNR in glioblastoma reported by Kälin et al. [2] combined with the potential pathophysiological roles of the apelinergic system in glioblastoma, suggest the apelinergic system could have a role in glioblastoma

A crucial step to investigating the role of the apelinergic system in glioblastoma is understanding the mRNA expression of $A P L N$ and $A P L N R$ in glioblastoma. This apelinergic system has been reported to be upregulated in several cancers, but there is less than a handful of reports on its expression in glioblastoma, and of these reports none are comprehensive. Thus, the aim of this chapter was to characterise the expression of APLN and APLNR in glioblastoma and glioblastoma-derived tissues. 


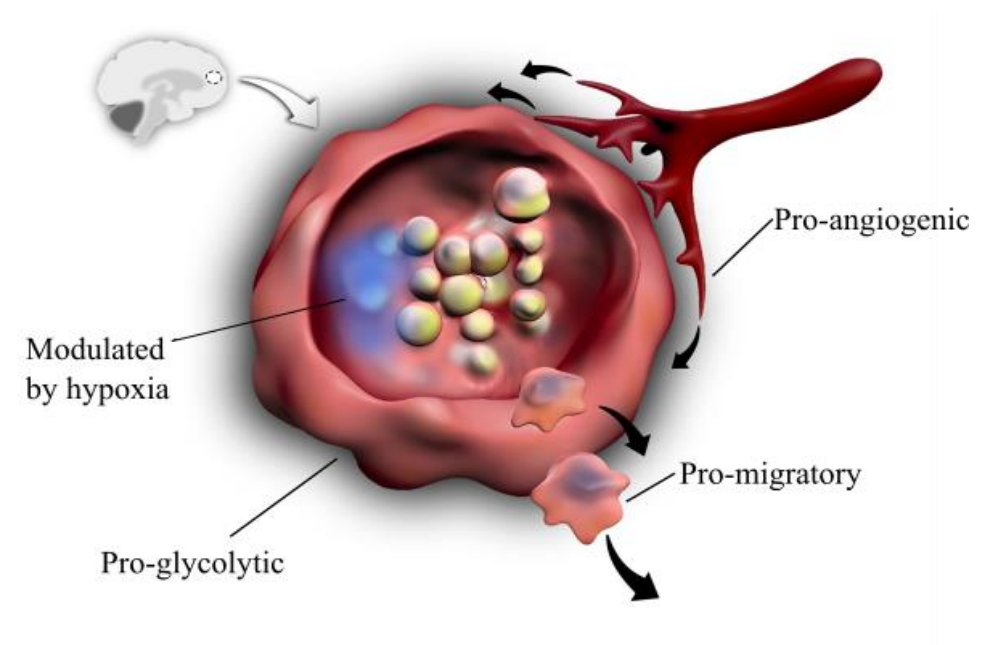

Figure 3.17. Summary of the roles of the apelinergic system in glioblastoma. The diagram demonstrates the potential physiological roles of the apelinergic system in glioblastoma. Apelin has been demonstrated to have pro-angiogenic and promigratory roles. Moreover, the apelinergic modulates glycolysis and is modulated by hypoxia. These processes are salient to glioblastoma pathophysiology and suggest the apelinergic system could have a role in glioblastoma biology. Figure illustrated by David Young.

\subsubsection{Analysis of $A P L N$ and $A P L N R$ mRNA expression in glioblastoma tissue and glioblastoma-derived cell lines}

It was hypothesised that $A P L N$ and $A P L N R$ mRNA expression would be elevated in samples of glioblastoma tissue derived from human patients in comparison to normal brain tissue, based on the report by Kälin et al. [2] that showed APLN and APLNR mRNA expression in the microvasculature and tumour cells surrounding necrosis in glioblastoma using in situ hybridisation [2]. Figure 3.1 showed $A P L N R$ and SLC2A1 mRNA expression was found to express equally and $A P L N$ mRNA expression was found to be increased in glioblastoma tissue over non-tumour human brain. This mRNA data had some limitations. The qPCR measurements performed in Chapter 3 examined the expression levels of APLN and APLNR mRNA in only a small sample of resected glioblastoma tissue and one sample control brain tissue. 
The second key assumption made by this study was that the HPRT mRNA expression level was an adequate normalisation control between different glioblastoma tissues and glioblastoma-derived cell lines. Some publications have reported that HPRT is valid as a housekeeping gene for testing mRNA expression in glioblastoma tissue samples [346], whereas others point to limitations in its use [400]. Ideally, a second housekeeping gene, such as TATA box-binding protein (TBP), could have been included with HPRT; however, due to the small amounts of tumour RNA available, only one housekeeping gene was selected for normalising the data.

To account for the low sample number public data RNAseq from the TCGA was analysed to account for these limitations. The data in Figure 3.3 corroborates the data shown in Figure 3.1 that there is an upregulation of $A P L N$ mRNA expression in glioblastoma. In addition, the data from Figure 3.3 shows that there is no difference in $A P L N$ or APLNR expression between glioblastoma subtypes. This was slightly unexpected as the presence of upregulated APLNR in cells surrounding necrosis reported by Kälin et al. [2]lead to the inference that APLNR may be upregulated.

There is currently no data describing the expression of $A P L N$ and $A P L N R$ in relation to various tumours in the category of diffuse astrocytoma and oligodendroglioma. During this project, it was identified that $A P L N$ expression is elevated in grade IV glioblastoma over the grade II and III tumours. In addition, APLNR expression was also elevated in grade IV over grade II and III tumours. It is unclear why $A P L N$ and $A P L N R$ are increased in comparison to lower grade tumours, but $A P L N R$ is not elevated when compared to normal tissue. Further analysis of control human brain tissue could enable a more clear understanding of endogenous APLNR expression in healthy brain tissue.

The increased $A P L N$ mRNA expression seen in glioblastoma that is not seen in lower grade glioma may be significant because the increased production of $A P L N$ could allude to an upregulation of apelinergic system signalling. The expression of APLN mRNA levels increases with the grade which is suggestive that upregulated $A P L N$ mRNA expression is due to features present in higher grade glioblastoma. This could be due to pathophysiological features such as 
hypoxia and aberrant microvasculature which are more prevalent in grade IV glioblastoma than the lower grade diffuse astrocytoma and oligodendroglioma.

It is unclear whether the upregulation of $A P L N$ in glioblastoma is due to the presence of blood vessels or hypoxia. $A P L N$ is well reported to be involved in angiogenesis [401], i.e. it has been noted to be expressed on endothelial cells in cancers such as breast cancer $[3,98]$ and $A P L N$ is also well reported to be upregulated by hypoxia $[3,6,10]$, To ascertain which of these physiological features contribute to APLN expression, the correlation between APLN and other genes was assessed. APLN and APLNR mRNA were compared to the expression of the glucose transporter gene $S L C 2 A 1$. Intriguingly, $S L C 2 A 1$ and $A P L N$ mRNA expression were found to correlate positively. Retrospectively, this gene may not have been the most optimal because both these genes are expressed in blood vessels $[66,362]$ and are modulated by hypoxia $[10$, 402]. However, because of limited sample quantities, multiple genes could not be used. To ascertain what causes the correlation of $S L C 2 A 1$ and $A P L N$ mRNA, public data was utilised to examine the correlation of $S L C 2 A 1$ and $A P L N$ with endothelial and hypoxia markers.

\subsubsection{The cause of $S L C 2 A 1$ and $A P L N$ correlation}

The correlation of SLC2A1 and APLN mRNA was present in glioblastoma and glioblastoma subtypes but not present in lower grade tumours. This could be ascribed to the increased presence of aberrant microvasculature or hypoxia. Therefore, the correlation of mRNA expression of the endothelial marker PECAMI and the hypoxia markers $C A 9$ and HIF $1 \alpha$ were compared with SLC2A1 and APLN.

The poor correlation of the hypoxia markers $C A 9$ and $H I F 1 \alpha(\mathrm{rs}=0.20, \mathrm{p}=0.0147)$ as well as between HIF1 $\alpha$ and the hypoxia modulated APLN and SLC2A1 was ascribed to the weak correlation of HIF $1 \alpha$ mRNA expression to hypoxia levels, which has been reported prior [391, 403]. Bache et al. [391] also reported that the genes CA9 and SLC2A1 correlated with several other hypoxia markers, but HIFl $\alpha$ did not. Though a reason for this was not given, it could be due to the post-translational regulation of HIF $1 \alpha$ by oxygen [404]. However, the assertion that SLC2A1 and CA9 are markers of hypoxia is valid. 
There was a moderate correlation seen between $S L C 2 A 1$ and $C A 9(\mathrm{rs}=0.61)$ which suggests that $S L C 2 A 1$ expression is more likely to be due to hypoxia. The weak correlation between $S L C 2 A 1$ and $A P L N(\mathrm{rs}=0.43$ ) and weak to moderate positive correlation between $A P L N$ and $C A 9$ (rs $=0.39$ ) suggest that the $A P L N$ increase could be due to hypoxia. Furthermore, there were weaker correlations between the endothelial marker PECAMI and APLN (rs $=0.35)$ and $S L C 2 A 1$ (rs $=0.05$ ) further substantiates the claim that the APLN SLC2A1 correlation is due to hypoxia. A correlation between PECAMI and APLN was expected, due to the apelinergic system's role in blood vessel formation $[19,405]$, but the lack of correlation between SLC2A1 and PECAM1 suggests the APLN SLC2A1 correlation is due to hypoxia.

Table 3.4. A summary of the correlations of APLN and SLC2A1 between hypoxic and endothelial markers.

\begin{tabular}{|c|c|c|c|c|}
\hline & CA9 & APLN & $S L C 2 A 1$ & PECAM1 \\
\hline CA9 & & & & \\
\hline APLN & $0.39 * * *$ & & & \\
\hline$S L C 2 A 1$ & $0.61 * * *$ & $0.43 * * *$ & & \\
\hline PECAM1 & $0.02 \mathrm{~ns}$ & $0.35 * * *$ & $0.05^{\mathrm{ns}}$ & \\
\hline
\end{tabular}

The correlation data is limited in that it is only suggestive of hypoxias role in APLN expression and does not show a direct causal link. A future experiment in this study will be to test the effect of hypoxia on glioblastoma-derived cell lines to confirm the effect of hypoxia on APLN mRNA expression. Without more detailed IHC orthotopic xenograft evidence showing the levels of APLN in the tumour colocalised to markers of hypoxia or vasculature it is difficult to confirm the cause of the SLC2A1 APLN correlation from mRNA; however, there is a strong suggestion that hypoxia is a key factor to be further investigated in this thesis. 


\subsubsection{Intratumoral expression of $A P L N$ and $A P L N R$ mRNA}

The mRNA expression of $A P L N$ and $A P L N R$ was assessed using the IVY GAP dataset in five intratumoral locations (Depicted in Figure 3.18). The APLN and APLNR mRNA expression of each region were compared then to the outermost region (leading edge or LE) of the tumour.

APLN expression was elevated in the microvasculature proliferations (MVP) and pseudopalisading cells surrounding necrosis (PAN); this corroborates literary reports [2]. The pseudopalisading cells surrounding necrosis are well known to be hypoxic $[175,205]$ as such $A P L N$ upregulation could be expected. Interestingly, $A P L N$ expression was also noted to be increased in the more centralised locations of the tumour (CT) when compared to the outside LE (Figure 3.8, Panel A). The CT predominantly contains 100-500: 1 tumour cells to normal cells thus suggesting that glioblastoma tumour cells express $A P L N$. Assessment of the hypoxia marker $C A 9$ revealed significantly higher expression in the CT and PAN but not in the IT or in MVP suggesting these regions are hypoxia and the elevated $A P L N$ expression may be due to hypoxia corroborating data described above.

$A P L N R$ expression varied in each region. There was a wide range of $A P L N R$ expression in the outermost region (LE) making comparisons to other regions difficult. Expression was statistically significantly elevated in the IT and MVP but not in the CT. Even more surprisingly, APLNR expression was not significantly different in pseudopalisading cells (PAN) compared to the LE which was reported in the literature [2]. This is suggestive that while APLN and $A P L N R$ are expressed in glioblastoma tumours further study in the localization and expression would be beneficial; especially by using techniques such as MALDI mass spectrometry imaging of glioblastoma tissue.

This data critically shows a potential dual role of APLN and APLNR being upregulated in both the MVP but also upregulated in tumour cells depending on the location. One of the most critical limitations of this data is that while overall trends of mRNA expression can be seen, the contribution of cell types to each region is not always clear. Different cell types may both simultaneously overexpress APLN (possibly due to hypoxia), or not express APLNR as was revealed during in the single cell RNAseq data (discussed further below). The heterogeneous 
nature of glioblastoma needs to be taken into account when considering the role of the apelinergic system in glioblastoma and this will require more in-depth analysis of $A P L N$ and APLNR expression in primary tissue samples.

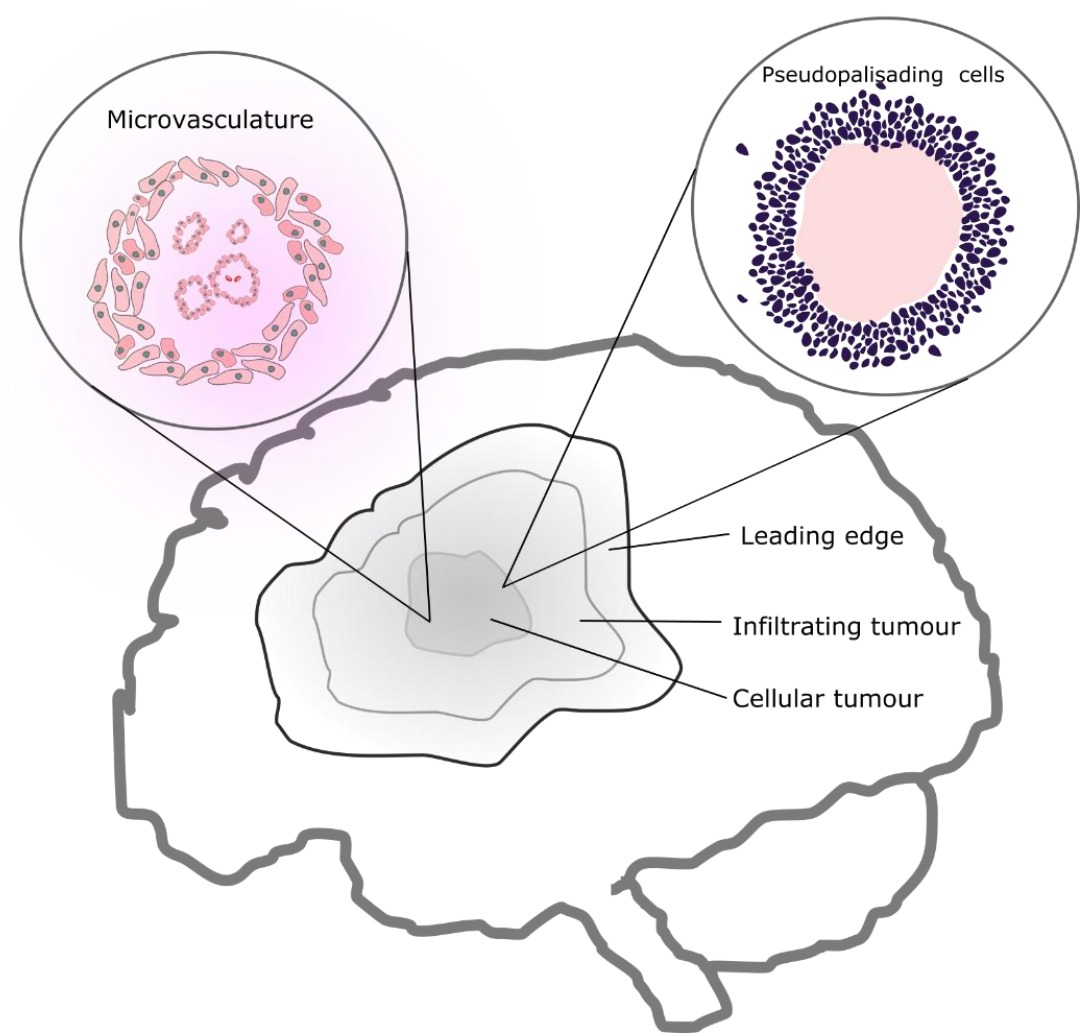

Figure 3.18. Regions assessed in the IVYGAP dataset. The brain outline contains a tumour with three different areas of the tumour, the leading edge, infiltrating tumour and cellular tumour. The features of microvasculature proliferations and pseudopalisading cells around regions of necrosis (labelled pseudopalisading cells are found centrally in the tumour but can vary. 


\subsubsection{Single cell mRNA expression of $A P L N$ and $A P L N R$}

Single Cell RNAseq data available publicly were analysed and surprisingly showed elevated, cell-specific expression of APLNR in astrocytes, tumour and vascular cells. While the upregulation of $A P L N R$ was somewhat expected based on IVY GAP data from above and literary evidence of the role of the apelinergic system in angiogenesis; the elevated expression of $A P L N R$ in astrocytes and tumour cells was intriguing and points to directions of future research. This data could lead to the hypothesis that only specific cells in the tumour may respond to secreted APLN. APLN was upregulated in several cell types including neoplastic cells, vascular cells, neurons, oligodendrocytes and astrocytes. Furthermore, single cell RNAseq data showed the tumour core had upregulated APLN mRNA expression; it's important to note that Darmanis et al. [386] also found significantly elevated expression of a range of hypoxia markers in the tumour core. This suggests that APLN expression in the tumour core may be regulated to some extent by hypoxia. The expression APLN in numerous cell types but specific overexpression of $A P L N R$ to particular cell types suggests that while several cells may secrete APLN, only certain cells may respond to APLN.

\subsubsection{The mRNA expression of the APLNR receptor in tumour-derived cell lines and immortalized cell lines}

To assess which cell lines expressed $A P L N$ and $A P L N R$ to comparable levels as seen in glioblastoma tissue, the mRNA expression of $A P L N$ and $A P L N R$ was measured in several primary and immortalised glioblastoma-derived cell lines. This could help determine which would be an optimal model of glioblastoma. APLN and APLNR mRNA expression in resected tumour tissue were measured at 100-fold higher than the primary cell lines and approximately $100-1000$ fold higher than immortalised cell lines. This loss APLNR and APLN was seen in all in vitro glioblastoma cell lines and may be ascribed to a combination of factors including removal of tissue from the specialised tumour microenvironment [406] and subsequent serumbased in vitro culture which has been reported to occur [407, 408]. This loss of expression of cancer-related genes due to cell culture is also not uncommon. For example, the 
overexpression of the endothelial growth factor receptor (EGFR) [409], has been reported in $40 \%$ of glioblastoma and of those, approximately $70 \%$ have variants of the EGFR receptor [410]. In vitro cell culture reduces this EGFR amplification [327]. The loss of salient gene expression is detrimental to making accurate biological hypotheses about cancers [411]. A critical future experiment will be to determine the expression of APLN and APLNR in recently patient-derived cell lines, followed by recapitulating the tumour in SCID/NOD mice and under in vitro culture conditions to see which maintains APLN and APLNR expression.

One of the primary or immortalised cell lines examined during this chapter was intended to be used as an in vitro model of glioblastoma for future studies in this thesis. However, none of the cell lines expressed $A P L N R$ in equivalent amounts to tumour tissue. Artificially increasing the $A P L N R$ expression to replicate what is measured seen in normal and tumorous tissue could be an appropriate recourse. The low expression of $A P L N$ is less detrimental to this study as this can be exogenously added at another time. The lack of receptor expression would potentially mean that the apelinergic system signalling pathway is not as active as in primary tissue and would not respond to exogenous APLN treatments in a sufficient manner. For future experiments artificially increasing the $A P L N R$ receptor expression via stable transfection may prove beneficial.

\subsubsection{Protein expression of APLNR in glioblastoma models}

\subsubsection{Western blot verification of APLNR antibody}

Two anti-APLNR antibodies were assessed using western blotting to ensure target specificity. The APLNR-Nterm antibody was found to have no immunoreactivity (IR) in the U87MG and HEK293 lysate but yielded the appropriate band in mouse heart (Figure 3.10). This was ascribed to the APLNR-Nterm antibodies being raised and verified for murine tissue and not the human cell lines used. Thus, the APLNR-Nterm antibody was not used.

The APLNR-Cterm antibody produced the manufacturer's expected band at $58 \mathrm{kDa}$. However, there was a second band for the APLNR receptor in the analysed cell lines. This was 
hypothesised to be due to glycosylation [42] which has also been reported by Puffer et al. [50] who demonstrated that the APLNR receptor has two N-glycosylation sites that yield different banding patterns depending on the glycosylation [50]. It was concluded that the APLNR-Cterm antibody was binding to the appropriate target and thus was carried forward for further validation of antibody specificity. The post-translational modification of the APLNR have been noted prior and have been hypothesised to affect the function of the APLNR [15]; this is an important area of future research but was beyond the scope of this study.

APLNR has been extensively reported to localise to blood vessels [2, 412, 413]. As such, to further confirm APLNR specificity, samples of the highly vascular infantile haemangioma were used to determine if the expression of APLNR was localised to blood vessels. APLNR IR localised with $C D 34$ and $\alpha-S M A$ IR, two markers commonly associated with blood vessels showing APLNR is appropriately associated with blood vessels suggesting the APLNR-Cterm antibody was binding to APLNR.

Finally, to validate the APLNR-Cterm antibody in the murine brain, the IHC expression of APLNR was investigated in regions of the murine brain where APLNR expression had been previously reported. APLNR IR was noted in the corpus callosum, hippocampal region and piriform cortex. This suggested APLNR specific binding.

It was concluded that the western blot data in conjunction with localisation of APLNR IR in the murine brain and infantile haemangioma tissue suggested the APLNR-Cterm antibody was specific to APLNR and validates the use of it in this study.

\subsubsection{APLNR expression in orthotopic xenograft glioblastoma tissue and tumour-derived cell lines}

APLNR protein expression was assessed in several glioblastoma tissue sources. APLNR IR was seen in orthotopic xenograft glioblastoma tissue (Figure 3.12, Panel A), but was more widely expressed than as reported by Kalin et al. [2], this may be ascribed to the differences in the orthotopic xenograft glioblastoma model and the murine GL261. The discrepancy in 
expression could also be due to the differences in technique, Kälin et al. [2] utilized in situ hybridisation. Ultimately without more tissue samples, further conclusions could not be made.

The APLNR protein was also assessed using immunocytochemistry (ICC) in glioblastoma tumour derived lines (Figure 3.12, Panels $\mathrm{C}$ and $\mathrm{E}$ ). There appears to be more perinuclear APLNR localization than membrane-bound as reported HUVECS [373]. This is important as lack of expression of the APLNR receptor on the membrane may limit signalling through the apelinergic system which could impact later functional studies.

Despite comparatively lower mRNA expression of $A P L N R$ than glioblastoma tumour samples, both 0906 and U87MG showed IR for the APLNR receptor. However, ICC is not a quantitative methodology, and as such, it is difficult to relate the IR seen in Figure 3.12 to mRNA expression seen in of APLNR in the same cell lines. ICC also is limited in that provides no information about the cell signalling or functional activity of the APLNR receptor which is essential for apelinergic system signalling.

There is a potential lack of APLNR signalling as evidenced by predominantly intracellular APLNR expression and low mRNA expression of APLNR. This suggests that artificially upregulating the APLNR receptor to ensure maximal apelinergic system signalling would beneficial when trying to determine the role of the apelinergic system in glioblastoma.

\subsubsection{The expression of APLNR in the GL261 model of glioblastoma}

APLNR levels were assessed in the orthotopic glioblastoma model GL261 to see whether GL261 tumours express APLNR and if APLNR IR localizes in the GL261 tumour to previously reported areas such as microvessels or regions around necrosis [2]. Despite finding APLNR expression in western blotting, control IH samples as well as in previously reported C57/BL6 mice brain regions, and glioblastoma-derived cells lines, tumour expression of APLNR was not detected in GL261 burdened mice brains.

APLNR antibody binding and expression were confirmed using several different formats suggesting a lack of APLNR IR in GL261 tumours was not necessarily due to technical issues. 
The lack of APLNR in the tumour could be ascribed to the GL261 cells low expression of APLNR mRNA and once injected into the murine brain continued to maintain low expression of APLNR. This would also corroborate with the positive APLNR expression seen in orthotopic xenograft glioblastoma tissue (Figure 3.12, Panel A) as the more recently isolated tissue would likely express higher levels of APLNR. This suggests that once tissue samples are removed from the tumour region, they may require special culture conditions to maintain expression of APLNR.

In the future, if an in vivo models is intended to be used, freshly derived cell lines or a cell line stably expressing APLNR may be beneficial to the further study of the role of the apelinergic system in glioblastoma. Future experiments would include, verification antibody binding via immunoprecipitation and mass spectrometry in tandem with further investigation of APLN and APLNR expression in orthotopic xenograft models.

\subsubsection{Future directions}

Due to unavailability of primary patient-derived tissue to generate orthotopic xenograft tissue tumours, it was difficult to fully characterise the expression of the APLN and APLNR in clinically representative samples. In the future, utilizing patient-derived samples to recapitulate tumours in SCID- NOD mice would be highly beneficial for further IHC studies. One example of this would be using orthotopic xenograft tissue to determine the localisation of APLNR and APLN expression in the varying regions of the tumour and correlating this to other markers; i.e. in the hypoxia markers such as $C A 9$ and blood vessel markers like $C D 34$. Furthermore assessing expression of APLN and APLNR in different cell types using IHC and examining for colocalisation of cell-specific markers such as the astrocyte marker Glial fibrillary acidic protein (GFAP) [414] and comparing this to APLN and APLNR expression.

One of the key limitations of this chapter was the predominantly mRNA based analysis of expression. It is well known that the levels of mRNA detected do not necessarily correlate with the protein expression [415-421]. If there had been more available xenograft or glioblastoma tumour tissue that retained high levels of APLNR and APLN expression, further 
immunohistochemical or western blotting characterisation of the expression and localisation of APLNR and APLN proteins would have been performed. This would enable a better understanding of the expression of the apelinergic system in glioblastoma.

If more patient samples were available an in-depth characterisation of the cell populations in these patient samples could also be performed using a fluorescence assisted cell sorter, isolating populations such as the glioblastoma stem cells. These population of cells are known to be present in glioblastoma [422], and it would be interesting to analyse the expression of APLNR or APLN on these cancer stem cells or if the apelinergic system expression was ubiquitous to all cells in the tumour.

It would be useful to examine the role of other apelinergic system peptides in glioblastoma since it is not known if the different apelinergic system peptides have different roles in glioblastoma tumour biology or cancer biology as a whole. The recently characterised apelinergic system peptide ELABELA [40] was briefly investigated during this thesis. Initially described in Chapter 1, ELABELA has been reported to have a significant role in early embryonic development [33, 85, 86] and has also been implicated in ovarian cancer [396]. Preliminary investigation in the present study (Chapter 8, Section A.4) demonstrated a low abundance of ELABELA mRNA expression in resected glioblastoma tissue with similar low expression levels in both primary cell lines and established immortalised cell lines. The low expression of ELABELA mRNA in resected glioblastoma tissue led to ELABELA research being classified as low priority for this current study but in the future may be worthy of further investigation.

\subsubsection{Choosing a cell model for investigation of the apelinergic system in glioblastoma}

The primary goal of this thesis was to investigate the role of the apelinergic system in glioblastoma. It is imperative that APLNR expression be comparable to levels measured in the original tumour so when the apelinergic system is modulated through the addition of exogenous APLN or blocking with an antagonist, a response is seen. All the cell lines tested during this 
chapter demonstrated a significant decrease in $A P L N$ and $A P L N R$ receptor mRNA expression when compared to resected glioblastoma tissue. There are several ways to remedy this for future experimentation.

One method would be to isolate fresh tumour cell lines from biopsied glioblastoma. There are some literature reports that suggest that more complicated and costly methodology may be used to propagate features seen in original tumours if good faithfulness to the original tumour phenotype is needed [329], this has been reported in glioblastoma tumour cells [313]. However, due to a lack of primary tissue with elevated $A P L N R$ and $A P L N$, this was not possible. Alternatively, a cell line consistently expressing elevated levels of the APLNR receptor would be beneficial. This could be an immortalised glioblastoma cell line stably transfected with the APLNR receptor to mimic the elevated expression of APLNR seen in tumours. Though an immortalised line will have potential limitations with regards to the changes induced by stable expression of the protein.

The choice of which cell type to upregulate with APLNR is difficult. Kälin et al. [2] demonstrated APLNR expression in tumour cells as well as microvasculature cells. The orthotopic xenograft data (Figure 3.12), as well as the IVY GAP data (Figure 3.8), also suggest there is a strong tumour cell expression of $A P L N R$. There is already substantial evidence to suggest the role of the apelinergic system is critical to tumour vascular biology, but there has been much less research on glioblastoma tumour cells. This suggests a glioblastoma tumour cell may be more advantageous to upregulate $A P L N R$ expression. Thus, a glioblastoma cell line such as U87MG with abundant $A P L N R$ would be best for in vitro investigations of the apelinergic system in glioblastoma.

\subsubsection{Final conclusions}

The research in this chapter indicates that mRNA expression of APLN but not APLNR is upregulated in glioblastoma. This was corroborated by publicly available datasets. In addition, $A P L N$ and $A P L N R$ expression were higher in grade IV glioblastoma than lower grade tumours. This suggests the apelinergic system may have a role in glioblastoma. Significantly, the 
elevation in $A P L N$ expression was noted in hypoxic regions of glioblastoma tissue and was hypothesised to be due to hypoxia modulating $A P L N$ which has been previously reported for other cell lines. This relationship will be further investigated later in this thesis.

This chapter also showed that $A P L N R$ and $A P L N$ expression is decreased in primary derived and immortalised cell lines suggesting that resection and in vitro culture reduces expression of $A P L N$ and $A P L N R$. Furthermore, this indicates a cell line expressing abundant APLNR like the parent glioblastoma tumour is critical for future experiments in this study. I suggest a basic transfected immortalised cell line model significantly upregulating the APLNR receptor will create an appropriate in vitro cell line for a study into the role of the apelinergic system in glioblastoma. This could also be used in conjunction with primary cell lines. This would allow for the initial characterisation of the role of the apelinergic system in glioblastoma and identify future research targets that could be further examined in more clinically relevant models in the future. 


\section{Chapter 4 Creation of an apelinergic system model in glioblastoma}

\subsection{Introduction}

\subsubsection{The selection of an in vitro model of the apelinergic system glioblastoma}

In the previous chapter (Chapter 3), it was shown that expression of $A P L N$ mRNA was higher in glioblastoma, compared to healthy brain tissue samples and, APLNR and APLN mRNA were positively correlated with grade of diffuse astrocytic and oligodendroglial glioblastoma tumours. This suggests that the apelinergic system is upregulated as glioblastomas develop and progress from less to more aggressive sub-types. Studies that have previously investigated the role of the apelinergic system in cancer biology have utilized several methods including immortalized cell lines [7], stably expressing cell lines or the derivation of primary cell lines from patient tissues which have then been either studied in vitro or injected into mice $[3,4,87$, 225]. However, currently there is no well-defined model for studying the apelinergic system in glioblastoma, and in particular the pro-migratory role of the apelinergic system in glioblastoma cells.

Results from the previous chapter also revealed that mRNA expression levels of APLNR and $A P L N$ were markedly lower in primary and immortalised cells derived from glioblastoma, compared to that in glioblastoma tissue samples. This suggests that the resection and 
subsequent in vitro culture of glioblastoma cell lines results in a reduction in the expression of the APLNR and APLN mRNA. This observation is concerning because it is possible that these cells do not adequately represent the tumour cells which are present in situ and are less likely to respond to modulation of the apelinergic system when tested.

The reduction of gene expression due to resection and in vitro culture has been well documented [423]. While other methods exist for maintaining expression of these genes in in vitro systems, they rely on the use of expensive mediums, supplements and the availability of fresh tissue samples. These are not always readily available, and primary cell lines are also difficult to obtain and often undesirable due to their inability to be readily transfected [424]. Therefore, a glioblastoma cell line that has been stably transfected with the APLNR gene may provide a more representative cell line of glioblastoma in order to test the apelinergic system.

\subsubsection{Chapter Aims}

The primary aim of this Chapter was to develop a glioblastoma cell line that over-expresses the APLNR gene for the purpose of investigating the role of the apelinergic system in glioblastoma. Following generation of the stably-transfected cell line, the aim was then to characterise the cell line to verify a similar activation of the apelinergic system pathway and localisation of $A P L N R$ receptors in glioblastoma as reported in the literature. These cell lines would then be used to examine the effects of the apelinergic system on cell migration in glioblastoma-derived cells.

Thus, the specific aims of this chapter were to develop and characterise a stable glioblastoma cell line that is responsive to the apelinergic system and use this cell line to APLNR examine the migratory role of APLN in glioblastoma-derived cell lines. 


\subsection{Methods}

\subsubsection{Transfection}

During this chapter, a commercially purchased plasmid from Origene (discussed in full in the general methods Chapter 2, Section 2.7) was stably transfected into the U87MG glioblastoma cell line and the Human embryonic kidney 293 (HEK293) cell line. The transformation and propagation of this plasmid in E. coli are described in detail in Chapter 2, Section 2.5. The transfection protocol is described below.

All transfections were performed with Lipofectamine 3000 transfection reagent (Invitrogen, Thermofisher Scientific, Auckland, NZ) as per manufacturer's instructions. The only deviation from manufactures protocol was a reduction of incubation time with Lipofectamine and DNA to $5-8$ hrs. The transfection protocol is as follows. Cells were plated and allowed to reach approximately 60 - 70\% confluence. P3000 reagent and $0.5 \mu \mathrm{g}$ of APLNR-GFP plasmid DNA were diluted in Optimem medium. Lipofectamine was diluted in Optimem medium and vortexed for $2-3 \mathrm{sec}$. The Lipofectamine and DNA /p3000 mix were combined and incubated for $5 \mathrm{~min}$ at $25^{\circ} \mathrm{C}$. Cells to be transfected were washed with base DMEM medium and replaced with cell culture medium supplemented with $10 \%$ FBS before transfection to remove penstrep. Cells were left for 5 - $12 \mathrm{hrs}$ in transfection medium depending on the cell line and then switched to complete cell medium without Lipofectamine/DNA and left for $24-48 \mathrm{hrs}$ before subsequent manipulation to generate the stable cell line. 


\subsubsection{G418 selection of cell lines}

G418 (Geneticin sulphate) (Fisher bioreagents, Thermofisher Scientific, Auckland, NZ) was used to select for transfected cells as the plasmid carries a neomycin resistance cassette. A concentration-response assay was generated for each cell line to ascertain the minimal G418 concentration that would kill untransfected cells. Cells were cultured in penstrep free media supplemented with between 0 and $1400 \mu \mathrm{g} / \mathrm{mL}$ of G418. Cell health was qualitatively assayed using light microscopy to look for evidence of rounding and loss of adherence to the culture surface. U87.APLNR cells are maintained with geneticin at $400 \mu \mathrm{g} / \mathrm{mL}$; geneticin is removed prior to use in experiments.

\subsubsection{Gene expression analyses}

To confirm the stable expression of APLNR mRNA in these cell lines, endpoint (RT-PCR) and Reverse transcription quantitative PCR (RT - qPCR) were performed. RT - qPCR was performed as described in Chapter 2, Section 2.2. RT - PCR was performed as per RT-qPCR with modifications to the primers used and the addition of a $3 \%$ agarose gel. Three pairs of primers were designed to the three regions of the APLNR-GFP transcript as seen in the schematic below (Figure 4.1). This was done to ensure that a complete transcript was integrated, and mRNA was being generated. To ensure the transcripts generated were specific to the targeted regions products were examined on a $3 \%$ low melting point agarose gel in $1 \mathrm{x}$ TAE. The ladder used was a GeneRuler low range ladder from Thermofisher Scientific. 


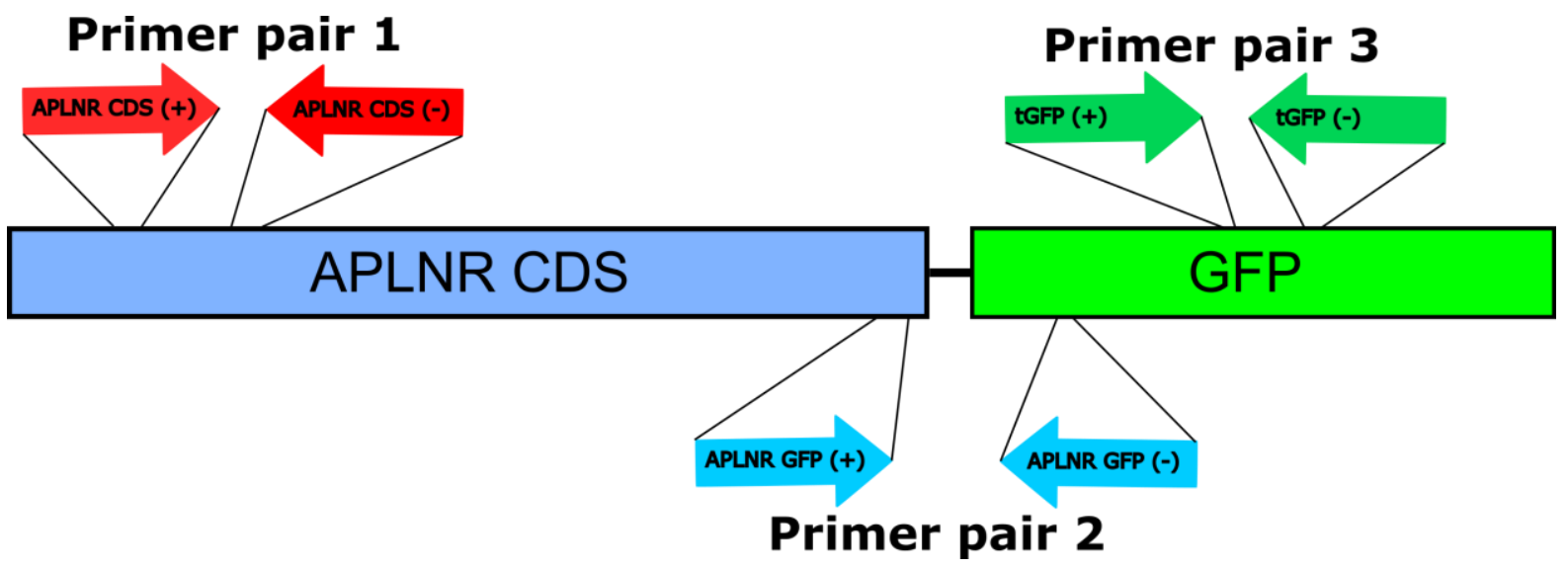

Figure 4.1. Schematic showing the areas of binding of the three pairs of APLNR-GFP directed primers. The $A P L N R$ coding sequence (APLNR-CDS) region is depicted in blue and shows the region of the transcript that codes for the $A P L N R$ receptor. The area in green labelled GFP shows the region coding for the GFP portion of the transcript. The three primer pairs are directed to both ends of the transcript and the region between (Primer pairs 1 - 3). Primer sequences are found in Chapter 2 Table 2.2 and are named as follows, primer pair 1: APLNR.Human.CDS, primer pair 2: APLNR.GFP and primer pair 3: tGFP.

\subsubsection{Testing literary reported apelinergic system signalling pathways}

During this Chapter, stable cell lines expressing an APLNR-GFP fusion protein were generated. However, it was unclear if the fusion protein would inhibit downstream signalling pathways. The APLNR receptor is complex, with several signalling pathways reported; such as, classical GPCR subunits $\mathrm{G \alpha}_{\mathrm{q} / 11}[56]$ and $\mathrm{G \alpha}_{\mathrm{i} / \mathrm{o}}[57,425]$ but also demonstrating receptor internalization which has been reported to be mediated through beta-arrestin [59]. As such the effect of exogenous [Pyr ${ }^{1}$ ]apelin-13 on APLNR-GFP internalisation was measured by flow cytometry and fluorescence microscopy. The apelinergic system has been reported to activate the MAP kinase pathway through $\mathrm{G} \alpha_{\mathrm{i}}[52,60]$. Treatment of $A P L N R$ expressing cells with APLN is known to activate the Extracellular Signal-regulated Kinase (ERK) pathway, resulting in an increase of phosphorylated ERK (pERK) [4, 46, 52, 267]. Activation of the APLNR-GFP receptor in HEK293.APLNR and U87.APLNR was measured by western blotting of phosphorylated ERK. 


\subsubsection{Flow cytometry}

Changes in fluorescence were measured using flow cytometry. HEK293.APLNR and U87.APLNR cells were treated with $2 \mu \mathrm{M}\left[\mathrm{Pyr}^{1}\right]$ apelin-13 overnight to determine if $\left[\mathrm{Pyr}^{1}\right]$ apelin-13 induced a change in surface receptor expression. Briefly, cells were plated in a 6 well plate at 80,000 - 100,000 cells per well and allowed to adhere overnight. Cells were then treated with $\left[\mathrm{Pyr}^{1}\right]$ apelin-13 at a $2 \mu \mathrm{M}$ concentration or vehicle in low serum medium $(1 \%$ FBS, 1\% Penicillin-streptomycin), then trypsinised and prepared for flow cytometry as previously described in Chapter 2, Section 2.8.1.

\subsubsection{Fluorescence Microscopy}

Cells were assessed on a confocal microscope as per Chapter 2 (2.1.3). The internalisation of the APLNR-GFP receptor was also assessed using fluorescence microscopy. Photos of HEK293.APLNR and U87.APLNR cells were taken on an Olympus IX51 inverted microscope (Olympus, Hamburg, Germany) fitted with phase contrast and fluorescence objectives and a DP70 digital camera. After which cells were treated as above (4.2.4.1) and micrographed after overnight incubation.

\subsubsection{Western blotting}

The apelinergic system modulates the MAP kinase pathway. ERK phosphorylation (pERK) was used as a measure of this activation. To achieve maximal pERK activation, U87MG, U87.APLNR and HEK293.APLNR cells were serum-starved overnight, after which they are treated with [Pyr $\left.{ }^{1}\right]$ apelin-13 $(1 \mu \mathrm{m})$ diluted in serum-free medium, cells were treated for $5 \mathrm{~min}$. Cells were lysed using RIPA (Appendix, B.1.18) supplemented with phosphatase inhibitor cocktail 2 (Cat \#: p5726, Sigma Aldrich, Auckland, NZ) and protease inhibitor cocktail (Cat \#: p8340, Sigma Aldrich, Auckland, NZ). All protein samples were normalised first to cell density and then by protein concentration as quantified by the BCA assay (as per Chapter 2, Section 2.9.2). Generic western blot methodology is fully described in Chapter 2, Section 2.9. 


\subsubsection{Migration assays}

The positive effect of exogenous [ $\left.\mathrm{Pyr}^{1}\right]$ apelin-13 treatment on cellular migration is well established in literature $[4,88,200,267,426]$ and was utilized as an output to test the validity of stable cell lines generated during this Chapter as models of glioblastoma. Migration assays are described in depth in Chapter 2, Section 2.3.2. Transwell assays were performed on U87MG and U87.APLNR cells as described in the literature [354, 427]. The full protocol is described in the Chapter 2, Section 2.3.2.2. Wound healing assays were performed with 0906, U87MG and U87.APLNR. The full protocol is described in the general methods Chapter 2, Section 2.3.2.1.

\subsection{Results}

\subsubsection{The creation of cell lines stably expressing $A P L N R$-GFP}

To develop a stably transfected cell line, the U87MG was transfected and alongside the readily transfectable HEK293 cell line as a control cell line. Initial transfections of U87MG yielded high toxicity after $24-48 \mathrm{hrs}$ incubation. The recommended manufacturer's transfection protocol was modified to decrease the transfection time from overnight to $5-8 \mathrm{hrs}$. The reduction in transfection time revealed that whilst the transfection rate appeared to be slightly lower, the cells did not take on the rounded "halo-like" appearance of those that were incubated overnight (Figure 4.2), this phenotype is an indicator of sick cells and would often die 2 - 3 days post-transfection. 


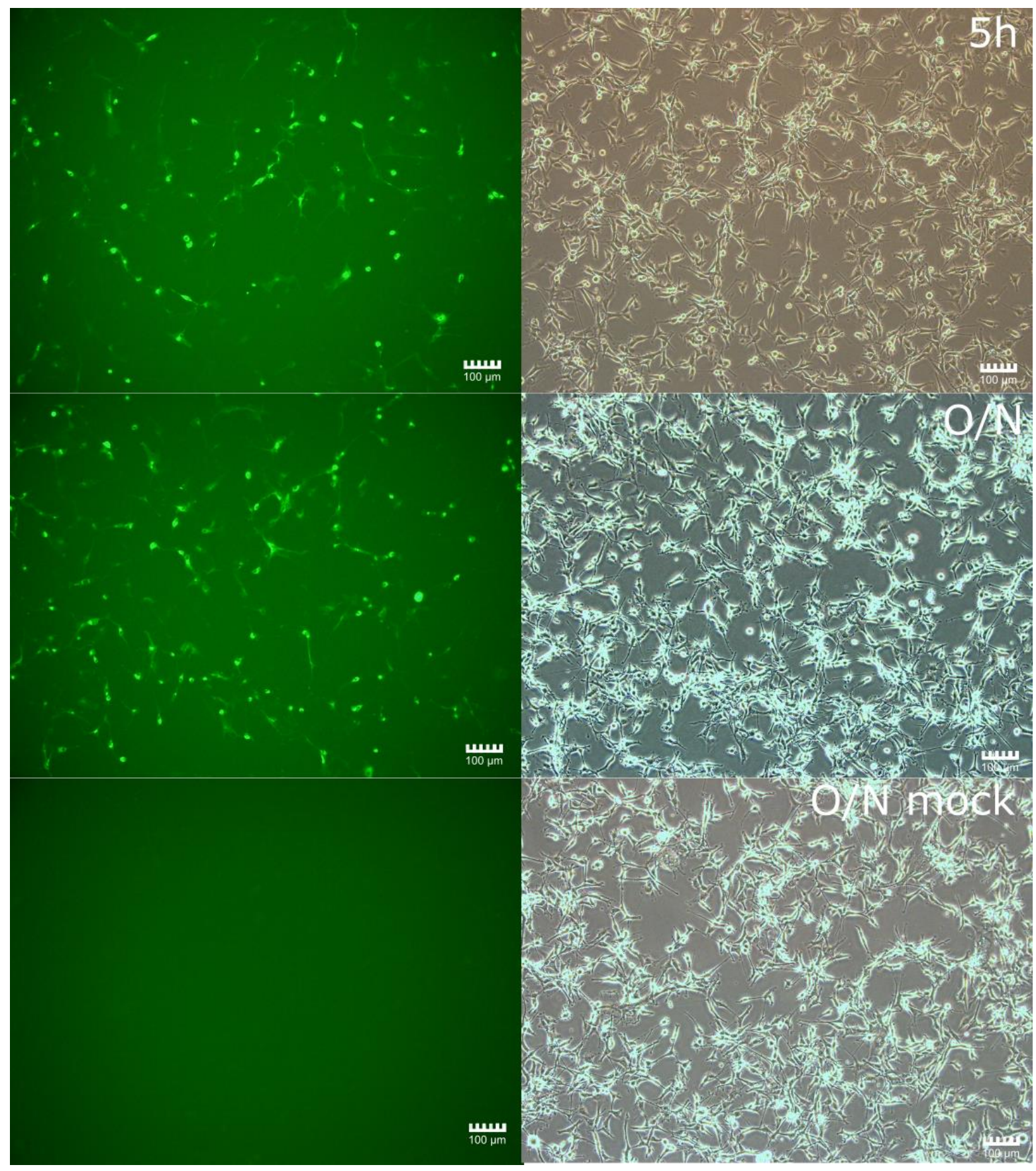

Figure 4.2. Effect of transfection time on transfection efficiency and morphology on U87MG cells. The left-hand panels depict a FITC image to demonstrate transfected cells and the corresponding phase contrast image in the adjacent right-hand panel to show all cells present. The U87 cells underwent a transfection incubation for either $5 \mathrm{hrs}$ (top) or overnight (middle \& bottom). The bottom panel is a mock transfection whereby DNA was omitted from the transfection method. All scale bars correspond to $100 \mu \mathrm{M}$. 
Following the modifications to the transfection procedures, the transfection efficiency of the U87MG cells was approximately 30\% (Figure 4.3). However, unfortunately, the transfection appeared to be transient, and fluorescence was lost after $2-3$ days in culture without further selection. To overcome this limitation, and to produce a homogeneous, stable population of U87MG cells expressing the APLNR receptor, an enrichment step was performed that made use of a geneticin (G418) antibiotic selection procedure. G418 blocks polypeptide synthesis resulting in cell death. Transfected cells are given resistance capabilities due to a neomycin resistance gene present on the APLNR-GFP plasmid. This gene modifies the properties of G418 resulting in the prevention of G418 binding to the ribosome and stopping translation. The selection of G418-resistant cells was performed for 10 - 20 days in progressively increasing concentrations of G418 to ensure the death of all non-plasmid expressing cells. It was found, however, that although transfected cells were resistant to G418, large populations of non-GFPexpressing cells were also resistant to G418. This phenomenon has been previously described [428], but it was not possible to ascertain whether the cause was biological or technical in the present study. An example of a population of cells prior to the GFP cell enrichment process is shown in Figure 4.3 below. 


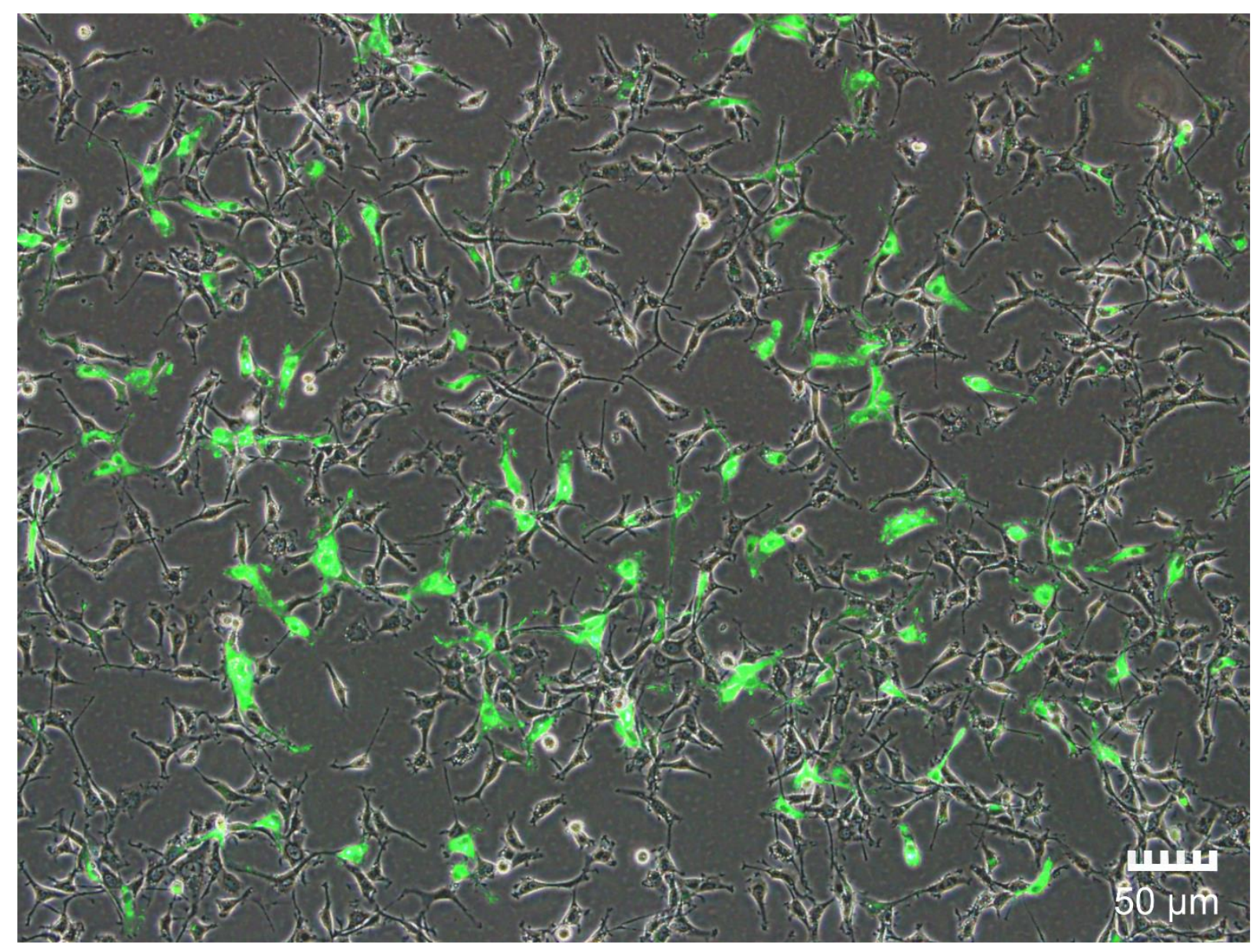

Figure 4.3. Representative image of U87MG cells transfected with APLNR (green cells) after iterative rounds of G418 selections with large populations of non-GFP, G418 resistant cells. An image of U87MG cells transfected with $A P L N R$ was taken on a FITC filter and superimposed over a phase contrast photo. Numerous non-GFP expressing cells can be seen compared to a smaller number of GFP positive cells. Scale bar corresponds to $50 \mu \mathrm{M}$.

Several methods were trialled to isolate the GFP-positive cells from heterologous cell population (summarised in Appendix A.3). A limiting dilution method was tested for developing a clonal stable cell line. This method involves diluting cell to a concentration of 0.5 cells per well to ensure a binomial distribution exists over a 96-well plate, i.e. wells contain either one cell or no cell. Wells are then checked via fluorescence microscopy for the presence of a single GFP-positive cell and are then selected for further subculture (expansion). Unfortunately, U87MG cells at a density of 0.5 cells per well did not survive. Therefore, the U87MG cells were serially diluted such that each cell of a 96-well plate contained 
approximately 250 to 2 cells per well. In addition, the cells were supplemented with conditioned medium to enhance growth at low densities and is used for the production of stable cell lines [429]. Conditioned medium was made using the non-transfected U87MG cell line in exponential growth phase grown for $24-48 \mathrm{hrs}$. The medium was removed, centrifuged at 300 RCF for 5 min to pellet any cellular debris, and $80 \%$ of the supernatant removed and used to make up the limiting dilution media. The conditioned medium was never frozen or filtered to prevent protein degradation or loss onto the filter membrane. The conditioned medium was incorporated into a mixture that consisted of $40 \%$ conditioned medium, $49 \%$ fresh medium, $1 \%$ penicillin-streptomycin and 10\% FBS and this was termed "limiting dilution media".

A summary of the procedure that was used to generate the U87MG stable cell line is summarised in Figure 4.4. In short, transfection of U87MG cells with the APLNR-GFP plasmid was performed, followed by several rounds of G418 selection at increasing concentrations from $800 \mu \mathrm{g} / \mathrm{mL}$ to $1600 \mu \mathrm{g} / \mathrm{mL}$. Once a population of G418-resistant, GFP-expressing cells were derived, the population of GFP-positive cells were enriched. Cells were diluted as described above with the fresh limiting dilution medium. Wells with randomly higher numbers of GFPexpressing, relative to GFP-negative, cells were transferred in 24-well plates for sub-cloning. Cells were left to grow until sufficiently high enough density was achieved to seed a $35-\mathrm{mm}$ dish. Wells with higher numbers of GFP-expressing, relative to GFP-negative, cells were transferred into 35-mm dishes. Once there was a sufficient number of GFP-expressing cells to perform another serial dilution into a 96-well plate, this process was repeated. Approximately five rounds of this limiting dilution method were performed before a sufficiently high proportion of GFP-expressing U87MG cells were achieved. These stably transfected U87MG cells are hereafter defined as U87.APLNR cells. HEK293 cells were found to be more resilient to single cell dilution, therefore the same process was used as described above with the exception that the cells were diluted to 0.5 cells/well rather than from 2 - 250 cells per well as with U87.APLNR cells. 

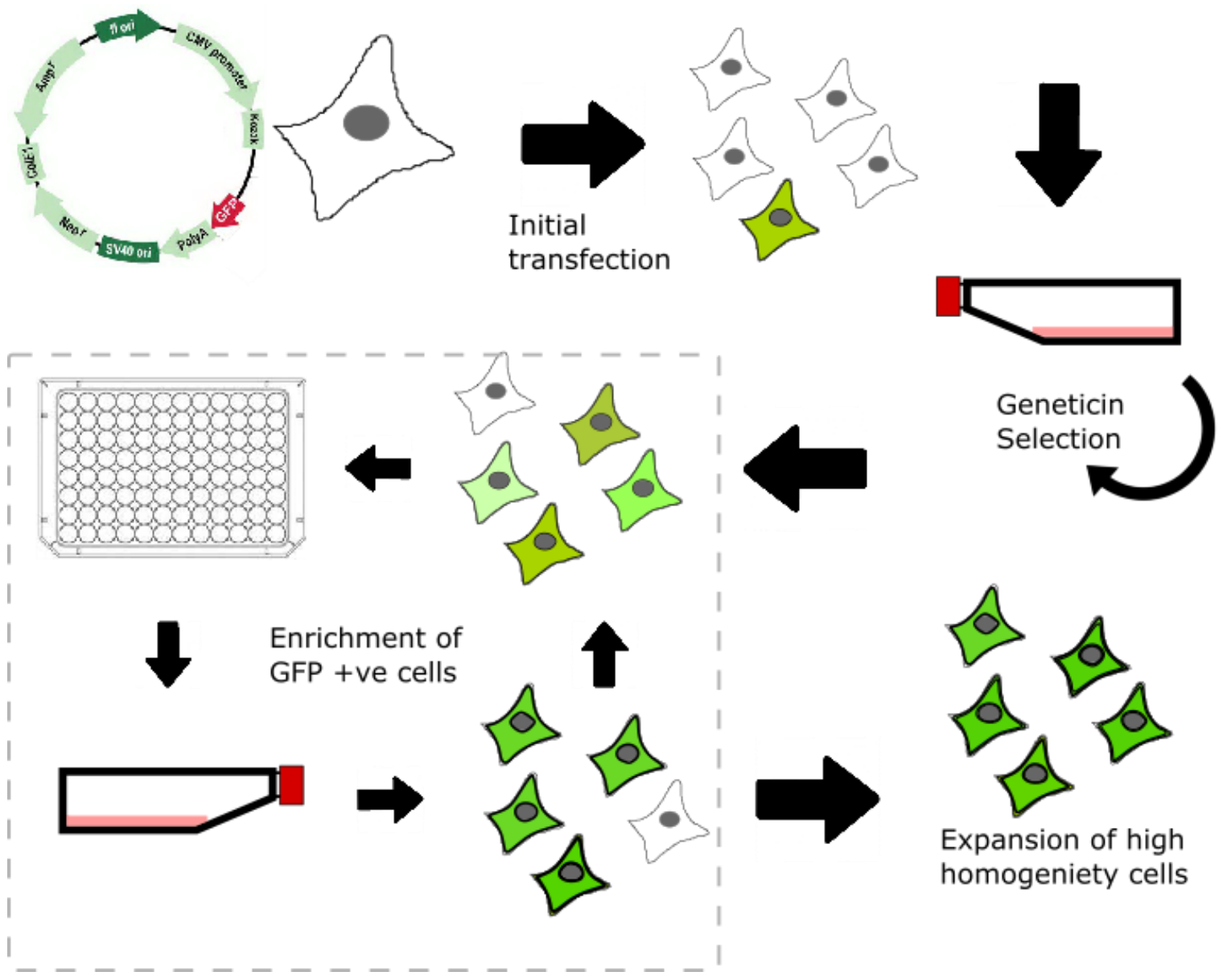

Expansion of high homogeniety cells

Figure 4.4. Flowchart of the single clonal cell line selection procedure. Cells are transfected with a plasmid containing APLNR and geneticin (G418)-resistance genes as well as GFP. After several rounds of selection in increasing concentrations of G418, G418-resistant cells then undergo multiple rounds of limiting dilution enrichment (delineated by the dotted box) until a high GFP expression was demonstrated. The G418-resistant stably transfected cells were then frozen and stored in liquid nitrogen.

After the enrichment procedure, the proportions of GFP expressing cells within the cell population were quantitatively and qualitatively analysed using flow cytometry and fluorescence microscopy, respectively. The cell populations were found to consist of a high percentage of GFP expressing cells. 
The HEK293.APLNR cells were enriched to monoclonal populations through limiting dilution. Flow cytometry analysis of GFP-expressing HEK293.APLNR cells showed approximately $100 \%$ of cells with GFP expression, confirming their monoclonal nature (Figure 4.5, green peak). The HEK293.APLNR cells were found to remain GFP-positive without maintenance concentrations of G418. In contrast, the U87.APLNR cell line required a G418 concentration of $400 \mu \mathrm{g} / \mathrm{mL}$ to maintain the stable transfection.

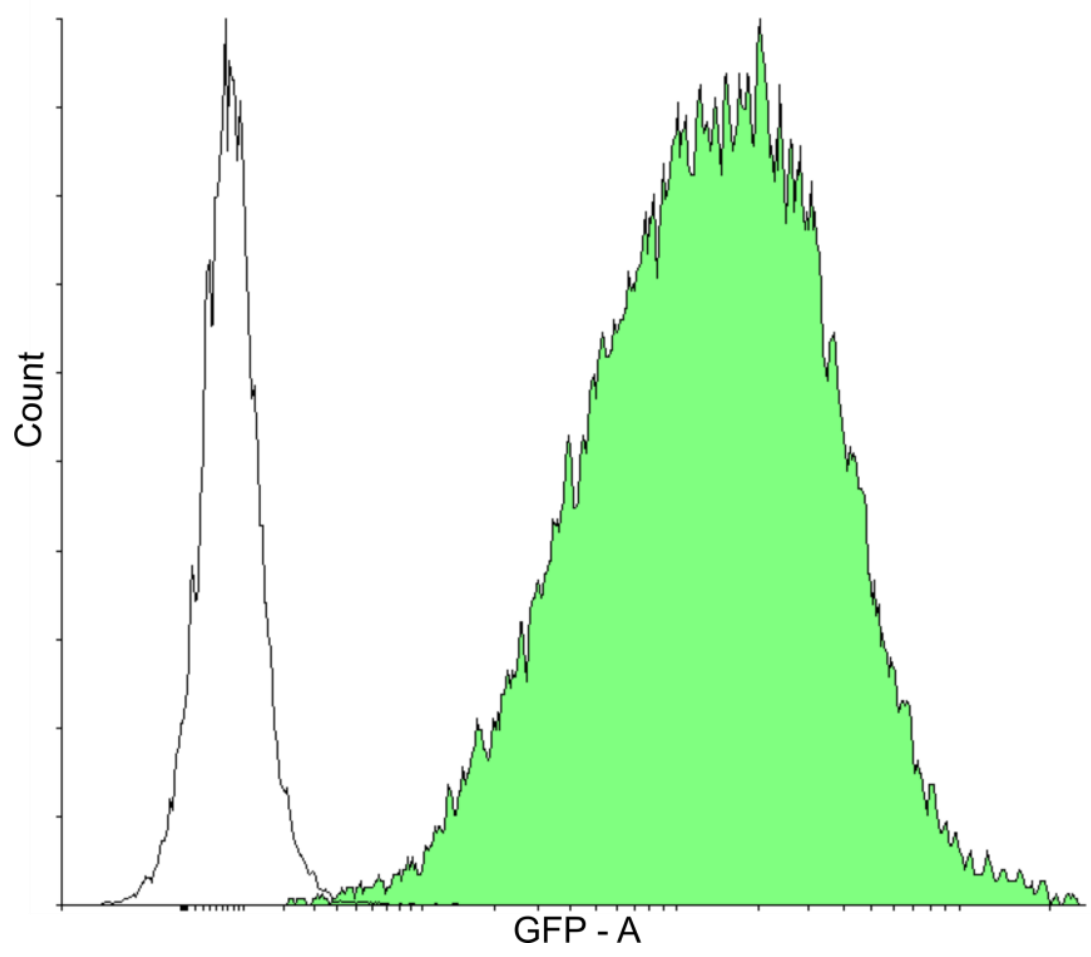

Figure 4.5. Representative figure of overlay histogram of 20,000 HEK293 (colourless peak) and 20,000 HEK293.APLNR (green peak) cells assessed by flow cytometry. The flow cytometer settings (e.g. gating) were identical for both cell samples, HEK293.APLNR and HEK293 were displayed on a separate histogram which was then overlaid. The $\mathrm{Y}$ axis denotes the count of cells and the $\mathrm{X}$ axis denotes the expression of GFP on a log scale.

Qualitative assessment of the transfected cell line populations using fluorescent microscopy revealed that the majority of cells within the U87.APLNR and HEK293.APLNR cell lines were GFP positive, and this was maintained over multiple passages suggesting stable expression of APLNR in both cell types (Figure 4.6; A-D). 
Quantitative assessment of the transfected cell lines by flow cytometry revealed the presence of two populations of GFP positive cells in the U87.APLNR cell line, one population, highly expressing GFP and one lower expressing population (Figure 4.6; F-G). In a single population of U87.APR cells, approximately $90 \%$ of cells were GFP-positive, compared to un-transfected U87MG cells (Figure 4.6; E). The presence of two populations of GFP-positive cells in the U87.APLNR cell line was perhaps not unexpected due to single U87.APLNR cells not surviving the culture conditions, and thus a polyclonal population of U87.APLNR cells are likely to be generated. It was assumed that the polyclonal nature of the U87.APLNR cell line, i.e. varied expression of $A P L N R$, would not be a significant limitation for studying the effects of the apelinergic system on these cell lines in future experiments. 



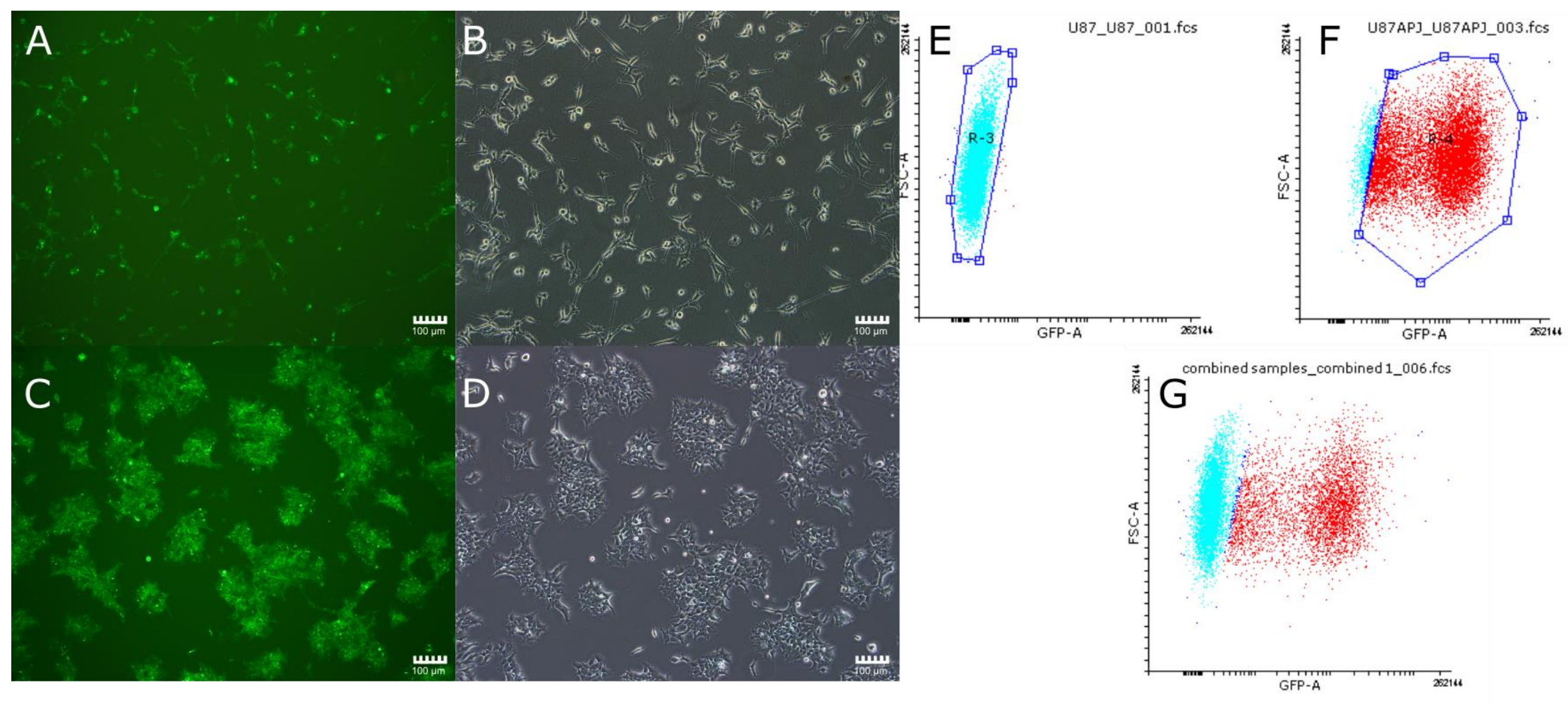


Figure 4.6. Qualitative and quantitative assessment of the purity of the U87.APLNR and HEK293.APLNR cell lines by fluorescence microscopy (A - D) and flow cytometry (E -G), respectively. Left-hand figures show fluorescence (A \& C) and phase contrast (B \& D) micrographs of U87.APLNR (A \& B) and HEK293.APLNR (C \& D) cell lines. Right-hand figures illustrate flow cytometric analysis of GFP expression in non-transfected U87MG cells (E), U87.APLNR cells (F) and combined populations of U87.APLNR and non-transfected U87MG cells (G). GFP non-expressing and expression cell populations are shown in blue and red, respectively. Total percentage of GFP expressing cells in the U87.APLNR cell line was approximately $90 \%$. All scale bars correspond to $100 \mu \mathrm{M}$ 


\subsubsection{Confocal analysis of stable cell lines}

The intracellular localisation of the $A P L N R-G F P$ protein was examined in U87.APLNR cell using a confocal microscope (Olympus AX71, Olympus, Germany) (Figure 4.7). A punctate, perinuclear expression was observed on the U87.APLNR cells (Figure 4.7, Panel B) which were also observed in the 0906 and U87MG glioblastoma cell lines (Chapter 3, Figure 3.12). There is also abundant expression on the plasma membrane which differed from previous data in Chapter 3, Figure 3.12. APLNR localisation was found to be mostly perinuclear in U87MG and 0906 in the previous chapter; however, U87.APLNR appears to have a more widespread membranous expression of $A P L N R$; this is can also be seen in the membrane protrusions that connect cells in Figure 4.7, Panel C indicated by white and red arrows. 



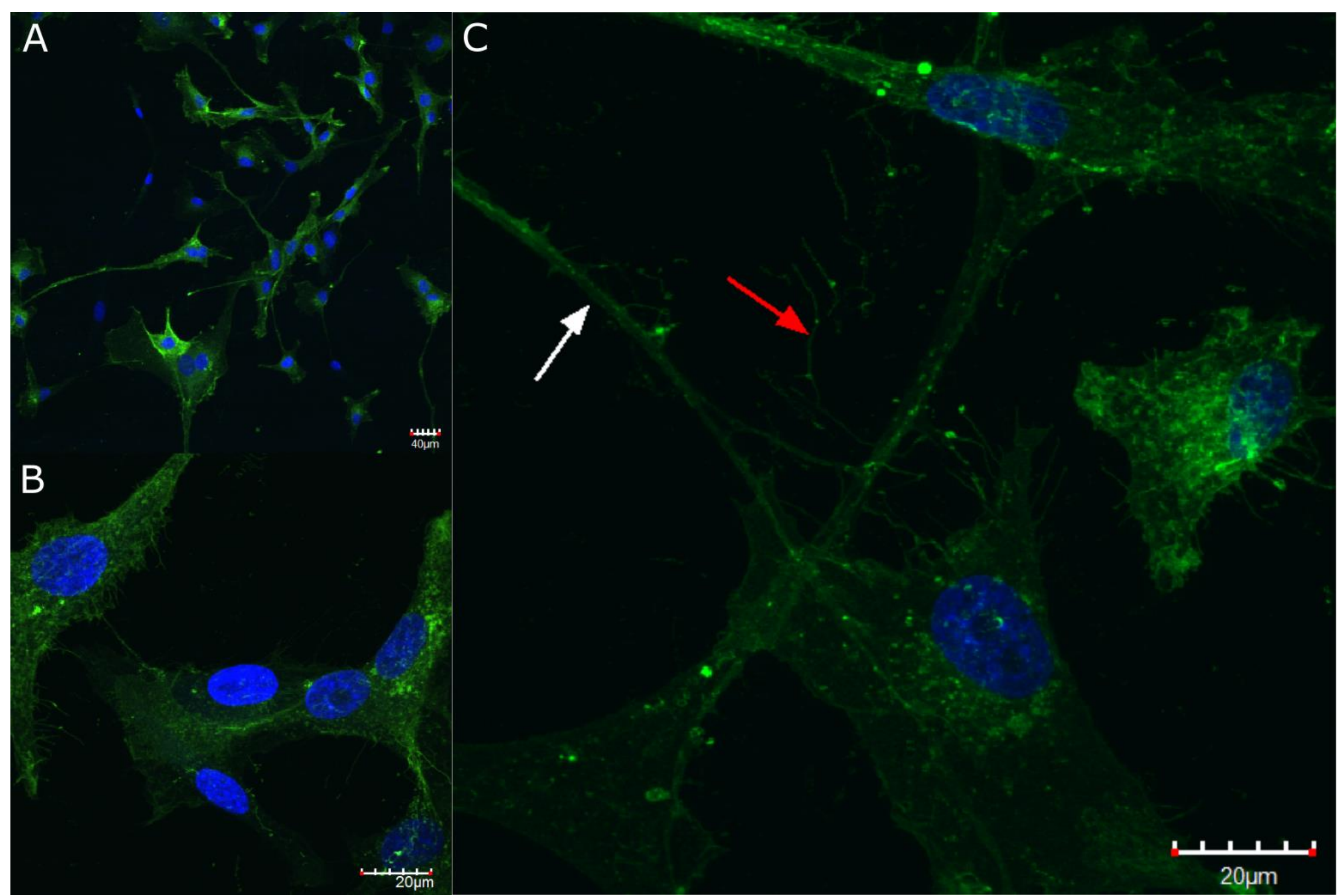


Figure 4.7. Localisation of GFP-labelled APLNR in the stably transfected U87.APLNR cell line using confocal analysis. All figures show Z-stack images throughout the total depth of the cells. Micrographs at low (20x; A), medium (60x; B) and high (100x; C) magnification of U87.APLNR cells are counterstained with the nuclear stain DAPI (blue). The white arrow indicates a thick membrane protrusion and the red arrow indicates a thinner membrane protrusion. 


\subsubsection{Western blot analyses of stable lines}

In an attempt to confirm the expression of the APLNR-GFP protein, the rabbit-raised polyclonal antibody (described and verified in Chapter 3, Section 3.3.8.1) against the Cterminus of human APLNR (hereafter APLNR-Cterm) was used to detect the APLNR-GFP protein.

$A P L N R$-Cterm antibody produced the expected banding pattern for endogenous APLNR in the U87MG and mouse heart lysate as well as in the U87.APLNR and HEK293.APLNR cell lines. This is indicated by the dark blue and light blue arrows (Figure 4.8, panel A).

However, no immunoreactivity for the APLNR-GFP protein was seen in the expected area (as denoted by the black arrows (Figure 4.8, panel A) in the U87.APLNR and HEK293.APLNR lysates. The lack of binding of the $A P L N R$-Cterm antibodies in stable cell line lysates may be due to the GFP tag located at the C-terminal of APLNR, the proximity of the GFP tag to the Cterminus may be interfering with antibody binding or recognition.

Furthermore, it was interesting to note that despite confocal and fluorescence imaging data showing an overexpression of $A P L N R-G F P$, purification for western blotting does not show a significant expression of APLNR-GFP protein (indicated by the red arrow) Figure 4.8, panel $\mathrm{B}$, despite adequate protein being loaded, as denoted by the yellow arrow indicating alphatubulin $(50 \mathrm{kDa})$. This was ascribed to isolation techniques of the protein as well as loss of endogenous GFP due to isolation and subsequent manipulation. The difference in APLNRGFP expression is not unexpected as U87.APLNR is a heterogeneous population of cells whereas HEK293.APLNR are a homogeneous population of cells. 

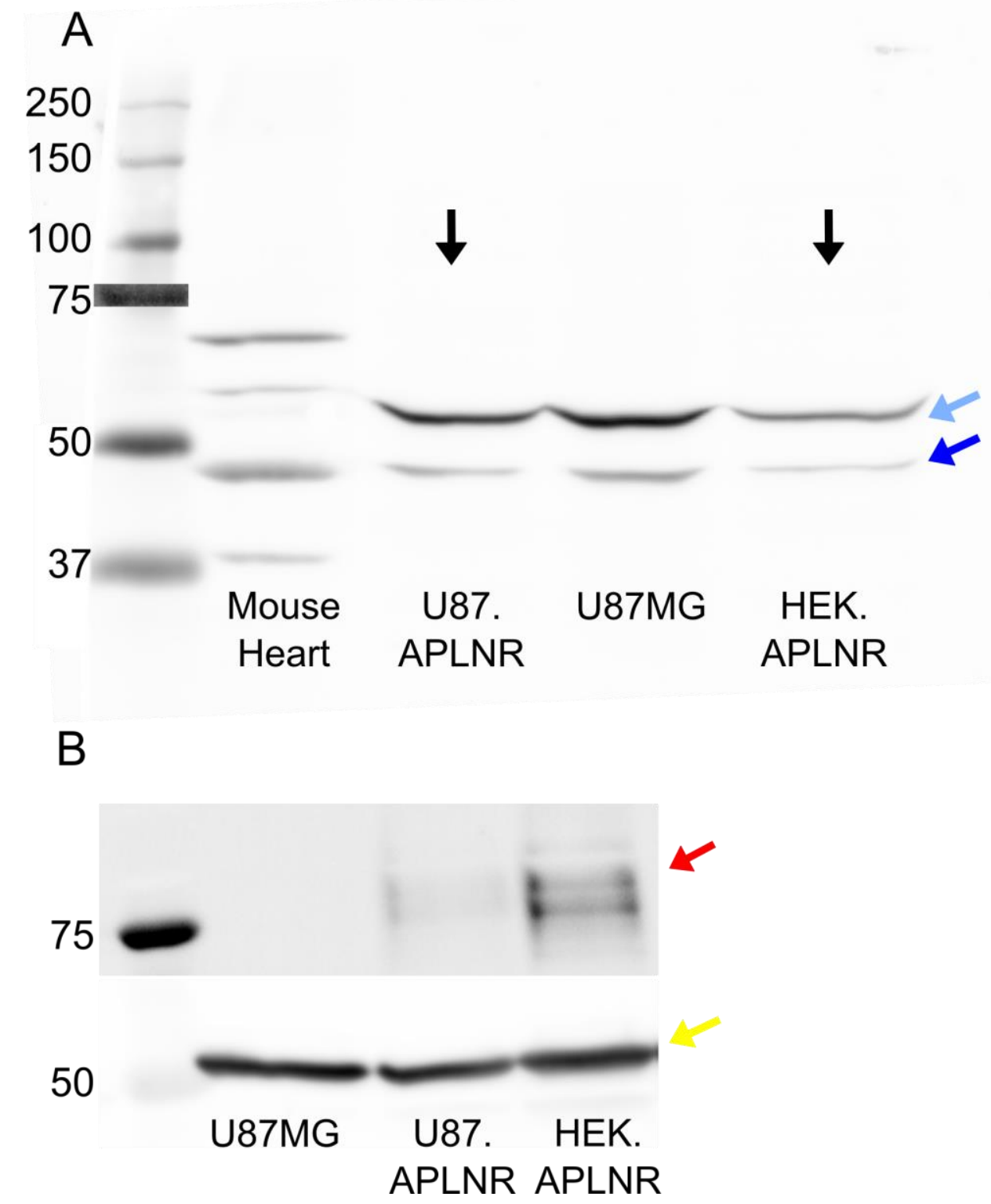

Figure 4.8. Representative western blots (WB) for endogenous APLNR and transfected APLNR-GFP. Panel A shows a WB of endogenous APLNR expression (indicated by the light blue and dark blue arrows) and a lack of immunoreactivity for the APLNR-GFP protein (expected location is indicated by the black arrows at $75 \mathrm{kDa}$ ) when probed with an antibody raised to the $\mathrm{C}$ terminus of APLNR. The WB was imaged using a CY5 conjugated secondary antibody. Panel B shows a WB of the APLNR-GFP protein detected by the endogenous GFP fluorescence at $75 \mathrm{kDa}$ (indicated by the red arrow) and the loading control alpha-tubulin (indicated by the yellow arrow) at $50 \mathrm{kDa}$. 


\subsubsection{Stable Expression of $A P L N R$ in U87.APLNR and HEK.APLNR Cell}

\section{Lines}

Several freeze-thaw procedures had been performed on the U87.APLNR and HEK293.APLNR cell lines with no apparent loss of GFP, suggesting the plasmid had stably integrated into both cell lines.

As described in Section 4.2.3, the three primer sets span the coding region for the APLNR gene, the junction spanning across the 3'-region of APLNR and the 5' region of GFP (APLNR-GFP) and GFP genes. The rationale for the primer design was to enable human brain tissue to be used as a positive (for $A P L N R$ ), and negative (for $A P L N R$-GFP and GFP) controls and to test that the success of the APLNR-GFP transfection. The results from the end-point PCR experiments are illustrated in Figure 4.9. Marked expression of all three transcripts (APLNR, APLNR-GFP and GFP) were present in both the U87.APLNR and HEK.APLNR cell lines. As expected, only expression of $A P L N R$ mRNA was detected in human brain samples.

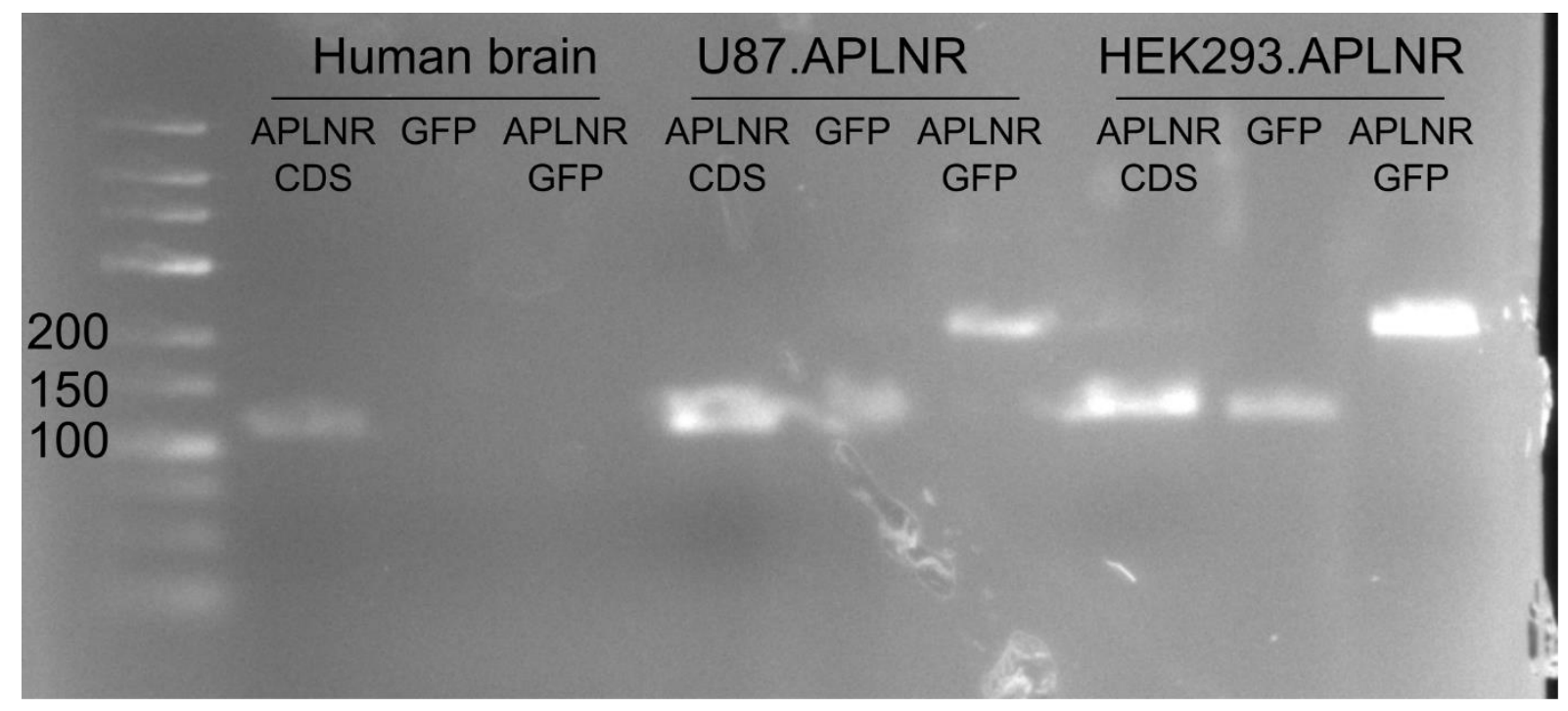

Figure 4.9. End-point-PCR bands representing the expression of APLNR (APLNR CDS), GFP and APLNR-GFP mRNA in human brain tissue (positive control for APLNR mRNA and negative control for GFP and APLNR-GFP mRNA), and U87.APLNR and HEK293.APLNR cell lines. The predicted sizes for APLNR CDS, GFP and APLNR-GFP cDNA were $111 \mathrm{bp}, 109 \mathrm{bp}$ and $183 \mathrm{bp}$, respectively. 
The results from the qPCR experiments are illustrated in Figure 4.10. The mRNA expression levels of $A P L N R$ in the U87.APLNR cell line was significantly higher than that in nontransfected U87MG cells (unpaired t-test $(2)=3.039, \mathrm{p}=0.0385$ ).

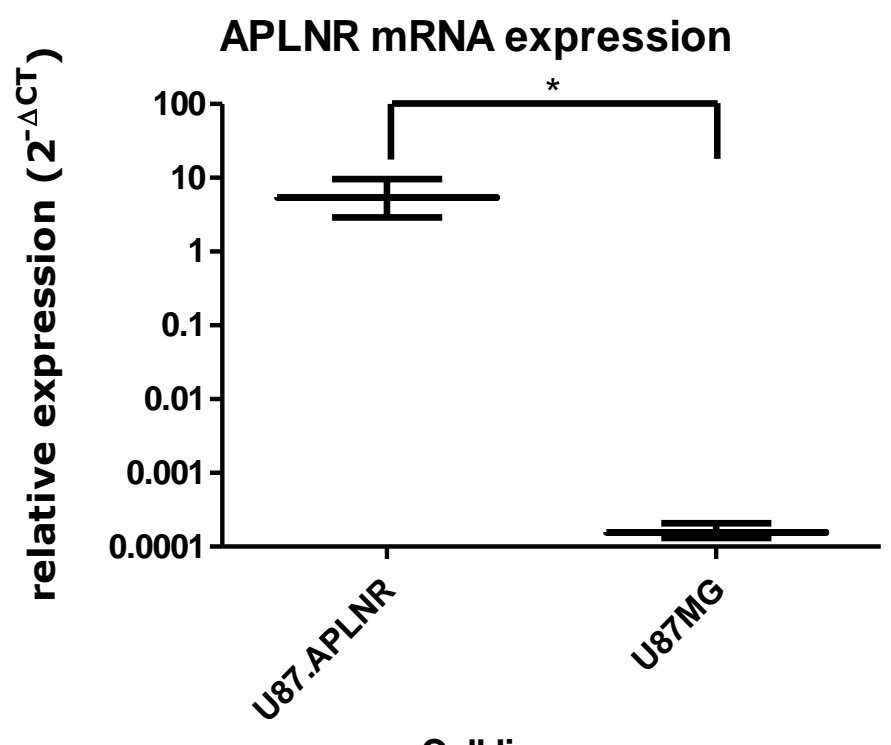

Figure 4.10. Quantitative analyses of APLNR mRNA expression levels in U87.APLNR cells and non-transfected U87MG cells. All data were normalized to HPRT and expressed as $2^{-\Delta \mathrm{CT}}$ presented on a $\log 10$ scale. Data are presented as a mean of three replicate experiments \pm SEM. Asterisk denotes a significant difference at $p$-value of 0.0385 .

\subsubsection{Analyses of APLN-Mediated Cellular Processes}

The intended use of the U87.APLNR cell line developed in this study was for functional experiments to elucidate the role of the apelinergic system in glioblastoma. To ensure an adequate response to the biological system being tested, an APLNR-GFP plasmid was chosen to be stably expressed. To further increase the utility of a stable cell line, an APLNR-GFP fusion protein was chosen over just the APLNR protein alone for transfection because fluorescence 
emitted from GFP allows for ease of visualisation confirmation, enabling a wider range of experiments.

However, it was unclear whether stable expression of $A P L N R$, particularly with fusion to GFP, would lead to active signalling pathways. There was the possibility that binding of the ligand to $A P L N R$ and/or subsequent signalling pathways would be interrupted due to the presence of a GFP tag. Due to the external N-terminus being essential for APLNR signalling [430, 431], a GFP tagged to the C-terminus of $A P L N R$ was purchased for these experiments. A similar $A P L N R$-GFP ligated plasmid was reported to be successfully transfected into $\mathrm{CHO}$ cells and exhibited $A P L N R$ function as measured by internalisation of the fluorescent receptor [432].

To test the activity of the transfected APLNR-GFP receptor, common literature reported pathways were tested for activation after addition of exogenous [ $\left.\mathrm{Pyr}^{1}\right]$ apelin-13.

\subsubsection{Effects of [Pyr $\left.{ }^{1}\right]$ apelin-13 on APLNR internalization in stable cell lines}

Stimulation of APLNR by [Pyr ${ }^{1}$ apelin-13 has been reported to cause cell surface receptor expression changes [432] that are reliant on a serine residue at position 348 [433] of the 350 amino acid $A P L N R$ protein. Thus, [Pyr1]apelin-13 was added to cultures of HEK293.APLNR and U87.APLNR cells to test whether receptor internalization occurred which would confirm that the GFP tagged to the C-terminus end of $A P L N R$ did not interfere with the function of $\mathrm{C}$ terminal serine 348 .

The effect of $\left[\mathrm{Pyr}^{1}\right]$ apelin-13 treatment on GFP fluorescence intensity was quantified are illustrated in Figure 4.11. [Pyr1] apelin-13 caused a statistically significant (paired t-test(2) $=13.52, \mathrm{p}=0.0054)$ in mean fluorescence in HEK293.APLNR cells (Figure 4.11, panel B), and whilst a similar trend was observed in U87.APLNR, this was not significant (paired ttest $(2)=1.563, \mathrm{p}=0.2586)($ Figure 4.11 , panel B). 

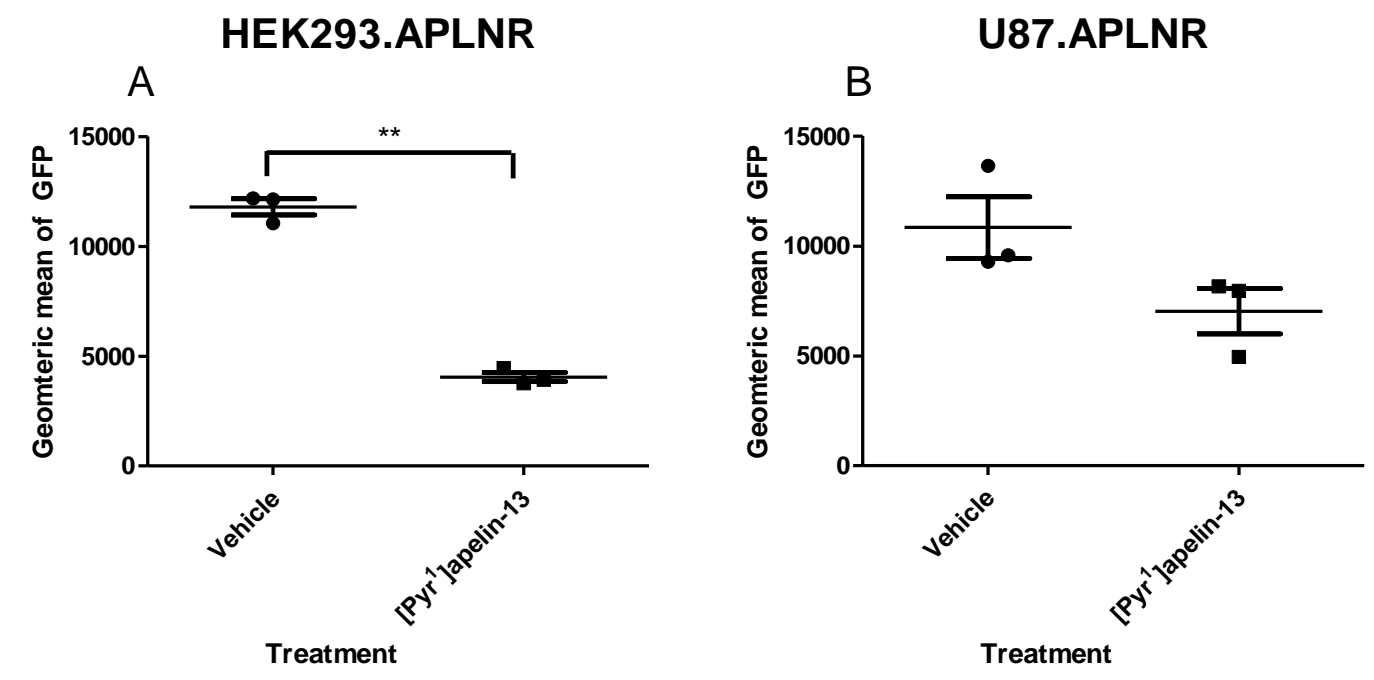

Figure 4.11. Geometric mean \pm SEM GFP expression in HEK293.APLNR (A) and U87.APLNR (B) cell lines in the absence (vehicle) or presence of [Pyr1]apelin-13. Geometric mean values were determined from 3 replicate experiments. Asterisks denote a significant difference $(\mathrm{P}=0.0054$, paired Student's t-test, $\mathrm{n}=3)$.

The effect of $\left[\mathrm{Pyr}^{1}\right]$ apelin-13 treatment on receptor localization, using fluorescence microscopy to reveal punctate cell surface or cytosolic (internalized) expression of GFP, in HEK293.APLNR and U87.APLNR cells were also examined and is illustrated in Figure 4.12. The fluorescence of GFP was clearly observed to shift from cell surface to cytosolic expression following $\left[\mathrm{Pyr}^{1}\right.$ ]apelin-13 treatment in HEK293.APLNR cells. Whilst this effect is also observed in U87.APLNR cells following [Pyr1]apelin-13posure, it was much less apparent. 


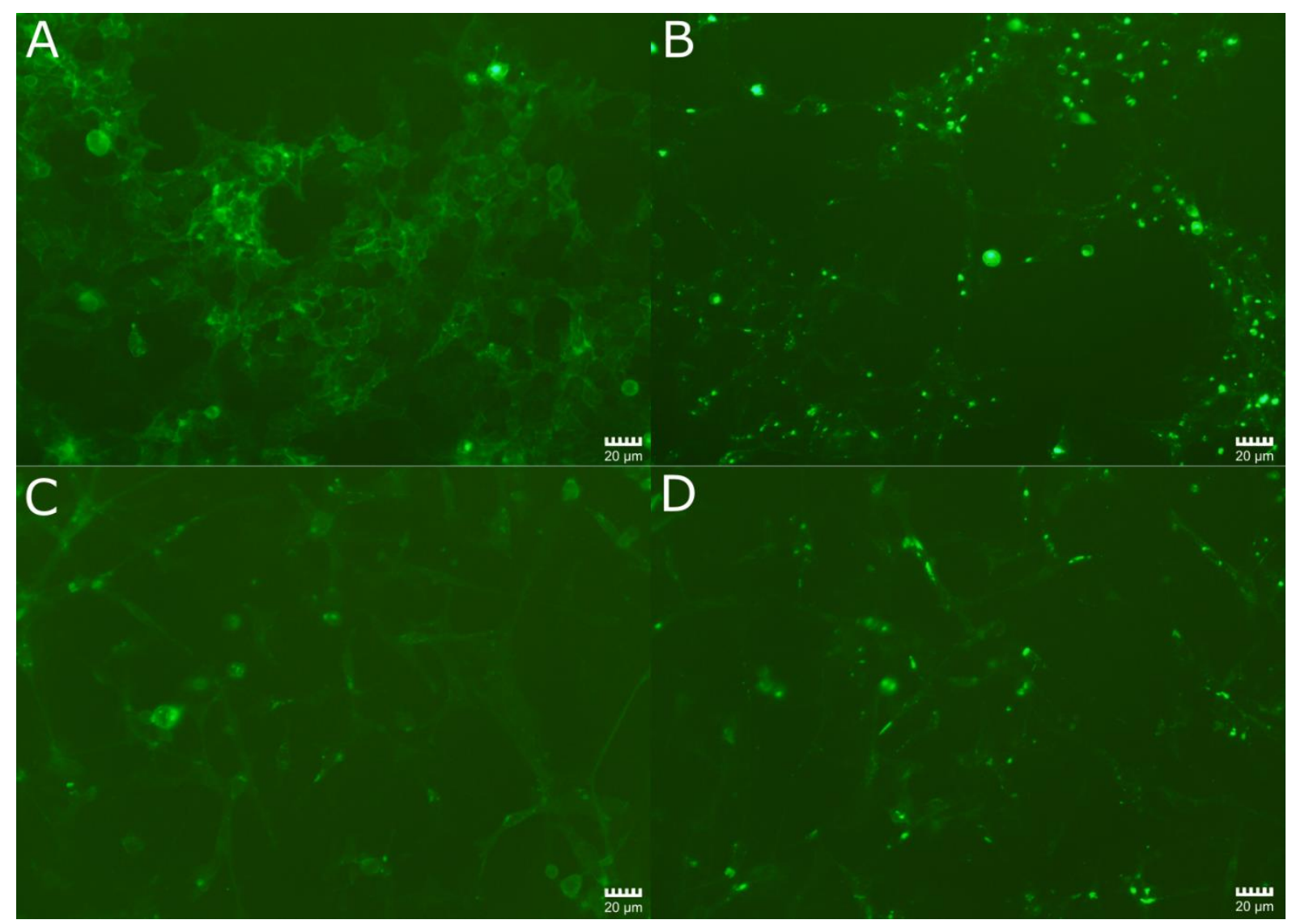

Figure 4.12. Representative images of GFP expression as a measure of APLNR localization in HEK293.APLNR $(\mathrm{A} \& \mathrm{~B})$ and U87.APLNR (C\&D) after overnight exposure to the vehicle alone (controls; A\&C) or [Pyr ${ }^{1}$ ]apelin-13 (B\&D). The change in receptor localization was noted over 3 independent experiments. 


\subsubsection{Effects of [Pyr1] apelin-13 on ERK phosphorylation in stable cell lines}

A pERK phosphorylation time course experiment, as described elsewhere [52], was repeated to confirm activation the of the apelinergic signalling pathway by exogenous [ $\mathrm{Pyr}^{1}$ ]apelin-13 treatment. The time course experiment was performed on the HEK293.APLNR rather than the U87.APLNR cell line because changes in pERK are easier to see. This is because the reduction of basal levels of pERK through serum starvation is more prevalent in HEK293.APLNR than in U87.APLNR. It was demonstrated that pERK activation in HEK293.APLNR was measurable by Western blotting after treatment with $\left[\mathrm{Pyr}^{1}\right]$ apelin-13 and that the optimal time for cell collection following the addition of $\left[\mathrm{Pyr}^{1}\right]$ apelin-13 was 5 min (Figure 4.14), as is consistent with the literature [52].

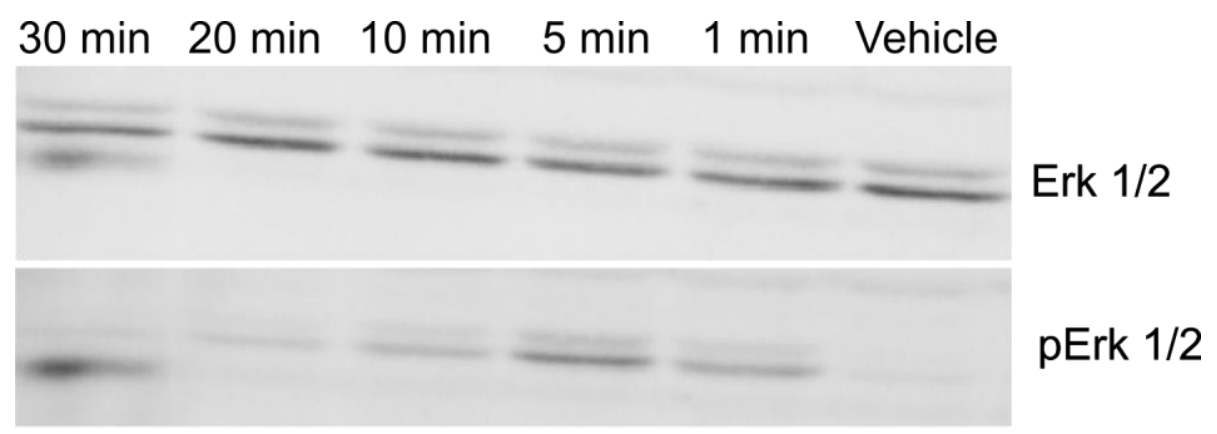

Figure 4.13. A western blot showing the effect of $\left[\mathrm{Pyr}^{1}\right]$ apelin-13 over time on ERK phosphorylation (pERK) in HEK293.APLNR cells. Cells were seeded at in a 24-well plate and were treated with [ $\mathrm{Pyr}^{1}$ ]apelin-13at differing time points after which cell lysates were extracted, and western blotted. The time taken for $\left[\mathrm{Pyr}^{1}\right]$ apelin-13 to phosphorylate ERK maximally in HEK.APLNR cells was approximately 5 min which is as reported in the literature [52]. 
Next, the effect of $\left[\mathrm{Pyr}^{1}\right]$ apelin-13 on pERK expression was tested on U87.APLNR and U87MG (negative control) cells at five minutes following $\left[\mathrm{Pyr}^{1}\right]$ apelin-13 or vehicle treatment and the results are illustrated in Figure 4.14. A clear difference in pERK due to [Pyr ${ }^{1}$ ]apelin13 treatment is not discernible. This was attributed to the constitutive activity of pERK in cancer cells that was unable to be abrogated by serum starvation, unlike HEK293.APLNR cells as seen above (Figure 4.13, vehicle lane). As such the changes in pERK expression were quantified using densitometry and are illustrated in Figure 4.15 as the pERK/ERK ratio, as well as pERK and ERK levels. An increase in the pERK/ERK and total pERK levels was observed in the U87.APLNR following [Pyr ${ }^{1}$ ]apelin-13 treatment, which was not observed in control U87MG cells (Figure 4.15).

U87.APLNR lysate
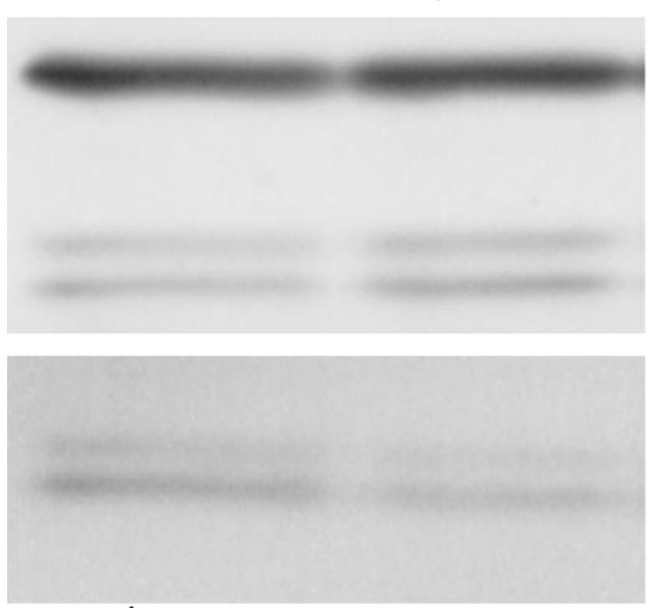

\section{$\left[\right.$ Pyr $^{1}$ ]apelin-13 Vehicle}

U87MG Iysate

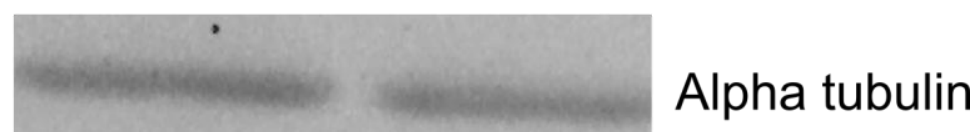

Erk $1 / 2$

\section{pErk $1 / 2$}

Figure 4.14 The effect of apelin-13 on pERK levels in U87.APLNR and U87MG cells. Cells were treated with apelin13 or vehicle for 5 min after which cell lysate was extracted and western blotted. The upper panels show total Erk and alpha tubulin. The lower panels show pERK levels. A clear change in the amount of pERK after [Pyr $\left.{ }^{1}\right]$ apelin-13 treatment in comparison to vehicle is not obvious. 


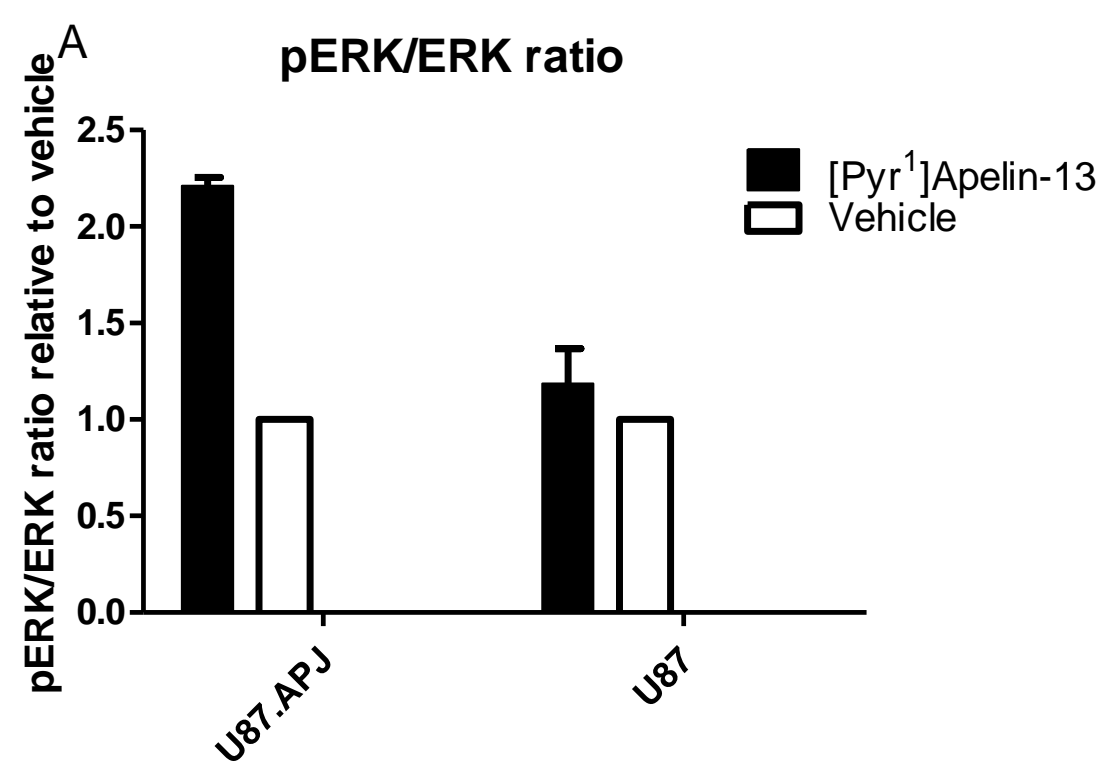

B

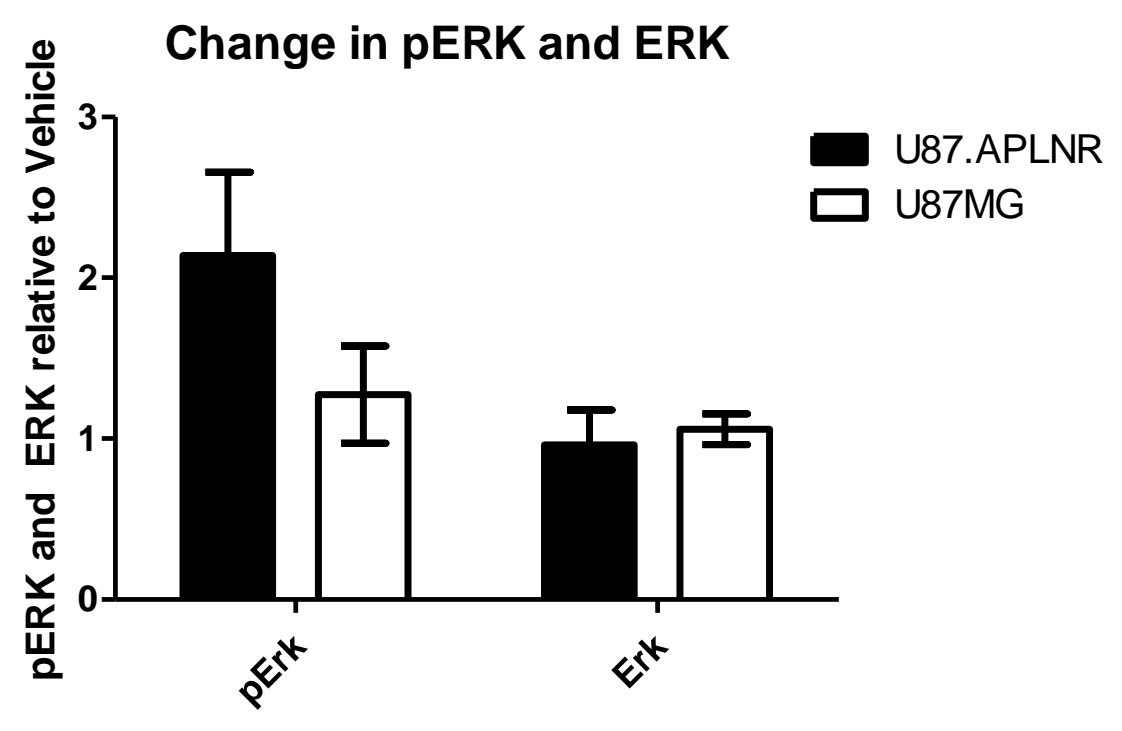

Figure 4.15. Change in phosphorylated ERK (pERK) after [ $\left.\mathrm{Pyr}^{1}\right]$ apelin-13 treatment in U87MG and U87.APLNR cells. Panel A shows the ratio of pERK/ERK normalised to alpha-tubulin expression and is shown relative to the vehicle control. Panel B shows total pERK, and ERK levels normalised to alpha-tubulin expression and are shown relative to the vehicle protein levels. Error bars are expressed mean $\pm \mathrm{SEM} n=2$. 


\subsubsection{Effects of $\left[\mathrm{Pyr}^{1}\right]$ apelin-13 on cell migration in stable cell lines}

The effect of $\left[\mathrm{Pyr}^{1}\right]$ apelin-13 on migration was tested in HEK293.APLNR and U87.APLNR cells lines to determine the validity of these cells lines as an appropriate model for studying the role of the apelinergic system in glioblastoma. Initially, the HEK293.APLNR cell line was tested as illustrated in Figure 4.16 using the wound healing assay. These results confirmed that $\left[\mathrm{Pyr}^{1}\right]$ apelin-13 induced a significant increase (paired t-test $(2)=6.721, \mathrm{p}=0.0214$ ) in the migration of HEK293.APLNR cells.

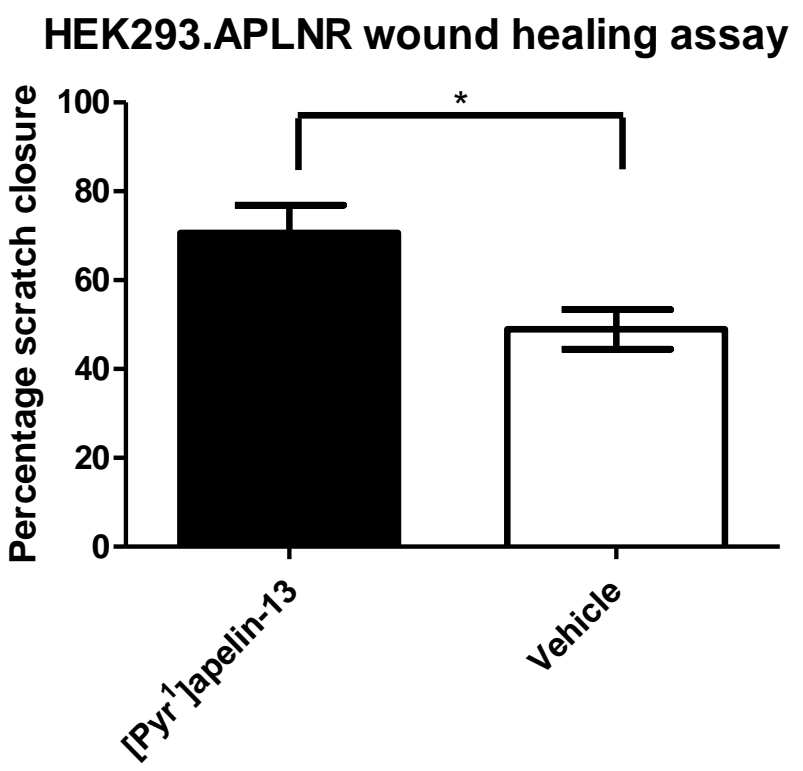

Condition

Figure 4.16. The effect of $\left[\mathrm{Pyr}^{1}\right]$ apelin-13 on HEK293.APLNR migration using the wound healing assay. $\left[\mathrm{Pyr}^{1}\right.$ ]apelin-13 $(1 \mu \mathrm{M})$ significantly increased HEK293.APLNR migration. (paired t-test $(2)=6.721, \mathrm{p}=0.0214$ ). The assay was performed independently 3 times in duplicate. Error bars are expressed mean \pm SEM

Thereafter, the U87.APLNR, U87MG and 0906 cell lines were tested using the wound healing assay following administration of $\left[\mathrm{Pyr}^{1}\right]$ apelin-13 alone or in combination with ML221 (APLNR inhibitor), and the results are illustrated in Figure 4.17. A small but statistically significant effect could be seen upon [ $\mathrm{Pyr}^{1}$ ]apelin-13 treatment of 0906 (Figure 4.17 , panel A) (paired t-test $(6)=5.015, \mathrm{p}=0.0024)$. No statistically significant effect could be seen upon $\left[\mathrm{Pyr}^{1}\right.$ ]apelin-13 treatment of U87MG or U87.APLNR. 

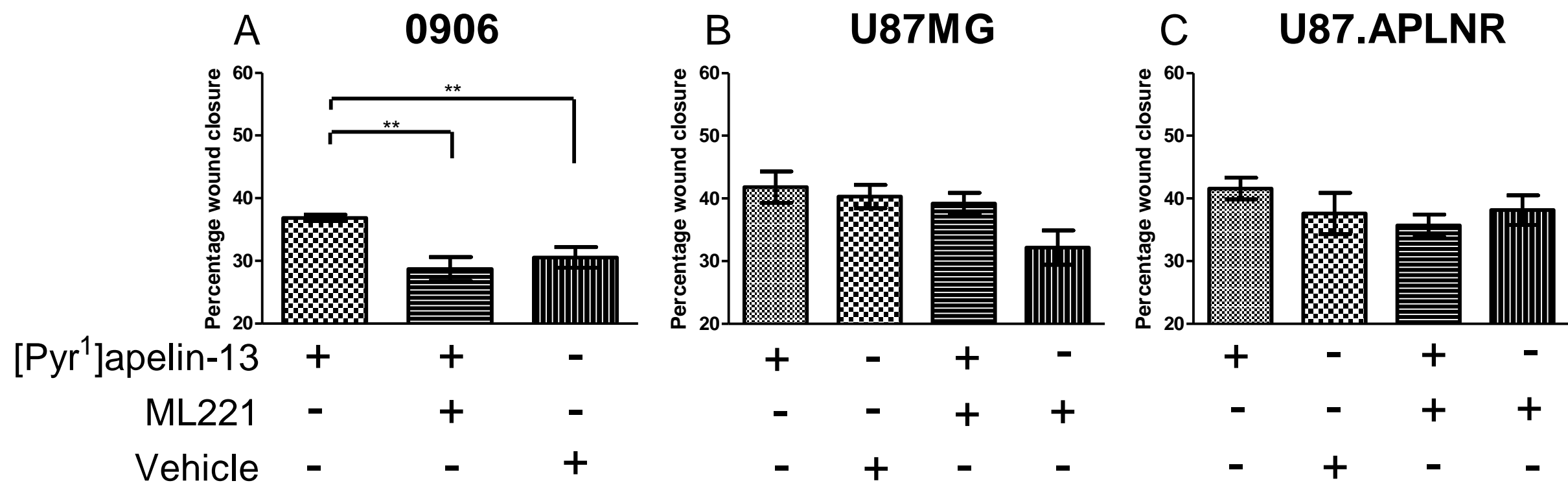

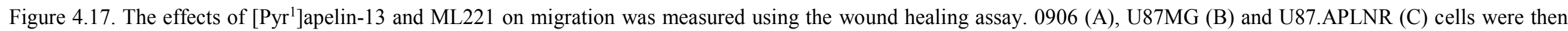

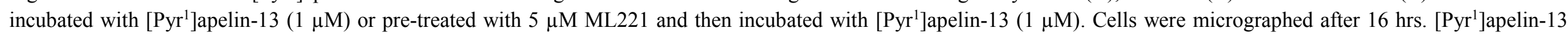

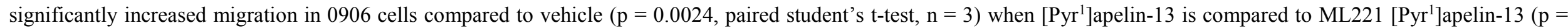

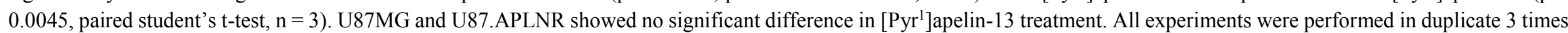
independently $(n=3)$ each replicate was graphed. The data is presented as mean \pm the SEM. 
Whilst the wound healing assay is beneficial due to it being a cheap and relatively easy measure of migration; it is arguably more a measure of chemokinetic movement rather than true chemotactic migration. Moreover, it is limited by technical limitations, in this case, the U87MG and U87.APLNR cells would not form a confluent monolayer, instead, growing in mounds when dense. There are several different migration assays available as alternatives, and the transwell assay was utilised to determine the chemotactic effects of $\left[\mathrm{Pyr}^{1}\right]$ apelin-13 on U87.APLNR and U87MG cells and these results are illustrated in Figure 4.18. Stable APLNR expression inhibits migration of cells in the U87.APLNR cell line, in comparison to U87MG cells endogenously expressing $A P L N R$. This effect is significantly abrogated by exogenous $\left[\mathrm{Pyr}^{1}\right]$ apelin-13 treatment (paired t-test $\left.(5)=7.465, \mathrm{p}=0.0007\right)$. No $\left[\mathrm{Pyr}^{1}\right]$ apelin-13 effect is seen in U87MG cells presumably because of the reduced expression of APLNR in nontransfected cells.
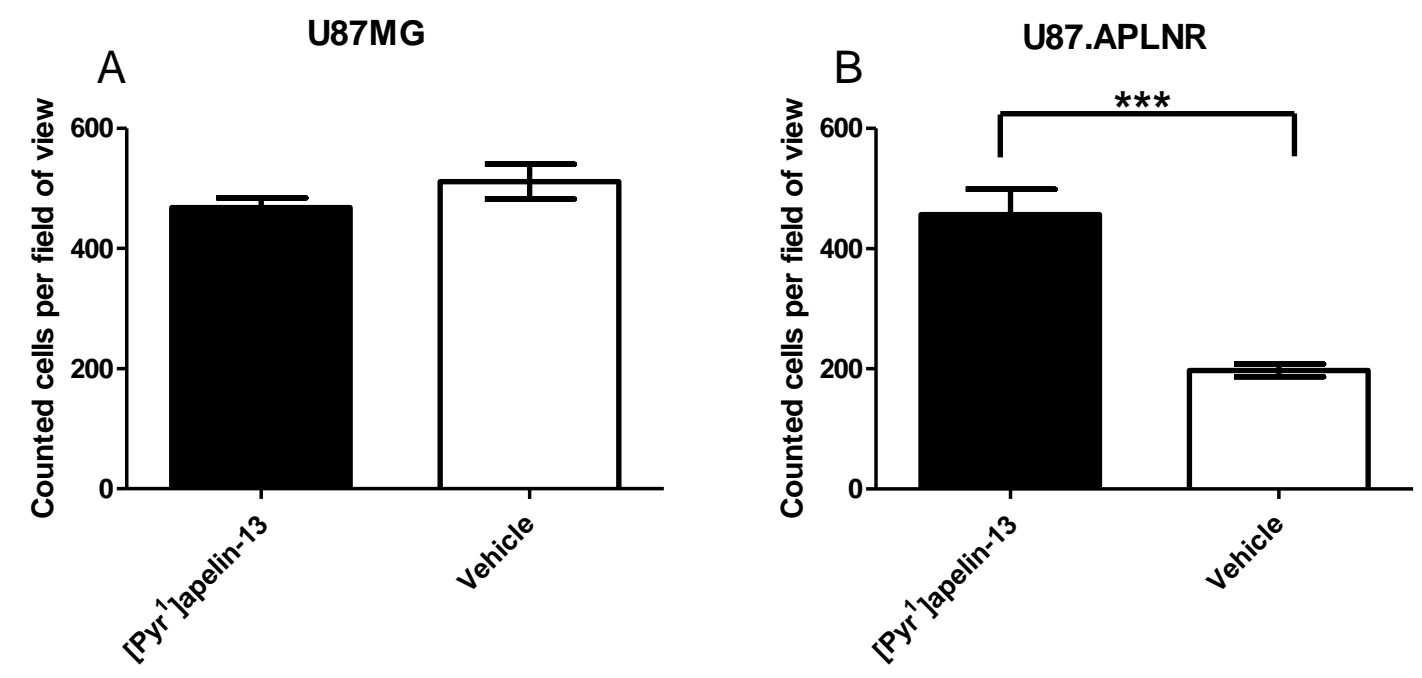

Figure 4.18: The effects of [ $\left.\mathrm{Pyr}^{1}\right]$ apelin-13 on the chemotactic migration of U87.APLNR and U87MG assessed using the transwell assay. [ $\mathrm{Pyr}^{1}$ ]apelin-13 was found to statistically increase migration in U87.APLNR cells (paired t-test $(5)=7.465, \mathrm{p}=0.0007$ ). Five randomised areas of the membrane were micrographed and total cells counted. Values are expressed as counted cells per field of view. All experiments were performed in duplicate 3 times independently $(n=3)$ each replicate was graphed. The data are presented as mean \pm the SEM. 


\subsection{Discussion}

\subsubsection{Summary of chapter}

In this chapter, two stable cell lines were created and assessed for expression of the APLNRGFP fusion protein. The stable transfection of glioblastoma cells (U87MG) proved to be challenging with deviation from manufacturer protocols required as well as optimisation of standard stable cell line generation techniques. Both cell lines were of high purity as confirmed through visual analysis and flow cytometry quantification. Both cell lines were shown by endpoint PCR to be expressing the full-length mRNA transcript, and elevated expression of APLNR was observed when comparing U87.APLNR and U87MG cell lines.

The U87MG cell line is one of the most widely studied immortalised glioblastoma cell lines [311] and is reported to be relatively easy to transfect which makes it an ideal cell line to study the role of the apelinergic system in glioblastoma. Chapter 3 revealed a significant reduction in the mRNA expression of $A P L N$ and $A P L N R$ in cultured cell lines compared to glioblastoma tumour tissue. Chapter 3 did report the presence of $A P L N R$ by immunocytochemistry and western blotting in immortalised in vitro cultured lines; however, this data was qualitative and did not reflect the total amount of $A P L N R$ present or the functional activity of the apelinergic system. To ensure a high degree of consistency regarding APLNR expression and an active apelinergic system, stable transfection of $A P L N R$ into the U87MG cell line was regarded as the best approach. Furthermore, it was hypothesised that stable APLNR transfection would replicate the expression of $A P L N R$ seen in resected tumour samples.

In this chapter, activation of previously-reported apelinergic system pathways was performed to ensure the model was representative of the apelinergic system. Finally, the functionality of the model system was tested by measuring its ability to regulate cell migration, which is reported in the literature to be modulated by APLN. The results from this study that APLN can cause migratory effects thus suggesting that the cell lines created would be appropriate as a model of the apelinergic system in glioblastoma. 


\subsubsection{Analysis of $A P L N R$ localization and expression}

The expression and localization of $A P L N R$ in the U87.APLNR cell line was analysed through visualisation of GFP using confocal microscopy and fluorescence microscopy. Expression of APLNR in untreated U87.APLNR cells were shown to be located in the periphery of the cell suggesting a cell membrane expression; however, following $\left[\mathrm{Pyr}^{1}\right]$ apelin-13 treatment, became punctate and localised to the cytosol. The internalisation of APLNR following activation by $\left[\mathrm{Pyr}^{1}\right]$ apelin-13 has been reported in the literature [373]. However, it has also been demonstrated that a strong expression exists exclusively in the plasma membrane [432, 434].

Protein expression, as demonstrated by western blotting in this study, indicated that the APLNR-Cterm antibody did not sufficiently bind the APLNR-GFP fusion protein. This is possibly due to the epitope of APLNR, to which the Cterm antibody recognises, being in close proximity to the region where the GFP protein is bound, thus preventing antibody binding. In the future, I would have liked to have purchased more APLNR antibodies from other companies to show the APLNR-GFP protein presence, but this was not financially viable. In addition, to ensure APLNR-GFP receptor expression is localised to the membrane, membrane fractions of U87.APLNR and HEK293.APLNR would be compared to U87MG and 0906 cells. This was shown by Kawahara et al. [434] in 3T3 cells transfected with a fusion APLNR-GFP protein [434].

\subsubsection{Analysis of commonly activated APLNR pathways.}

Receptor internalization of APLNR has been reported to occur due to the activation of the betaarrestin pathway involving amino acids at the $\mathrm{C}$ terminus of $A P L N R$ receptor [433]. The data presented shows that upon exogenous APLN treatment, receptor internalization can be seen observed in HEK293.APLNR, and to a lesser extent in U87.APLNR, cells. This suggests that the GFP tag does not interfere with receptor internalization. Internalization of $A P L N R$ was lower in U87.APLNR cells than HEK293.APLNR cells when measured by flow cytometry. This can also be seen when comparing the fluorescence micrographs of U87.APLNR and HEK293.APLNR treated with $\left[\mathrm{Pyr}^{1}\right]$ apelin-13 where more punctate and cytosolic expression 
of GFP is seen when treating HEK293.APLNR but not as much in U87.APLNR. This was attributed to the difference in homogeneity of the HEK293.APLNR population while U87.APLNR is a more heterogeneous population with different levels of APLNR-GFP expression in each cell type. Irrespective, the data still indicates that signalling mediated by the beta-arrestin pathway is active in both cell types.

The MAP kinase pathway is commonly activated upon APLN treatment and may be measured through phosphorylation of ERK by Western blotting. The U87.APLNR and HEK293.APLNR cell lines both demonstrated ERK phosphorylation in response to exogenous $\left[\mathrm{Pyr}^{1}\right.$ ]apelin-13 exposure. It was difficult to assess the changes in pERK with U87MG cell lines because the MAP kinase pathway is constitutively activated in cancer cell lines and pERK could not be reduced by serum starvation. Nonetheless, the changes noted in pERK/ERK ratio do suggest that the $\mathrm{G} \alpha$ subunit signalling is active.

These data suggest that apelinergic signalling is active in the U87.APLNR and HEK293.APLNR cell lines. In the future, other assays such as cyclic adenosine monophosphate (cAMP) inhibition could be performed to confirm other activated pathways.

\subsubsection{Migratory role of the apelinergic system in glioblastoma}

Migration has been reported several times to be modulated by the apelinergic system. Stable APLNR-GFP expression appeared to reduce migration in the transwell assay using U87.APLNR cells, compared to U87MG cells. The addition of exogenous [ $\mathrm{Pyr}^{1}$ ]apelin-13 abrogated this effect. When comparing $\left[\mathrm{Pyr}^{1}\right]$ apelin-13 treatment to the vehicle control, $\left[\mathrm{Pyr}^{1}\right.$ ]apelin-13 appeared to have a pro-migratory effect in U87.APLNR but not U87MG. It is unclear why stable $A P L N R$-GFP expression caused a reduction in migration. It is plausible that the effect is due to the apelinergic system signalling; however, it is also likely that this reduction of migration is due to the over-expression of the APLNR-GFP receptor. Further investigation would be required to determine the cause of the reduced migration seen.

A small, statistically significant, $\left[\mathrm{Pyr}^{1}\right]$ apelin-13-induced, induction of migration was seen in 0906 cells but not U87MG and U87.APLNR cells (Figure 4.17). U87MG cells were 
challenging to perform wound healing assays with due to their tendency to aggregate and form spheroids when nearing confluency, thus generating results that indicated a lack of migration. It is also plausible that an effect in the wound healing assay is not measurable because U87MG cells do not lend themselves to wound healing assays due to their tendency to form spheroids at high density, and at lower densities U87MG cells tend to not migrate into the wound space as readily due to the space already being present around them. The wound healing assay tends to favour cell lines that form monolayers such as HEK293 or 0906 cells of which migratory effects were also tested in this chapter.

From data presented herein, the biological role of the apelinergic system and its pro-migratory role, cannot be definitively confirmed in glioblastoma cell lines. It is possible that the apelinergic system has a mild pro-migratory role in glioblastoma tumour cells. It is more convincing that exogenous $\left[\mathrm{Pyr}^{1}\right.$ ] apelin-13 treatment in cells stably expressing APLNR results in migration as seen in the transwell assay and in wound healing assays using HEK293.APLNR. Moreover, based on the pro-migratory effects of [ $\mathrm{Pyr}^{1}$ ]apelin-13 observed in U87.APLNR and HEK293.APLNR cells lines, both cell lines are appropriate models to probe the role of the apelinergic system.

\subsubsection{Future directions}

Cells were cultured in a monolayer format, however, given more time alternative culturing methods would have been trialled such as a spheroid culture system. Spheroid cultures make use of non-adherent plates to induce the formation of multicellular tumour spheroids which are more representative of the original tumour structure even maintaining EGFR expression which is often lost through standard monolayer culture [435]. While spheroid cultures could have been generated and were initially trialled with U87MG and U87.APLNR cells (Appendix Section A.2), it was decided not to work with the spheroid cultures unless time and expense allowed. This would have opened the use of several other migration assays that utilize spheroids including the spheroid outgrowth assay. 
Additionally, I would have liked to extend the in vitro model generated in this chapter by injecting the U87.APLNR cells into SCID/NOD mice by either subcutaneous or stereotactic cranial injections and assessed the presentation of resultant tumours via haematoxylin and eosin staining but this was beyond the scope of the study.

In this chapter, U87.APLNR cells exhibited a loss in migration due to stable transfection. A truncated receptor was generated from the purchased plasmid (details described in the Appendix section A.1) which would have been beneficial as a control to the experiment in Figure 4.18. This truncated receptor was intended as a control to demonstrate APLN-APLNR specific signalling and demonstrate the effect of stable transfection. In short, a portion of the $A P L N R$ receptor sequence was removed, the ends were blunted and re-ligated together to create a fusion protein that would express part of the APLNR receptor and the GFP tag. The truncated receptor was not utilised due to insufficient time available to create a stable cell line and repeat all the experiments using this model. In addition, preliminary transfections of the truncated receptor exhibited high cytotoxicity suggesting the protein generated may be toxic. This truncated receptor would have been used in conjunction with the above-generated cell lines to better characterize the receptor dynamics and signalling processes of the apelinergic system.

To further verify the stably transfected cell lines signalling through the apelinergic system, internalisation studies utilising sucrose to prevent clathrin-mediated endocytosis in U87.APLNR and HEK293.APLNR cells would have been performed. This would have confirmed if changes in GFP expression during flow cytometry represented endocytosed receptors [436]. Furthermore, co-localisation studies examining APLNR expression with endosome/exosome markers would have been used to see if the punctate cytosolic expression was due to shuttling of the receptor to or from the membrane. It was difficult to determine if internalized receptors were marked for degradation and replaced by newly synthesised proteins or recycled to the membrane. Cycloheximide studies were attempted but were not successful.

Future studies examining the inhibitory effects of ML221 would have also been beneficial. This could have been performed using internalisation studies as described above to see if 
ML221 blocked these effects. This would have ensured signalling was mediated through the APLNR.

If more funding was available, further experimentation on the apelinergic system's involvement in cell migration using transwell assays would have been conducted. Specifically, examining the primary, 0814, 0906 and 1003 cell lines or better still, patient isolated biopsies of glioblastoma cells expressing elevated amounts of $A P L N R$. In addition, siRNA would have been used to knock-down $A P L N R$ expression to ensure the effect was mediated through the $A P L N R$ receptor. A transwell assay chamber with a Matrigel-coated membrane could also have been used to examine the ability of the $\left[\mathrm{Pyr}^{1}\right]$ apelin-13-treated cells to digest the Matrigel and migrate through the membrane pores as a measure of invasion.

\subsubsection{Conclusion}

The generation of U87.APLNR cell line was successful. The U87.APLNR model was considered beneficial due to the high expression of the APLNR receptor. Furthermore, changes seen due to exogenous $\left[\mathrm{Pyr}^{1}\right]$ apelin-13 addition in phosphorylated ERK levels and promigratory effects suggested that the apelinergic system was functional in the U87.APLNR cell line. Thus, it is an effective cell line for investigating the role of the apelinergic system in glioblastoma. The HEK293.APLNR cell line provides an excellent control cell line for studies and in addition will be beneficial for the testing of aptamers potentially generated in later chapters. Several glioblastoma cell lines will also be used during this thesis to corroborate data generated by these stable lines; this includes the primary glioblastoma cell line 0906. 0906 was selected due to its relatively higher mRNA expression of apelinergic system components as well as response to exogenous $\left[\mathrm{Pyr}^{1}\right]$ apelin-13 in the wound healing assay which makes it ideal for functional experiments and RT-qPCR experiments.

In summary, for the purposes of this thesis, the optimal choice of model is a combination of stably transfected cell lines and glioblastoma-derived primary cell lines. These would allow for initial fundamental investigations into the role of the apelinergic system in glioblastoma that could be followed up in more clinically relevant in vivo models potentially using the stable cell 
line generated. Two cell lines were generated stably expressing the APLNR receptor. These both showed an increase in ERK phosphorylation and changes in surface receptor expression in response to exogenous [ $\mathrm{Pyr}^{1}$ ]apelin-13 suggesting active apelinergic system signalling. Finally, apelin was demonstrated to be potentially pro-migratory in 0906 and U87.APLNR cells, also suggesting an active apelinergic system. These results suggested that the selected cell lines were reasonable models for use in subsequent experiments. This chapter also presented evidence that suggests APLN may have a potentially pro-migratory role in glioblastoma tumour cells, all though this requires further study. 


\section{Chapter 5 The role of the apelinergic system in cell}

\section{stress}

\subsection{Introduction}

\subsubsection{Role of the apelinergic system in stress response in glioblastoma}

During the genesis of this study, it was hypothesised that the apelinergic system might be involved in the response of glioblastoma cells to stress. In this chapter, glioblastoma cells were exposed to a number of stressors which are common-place within a tumour environment, such as nutrient deprivation, hypoxia and therapeutic treatment [181]. In response to these stressors, glioblastoma cells have been reported to modulate molecular responses which compensate and inevitably lead to the survival of the cell [437-439]. A typical example of this is the upregulation of hypoxia-inducible factors (HIFs) in response to hypoxia, which subsequently modulates further molecular pathways [440, 441]. There are many examples of down-stream genes being modulated due to common tumour stressors such as hypoxia or glucose deprivation (GD) $[249,442,443]$. Examples of this include astrocyte-elevated gene-1 (AEG1) [444], prominin-1 (PROM1) [445, 446] and doublecortin (DCX) [447]. These stress response mechanism are likely to improve survival and thus, disease progression in an otherwise suboptimal environment [181, 438, 441, 448].

Components of the apelinergic system have been reported to be upregulated under conditions of hypoxia and GD (discussed in length below) warranting further investigation into the response of the apelinergic system to cell stressors. Whilst a protective role by the apelinergic 
system in response to stress has been previously studied; it has not yet been investigated within cancer biology. The potential mechanisms through which the apelinergic system may facilitate a protective effect have been reviewed elsewhere [185], and a review of apelinergic system anti-apoptotic effects is shown in Chapter 1, Table 1.6. However, the study described in this chapter focussed on the protective response of the apelinergic system to specific stress stimuli (see below) hypothesising that the apelinergic system may be involved in the response of glioblastoma cells to these stressors.

\subsubsection{Hypoxia}

The apelinergic system has been shown to be modulated by hypoxia. As hypoxia is intrinsic to glioblastoma pathogenesis [440, 449], it is plausible that hypoxia-mediated APLN or APLNR expression may play a role in mitigating hypoxia in glioblastoma cells. The relationship between hypoxia and HIFla in glioblastoma is well established [450]. Moreover, the modulation of $A P L N$ expression by HIF1 $\alpha$ protein is also well established [10, 82]. Furthermore, hypoxia-mediated up-regulation of $A P L N R$ expression has also been noted in a number of cell lines (reviewed in Chapter 1 Table 1.7). Importantly, there have been several reports of the apelinergic system protecting against hypoxia-mediated cell death. For example, APLN-13 has been reported to protect against cobalt chloride (a known HIFla inducer) mediated death in Müller cells [250] and rat retinal pericytes [200]. APLN-13 has also been shown to protect against hypoxia-mediated cell death in RAW264.7 cells [234] and neonatal cardiomyocytes [230].

However, the involvement of the apelinergic system in the response of glioblastoma cells (or glioblastoma-derived cells) to hypoxia, has not been investigated previously. In this chapter, investigations were performed in glioblastoma cell lines to determine the effects of hypoxia on expression levels of APLN and APLNR mRNA in glioblastoma-derived cells. Furthermore, I hypothesised that APLN-13 would protect against hypoxia-mediated cell death in the U87.APLNR cell line. 


\subsubsection{Glucose deprivation}

The apelinergic system has also been reported to modulate glucose metabolism [25, 26]. Importantly, $A P L N$ and $A P L N R$ mRNA expression have been reported to increase due to complete GD in cardiomyocytes [194, 251] and Müller cells [250]. Furthermore, APLN has been reported to protect against cell death resulting from GD [234, 250, 251]. The research in this chapter investigated the response of the apelinergic system to GD in glioblastoma-derived cancer cell lines. Additionally, the effect of combined oxygen and glucose deprivation (OGD) on the apelinergic system in glioblastoma-derived cancer cell lines was also assessed because tumours often experience both conditions simultaneously and the interplay between hypoxia and glucose metabolism is critical in glioblastoma [399]. To study the response of the apelinergic system in glioblastoma-derived cells under GD and OGD conditions, glucose was withdrawn, and the effects on mRNA expression levels of APLN and APLNR were investigated. Finally, the effect of [Pyr1] apelin-13 on GD and OGD treated glioblastoma-derived cells was investigated.

\subsubsection{Chemotherapeutic compounds}

There have been no reports of the apelinergic system responding to, or protecting against, stress induced by chemotherapeutics. However, the body of evidence describing a protective role of the apelinergic system against cell death (summarised in Chapter 1, Table 1.6) suggests a potential role. Furthermore, anti-cancer agents like temozolomide (TMZ) and doxorubicin induce cell stress through mechanisms such as oxidative damage and endoplasmic reticulum (ER) stress. The apelinergic system has been reported to attenuate oxidative stress [259, 451] and protect against ER stress-mediated apoptosis [190, 258, 452]. Thus, the changes in mRNA expression levels of $A P L N$ and $A P L N R$ and the protective role of [Pyr1]apelin-13 in glioblastoma-derived cells undergoing TMZ or doxorubicin-mediated cell death, were also investigated in this chapter. 


\subsubsection{Chapter aims}

The primary aim of this chapter was to investigate the responses of the apelinergic system to cell stressors invoked by hypoxia, GD, OGD, TMZ and doxorubicin treatment in cultured glioblastoma cells. Specifically, the responses assessed were changes in expression levels of APLN, APLNR, solute carrier family 2 member 1 (SLC2A1) and Prominin 1 (PROM1) genes. The second aim of this chapter was to elucidate whether the apelinergic system, through the action of [Pyr1] apelin-13, protects against cell death induced by the aforementioned stressors.

The gene $S L C 2 A 1$ was determined to be the most appropriate marker of hypoxia as the HIF $1 \alpha$ gene is post-translationally regulated by oxygen levels. This is discussed in detail full below (5.4.1). As SLC2A1 mRNA is upregulated by HIFla binding to the SLC2A1 promoter [453], the SLC2A1 gene can act as an indirect indicator of hypoxia. This has been confirmed in hypoxic-regions within glioblastoma tumours [183] and in hypoxia-treated U87MG cells [454]. Moreover, a recent study revealed that the expression of $S L C 2 A 1$, but not $H I F 1 \alpha$, mRNA levels were correlated with a suite of other hypoxia-related markers (CA9, HIF2 $\alpha, V E G F$ and $O P N$ ) when comparing glioblastoma tissue to adjacent tissues in the brain [391]. The gene PROMI was examined due to its critical role in glioblastoma biology. Initially, PROMI was reported as a cancer stem cell (CSC) marker; however, this remains controversial [239, 455, 456]. Irrespective of its status as a CSC marker, PROMI mRNA expression has been reported to be upregulated under hypoxic conditions [449, 457]. It has also been reported to be a marker of bioenergetic stress in cancers [456, 458] including glioblastoma [238, 333, 445]. The presence of PROMI has been associated with several pathophysiological functions in glioblastoma [459] including mediating glioma cell resistance to nutrient deprivation [238]. These literary reports suggest that the PROMI gene is a suitable marker in glioblastoma biology. 


\subsection{Materials and Methods}

\subsubsection{Experimental design}

The experiments performed in this chapter utilise the immortalised cell lines U87MG, GL261 and LN18 and the primary cell line 0906. Stressors of hypoxia, GD, OGD and chemotherapeutic treatments were applied to some or all of these cell lines, and three response measures were assessed. Quantitative PCR was utilised to detect changes in expression levels of APLN, APLNR, SLC2A1 and PROM1 mRNA. Haemocytometer cell counts were used to detect changes in stressor-mediated cell death. In some cases, the addition of [Pyr1] apelin-13 to the cell culture medium occurred to elucidate a possible protective role against the aforementioned stressors. Finally, wound healing assays were performed to assess the effect of hypoxia on cell migration.

\subsubsection{Cell culture methodologies}

Standard cell culture methodologies and details of each individual cell line have been described previously in Chapter 2, Section 2.1. Several cell lines were used as models during this chapter and are described herein.

\subsubsection{Hypoxia experiments}

To measure the effects of hypoxic conditions on gene expression, cells were incubated under the conditions of $1 \% \pm 0.5 \% \mathrm{O}_{2}, 5 \% \mathrm{CO}_{2}$ and $94 \% \mathrm{~N}_{2}$ in a specialized hypoxia chamber $(\mathrm{C}$ Chamber, Biospherix LTD, Redfield, NY) for 24 hrs. Plates of normoxic cells were incubated adjacent to the hypoxia chamber in a $37{ }^{\circ} \mathrm{C}$ incubator under atmospheric oxygen and $5 \% \mathrm{CO}_{2}$ conditions. After $24 \mathrm{hrs}$ incubation, the media was aspirated, and $300 \mu \mathrm{L}$ of TRIzol reagent was added to each well. Total RNA was extracted from the cells and processed for qPCR as previously described (Chapter 2, Section 2.2). 
To assess the protective effects of [Pyr1]apelin-13, numbers of live cells incubated under hypoxia and normoxia conditions were counted as described below. It was apparent from preliminary data (Appendix A.6 Figure A.6.3) that the hypoxic conditions used in this study were not sufficient to cause cell death in $24 \mathrm{hrs}$ even under low serum concentrations (1\% FBS). Therefore, subsequent experiments were performed in the absence of FBS; with low FBS concentrations present as a control to demonstrate the effects of hypoxia on serum supplemented cells. Cells were then treated for $24 \mathrm{hrs}$ with [Pyr1]apelin-13 $(1 \mu \mathrm{M})$ or vehicle alone and incubated under either hypoxic $\left(1 \% \pm 0.5 \% \mathrm{O}_{2}\right)$ or normoxic conditions.

\subsubsection{GD and OGD experiments}

To measure the effects of GD and OGD on gene expression levels, cells were cultured in standard glucose-containing media or media with glucose omitted under normoxic conditions. In the case of OGD, cells were also cultured under hypoxic conditions (as described above). Standard glucose conditions and culture media varied between immortalised and primary cell lines. The immortalised cell lines (i.e. U87MG, U87.APLNR, GL261 and LN18) were cultured in DMEM medium containing $25 \mathrm{mM}$ glucose, whilst the 0906 primary cell line was cultured in RPMI medium containing $11 \mathrm{mM}$ glucose. After $24 \mathrm{hrs}$ of culture, cell media was removed from cells and replaced with $300 \mu \mathrm{L}$ of TRIzol. Total RNA was extracted from the cells and processed for qPCR as previously described (Chapter 2, Section 2.2.1).

Measuring the effect of [Pyr1] apelin-13 on GD and OGD-treated cells in regards to cell number was more complicated because complete GD leads to low numbers of adherent cells for haemocytometer counts (i.e. 1 - 5 cells per field of view) which is too low for accurate measurement. As such, glucose concentrations were optimised as discussed below (Section 5.3.2.2). The concentration of glucose was adjusted by adding filtered sterilised D-Glucose $\left(100 \mathrm{mM}\right.$ stock in $\left.\mathrm{ddH}_{2} \mathrm{O}\right)$ to glucose-free medium.

\subsubsection{Chemotherapeutic treatment experiments}

The effects of the chemotherapeutic agents doxorubicin and temozolomide on APLNR, APLN and PROM1 mRNA expression in glioblastoma-derived cell lines (i.e. LN18, U87MG and 0906) were measured. A preliminary experiment was performed in conjunction with a 
colleague (Dr Ryan Steel) to determine expression levels of the aforementioned genes in the U87MG and LN18 cell lines after $24 \mathrm{hrs}$ of exposure to $100 \mathrm{nM}$ doxorubicin in vitro. Due to time constraints, as well as difficulty in detecting the low abundance of APLNR transcripts in U87MG and LN18 cell lines, only the 0906 cell line was utilised for determining the effects of TMZ on APLNR, APLN and PROM1 gene expression levels. The rationale for treating the 0906 cells with $800 \mu \mathrm{M}$ of TMZ or $500 \mathrm{nM}$ of doxorubicin is described below.

The effect of [Pyr1]apelin-13 on doxorubicin and TMZ-induced cell number was tested. Initially, cell viability experiments were performed on U87MG and LN18 cell lines following treatment of $100 \mathrm{nM}$ doxorubicin using the propidium iodide (PI) dye test. This concentration proved to be insufficient to induce cell death, but higher concentrations masked the ability of the flow cytometer to detect the PI dye (Appendix A.5, Figure A.5.3). To mitigate this, haemocytometer cell counts were instead performed on adherent cells to enable higher concentrations of doxorubicin to be used. Doxorubicin concentrations were titrated to find the appropriate concentration for cell death (Appendix A.5, Figure A.5.1), and $500 \mathrm{nM}$ was selected as an appropriate concentration that elicited some but not total cell death.

Early experiments using the apelinergic system inhibitor ML221 on U87MG and LN18 cell lines were inconclusive. Furthermore, Chapter Three experiments revealed that all cell lines tested had low expression levels of APLNR. This caused concerns about cell line responsiveness to exogenous [Pyr1]apelin-13 because the APLNR receptor is required to respond to exogenously added [Pyr1]apelin-13. Thus, subsequent experiments utilised the U87.APLNR cell line (described in Chapter 4) to ensure the apelinergic system was at least active to elicit an appropriate response in this glioblastoma cell line.

Therefore the experiments presented below were performed on U87.APLNR cells treated with $500 \mathrm{nM}$ of doxorubicin and $800 \mu \mathrm{M}$ of TMZ. The TMZ concentration used was selected from previous studies by others, and doxorubicin concentration was determined by titration [323, 460-462] (Appendix A.5, Figure A.5.1). To control for the effects of TMZ and doxorubicin a "No treatment" control was included which was cells with no vehicle, doxorubicin, TMZ or [Pyr1]apelin-13; the vehicle group was treated with 1x PBS. 


\subsubsection{Methodologies Performed}

\subsubsection{Quantitative PCR}

For all qPCR experiments described below, immortalised cell lines U87MG, GL261 and LN18 and the primary cell line 0906 were used. The change in gene expression levels was quantified using a qPCR method described in full in Chapter 2, Section 2.2. Relative gene expression levels were analysed using the $2^{-\Delta \Delta \mathrm{CT}}$ method $[344,345]$ fully described in Chapter 2, Section 2.2.6.1. All data are expressed as $2^{-\Delta \Delta C \mathrm{~T}}$ values on a linear scale.

To assess if the change in gene expression for a gene of interest was significant, the delta CT $(\triangle \mathrm{CT})$ values for the treated and untreated condition of each cell line (0906, GL261, LN18 and U87MG) was analysed using a unpaired Student's t-test, using PRISM 5.0 (Graph Pad) as described elsewhere [463]. There are several data requirements before a Student's t-test may be used. Firstly, the data must exhibit a normal (Gaussian) distribution, and the D'Agostino \& Pearson omnibus normality test was to test this. Data for all genes were tested individually under each treatment condition, and all genes but $A P L N R$ were normally distributed. Thus, all genes including APLNR data was also graphed using a box plot to look at the symmetry and spread of data and compared to other genes where normality was detected, to ensure no major outliers or irregularities in the spread of data [464]. These analyses reported in Appendix, Section A.9. Asterisks were used to denote any significant differences in gene expression levels: $\mathrm{p}<0.001=* * *, \mathrm{p}=0.001$ to $0.01=* *, \mathrm{p}<0.01$ to $0.05=*$.

\subsubsection{Haemocytometer Cell Counting Method}

As briefly discussed above, two methodologies for measuring cell death were trialled. Initially, PI staining was performed as follows. Cells were seeded at 40,000 cells per well in a twentyfour well plate and incubated under normoxic conditions for $5 \mathrm{hrs}$ prior to subjection to test treatments or conditions for $24 \mathrm{hrs}$. To prepare cells for flow cytometry, media from each well was transferred to flow cytometer tubes. Adherent cells were washed with $300 \mu \mathrm{L}$ of $1 \times$ PBS and the washings were added to the cell media. Cells were removed from the well surface with 
the addition of $200 \mu \mathrm{L}$ of TrypLE. Following a 4 min incubation at $37^{\circ} \mathrm{C}, 800 \mu \mathrm{L}$ of cell media supplemented with $10 \%$ FBS was added to each well to inactivate the TrypLE. The entire 1 $\mathrm{mL}$ volume was added to the flow cytometer tube containing the initial media and wash solution. The tubes were centrifuged at $300 \mathrm{RCF}$ for $5 \mathrm{~min}$; the supernatant was discarded. A $300 \mu \mathrm{L}$ aliquot of FACS buffer (1x PBS $+1 \%$ FBS) was added to wash the cell pellet. Following a second centrifugation step, the pellet was resuspended in $300 \mu \mathrm{L}$ of FACS buffer. The staining step was performed by adding $20 \mu \mathrm{L}$ of PI $(20 \mu \mathrm{g} / \mathrm{mL}$ in $1 \mathrm{x}$ PBS $) 20 \mathrm{sec}$ prior to running the sample through the flow cytometer. Results showed very low percentages of cell death $(1-10 \%$ cell death) which made determining concordant changes in cell viability difficult. It was concluded that this method was suboptimal in this case.

As an alternative method, haemocytometer cell counts were then tested to determine whether cell death could be extrapolated from measures of remaining adherent cell counts relative to the starting number of cells. For all haemocytometer counts, cells were seeded at a density of 80,000 per well in six well-plates and cultured for $5 \mathrm{hrs}$ under normoxic conditions prior to the respective treatments as discussed above. Following a $24 \mathrm{hr}$ incubation period, adherent cells were collected using a similar procedure as described above for cell prepared for flow cytometry, with the exception that cells were transferred to $1.8 \mathrm{~mL}$ microcentrifuge tubes. The final suspension volume was in $100 \mu \mathrm{L}$ of serum-free cell culture media [465]. Initially, cell counts were performed using the trypan blue exclusion test to separately measure the live and dead cells in both the adherent and non-adherent populations of cells. However, as with the flow cytometry tests, the number of dead cells in both the adherent and non-adherent population was minimal ( $1-5$ cells per field of view). Thus, it was only possible to accurately count the clear (intact) cells. To assess if the differences in cell number were significant the paired Student's t-test was employed using PRISM 5.0 (Graph Pad). The limitations of using numbers of healthy cells relative to the starting number of cells are acknowledged and discussed in full below (Section 5.4.5). 


\subsubsection{Wound healing assay}

The 0906 cell line was used for wound healing assays because the cells grow efficiently in a monolayer, unlike U87MG and U87.APLNR cell lines, thus providing more technical accuracy and consistency at the expense of not expressing as high a level of APLNR. Differences in migration were asses using the paired Student's t-test with PRISM 5.0 (Graph Pad). Wound healing assays were performed as described in detail in Chapter 2 section 2.3.2.1.

\subsubsection{Treatments}

The preparation of [Pyr1]apelin-13 and ML221 solutions are detailed in Chapter 2, Sections 2.2.3.1 and 2.2.3.2, respectively. [Pyr1] apelin-13 and ML221 was used at concentrations of 1 $\mu \mathrm{M}$ and $5 \mu \mathrm{M}$, respectively. Cells were incubated in minimal FBS concentrations $0 \%(\mathrm{v} / \mathrm{v})$ FBS supplementation to minimize the confounding effects of FBS [466-468].. In most experiments, the incubation time was no longer than $24 \mathrm{hrs}$ due to stability concerns of the APLN peptide [348]. However, doxorubicin and TMZ haemocytometer count experiments were performed over $48 \mathrm{hrs}$ with a 50\% medium change at $24 \mathrm{hrs}$ to ensure the presence of [Pyr1]apelin-13. The experiments were performed in serum-free conditions ( $0 \% \mathrm{FBS})$. Doxorubicin was used at $500 \mathrm{nM}$ and TMZ at $800 \mu \mathrm{M}$ concentrations. Medium and non-adherent cells were removed at $48 \mathrm{hrs}$ and the remaining cell detached and counted by haemocytometer. 


\subsection{Results}

\subsubsection{Effects of hypoxia on the apelinergic system}

\subsubsection{Changes in gene expression under hypoxic conditions}

The mRNA expression levels of APLNR, APLN, SLC2A1 and PROM1 in four glioblastomaderived cell lines cultured under hypoxic condition are depicted in Figure 5.1. The results are presented as normalised against the expression levels of the control cells cultured under normoxic conditions. Overall, hypoxia led to the statistically significant upregulation of $A P L N$ mRNA expression in U87MG (unpaired t-test $(4)=9.599, \mathrm{p}=0.0007$ ) and LN18 (unpaired ttest $(4)=8.844, \mathrm{p}=0.0009$ ) (Figure 5.1). The 0906 cell line did upregulate APLN expression but not significantly so (unpaired t-test $(4)=1.835, \mathrm{p}=0.1404$ ). The GL261 cell lines did not exhibit $A P L N$ mRNA expression levels higher than control cells (unpaired t-test $(4)=0.8692$, $\mathrm{p}=0.4338$ ), and this may be attributed to its low abundance of $A P L N$ transcripts as indicated by significantly lower expression levels than that of the reference gene (HPRT; i.e. high mean $\triangle \mathrm{CT}$ value; see Table 5.1. The expression levels of APLNR mRNA were not altered by hypoxia in any of the cell lines tested, however, there was a trend (unpaired t-test $(4)=2.603, p=0.0599$ ) of $A P L N R$ mRNA up-regulation in U87MG cells under hypoxic conditions. It is important to note that the U87MG, GL261 and LN18 cell lines have a low transcript abundance of APLNR which makes detection difficult ( $\triangle \mathrm{CT}$ range: $12-20$, Table 5.1). Hypoxia caused an increase in SLC2A1 mRNA levels in U87MG (unpaired t-test(4) $=21.05, \mathrm{p}=<0.0001$ ) and GL261 (unpaired t-test $(4)=3.153, \mathrm{p}=0.0344$ ) cells. Notably, the U87MG cell line exhibited a $\sim 35$ fold increase in $S L C 2 A 1$ mRNA levels in response to hypoxia. Finally, the expression levels of PROM1 mRNA was upregulated in only the U87MG (unpaired t-test $(4)=7.593, \mathrm{p}=0.0016$ ) cell line due to hypoxia. 

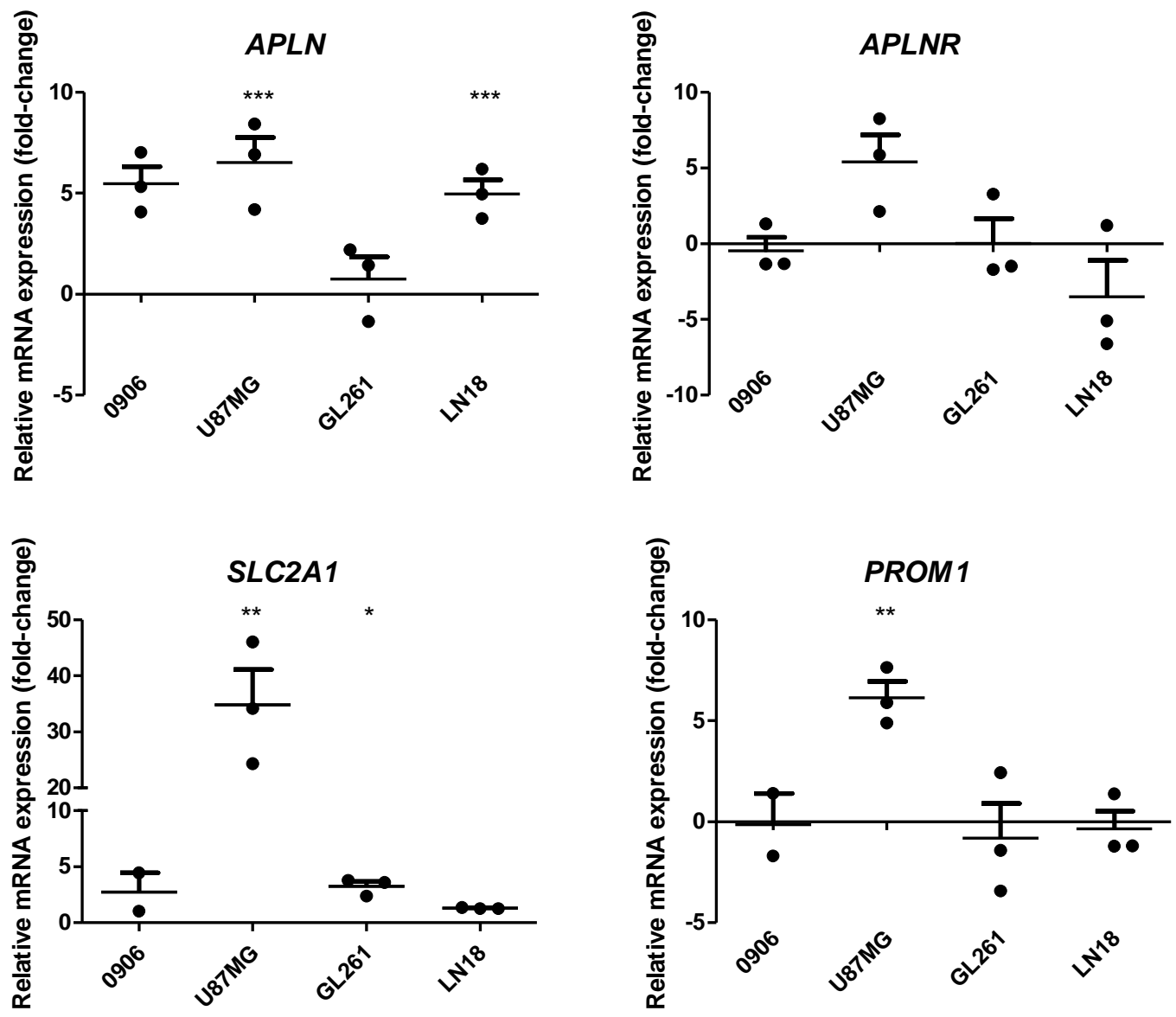

Figure 5.1. The effects of hypoxia on expression levels of APLN, APLNR, SLC2A1 and PROM1 mRNA. Cells were cultured under hypoxic $\left(1 \% \pm 0.5 \% \mathrm{O}_{2}\right)$ and normoxic conditions for $24 \mathrm{hrs}$. All data is normalized to a reference gene (HPRT) and expressed relative to the same cell line under normoxic conditions. Data are presented on a linear scale as mean $2^{-\Delta \Delta \mathrm{CT}}$ values \pm SEM. Each mean value was calculated from three biological replicates for each cell line. Significant changes in gene expression were ascertained by comparing the $\Delta \mathrm{CT}$ values using a Student's t-test. Asterisks were used to denote any significant differences in gene expression levels: $\mathrm{p}<0.001=$ $* * *, \mathrm{p}=0.001$ to $0.01=* *, \mathrm{p}<0.01$ to $0.05=*$. 
Table 5.1. The average delta cycle threshold $(\triangle \mathrm{CT})$ values for all genes under hypoxic and normoxic conditions. Average $\triangle \mathrm{CT}$ values were calculated by subtracting the CT value of the reference gene (HPRT) from the CT value of gene of interest) for each gene measured in four glioblastoma cell lines (U87MG, LN18, GL261 and 0906) treated under hypoxic and normoxic conditions.

\begin{tabular}{crrrrrrrr}
\hline \multicolumn{8}{c}{ Average delta CT's of genes under hypoxia and normoxia } \\
\hline Gene & \multicolumn{2}{c}{$A P L N$} & \multicolumn{2}{c}{$A P L N R$} & \multicolumn{2}{c}{$S L C 2 A 1$} & \multicolumn{2}{c}{ PROM1 } \\
Condition & Hypoxia & Normoxia & Hypoxia & Normoxia & Hypoxia & Normoxia & Hypoxia & Normoxia \\
U87MG & 6.12 & 8.77 & 17.13 & 19.35 & 3.48 & 8.56 & 14.42 & 17.02 \\
LN18 & 8.21 & 10.49 & 20.20 & 18.60 & 4.19 & 4.55 & 15.29 & 15.27 \\
GL261 & 16.10 & 16.50 & 12.23 & 12.35 & -4.84 & -3.17 & 13.89 & 13.55 \\
$\mathbf{0 9 0 6}$ & 9.14 & 11.56 & 8.86 & 8.70 & 3.81 & 4.72 & 9.88 & 9.75 \\
\hline
\end{tabular}

\subsubsection{Role of APLN in protecting against hypoxia-induced cell stress}

The effects of [Pyr1]apelin-13 on cell number in hypoxia-treated U87MG and U87.APLNR cells were compared in four to five replicate experiments, and the results are illustrated in Figure 5.2. The supplementation of culture media with 1\% FBS caused cell numbers to remain static or increase, to ensure the effects of hypoxia were not masked by FBS all experiments were performed in the absence of FBS. Under hypoxic conditions in both cell lines, and under normoxic conditions in the U87MG cells, the addition of [Pyr1]apelin-13 did not result in a higher number of live cells. However the addition of [Pyr1]apelin-13 to U87.APLNR cells under normoxic conditions appeared to cause an increase (paired t-test $(4)=2.761, p=0.0508$ ) in live cell number. This experiment was performed in duplicate $(\mathrm{n}=5$ independent experiments), with each coloured set of points on the graph representing a single experiment (Figure 5.2). Each experimental pair of points in the U87.APLNR cells cultured under normoxic conditions showed a pro-survival trend in the presence of APLN. It was noted this trend was not present in normoxic cultured U87MG cells (Figure 5.2). It was concluded that while there was a potential protective effect seen under normoxic conditions, $\left[\mathrm{Pyr}^{1}\right]$ apelin-13 did not modulate cell number in hypoxia-treated cells. 


\section{Hypoxia}

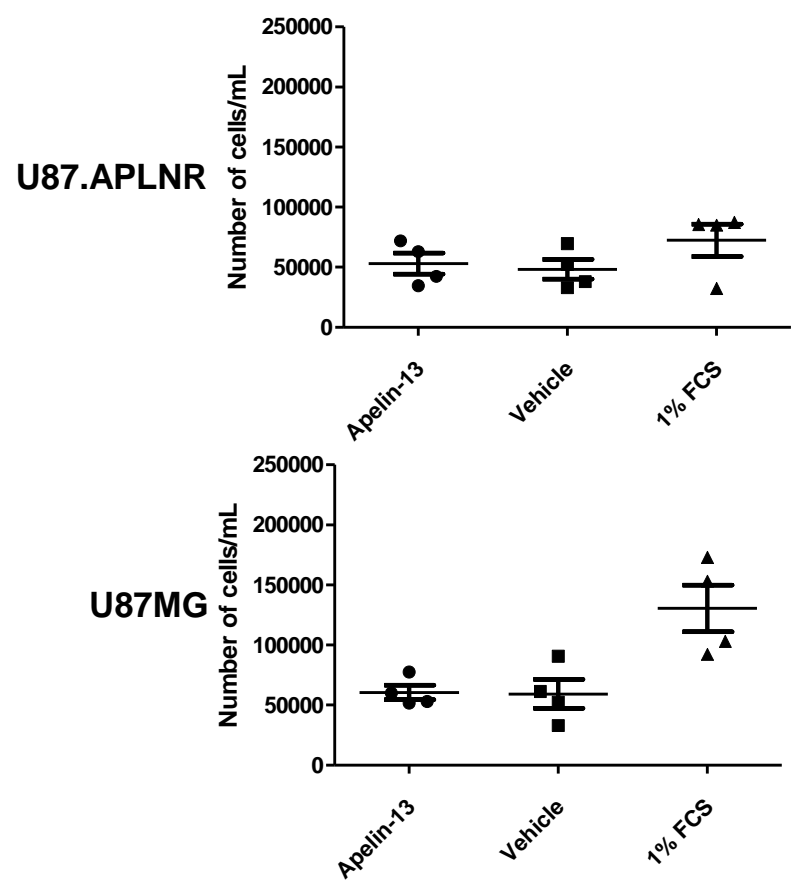

Normoxia

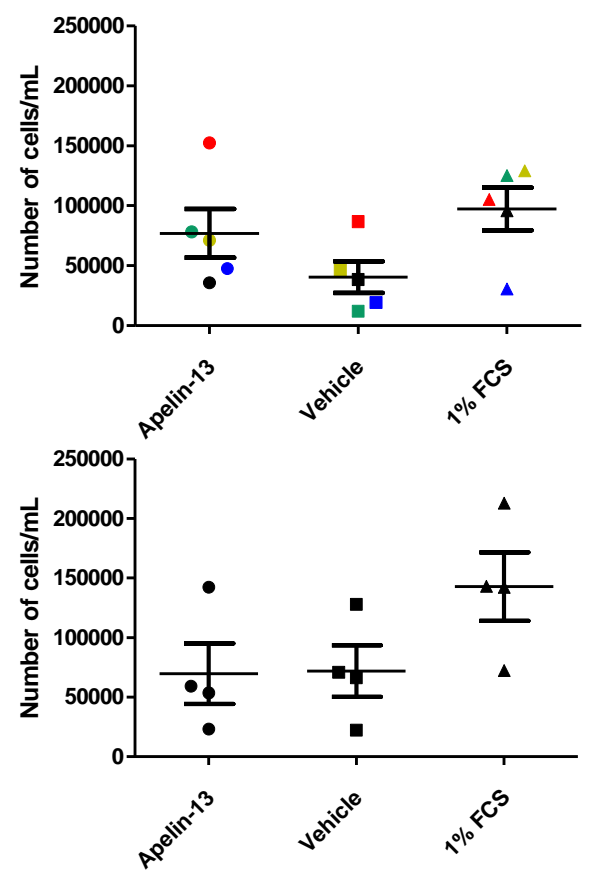

Figure 5.2. The effect of [Pyr1]apelin-13 on the survival of U87MG and U87.APLNR cell lines cultured in hypoxic and normoxic conditions. The U87.APLNR and U87MG cells were cultured under hypoxia $(1 \% \pm 0.5 \%$ $\mathrm{O}_{2}$ ) or normoxia for $24 \mathrm{hrs}$ under serum-free conditions with or without $1 \mu \mathrm{M}$ of [Pyr1]apelin-13 or in the presence of serum (1\% FBS). Each individual point represents the mean value of duplicates with four to five replicate experiments performed. Data are expressed mean \pm SEM. Each individual experiment for U87.APLNR cells cultured under normoxic conditions is coloured separately to illustrate the apparent trend. 


\subsubsection{The effect of hypoxia on glioblastoma cell migration}

Results within this chapter revealed that hypoxia upregulates $A P L N$ mRNA expression levels (Figure 5.1) which were hypothesised to augment the migratory response of these cells. To test this hypothesis, the effect of hypoxia on the migration of 0906 cells in the wound healing assay was tested (Figure 5.3). Interestingly, hypoxia had an anti-migratory effect (paired ttest $(2)=6.656, p=0.0218)$, rather than a pro-migratory effect, on 0906 cells. Addition of an apelinergic pathway inhibitor (ML221) was used to indicate whether these effects were facilitated via the APLNR however it appears that endogenous APLN was not involved in the anti-migratory effects (Figure 5.3, Panel B).
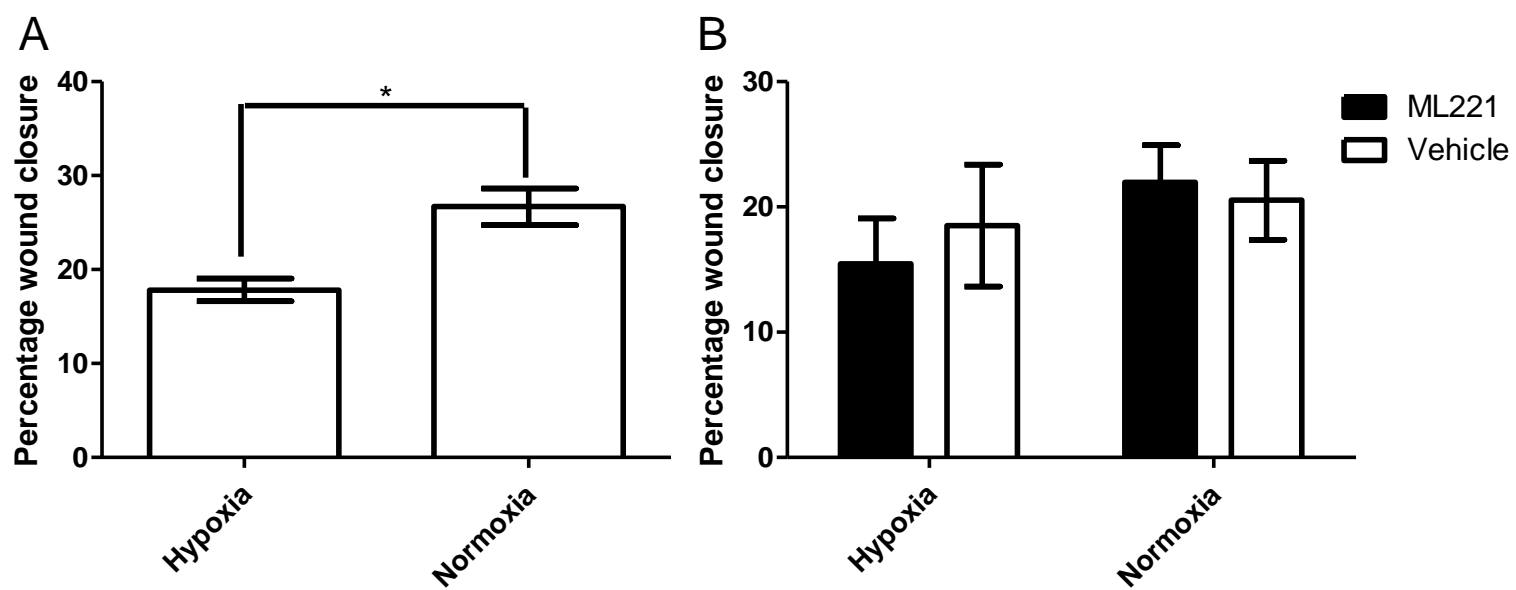

Figure 5.3. The effects of apelinergic inhibitor (ML221) on the migration of 0906 cells in hypoxic and normoxic conditions. Wound recovery was monitored under normoxic and hypoxic conditions in 0906 cells (Panel A). The inhibitor ML221 $(5 \mu \mathrm{M})$ was tested for its involvement in cell migration (Panel B). Data are expressed as mean \pm SEM, and each column represents three independent replicate experiments. Differences between groups are denoted by an asterisk $*=($ paired t-test $(2)=6.656, \mathrm{p}=0.0218)$. 


\subsubsection{Changes in gene expression during glucose deprivation and combined oxygen-glucose deprivation in glioblastoma-derived tumour cells}

The effect of GD on APLN, APLNR, PROMI and SLC2A1 mRNA expression in four glioblastoma cell lines are illustrated in Figure 5.4. All data presented is relative to control cells that were cultured in standard glucose concentrations under normoxic conditions. A significant reduction in APLN expression was evident both in U87MG (unpaired t-test(4) $=41.56, \mathrm{p}<$ 0.0001 ), LN18 (unpaired t-test $(4)=12.49, \mathrm{p}=0.0002$ ). GL261 cells showed a trend in response to GD (unpaired t-test $(4)=2.702, \mathrm{p}=0.0540$ ), whilst expression levels in 0906 cells showed no response. Interestingly, mRNA expression levels of APLNR increased in U87MG cells (unpaired t-test $(4)=14.35, \mathrm{p}=0.0001$ ) and appeared to increase in LN18 cells (statistical analyses not being performed due to $\mathrm{N}=2$ replicate experiments) in response to GD whilst expression levels in 0906 and GL261 cells again exhibited no response to GD. It should be noted that detection of $A P L N R$ in LN18 cells was challenging due to the low transcript abundance $(\triangle \mathrm{CT}=18.6)$ (Table 5.1). In contrast, SLC2A1 mRNA was significantly elevated in U87MG cells (unpaired t-test(4) $=9.825, \mathrm{p}=0.0006$ ), GL261 cells (unpaired t-test(4) $=3.240, \mathrm{p}=0.0317$ ) and LN18 (unpaired t-test $(4)=3.820, \mathrm{p}<0.0188$ ) but not 0906 cell lines in response to GD (statistical analyses not being performed on 0906 cells due to $\mathrm{n}=2$ replicate experiments). There was an upregulation in PROM1 mRNA expression following GD in only U87MG cells (unpaired t-test(4) $=7.593, \mathrm{p}=0.0016$ ) LN18, GL261 and 0906 showed no change in $P R O M 1$ in response to GD. 

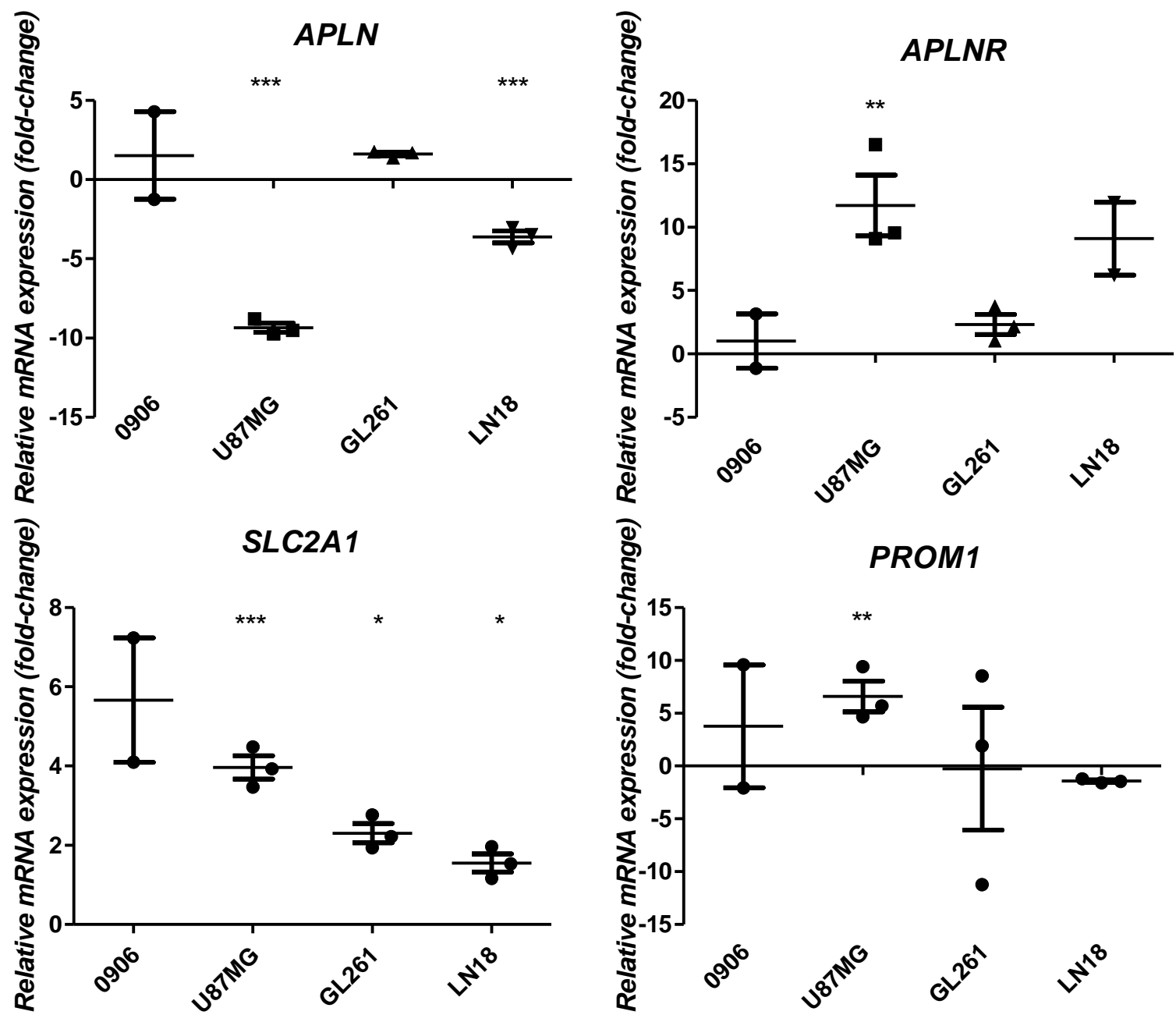

Figure 5.4. The effects of glucose deprivation on APLN, APLNR, SLC2A1 and PROM1 mRNA expression in four glioblastoma cell lines. All data were normalized to a reference gene (HPRT) and expressed relative to the cell line under euglycemia conditions. Data are expressed as the mean \pm SEM from three independent replicate experiments. The exception to this is APLNR mRNA levels in 0906 and LN18 cells where two replicate experiments were performed, in these cases statistics were not performed, and the range of data points are shown. Significant changes in gene expression were ascertained by comparing the $\Delta \mathrm{CT}$ values using a Student's t-test. Asterisks were used to denote any significant differences in gene expression levels: $p<0.001=* * *, p=0.001$ to $0.01=* *, p<0.01$ to $0.05=*$. Student's t-tests were only performed where $\mathrm{n}>2$. 


\subsubsection{The effect of combined oxygen and glucose deprivation (OGD) on mRNA expression by glioblastoma-derived cell lines}

The effects of OGD on mRNA expression levels of APLN, APLNR, SLC2A1 and PROM1 in four glioblastoma cell lines are depicted in Figure 5.5. There was increase of APLN mRNA expression in response to OGD in U87MG (unpaired t-test(4) $=6.940, p=0.0023$ ), LN18 (unpaired t-test $(4)=4.539, \mathrm{p}<0.0105$ ) and GL261 (unpaired t-test $(4)=3.106, \mathrm{p}=0.0360$ ) but not 0906. Interestingly, expression levels of $A P L N R$ mRNA did not change in response to OGD. Whilst the 0906 cells no response of $S L C 2 A 1 \mathrm{mRNA}$ levels following OGD, all other cell lines showed significant increases of 150-fold in U87MG (unpaired t-test(4) $=36.72, \mathrm{p}<$ 0.0001 ), 14-fold in GL261 (unpaired t-test(4) $=8.775, \mathrm{p}=0.0009$ ) and 4-fold in LN18 (unpaired t-test $(4)=17.83, \mathrm{p}<0.0001$ ) cells. Expression levels of PROM1 mRNA only increased in U87MG cells (unpaired t-test $(4)=41.56, \mathrm{p}<0.0001$ ) in response to OGD, and remained unchanged in the GL261, LN18 and 0906 cell lines. 

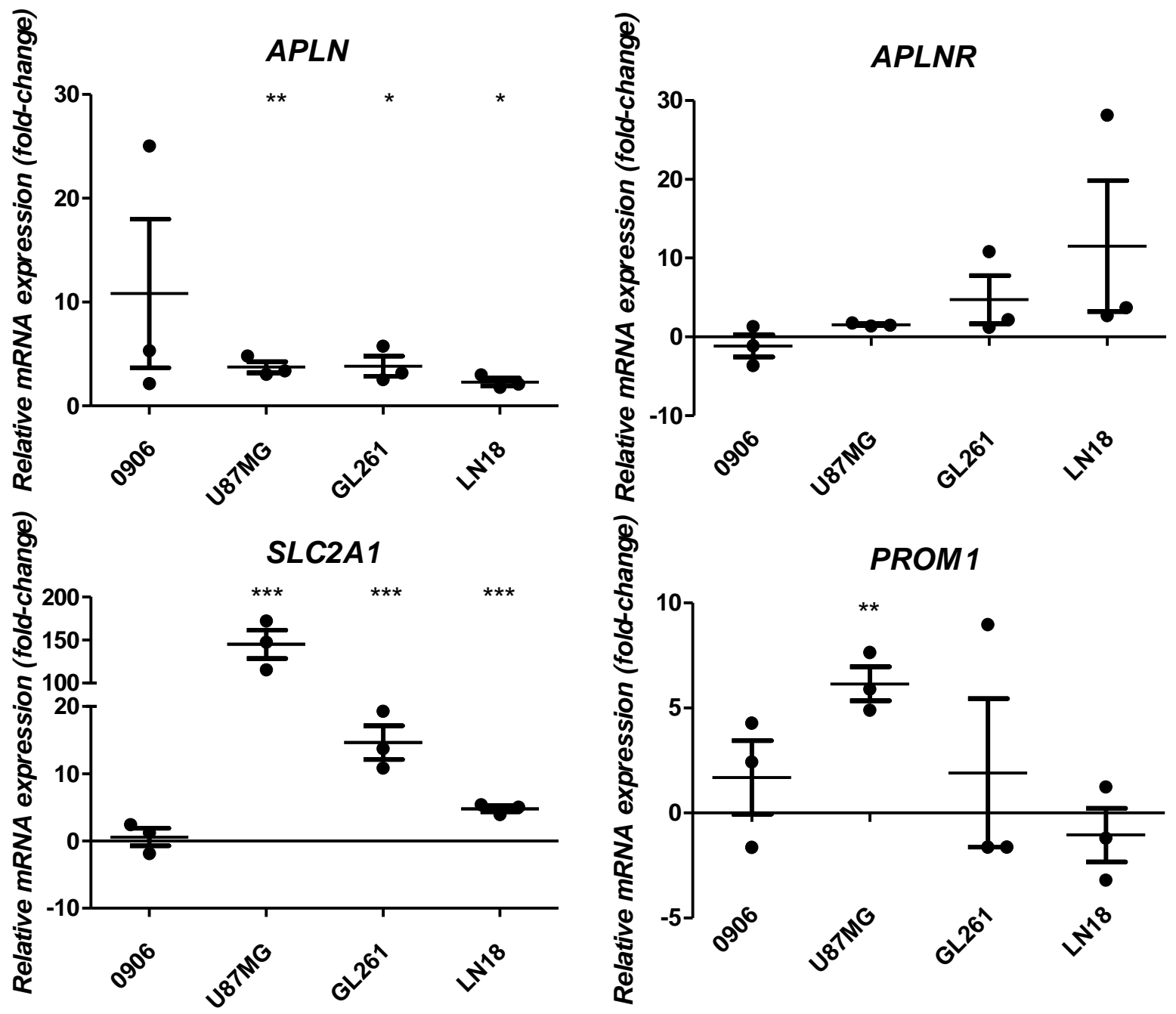

Figure 5.5. The effects of combined hypoxia and glucose deprivation on mRNA expression levels of $A P L N$, $A P L N R, S L C 2 A 1$ and PROM1 in four glioblastoma cell lines. All data are normalized to a reference gene (HPRT) and expressed relative to the same cell line under normoxic euglycemia conditions. Data are expressed as the mean \pm SEM from three independent replicate experiments for each cell line. Significant changes in gene expression were ascertained by comparing the $\Delta \mathrm{CT}$ values of the treated and untreated groups using a Student's t-test. Asterisks were used to denote any significant differences in gene expression levels: $\mathrm{p}<0.001=* * *, \mathrm{p}=$ 0.001 to $0.01=* *, \mathrm{p}<0.01$ to $0.05=*$. 


\subsubsection{Optimization of an assay to determine the effect of [Pyr1]apelin- 13 in conditions of GD and OGD.}

To test the protective effects of [Pyr1]apelin-13 on under conditions of GD and OGD, glioblastoma cells were cultured in the presence or absence of [Pyr1]apelin-13 with GD or OGD stress. This was tested in the U87.APLNR cell line using the U87MG cell line as a control. Several preliminary experiments were undertaken to choose optimal culturing conditions that would elicit sufficient GD- and OGD-induced cell death without an excessive loss of cell numbers.

A preliminary experiment in which U87MG cells were cultured in low glucose and euglycemic conditions ( $2 \mathrm{mM}$ glucose, $1 \%$ FBS) in the presence or absence of the APLNR inhibitor (ML221) is illustrated in (Figure 5.6). Neither glucose concentration nor the presence of ML221 had any effect on the proportion of dead cells. Cell death in this preliminary experiment was measured using PI viability. The low numbers of dead cells $(<10 \%$ cell death) suggested inaccurate measurements. As such, haemocytometer cell counts were performed on the adherent cells to assay better assess if cell number was static or if cells were dying and losing adherence. Additionally, this preliminary experiment also highlighted the need to generate the U87.APLNR cell line (see Chapter 4) to ensure that any lack effect due to the addition of apelinergic system modulators such as [Pyr1]apelin-13 was not merely due to an insufficient apelinergic system within the immortalised cell line. 


\section{U87MG OGD}

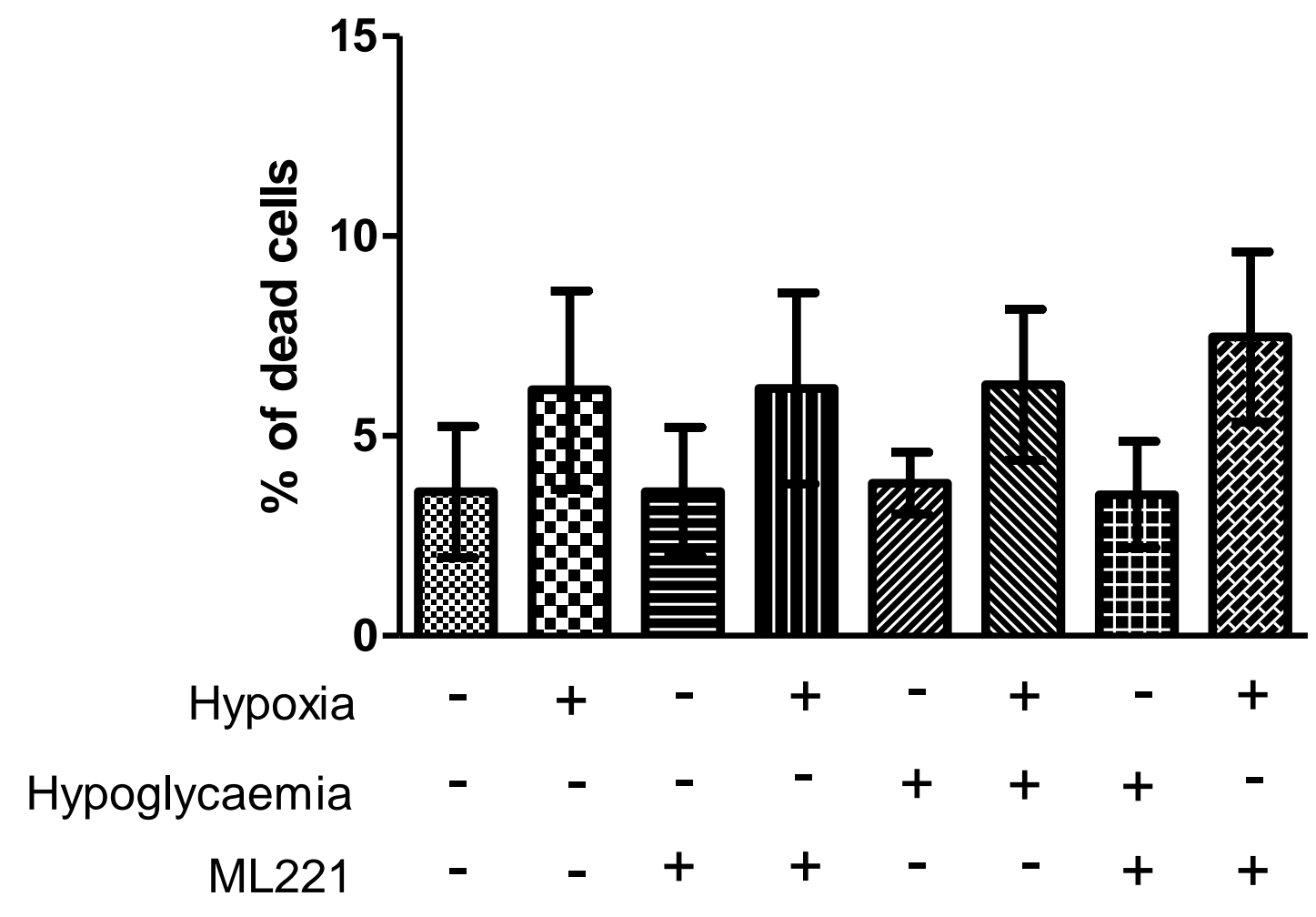

Figure 5.6. The effects of low glucose concentrations and the APLNR inhibitor (ML221) on U87MG cells incubated under normoxic and hypoxic conditions. U87MG cells were cultured during normoxic or hypoxic conditions in standard $(25 \mathrm{mM})$ or low $(2 \mathrm{mM})$ glucose conditions in combination with the presence or absence of ML221 $(5 \mu \mathrm{M})$. Cell viability was measured using propidium iodide viability staining and flow cytometry. All experiments were performed in triplicate ( $\mathrm{n}=3$ independent experiments), and values are expressed as mean \pm SEM.

A preliminary experiment was performed to assess the ability of [Pyr1]apelin-13 to protect against GD in U87.APLNR and U87MG cells (Figure 5.7, Panel A). The data indicated that [Pyr1] apelin-13 protected U87.APLNR, but not U87MG, cells but considering the marked loss of cells due to GD ( 2000 cells counted from 80,000 cells seeded), the ability to make accurate measurements was dubious. To prevent such a marked effect of GD on cell death, glucose concentrations were titrated against cell number, and the optimal glucose concentration for 
U87MG cells was selected as $0.25 \mathrm{mM}$ as it elicited a substantial loss of cells without too drastic a reduction to ensure accurate cell counts (Figure 5.7, Panel B).
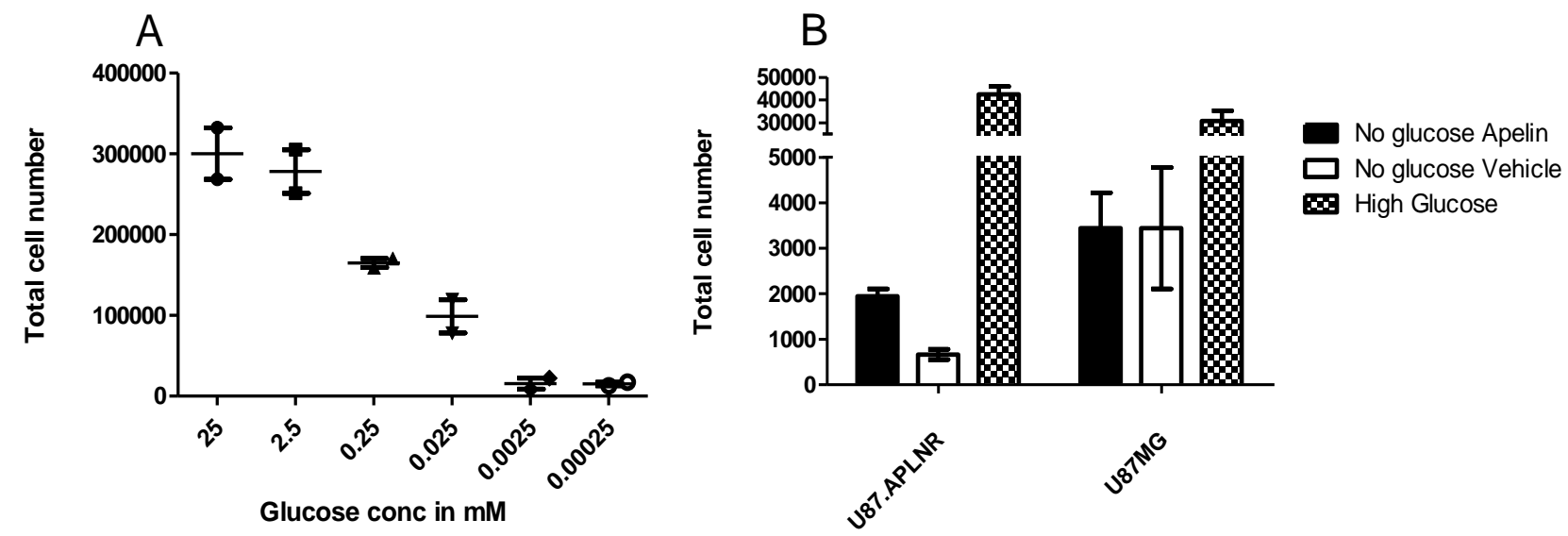

Figure 5.7. The effects of [Pyr1]apelin-13 on cell death induced by glucose deprivation (GD) in U87.APLNR and U87MG cells (A) and the correlation between glucose concentrations and total cell number (B). The optimal glucose concentrations needed for some cell death but not a total loss of U87MG cells was also determined by titration after $24 \mathrm{hrs}$ in culture in differing glucose concentrations and adherent cells counted using a haemocytometer (Panel B) ( $\mathrm{n}=1$ in duplicate). Cells were cultured in GD or normal glucose concentrations (25 $\mathrm{mM})$ for $24 \mathrm{hrs}$ with or without [Pyr1] apelin-13 $(1 \mu \mathrm{M})$, after which haemocytometer cell counts were performed on the adherent cells $(n=1)$. Values are expressed as the mean \pm SEM.

It was hypothesized that APLN might protect against GD-mediated cell death, but a less aggressive GD environment may be required to observe this. The previous experiment was therefore repeated but in this modified experiment, U87.APLNR and U87MG cells were cultured in $0.25 \mathrm{mM}$ glucose. Following a $24 \mathrm{hrs}$ incubation under normoxic and hypoxic conditions with no serum supplementation, in the presence and absence of [Pyr1] apelin-13, the numbers of adherent cells were counted.

Over several attempts, no substantial loss of cell number was noted when compared to standard glucose conditions. Moreover, no [Pyr1] apelin-13effect was seen in U87MG or U87.APLNR cells stressed with GD or OGD. . Furthermore, inter-experimental variation was difficult to control and was unclear why; suggestions are made in the discussion below. Overall, 
exogenous [Pyr1]apelin-13 addition was without effect, even in the case of the stably transfected cells with overexpressed APLNR.

\subsubsection{The effects of chemotherapy drugs on $A P L N$ and $A P L N R$ mRNA}

Chemotherapeutic-mediated stress was examined in the cell lines utilised in this study. The mRNA expression levels of $A P L N, A P L N R$ and PROM1 in three glioblastoma cell lines (U87MG, LN18 and 0906) after treatment with doxorubicin (Figure 5.8) and TMZ (Figure 5.9).

Initially, the change in gene expression after doxorubicin treatment (100nM) was assessed in LN18 and U87MG cells. Detection was challenging due to low transcript abundance especially with regards to APLNR. After $24 \mathrm{hrs}$ of incubation, APLN mRNA expression was decreased in U87MG (unpaired, t-test(4) $=3.722, \mathrm{p}=0.0204$ ) but not in LN18. APLNR mRNA expression appeared to be increased in U87MG but decreased in LN18, but this was not statistically significant. There was an upregulation of PROM1 mRNA in U87MG (unpaired, ttest $(4)=6.368, p=0.0031$ ) which was consistent with reports by others [469] but not LN18. 

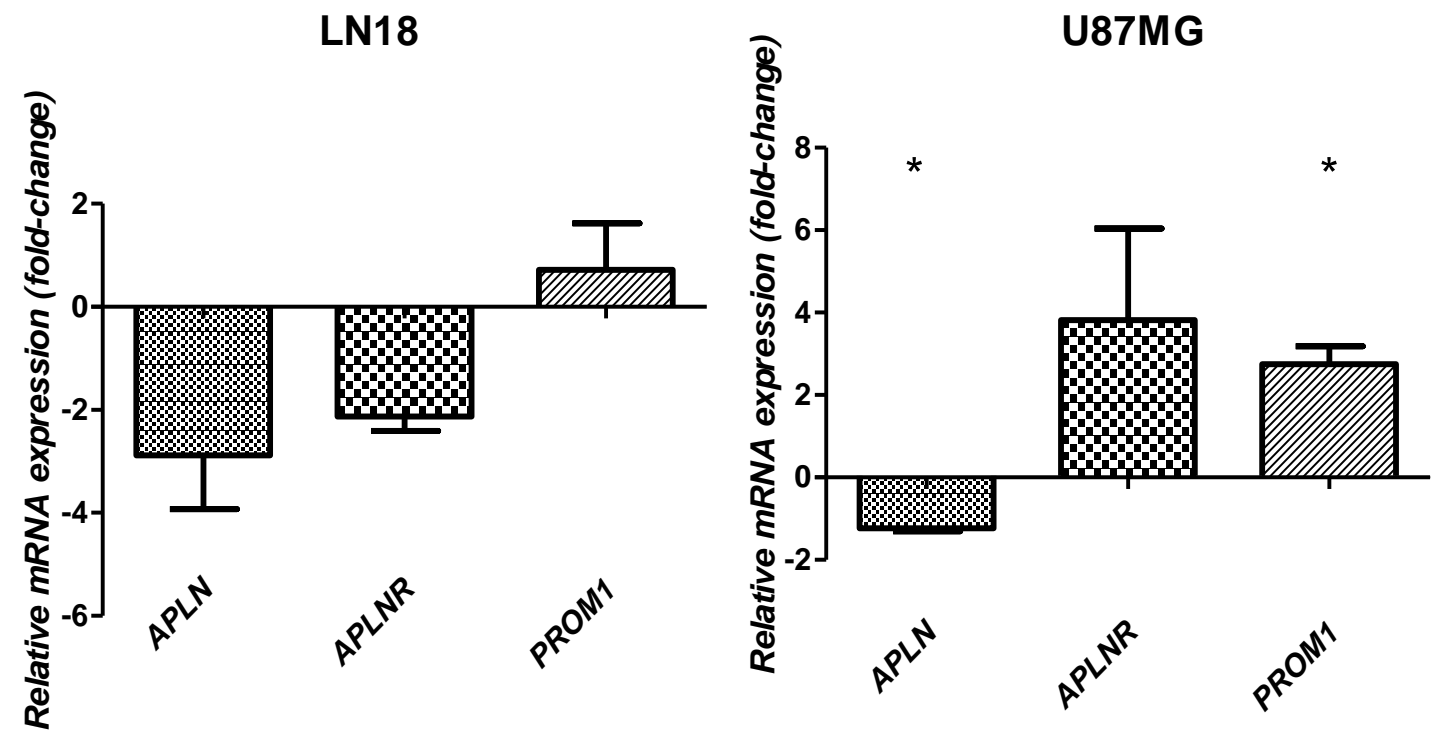

Figure 5.8. The effects of doxorubicin treatment on expression levels of PROM1, APLN and APLNR mRNA in U87MG and LN18 cells. Cells were cultured for $24 \mathrm{hrs}$ in doxorubicin $(100 \mathrm{nM})$. All data are normalized to HPRT and expressed relative to the vehicle-treated control cell line. Values are presented as mean \pm SEM of three replicate experiments. Significant changes in gene expression were ascertained by comparing the $\triangle \mathrm{CT}$ values of the treated and untreated groups using a Student's t-test. Asterisks were used to denote any significant differences in gene expression levels: $\mathrm{p}<0.001=* * *, \mathrm{p}=0.001$ to $0.01=* *, \mathrm{p}<0.01$ to $0.05=*$.

In order to remedy the difficulties in detection due to low transcript abundance, the 0906 primary glioblastoma cell line, which has high expression of APLNR and APLN mRNA expression (See Chapter 3), was also tested for chemotherapeutic stress effects (Figure 5.9). Unpaired Student's t-tests were performed on all data except PROMI expression due to low n. There were no statistically significant changes in gene expression due to doxorubicin or TMZ treatment. 

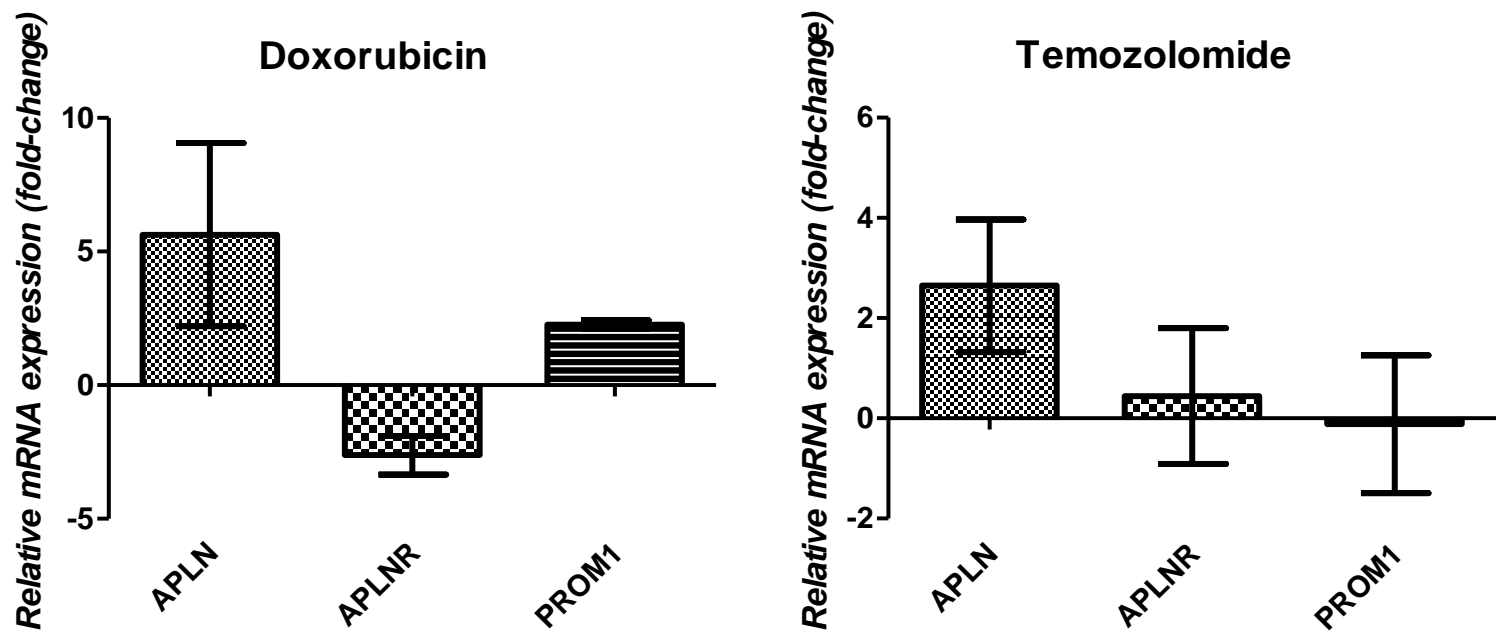

Figure 5.9. The effects of doxorubicin and TMZ treatment on expression levels of PROMI (2n), APLN (3n) and $A P L N R(3 \mathrm{n})$ in 0906 cells. The concentrations of doxorubicin and TMZ used on 0906 cells were $500 \mathrm{nM}$ and $800 \mu \mathrm{M}$. Results are presented as mean \pm SEM values.

The lack of consistent changes in the mRNA expression of APLN and APLNR is suggestive that the apelinergic system is not critical to responding to chemotherapeutic stress. In addition to mRNA expression, the effect of exogenous [Pyr1]apelin-13 on the U87MG and U87.APLNR cell lines following treatment with doxorubicin and TMZ was investigated.

\subsubsection{Investigating a protective role of apelin on the doxorubicin- mediated death of U87.APLNR cells}

A protective role of the apelinergic system in doxorubicin-mediated cell death was investigated. The effects of [Pyr1]apelin-13 on doxorubicin-treated U87.APLNR cells are illustrated in Figure 5.10. Cells were incubated for $48 \mathrm{hrs}$ in the presence of doxorubicin with either [Pyr1]apelin-13 or a vehicle control to determine if [Pyr1]apelin-13 had any protective effects against doxorubicin. A 50\% media change at $24 \mathrm{hrs}$ to enable accumulation of cell death to enable significant changes in cell number. Doxorubicin treatment decreased the number of adherent U87.APLNR cells in vehicle (paired t-test $(5)=4.329, \mathrm{p}=0.0075$ ) and [Pyr1]apelin13 groups (paired t-test $(5)=3.923, p=0.0111$ ) compared to the no treatment control. This 
cytotoxic effect was moderately abrogated by [Pyr1]apelin-13 (paired t-test $(5)=3.961, \mathrm{p}=$ 0.0107). Thus, [Pyr1]apelin-13 protected the cells against doxorubicin-mediated stress.

\section{Doxorubicin treated U87.APLNR}

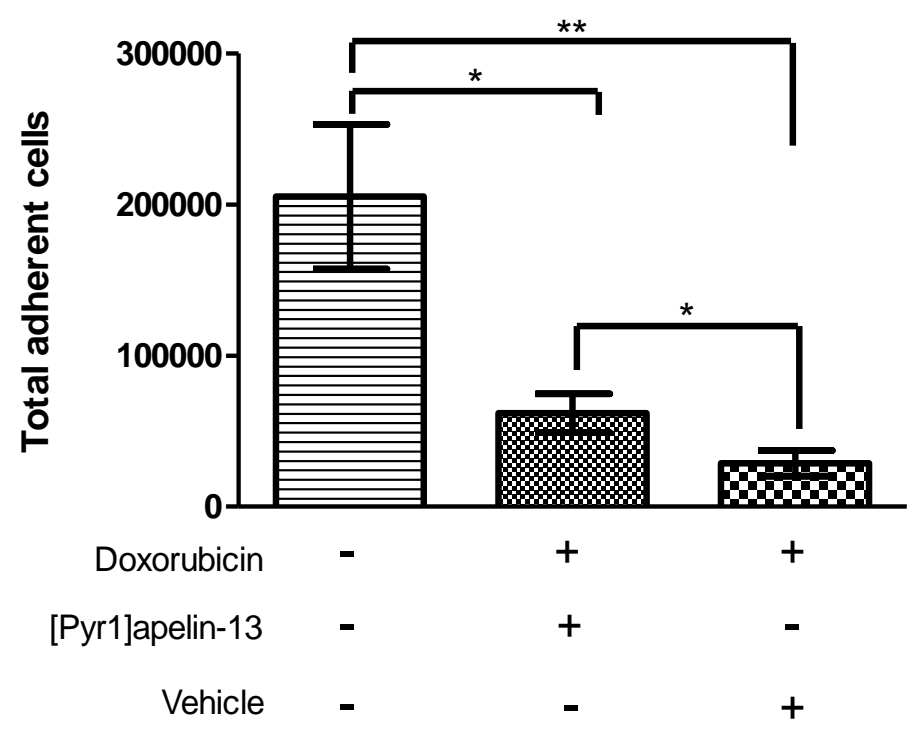

Figure 5.10. The effects [Pyr1] apelin-13 on doxorubicin-mediated death in U87.APLNR cells. The concentrations of doxorubicin and [Pyr1] apelin-13 used were $500 \mathrm{nM}$ and $1 \mu \mathrm{M}$, respectively. Non-adherent (dead) cells were removed, and the remaining adherent (live) cells were counted by haemocytometer. Results are presented as mean \pm SEM values from five independent experiments. Significant differences between treatment groups are denoted by asterisks. Changes in adherent cells were assessed using a paired Student's t-test. Asterisks were used to denote any significant differences, $* *=0.0075, *=0.0111,,^{*}=0.0107$ (vehicle vs [Pyr1]apelin-13). 


\subsubsection{Investigating a protective role of apelin temozolomide-mediated death of U87.APLNR cells}

A protective role of the apelinergic system in TMZ-mediated cell death was also investigated as TMZ is a more clinically relevant compound. The effects of [Pyr1] apelin-13 in TMZ-treated U87MG and U87.APLNR cells are illustrated in Figure 5.11. Whilst there were no TMZmediated effects on the loss of U87MG or U87.APLNR cells, the addition of [Pyr1] apelin-13 increased (paired t-test $(5)=3.274, \mathrm{p}=0.0221$ ) the survival rate of U87.APLNR cells, regardless of TMZ treatment. This suggests [Pyr1]apelin-13 may have a mitogenic role since cell number was higher than the cells that did not receive TMZ.

U87MG

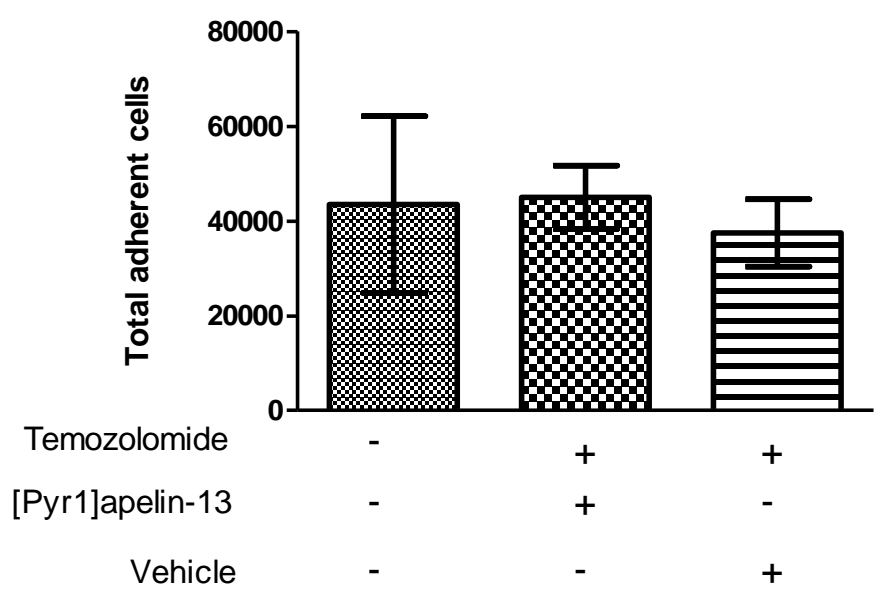

U87.APLNR

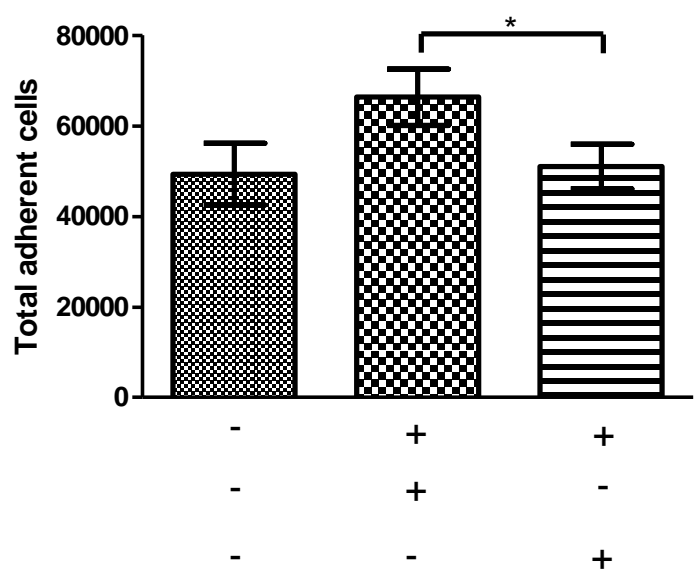

Figure 5.11. The effects of [Pyr1]apelin-13 on the cell number of temozolomide (TMZ)-treated U87MG and U87.APLNR cells. The concentrations of TMZ and [Pyr1] apelin-13 were $800 \mu \mathrm{M}$ and $1 \mu \mathrm{M}$. Non-adherent (dead) cells were removed, and the remaining adherent (live) cells were counted by haemocytometer. Results are presented as mean \pm SEM values from five independent experiments. Significant differences between treatment groups are denoted by asterisks $(* \mathrm{p}=0.0221)$. 


\subsection{Discussion}

Cancers often upregulate genes as part of a response to stress stimuli; this enables tumour cell survival $[181,470]$. During the inception of this thesis, it was hypothesised that components of the apelinergic system might be upregulated under conditions of cell stress. This chapter sought to characterize the changes in gene expression of $A P L N$ and APLNR under several cell stress stimuli that glioblastoma tumour cells are known to endure in vivo. Individually, these are hypoxia, GD, OGD and chemotherapy treatment. Changes in gene expression of these aforementioned genes could be used as a proxy to indicate the involvement of the apelinergic system in the mediation of stress responses of the glioblastoma cells. Previous reports had suggested a protective role for the apelinergic system in cancer [185]. Thus, in this chapter, glioblastoma-derived cells were cultured under stress conditions with and without [Pyr1] apelin-13, and cell numbers, as a measure of cell survival, were assessed to test this.

\subsubsection{The role of the apelinergic system in glioblastoma response to hypoxia}

During these experiments it was critical to have a gene that was modulated by hypoxia and the gene $S L C 2 A 1$ is an appropriate indicator of hypoxia in glioblastoma [391]. Historically, the transcription factor HIF1 $\alpha$ has been used; however, it has been reported several times in the literature that hypoxia-mediated upregulation of $H I F 1 \alpha$ is not necessarily detectable at a mRNA level [471]. This has also been reported extensively in glioblastoma literature [212, 403, 472-477]. This lack of change of HIF1 $\alpha$ at an mRNA level could be attributed to the posttranslational regulation of HIF1 $\alpha$, with most regulatory pathways modulating HIF $1 \alpha$ protein levels or binding affinity rather than mRNA levels [478, 479]. Thus, the up-regulation of HIF $1 \alpha$ is better measured at a protein level than at mRNA level but was unable to be completed during this study. 
Interestingly, hypoxia only upregulated $S L C 2 A 1 \mathrm{mRNA}$ expression levels in U87MG and GL261 cells, but not in 0906 and LN18 cells. In concordance with other studies in U87MG cells [454], U87MG incubated in hypoxia conditions increased SLC2A1 mRNA expression levels by 35 -fold.

PROM1 is critical to glioblastoma pathogenesis, and hypoxia has been reported to increase PROM1 mRNA expression levels in glioblastoma cells [445, 457]. Changes in PROM1 mRNA expression in response to hypoxia were inconsistent between glioblastoma cell lines with only the U87MG cells showing increased PROM1 mRNA levels. Whilst variation between cell lines was not unexpected, the marked induction of PROM1 transcription in only U87MG cells was intriguing and may suggest that specific glioblastoma cell lines are more appropriate to investigate the relationship of hypoxia in glioblastoma pathogenesis. Alternatively, it could suggest that recently-resected glioblastoma cells are the only appropriate in vitro cell line to use for this purpose.

APLNR mRNA levels were not significantly upregulated in any of the cell lines tested; however, U87MG cell did exhibit a trend towards upregulation. Whilst upregulation of $A P L N R$ due to hypoxia has been reported previously in other cell lines (Summarised in Chapter 1 , Table 1.7), it has not been reported in tumour cells and did not occur in cell lines tested. It is important to consider that U87MG cells exhibited increased APLNR and PROM1 mRNA expression levels as well as a marked increase in SLC2A1 mRNA expression. As SLC2A1 and PROM1 are indicators of a hypoxic response and glioblastoma pathogenesis, respectively, it could be argued that U87MG cells provide a more accurate model of hypoxia in glioblastoma.

Most significantly, hypoxia-induced $A P L N$ mRNA expression occurred to varying degrees in two of the four glioblastoma cell line tested. This has not been reported previously in glioblastoma cells and is suggestive of an intact hypoxic-sensitive response element located upstream of the $A P L N$ gene as has been reported for other established cancer cell lines [3, 10]. While there was an upregulation of $A P L N$ mRNA in 0906 cells, this was not significant in the unpaired Student's t-test likely due to the variation in biological replicates. However, when examined using the paired Student's t-test, the response to hypoxia was statistically significant 
(paired t-test(2) $\mathrm{t}=10.66, \mathrm{p}=0.0087$ ). The failure of hypoxia to regulate Apln mRNA expression in the GL261 mouse glioblastoma cell line was unexpected and may suggest species differences in the role of the apelinergic system in responding to hypoxia-mediated stress.

In the future, additional experiments examining the expression of $A P L N$ protein under differing hypoxic conditions will be essential to ascertain under what conditions APLN is produced in glioblastoma. This should be performed in freshly resected glioblastoma tumour derived cell lines.

\subsubsection{Protection by the apelinergic system in hypoxia-mediated stress}

The apelinergic system has been implicated to have an anti-apoptotic role in several diseases, but evidence for APLN-13 protecting against cell death in cancer cells is limited [87]. The purpose of the upregulation of $A P L N$ in response to hypoxia was hypothesised to be a protective mechanism against hypoxia-mediated stress, but no effect of [Pyr1]apelin-13 on hypoxiatreated cells was observed in the present study. This lack of effect could be attributed to the apelinergic system not having a protective role against hypoxia-mediated cell stress or due to an insufficient incubation time under hypoxic conditions as there was no significant reduction of cell number after hypoxic incubation for $24 \mathrm{hrs}$. Unfortunately, an extension of hypoxia culture time was not possible due to concerns of degrading [Pyr1] apelin-13. Moreover, if a media change was desired at $24 \mathrm{hrs}$ (in total hypothetical incubation time of $48 \mathrm{hrs}$ ), this would cause a sudden influx of oxygen which may mask results.

\subsubsection{Effects of hypoxia on cell migration}

In Chapter 4, it was established that apelin could have a pro-migratory effect on glioblastoma cells. It was hypothesised that the upregulation of $A P L N$ expression due to hypoxia in two to three out of the four glioblastoma cell lines increase their migratory capability as a mechanism to bring cells toward the hypoxic environment. This hypothesis was made because a promigratory role for hypoxia has already been reported for U87MG cells using multi-well insert transmembrane assays [211, 480, 481]. 
The 0906 cell line was selected as the glioblastoma cell line to test the effects of the apelinergic system on hypoxia-induced cell migration utilising a wound healing assay. The inhibition of the migration observed in the present study in response to hypoxic conditions was unexpected. The pro-migratory effects of hypoxia as reported in the literature may be a chemotactic response that is not likely to be measured in a wound healing assay, which measures chemokinetic migration.

Upon retrospective thought, it was also realised that the putative secretion of APLN due to hypoxia may, in fact, not recruit glioblastoma tumour cells such as the ones used during this study but would instead recruit cells that may alleviate hypoxia such as vascular cells.

Due to the limitation stated above, the role of the apelinergic system in hypoxia-induced cell migration is inconclusive. In retrospect, the transwell assays would have been a more effective assay to investigate migration by using hypoxia cultured glioblastoma tumour cell conditioned media as a chemoattractant. A short interfering RNA (siRNA) approach to removing APLN from conditioned media could be used; then both APLN present and absent conditioned media could be used in the transwell assay. In addition, testing other cell lines migratory capacity in response to APLN, i.e. vascular cells such as endothelial or pericytes cells would be advantageous.

\subsubsection{Future directions for testing the role of the apelinergic system in hypoxia-induced stress response.}

In hindsight, it was apparent that the apelinergic system may not hold a role in cell survival during hypoxic stress; however, there may have been other roles for hypoxia-induced APLN expression that could have been assayed. Glioma cells often respond to cell stress through the process of autophagy to mitigate cell death, choosing to degrade damaged or redundant organelles to survive [482]. Autophagy has also been implicated to have an essential role in the progression of glioblastoma [482]. Hypoxia has been reported to induce autophagy in glioblastoma cells [209, 483] and the apelinergic system has been reported to regulate autophagy [90, 251]. Measuring autophagic markers such as autophagosome formation after 
hypoxic treatment of glioblastoma cells cultured in the presence and absence of [Pyr1]apelin13 may reveal more insights into the role of the apelinergic system in regulating hypoxiamediated responses.

Furthermore, retrospective contemplation lead to future consideration of using western blotting to monitor changes in intracellular APLN and HIF $1 \alpha$ protein levels, as well as secreted APLN, but may have required primary glioblastoma cell lines obtained from biopsied tissue which exhibits a higher endogenous expression of $A P L N$. The use of freshly derived glioblastoma cells provides a model more analogous to in situ glioblastoma tumours.

The link between hypoxia, APLN and angiogenesis is well established [10, 405, 484]; however, the role of the apelinergic system in alternative mechanisms of tumour vascularization [485] is yet to be investigated. One such mechanism that was hypothesised during this thesis that hypoxia-induced APLN expression may have a role in vascular mimicry, which is the process where tumour cells form vessel-like networks $[485,486]$. Vascular mimicry is well described in glioblastoma [487, 488]. Other hypoxia-meditated angiogenic peptides such as VEGF have been noted to have effects on vascular mimicry [489]. The Matrigel tube formation assay [350] has been used by researchers to determine the angiogenic potential of glioblastoma tumour cells by seeding tumour cells onto a Matrigel surface and observing the structures formed [486, 487, 489]. The vascular mimicry assay used in the present study was limited by insufficient time to perform repeated measurements to reduce variation in the responses. These results are presented in the Appendix (Appendix A.7). If optimised, this assay would have given some indication of the role of the apelinergic system in vascular mimicry, especially under hypoxic conditions, and this avenue may be worth further investigation. 


\subsubsection{The role of the apelinergic system on glucose deprivation and combined oxygen-glucose deprivation in glioblastoma}

\subsubsection{The effect of GD on gene expression levels}

Glucose metabolism is a critical process in glioblastoma because not only is glucose required for the rapid growth of glioblastoma tissue [240] but elevated glucose levels have been associated with poor outcomes in patients with glioblastoma [490-492]. It has been suggested that targeting of glucose metabolism could be a viable treatment methodology $[362,397]$ with several reports even suggesting approaches such as ketogenic diets that reduce carbohydrates may help with treating glioma [493-498]. The role of the apelinergic system in both mediating glucose metabolism uptake $[58,337]$ and the response to GD in cardiomyocytes $[194,251]$ and retinal glial cells [250] makes understanding the role of the apelinergic system under GD in glioblastoma important.

The changes in gene expression due to GD were measured (Figure 5.4) and found to be highly variable, with the exception of SLC2A1 which was upregulated in all cell lines except 0906. The upregulation of $S L C 2 A 1$ due to hypoxia has been reported previously [499, 500]. Interestingly, $A P L N$ and $A P L N R$ expression were inversely correlated in U87MG cells. The decrease in APLN expression and a concomitant increase in APLNR expression in these cells may suggest a regulatory feedback loop between ligand and receptor. Conversely, due to the lack of response in other cell lines, this could be an artefact. Nevertheless, the correlation between APLN and APLNR appear to be unrelated to the expression of PROM1 and SLC2A1 mRNA, if $P R O M 1$ and $S L C 2 A 1$ are considered indicators of response to GD. The U87MG cell line, however, responded strongly to both GD and OGD by upregulating SLC2A1 and PROMI expression, potentially indicating a more relevant response to what may occur in the original tumour. A firm conclusion is difficult to make from the results above. Recently-resected primary glioblastoma cell lines could replace the immortalised cell lines as a more representative form of glioblastoma to determine changes in expression levels of key genes after culturing in conditions of GD and OGD. 


\subsubsection{Effects of combined hypoxia and GD on gene expression}

The condition of OGD is common in glioblastoma biology because the poor functionality of the vasculature and rapid growth leads to areas that exhibit simultaneous oxygen and glucose deprivation. In addition, hypoxia modulates glucose metabolism with reports of glycolysis begin favoured over oxidative metabolism in glioblastoma tumour cells [501, 502] via activation of the HIF1 $\alpha$ transcription factor [253].

The apelinergic system could be critically involved in this axis between hypoxia and glucose deprivation. HIFla is also known to modulate APLN expression [10] and, based on the increase of $A P L N$ due to hypoxia, HIF1 $\alpha$-induced upregulation of $A P L N$ is likely to occur in glioblastoma cells as well. Under OGD conditions, APLN mRNA was upregulated in U87MG, GL261 and LN18 cells under hypoxic conditions. However, this did not occur in GD conditions alone. This could suggest that $A P L N$ mRNA production is driven by hypoxia and not modulated by changes in glucose concentration.

The expression levels of SLC2A1 increased 2 - 5 fold in U87MG and GL261 cells exposed to hypoxia and GD, as reported in the literature [499, 500]. However, under OGD conditions, SLC2A1 expression increased over 100-fold in U87MG cells and over 10-fold in GL261 cells suggesting a synergistic effect under OGD conditions. This was not unexpected has HIF1 $\alpha$ modulates SLC2A1 mRNA expression [453, 503]. However, there was no similar evident change in APLN, and APLNR mRNA expression in response respond to GD and OGD mediated stress apart from the upregulation of $A P L N$ under OGD conditions. It appears unlikely that there is a direct relationship between $A P L N$ and $A P L N R$ and conditions involving low glucose.

\subsubsection{Role of apelin protecting against GD and OGD cell death}

\subsubsection{The optimization of low glucose conditions}

Experiments were performed to determine if the apelinergic system had any effect on cell survival in stressed glioblastoma cell lines exposed to GD and OGD. The optimization of GD conditions was challenging in comparison to hypoxic conditions. Initially, low glucose 
conditions were tested on U87MG cells with low serum supplementation (1\% FBS). The low glucose $(2 \mathrm{mM})$ conditions used initially was $\sim 1 / 10^{\text {th }}$ the standard glucose concentration in the medium, yet it yielded no significant changes in cell viability over 24 hours. The lack of a further response in the form of cell death in glioblastoma cells cultured in low glucose concentrations suggested that the low 1\% FBS conditions were masking cell death. To remedy this, the appropriate glucose conditions was determined to obtain a reasonable level of cell death and was found to be $\sim 0.25 \mathrm{mM}$. A serum-free, glucose-free experiment was performed on U87.APLNR and U87MG cells but cell death was too high to enable accurate cell counts. Thus it was concluded that a serum-starved medium containing a reduced $(0.25 \mathrm{mM})$ glucose concentration would be optimal to induce cell death without too substantial a reduction in cell numbers.

Using the optimised conditions described above, no significant effects on cell number due to GD or combined OGD were observed after 24 hours in U87MG and U87.APLNR cells. This lack of a glucose-mediated effect suggests there were technical flaws in the experimentation that were not accounted for since others have reported a clear and significant effect of low glucose on cell growth and viability [240, 242, 244, 504-506].

The GD and OGD aspects of this investigation were challenging in deciding the right glucose concentration and serum concentration. Retrospectively there were several changes that could have been made to better identify the role of the apelinergic system in glioblastoma cells under GD and OGD stress. Initially, during this chapter, PI viability was used as a measure of cell death. This was later modified to cell counting as for this yields more information than just the proportion of dead cells which doesn't necessarily take factors such as quiescent cells into account. Other measures of GD responses should also have been used such as cycle analyses, ROS levels [240], Akt phosphorylation [247, 507], lactate dehydrogenase release [505], AMPK activation [508] and appearance of autophagy markers [209]. Some of these outcomes have already been measured regarding the apelinergic system under GD conditions in other cell lines. For example, under GD conditions, autophagy markers were reduced in cardiomyocytes [251] and measurement of viability markers indicated protection against cell 
death in retinal glial cells [250]. A more comprehensive study of the effects of the apelinergic system on different responses of glioblastoma cells may beneficial in future studies.

Adequate incubation time is critical to allow the accumulation of sufficient changes in the culture to be measured, and a $24 \mathrm{hrs}$ culture time might have been insufficient. For future experiments, an extended incubation time could be used for GD experiments.

The medium composition used during GD experiments is essential to assuring precise conditions of cell stress that leads to cell death, but this consistency was not seen during the experiments conducted herein. The media used was glucose-free DMEM to allow for glucose concentration adjustments. Care was taken to use medium free of alternative carbon sources such as sodium pyruvate. In addition, even though confounding factors such as FBS and glutamine have been reported not to affect the viability of GD U87MG, LN18 and LN229 cells [240], it was decided that FBS concentration should be minimized to reduce its role in protecting cells from death or influencing cell proliferation [509]. Serum concentrations used previously in studies of GD in glioblastoma cells have varied considerably, ranging between $0 \%$ [507], $1 \%$ [248] up to $10 \%$ FBS [240, 242, 247]. The majority of papers utilized $10 \%$ FBS and glucose-free conditions but made use of a dialyzed FBS to remove the glucose present in FBS [242] which appears to be critical to GD experiments. The use of low serum (1\% FBS) in initial experiments may have confounded earlier results. In the future, dialyzed serum with no glucose should be used rather than the minimal glucose and no serum approach of this study. The culture conditions were likely the primary issue, in the future, a more defined cell medium would be used in conjunction with several other measures as described prior.

The $2 \mathrm{D}$ culture system used in this study was not replicative of the tumour environment. The U87.APLNR cell line could be used in a 3D model such as a spheroid. Spheroids are more reflective of the original tumour phenotype with features such as concentration gradients of nutrients and have been used prior within the context of studying glucose metabolism in glioblastoma [510]. Comparing glucose metabolism throughout spheroids made up of U87MG or U87.APLNR cells may have revealed more about the apelinergic system role in glucose metabolism. Alternatively, spheroids generated from patient isolated tissue could be used. 
A conclusive role of the apelinergic system in GD glioblastoma cells was unable to be established based on the results of these studies. The lack of consistent gene expression changes between cell lines to the tested stressor made it difficult to assess. Moreover, 0906 and GL261 cells exhibited no changes in APLN or APLNR under GD. This lack of consistency may suggest that there is no role for the apelinergic system under GD conditions. However, further research using previously described experiments would help elucidate the apelinergic system's role in GD and OGD. The data in this chapter, though not comprehensive, suggest the apelinergic system does not have a central role in promoting cell survival under conditions of hypoxia or GD and OGD. The upregulation of APLN mRNA expression in hypoxic and OGD conditions is suggestive that the apelinergic system has some role in glioblastoma tumour biology with regards to hypoxia, but further investigation is clearly needed.

\subsubsection{The effects of anti-cancer agents and the apelinergic system}

\subsubsection{Conclusions and future experiments on chemotherapy stress effects on the apelinergic system}

The reported anti-apoptotic role of the apelinergic system [185] suggests that it may influence cell survival under anti-cancer treatment stress. The apelinergic system has been shown in one study to respond to DNA damage by regulating the DNA damage repair enzyme 8-oxoguanine glycosylase (OGG1) [203]. However, no other evidence has been reported of a direct link between the apelinergic system and DNA-damaging agents. The mRNA expression results, however, show that there was no consistent significant change in APLN and APLNR mRNA expression following doxorubicin treatment with the exception of $A P L N$ downregulation in U87MG cells. The PROM1 gene has been reported as a stress marker in glioblastoma [333, 445] and was significantly upregulated in U87MG (2-fold) but not LN18 suggesting there was a low cellular response to doxorubicin. Moreover, there was no concomitant upregulation of $A P L N R$ and downregulation of $A P L N$ which suggests that the apelinergic system may not have a role in response to doxorubicin. Further analysis of cell lines expressing APLN and APLNR at more similar levels to original tumour tissue may be beneficial. The LN18 and U87MG cell 
lines were established and cultured in serum for long periods of time; whereas, the 0906 cell line arguably has a phenotype more akin to the original tumour due to its more recent origin from the parent resected tumour. This is reflected in a higher expression of apelinergic system components (Chapter 3). However, there were no significant changes in 0906 cells under TMZ or doxorubicin treatment. It would be interesting to follow up this result by measuring mRNA levels of $A P L N$ and $A P L N R$ in response to chemotherapeutic agents in other recently resected glioblastoma-derived cell lines. Furthermore, correlating APLN and APLNR expression at a protein or mRNA to other markers of DNA damage such as gamma-H2AX would be beneficial.

Because APLNR expression would be necessary for detecting APLN mediated effects, the stably transfected U87.APLNR cell line was used to see if [Pyr1]apelin-13 protected against anti-cancer treatment induced cell death. The U87.APLNR cells were treated for 48 hrs with high dose doxorubicin $(500 \mathrm{nM})$, which resulted in significant cell death that was reduced by [Pyr1]apelin-13 treatment. This is suggestive that exogenous [Pyr1]apelin-13 may have a protective role in doxorubicin-mediated cell stress. Following treatment of U87.APLNR cells with TMZ, the addition of [Pyr1]apelin-13 also improved cell survival, a result not seen with U87MG cells. Due to the general lack of toxicity seen after TMZ treatment, the increase in cell numbers could suggest that apelin has a protective effect when treated with chemotherapeutic agents or that apelin has a pro-mitogenic role. Previous data from our lab demonstrated no proliferative effects of apelin in GL261 cells, measured by MTT assays and phosphohistone 3 $\left(\mathrm{PH}_{3}\right)$ staining (Ryan Steel and Abraham Mains, unpublished data). In retrospect, further investigation of the mitogenic capacity of apelin in glioblastoma tumour cells may have been worth assessing as other labs have demonstrated a mitogenic effect of apelin in tumour cell lines [9]. A more definitive proliferation experiment could have made use of low serum medium (as opposed to 0\% FBS), treating U87.APLNR cells with [Pyr1]apelin-13 and measuring a proliferative marker such as Ki67 or bromodeoxyuridine pulse labelling.

High concentrations of TMZ $(800 \mu \mathrm{M})$ had little cytotoxic effect on U87.APLNR and U87MG cells. The lack of TMZ toxicity at high concentrations was unexpected as TMZ is reported to be effective at lower concentrations than what was used during this study [511]. It could be asserted that because $\mathrm{TMZ}$ is a prodrug that, once metabolised in the liver, generates the active 
compound methyltriazenoimidazole-carboxamide (MITC). Thus hepatic activation is hypothetically required for TMZ use [512]. However, TMZ is also advantageous in that it spontaneously converts to its active form in solution at a neutral and alkaline $\mathrm{pH}$ [513-515] thus not requiring hepatic activation for its use in in vitro experiments. In the future, testing for the active form of TMZ would be beneficial to ensure activity, in addition to this testing TMZ and doxorubicin activity with other glioblastoma cell lines with high APLNR expression would be beneficial.

Two hypotheses can be put forward to explain the effects seen in the present study. It is plausible that apelin may have an anti-apoptotic role in response to DNA damaging agents. Alternatively, and more likely, the low serum conditions (which all experiments were performed under) created an environment in which apelin was effective in preventing cell death. This would explain the protective effect observed in doxorubicin and TMZ treated cells despite a lack of TMZ-induced cytotoxicity. This can be further corroborated by the trend for increased cell numbers $(n=5, p=0.0508)$ seen under low serum conditions in Figure 5.2. Furthermore, the reason an [Pyr1] apelin-13-induced increase in cell number was observed after TMZ (Figure 5.11) and doxorubicin (Figure 5.10) treatment but not under low serum levels in normoxic conditions (Figure 5.2) may be accounted for by the different incubation times of 48 hrs and 24 hrs, respectively. Any [Pyr1] apelin-13 mediated anti-apoptotic response may not be a specific response to DNA damage but rather a response to serum deprivation-induced cellstress. This conclusion is supported by several reports stating that apelin protects against serum deprivation-induced cancer cell apoptosis [46, 201, 252]. Perhaps these [Pyr1]apelin-13 effects are a general ubiquitous stress response rather than anti-cancer specific protection by apelinergic system signalling in response to chemotherapeutic treatment.

While cell number was focused on during this study to identify APLN effects, the measurement of other responses to DNA damage would have been more appropriate to this investigation. For example, APLN inhibits ROS production [516], and ROS are commonly induced by multiple different stress stimuli including chemotherapeutic compounds [517, 518]. Another crucial measure would be the induction of autophagy markers as discussed earlier. TMZ has been shown to induce autophagy in glioma cells [461], making autophagy a worthwhile topic 
of future investigation. Finally, the apelinergic system has been implicated in modulating endoplasmic reticulum stress response $[258,452]$ which is often induced by drugs such as TMZ [256]; examining expression of ER stress markers such as BIP (Binding immunoglobulin protein) and CHOP (CCAAT-enhancer-binding protein homologous protein) could prove to be worthwhile future direction.

\subsubsection{Retrospective changes to this chapter}

Retrospectively, there were several changes that would have been made to the experimental design in this chapter if it were to be redone. While using RT-qPCR to identify potential roles for the apelinergic system under stress was a promising approach, the measurement of adherent cells by haemocytometer counts to assess the effect of [Pyr1]apelin-13 was not appropriate. The reason for using cell counts was because during the initial stages of this study, it was unclear what the role of the apelinergic system in glioblastoma may be and it was felt that this assay would yield information of whether cell number was increasing, static or decreasing and this would suggest what conditions to investigate further. However, when U87.APLNR and U87MG cells were placed under stressor conditions (doxorubicin excluded) consistent and significant changes in cell number were challenging to measure. Moreover, haemocytometer cell counts of adherent cells are limiting in that it takes a snapshot of the cells at a specific time and does not yield information about cell health, proliferation or overall activity (i.e. if cells are senescent or active). Adherent cells are also only a proxy for cell death and do not indicate the health of the cells lost. In addition, cell counting is strongly influenced by technical variances in experimentation. If I were to redo the investigation I would likely eschew variable measures of cell death such as cell counting and utilize other measures of cell health such as apoptotic markers, autophagy markers and markers of DNA damage (discussed in full below) which are able to be used in more high throughput approaches such as flow cytometry and do not rely on cell death to make measurements. I would continue to treat with and without [Pyr1]apelin-13 to see if apelin had effects on these measures. During this thesis, most incubations were capped at $24 \mathrm{hrs}$ to ensure [Pyr1] apelin-13 was present in culture and did not degrade. Retrospectively it may take more time for cell death to occur in glioblastoma cells by 
stressors such as hypoxia and glucose deprivation, as such, incubation times would be extended to $48-72$ hrs with media changes to ensure [Pyr1]apelin-13 was present at all times.

\subsubsection{Overall chapter conclusions}

During this chapter, the role of the apelinergic system under several cell stress conditions was examined in depth explicitly assessing upregulation of APLNR or APLN mRNA expression and [Pyr1]apelin-13 mediated pro-cell survival effects. It was hypothesised that once a significant effect was identified, this could be further investigated to determine the role of the apelinergic system. There were no significant effects of the apelinergic system, with the exception of the hypoxia-mediated $A P L N$ mRNA upregulation. Taking into account data from previous chapters and reports of paracrine signalling systems the apelinergic system is involved in [2], it was hypothesised that upregulation due to hypoxia might form the basis of an apelin signalling pathway as depicted below in Figure 5.12. I hypothesise that cell dense regions or areas lacking in competent blood vessels such a pseudopalisading necrotic regions experience hypoxia and secrete APLN which diffuses to other regions activating cell lines to migrate towards APLN rich regions (potentially to alleviate hypoxia). The importance of this conclusion is enhanced by the in silico analysis of single cell RNAseq data (Chapter 3, Fig 3.9) showing that astrocytes, vascular cells and neoplastic cells have a higher expression of APLNR, potentially indicating higher responsiveness to $A P L N$. In addition, the tumour core has increased levels of $A P L N$ expression that correlates to increased hypoxia markers further indicating hypoxia-induced $A P L N$ may play a critical role in glioblastoma pathogenesis. 


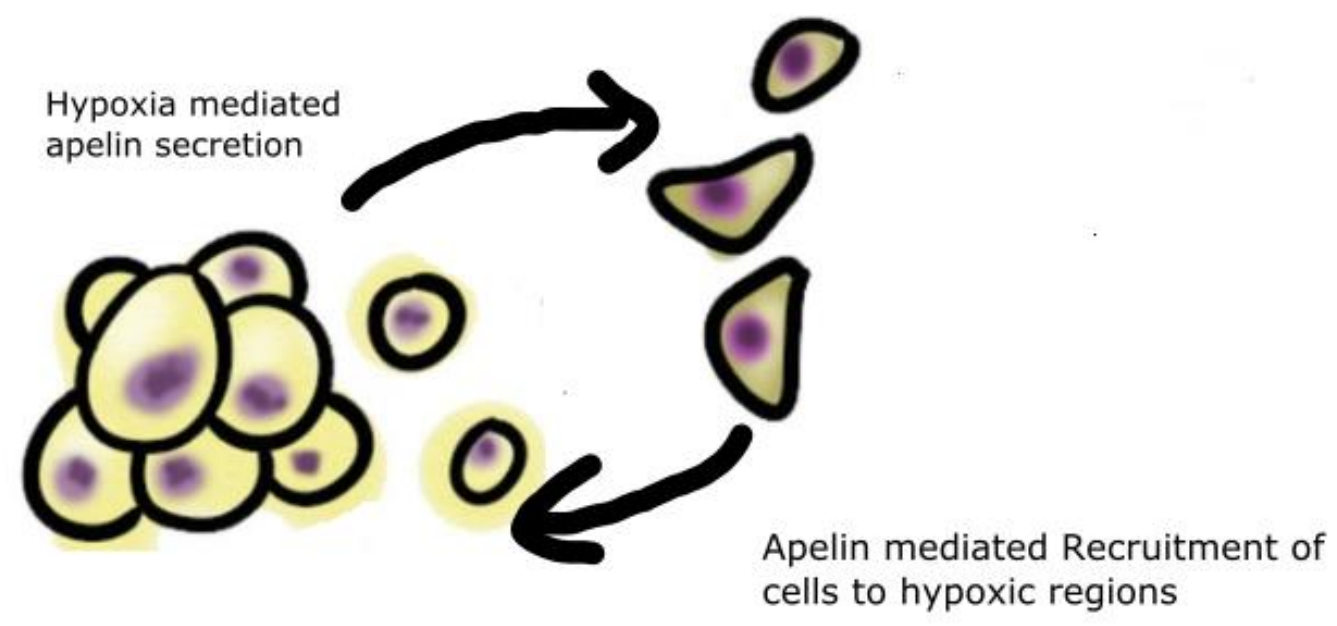

Figure 5.12. A possible role for hypoxia-induced APLN. The cell dense hypoxic regions denoted by the yellow shading could secrete APLN due to hypoxic conditions, this may then in turn diffuse to other areas (denoted as pink cells) which recruits specific cells from non-hypoxic regions to hypoxic areas.

The data presented, though not comprehensive, do not suggest a protective role for the apelinergic system in glioblastoma response to stress but do suggest the general protective effect, similar to what is reported in the literature, is present in glioblastoma cells. Significantly, hypoxia-mediated upregulation of $A P L N$ mRNA was seen in glioblastoma-derived cells. While a clear role for the apelinergic system under cell stress was not identified, the data from this chapter suggests that further research into hypoxia-mediated signalling mechanisms which the apelinergic system may be a part of is a promising direction. To the best of my knowledge, this is the first suggestion of this type of model for the apelinergic system in glioblastoma and presents an intriguing paradigm for further investigation. 



\section{Chapter 6 Generation of an aptamer to apelin-13}

\subsection{General introduction: The generation of an aptamer against apelin 13}

The previous chapters have explored the overall aim of this thesis which was, to assess the role of the apelinergic system in glioblastoma. Many reports are indicating the potential of serum APLN-13 as a biomarker for diseases like chronic heart failure [288] and several cancers [280] such as lung cancer [91]. Serum APLN is also a valid predictor of bevacizumab efficacy in cancer patients $[93,405]$. The breadth of literature implicating serum apelin as a biomarker of disease suggests an assay that can accurately detect and quantify serum apelin would be highly beneficial.

The current methods of serum APLN detection appear inconsistent and have room for improvement [12]. Currently, there are several antibodies available to quantify APLN in serum in an enzyme-linked immunosorbent assay (ELISA) format, but they lack the desired specificity due to the antibodies being unable to discriminate between the different APLN isoforms [283]. Isoform recognition is critical because each APLN isoform can have different physiological effects; such as, on receptor internalisation [59]. Therefore, the detection of these specific isoforms is crucial to advancing the understanding of apelinergic system biology. Finally, there is some discrepancy between ELISA and mass spectrometry (MS) reported concentrations of serum APLN, suggesting that a more consistent and specific detection method would be beneficial. These discrepancies are described in detail in Chapter 1, Section 1.5.2 but are briefly summarised here. 
Most literary reports attempting to quantify APLN in serum tend to utilise the ELISA assay from Phoenix pharmaceuticals which detects APLN at a possible range of $0.07-0.79 \mathrm{ng} / \mathrm{mL}$ [284]; all reviewed literature report detection of APLN in serum within the manufacturer prescribed range, but none of the reports are concordant with MS data. Of the reports of serum APLN detection, one report could not detect serum apelin by MS [283] and a second report detected apelin at $0.007-0.023 \mathrm{ng} / \mathrm{mL}$ [281]; which is lower than the detection threshold of the Phoenix pharmaceuticals kit. It is doubtful that MS could not accurately detect what ELISA assays could, which brings into question previously reported concentrations of APLN. An aptamer against apelin-13 would be highly beneficial to detect apelin in serum because of the several advantages aptamers hold over antibodies.

Aptamers are highly advantageous compared to traditional antibodies for many reasons, which have been detailed in several reviews [519-522] and as detailed in Chapter 1, Section 1.5.2. Some of the critical benefits of aptamers are: they have limited between batch variation are readily chemically synthesised and can be raised to a high sensitivity and specificity for detection of biomarkers [523]. Aptamers tend to degrade In vivo more readily than antibodies, but their pharmacokinetics can be modified easily by adding different functional groups. In comparison, antibodies also degrade and are not as resistant to $\mathrm{pH}$ and temperature shifts as aptamers are. These properties present aptamers favourably as a new alternative to antibodies in sensors and other applications.

This chapter will provide a concise review of the methodology of aptamer generation, and the results of methods trialled in this thesis to generate an apelin-13 aptamer will be discussed. The chapter will end with a discussion about why this SELEX process did not work and how future aptamer generation would be performed. 


\subsection{A review of aptamer technology}

\subsubsection{Overview of aptamer generation}

Aptamers are single-stranded oligonucleotide or peptide molecules that are selected to bind with high affinity and specificity to a target. Aptamers, like antibodies, can and have been used extensively for diagnostic applications [524, 525]. More recently, aptamers have also been trialled in therapeutic applications with several aptamers in varying stages of clinical trials [526].

Aptamers are generated using the process of SELEX (Systematic Evolution of Ligands by EXponential enrichment) summarised in Figure 6.1. The SELEX process was partially developed by three separate lab groups [527], in 1989 [528] and 1990 [529, 530].

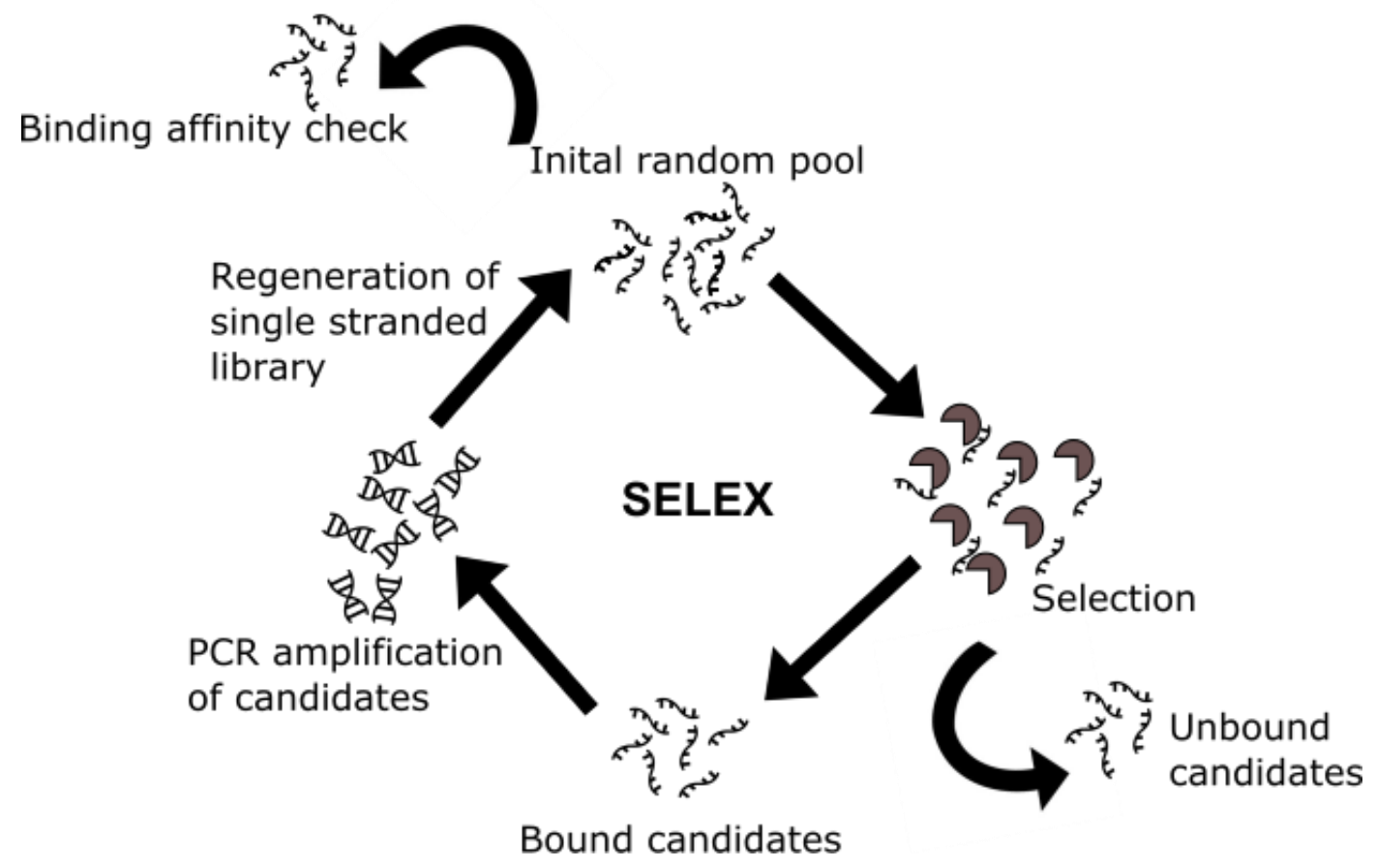

Figure 6.1. A summary of the process of SELEX. SELEX starts with an initial random pool of aptamer candidates coincubated in the selection stage with a target of interest. Unbound candidates are removed and candidates demonstrating affinity are amplified by PCR. The single stranded library is then regenerated for further rounds of SELEX. In each round, a small percentage of library is removed for testing the affinity of the library to the target. 


\subsubsection{Selection}

SELEX begins with designing an initial random candidate pool of approximately $1 \times 10^{14}$ different oligonucleotide sequences. Each sequence has a core random sequence region (40 to 80 nucleotides in length) that is flanked by known primer sequences. The library is incubated with the desired target molecule to allow for candidate oligos that demonstrate an affinity to the target to bind.

Selection can be performed in many ways and on a wide range of targets such as whole cells, viruses, proteins and toxins. Commonly this has been done by immobilising proteins on a nitrocellulose membrane [531] or linking proteins to columns but has extended to conjugating targets to paramagnetic beads (PMBs). More complex methods use cells [532] or bacteria to express target molecules. It is also possible to perform whole-cell SELEX [533] against entire cells to generate an aptamer specific to a particular cell type.

After allowing oligos to bind to the target molecule, the unbound oligos are washed away with several wash steps. To increase selection stringency that ensures the oligos demonstrating the highest affinity are retained within the selection pool, the washing step is usually varied in successive rounds (i.e. by increasing wash volume, wash time or the number of washes) [534]. Increasing stringency of washing is a crucial step as the stringency has a direct effect on the number of candidates oligos remaining and the specific affinity of these candidates to the target molecule [535]. During this study, a PMBs approach was taken.

\subsubsection{PCR amplification}

Following selection and washing, bound candidates are eluted and amplified using PCR. The amplification of aptamer candidates is one of the most crucial steps in SELEX as this step allows the amplification of oligos from the previous round of selection to make up the new selection pool. This amplification step is also where errors may occur; such as, the potential generation of nonspecific products which can interfere with subsequent rounds of SELEX. These nonspecific products can be generated through primer-primer interactions. However, a 
more specific issue with complex template libraries such as those involved in SELEX is the hybridisation of library sequences which leads to the formation of chimeric products [536]. The formation of chimeric products is due to the variable regions of aptamer sequences having homology with other sequences which allows annealing of two candidates to occur and thus

priming a PCR reaction. This by-product formation is detrimental to SELEX as it can lead to spurious products overwhelming the selected sequences of interest $[537,538]$. One way of reducing the generation of spurious products in complex template libraries is using emulsion PCR [539]. Emulsion PCR is the process whereby, rather than utilising a bulk PCR reaction which enables oligos to miss-prime and generates spurious products [536]; the PCR reaction is dispersed into small droplets of PCR buffer in a continuous phase of mineral oil. The mineral oil contains surfactants that enable the droplets to remain stable under PCR cycling. Each droplet contains one oligo with the reagents required for the PCR reaction which prevents intermolecular interactions and improves the efficacy of PCRs during SELEX. Emulsion PCR was trialled during this thesis but eschewed in favour of using quantitative PCR to determine the optimal cycles for each round thus preventing the generation of spurious products (detailed below 6.3.3).

\subsubsection{Generation of single-stranded oligos}

Aptamer selection is undertaken using only single-stranded oligos. Therefore, the generation of single-stranded DNA (ssDNA) following PCR amplification is the next important step in SELEX. There are several ways to generate ssDNA for use in SELEX that have been reviewed in full previously [540] but will be briefly reviewed here. One of the more common methods utilises a biotin-streptavidin approach [541]. Eluted oligos are PCR amplified using a biotin labelled primer, then the biotin labelled dsDNA is mixed with magnetic streptavidin beads and captured on a magnet. The non-biotinylated strand is denatured from the DNA duplex using alkaline denaturation and removed with the supernatant. Alternatively, an enzymatic approach to ssDNA generation may be used such as using lambda exonuclease [542]. During the initial dsDNA PCR, one of the strands is phosphorylated allowing it to be then recognised and degraded by the lambda exonuclease, leaving a single-stranded product. 
It is also possible to separate the different strands by size using a high resolution denaturing polyacrylamide gel $[542,543]$. PCR amplification is performed with one primer containing a terminator and an extra set of 20 nucleotides to increase the unwanted strand size, this then allows size discrimination on a gel and subsequent isolation via gel extraction [542].

Another useful method is asymmetric PCR which utilises a biased molar ratio of forward to reverse primer to promote the formation of the desired ssDNA product. Once the reverse primer is exhausted the predominant forward primer produces more strands of the desired DNA while the amount of reverse primer product remains the same. The use of asymmetric PCR has been used to generate aptamers several targets, indicating its robustness [544]. The process requires optimisation of the ratio of primers, which varies from 100:1 to 20:1 [545], as well as the amount of starting template. It is highly beneficial due to its cost-effective nature but requires a significant amount of optimisation and is less efficient than standard PCR [545]. Uniquely, some authors have merely diluted the initial bulk PCR reaction and directly placed it into a new PCR reaction with a single primer or an antisense oligo to block one of the primers, thus, facilitating single strand production [529]. Asymmetric PCR was utilised during this project.

\subsubsection{Monitoring of library affinity}

Following the regeneration of the single-stranded library, a sample is taken and its binding affinity tested in comparison to the non-specific initial library [546]. It is essential to periodically assess library binding affinity to the target molecule during successive rounds of SELEX to ensure specificity is increasing with each selection round. Once affinity is several folds higher than the un-selected library, sequencing of candidate oligos can begin.

The monitoring of library affinity can be done through several different methods which have been reviewed elsewhere [547]. The most common binding assays trialled in this thesis were dot blot assays and flow cytometry, but recently there have been some papers using a qPCR method [548]. These three methods of assessing binding affinity are summarised below.

The most common method of measuring library enrichment is flow-cytometry [533]. It is highly beneficial due to the single bead (or cell) interrogation yielding a wealth of information 
$[533,549]$. This process usually involves labelling the aptamer library with a fluorescent molecule such as fluorescein, binding the library to the target molecule and running the sample on a flow cytometer. Enriched aptamer libraries will bind with a higher affinity to the target than to control targets and display a fluorescent shift as depicted in Figure 6.2.
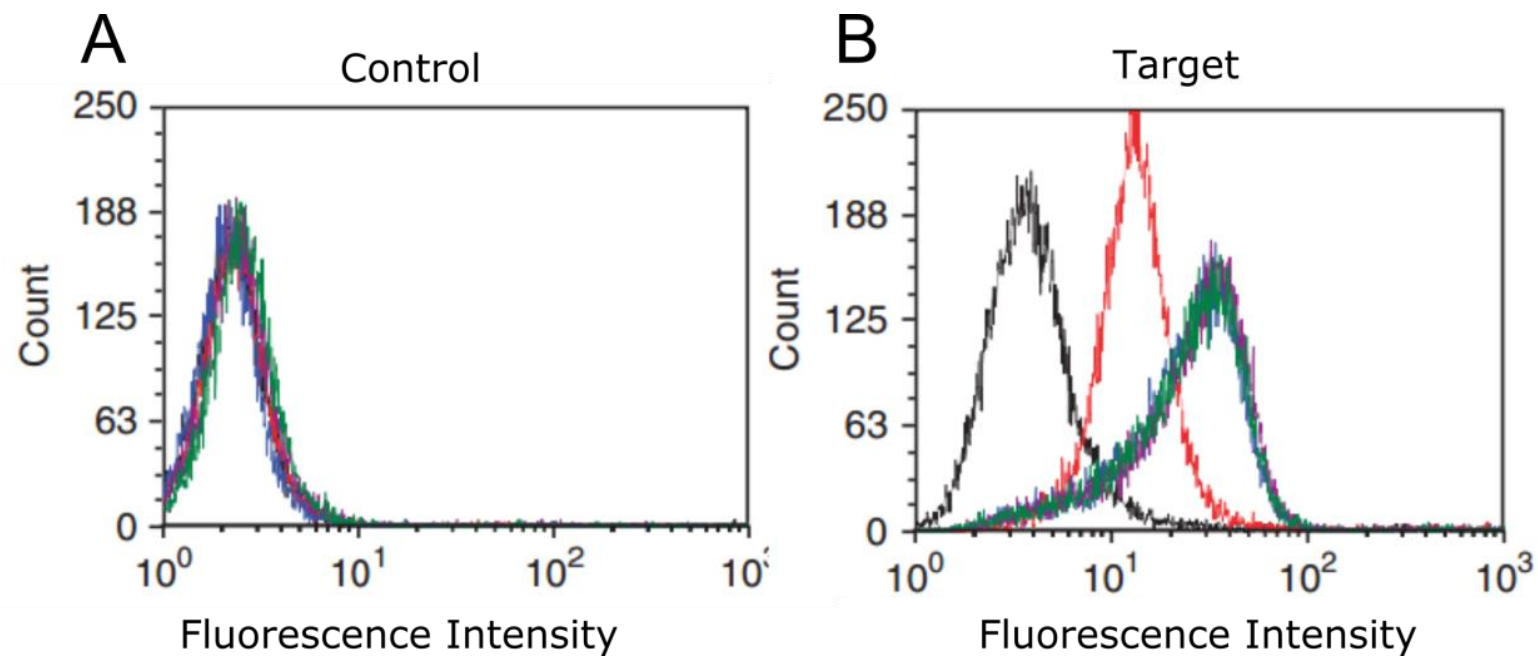

Figure 6.2. An example of a fluorescence intensity shift due to aptamer binding, reproduced from Sefah et al. [533]. Panel A depicts libraries after differing rounds of SELEX bound to non-target cells. Panel B depicts the same libraries as in panel A bound against the target. The black peak in panel B represents the unselected library, and the peaks to the right represent selected libraries with increased affinity for the target. (Reproduced with permission from Nature Publishing Group).

A dot blot assay is another conventional method used to measure library enrichment [550, 551]. The assay is carried out by dotting different concentrations of target molecule onto a nitrocellulose membrane and probing with an aptamer library labelled with a detection mechanism such as a fluorescent molecule or enzyme.

Quantitative PCR (qPCR) binding assays are another measure of library enrichment that is highly sensitive and cost-effective $[548,552,553]$. A qPCR binding assay involves generating an aptamer standard curve and using this to quantify the amount of aptamer that has bound to its target compared to a control library and negative control target [548]. High-affinity libraries will appear sooner on a qPCR trace as there is more aptamer bound to the target. 
Following the measurement of binding the remaining regenerated single-stranded library reenters the SELEX process. Iterative rounds of SELEX are performed, with increasing stringency as mentioned previously, until a library is sufficiently enriched for its affinity for the target.

\subsubsection{Chapter aim}

The present study attempts to generate a DNA aptamer to the apelin-13 peptide, the most common isoform of apelin found in the serum [281]. The development of an aptamer to apelin13 would be a promising tool to later develop a method to detect apelin-13 in serum. In this chapter, several methods for each step of aptamer generation were investigated and outlined below. 


\subsection{General methods}

\subsubsection{Ligand coupling \& Verification}

\subsubsection{MALDI-TOF mass spectrometry verification of apelin-13}

Matrix-assisted laser desorption ionisation - time of flight analysis (MALDI-TOF) was utilised to confirm the structure and purity of commercially purchased apelin-13 (Cayman Chemicals, Item no 13523). Alpha-cyano-4-hydroxycinnamic acid ( $\alpha$-CHCA) was used as the matrix for this analysis. Fresh matrix solution was prepared by the addition of $10 \mathrm{mg}$ of $\alpha$ CHCA to $1 \mathrm{~mL}$ of $0.1 \%(\mathrm{v} / \mathrm{v})$ trifluoroacetate (TFA) and $50 \%(\mathrm{v} / \mathrm{v})$ acetonitrile $(\mathrm{ACN})$. The solution was vortexed for $45 \mathrm{sec}$ and centrifuged at 13,600 RCF for $5 \mathrm{~min}$ to pellet the undissolved $\alpha$-CHCA. The supernatant was removed and placed into a fresh microcentrifuge tube. Apelin-13 (50 $\mu \mathrm{g}$ ) was diluted to $0.1 \mu \mathrm{g} / \mathrm{mL}$ in diluent $\left(70 \% \mathrm{ACN}, 30 \% \mathrm{ddH}_{2} \mathrm{O}\right.$, acidified with $0.1 \%$ (v/v) TFA) and purified using a perfect pure C-18 zip tip (Sigma Aldrich, Auckland) according to manufacturer's protocol. TFA $(1 \mu \mathrm{L})$ was added to $20 \mu \mathrm{L}$ of eluted apelin sample, and the apelin sample was then combined with the pre-prepared fresh matrix solution in a 1:10 ratio and then $1.5 \mu \mathrm{L}$ of this was spotted onto a MALDI plate and allowed to air dry for $1 \mathrm{hrs}$. Spectrum data was acquired using a Voyager DE Pro MALDI-TOF mass spectrometer (Applied Biosystems, Foster City, CA, USA). Five individual mass spectra were obtained and analysed.

\subsubsection{Ligand coupling}

Apelin-13 peptide was conjugated to epoxy residue coated PMBs as per the manufacturer's instructions (Life technologies, Thermofisher Scientific, Auckland). It was acknowledged that apelin-13 may spontaneously form the pyroglutamated form but that this was not a drawback because an aptamer to apelin-13 should recognize both forms of apelin-13. The epoxy groups on the PMB bind amino and sulfhydryl groups of proteins permanently binding the protein to the beads. Both the target (apelin conjugated) and control beads (no peptide) were incubated 
for $1 \mathrm{~min}$ with glycine $(100 \mathrm{mM})$ made up in $0.1 \mathrm{M}$ sodium phosphate buffer ( $\mathrm{pH} 7.4)$ to block reactive epoxy groups.

\subsubsection{Aptamer selection}

A custom designed oligo library (40-nucleotide random region flanked by two 20-nucleotide primer region) was commercially purchased from IDT (Integrated DNA Technologies, Singapore). The library was initially prepared to a $2 \mu \mathrm{M}$ working concentration in binding buffer (1x PBS supplemented with $1 \mathrm{mM} \mathrm{MgCl} 2$ and $0.05 \%$ Tween 20), denatured by heating to $95{ }^{\circ} \mathrm{C}$ for $5 \mathrm{~min}$ and snap-chilled on ice for $10 \mathrm{~min}$ to allow the ssDNA to form their most stable secondary structures. Aptamer sequences are provided below in Table 6.1.

Table 6.1. Details of aptamer library and primer sequences. $\mathrm{N}$ refers to a random nucleotide.

\begin{tabular}{ll}
\hline Name & Sequence \\
\hline Ap2-fwd-FAM primer & /56-FAM/TCGCACATTCCGCTTCTACC \\
Ap2-library & TCGCACATTCCGCTTCTACC-N40-CGTAAGTCCGTGTGTGCGAA \\
Ap2-fwd primer & TCGCACATTCCGCTTCTACC \\
Ap2-rev primer & TTCGCACACACGGACTTACG \\
\hline
\end{tabular}

Target (apelin-13 conjugated) and control beads were washed in binding buffer. Apelin-13 conjugated PMBs in the binding buffer was made up at a concentration of $2 \times 10^{6} \mathrm{PMBs} / \mu \mathrm{L}$ with $7 \mu \mathrm{L}$ used for the initial rounds and $4 \mu \mathrm{L}$ for subsequent rounds as detailed below (Table 6.2). The target beads were added to a $15 \mathrm{~mL}$ falcon tube and washed with binding buffer (500 $\mu \mathrm{L}) 2$ times and resuspended in $1 \mathrm{~mL}$ of binding buffer. $1 \mathrm{~mL}$ of the denatured oligo was added to the resuspended PMBs (final concentration $1 \mu \mathrm{M}$ ) and incubated on a Hula mixer (Invitrogen, Thermofisher Scientific, Iowa, USA) for 30 min to allow for selection (Hula mixer settings: Orbital, 10 RPM; reciprocal, $90^{\circ}$ for $26 \mathrm{sec}$ ). 
Once bound, the beads were held magnetically using a magnetic separation rack (Invitrogen, Thermofisher scientific, Auckland) and washed twice using $1 \mathrm{~mL}$ of binding buffer. The number and volume of washes were increased in successive rounds to increase the stringency of selection and summarised in Table 6.2.

For every second round of SELEX commencing at the third round of selection, the oligos from the selection pool were incubated with the control beads $(35 \mu \mathrm{L}$ of PMB's blocked with glycine) for $30 \mathrm{~min}$. The PMBs were then magnetically bound and the supernatant collected and applied to the target beads for the subsequent round of positive selection.

After four rounds of SELEX, salmon sperm (Sigma Aldrich, Auckland) was added to the binding buffer in the selection step at $100 \mu \mathrm{g} / \mathrm{mL}$ to act as a competitive binder [554]. Following the washing steps, the beads were suspended in $100 \mu \mathrm{L}$ of Tris-EDTA buffer and heated to $95{ }^{\circ} \mathrm{C}$ for $10 \mathrm{~min}$ to elute bound oligos. Eluted oligos were then used for library amplification. 
Table 6.2. Summary of selection stringencies applied during SELEX. During SELEX the stringency of each round is increased to select for tighter binding oligo candidates. This is done through modulation of wash volumes, wash numbers and the addition of salmon sperm as a competitive binding agent.

\begin{tabular}{|c|c|c|c|c|}
\hline $\begin{array}{l}\text { Selection } \\
\text { round }\end{array}$ & Selection type & $\begin{array}{c}\text { Wash volume } \\
(\mathrm{mL})\end{array}$ & $\begin{array}{l}\text { Number of } \\
\text { washes }\end{array}$ & $\begin{array}{l}\text { Volume of beads } \\
\text { added }(\mu \mathrm{L})\end{array}$ \\
\hline 1 & Apelin-positive & 1 & 2 & 7 \\
\hline 2 & Apelin-positive & 1 & 2 & 7 \\
\hline 3.0 & Glycine-negative & - & - & 35 \\
\hline 3.1 & Apelin-positive & 1 & 2 & 7 \\
\hline 4.0 & $\begin{array}{c}\text { Apelin-positive }+ \\
\text { salmon sperm DNA }\end{array}$ & 1 & 2 & 7 \\
\hline 5.0 & Glycine-negative & - & - & 35 \\
\hline 5.1 & $\begin{array}{c}\text { Apelin-positive }+ \\
\text { salmon sperm DNA }\end{array}$ & 1 & 2 & 7 \\
\hline 6.0 & $\begin{array}{c}\text { Apelin-positive }+ \\
\text { salmon sperm DNA }\end{array}$ & $1,3,3$ & Once each & 7 \\
\hline 7.0 & Glycine-negative & - & - & 35 \\
\hline 7.1 & $\begin{array}{c}\text { Apelin-positive }+ \\
\text { salmon sperm DNA }\end{array}$ & $1,6,6$ & Once each & 7 \\
\hline 8.0 & $\begin{array}{c}\text { Apelin-positive }+ \\
\text { salmon sperm DNA }\end{array}$ & $1,6,6$ & Once each & 7 \\
\hline 9.0 & Glycine-negative & - & - & 35 \\
\hline 9.1 & $\begin{array}{c}\text { Apelin-positive }+ \\
\text { salmon sperm DNA }\end{array}$ & $6,6,6$ & Once each & 4 \\
\hline 10 & $\begin{array}{c}\text { Apelin-positive }+ \\
\text { salmon sperm DNA }\end{array}$ & $6,6,6$ & Once each & 4 \\
\hline
\end{tabular}




\subsubsection{Library amplification}

PCRs were undertaken using a 2-step protocol using Bioline Sensimix (Quantace, Total lab science, Auckland, NZ). An initial $95^{\circ} \mathrm{C}$ denaturation was performed for $5 \mathrm{~min}$, after this $15-$ 25 cycles of $95{ }^{\circ} \mathrm{C}$ denaturing for $10 \mathrm{sec}$ to $55^{\circ} \mathrm{C}$ annealing for $15 \mathrm{sec}$ was performed. PCRs were set up as $50 \mu \mathrm{L}$ reactions. The purified un-diluted template was added to the PCR reaction in the maximal amount according to manufacturer's instructions. Forward and reverse primers were used at a $200 \mathrm{nM}$ concentration unless stated otherwise. All PCRs were run on a Biorad CFX 96 thermocycler (Biorad, Auckland NZ).

Initially, a test PCR amplification was performed to determine the optimal PCR cycle number, this was determined as the cycle before the peak fluorescence value (Figure 6.3, red arrow). After the peak fluorescence point the likely hood of non-specific products forming increases. Once the test PCR was completed, a bulk amplification was performed on half of the eluted oligos, using the optimally determined number of cycles.

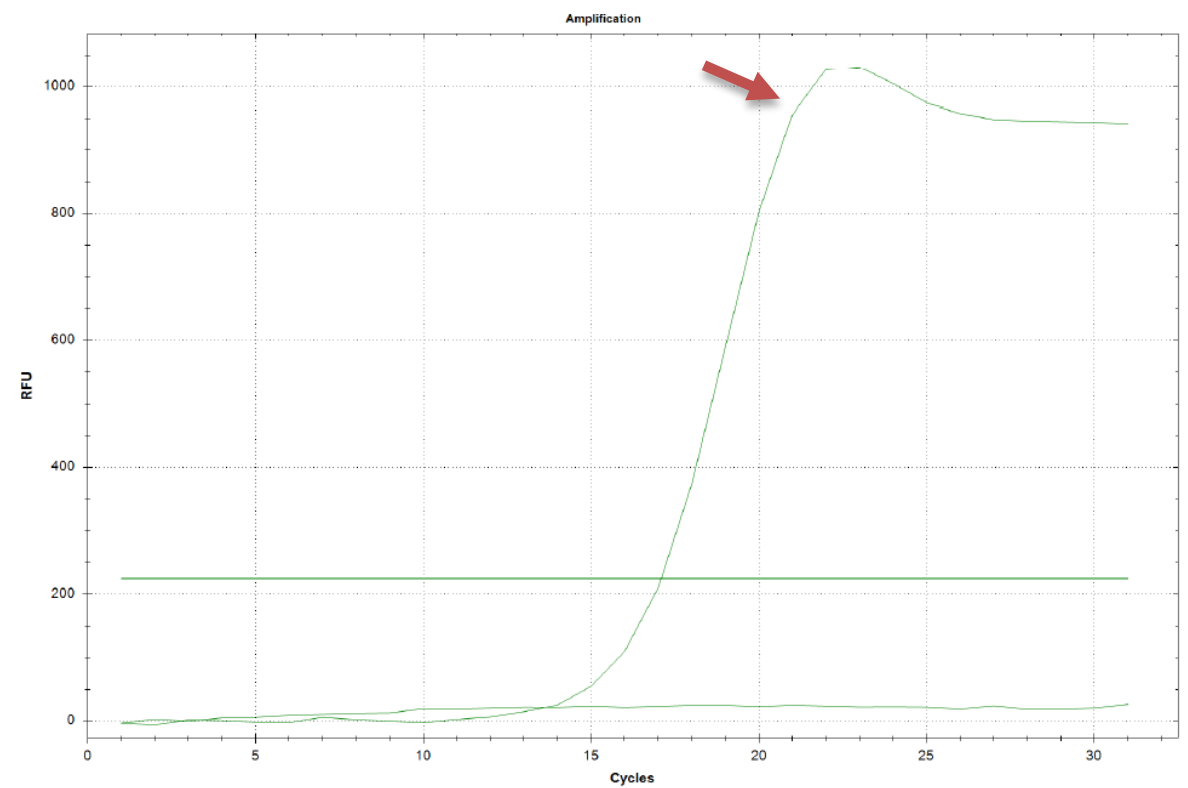

Figure 6.3. Amplification of eluted aptamer candidates using qPCR. Panel A shows a qPCR trace, the red arrow indicates the optimal amplification point to stop the reaction which corresponds to approximately 21 cycles. 
The bulk reaction is then run at $100 \mathrm{~V}$ for $40 \mathrm{~min}$ on a $3 \%(\mathrm{w} / \mathrm{v})$ agarose gel prepared using $1 \mathrm{X}$ TAE buffer and the appropriate band excised and purified using an optimised version of the crush and soak extraction [555]. Briefly, the target band from the agarose gel was excised, fragmented into smaller pieces before being crushed in equal (w/v) of ultrapure water. The gel was then incubated at $70{ }^{\circ} \mathrm{C}$ for $2 \mathrm{hrs}$ after which the suspension was centrifuged at 17,000 $\mathrm{RCF}$ for 10 min to remove any agarose debris, the supernatant containing the DNA was then transferred to a clean tube.

\subsubsection{Generating ssDNA library using asymmetric PCR}

Once bulk PCR is performed, a test asymmetric PCR is performed to determine the optimal number of cycles for asymmetric PCR. To achieve asymmetric PCR product generation, a biased 20:1 molar ratio of primers was used (forward $(1 \mu \mathrm{M})$ to reverse $(50 \mathrm{nM})$ primer). All PCR cycling conditions were kept identical as described previously except PCRs being run on a Biorad iCycler thermocycler (Biorad, Auckland NZ).

Optimal cycle number was determined by utilising a Fluorescein amidite (FAM) labelled fluorescent primer to label the single-stranded product and provide a standard (Figure 6.4). Several reactions were run at differing numbers of cycles and then run on a $3 \%(\mathrm{w} / \mathrm{v})$ agarose gel prepared using 1x TAE buffer. The gel was scanned with a FLA5000 laser gel scanner (Fujifilm Medical Systems, USA). The gel was examined for smearing above the target product band; this indicates non-specific product formation from over-amplification. Also, the size of the desired product was checked. 


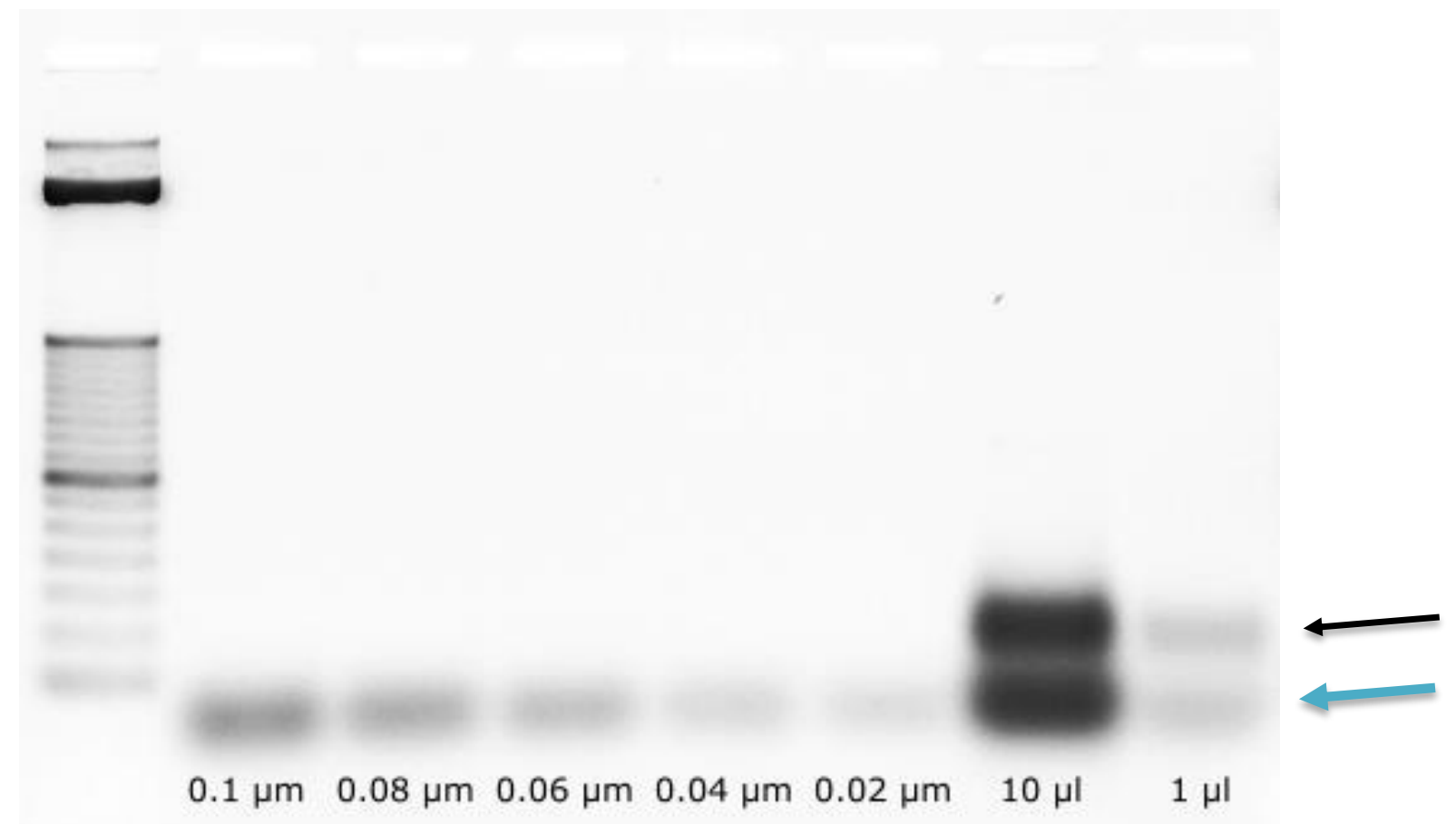

Figure 6.4. Representative figure showing an example of asymmetric PCR products and FAM dilution standards. The black arrow indicates the desired product, the blue arrow indicates unincorporated primer. The concentrations along the bottom indicate the amount of FAM primer in the standard.

Following this, bulk asymmetric PCR amplification was performed at the optimal cycle number. The resulting product was desalted using a NAP5 column (Illustra, GE Healthcare, United Kingdom) based on size exclusion using Sephadex G25 medium. The resulting sample was re-eluted using fresh binding buffer. After desalting, the library was run on a 3\% agarose gel with FAM primer standards to determine the quantity of library present through band densitometry (Figure 6.4). This desalting process was performed from Round 3 onwards, before this a crush and soak purification was performed as described above. 


\subsubsection{Binding affinity assays}

Binding affinity assays were performed following select positive selection rounds using the regenerated single-stranded library to ensure enrichment of the library to the target was occurring.

\subsubsection{Quantitative - PCR based binding assay}

Binding affinity was initially assayed using qPCR to detect the amount of bound ssDNA present on the target molecule. The oligo library from each selection round was diluted to several different concentrations and incubated for 45 mins with a fixed amount of target and control beads for comparison. Following the incubation, the beads were bound using a magnet, washed twice using binding buffer and resuspended in $100 \mu \mathrm{L}$ of ultrapure water. The beads with the bound aptamers were heated at $95{ }^{\circ} \mathrm{C}$ for 10 min to elute the aptamers, $2 \mu \mathrm{L}$ of the resulting supernatant was used in the PCR performed as described prior. The amount of relative bound aptamer was calculated by the following equation:

$$
2 \text { - (Test aptamer library - round } 0 \text { aptamer library) }
$$

\subsubsection{Dot blot assay}

A dot blot assay was utilised to study the binding affinity of the oligos in the selection pool using a previously reported method [556]. Serial titrations of apelin-13 peptide were prepared, $1 \mu 1$ volumes were blotted on a water dampened nitrocellulose membrane $(0.45 \mu \mathrm{M}$ pore size, Whatmann) and incubated for 2 hrs at RT to allow for the apelin-13 peptide to bind the nitrocellulose. The membrane was then blocked for $1 \mathrm{hrs}$ using a mixture of $2 \%(\mathrm{w} / \mathrm{v})$ skim milk powder (Pams, PaknSave) and $0.1 \mathrm{mg} / \mathrm{mL}$ sheared salmon sperm dissolved in $1 \mathrm{x}$ binding buffer. The aptamer library labelled with FAM was prepared using $1 \mathrm{x}$ binding buffer. Following blocking, the membrane was incubated with the FAM labelled oligo library for 60 min at RT with mild agitation. After the incubation step, the membrane was washed once with 
1x PBS and scanned using the FITC filter on a Fujifilm FLA5000 fluorescence scanner (Fujifilm Medical Systems, USA) at $400 \mathrm{~V}$.

\subsubsection{Flow cytometry binding assay}

To determine the binding affinity of oligos to the apelin-13 conjugated PMBs, the FAM-labeled oligo library was incubated with the target and control PMB's in a similar fashion as described above (6.3.5.1). Following co-incubation of oligo library with the respective beads, the beads were washed $2 \mathrm{x}$ using binding buffer as described previously (6.3.5.1). Following the final wash, the beads were run on a FACS Canto II (BD Biosciences, USA) and the fluorescence was examined using a FITC filter.

\subsection{Results}

\subsubsection{Verification of apelin integrity}

To ensure the correct size and integrity of the apelin-13 peptide MALDI-TOF was used. MALDI spectrum acquired was examined for the presence of a $1550.8 \mathrm{Da}$ target protein to confirm apelin-13.

The spectrum below (Figure 6.5) shows the presence of a peak at 1550.8; there was an additional 1533.8 Da peak that may represent a degraded product as previously described [557]. Alternatively, the second peak at 1533.8 may be apelin-13 that has been reported to spontaneously cyclize to $\left[\mathrm{Pyr}^{1}\right]$ apelin-13 $[68,348]$. $\left[\mathrm{Pyr}^{1}\right]$ apelin-13 is reported to have a mass to charge ratio of 1533.8 [281], 


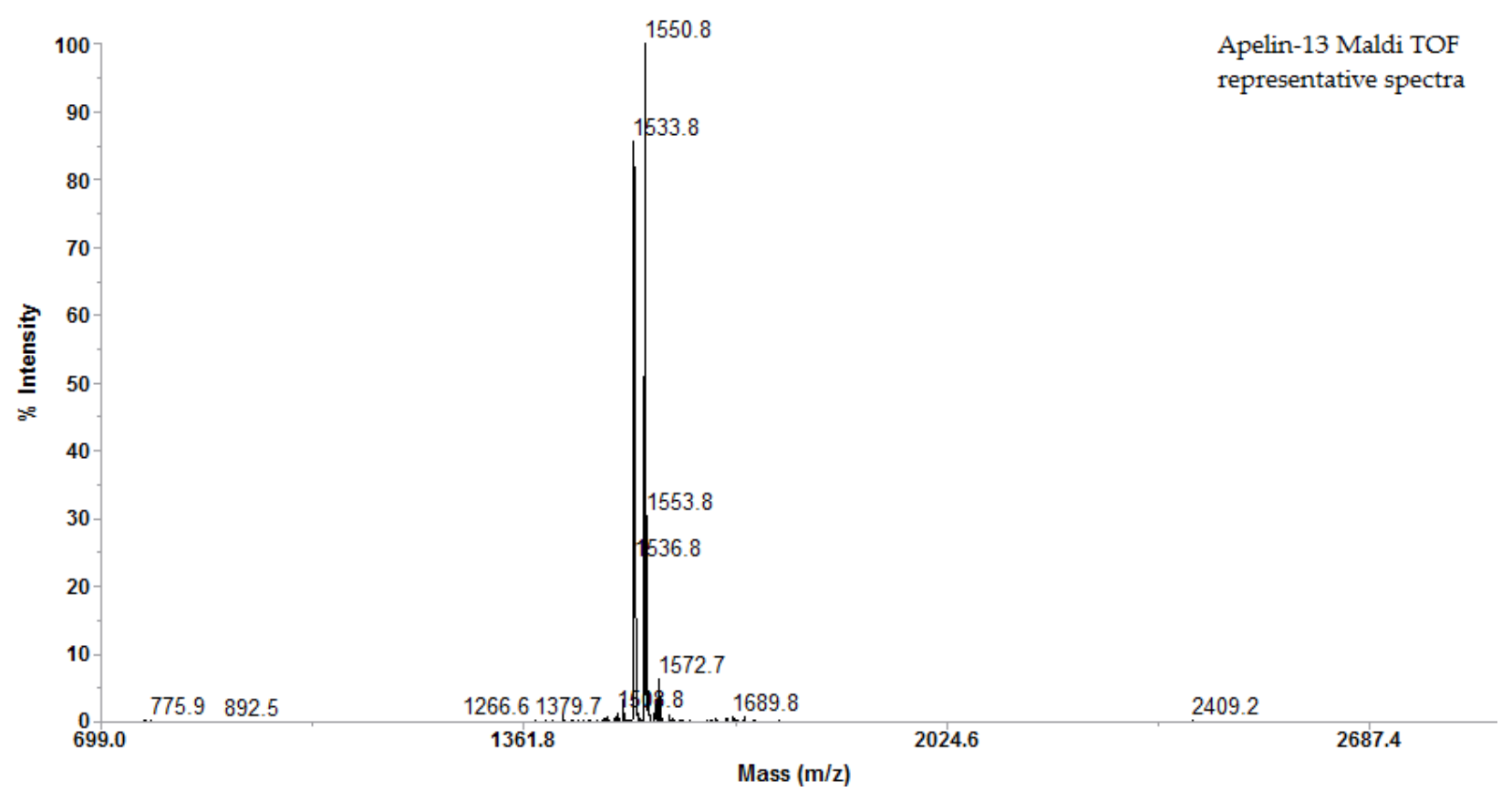

Figure 6.5. Representative image of apelin-13 MALDI-TOF spectrum. Apelin-13 is represented by a peak at 1550.8 and an unknown peak at 1533.8 .

\subsubsection{PCR amplification of candidates and regeneration of single-stranded library}

There are two steps where amplification of the aptamer library was required. The first step was after selection when the eluted oligos were amplified and the second was the regeneration of the single-stranded library. Efficient PCR amplification of the eluted oligos is essential as nonspecific product formation is highly detrimental to the amplification of aptamer libraries and thus the efficacy of SELEX is reduced [538].

\subsubsection{Emulsion PCR trial}

To prevent the non-specific product formation that occurs during conventional PCR emulsion PCR was trialled during this study. Ultimately, emulsion PCR was never used due to several issues during the optimisation process. The primary issue was ensuring the droplets generated were consistently monodispersed. An example emulsion showed below (Figure 6.6, Panel A) 
was obtained from a dropwise addition of the aqueous phase (PCR mix) into a continually stirring oil mix [539] using a syringe pump to control the flow rate of the aqueous phase thus modulating droplet size. Droplets were measured using an automated analysis pipeline derived from [558] implemented in ImageJ [559]. An example of the output from the pipeline can be seen in Figure 6.6, panel B, measurements from the output were then used to asses droplet size dispersion. It was found that emulsions generated in this way were not monodispersed as desired and would be ineffective for emulsion PCR. To correct the polydispersed emulsion, a microfluidic approach was trialled.

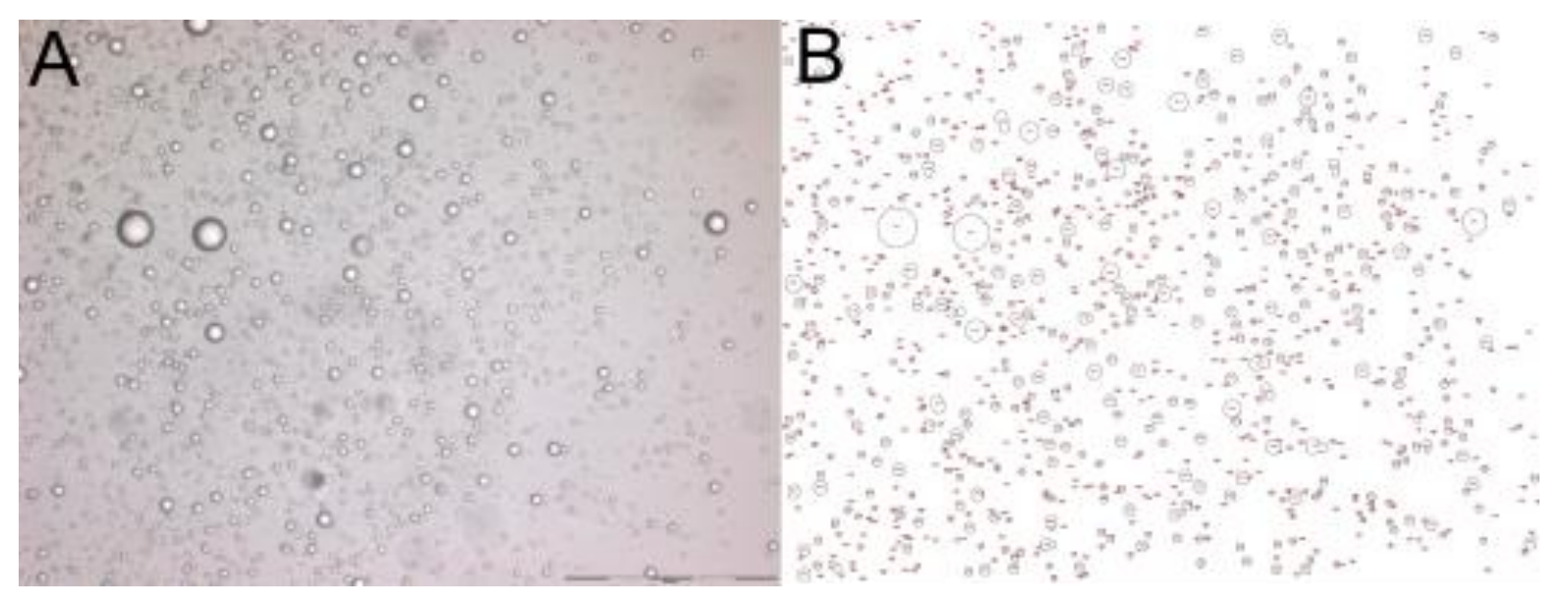

Figure 6.6. An example of a polydispersed emulsion generated for emulsion PCR. Panel A shows a raw image of an emulsion; the scale bar refers to $200 \mu \mathrm{M}$. Panel B shows the output of the ImageJ pipeline measuring the size of each droplet.

A flow-focusing droplet generator was purchased commercially (Micronit Microfluidics, Dortmund, De). Oil was pumped in from two junctions into a flow focusing pore where the aqueous phase meets the two oil channels resulting in a focusing effect that generates droplets. The flow rates of the droplets were optimised to generate a monodispersed emulsion. Ultimately a more monodispersed emulsion was generated using the microfluidic setup, but the consistency of droplet generation was a limiting factor.

The second and primary issue of emulsion PCR was the thermostability of the emulsion droplets. Commonly detergents are added to the oil mix to act as surfactants which prevent collapse or coalescence of the PCR droplets during thermocycling [560]. Commonly a mix of three detergents: (4.5\% (v/v) Span 80 (Sigma-Aldrich, Auckland, NZ), 0.4\% (v/v) Tween 80 
(Sigma-Aldrich, Auckland, NZ) and 0.05\% (v/v) Triton x-100 (Sigma-Aldrich, Auckland, NZ) [539] is used. However, this surfactant mix was found not to be thermostable as the droplets coalesced after thermocycling. Different compositions of the above surfactants were trialled but were unsuccessful. Instead, other fluorinated surfactants [561] were attained for use, but ultimately the optimisation process was too time consuming and was eschewed for the use of other methods. The final issue of emulsion PCR was the recovery of DNA products from the oil-water emulsion using the water-saturated ether method [536]. The water-saturated ether method was found to be inefficient and resulted in very low-quality yields. Ultimately, a quantitative PCR approach as described in 6.3.3 was used to determine the optimal cycle number and prevent the generation of spurious products instead of emulsion PCR. This method has been reported in the literature $[553,562]$ and was found to be effective. In the future, if emulsion PCR were intended to be used, a different commercial droplet generator would be used alongside a different surfactant mix which contained fluorinated oil.

\subsubsection{Measurements of library binding affinity with different methods}

Binding affinities were tested using three different methods, but no enrichment could be detected through any method after ten rounds of SELEX. 


\subsubsection{Flow cytometry}

Initially, flow cytometry was used to detect apelin binding of the SELEX libraries; there was no enrichment seen after round 3 of SELEX. Figure 6.7 shows an increase in binding of the library to the apelin beads, i.e. a small shift in the peak was seen with the round 3 SELEX library (Figure 6.7, red peak), but round 4 of SELEX shows a decrease back to the initial library level of random binding. As a control experiment a fluorescent apelin antibody was used to detect the apelin presence on the beads, but again the difference in fluorescent peak shift was unable to be detected. Ultimately qPCR detection methods were utilised to quantify binding.

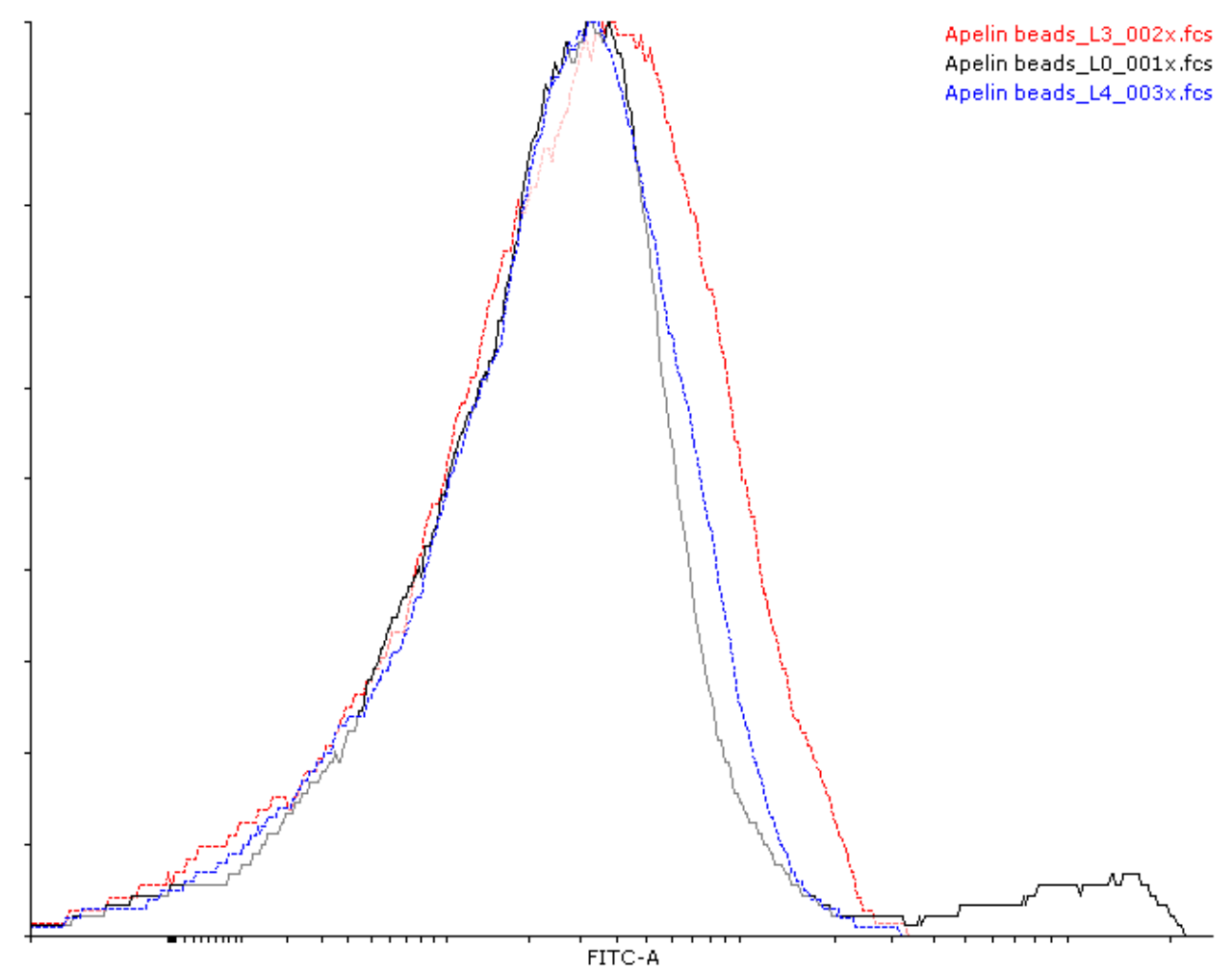

Figure 6.7. Representative image of apelin-13 conjugated PMBs bound with the round 0, 3 and 4 library run on a flow cytometer to test binding. The black peak represents the SELEX round 0 (L0) library; red represents the SELEX round 3 (L3) and the blue represents SELEX round 4 (L4). Purified FAM labelled samples of each library were incubated with the same concentration of apelin beads and run on a flow cytometer using the FITC filter. 


\subsubsection{Dot blotting}

Dot blotting was also trialled to detect library enrichment using the FAM labelled aptamer library. To determine the minimal detection capability of the Fujifilm FLA5000 gel scanner, dilutions of the FAM labelled primer were blotted onto a nitrocellulose membrane, and the membrane scanned at a range of voltages (Figure 6.8). Figure 6.8 shows that FAM signal is detectable at low picomole amounts. To determine the library binding affinity for the target apelin-13, apelin-13 was dotted onto a nitrocellulose membrane, and FAM labelled aptamer libraries were used to probe the membrane. No enrichment or aptamer binding could be detected by dot blotting using the scanner on a range of voltages from $400 \mathrm{~V}-700 \mathrm{~V}$.

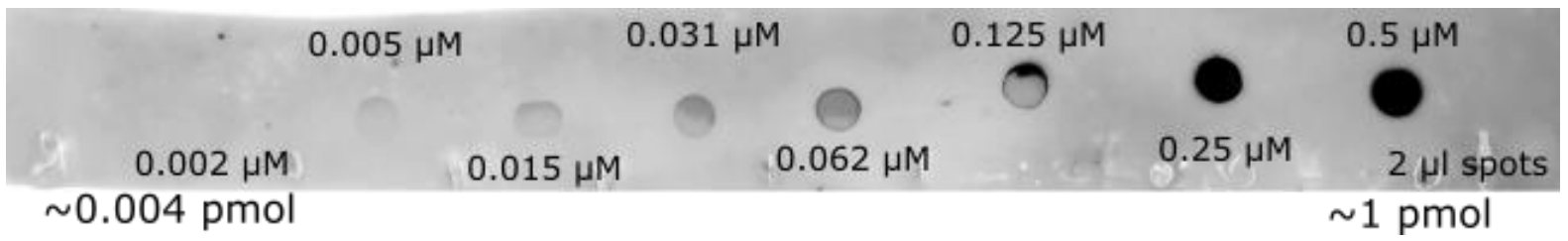

Figure 6.8. Optimization of dot blot detection levels through serial dilution of FAM primer scanned at $600 \mathrm{~V}$. A range of FAM primer concentrations dotted on to nitrocellulose were scanned at a range of different voltage settings to determine the detection limits of the FUJIFILM FLA5000 flatbed scanner.

As a control experiment apelin-13 antibody (Abcam, Auckland, NZ) was used as a positive control for binding, but this antibody could not detect the apelin-13 peptide. While the antibody was polyclonal and raised to the $\mathrm{C}$ terminus of the 77-amino acid apelin prepropeptide (of which apelin-13 is derived from), it is entirely possible that it is not able to pick up apelin-13 as it is only verified to the full 77 amino-acid prepropeptide. 


\subsubsection{Quantitative PCR detection of aptamer enrichment}

Quantitative PCR detection of aptamer library affinity was attempted. While the qPCR method appeared sensitive, results were highly inconsistent. Figure 6.9 shows a concentrationdependent increase in aptamer bound to beads. Representative results as seen below (Figure 6.9) indicated binding, but this was not replicable over three independent experiments.

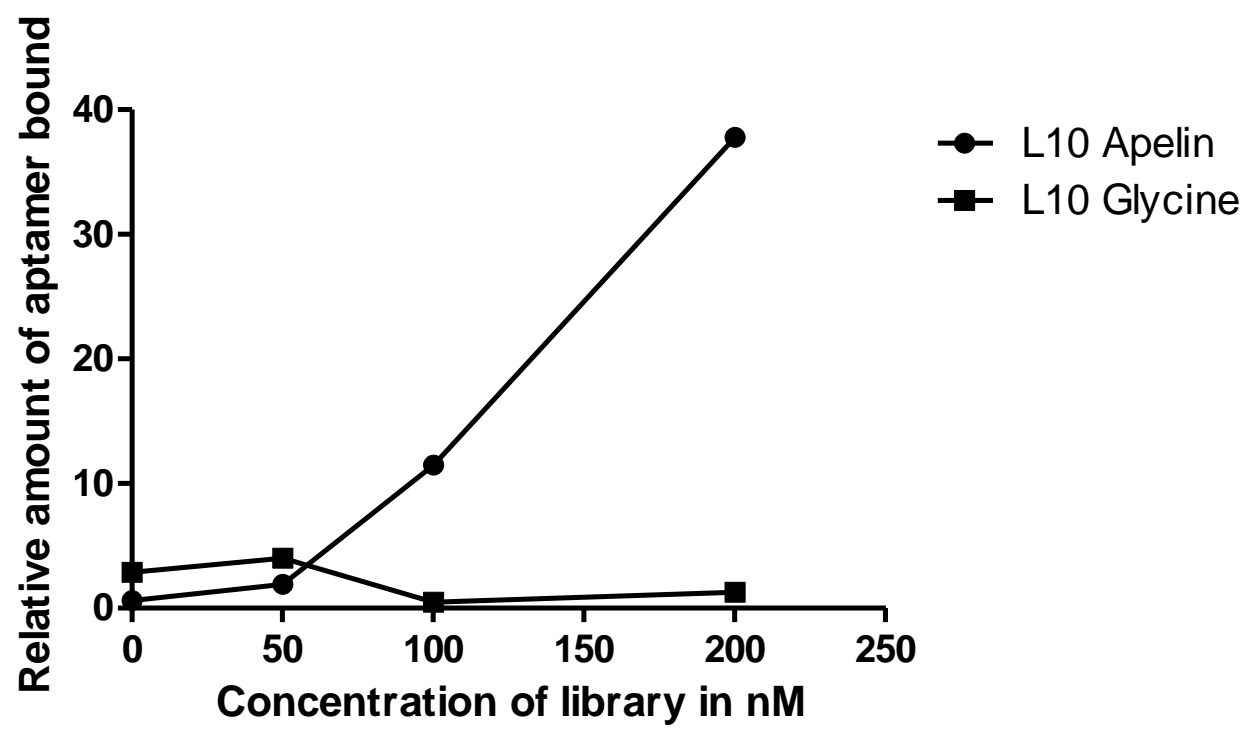

Figure 6.9. The binding affinity of the round 10 library to apelin and glycine beads. Differing concentrations $(0-$ $250 \mathrm{nM}$ ) of aptamer library from rounds 0 (L0) and 10 (L10) of SELEX were incubated with apelin and glycine beads for 30-45 minutes after which beads were washed twice, and bound aptamers were heat eluted in $100 \mu \mathrm{L}$ of $\mathrm{ddH}_{2} \mathrm{O} .2 \mu \mathrm{L}$ of the resulting eluate was used as a template in a PCR with the same cycling conditions as described in the methods. The relative bound aptamer was calculated by the following equation: $2^{\text {-(cq L10-cq L0) }}$.

In conclusion after ten rounds of SELEX, no enrichment of the library was detected. Potential reasons for this and future directions are described below. 


\subsection{Discussion}

In this chapter, ssDNA oligos were used to select aptamers capable of binding to the apelin-13 peptide. Apelin-13 was conjugated to PMB's and used to capture oligos from the selection pool that demonstrated some affinity for the target peptide. Ten rounds of selection were undertaken, and no aptamers capable of binding apelin-13 with high affinity were isolated.

\subsubsection{Coupling of apelin-13 to paramagnetic beads}

During this SELEX process the coupling of apelin-13 to the beads was performed as described above (6.3.1) but the coupling efficiency was unable to be quantified. Ensuring accurate protein loading and knowledge of the amount of protein on the beads is essential. Several methods to detect coupling were trialled but were unsuccessful. Spectrophotometry was used to detect the amount of apelin-13 before and after PMB binding using a Nanodrop ND-1000 (Thermofisher, Auckland) by measuring absorbance at $280 \mathrm{~nm}$; however, this was not effective due to the lack of tyrosine and tryptophan groups on apelin-13. Measuring the amount of apelin-13 remaining after coupling to PMBs was also trialled using BCA assays but gave no conclusive evidence of apelin-13 coupling. This was ascribed to coupling reagents that were present during the apelin13 coupling to the PMB inhibiting subsequent BCA reactions. Finally, when comparing apelin beads to negative control beads using the BCA assay, the negative control beads also gave a strong positive result (most likely due to the bond present between the inactivating glycine and the PMBs) making comparing coupling efficiency between beads challenging. The assumption had to be made that apelin-13 was bound to the beads based off successfully coupling BSA to beads, which was successfully quantified using spectrophotometry to measure the change in protein concentration of a BSA solution before and after PMB binding. In the future, a better quantitation method to ensure binding of the apelin-13 peptide to beads would be used.

The second drawback of conjugating the apelin-13 to the PMBs was the possibility of the beads interfering with the ability of oligos to bind to the apelin-13. This concept of the solid support matrix that the target is bound to interfering with SELEX has been reported prior with potential effects such as the support matrix non-specifically adsorbing oligos or blocking oligo-target 
interactions through steric hindrance [563-565]. In addition, when binding the target peptide to the epoxy residue coated beads, the apelin-13 binds to the epoxy groups via amino and sulfhydryl groups on the apelin; the proximity of the bead to the peptide and the different orientations make it possible that the precise structural conformation that was generated for apelin-13 upon binding was not the native conformation; thus, selected aptamers may not bind to the native apelin-13 when tested again after selection. The proximity of the apelin-13 molecule to the bead also may not have permitted efficient SELEX; this could have been solved by utilising a longer linker that attaches the target molecule to the bead.

The selection and subsequent partitioning of bound candidates from the target is a critical stage of SELEX. Commonly, targets must be bound onto solid supports for SELEX, an example of this is the paramagnetic bead methodology; however, as stated prior, solid support methods have limitations. In the future, a different form of SELEX called capillary electrophoresis SELEX (CE-SELEX) would have been used. CE-SELEX involves performing SELEX with the target molecule in free solution with the oligo library. Partitioning of the bound oligo-target complexes and unbound oligos is performed using capillary electrophoresis [566].

\subsubsection{PCR amplification of libraries and the use of evolutionary methods}

After selection, PCR amplification of the eluted oligos was performed followed by gel extraction using a crush and soak protocol. Traditional column based purification kits were not used because they are less efficient at purifying small size DNA products than the crush and soak and the crush and soak method is more cost-effective, and rapid consistent for smaller size products. However, gel purification by this method is not as efficient as other methods and can result in the loss of some of the library. Utilizing a biotin labelled primer approach as described above (6.2.4) would enable more efficient recovery of the bound oligo free of contaminating primers.

QPCR was used instead of standard PCR as a method to prevent over-amplification of aptamer libraries in this selection [537] by monitoring the progression of the PCR and halting it just prior to peak fluorescence; spurious products are minimised as they generally form in the cycles 
following this point [537]. One of the limitations of the PCR amplification of complex libraries is the biasing of specific favourable sequences in the library during PCR which then leads to less variability in the library and ultimately poorer SELEX [567-569]. PCR is unavoidable because amplification of libraries is required. One approach to reducing bias is using an evolutionary approach such as error-prone PCR to induce mutations in aptamer libraries thus giving more variation to enriching libraries to select a better target [570]. While using repeated rounds of SELEX to enrich the aptamer library is plausible, in future a mutagenesis step will be used to enrich the probability that a candidate is found.

Asymmetric PCR followed by NAP5 column purification was used to regenerate singlestranded DNA libraries, this process is affected by the same PCR limitations as described above. Furthermore, it was difficult to produce large enough and pure (free of primer) samples of the single-stranded library. The presence of free primer could have detrimentally affected SELEX; this could have also been remedied by using a biotin labelled primer approach or an enzymatic approach such as using lambda exonuclease [571]. Biotin-streptavidin based singlestranded library generation would enable the production of a higher concentration of library with no contaminating primers and would limit the use of PCR.

\subsubsection{Binding assays}

One of the major issues during this series of SELEX rounds was the lack of positive control for apelin-13 binding during enrichment experiments. The antibody available did not bind to apelin-13 (data not shown). A more specific anti-apelin-13 antibody would have enabled better optimisation of the flow cytometry and dot blotting assays and would have provided a positive control of binding.

During SELEX, a FAM conjugated library was utilised with the intention of detecting the aptamer bound in different fluorescent assays. It is possible that there was not enough of a signal generated from the FAM molecule on the oligos when binding to apelin-13 or apelin conjugated PMBs because, at the earlier stages of selection, there is a lower binding affinity. To remedy this, instead of FAM labelling, a non-labelled library would be used. During affinity 
testing assays this library would then be labelled using biotin. If aptamer candidates were labelled with biotin, detection via dot blot would be more effective because streptavidinconjugated horseradish peroxidase molecules may be used to amplify the low signal as reported by Savory et al. [553]. Furthermore, it was difficult to generate high concentration ssDNA libraries (i.e $>300 \mathrm{nM}$ ) using asymmetric PCR. Using a biotinylated primer method would allow for the generation of high concentrations of ssDNA library during single-stranded library amplification.

\subsubsection{Future directions}

After the development of this aptamer, the first study performed would be to determine if an apelin-13 aptamer could detect serum apelin in the blood of diabetics compared to nondiabetics. This difference in serum apelin levels between diabetics and non-diabetics has been well documented [13]. To ensure that aptamers are resistant to serum degradation, promising aptamer candidates would be trialled in serum degradation assays as described in the literature [572], with the aim to use chemical modification to ensure resistance to serum degradation.

\subsubsection{Conclusions}

The modified aptamer selection methods described would increase the probability of a successful aptamer being generated in future attempts. This initial pilot study served as a preliminary investigation into the current detection methods of apelin-13 and reported the value of generating an apelin-13 aptamer. 



\section{Chapter 7 General Discussion}

\subsection{The potential role of the apelinergic system in glioblastoma}

Glioblastoma remains a highly recalcitrant malignancy with a dismal prognosis despite extensive research towards developing more effective treatments [573]. In recent years there has been a push to understand better the molecular pathogenesis of glioblastoma [574], which includes understanding how different signalling systems contribute to the disease.

In the last five years the apelinergic system has been reported to play an important role in tumour biology $[6,87,88,90,95,225]$; however, the role of the apelinergic system in glioblastoma is not well studied.

During the inception of this thesis, it was noted that the apelinergic system was involved in physiological processes that could contribute to glioblastoma tumour pathophysiology (reviewed in Table 7.1). Thus, the primary goal of this thesis was to investigate the role of the apelinergic system in glioblastoma biology. There were three aims to the study: firstly, to assess gene expression levels of the ligand and receptor of the apelinergic system (i.e. APLN and $A P L N R)$ in glioblastoma tissues; secondly, to generate appropriate model(s) for studying the apelinergic system in glioblastoma and; thirdly, to investigate the response of the apelinergic system to cellular stress as a potential mechanism of stress protection. 
Table 7.1. A summary of the physiological roles of the apelinergic system that could contribute to glioblastoma pathophysiology.

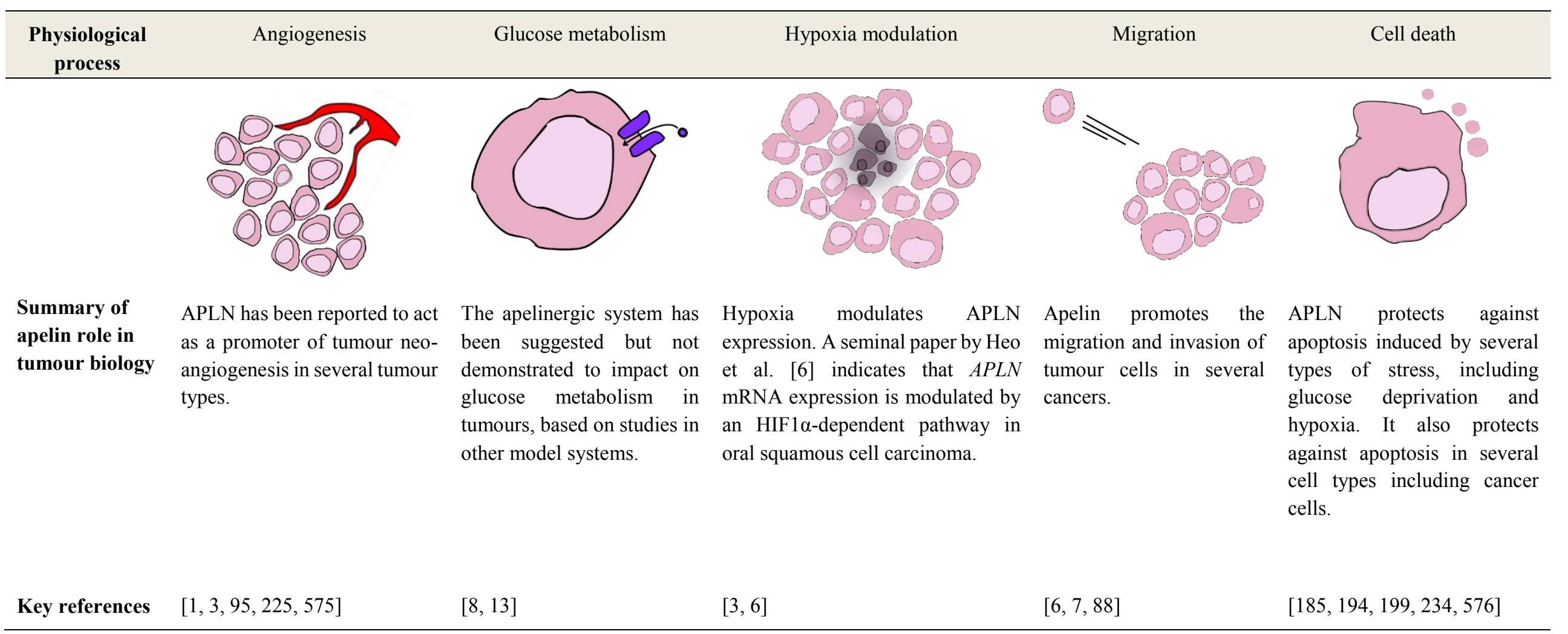




\subsection{Characterisation of the mRNA expression of $A P L N$ and $A P L N R$ in glioblastoma}

\subsection{1 $A P L N$ and $A P L N R$ mRNA levels in glioblastoma}

The analysis of gene expression in cancers has been a mainstay of cancer biology for decades [577] and has led to a deeper understanding of tumour classification, pathogenesis and patient prognosis [578, 579]. Over the last decade, the upregulated expression of APLN and APLNR has been noted in several cancers (reviewed Chapter 1, Table 1.3) but only in the blood vessels and surrounding necrotic regions in glioblastoma [2]. It was not clear if the expression of $A P L N$ or $A P L N R$ in glioblastoma was unique to glioblastoma or contributed to its pathogenesis.

Accordingly, expression of $A P L N$ and $A P L N R$ mRNA were measured in patient-derived samples of glioblastoma. APLNR mRNA expression was similar to that in normal brain tissue, but $A P L N$ mRNA expression was elevated in glioblastoma. This was corroborated by the analysis of public data from the cancer genome atlas (TCGA) using the data portal GlioVis [382]. It was concluded from this analysis that $A P L N$, but not APLNR, mRNA expression is likely to be upregulated in glioblastoma. This may suggest APLN has a role in glioblastoma; however, could also be accounted for by the extensive vasculature of glioblastoma.

\subsubsection{1 $\quad A P L N$ and $A P L N R$ mRNA levels in lower grade glioma}

$A P L N$ and $A P L N R$ mRNA expression were examined in different astrocytic and oligodendroglial tumours using public datasets to determine if the overexpression of $A P L N$ was unique to glioblastoma. APLN mRNA expression was found to correlate with the numerical grade of tumour, with the highest expression of $A P L N$ in grade IV glioblastoma. However, there was no difference in $A P L N$ mRNA expression among the different molecular subtypes of glioblastoma. These data indicate that upregulated APLN expression is unique to glioblastoma. This upregulation of $A P L N$ may be ascribed to the pathological features more commonly found in glioblastoma than lower grade tumours; such as microvasculature proliferations and 
pseudopalisading necrosis. It was surprising in the present study that APLNR mRNA expression was upregulated when compared to lower grade tumour but not compared to normal tissue, although this may be ascribed to the low number of normal tissues available for analyses. It is also prudent to note that the difference in $A P L N$ transcript counts between grade IV, and lower grade tumours is 2.12 ( $\log 2$ of normalised transcript counts), whereas APLNR had a difference of 0.58 ( $\log 2$ of normalised transcript counts). While a statistical difference is present, it is unclear whether the difference holds any biological significance.

The elevated mRNA expression of $A P L N$, and to a lesser extent $A P L N R$, suggest that the apelinergic system may play a role in glioblastoma. Alternatively, the upregulation of APLN and APLNR could simply be ascribed to the endothelial cells (ECs) that make up microvasculature proliferations found in glioblastoma (ECs are reported to express APLN and $A P L N R$ ) potentially contributing to the higher expression of APLN and APLNR seen in the tumour samples. If the changes in mRNA expression are the consequence of an increased number of ECs present in glioblastoma rather than tumour cells, then the expression of APLN and APLNR is less likely to contribute to the pathogenesis of glioblastoma directly and rather, is just part of its known role in blood vessel formation.

\subsubsection{APLNR and $A P L N$ mRNA levels in glioblastoma-derived cell lines}

The data in Chapter 3 showed that both $A P L N$ and $A P L N R$ expression levels were significantly lower in glioblastoma-derived cell lines compared with resected glioblastoma tissue. The cause of this low expression is unclear. One possible explanation is that the removal and sub-culturing of glioblastoma cells reduces $A P L N$ and $A P L N R$ mRNA expression over time. There are numerous reports of serum cultures reducing expression of salient genes [313, 324, 327, 329, 580]. However, without a direct comparison of $A P L N$ and APLNR mRNA expression between freshly derived glioblastoma tumour cells and resected glioblastoma tumour cell lines at different time points after resection; it is difficult to determine the validity of this.

It is plausible that the glioblastoma cell lines tested simply have a low expression of APLNR and $A P L N$. Sorli et al. [3] reported that APLNR mRNA is undetectable in the well-established 
TS/A breast cancer cell line and that $A P L N$ mRNA was only detectable at a high cycle threshold (Ct of 34). However, subsequent studies have shown higher levels of APLN and APLNR mRNA in another breast cancer cell lines (Hs 578T) as well as breast cancer tissue $[1,98]$. This may suggest that while primary tumour cell lines and tumour tissue may express high levels of $A P L N$ and $A P L N R$, established cell lines may lose expression.

Finally, the low expression of $A P L N$ and $A P L N R$ in glioblastoma-derived cell lines may be attributed to the possibility that glioblastoma tumour cells do not express abundant amounts of $A P L N$ and $A P L N R$ and other cell types (such as vascular cells) that make up glioblastoma express high levels of $A P L N$ and $A P L N R$. Others have reported mRNA expression of $A P L N$ and APLNR at more readily detectable levels in colorectal cancer lines [87], and APLN mRNA expression was more readily detected in breast cancer lines $[1,98]$. To answer the question of what cell types within glioblastoma express high $A P L N$ or $A P L N R$, public RNAseq data were analysed.

\subsubsection{APLN and APLNR mRNA levels in different intratumoral regions and cell types}

In Chapter 3 public datasets were analysed to determine the intratumoral mRNA expression of $A P L N$ and $A P L N R$ in a glioblastoma tumour (IVY GAP dataset) and cell type-specific expression in glioblastoma (Darmanis et al. [386] single cell RNAseq). Figure 7.1 is a summary of the salient information from these datasets. The IVY GAP dataset (represented in Figure 7.1 as a solid glioblastoma tumour divided into three layers) gives an intriguing picture of where $A P L N$ and $A P L N R$ expression is localised in a glioblastoma tumour. The three layers: the external leading edge (LE) the middle infiltrating tumour (IT) and the cellular tumour (CT) each have different ratios of tumour cells to normal cells, enabling some interpretation of whether tumour cells express $A P L N$ or $A P L N R$. The LE has a ratio of 1-3:100 tumour to normal cells while the more centrally located CT has a ratio of 100-500:1 tumour to normal cells. The CT was found to have a statistically higher expression of APLN than the LE and a higher but not statistically significant expression of $A P L N R$. This data may suggest that glioblastoma tumour cells express higher $A P L N$ over normal cells. It is also plausible that the increase of 
APLN in the more central areas of a tumour is due to hypoxia as the hypoxia marker $C A 9$ is also highly expressed in these central tumour regions. This suggests that it is possible the tumour cells express $A P L N$ in a hypoxic environment.

The hypoxia-mediated modulation of $A P L N$ expression is reported in other tumours $[3,6]$ but not in glioblastoma. During analyses of the IVYGAP dataset, it was found that APLN was overexpressed in the pseudopalisading tumour cells surrounding necrotic regions (PAN) which are commonly hypoxic [175] further suggesting that hypoxia modulates $A P L N$ expression in glioblastoma.

Finally, the microvasculature proliferations (MVP) of glioblastoma were also found to express high mRNA expression of $A P L N$ and $A P L N R$, potentially due to the well-documented role of the apelinergic system in angiogenesis [225].

It was unclear from the IVYGAP dataset what cells expressed APLN and APLNR. To definitively answer which cell types express $A P L N$ and $A P L N R$, a single cell RNAseq dataset was utilised. While $A P L N$ was overexpressed in several cell types and correlated with hypoxia markers in the centre of a tumour. It was found that $A P L N R$ was overexpressed in three specific cell types. APLNR was upregulated as expected in vascular cells, but also in astrocytes and neoplastic cells (as summarised below in Figure 7.1.).

Combined, these two data sets suggest a potentially more complex role for the apelinergic system in glioblastoma. The more widespread expression of APLN and the more selective overexpression of $A P L N R$ to specific cell types of glioblastoma could suggest that only certain cells will respond to APLN by expressing APLNR. Furthermore, the data support the assertion that hypoxia is a central driver of APLN expression in glioblastoma. I hypothesise this may form the basis of a paracrine signalling system that involves apelin secretion by several cell types while only vascular, neoplastic and astrocytic cells may respond to this. This hypothesis requires further research to confirm.

The idea of specific cell-to-cell interactions via the apelinergic system was echoed recently in the first paper investigating the role of the apelinergic system in glioblastoma in nearly a decade 
[270]. The publication provided a leap in the field's understanding of the APLN/APLNR signalling axis in glioblastoma, reporting that endothelial cells secrete APLN and that this is likely to maintain glioblastoma stem cell (GSC) populations, as is shown in Figure 7.1. Furthermore, the authors noted that GSCs heterogeneously expressed APLNR, but not APLN mRNA. Significantly, the authors reported that a reduction of APLNR expression moderately reduced the ability of GSCs to form tumours [270]. However, the authors do not discuss the potential contribution of hypoxia to the apelinergic system in glioblastoma.

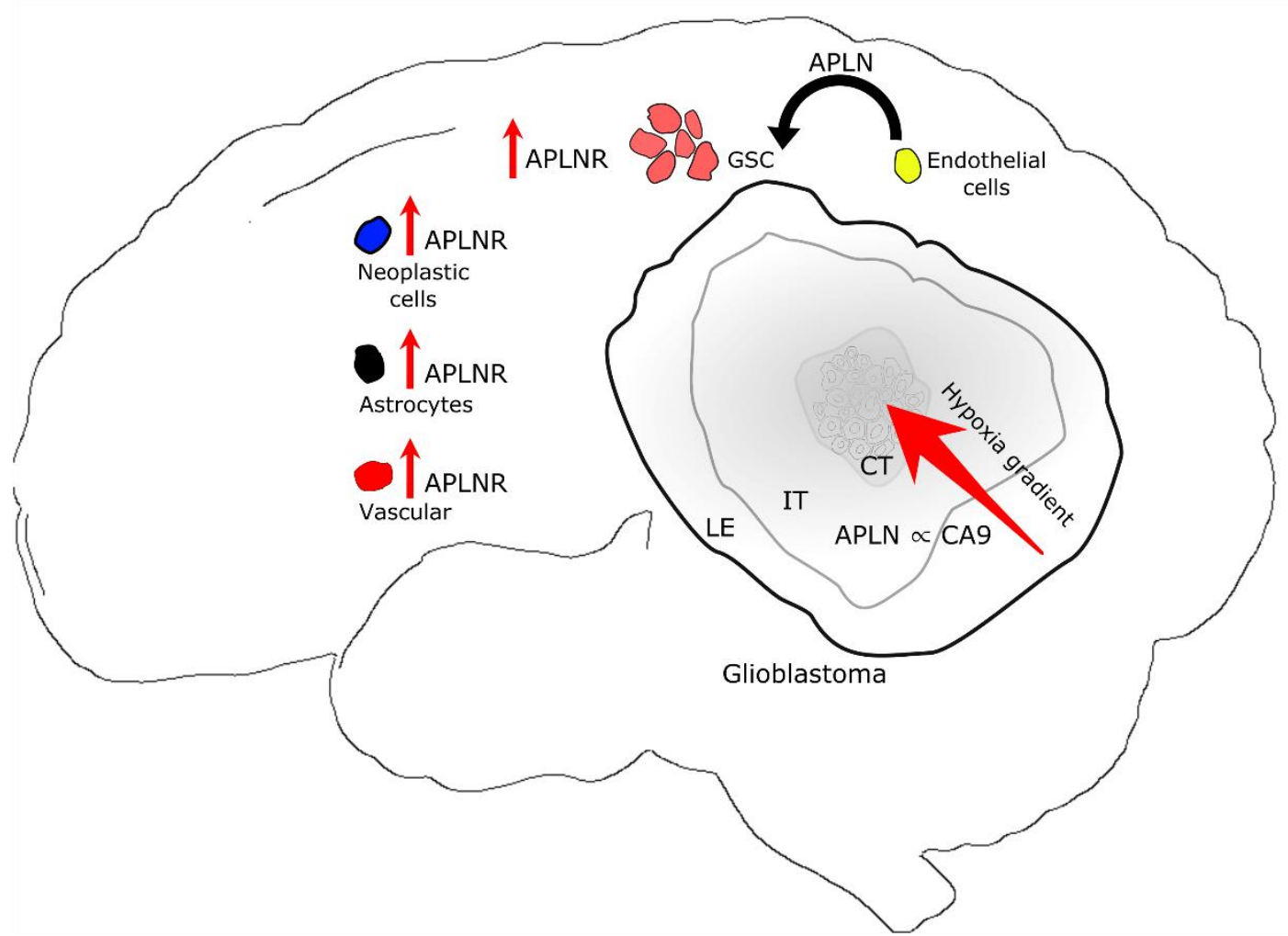

Figure 7.1. Summary of expression data from Chapter 3 regarding the intratumoral expression of APLN and $A P L N R$. Data from the IVYGAP dataset shows that $A P L N$ expression increases closer to the centre of the tumour. This coincides with the hypoxia marker CA9 suggesting $A P L N$ expression increases along a hypoxia gradient. Data from Darmanis et al. 2017 shows widespread expression of $A P L N$ in cell lines but specific upregulation of APLNR to neoplastic, vascular and astrocytic cells. Harford-Wright et al. [270] showed that endothelial cells secreting apelin stimulate GSCs. 


\subsubsection{Correlation between $S L C 2 A 1$ and $A P L N$ mRNA levels}

During the initial stages of this study, it was unclear what the mRNA expression of $A P L N$ and $A P L N R$ expression in glioblastoma tumour tissue would be, as such, $S L C 2 A 1$ was used as another example of a cancer-related gene. Moreover, because $S L C 2 A 1$, like $A P L N$, is modulated by hypoxia and is expressed in glioblastoma tumour cells [359], it provided a useful gene to compare $A P L N$ expression against. It was noted that SLC2A1 and APLN mRNA expression levels were directly correlated and may be attributed to hypoxia (thus upregulating $S L C 2 A 1)$ or blood vessel presence, since blood vessels of the brain express $S L C 2 A 1$ [359, 362, 581]. By comparing to $C A 9$ (a marker of hypoxia) and $P E C A M 1$ (an endothelial cell marker), the correlation of $A P L N$ and SLC2A1 was attributed to hypoxia. This was not wholly unexpected as hypoxia does contribute greatly to the pathogenesis of glioblastoma. However, Heo et al., 2012 performed a similar analysis using immunohistochemistry (IHC) to investigate the expression of CA9 and APLN in oral squamous cell carcinoma (OSCC) but found no correlation [6], despite strong IHC expression of APLN and APLNR in OSCC. The authors did, however, show that hypoxia induces APLN expression in different oral cancer cell lines. This could suggest that while the relationship between hypoxia and APLN is ubiquitous, it may be more relevant to the pathogenesis of glioblastoma.

In Chapter 5, it was shown that the APLN mRNA expression was ubiquitously upregulated in response to hypoxia in several glioblastoma cell lines. This is congruent with several studies $[6,10,82,83]$. The upregulation of $A P L N$ mRNA in glioblastoma cells due to hypoxia has not previously been reported, and its role in glioblastoma has yet to be determined, but its potential importance is clear. The potential roles of hypoxia-mediated apelin expression are discussed further below (Section 7.4.2.1). 


\subsubsection{Future directions and limitations of Chapter 3}

The evidence from this thesis suggests that $A P L N$ is overexpressed in glioblastoma tumours and that this may be directly related to hypoxia. Further studies need to be conducted to address the limitations of this study and satisfy further lines of questioning. The major limitation of this chapter was that insufficient human glioblastoma material was available.

Subsequent studies should focus on evaluating protein levels of APLN and APLNR in human glioblastoma samples. This would require extensive IHC, or, better still, matrix-assisted laser desorption ionization imaging mass spectrometry (MALDI-IMS) analyses of glioblastoma tissue resected from patients. Analyses of a tissue bank of glioblastoma using MALDI-IMS would enable an in-depth characterisation of the localisation and protein levels of APLN and APLNR in glioblastoma. Furthermore, it would be capable of characterising and quantifying the different isoforms of APLN in tumour sections.

Additionally, a more in-depth IHC analysis of the levels of APLNR and APLN alongside other cellular markers such as CD146 (endothelial marker), Sox-2 (stem cell marker), CA9 (hypoxia marker) and CD14 (macrophage marker) would identify co-localisation and perhaps interplay between these genes.

The hypothesis that in vitro cell culture results in deficient $A P L N$ and $A P L N R$ expression that is not representative of the in vivo tissue also warrants testing. To achieve this, glioblastoma resected tissue could be propagated in SCID/NOD mice and cells could be frozen as well as established in long-term in vitro cultures. A comparison of the APLN and APLNR levels measured at both a protein and gene level would confirm this.

Collectively, these experiments would enable a better understanding of the presence of the apelinergic system in glioblastoma. 


\subsection{Selection and development of an in vitro glioblastoma cell model}

Generating a cell line to model the apelinergic system in glioblastoma was challenging. Ostensibly, an appropriate cell line to utilise as a model should have similar mRNA expression of $A P L N$ and $A P L N R$ to resected glioblastoma tissue. As detailed above, Chapter 3 results revealed that both APLNR and APLN mRNA levels were significantly lower in glioblastomaderived primary and immortalised cell lines compared with resected glioblastoma tissue. The low mRNA expression of $A P L N R$ was particularly concerning if these glioblastoma-derived cell lines were to be used as models of the apelinergic system in glioblastoma. Reduced receptor expression would like cause non-responsiveness of cells to exogenous apelin-13 or other modulations of the apelinergic system.

Despite low APLNR expression in glioblastoma-derived cells, positive APLNR immunoreactivity (IR) was noted in both 0906 and U87MG cell lines using IHC. A discrepancy between mRNA and protein levels has been reported previously in colon cancer cells where APLNR mRNA expression levels were low but measurable while protein levels were undetectable despite using three different detection antibodies [87]. Whilst this result is the reverse of that in my study, it highlights the need to understand the limitations of the methodologies used and the reagents available. The interpretation of the Picault studies could be either an insensitive APLNR antibody that does not accurately reflect APLNR protein levels or that the APLNR receptor is not synthesised. When downstream effects of APLNR activation was tested in Chapter 4, it was noted that exogenous apelin-13 treatment caused no increase in phosphorylated ERK or transwell migration by U87MG cells but did so in U87.APLNR. Given the positive APLNR IR detected in U87MG cells, this suggests that the APLNR antibody used in IHC was not binding to the specific target and that the low mRNA expression was indeed an accurate reflection of APLNR levels.

Verification of the APLNR antibody used in the IHC work was attempted using western blotting and generated the expected bands in control cell lines. Additionally, APLNR IR 
localised to the blood vessels of infantile haemangioma as expected. Finally, APLNR IR was localised to pre-reported regions of the mouse brain, suggesting that the APLNR antibody was binding appropriately. No clear conclusion could be made about the veracity of antibody binding; irrespective of this, it was clear that there was no signalling occurring through the U87MG endogenous APLNR receptor.

To mitigate the loss $A P L N R$ mRNA expression in tumour-derived cell lines as well as the lack of a response to exogenous APLN, either fresh tissue cell lines had to be used or a cell line stably expressing APLNR had to be generated. Due to no primary tissue being available to generate primary cell lines, this study generated a glioblastoma cell line stably transfected with a GFP-tagged APLNR. These stable cell lines were validated through the observation of the GFP-tagged receptor internalisation in response to exogenous $\left[\mathrm{Pyr}^{1}\right.$ ]apelin-13 treatment. In addition, $\left[\mathrm{Pyr}^{1}\right]$ apelin-13 treatment led to increased migration in the transwell assay as well as increased phosphorylated ERK levels [59, 432, 582]. This validates the U87.APLNR cell line as an appropriate model for testing the role of apelinergic system on several aspects of glioblastoma and thus, may accurately recapitulate the in vivo behaviour and signalling of tumour cells in their niche compared with the isolated glioblastoma cell lines.

Throughout this study, it became increasingly apparent that the heterogeneous nature of glioblastoma made replicating $A P L N$ and $A P L N R$ expression seen in glioblastoma difficult. The different cell types present in glioblastoma exhibit different expression levels of APLN and $A P L N R$ respectively and this may mean each cell line contributes to glioblastoma pathogenesis to different extents. This was exemplified by the data showing different expression levels of $A P L N R$ and $A P L N$ in the various cell types. Furthermore; the tumour microenvironment may play a more critical role than previously realised; as indicated by the intratumoral data from the IVYGAP dataset and in the literature [270]. In the future, it is likely that an in vivo model or 3D in vitro model that uses multiple cell types will critical for further study of the apelinergic system in glioblastoma. Moreover, care must be taken to ensure that all composite cells of the glioblastoma tumour microenvironment are present to achieve a model that truly replicates the original tumour. 


\subsubsection{Alternative models for studying the apelinergic system in glioblastoma}

In the present study, $A P L N R$ was upregulated in U87MG glioblastoma tumour cells. This is more analogous to glioblastoma as reflected by the upregulation of APLNR in neoplastic cells (alongside vascular cells and astrocytes) in the single cell RNAseq data reported in Chapter 5. This was highly beneficial in dissecting the potential roles of the apelinergic system in vitro. However, the U87.APLNR model was limiting for two reasons.

U87MG cells were used as a negative control for the present study. Ideally, a more suitable control would have been to use a cell line stably transfected with an inactivated APLNR receptor similarly fused to the GFP protein or a total knockout cell line generated via the use of siRNA methods as a negative control. Significant effort was spent trying to generate a truncated version of the APLNR-GFP plasmid (Appendix Chapter 8). However, preliminary transfections of the truncated construct demonstrated potential cytotoxicity, and there was insufficient time available to address these issues. As such, the untransfected U87MG cell line was used as a negative control for studies with the APLNR antagonist ML221 to demonstrate APLNR-mediated effects. Future experiments should include the establishment of a inactivated $A P L N R$, or siRNA-based $A P L N R$-negative, U87MG control cell line.

APLN has been suggested as a paracrine signalling factor [3] and as such, is likely to interact with other cell types. A limitation of any in vitro cell model for studying the role of the apelinergic system in glioblastoma is that a single cell line does not reflect the multicellular nature of glioblastoma. A recent study by Harford-Wright et al. [270] also suggested a paracrine role for the apelinergic system between endothelial cells and GSCs. The data from Chapter 3 shows that the apelinergic system is likely modulated by hypoxia and presents the idea that certain cells (potential tumour or vascular cells) may respond more readily to secreted APLN. This would infer that a model which replicates the heterogeneity of glioblastoma would be appropriate. One example of this is the model used by Inda et al. [583] which utilised a mixture of engineered cell lines at known proportions to replicate a heterogeneous system [583, 584]. Models such as this may still require the use of APLNR-transfected cell lines such as generated in this thesis. Alternatively, the direct isolation of tumour cells from glioblastoma 
tumours and the subsequent culture of these cells in serum-free medium supplemented with growth factors such as epidermal growth factor may help maintain APLNR and APLN expression at tumour-relevant levels [270, 313].

Irrespective of which types of cells are used, a shift from 2D to 3D cultures appears critical [219]. Another such 3D model is the 3D organoid system that is generated by suspending tumour cells in Matrigel; this enables the generation of a model of glioblastoma that replicates hypoxic gradients and glioblastoma tumour cell heterogeneity as found in vivo [585]. In vivo models have also been used to more accurately reflect the biology of the multicellular microenvironment of glioblastoma [270].

During this study, an in vitro cultured 2D model was selected as it provided a simple system for the initial investigation into the role of the apelinergic system at the time of the inception of this study. However, it is now clear from the data presented in this thesis as well as newly published reports that a 3D or in vivo model would be more appropriate. The data from Chapter 3 suggest that tumour cells can express high levels of $A P L N R$ expression (equivalent to normal tissue) and that in vitro culture of glioblastoma-derived lines results in a reduction of APLNR and $A P L N$ expression, indicating that upregulating $A P L N R$ expression in U87MG cells was an appropriate choice. To the best of my knowledge, this study is the first instance in which expression of $A P L N R$ has been upregulated, instead of $A P L N$, in a tumour cell line (see Table 3.3). Stably transfected cell lines such as the U87.APLNR model may prove beneficial for future studies when used together with multicellular models or in vivo implantation technology. 
Table 7.2. Summary of key papers that use stable transfection to modulate endogenous $A P L N$ or $A P L N R$ expression to study the apelinergic system in tumour biology.

\begin{tabular}{|c|c|c|c|}
\hline Disease modelled & Genetic change & Result & Reference \\
\hline $\begin{array}{l}\text { Role of apelin in } \\
\text { breast cancer }\end{array}$ & $\begin{array}{l}\text { Upregulated } A P L N \text { expression } \\
\text { via stable transfection in non- } \\
A P L N \text { or } A P L N R \text { expressing } \\
\text { TS/A breast cancer cells. }\end{array}$ & $\begin{array}{l}\text { Apelin overexpression } \\
\text { leads to a more rapid } \\
\text { onset of more vascular } \\
\text { tumours. }\end{array}$ & [3] \\
\hline $\begin{array}{l}\text { Effects of apelin on } \\
\text { blood vessel growth } \\
\text { in colon tumours. }\end{array}$ & $\begin{array}{l}\text { Upregulated } A P L N \text { expression } \\
\text { by stable transfection in } \\
\text { Colon26 mouse colon cancer } \\
\text { cells. The authors do not } \\
\text { directly address the expression } \\
\text { of } A P L N R \text { in Colon } 26 \text { lines. }\end{array}$ & $\begin{array}{l}\text { Overexpression of apelin } \\
\text { inhibited the growth of } \\
\text { colon } 26 \text { tumours, but the } \\
\text { blood vessels produced } \\
\text { by colon } 26 \text { apelin } \\
\text { overexpressing cells } \\
\text { were much larger } \\
\text { calibre. }\end{array}$ & {$[358]$} \\
\hline $\begin{array}{l}\text { Investigating effects } \\
\text { of apelin on } \\
\text { lymphangiogenesis in } \\
\text { melanoma }\end{array}$ & $\begin{array}{l}\text { Upregulated } A P L N \text { expression } \\
\text { by stable transfection in B16 } \\
\text { mouse melanoma cells. Authors } \\
\text { did not address the endogenous } \\
\text { expression of } A P L N \text { and } A P L N R \\
\text { in B16 cells. }\end{array}$ & $\begin{array}{l}\text { Overexpression resulted } \\
\text { in increased tumour size } \\
\text { and higher vessel density } \\
\text { in tumours. }\end{array}$ & [4] \\
\hline $\begin{array}{l}\text { Investigating the role } \\
\text { of apelin in non-small } \\
\text { cell lung cancer } \\
\text { (NSCLC) }\end{array}$ & $\begin{array}{l}\text { Upregulated } A P L N \text { expression } \\
\text { by stable transfection of APLN } \\
\text { in low endogenous apelin } \\
\text { expression NSCLC lines H358 } \\
\text { and H1975. Authors did not } \\
\text { address endogenous expression } \\
\text { of } A P L N R \text { nor did they } \\
\text { demonstrate the contribution of } \\
\text { overexpressing cells to tumour } \\
\text { growth. }\end{array}$ & $\begin{array}{l}\text { Overexpression resulted } \\
\text { in accelerated tumour } \\
\text { growth and higher } \\
\text { microvessel density. }\end{array}$ & [1] \\
\hline
\end{tabular}




\subsection{Investigating the role of the apelinergic system in glioblastoma}

At the beginning of this $\mathrm{PhD}$ study, there was no information available on the role of the apelinergic system in glioblastoma. Figure 7.1 illustrates several potential roles of the apelinergic system in glioblastoma based on previous studies that involved the apelinergic system with end measures such as migration, angiogenesis, glucose metabolism, modulation by hypoxia and protection against cell death [8]. Of the five roles reviewed in Figure 7.1, angiogenesis is currently the most probable within glioblastoma because glioblastoma exhibits large microvascular proliferations and the apelinergic system has been reported to have a significant role in angiogenesis in other tumour types [93, 225, 405, 586]. However this information was not known at the initiation of my studies. Moreover, the appropriate cell lines and models were not available to study the contribution of the apelinergic system to glioblastoma and, as such, was not included in the study. Whilst, inclusion of this end measure would have been valuable as well as validated the techniques and tools used in this study, the role of the apelinergic system in the lesser studied aspects of glucose metabolism, hypoxia, migration and cell death were investigated herein.

\subsubsection{Role of apelinergic system in migration in glioblastoma cells}

Glioblastoma is a highly invasive neoplasm making resection difficult and recurrence inevitable [263, 587]. Identifying the mechanisms by which invasion occurs is critical to treatment. The process of invasion involves several distinct steps, one of which is cell migration. The apelinergic system has been reported to have a pro-migratory role in several cell lines, including highly relevant cell lines such as vascular smooth muscle cells that make up the blood vessels [588] [266, 267], MCF-7 breast cancer cells [97], endothelial cells [231] and other tumour cell lines $[4,6]$. There were no reports on effects of the apelinergic system on migration in glioblastoma cells. As increased motility of cells directly contributes to the invasive nature of glioblastoma, migration was used as an initial test of the U87.APLNR cell model as well as to assess the role of the apelinergic system in cell migration. 
Initially, migration was measured using the wound healing assay. The addition of exogenous apelin-13 caused a small statistically significant increase in migration of the primary glioblastoma line 0906 but not U87.APLNR or U87MG. This was due to the U87 cell lines not being technically appropriate for wound healing assays as they do not form monolayers. To remedy this, the transwell migration assay was used.

Exogenous apelin-13 caused the enhanced migration of U87.APLNR cells; however, for unknown reasons, the baseline migration level of untreated U87.APLNR cells were less than that of untreated U87MG cells. Interestingly, $\left[\mathrm{Pyr}^{1}\right]$ apelin-13 restored the migration rate of U87.APLNR cells but had no effect on U87MG cells. This confirms stable transfection of the APLNR gene that is capable of synthesising bioactive APLNR in the U87.APLNR cells. Taken together with the 0906 data, this suggests that $\left[\mathrm{Pyr}^{1}\right.$ ]apelin-13 had a pro-migratory effect in glioblastoma cells as in other cancer cells. Alternatively, this could suggest that stable APLNRGFP expression results in abrogated migration which exogenous $\left[\mathrm{Pyr}^{1}\right.$ ]apelin-13 treatment restores. To nullify that later possibility, a control cell line expressing inactivated APLNR-GFP construct would need to be generated. It is unclear whether the abrogation of migration due to APLNR-GFP transfection was a significant biological effect or simply a non-specific effect of stable cell line generation as has been reported previously [589-592].

Further studies on the effects of apelin-13 on migration should concentrate on using recently resected primary tumour-derived cell lines in the transwell assay. The transwell assay would be highly beneficial for assessing the contribution of the apelinergic system to invasion in glioblastoma. Furthermore, it is plausible that the migratory effects of [ $\mathrm{Pyr}^{1}$ ]apelin-13 may be small in tumour cells but large in other cells like vascular cells with a higher number of APLNR present. Future experiments should involve assessing multiple cell lines and cell types. Together, the data presented in this thesis suggests that apelin has a pro-migratory effect on tumour cells. 


\subsubsection{Role of the apelinergic system in cell stress response.}

During this thesis, it was hypothesised that the apelinergic system could protect glioblastoma against cell stressors present in the tumour microenvironment. Glioblastoma, like most solid tumours, undergoes many stressors such as hypoxia, nutrient deprivation and exposure to cytotoxic therapeutic agents [181]. The apelinergic protects against several types of cell stress (reviewed in Chapter 1, Table 1.6). It has also been reported to be modulated by hypoxia and glucose metabolism $[3,194]$ as well as directly modulate glucose metabolism. This makes an investigation of the role of the apelinergic system under these conditions intriguing and potentially difficult. Furthermore, no information was available on the effects of chemotherapeutic compounds on APLN and APLNR expression levels. The number of reports of the apelinergic system protecting against different forms of cell stress has led to the hypothesis that APLN may play a role in enabling the recalcitrant glioblastoma to survive in suboptimal environments.

Genes such as $A E G-1$ [444] and $c-M Y C$ [593] have been reported to dysregulate in response to hypoxia or glucose deprivation in glioblastoma and play a role in protecting glioblastoma cells against cell death. To assess the possible roles of the apelinergic system to cell stress, mRNA expression levels of four key genes (i.e. APLN, APLNR, PROM1, and SLC2A1) were measured under the stress conditions of hypoxia, glucose deprivation, combined hypoxia and glucose deprivation, and chemotherapeutic treatment. Following the assessment of gene expression, the ability of $\left[\mathrm{Pyr}^{1}\right]$ apelin-13 to protect against cell death was also assessed under the same conditions. By assessing the dysregulation of genes due to various stressors, I could address the impact of the apelinergic system under these conditions. Reduction of cell death was hypothesised as a likely outcome of apelin treatment due to the volume of literature suggesting apelin has a protective role. 


\subsubsection{Role of the apelinergic system under hypoxia-mediated stress}

Oxygen is a critical nutrient for cells, and the deprivation of oxygen leads to significant cell stress. Cells respond to this stress in numerous ways, including the upregulation of certain genes through transcription factors called hypoxia-inducible factors (HIFs) [594]. Hypoxia has been reported to increase $A P L N$ expression in several cell lines, putatively through HIF-1 $\alpha$ binding to a hypoxia response element (HRE) in the apelin gene [10, 224]. This is notable for this study because hypoxia is also a master regulator of glioblastoma progression [206, 221]. In addition, hypoxia-induced $A P L N$ expression has been implicated in a number of non-cancer related $[28,222,250,258]$ and cancer-related $[3,6]$ physiological processes, but it has not yet been investigated in glioblastoma.

In Chapter 3, it was shown that $A P L N$ positively correlated with the hypoxia markers $C A 9$ and $S L C 2 A 1$, suggesting the potential modulation by APLN in response to hypoxia. In Chapter 5 it was also noted that hypoxia upregulated $A P L N$ mRNA expression in glioblastoma cells. The upregulation of $A P L N$ coincided with $S L C 2 A 1$ which has been reported by others as an appropriate marker of hypoxia [391]. The effects of hypoxia on APLN, APLNR, PROM1, and $S L C 2 A 1$ in the various cell lines tested are summarised in Table 7.2 below. 
Table 7.3. Summary of effects of hypoxia on APLN, APLNR, SLC2A1 and PROM1 mRNA expression in four glioblastoma cell lines. $\uparrow=$ upregulation, $-=$ no change, $\downarrow=$ downregulation. One arrow indicates $<5$ fold change. Two arrows indicate $5-10$ fold change and three arrows indicate a $>10$ fold change. A star indicates a statistically significant effect change in gene expression.

\begin{tabular}{lllll}
\hline \multicolumn{5}{c}{ Hypoxia } \\
& $A P L N$ & $A P L N R$ & $S L C 2 A 1$ & PROM1 \\
\hline 0906 & $\uparrow *$ & - & $\uparrow$ & - \\
U87MG & $\uparrow \uparrow *$ & $\uparrow$ & $\uparrow \uparrow \uparrow *$ & $\uparrow^{*}$ \\
GL261 & $\uparrow$ & $\downarrow$ & $\uparrow *$ & - \\
LN18 & $\uparrow^{*}$ & $\downarrow$ & $\uparrow$ & - \\
\hline
\end{tabular}

Initially, it was hypothesised that the increase in $A P L N$ mRNA expression due to hypoxia might protect against hypoxia-mediated cell death, as $A P L N$ has been reported by others to be antiapoptotic under hypoxia-mediated stress [224, 250]. However, evidence shown in Chapter 5 suggests that apelin-13 confers no protective effect in glioblastoma cells against hypoxiamediated cell death. Apelin-13 did show a trend towards protecting against cell death in serumdeprived U87.APLN cells, which was not observed in the serum-deprived U87MG control cells. This was significant due to other reports of apelin-13 protecting against serum starvation. Although the difference was not significant, a longer incubation time of $48 \mathrm{hrs}$ is likely to have had a larger effect. This was not performed due to concerns of apelin-13 stability in culture.

It was hypothesised that the observed increase in $A P L N$ mRNA expression due to hypoxia might have a non-protective functional role in glioblastoma tumour biology. Due to the volume of literature implicating APLN in pro-migratory roles as well as the data from Chapter 4 which demonstrated a potential pro-migratory effect, the effects of hypoxia on glioblastoma tumour cell migration was investigated.

Hypoxia has been previously reported to be pro-migratory in glioblastoma cells using transwell migration assays $[211,399,595]$. To determine if the effects of hypoxia on cell migration are similar in a wound healing assay, 0906 cells were used. Additionally, signalling by the apelinergic system was blocked using the APLNR inhibitor ML221 to determine if any migratory effects seen in the wound healing assay were mediated through the APLNR receptor. Surprisingly, it appeared hypoxia inhibited the chemokinetic movement of glioblastoma cells 
into the wound. However, this could not be attributed to hypoxia without follow up experiments utilizing hypoxia conditioned media in conjunction with a chemotactic assay such as the transwell assay. In addition, ML221 had no effect on migration, which is suggestive that hypoxia-induced apelin may not have a role in glioblastoma tumour cell migration. However, this would also require follow up experiments to confirm using the transwell assay.

In retrospect, investigating the role of the hypoxia-mediated increase in apelin expression and its effect on migration requires a different approach. The hypoxia-mediated increase in apelin expression at the protein level and the level of hypoxia-mediated APLN secretion into the cell medium should first be verified using western blotting or mass spectrometry. Since most studies in the literature utilize the transwell assay for cell migration assessment, transwell assays could be performed using conditioned medium generated from cells cultured under hypoxic conditions. These results could be compared with those produced using an apelindeficient conditioned medium from cells treated with a specific siRNA to inhibit APLN generation before being cultured under similar hypoxic conditions.

The data from Chapters 3 and 5 regarding the effect of hypoxia on the apelinergic system suggest that while there was clearly is a correlation between hypoxia and APLN mRNA expression, further experiments are required to determine the specific role of hypoxia-mediated $A P L N$ expression. The public single-cell RNA sequencing data analysed in Chapter 5 indicated that $A P L N R$ is overexpressed in specific cell types (vascular, neoplastic, and astrocytic cells) and that several different cell types express a baseline level of $A P L N$. In addition, there is a higher expression of $A P L N$ in the tumour core than in the periphery of a tumour which correlated with markers of hypoxia [386]. This elevated APLNR expression in certain cell types may suggest a cell-specific response to hypoxia-mediated APLN. A more appropriate avenue to investigate would be the role of hypoxia-induced apelin expression in the relationship between glioblastoma tumour cells and non-tumour cells (such as vascular cells) by using 3D models, such as multicellular spheroids [596]. Multicellular spheroids have been used in a variety of assays, including the investigation of the interaction of endothelial and tumour cells [597] and the assessment of tumour cell invasion [598]. The use of single-cell-type spheroids was initially examined in the present study (Chapter 8, Appendix A.2) using the U87.APLNR 
cell line. The early results showed promise, but these experiments were ultimately not continued due to time constraints, and a lack of an appropriate APLNR inactivated control cell line.

\subsubsection{Limitations and future directions of the hypoxia studies}

In retrospect, one of the issues that was not examined by this study was the severity of the hypoxia under which the glioblastoma tumour cells were cultured. Hypoxia in brain tumours is considered to exist at oxygen levels between 0.5 and $10 \%[599,600]$. To simplify experimentation, a low oxygen level of $1 \pm 0.5 \%$ was selected; however, the biological relevance of this oxygen concentration is debatable. Areas of pseudopalisading necrosis in glioblastoma cells only display moderate levels of hypoxia $\left(\mathrm{pO}_{2}=2.5-5 \%\right)$, [601], indicating that the levels of $\mathrm{O}_{2}$ used in this work may have been more severe than necessary. Thus, the results obtained may not reflect the in situ conditions in a tumour, particularly since tumours generally have a gradient of $\mathrm{O}_{2}$ with the lowest $\mathrm{O}_{2}$ levels in the core. In the future, it will be important to examine the role of the apelinergic system under hypoxic conditions using an in vivo model or a spheroid cultured at normoxic conditions, either of which would enable a hypoxic gradient to be established. Furthermore, correlating oxygen concentration with $A P L N$ expression at both a mRNA and protein level would be critical.

The apelinergic system has repeatedly been implicated in modulating tumour vascular biology $[372,405]$, including via hypoxic mechanisms [357]. Here, hypoxia-induced apelin secretion was hypothesised to induce glioblastoma tumour cells to form blood vessel-like structures through a process called vascular mimicry. Alternative mechanisms of vascularization, including vascular mimicry, have been previously reported in glioblastoma [487, 489] but have not been investigated within the context of the apelinergic system. Vascular mimicry in glioblastoma was proposed to be mediated through several angiogenic peptides [486]; furthermore, it was hypothesised that apelin, an angiogenic peptide, may be involved in this process. The involvement of vascular mimicry was assessed in the present study using Matrigel cell culture models [350]. Apelin appeared to have no effect on vascular tube formation in Matrigel, but the results produced by the Matrigel tube formation assays were variable and 
inconclusive. Unfortunately, there was insufficient time to overcome these methodological shortcomings to obtain a definitive answer. Thus, analysing the role of the apelinergic system in vascular mimicry within glioblastoma may still be a beneficial area of investigation for future work.

\subsubsection{Role of the apelinergic system under conditions of glucose deprivation and combined hypoxia/glucose deprivation}

Glioblastoma cells often experience conditions of low nutrient supply, such as GD, which can lead to cell stress $[181,242,504]$. The apelinergic system has been reported to protect against a wide range of stress stimuli [185]. Contradictory evidence of the effects of GD on expression of APLN and APLNR (Zhang et al., 2009; Wang et al., 2012; Jiao et al., 2013) highlighted the importance of further studies in this area, particularly as the apelinergic system modulates glucose uptake [24, 397]. Given that hypoxia increases $A P L N$ expression in glioblastomaderived cells and hypoxia also influences glucose metabolism in glioblastoma, there may be a link between hypoxia-induced $A P L N$ and glucose metabolism [177, 501]. It is important therefore, to investigate the apelinergic system under conditions of combined oxygen and glucose deprivation (OGD). The mRNA expression levels of PROM1, SLC2A1, APLN, and $A P L N R$, were measured under GD or OGD condition and these results are summarised in Table 7.4 .

SLC2A1 mRNA expression has been widely reported to be upregulated by GD and OGD and as such was used as a positive control to demonstrate GD effects [499, 500]. SLC2A1 was upregulated in all cell lines under conditions of GD with the exception of LN18 whereby there was only a slight induction. Under OGD conditions, all cell lines except 0906 significantly upregulated $S L C 2 A 1$. This may suggest that culture conditions are effective and that each cell line has a different degree of response to GD and OGD conditions. This could further suggest certain cell lines are more appropriate to generate conclusions depending on the degree of response to GD and OGD as measured by induction of SLC2A1. Alternatively, it is also plausible that freshly derived glioblastoma cell lines are the most faithful indicator of glioblastoma tumour response under GD and OGD conditions. 
The changes in APLN mRNA varied between GD and OGD conditions. APLN was downregulated under GD conditions but was upregulated in three of the four cell lines under OGD conditions. The biological significance of the downregulation of APLN under GD was unclear but has been reported prior in retinal glial cells [250]. However, it is of note that the authors showed a strong decrease of $A P L N$ at four and six-hour time points while at twelve hours the mRNA expression of $A P L N$ was still lower than control but had increased relative to the four- and six-hour time points. This was also seen for APLNR under GD conditions. This may suggest the appropriate time points for examining glucose-mediated changes in $A P L N / A P L N R$ expression was shorter than studied in this thesis and in future several other time points should be investigated. The upregulation of APLN under OGD, but not GD, was attributed to the presence of a hypoxic response similar to that observed when glioblastoma cells are treated with hypoxia alone. This may supersede any glucose-mediated changes in mRNA expression. APLNR expression was hypothesised to be downregulated when treated with GD alone as reported prior [250].; however, APLNR mRNA expression was slightly elevated in LN18 and showed significant upregulation in U87MG. This upregulation of APLNR was not present under OGD conditions.

The lack of concordant changes in gene expression due to GD and OGD (with the exception of $A P L N$ under OGD conditions) may suggest that the apelinergic system may not hold a key role under conditions of GD or OGD. Alternatively, this difference in mRNA changes to reported changes could be ascribed to time point. If several other earlier time points were measured, there might have been a more congruent effect.

Finally, the difference in responses between cell lines was highly intriguing. While this cell to cell line difference is not unexpected, the question needs to be asked which cell line is more appropriate to examined OGD/GD effects. For example, U87MG cells consistently responded to conditions of GD and OGD with significant upregulation of SLC2A1 and PROM1 levels. PROM1 is a marker of bioenergetic stress [445] and is upregulated by hypoxia [457, 602]. $S L C 2 A 1$ levels have been reported to increase in response to both hypoxia [453] and GD [500]. U87MG is the only cell line that consistently showed upregulated SLC2A1 and PROMI (in most cases) in response to these stressors, begging the question, are results derived from 
U87MG are more relevant than other cell lines tested. An appropriate follow-up to this hypothesis would be to examine the effect of $\left[\mathrm{Pyr}^{1}\right]$ apelin-13 on glucose deprived glioblastoma cells including U87MG by examining alternative metabolism and cell health measures such as autophagy markers or production of reactive oxygen species [240].

Table 7.4. Summary of mRNA expression changes due to glucose deprivation and combined hypoxia/glucose deprivation. $\uparrow=$ upregulation, $-=$ no change, $\downarrow=$ downregulation. One arrow indicates $<5$ fold change. Two arrows indicate $5-10$ fold change and three arrows indicate a $>10$ fold change.

\begin{tabular}{|c|c|c|c|c|c|c|c|c|}
\hline & \multicolumn{4}{|c|}{ Glucose deprivation } & \multicolumn{4}{|c|}{ Hypoxia + Glucose deprivation } \\
\hline & $A P L N$ & APLNR & $S L C 2 A 1$ & PROM1 & APLN & APLNR & $S L C 2 A 1$ & PROM1 \\
\hline 0906 & - & - & 个* & - & $\uparrow^{*}$ & - & $\uparrow$ & $\uparrow$ \\
\hline U87MG & $\downarrow^{*}$ & 个个* & 个* & $\uparrow$ & $\uparrow^{*}$ & $\uparrow$ & 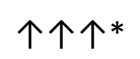 & 个个* \\
\hline GL261 & $\uparrow$ & $\uparrow$ & $\uparrow *$ & - & $\uparrow^{*}$ & $\uparrow$ & 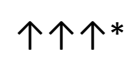 & - \\
\hline LN18 & $\downarrow^{*}$ & 个* & $\uparrow$ & $\downarrow$ & $\uparrow$ & $\uparrow *$ & 个* & $\downarrow$ \\
\hline
\end{tabular}

Exogenous [Pyr ${ }^{1}$ ]apelin-13 was added to U87.APLNR and U87MG cells under conditions of OGD or GD to assess cell survival. Through several steps of optimisation, serum-free and low glucose $(0.25 \mathrm{mM})$ conditions were determined to be optimal for testing the effects of apelin on cell survival. However, there were significant issues with repeatability when treating cells under these conditions. Retrospectively, it was noted that previous work [240, 242, 247] reported using a high concentration of dialyzed FBS, which contains little glucose in contrast to normal FCS, in combination with no supplementary glucose $(0 \mathrm{mM})$. It is plausible that the initial tests performed at low concentrations of FCS and a complete absence of glucose $(0 \mathrm{mM})$ were skewed by the presence of glucose in the FCS leading to the selection of $0.25 \mathrm{mM}$ glucose which was not an appropriate condition to test GD. Future experiments on the role of apelin under conditions of OGD or GD should utilise dialyzed FBS.

One limitation of these experiments was the measurement of cell number as opposed to other measures of cell health (such as autophagy markers or cell cycle analysis). Cell number was assessed as an initial step to screen for significant changes as it was unclear what the role of the apelinergic system under conditions of GD and OGD would be. It is plausible that the time 
points analysed and conditions used meant that measuring cell number was less optimal than other measures like lactate dehydrogenase (LDH) release [505].

Overall, the evidence presented in Chapter 5 suggests that the apelinergic system may not have a pro-survival role in glioblastoma in response to GD or OGD conditions. In the future, examination of other measurements of glioblastoma cell health under GD conditions, such as autophagy (expanded upon below) and LDH release, may provide further insight into the role of the apelinergic system in glioblastoma under GD and OGD conditions.

\subsubsection{Role of the apelinergic system in response to chemotherapeutic agents}

In Chapter 5, the apelinergic system was examined in glioblastoma cells treated with the chemotherapeutic compounds doxorubicin and temozolomide. It was hypothesised that if the apelinergic system was involved in cell stress responses to chemotherapeutic agents, there would be an induction of $A P L N$ or $A P L N R$ mRNA expression in treated cells. There were no consistent changes in $A P L N$ or $A P L N R$ mRNA expression in doxorubicin-treated 0906, U87MG, or LN18 cells. PROM1 upregulation is a marker of bioenergetic stress [445] and chemotoxic stress [333, 603]. There was a statistically insignificant trend of higher PROMI levels in response to doxorubicin treatment observed in all cell lines, as reported previously [333]. The changes in PROM1 mRNA expression were not accompanied by a concomitant change in APLN or APLNR mRNA expression. The lack of induction of APLN and APLNR mRNA combined with the consistent induction of PROM1 mRNA expression from doxorubicin suggests that the apelinergic system does not respond to doxorubicin-induced stress. Temozolomide (TMZ) was also used to induce cell stress in the 0906 cell line, after which APLNR, APLN, and PROM1 mRNA expression levels were measured. Although the 0906 cells appeared to have upregulated $A P L N$ mRNA in response to treatment with either $\mathrm{TMZ}$ or doxorubicin, these changes were small and did not suggest a significant biological response to either drug.

As stated earlier, all primary and established cell lines tested herein had significantly lower expression levels of APLN and APLNR compared with resected glioblastoma tissue. Therefore, 
cell survival experiments were performed in the U87.APLNR cell line with U87MG cells as a control. The addition of exogenous $\left[\mathrm{Pyr}^{1}\right]$ apelin-13 to U87.APLNR cells moderately but significantly protected against doxorubicin-mediated cell death. This result suggests that $\left[\mathrm{Pyr}^{1}\right]$ apelin-13 may have a protective effect against doxorubicin-induced cell stress. The effect of exogenous apelin-13 on TMZ-treated U87.APLNR cells was also tested. TMZ was not toxic to U87.APLNR or U87MG cells. Upon exogenous [ $\mathrm{Pyr}^{1}$ ]apelin-13 treatment, the cell number increased in the U87.APLNR but not U87MG, cell line. Similar results were observed when U87.APLNR, but not U87MG, cells were treated with $\left[\mathrm{Pyr}^{1}\right]$ apelin-13 under serum-starved conditions, suggesting the apelinergic system may play a small role in protecting against serum deprivation. The role of the apelinergic system in protecting against serum-deprivation has been reported previously [46, 201, 252]. Alternatively, a pro-mitogenic role could be suggested for $\left[\mathrm{Pyr}^{1}\right]$ apelin-13 in glioblastoma cells; however, previous data from our lab has not suggested a mitogenic role. Overall, the data presented in Chapter 5 suggest it is unlikely that the apelinergic system has a major protective role against chemotherapeutic treatment. It is more likely that the protective effects observed under these conditions are due to the apelinergic system providing some protection against serum deprivation.

\subsubsection{Limitations and future directions of Chapter 5}

During this thesis, cell health was only assessed by the percentage of dead cells or total adherent cells remaining; this was a limitation as in some experiments consistent cell death was not able to be measured. For example, TMZ treatment did not induce significant cell death for its effects on cell health to be measured in these assays. In the future, examining other measures of cell health, such as cell cycle analysis or autophagy levels, may provide better insight into the role of the apelinergic system in glioblastoma exposed to external stresses. Autophagy is the process whereby cells destroy their own organelles and proteins for re-use in cellular metabolism and acts as a protective mechanism in tumour cells [604]. Glioblastomas rely heavily on autophagy, often initially responding to therapies through autophagy [482]. The apelinergic system has been reported to induce autophagy in A549 lung adenocarcinoma cells [90], mesenchymal cells [605], and rat smooth muscle cells [265], but, conversely, apelin treatment reduces GD-induced 
autophagy in myocardial cells [251]. Future experiments could investigate the role of apelin in autophagy regulation in glioblastoma by examining the autophagy marker microtubuleassociated protein 1 light chain 3 (LC3) by western blotting.

One of the other major limitations of this chapter was the use of an in vitro single cell line model rather than an in vivo or in vitro model utilising glioblastoma-derived tissue samples to accurately recapitulate the tumour microenvironment with the multicellular interactions occurring [606]. During this study, I hypothesised the apelinergic systems role in glioblastoma might be critical within the multicellular tumour microenvironment as suggested by the data presented in Chapter 3 and recent evidence presented by Harford-Wright et al., 2017. This is particularly relevant when examining previous reports of the apelinergic system facilitating cell to cell interactions in other systems. This includes; endothelial cells in glioblastoma modulating GSC growth [270], endothelial cells maturing astrocytes through the apelinergic system [607] and facilitating the T cell-mediated killing of tumour cells [102]. This indicates it is critical for a multicellular model to be used in the future.

The use of 3D models either in vivo or in vitro is also critical within the context of examining the response of glioblastoma to stressors (alternative models are discussed above in 7.3.1). The response of glioblastoma to stressors such as GD and hypoxia occurs in particular regions of the tumour with low blood flow due to ineffective vasculature and high cell density. This suggests that the $2 \mathrm{D}$ conditions of culture and stress used in this thesis are not necessarily replicative of the tumour environment. Furthermore, within the context of chemotherapeutic treatments, it has been identified that 3D cultures more reliably predict clinical efficacy and relevance of treatments than 2D cultures [331]. This makes extrapolating results from this study to an in vivo setting difficult. Contrary to this, it could be asserted that this study was necessary to identify the basic cellular responses of the apelinergic system in a reductionist system and thus provide direction for future research. Irrespective, future directions when studying the apelinergic system should involve examining intercellular interactions in a 3D multicellular model. 


\subsection{The future of the apelinergic system in glioblastoma}

\subsubsection{The apelinergic system in modulating glioblastoma pathological angiogenesis}

During the inception of this thesis, it was noted based on previous evidence that the role of the apelinergic system in pathological angiogenesis was highly likely. Initial studies by Sorli et al., 2007 showed that stable expression of $A P L N$ in breast carcinoma lines lead to a highly vascular recapitulated breast cancer tumour $[2,3]$. Moreover, several other subsequent reports in following years suggested a likely role for the apelinergic system in tumour pathological angiogenesis $[1,10,358]$. With this in mind, it was elected not to study this aspect of glioblastoma for several reasons surrounding difficulties in procuring and generating an appropriate model of pathological angiogenesis. These included lack of available animal models, specialised reagents and matrices as well as the procurement of the appropriate endothelial lines. In the future, this line of investigation would have been appropriate to pursue.

\subsubsection{The potential intercellular signalling role of the apelinergic system}

The primary goal of this thesis was to investigate the role of the apelinergic system in glioblastoma. At the inception of this study, there was only one published report regarding the role of the apelinergic system in glioblastoma [2]. A decade later, a second paper regarding the apelinergic system in glioblastoma was published [270]. The results from this thesis combined with published material suggest that the apelinergic system is important in glioblastoma. However, the study herein chose to investigate the protective role. In retrospect, it seems more likely that the apelinergic system may have an intercellular signalling role or a pathological angiogenesis role in glioblastoma biology. It has been postulated previously that the upregulation of apelin expression by hypoxia may be part of a paracrine signalling process that involves endothelial cells [2, 3, 87]. Harford-Wright (2017) established a similar cell-to-cell contact role for the apelinergic system between endothelial cells and GSCs, postulating the importance of APLN in maintaining the glioblastoma vascular niche [270]. However, this work 
did not implicate hypoxia, which has been reported as critical to the GSC niche [218, 449, 608]. This thesis reports a strong link between APLN mRNA expression and hypoxia in several instances, such as following the investigation of publicly available data in Chapter 3 and in the experimental data presented in Chapter 5. Furthermore, data from Chapter 4 suggest that APLN may have some role in inducing the migration of tumour cells. Finally, the discovery that APLNR is overexpressed in certain cell lines (Chapter 3 Figure 3.9) lead to the hypothesis that APLN may recruit cells such as astrocytes, vascular cells, and neoplastic cells that have upregulated $A P L N R$ mRNA expression.

The data reported in this thesis, within the context of the published literature surrounding the apelinergic system and tumour biology, illustrates clear future directions for investigation. These would most likely focus on the role of the apelinergic system in the multicellular interactions of glioblastoma. However, the apelinergic system could have several potential roles in glioblastoma, some of which are summarised below (Figure 7.2.).

The recent report by Harford-Wright et al. [270] illustrates a link between glioblastoma stem cells and the vasculature of glioblastoma (depicted below in Figure 7.2.). Cancer stem cells (CSCs) such as GSCs require particular microenvironments (niches) to support their survival. There are multiple descriptions of stem cell niches $[608,609]$ that are characterised by key features such vasculature [271, 422, 610] and, interestingly, hypoxia [449]. However, the hypoxic niche was not discussed by Harford-Wright et al. [270]; this is an important omission as hypoxia supports GSCs survival and proliferation [449, 609]. The links between the apelinergic system and hypoxia in glioblastoma presented in this thesis and a number of published papers on the apelinergic system as a key regulator of pathological blood vessel formation $[358,575]$ suggests it is plausible that the apelinergic system has a significant role in the GSC niche. 
During this study, it was established that glioblastoma tumour cells upregulate $A P L N$ expression in response to hypoxia and above it was postulated that this hypoxia-mediated upregulation might have a signalling role but was not clear what the signalling role may be. Glioblastoma tumours present with numerous regions of hypoxia such as in areas of pseudopalisading necrosis [205] (as depicted below in Figure 7.2) where tumour cells migrate away from regions of necrosis. These hypoxic tumour cells may secrete apelin to recruit other cells to the hypoxic regions. Preliminary experiments were performed during this thesis to determine potential migratory roles for hypoxia-mediated APLN expression; (Chapter 5), but there was insufficient time and resources to finish this line of investigation. For example, there was insufficient time to optimize mass spectrometry to assess concentrations of APLN in cell media under hypoxic conditions and, furthermore, generate cell lines that do not produce APLN under hypoxic conditions.

While there was insufficient time to make conclusions on the role of hypoxia-mediated APLN, these preliminary experiments provide important directions for future research. It is clear that there is much research to be done with the apelinergic system in glioblastoma. There are several other recent publications that also suggest other potential apelinergic system roles in glioblastoma that should be investigated, as detailed below. 


\subsubsection{The immune aspects of the apelinergic system}

Immune cells such as macrophages have an important role in glioblastoma [611] and the apelinergic system may have a role in the modulation of immune cells [612]. Although the immune system fell outside the scope of this thesis, several reports on the apelinergic system and the immune system have indicated a potential association. APLNR has been reported to be up-regulated in hypoxia-treated RAW 264.7 macrophage cells, and apelin is reported to protect RAW 264.7 cells from apoptosis and stimulate their migration [234]. The immune system [613], including macrophages, have been proposed to be involved in the maintenance and progression of glioma [614]. This indicates a potential avenue of investigation.

Moreover, it was recently reported that the APLNR interacts with the transcription factor Janus kinase 1 (JAK1) to modulate interferon gamma and that APLNR abrogation reduces the efficacy of immune therapy [102]. This is contentious, as others report an increased efficacy of immune therapy when treating with apelin and $\alpha$-galactosylceramide-pulsed dendritic cells through apelin inducing the formation of mature non-leaky blood vessels [225]. This illustrates it is important to consider the immune system when studying the role of the apelinergic system in glioblastoma tumour biology, as such this is included below in Figure 7.2. 


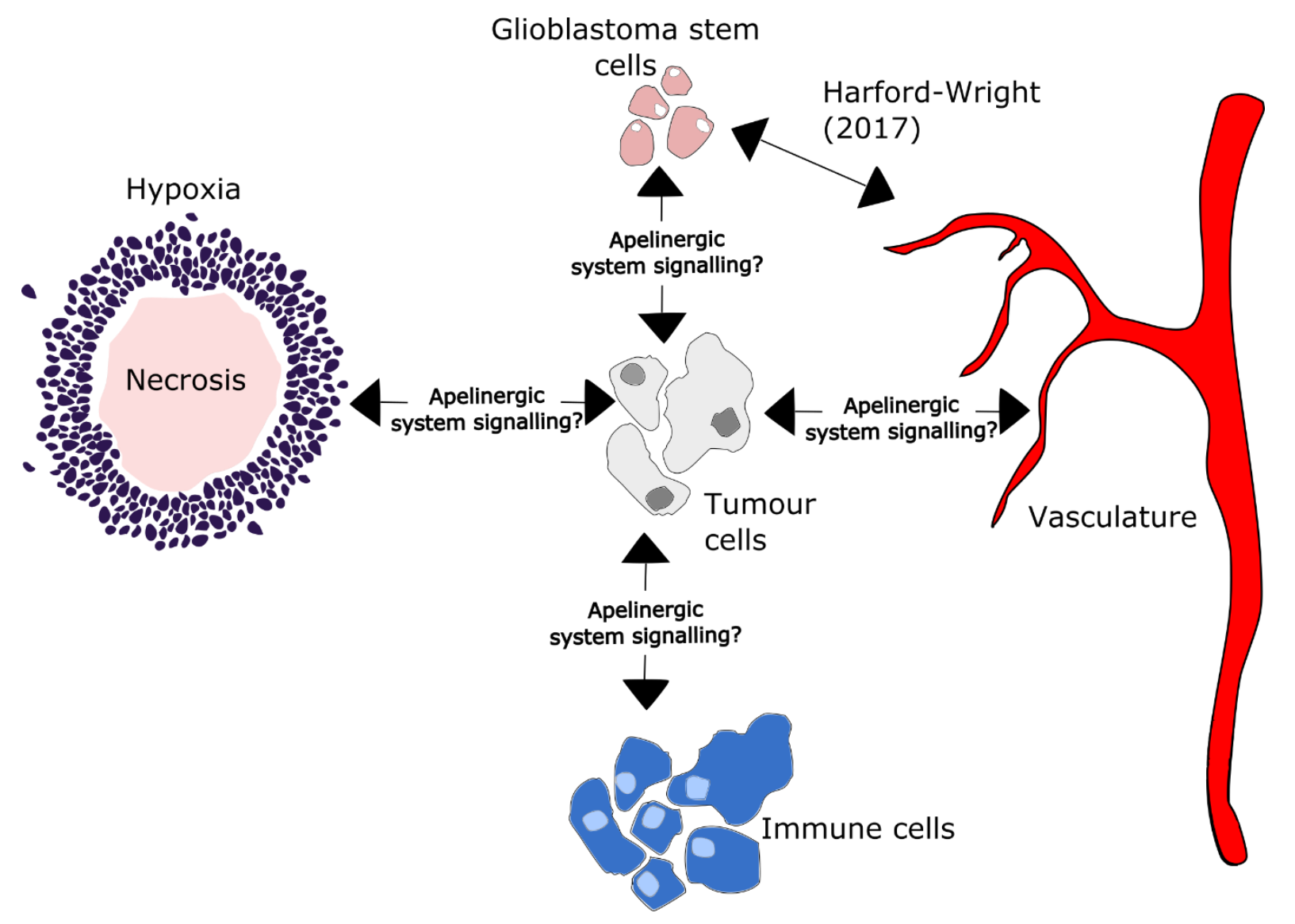

Figure 7.2. The potential roles of the apelinergic system in glioblastoma. The results of this study combined with literature suggest potential apelinergic system-cell interactions that could occur in glioblastoma, indicating potential future research directions. Harford-Wright et.al. [270] have illustrated a clear link between glioblastoma stem cells and endothelial cells (represented by the vasculature). The potential link between tumour cells and immune cells via the apelinergic system is not yet identified but recent report by Patel et al. [102] suggested the apelinergic system has a role in modulating the immune system and this can affect response to immunotherapies. During this study, the potential of role of the apelinergic system under hypoxic conditions was discussed in depth and provides another significant avenue for further research in the future (hypoxia is denoted as pseudopalisading necrosis in this diagram as a common area where hypoxic cells are noted in glioblastoma). 


\subsubsection{Targeting serum apelin-13 as a biomarker.}

Serum apelin-13 has shown promise as a biomarker in several diseases [7, 272, 275]. A secondary goal of this thesis was to attempt to generate an aptamer to apelin-13 in the hope this may be utilised in the future to detect apelin-13 in serum. In this thesis, an unsuccessful attempt was made to generate a DNA aptamer selective for apelin-13. While it was not clear why the attempt was unsuccessful, the benefits of generating an aptamer to apelin-13 were discussed. In particular, that aptamers can be raised to a very high specificity and affinity for the serum detection of proteins, suggests serum apelin may be a future sensitive biomarker for several diseases (fully reviewed in Chapter 1). Chapter 1 also reviewed the published data surrounding serum quantitation of apelin and found there to be discrepancies in the reported quantities between sensitive methods, such as mass spectrometry, and less sensitive methods, such as ELISA; this sentiment was recently echoed in a review [12]. An anti-apelin aptamer-based quantitation method would be highly beneficial because aptamers could allow for the sensitive detection of apelin in addition to its specific isoforms. 


\subsection{Final conclusions}

The primary goal of this thesis was to use in vitro models to investigate the role of the apelinergic system in glioblastoma, specifically focusing on the potential of the apelinergic system to protect against cell stress.

The mRNA expression of $A P L N$ and $A P L N R$ were assessed in glioblastoma by utilizing two approaches: performing direct experiments on tissue samples and analysing public databases. Interestingly, it was revealed that $A P L N$ was upregulated in glioblastoma. Public database analysis was further extended to other diffuse astrocytic and oligodendroglial tumours, and the results demonstrated the unique upregulation of $A P L N$ in glioblastoma over lower grade CNS tumours.

Numerous glioblastoma-derived in vitro cell lines were experimentally assessed in this thesis to determine the expression of $A P L N$ and $A P L N R$. As the cell lines tested did not mimic the expression of $A P L N R$ seen in resected primary tissue, it was concluded that a better model cell line was needed. An appropriate cellular model, the stably transfected U87.APLNR cell line was developed, and this model proved beneficial for investigating the effects of apelin on these glioblastoma tumour cells artificially expressing high levels of APLNR. Ultimately, however, this cell line has limitations because it lacks the multicellular tumour microenvironment in which $A P L N$ and APLNR are expressed in certain cell types in vivo.

The role of the apelinergic system in response to stress conditions induced by hypoxia, GD, OGD, and chemotherapy drugs in glioblastoma was evaluated. It was concluded that there were few apelinergic responses to these specific stresses, as no consistent mRNA response to stress was seen nor were any significant protective effects noted. However, the consistent upregulation of $A P L N$ under hypoxic conditions in glioblastoma was robustly established and is novel.

The data presented in this thesis regarding the role of the apelinergic under cell stress are limited in that the in vitro glioblastoma models used did not adequately represent the growth of tumour cells in their multicellular niche which the apelinergic system potentially works 
within. As such, extrapolation from cell culture to glioblastoma in vivo is hugely challenging. The data in this thesis requires further validation in either $3 \mathrm{D}$ tumour models using freshly resected glioblastoma tumour tissue.

However, based on a combination of the presented data and the published literature, this thesis outlines areas of future research that are likely to be important for determining the role of the apelinergic system in glioblastoma biology as well as the optimal models and reagents required. An attempt to develop an aptamer to apelin-13 was unsuccessful; however, these preliminary experiments will pave the way for future aptamer development, which could provide a better means of accurately measuring apelin-13 levels in serum.

Finally, this thesis amalgamated a wealth of apelinergic system knowledge relevant to glioblastoma tumour biology and combined with novel data generated herein, presents future directions for researching the role of the apelinergic system in glioblastoma tumour biology 



\section{Appendix A General Appendices}

\section{A.1 Creation of a truncated APLNR receptor}

To ensure that exogenous apelin-13 added to cell culture experiments was mediating its effects through APLNR, an antagonist to APLNR (ML221) was added, or the untransfected cell line was assayed. A better control would have been a truncated form of the APLNR receptor that still expressed functional GFP; this would control for stable expression of a protein with GFP without allowing signalling through APLNR. A truncated APLNR construct was generated but was not utilised due to the time it would take to generate a stably expressing cell line and the apparent cytotoxicity of the expressed product (data not shown).

The in silico digest programme NEBcutter2 (URL: http://nc2.neb.com/NEBcutter2/) was used to determine an area of the APLNR coding sequence outside of the ribosomal binding sequence and the Kozak consensus that could be cut using a commercial restriction enzyme. The enzyme would allow the generation of a non-functional APLNR protein with a GFP tail. Ideally, a frameshift mutation would have been generated using a single restriction enzyme to cut the plasmid in the middle of the open reading frame (ORF) generating an overhang, followed by using T4 polymerase to fill in the gap and then re-ligating the blunt ends. This was not possible due to there being no specific restriction sites in the ORF. Instead, a section of the ORF was removed using two enzymes, and the ends filled in and ligated back together.

To generate the truncated protein, $4 \mu \mathrm{g}$ of DNA was added to a restriction digest with $2.5 \mu \mathrm{L}$ of XhoI (New England Biolabs, MA, USA) and EcoRV (New England Biolabs, MA, USA) in a $50 \mu \mathrm{L}$ reaction. The digestion was then performed for $60 \mathrm{~min}$ at $37^{\circ} \mathrm{C}$. The product was run 
on a $1 \%$ gel to confirm the correct fragment was produced. The desired product was then excised and extracted using a Life Technologies Pure Link Gel (Life Technologies, Auckland) extraction kit with an additional isopropanol wash. The resulting extraction was cleaned and concentrated using a Zymo DNA (Genesearch, Christchurch) purification kit and quantified on a Nanodrop 1000-D (Thermofisher Scientific, Auckland) to confirm presence and purity of DNA. Following this, $1 \mu \mathrm{L}$ of the product was run on a $1 \% 1 \mathrm{x}$ TAE agarose gel for $60 \mathrm{~min}$ at $100 \mathrm{~V}$ to confirm the presence of the appropriate-sized DNA fragment.

The overhanging ends were blunted using T4 DNA polymerase. Three $\mu \mathrm{g}$ of purified DNA was added to the reaction volume of $50 \mathrm{uL}$. The reaction was allowed to proceed for $5 \mathrm{~min}$ and terminated at $75{ }^{\circ} \mathrm{C}$ for $10 \mathrm{~min}$. The column was cleaned using a Zymo DNA purification column. A large volume overnight ligation was performed with $1.6 \mu \mathrm{g}$ of DNA and $3 \mu 1$ of T4 ligase in a total volume of $160 \mu \mathrm{l}$ to favour intra-molecular ligation. The ligation was once more cleaned using a Zymo DNA purification column to remove salts for electroporation and transformed into electrocompetent DH5 $\alpha$ E.coli (See preparation: B.1.6). 
Initially, to screen the transformants, a colony PCR assay was performed which utilised the manufacturer-provided vector primers alongside an internal gene primer. This PCR was unable to be optimised and generated non-specific product (data not shown). As such, the correct plasmid was confirmed once more using a restriction digest assay with BSA 1 (New England Biolabs, MA, USA), as shown in Figure A.1.1, Panel A. BSA I was selected for its fragmentation pattern which would generate two equal sized products for the truncated APLNR receptor; this would appear as one band due to size homology of the two fragments. Four products would be generated when BSA I digests the standard APLNR plasmid. Only three bands will be discernible due to size homology of two of the restriction products. This can be seen in Figure A.1.1, Panel B. Following identification of the appropriate transformants, the truncated plasmid was confirmed by Sanger sequencing (Macrogen, Seoul); this can be seen in Figure A.1.1 Panel C.

The purified plasmid was transformed into chemically competent DH5 $\alpha$ E.coli cells as per Chapter 2, Section 0, expanded in LB broth supplemented with $100 \mu \mathrm{g} / \mathrm{mL}$ ampicillin and miniprepped using a Life Technologies Pure Link plasmid Miniprep kit following manufacturer's instructions. The plasmid was then transfected into U87MG cells as per Chapter 4, Section 4.2.1, to create a control cell line. Initial results indicated increased toxicity in the truncated plasmid over the standard APLNR-GFP plasmid. It was unclear as to whether this was due to protein misfolding or other technical factors during the transfection procedure. Ultimately, there was insufficient time to establish the truncated U87.APLNR cell line. 


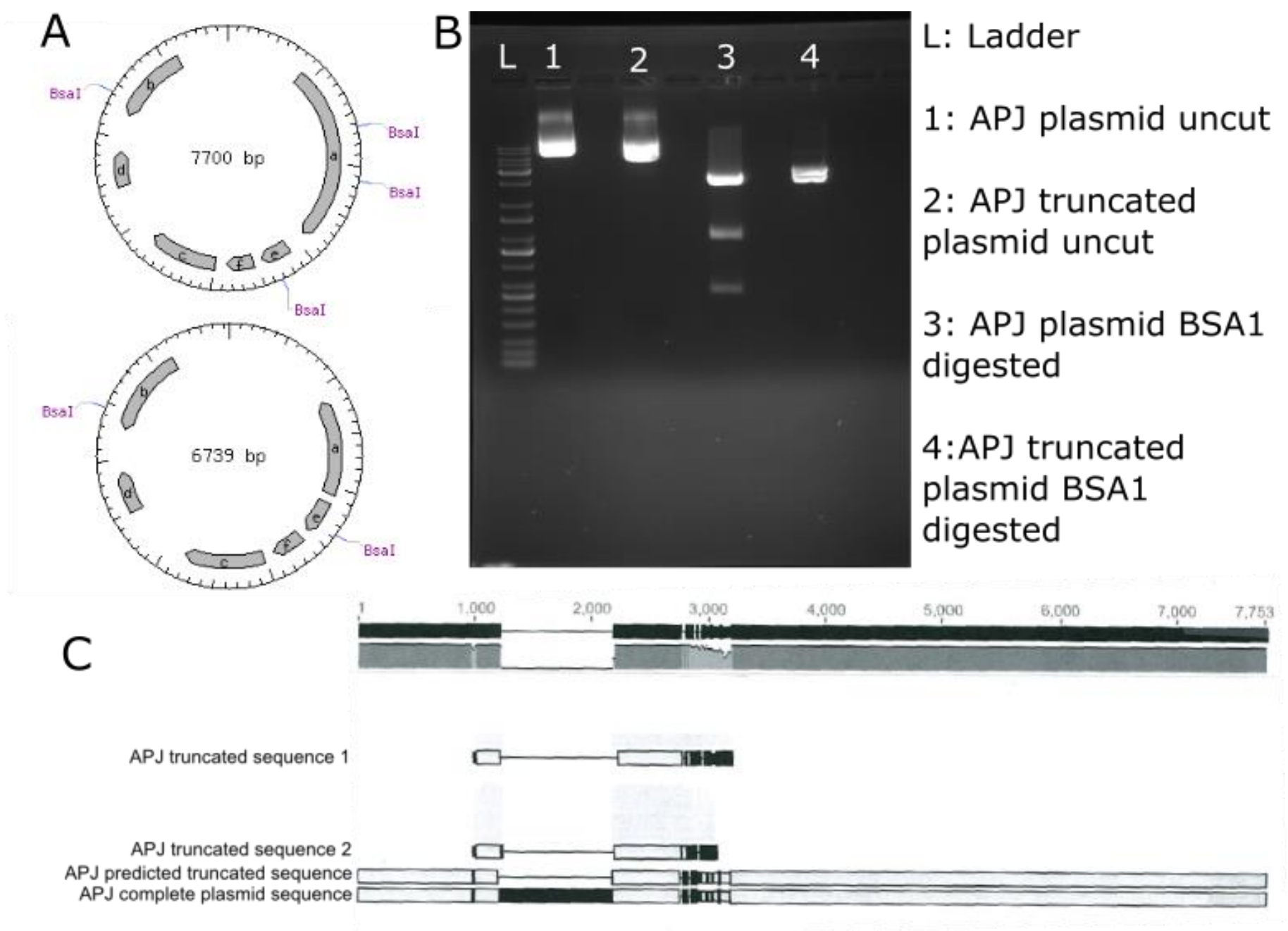

Figure A.1.1. The generation of the truncated APLNR plasmid and sequence confirmation. Panel A shows in silico restriction digest cut points performed on the uncut plasmid (7700 bp) and the predicted truncated receptor (6739 bp). Gel analysis (panel B) was as predicted with 3 fragments present for the standard APLNR plasmid (Panel B, Lane 3) and 2 bands discernible for the truncated APLNR receptor (Panel B, Lane 4). In addition, uncut plasmids were run of both the truncated plasmid and standard plasmid; these exhibited a slight mobility shift (Panel B, Lanes 1, 2). Finally, Sanger sequencing was used to confirm product formation. Sequencing matched the predicted outcomes as seen in Panel C. APLNR truncated sequence 1 and 2 refer to the 2 samples of the same plasmid sent for sequencing and predicted sequences are shown below. The sequence of the predicted truncated receptor matches the sequences returned. 


\section{A.2 Spheroid generation using U87.APLNR}

During this thesis, the discovery was made that hypoxia can modulate apelin expression in glioblastoma-derived cell lines. Hypoxia is a key feature of glioblastoma and presents intermittently in areas of hypercellularity and poor vascularisation [390]. Modelling this feature of glioblastoma is challenging. One such way to achieve this is using a spheroid culture. Spheroid cultures are 3D multicellular structures that more accurately reflect the original tumour phenotype of glioblastoma through allowing dense cellular cores, multicellular interactions and even preserving features such as EGFR amplification [435]. The use of spheroid culture in glioblastoma has been reviewed by Jensen et al. [615].

Different spheroid culture methodology was trialled during this thesis using low adherent surfaces such as non-tissue culture treated plastics, agar coated surfaces and commercial spheroid culture plates (data not shown). Ultimately the Nunclon Sphera flasks (Nunc, Thermofisher Scientific, Auckland, NZ) were utilised to generate spheroids because of their consistency and ease of use. During initial culturing, there was an apparent difference in the size of U87MG and U87.APLNR spheroids. These differences are seen in Figure A.2.1 panel D and E and graphically represented in Figure A.2.1 panel B and C. The difference in size was not able to be attributed to APLNR signalling and may be due to other factors such as a difference in growth rates. This difference in growth rates meant that any experiment performed could not use the U87MG cell line as a control of APLNR expression because the difference in growth rates adds confounding factors that could not be readily corrected for. Ideally, the truncated APLNR plasmid would have been used to generate a second cell line that would act as a control. Without an appropriate control such as the truncated APLNR-GFP cell line (Described above Section Appendix A) or a siRNA APLNR, knockout line spheroid culture was not used. 
The U87.APLNR spheroid model could be a good model of hypoxia and the apelinergic system because the spheroid generates a natural oxygen gradient with regions of hypoxia $[616,617]$. The spheroid model could be more beneficial especially if combined with techniques such as cryosectioning to examine the cross-section of spheroids. Live imaging using laser scanning confocal was attempted (Figure A.2.1, Panel A) but was limited in that the spheroid could not be imaged to a sufficient depth. Instead, a two-photon microscope approach may be better, but none was available at VUW. 

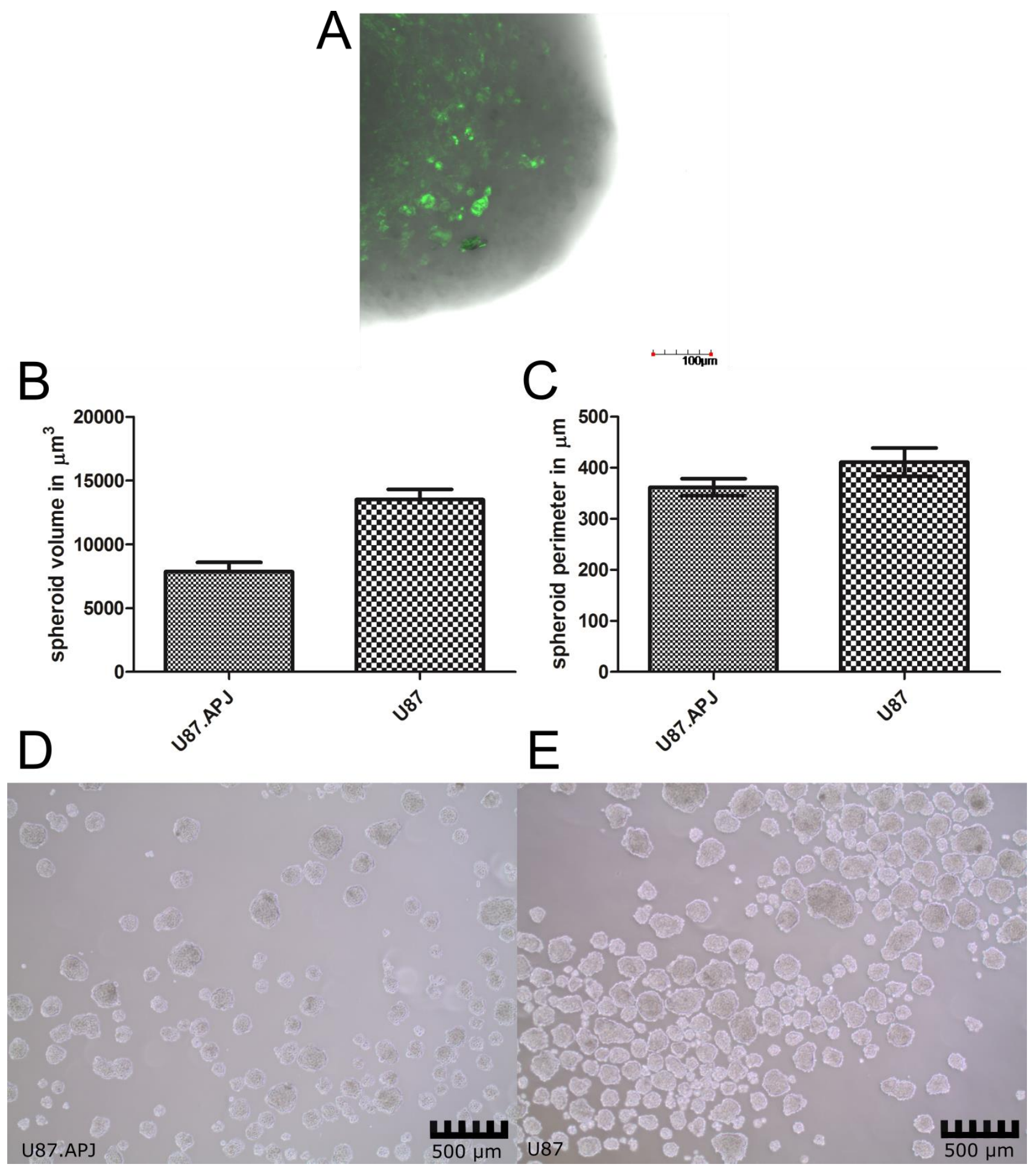

Figure A.2.1. Preliminary analysis of the morphology of U87MG and U87.APLNR spheroids. Spheroids were generated through use of specialized Nunclon spheroid forming plates exhibiting differing morphology. Panel A demonstrates an U87.APLNR spheroid in Z-stack image taken on laser scanning confocal. The average volume (Panel B) and perimeter (Panel C) of U87.APLNR $(\mathrm{n}=3)$ and U87MG $(\mathrm{n}=3)$ spheroids. Example photos of spheroids in culture; Panel D, U87.APLNR spheroids, and Panel E, U87MG spheroids. 


\section{A.3 Summary of methods trialled for the generation of a clonal stable cell line}

Several methods exist to select individual populations of GFP-positive G418-resistant cells to generate a monoclonal stable cell line [618]. Some of these methods were trialled in this thesis, and a complete list of all the possible methods are summarised below (Table A.3.5). Except for cloning rings, filter paper and FACS, all these methods were trialled. Alongside the methodology summary, the benefits and drawbacks are listed to aid in future research.

Table A.3.5. A summary of the potential methods able to create a clonal stable cell line.

\begin{tabular}{lll}
\hline Method & Pros & Cons \\
\hline $\begin{array}{l}\text { Cloning rings - isolates a } \\
\text { colony in a small cylinder to } \\
\text { trypsinise the colony of }\end{array}$ & $\begin{array}{l}\text { Efficiently isolates single } \\
\text { colonies, reusable, consistent }\end{array}$ & $\begin{array}{l}\text { Only isolates islands of cells, not necessarily clonal } \\
\text { populations of cells. Expensive to purchase cloning } \\
\text { interest off [619]. }\end{array}$ \\
& $\begin{array}{l}\text { rings. Use of grease can contaminate culture and } \\
\text { cause issues with downstream manipulation. } \\
\text { Technically difficult. }\end{array}$
\end{tabular}

Mechanical removal of colonies (toothpicks or pipette tips)[620]

Semi-solid media selection (agarose, methylcellulose $[621,622])$

Limiting dilution with the use of conditioned media [623-625]

Flow cytometry assisted sorting (FACS)[626]

Filter paper method coating filter paper disk in trypsin and laying over the colony to remove $[619,620]$
Accurate, cost-effective, rapid

Rough on cells, trying to obtain enough cells to reseed cultures, not good with sensitive cells, challenging to get a full colony.

Accurate, relatively costeffective

Easy to perform, cost-effective

Time-consuming, inefficient, requires multiple passages of the cells.

Effective, efficient

Requires a significant number of starting cells, is rough on cells. Sorting requires specialised equipment. Expensive and requires skilled operators.

Cost effective and allows

Not accurate, technically challenging

Time-consuming to optimise, cells that don't grow well in solid media cannot be used.

removal of whole colonies 


\section{A.4 Preliminary investigations into the APELA peptide}

The apelinergic system includes some peptides that have been recently discovered, including apelin receptor early endogenous ligand (APELA) otherwise known as toddler or ELABELA [33]. APELA is generated as a 54 amino acid polypeptide $[33,86]$ and cleaved to a 33 amino acid active form. APELA binds the APLNR receptor and a hitherto unknown other receptor [85]. The peptide structure is highly conserved in some species with the final 11 amino acids of APELA highly conserved in vertebrate species [33]. APELA is critical to cardiac development [33] and is secreted by human embryonic stem cells [85]. The mRNA expression of APELA has never been measured in glioblastoma tissue samples. A preliminary experiment examining the mRNA expression of APELA in resected glioblastoma tumour tissue samples and several in vitro cultured cell lines was performed (Figure A.4.1).

Figure A.4.1 indicates that APELA was expressed at significantly lower amounts than housekeeping gene HPRT. Data from Chapter 3 showed abundant expression of APLN and $A P L N R$ in resected tissue, but not in tissue-derived cell lines. APELA was also expressed at low concentrations in resected tissue samples when compared to the housekeeping gene HPRT. Most of the in vitro glioblastoma cell lines expressed less APELA than resected tumour except 0906 which showed ten-fold higher mRNA expression of APELA; the reason for this was unknown. 


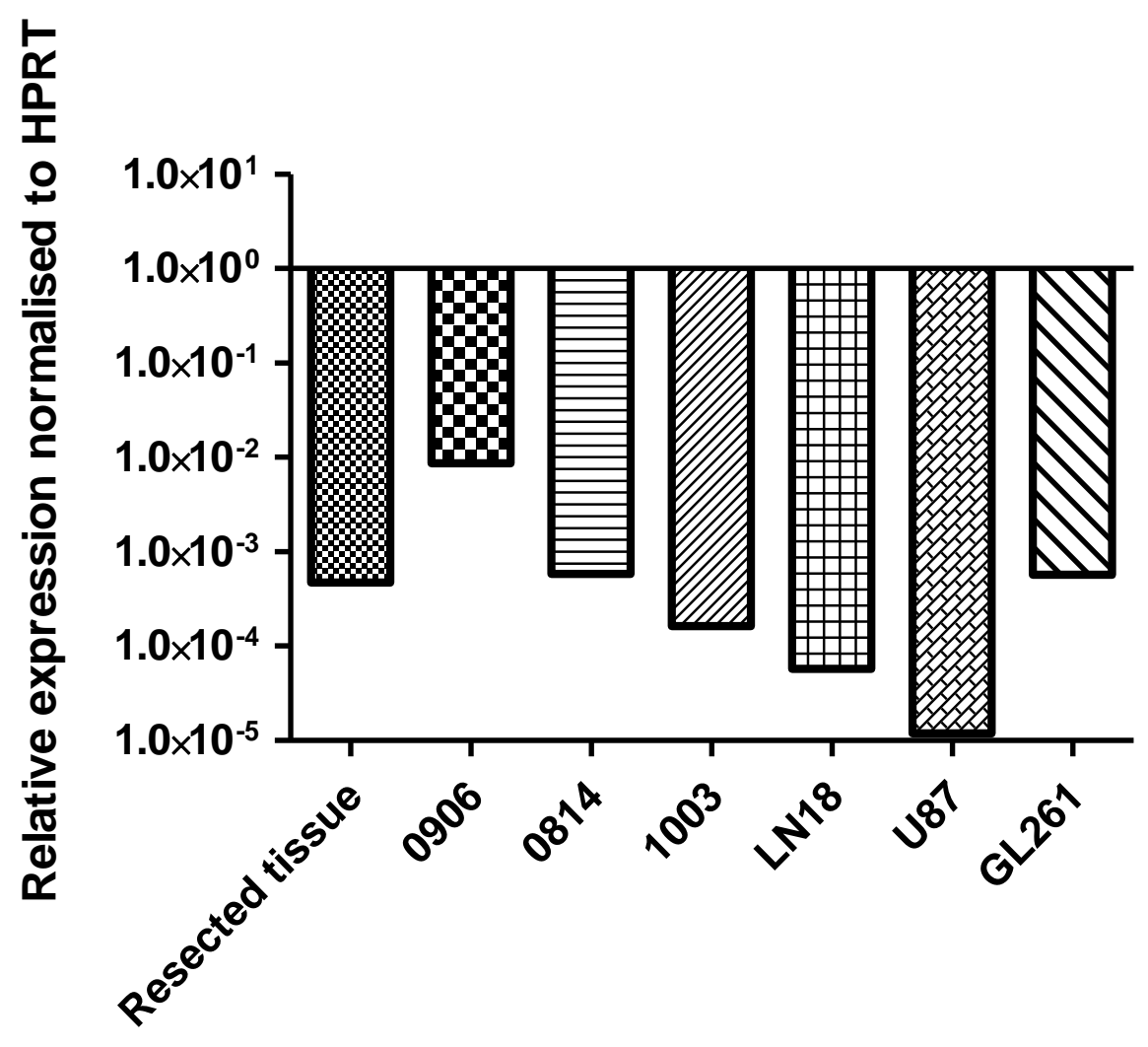

Cell lines

Figure A.4.1. The mRNA expression of APELA in glioblastoma tissue samples. Expression of APELA was measured in resected glioblastoma tissue and tissue-derived cell lines: 0906, 0814 and 1003. All tissue-derived cell lines were derived from primary glioblastoma tissues and were tested within 20 passages of isolation. Isolation from primary tissue was not performed by the Day lab. Data are expressed as relative fold expression (2- $\triangle \mathrm{CT})$ normalised to HPRT and expressed on a $\log _{10}$ scale. The APELA peptide was expressed in low abundance across all tissues.

Preliminary experiments were performed using the primary glioblastoma cell lines 0906 and 0814 to examine the effects of hypoxia on mRNA levels of APELA (Figure A.4.2). 0906 was used due to its high expression of APELA. To ensure that results from 0906 cells were not anomalous, 0814 was also used. Under hypoxic conditions, APELA expression was varied indicating that hypoxia may not modulate APELA expression.

Of interest was the consistent decrease in APELA expression under conditions of low or no glucose. This would be a good topic for future directions of the research. 

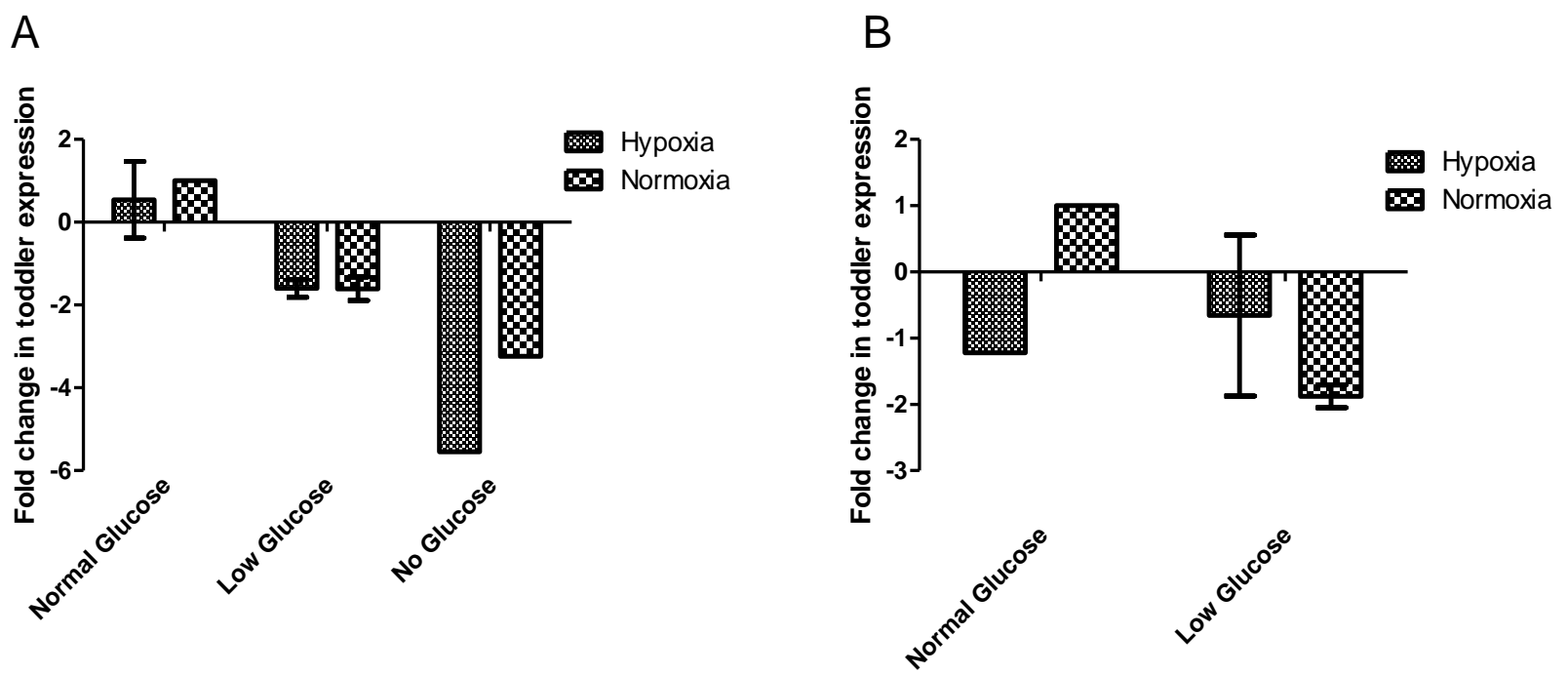

Figure A.4.2. A preliminary experiment examining the effects of oxygen and glucose deprivation on the primary cell line 0906 (Panel A) and 0814 (Panel B).All data are expressed as mean with SEM). Hypoxic conditions were $1 \% \mathrm{O}_{2}$. Cells were cultured in RPMI medium with a normal glucose concentration of $11 \mathrm{mM}$ or low concentration of $1 \mathrm{mM} . \mathrm{n}=1-3$.

APELA has been implicated to have a role in migration [40]. As a preliminary test, wound healing assays were performed using the 11 amino acid and 33 amino acid isoforms of APELA to see if any effects were present (Figure A.4.3). The preliminary data indicated toddler 11 is not pro-migratory. Definitive conclusions could not be made regarding Toddler 33 due to small sample size.

Initially, it was intended to characterise the effects of APELA in glioblastoma cell lines. However, due to the low expression of APELA in resected glioblastoma tumour tissue, it was deemed a low priority and would have been continued had time permitted. 

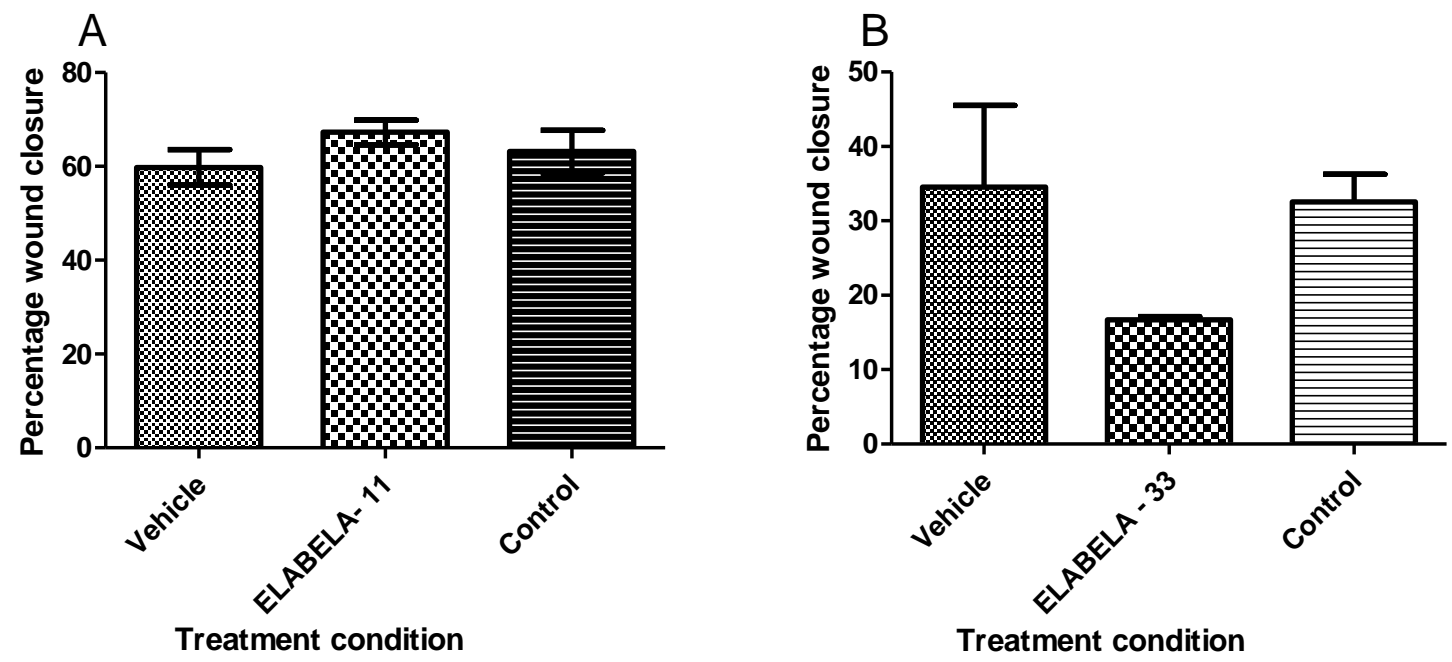

Figure A.4.3. The effect of APELA-11 (Panel A) $(n=3)$ and APELA-33 (Panel B) $(n=1)$ on HEK293.APLNR migration. As a preliminary investigation, migratory effects were examined on HEK293.APLNR due to their ease of growth for wound healing assays. 


\section{A.5 Optimization of concentrations of doxorubicin and TMZ on glioblastoma cell lines}

Temozolomide (TMZ) was used as a chemotherapeutic to investigate the role of the apelinergic system under anti-cancer agent stress. Cell lines used in this study did not exhibit cytotoxicity to TMZ $(800 \mu \mathrm{M})$. The TMZ concentration used was selected from previous studies by others [323, 460-462], and it was unclear why no cytotoxicity was seen.

During this thesis, preliminary investigations were also performed using the topoisomerase inhibitor doxorubicin. To determine the optimal concentration of doxorubicin, a titration of doxorubicin concentration was performed, and cell death was assessed by visual observation of cultures (Figure A.5.1). Also, $5 \mu \mathrm{M}$ of ML221 was added to see if there was any effect of the inhibitor. Cells treated with doxorubicin initially became enlarged (Figure A.5.2) and at high concentrations $(500 \mathrm{nM})$ died over the course of two days. 


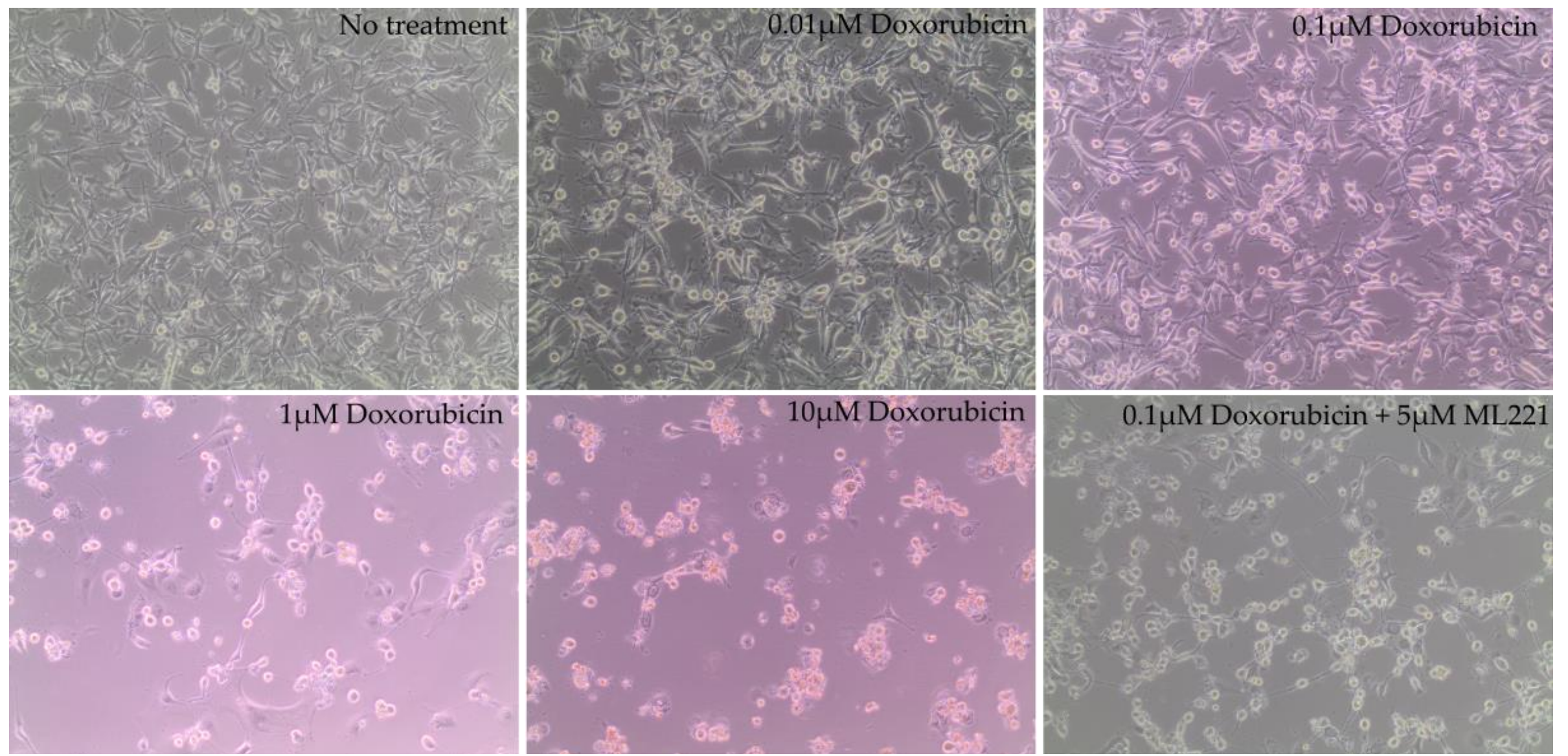

Figure A.5.1. Titration of the optimal doxorubicin concentration. Cells were treated with different concentrations of doxorubicin in low serum (1\% FBS) medium for $72 \mathrm{~h}$. ML221 (5 $\mu \mathrm{M})$ was added to determine if there was any effect. Cell death was assessed by qualitatively analysing the presence of adherent live cells and floating dead cells. 


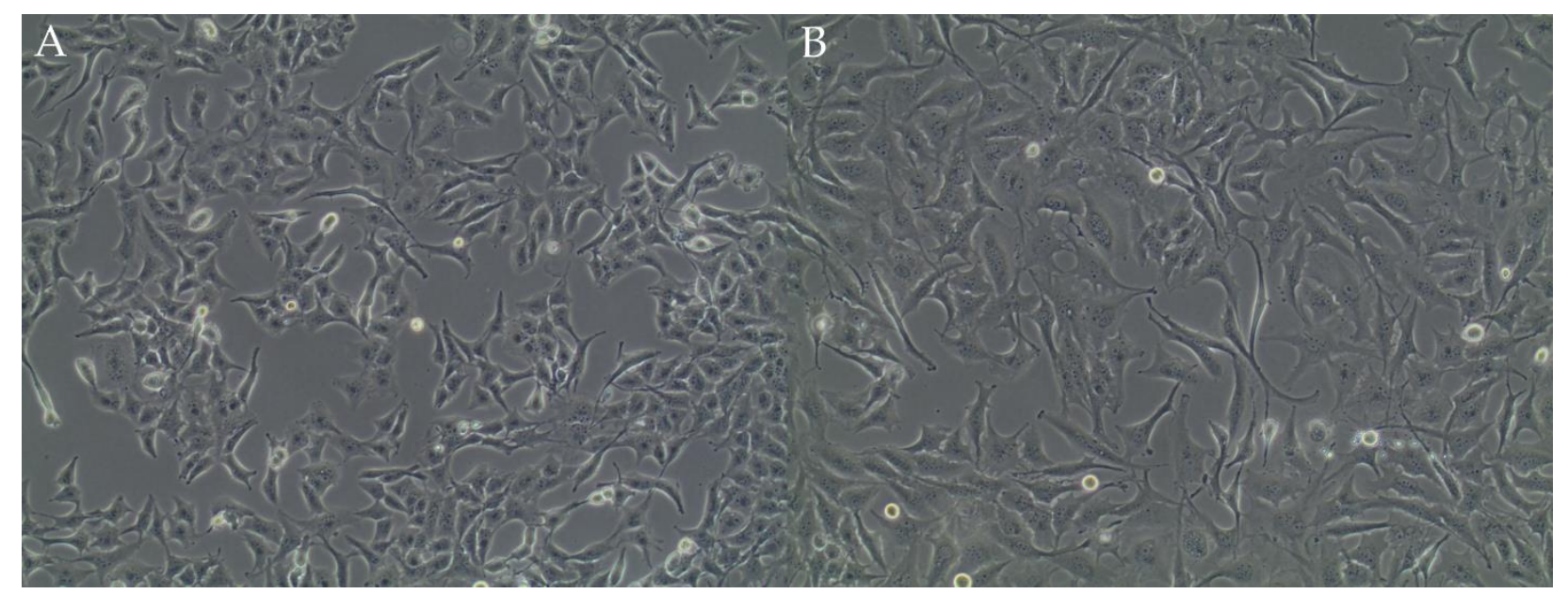

Figure A.5.2. Effect of doxorubicin on cell morphology. Treatment of U87MG glioblastoma cells control (panel A) and doxorubicin $(100 \mathrm{nM})$ (panel B) caused a significant effect on cell size but did not necessarily lead to cell death. Magnification using 10x objective.

The effect of apelin-13 on cells treated with doxorubicin was initially measured by flow cytometry using PI staining. It was noted that doxorubicin caused a dose-dependent increase in fluorescence (Figure A.5.3). At concentrations above $100 \mathrm{nM}$, doxorubicin shifted the emission spectrum, causing the fluorescent signal to spill over into the PI channel. Therefore, $100 \mathrm{nM}$ doxorubicin was used for initial flow cytometry experiments in this study (Figure A.5.4). 


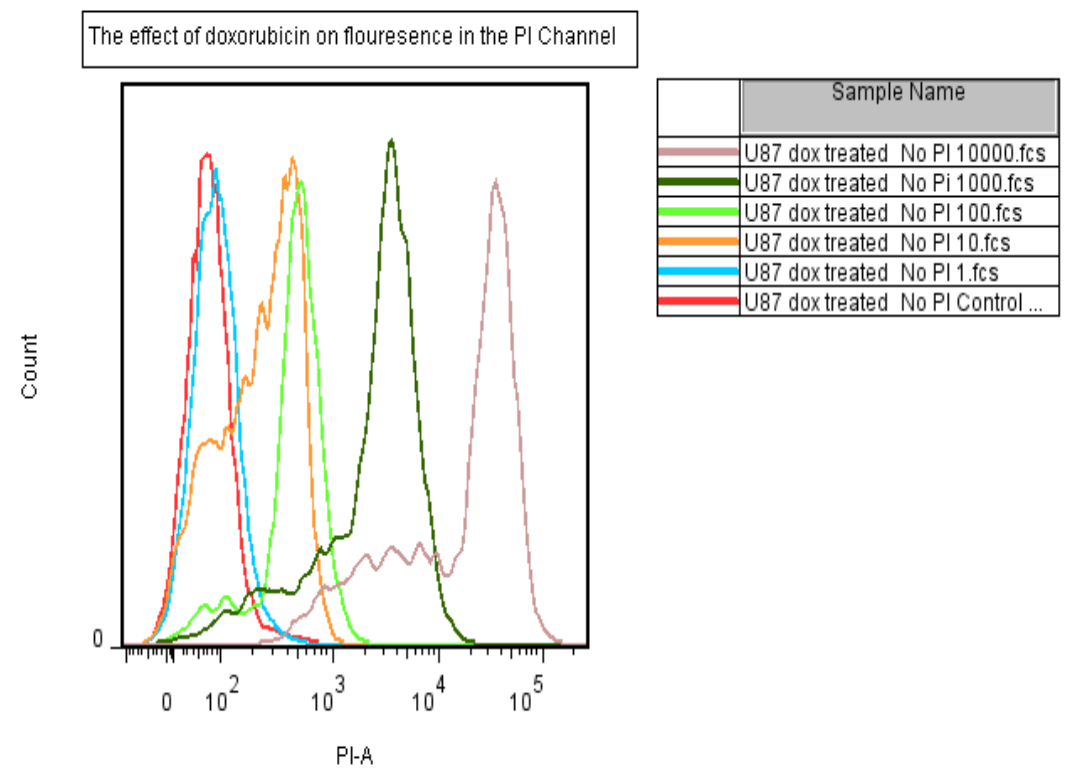

Figure A.5.3. The effect of endogenous doxorubicin fluorescence on the PI channel. Excess doxorubicin concentration causes the fluorescence to bleed over into the PI channel, thus making PI uptake viability determinations difficult. Cells were treated with the indicated concentrations of doxorubicin in $\mathrm{nM}$ for 24 hrs and analysed on a FACS Canto II flow cytometer.

The effect of the APLNR antagonist, ML221, was tested in U87MG cells treated with $100 \mathrm{nM}$ doxorubicin for $24 \mathrm{hrs}$ and cell viability was measured using PI staining (Figure A.5.4). There was no significant cell death from the doxorubicin treatment, and treatment with ML221 also had no significant effect on cell death on its own or in combination with doxorubicin (Figure A.5.4). As such, all further experiments studying the role of the apelinergic system in doxorubicin-treated cells (discussed in Chapter 5) were done using cell counts and higher concentrations of doxorubicin. 


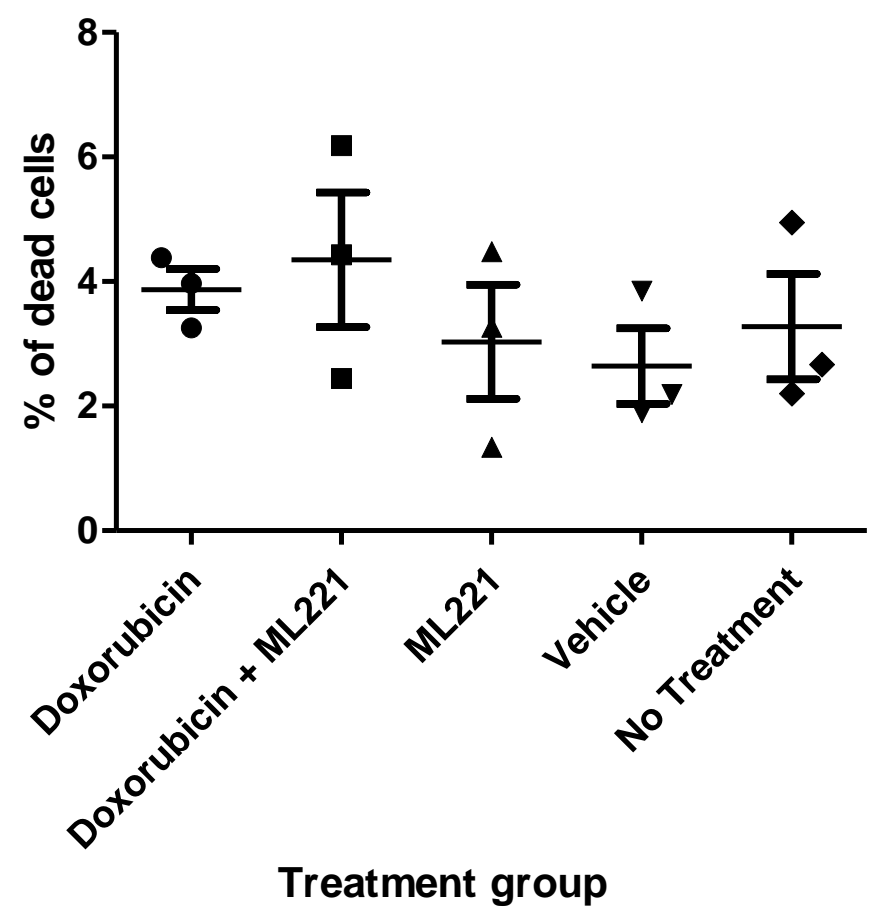

Figure A.5.4. The effect of APLNR inhibition on cell viability of U87MG cells treated with low dose (100 nM) doxorubicin. M1221 was used at $5 \mu \mathrm{M}$. Dead cells were determined by PI staining. Vehicle is DMSO at $<0.1 \%$ concentration. Data are presented as the mean \pm SEM ( $n=3$ experiments). 


\section{A.6 Optimization of hypoxia and glucose deprivation conditions.}

The effect of the apelinergic system under hypoxia-mediated cell stress was first tested in HEK293.APLNR (Figure A.6.1). HEK293.APLNR cells were selected as a control cell line because it was hypothesized that hypoxia would have a greater effect on cell viability than in glioblastoma tumour lines. Cell death was assessed using PI viability staining. Cells were cultured for $48 \mathrm{hrs}$ under hypoxic and normoxic conditions and treated with apelin at $1 \mu \mathrm{M}$ concentration or vehicle control. The data show a trend for a very small decrease in cell death when cells were treated with apelin $(\mathrm{n}=3, \mathrm{P}=0.1508$, Paired Student's $t$-test).

Hypoxia

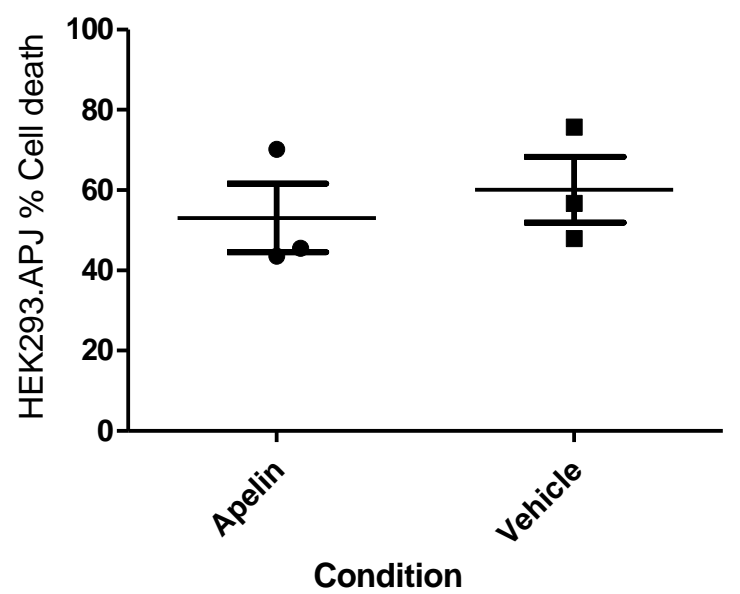

Normoxia

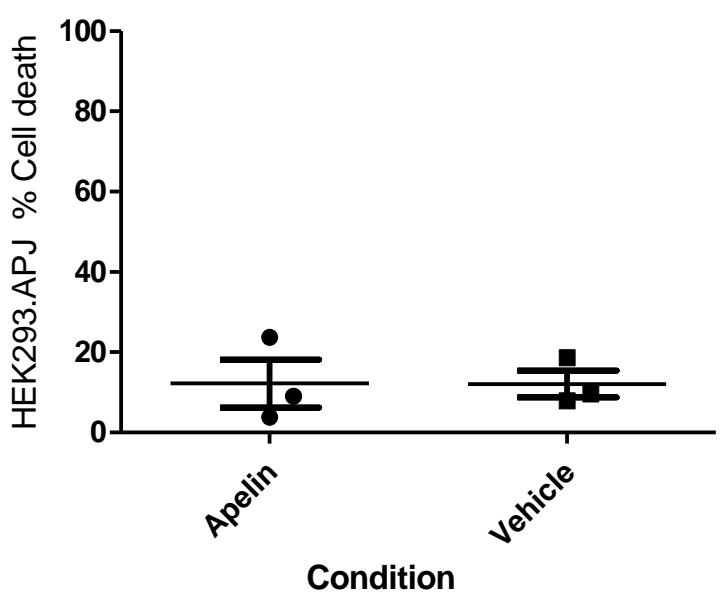

Figure A.6.1. The effect of hypoxia $\left(1 \% \mathrm{O}_{2}\right)$ on HEK293.APLNR cell viability. Apelin treatment of HEK293.APLNR cells under hypoxia showed a trend towards apelin protecting against hypoxia-mediated cell death $(n=3$, $p$-value $=0.0508$ paired Student's $t$-test $)$. Data are expressed as a dot plot of individual experimental replicates with SEM. Cell viability was measured using PI staining and flow cytometry. Each experiment was performed in duplicate $(\mathrm{n}=$ three biological preparations). Experiments were performed under no serum conditions (0\% FBS) for $48 \mathrm{hrs}$. 


\section{Effect of glucose deprivation on HEK293.APJ}

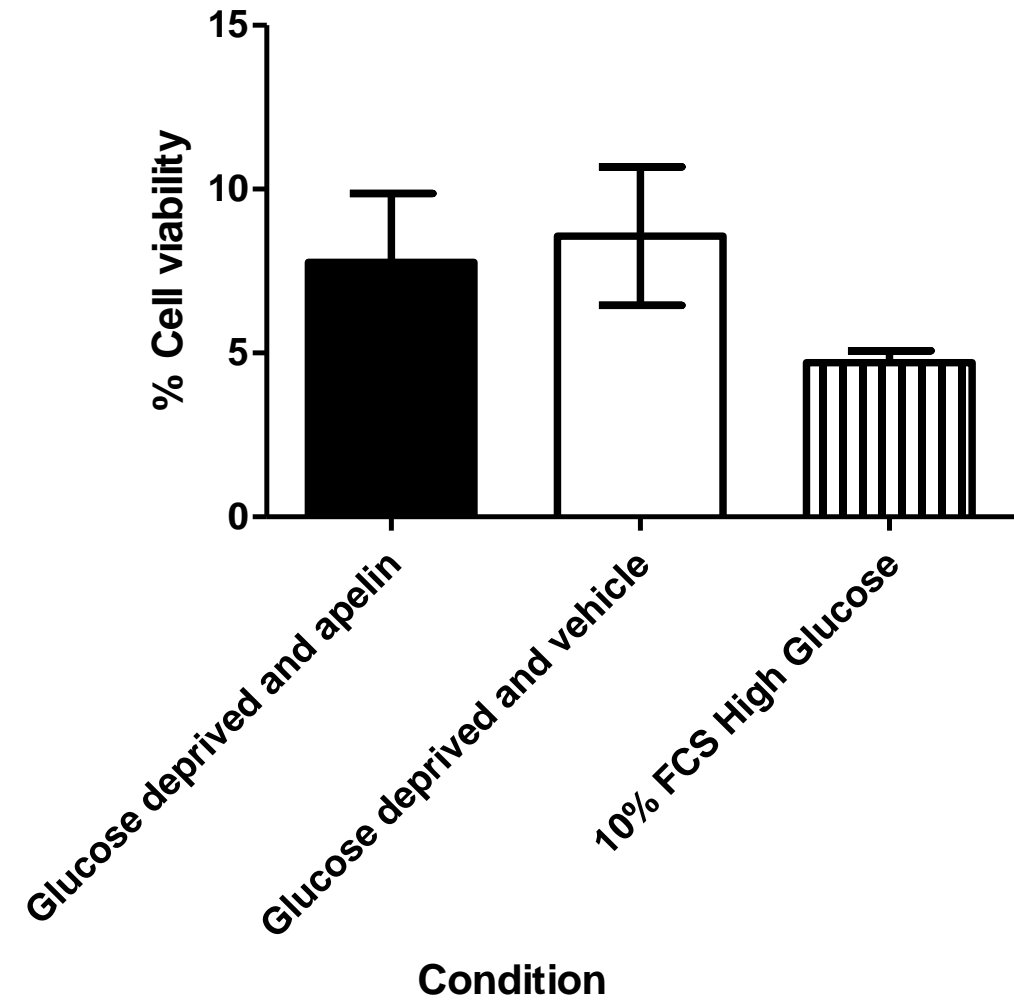

Figure A.6.2.The effect of GD on HEK293.APLNR cells. Cells were cultured for $24 \mathrm{hrs}$ at $1 \%$ FBS no-glucose $(0 \mathrm{mM})$ conditions with Apelin $(1 \mu \mathrm{M})$ or vehicle. Cell viability was measured using PI staining on a flow cytometer. There were no significant effects on cell death due to GD $(n=2)$.

To examine if the variability and low cell death seen due to low glucose and oxygen deprivation was due to glioblastoma cell lines, the HEK293.APLNR cell line was used to investigate GD effects. It was assumed that HEK293.APLNR would be sensitive to GD. Cell viability was measured using PI staining and flow cytometry. The data in Figure A.6.2 indicated that the GD treatment did not significantly induce cell death and that there were confounding technical issues. 
Preliminary experiments were performed on cell lines in hypoxic conditions $\left(1 \% \pm 0.5 \% \mathrm{O}^{2}\right)$ (Figure A.6.3, Panel A) and at a lower glucose concentration of $2 \mathrm{mM}$ (Figure A.6.3, Panel B) with 1\% FBS supplementation. None of the conditions elicited sufficient cell death in comparison to control conditions. As such it was concluded that the glioblastoma cell lines were not under enough stress to induce cell stress.
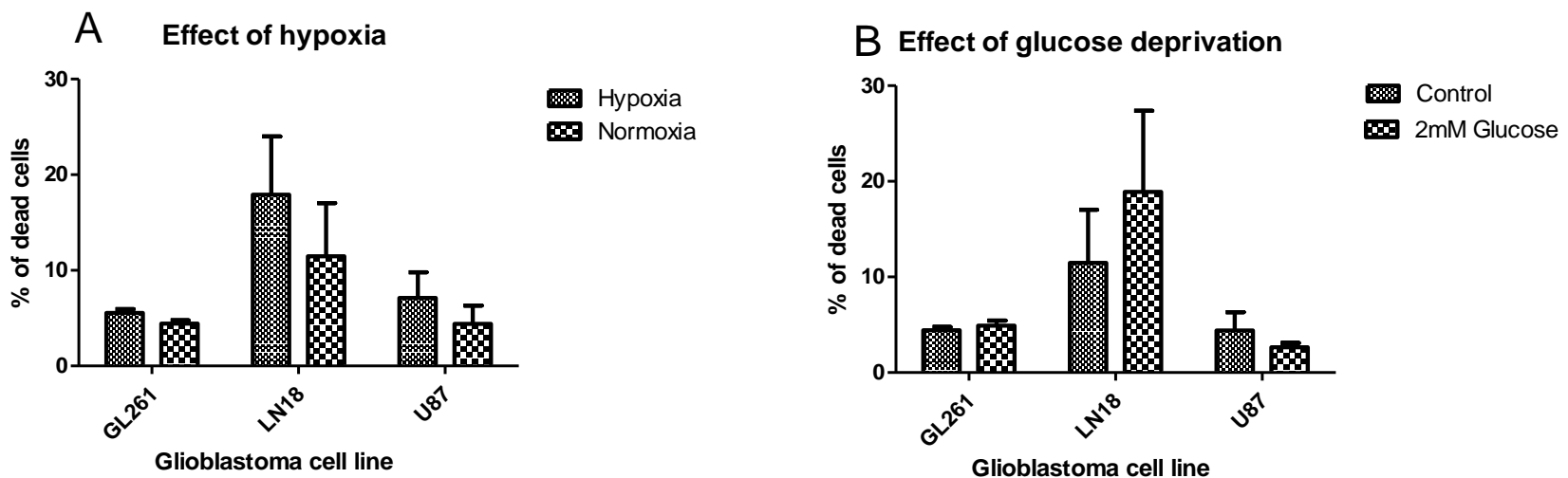

Figure A.6.3. Effect of oxygen and glucose deprivation on viability of glioblastoma cell lines ( $\mathrm{n}=3$ experiments). Values were expressed as a mean with error as SEM. Cell viability was assayed PI viability on a flow cytometer. All experiments were performed under 1\% FBS conditions over $24 \mathrm{hrs}$. Cell viability was assayed by PI staining on a flow cytometer. All experiments were performed under 1\% FBS conditions over $24 \mathrm{hrs}$. 


\section{A.7 The role of the apelinergic system in vascular mimicry}

The apelinergic system is reported to have a direct role in angiogenesis [401], and increased expression of the APLNR receptor has been reported in the microvasculature proliferations of glioblastoma [2]. The role of the apelinergic system in alternative mechanisms of neovascularization such as vascular mimicry (VM) has not been investigated, and some preliminary experiments were conducted in this thesis. VM involves tumour-derived cells forming a functional vascular network in the absence of true endothelial cells. This is seen to occur in glioblastoma and correlates with the grade of cancer [627],[628]. VM has also been postulated as a potential treatment target $[629,630]$.

The vascular mimicry (VM), Matrigel formation assay, has been reported in the literature prior [350] and was utilised to examine the role of the apelinergic system in VM. The assay works by seeding cells on a Matrigel-coated surface; cells which have vasculogenic potential form vessel-like structures. This assay is commonly used for endothelial cells but can also be used for tumour cells. Matrigel is a mix of extracellular matrix proteins derived from a mouse sarcoma cell line [631]. It is provided in a gel form and is used as a surface for cells to grow on and infiltrate. Matrigel has been used for many years to demonstrate vasculogenic activity of endothelial cells, usually using human umbilical vein endothelial cells (HUVECs) [632] but can also be used to demonstrate VM of tumour cells [350]. In this thesis, preliminary investigations were performed to see if the apelinergic system had any role in VM.

VM was assessed as described in [350]. Briefly, Matrigel was thawed on ice, and $50 \mu \mathrm{L}$ was pipetted into the well of a 96-well plate. The Matrigel was allowed to set for $45 \mathrm{~min}$ at $37{ }^{\circ} \mathrm{C}$. Cells were then seeded on to the Matrigel using DMEM medium supplemented with 1\% FBS and were incubated on the Matrigel overnight. 
There was no difference seen in tubule number (Figure A.7.1, panel A) and length (Figure A.7.1, panel B) between U87.APLNR and U87MG cells. A preliminary apelin treatment $(\mathrm{n}=$ 1) was also performed, but this showed no effect in U87.APLNR and U87MG cells (Figure A.7.1, panel C). Also, under hypoxia treatment, there was no effect seen $(n=2)$ (Figure A.7.1, Panel D).
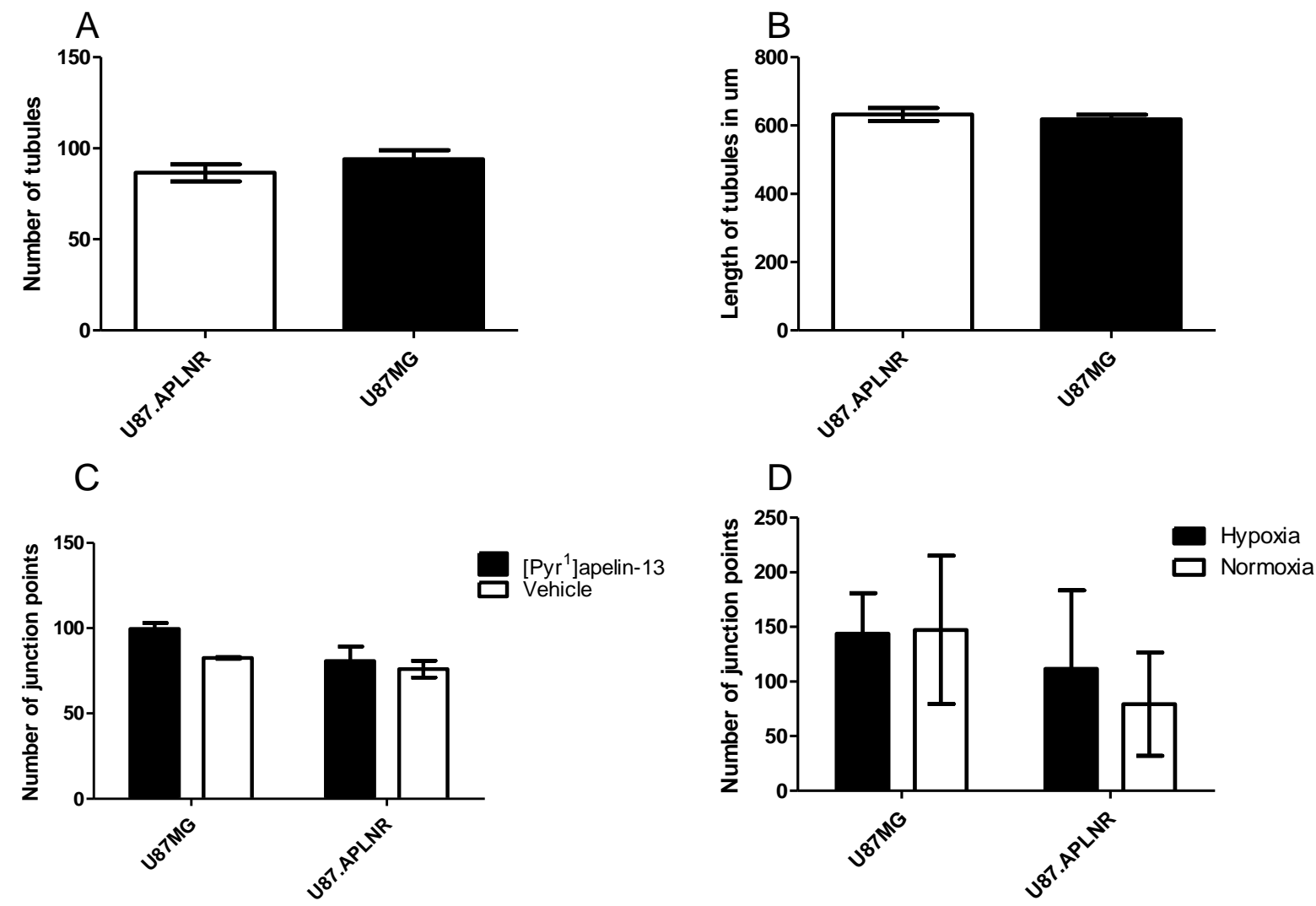

Figure A.7.1. An Initial investigation into the role of the apelinergic system on vasculogenic mimicry. The total number of tubules (Panel A) and mean tubule lengths (Panel B) were compared between U87MG $(n=6)$ and U87.APLNR $(n=8)$, then the effect of apelin (Panel C) $(n=2)$ and hypoxia (Panel D) $(n=1$ in duplicate) was investigated in U87MG and U87.APLNR. Matrigel was added to wells of a 96 well plate and allowed to adhere after which cells and treated with either apelin $(1 \mu \mathrm{M})$ or hypoxia $\left(1 \% \pm 0.5 \% \mathrm{O}_{2}\right)$, cells were left to incubate for $14 \mathrm{hrs}-17 \mathrm{hrs}$ and micrographed. Error bars are mean \pm SEM. 
Preliminary VM data was not suggestive of an apelin or hypoxia effect. These results were technically variable as shown by the error bars in Figure A.7.1, Panel D, and it is difficult to ascertain the cause. This variability can be seen visually in Figure A.7.2 which shows two biological replicates of U87MG cells seeded at the same density on Matrigel under hypoxic conditions $\left(1 \% \pm 0.5 \% \mathrm{O}_{2}\right)$ but exhibiting vastly different tube structures. Figure A.7.2, Panel A shows U87MG forming very thin tubule structures while Panel B shows much thicker tubules in fewer numbers with larger "node" formations than Panel A. This inferred that more time was required to optimise assay conditions. Moreover, no conclusions could be made.
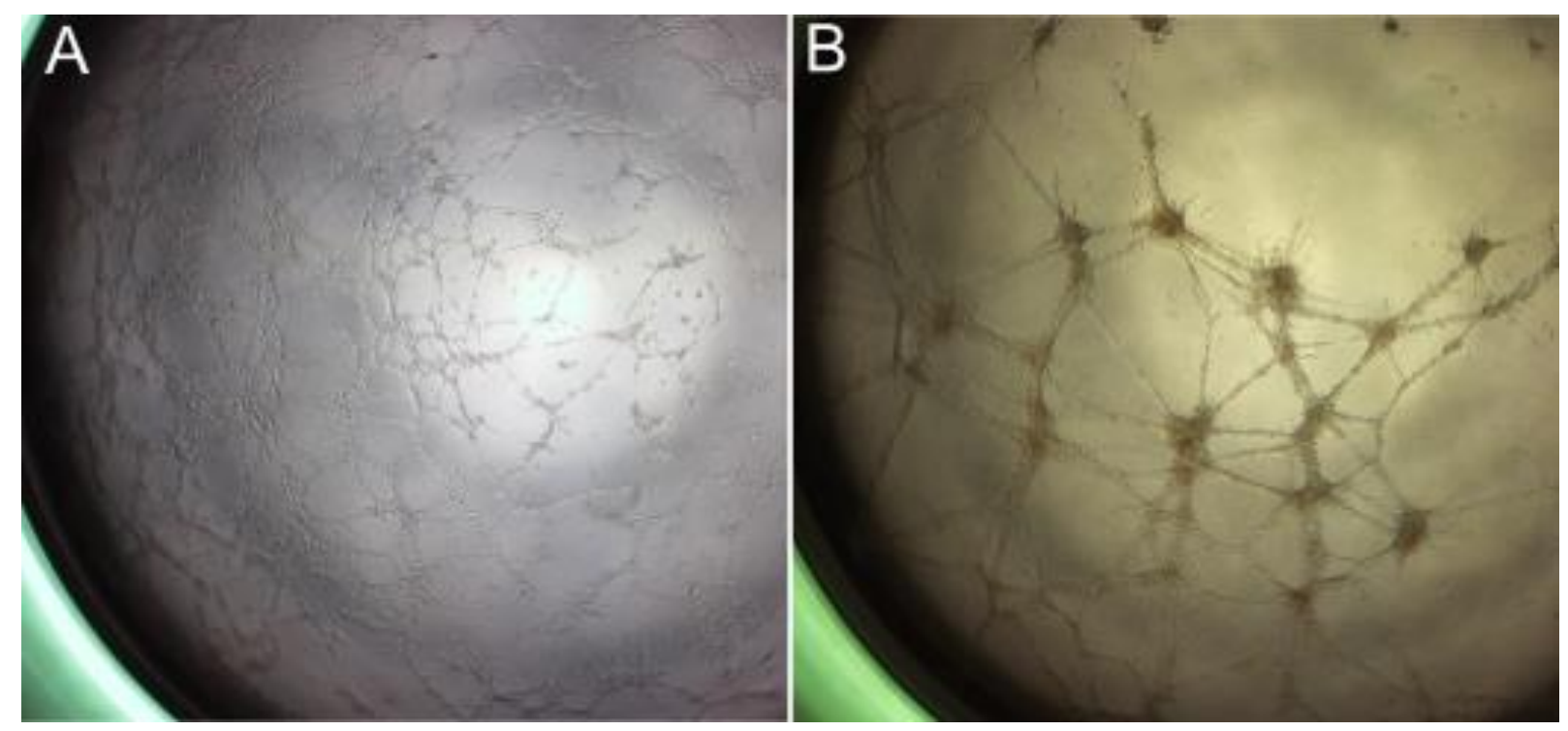

Figure A.7.2. Comparison of two separate experimental replicates of un-transfected U87MG cultures under hypoxic conditions during the Matrigel tube formation assay. Cells were normalised to a fixed seeding cell density. 


\section{A.8 Other correlations of Apelin and APLNR}

During this thesis, several other in silico analyses of public data was performed looking for correlations between apelin, APLNR and various molecular markers such as MGMT status, IDH status and statistics such as patient survival. An example of this is the Kaplan-Meier survival analysis shown in Figure A.8.1. The graph stratifies patients from the TCGA_GBM data set by high and low apelin expression, as defined by the median expression, and plots this against survival time. This shows no statistically significant evidence of apelin expression correlating with increased or decreased survival (Figure A.8.1). This also includes, when stratifying, for different subtypes and APLNR expression.

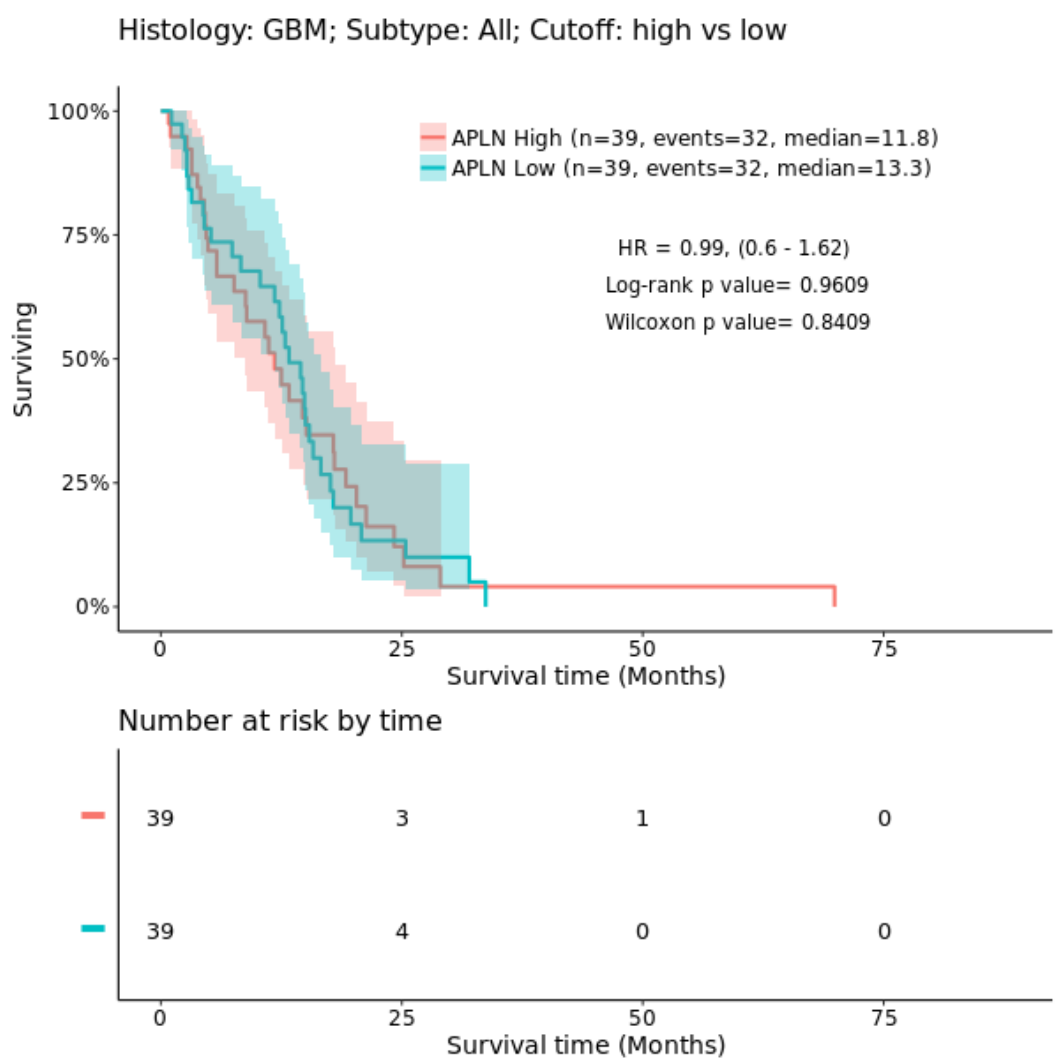

Figure A.8.1. A representative example of a Kaplan-Meier survival plots derived from GlioVis using the TCGA_GBM dataset. Kaplan-Meier survival analysis is plotted using a high vs low expression of apelin with no stratifying for the subtype. The confidence intervals are overlaid over the survival plots, and all subtypes and plotted numbers are tabulated below. 


\section{A.9 Delta cycle threshold ( $\triangle \mathrm{CT})$ statistical analysis of qPCR data}

\section{A.9.1 Testing normality}

During Chapter 5, Student's t-tests were used to determine if the difference in gene expression between treated and untreated groups was significant. A key assumption of the t-test is normality. To assess normality, the delta cycle threshold $(\Delta \mathrm{CT})$ was calculated for each target gene with each treatment group. This was calculated by subtracting the housekeeping gene cycle threshold (CT) from the target gene $\mathrm{CT}$. i.e. $\triangle \mathrm{CT}=$ target gene $\mathrm{CT}$ - housekeeping gene CT. The assumption of normality was tested using the D'Agostino \& Pearson omnibus normality test Table A.9.1. Normality tests are greatly skewed by low sample sizes (i.e. $<30$ ) therefore, data were also graphed using a scatterplot to assess the symmetry of expression (Figure A.9.1) and compared to the normality test results in Table A.9.1 to further assess if data sets were normal. This section provides a summary of the $\triangle \mathrm{CT}$ comparisons of RT-qPCR data from Chapter 5.

Table A.9.1. A table summarising the normality of each gene for each treatment group. Yes or no refers to if the data set passed normality test (alpha $=0.05)$.

\begin{tabular}{lllll}
\hline \multicolumn{4}{c}{ Summary of D'Agostino \& Pearson } & omnibus normality test results \\
\hline & APLN & APLNR & SLC2A1 & PROM1 \\
Hypoxia & Yes & No & Yes & Yes \\
Hypoglycaemia & Yes & Yes & Yes & Yes \\
Hypoxia + hypoglycaemia & Yes & No & Yes & Yes \\
\hline
\end{tabular}



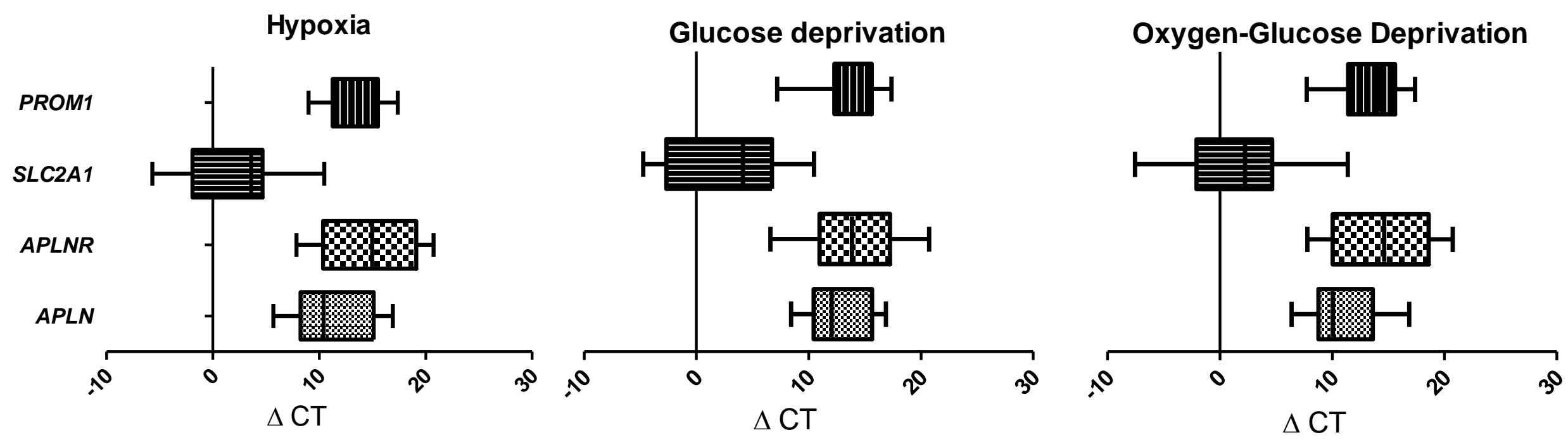

Figure A.9.1. Comparing the spread of gene expression to assess normality. Each delta cycle threshold $(\Delta \mathrm{CT})$ was calculated by subtracting the housekeeping gene cycle threshold (CT) from the target gene cycle threshold. Within each gene, the treated and untreated $\Delta \mathrm{CT}$ values were concatenated and the data presented as a boxplot. 


\section{Appendix B Recipes \& Reagents}

\section{B.1 LB and Agar plates}

\section{B.1.1 Lysogeny Broth (LB)}

The Miller's variant of LB was made for all routine culturing using DH5 $\alpha$ E.coli.

10 g Tryptone

$5 \mathrm{~g}$ Yeast extract

$10 \mathrm{~g} \mathrm{NaCl}$

Distilled water to $800 \mathrm{~mL}$. Dissolve solids in the water and $\mathrm{pH}$ LB to 7.6.

Adjust volume to 1L.

\section{B.1.2 LB-Agar}

$500 \mathrm{~mL}$ of the above LB was added to a $1 \mathrm{~L}$ Schott bottle and to this, $7.5 \mathrm{~g}$ of agar (Oxoid, Thermofisher Scientific, Auckland, NZ) was added. The suspension was then autoclaved to allow for the complete dissolving of agar. If required, ampicillin was added at a final concentration of $100 \mu \mathrm{g} / \mathrm{mL}$ once the agar was able to be handled.

\section{B.1.3 TAE agarose gels}

PCR products were visualised on 1-3\% agarose gel prepared using 1x Tris, acetic acid EDTA (TAE) buffer containing $0.1 \% \mathrm{EtBr}$. 


\section{B.1.4 50x TAE Stock Solution of TAE Buffer}

Tris base: $\quad 242 \mathrm{~g}$

Glacial Acetic Acid: $\quad 57.1 \mathrm{~mL}$

$0.5 \mathrm{M}$ EDTA $\quad 100 \mathrm{~mL}$

Add ddH2O to 1 Litre and adjust $\mathrm{pH}$ to 8.5 using $\mathrm{NaOH}$.

\section{B.1.5 Super optimal broth with Catabolite repression (SOC) medium}

$\begin{array}{lll}\text { Tryptone } & 2 \%(\mathrm{w} / \mathrm{v}) & 20 \mathrm{~g} \\ \text { Yeast extract } & 0.5 \%(\mathrm{w} / \mathrm{v}) & 5 \mathrm{~g} \\ \mathrm{NaCl} & 10 \mathrm{mM} & 0.584 \mathrm{~g} \\ \mathrm{KCl} & 2.5 \mathrm{mM} & 0.186 \mathrm{~g} \\ \mathrm{MgCl}_{2} & 10 \mathrm{mM} & 0.951 \mathrm{~g} \\ \text { Glucose } & 20 \mathrm{mM} & 3.603 \mathrm{~g} \\ \text { Made up in 1L ddH20, pH to } 7.0 . & & \end{array}$




\section{B.1.6 Preparation and transformation of electrocompetent cells}

For the generation of the truncated APLNR construct, the Dh5 $\alpha$ E.coli strain was made electrocompetent. A $3 \mathrm{~mL}$ culture was inoculated with Dh5a E.coli from a glycerol stock and grown overnight. The following day, $50 \mathrm{~mL}$ of $\mathrm{LB}$ supplemented with $10 \mathrm{mM} \mathrm{MgCl} 2$ was inoculated with $100 \mu \mathrm{L}$ from the overnight culture and allowed to grow at $37{ }^{\circ} \mathrm{C}, 200 \mathrm{rev} / \mathrm{min}$ until an $\mathrm{OD}_{600}$ of $0.35-0.4$ was achieved. Cells were cooled on ice for $15 \mathrm{~min}$ to halt cell growth after which cultures were transferred to $50 \mathrm{~mL}$ Falcon tubes and centrifuged at 2700 $\mathrm{RCF}$ for $15 \mathrm{~min}$ at $4{ }^{\circ} \mathrm{C}$. Following this, all work was done on ice. The cells were then resuspended gently in $50 \mathrm{~mL}$ of ice-cold, sterile $\mathrm{ddH}_{2} \mathrm{O}$ after which the cells were pelleted via centrifugation (2700 RCF for 15 min at $4{ }^{\circ} \mathrm{C}$ ). Cells were resuspended in $25 \mathrm{~mL}$ of ice-cold $10 \%$ glycerol and pelleted again via centrifugation. Finally, cells were washed with $10 \mathrm{~mL}$ of ice-cold $10 \%$ glycerol and pelleted by centrifugation. The supernatant was removed and the pellet resuspended in a minimal amount $(300-500 \mu \mathrm{L})$ of ice-cold sterile $10 \%$ glycerol. The $\mathrm{OD}_{600}$ of a $1 / 100$ dilution was then measured $\left(\mathrm{OD}_{600}\right.$ of $1.0=5 \times 10^{8}$ cells $\left./ \mathrm{mL}\right)$ and the final concentration of cells adjusted to $1.5-2.0 \times 10^{10}$ cells $/ \mathrm{ml}$ and aliquoted in $60 \mu \mathrm{L}$ aliquots in pre-chilled $1.5 \mathrm{~mL}$ microcentrifuge tubes. Cell aliquots were snap frozen in a metal tube block pre-chilled to $-80^{\circ} \mathrm{C}$ and stored at $-80^{\circ} \mathrm{C}$ until needed.

\section{B.1.7 Transformation of electrocompetent cells}

Electrocompetent cell aliquots were removed from $-80{ }^{\circ} \mathrm{C}$ storage and thawed on ice. Once thawed, 10-50 ng of plasmid DNA was added to the cells. Cells and DNA were then gently mixed by tapping and transferred to a pre-chilled, sterile $2 \mathrm{~mm}$ gap electroporation cuvette. Cells were then electroporated at $2.5 \mathrm{kV}, 25 \mu \mathrm{F}, 100 \Omega$ using a Gene Pulser Xcell Electroporation System (Bio-Rad, CA, USA), following which $900 \mu \mathrm{L}$ of room temperature SOC media was immediately added to the cuvette and gently inverted to mix. Cells were incubated at $37^{\circ} \mathrm{C}, 200 \mathrm{rev} / \mathrm{min}$ for $60 \mathrm{~min}$ to allow cell recovery. Finally, cells were plated on LB agar plates containing $100 \mu \mathrm{g} / \mathrm{mL}$ ampicillin. $100 \mu \mathrm{l}$ of undiluted, as well as $1 / 10$ and $1 / 100$ dilutions of cells, were plated initially to determine the optimal dilution for single colonies to 
be obtained on agar plates. The remainder of the transformation was mixed in a 1:1 ratio with sterile $80 \%$ glycerol and stored at $-80{ }^{\circ} \mathrm{C}$ until needed.

\section{B.1.8 Alkaline lysis buffer reagents}

\section{B.1.8.1 Alkaline Lysis Buffer I (Aka GTE buffer)}

$\begin{array}{ll}\text { Glucose } & 50 \mathrm{mM} \\ \text { Tris, } \mathrm{pH} 8.0 & 25 \mathrm{mM} \\ \text { EDTA, pH } 8.0 & 10 \mathrm{mM}\end{array}$

\section{B.1.8.2 Alkaline Lysis Buffer II}

Alkaline lysis buffer II is used at a final concentration of $0.2 \mathrm{M} \mathrm{NaOH}, 1 \%$ sodium dodecyl sulphate (SDS). Buffer II is prepared fresh before use with stock solutions of $3 \mathrm{M} \mathrm{NaOH}$ and $10 \%$ SDS. The buffer was not chilled after preparation to prevent precipitation of the SDS.

\section{B.1.8.3 Alkaline Lysis buffer III (5 M potassium acetate)}

Prepare $60 \mathrm{~mL}$ of $5 \mathrm{M}$ potassium acetate. Add $11.5 \mathrm{~mL}$ of glacial acetic acid and $28.5 \mathrm{~mL}$ of deionised water. This solution will be $3 \mathrm{M}$ with respect to potassium and $5 \mathrm{M}$ with respect to acetate. Store at $4^{\circ} \mathrm{C}$.

\section{B.1.9 Alkaline Lysis Miniprep protocol (from Sambrook et al. 3rd edition)}

\section{B.1.9.1 Alkaline lysis procedure}

The $\mathrm{O} / \mathrm{N}$ (Overnight) culture $(1.5 \mathrm{~mL})$ was centrifuged in a $2 \mathrm{~mL}$ microcentrifuge tube at 16,000 RCF for $1 \mathrm{~min}$. The supernatant was completely aspirated. The pellet was resuspended in STE (sodium Tris EDTA) buffer: $0.1 \mathrm{M} \mathrm{NaCl}, 10 \mathrm{mM}$ Tris-HCl (pH 8.0), 1 mM EDTA (pH 8.0 ) at $0.25 \mathrm{x}$ original culture volume and centrifuged at $16,000 \mathrm{RCF}$ for $1 \mathrm{~min}$ and the supernatant aspirated. 
The cell pellet was resuspended in $100 \mu \mathrm{L}$ of GTE buffer $(50 \mathrm{mM}$ glucose, $25 \mathrm{mM}$ Tris-Cl, and $10 \mathrm{mM}$ EDTA, pH 8) with vigorous vortexing if required. After $5 \mathrm{~min}, 200 \mu \mathrm{L}$ of lysis solution $(0.2 \mathrm{M} \mathrm{NaOH}, 1 \% \mathrm{SDS})$ was added, and the tube was inverted to mix. The contents were then placed on ice and immediately $150 \mu \mathrm{L}$ of $5 \mathrm{M}$ potassium acetate solution ( $\mathrm{pH} 4.8$ ) was added. The tube was stored on ice for $5 \mathrm{~min}$, then centrifuged at 16,000 RCF for $5 \mathrm{~min}$ at $4^{\circ} \mathrm{C}$. The supernatant was transferred to a new tube. The nucleic acids were then precipitated with $0.5 \mathrm{~mL}$ of cold isopropanol for $10 \mathrm{~min}$ and centrifuged at $13,000 \mathrm{RCF}$ for $5 \mathrm{~min}$ at $4{ }^{\circ} \mathrm{C}$. The isopropanol supernatant was aspirated off, and cold 70\% EtOH (1 mL) was slowly added down the side of the tube to the pellet and left for $3 \mathrm{~min}$, followed by centrifugation at 13,000 rpm for $5 \mathrm{~min}$ at $4^{\circ} \mathrm{C}$. The ethanol step was repeated. All the ethanol was aspirated and the DNA air dried. The pellet was then dissolved in $50 \mu \mathrm{L}$ of TE buffer $(10 \mathrm{mM}$ Tris-Cl, $1 \mathrm{mM}$ EDTA, $\mathrm{pH}$ 7.5).

\section{B.1.9.2 RNAse treatment and phenol-chloroform-isoamyl alcohol (PCIA) extraction}

If RNA removal was required, an RNAse treatment was performed followed by a PCIA extraction. After the DNA was precipitated as above using isopropanol, the pellet was dissolved in $0.4 \mathrm{~mL}$ of TE buffer. RNAse A solution was added and the solution incubated at $37^{\circ} \mathrm{C}$ for 20 to 30 min to digest the remaining RNA. Phenol: chloroform: isoamyl alcohol (25:24:1) (400 $\mu \mathrm{L}$ ) was added and the mixture vortexed vigorously for $30 \mathrm{sec}$, then centrifuged at 13,000 rpm for $5 \mathrm{~min}$ at $4^{\circ} \mathrm{C}$. The phenol was buffered at $\mathrm{pH} 7.0$ before use with Tris buffer. The supernatant was then removed and transferred to a new microcentrifuge tube. Chloroform: isoamyl alcohol $(0.5 \mathrm{~mL})$ was added to the aqueous phase to remove any residual phenol. The solution was vortexed briefly ( $5 \mathrm{~s}$ ), then centrifuged at $13,000 \mathrm{rpm}$ for $5 \mathrm{~min}$ at $4{ }^{\circ} \mathrm{C}$ and the supernatant transferred to a fresh tube.

A 1/10th volume of $3 \mathrm{M}$ sodium acetate ( $\mathrm{pH}$ 5.5) was added with $1 \mathrm{~mL}$ of ice-cold absolute ethanol to precipitate the plasmid DNA. The tube was left on ice for $10 \mathrm{~min}$, then centrifuged at $13,000 \mathrm{rpm}$ for $4 \mathrm{~min}$ at $4^{\circ} \mathrm{C}$. All the ethanol was removed, then $1 \mathrm{~mL} 70 \%$ ethanol was added down the side of the tube to the pellet and left for 3 minutes, followed by centrifugation 
at 13,000 rpm for $4 \mathrm{~min}$ at $4^{\circ} \mathrm{C}$. The ethanol was removed, and the pellet was air dried. The pellet was then resuspended in $20-100 \mu \mathrm{L}$ of TE buffer.

\section{B.1.10 1 x Phosphate Buffered Saline (PBS) pH 7.4}

$\begin{array}{lll}\mathrm{NaCl} & 137 \mathrm{mM} & 8 \mathrm{~g} / \mathrm{L} \\ \mathrm{Na}_{2} \mathrm{HPO}_{4} & 10.1 & 1.42 \mathrm{~g} / \mathrm{L} \\ \mathrm{KCl} & 1.6 \mathrm{mM} & 0.2 \mathrm{~g} / \mathrm{L} \\ \mathrm{KH}_{2} \mathrm{PO}_{4} & 1.8 \mathrm{mM} & 0.24 \mathrm{~g} / \mathrm{L}\end{array}$

Made up in $1 \mathrm{~L}$ ddH20, $\mathrm{pH}$ to 7.4 .

PBS was made by dissolving the salts in $800 \mathrm{ml}$ of $\mathrm{ddH}_{2} \mathrm{O}$, adjusting the $\mathrm{pH}$ to 7.4 using concentrated $\mathrm{HCl}$ adjusted to $1 \mathrm{~L}$ then sterilising by autoclaving. If required 10x PBS was made up which followed the same procedure however utilised 10x of each reagent.

\section{B.1.11 TE (Tris-EDTA) buffer $(100 \mathrm{~mL})$}

$\begin{array}{lll}\text { EDTA } & 1 \mathrm{mM} & 0.2 \mathrm{~mL} \text { of } 0.5 \mathrm{M} \text { EDTA } \\ \text { Tris base } & 10 \mathrm{mM} & 1 \mathrm{~mL} \text { of } 1 \mathrm{M} \text { Tris base } \\ \text { Made up in } & 100 \mathrm{~mL} \text { with ddH20, } \mathrm{pH} \text { to } 8.0 .\end{array}$

\section{B.1.12 1x Tris buffered saline (TBS)}

$\begin{array}{lll}\mathrm{NaCl} & 150 \mathrm{mM} & 8.76 \mathrm{~g} / \mathrm{L} \\ \text { Tris base } & 50 \mathrm{mM} & 6.0 \mathrm{~g} / \mathrm{L} \\ \text { Made up in } & 1 \mathrm{~L} \text { ddH20, } \mathrm{pH} \text { to } 7.4 .\end{array}$

TBS was made by dissolving the salts in $800 \mathrm{~mL}$ of $\mathrm{ddH}_{2} \mathrm{O}$, adjusting the $\mathrm{pH}$ to 7.6 using concentrated $\mathrm{HCl}$ adjusted to $1 \mathrm{~L}$ then sterilised by autoclaving.

\section{B.1.13 1x TBST}

Add $1 \mathrm{~mL}$ of Tween 20 to $1 \mathrm{~L}$ of $1 \mathrm{x}$ TBS 


\section{B.1.14 Stripping buffer}

$\begin{array}{ll}\text { SDS } & 20 \mathrm{~g} / \mathrm{L} \\ 0.05 \text { M Tris, pH 6.8 } & 992.5 \mathrm{~mL} / 1 \mathrm{~L} \\ \beta \text {-mercaptoethanol } & 7.5 \mathrm{~mL}\end{array}$

\section{B.1.15 0.5 M Na2EDTA}

$\mathrm{Na}_{2}$ EDTA $\quad 186.12 \mathrm{~g}$

EDTA salt was added to $700 \mathrm{ml}$ of $\mathrm{ddH}_{2} \mathrm{O}$ slowly under constant stirring with the addition of $\mathrm{NaOH}$ pellets to ensure the $\mathrm{pH}$ was alkaline. The $\mathrm{pH}$ was checked to be at 8.0 and adjusted; it was then aliquoted and sterilised by autoclaving.

\section{B.1.16 Western transfer buffer}

$\begin{array}{lc}\text { Tris } & 3.03 \mathrm{~g} / \mathrm{L} \\ \text { Glycine } & 14.4 \mathrm{~g} / \mathrm{L} \\ \text { SDS } & 0.38 \mathrm{~g} / \mathrm{L} \\ \text { Methanol } & 200 \mathrm{~mL} \\ \text { Made up fresh prior to transfer }\end{array}$

\section{B.1.17 5x Running Buffer}

$\begin{array}{ll}\text { Tris } & 15.0 \mathrm{~g} / \mathrm{L} \\ \text { Glycine } & 72 \mathrm{~g} / \mathrm{L} \\ \text { SDS } & 5 \mathrm{~g} / \mathrm{L}\end{array}$

Made up to $1 \mathrm{~L}$ with ddH20

\section{B.1.18 RIPA buffer}

$\begin{array}{ll}\text { Tris } & 1.2 \mathrm{~g} / \mathrm{L} \\ \mathrm{NaCl} & 8.766 \mathrm{~g} / \mathrm{L} \\ \text { EDTA } & 0.37 \mathrm{~g} / \mathrm{L} \\ \text { Triton-X-100 1\% } & 10 \mathrm{~mL} \\ \text { SDS } & 1.0 \mathrm{~g} / \mathrm{L} \\ \text { Sodium Deoxycholate } & 10 \mathrm{~g} / \mathrm{L} \\ \text { Made up to } 800 \mathrm{ml} \mathrm{pH} \text { to } 7.5 \text { and aliquoted. }\end{array}$




\section{B.1.19 5x Reducing Buffer}

$\begin{array}{ll}1.5 \mathrm{M} \text { Tris pH } 6.8 & 1 \mathrm{~mL} \\ 10 \% \text { SDS } & 5 \mathrm{~mL} \\ 80 \% \text { Glycerol } & 3.2 \mathrm{~mL} \\ 1 \% \text { Bromophenol Blue } & 0.8 \mathrm{~mL}\end{array}$

\section{B.1.20 10\% SDS (100 mL)}

Dissolve $10 \mathrm{~g}$ of SDS into $80 \mathrm{~mL}$ of $\mathrm{ddH}_{2} \mathrm{O}$, heat to $68^{\circ} \mathrm{C}$ to dissolve under constant stirring. Then add $\mathrm{ddH}_{2} \mathrm{O}$ to $100 \mathrm{~mL}$.

\section{B.1.21 $10 \%$ separating gel ( 2 gels)}

$1.5 \mathrm{M}$ Tris $\mathrm{pH} 8.8$

$10 \%$ SDS

N,N,N," -tetramethylethylenediamine

(TEMED)

$30 \%$ acrylamide/bis-acrylamide

$10 \%$ ammonium persulfate (APS)

$\mathrm{ddH}_{2} \mathrm{O}$
$5 \mathrm{~mL}$ $200 \mu \mathrm{L}$ $10 \mu \mathrm{L}$

$6.66 \mathrm{~mL}$ $100 \mu \mathrm{L}$ $8 \mathrm{~mL}$

\section{B.1.22 4\% stacking gel (2 gels)}

$\begin{array}{ll}0.5 \mathrm{M} \text { Tris pH } 6.8 & 2.5 \mathrm{~mL} \\ \text { 10\% SDS } & 100 \mu \mathrm{L} \\ \text { N,N,N," - tetramethylethylenediamine } & 10 \mu \mathrm{L} \\ \text { (TEMED) } & \\ \text { 30\% acrylamide/bis-acrylamide } & 1.33 \mathrm{~mL} \\ \text { 10\% ammonium persulfate (APS) } & 50 \mu \mathrm{L}\end{array}$




\section{Bibliography}

[1] Berta J, Kenessey I, Dobos J, et al. Apelin Expression in Human Non-small Cell Lung Cancer. J Thorac Oncol 2010; 5: 1120-1129.

[2] Kälin RE, Kretz MP, Meyer AM, et al. Paracrine and autocrine mechanisms of apelin signaling govern embryonic and tumor angiogenesis. Dev Biol 2007; 305: 599-614.

[3] Sorli SC, Le Gonidec S, Knibiehler B, et al. Apelin is a potent activator of tumour neoangiogenesis. Oncogene 2007; 26: 7692-7699.

[4] Berta J, Hoda MA, Laszlo V, et al. Apelin promotes lymphangiogenesis and lymph node metastasis. Oncotarget 2014; 5: 4426-37.

[5] Lv D, Li L, Lu Q, et al. PAK1-cofilin phosphorylation mediates human lung adenocarcinoma cells migration induced by apelin-13. Clin Exp Pharmacol Physiol 2016; 43: 569-579.

[6] Heo K, Kim YH, Sung HJ, et al. Hypoxia-induced up-regulation of apelin is associated with a poor prognosis in oral squamous cell carcinoma patients. Oral Oncol 2012; 48: 500-506.

[7] Feng M, Yao G, Yu H, et al. Tumor apelin, not serum apelin, is associated with the clinical features and prognosis of gastric cancer. BMC Cancer 2016; 16: 794.

[8] Kälin S, Kälin RE. Apelin and Cancer. In: Reizes O, Berger NA (eds) Adipocytokines, Energy Balance, and Cancer. Springer International Publishing, pp. 137-160.

[9] Yang Y, Lv SY, Ye W, et al. Apelin/APJ system and cancer. Clin Chim Acta 2016; 457: 112-116.

[10] Ciumas M, Montagne K, Agrapart M, et al. Hypoxia-Induced Apelin Expression Regulates Endothelial Cell Proliferation and Regenerative Angiogenesis Me. Circ Res 2008; 103: 432-440.

[11] Tatemoto K, Hosoya M, Habata Y, et al. Isolation and characterization of a novel endogenous peptide ligand for the human APJ receptor. Biochem Biophys Res Commun 1998; 251: 471-6.

[12] Shin K, Kenward C, Rainey JK. Apelinergic System Structure and Function. Compr Physiol 2017; 8: 407-450.

[13] Bertrand C, Valet P, Castan-Laurell I. Apelin and energy metabolism. Front Physiol 2015; 6: 1-5. 
[14] Roberts EM, Newson MJF, Pope GR, et al. Abnormal fluid homeostasis in apelin receptor knockout mice. J Endocrinol 2009; 202: 453-462.

[15] O'Carroll A-M, Lolait SJ, Harris LE, et al. The apelin receptor APJ: journey from an orphan to a multifaceted regulator of homeostasis. J Endocrinol 2013; 219: R13-R35.

[16] Falcão-Pires I, Ladeiras-Lopes R, Leite-Moreira AF. The apelinergic system: a promising therapeutic target. Expert Opin Ther Targets 2010; 14: 633-45.

[17] Narayanan S, Harris D, Maitra R, et al. Regulation of the Apelinergic System and Its Potential in Cardiovascular Disease: Peptides and Small Molecules as Tools for Discovery. J Med Chem 2015; 58: 7913-7927.

[18] Kalea AZ, Batlle D. Apelin and ACE2 in cardiovascular disease. Curr Opin Investig Drugs 2010; 11: 273-82.

[19] Kidoya H, Naito H, Takakura N. Apelin induces enlarged and nonleaky blood vessels for functional recovery from ischemia. Blood 2010; 115: 3166-74.

[20] Castan-Laurell I, Dray C, Attané C, et al. Apelin, diabetes, and obesity. Endocrine 2011; 40: 1-9.

[21] Yue P, Jin H, Aillaud M, et al. Apelin is necessary for the maintenance of insulin sensitivity. Am J Physiol Endocrinol Metab 2010; 298: E59-67.

[22] Attane C, Foussal C, Le Gonidec S, et al. Apelin Treatment Increases Complete Fatty Acid Oxidation, Mitochondrial Oxidative Capacity, and Biogenesis in Muscle of Insulin-Resistant Mice. Diabetes 2012; 61: 310-320.

[23] Daviaud D, Boucher J, Gesta S, et al. TNFalpha up-regulates apelin expression in human and mouse adipose tissue. FASEB J 2006; 20: 1528-30.

[24] Dray C, Knauf C, Daviaud D, et al. Apelin stimulates glucose utilization in normal and obese insulin-resistant mice. Cell Metab 2008; 8: 437-45.

[25] Zhu S, Sun F, Li W, et al. Apelin stimulates glucose uptake through the PI3K/Akt pathway and improves insulin resistance in 3T3-L1 adipocytes. Mol Cell Biochem 2011; 353: 305-313.

[26] Xu S, Han P, Huang M, et al. In vivo, ex vivo, and in vitro studies on apelin's effect on myocardial glucose uptake. Peptides 2012; 37: 320-6.

[27] Yue P, Jin H, Xu S, et al. Apelin decreases lipolysis via G(q), G(i), and AMPKDependent Mechanisms. Endocrinology 2011; 152: 59-68.

[28] Han S, Wang G, Qi X, et al. A possible role for hypoxia-induced apelin expression in enteric cell proliferation. Am J Physiol Regul Integr Comp Physiol 2008; 294: R18329. 
[29] Azizi M, Iturrioz X, Blanchard A, et al. Reciprocal regulation of plasma apelin and vasopressin by osmotic stimuli. J Am Soc Nephrol 2008; 19: 1015-1024.

[30] Piairo P, Moura RS, Nogueira-Silva C, et al. The apelinergic system in the developing lung: Expression and signaling. Peptides 2011; 32: 2474-2483.

[31] Chun HJ, Ali Z a., Kojima Y, et al. Apelin signaling antagonizes Ang II effects in mouse models of atherosclerosis. J Clin Invest 2008; 118: 3343-3354.

[32] Yu QC, Hirst CE, Costa M, et al. APELIN promotes hematopoiesis from human embryonic stem cells. Blood 2012; 119: 6243-6254.

[33] Chng S, Ho L, Tian J, et al. ELABELA: A hormone essential for heart development signals via the apelin receptor. Dev Cell 2013; 27: 672-680.

[34] Aniello CD, Lonardo E, Iaconis S, et al. G Protein-Coupled Receptor APJ and Its Ligand Apelin Act Downstream of Cripto to Specify Embryonic Stem Cells Toward the Cardiac Lineage Through Extracellular Signal-Regulated Kinase/p70S6 Kinase Signaling Pathway. Circ Res 2009; 105: 231-238.

[35] D'Aniello C, Fiorenzano A, Iaconis S, et al. The G-protein-coupled receptor APJ is expressed in the second heart field and regulates Cerberus-Baf60c axis in embryonic stem cell cardiomyogenesis. Cardiovasc Res 2013; 100: 95-104.

[36] Kurowska P, Barbe A, Różycka M, et al. Apelin in Reproductive Physiology and Pathology of Different Species: A Critical Review. Int J Endocrinol 2018; 2018: 1-12.

[37] Roche J, Rame C, Reverchon M, et al. Apelin (APLN) and Apelin Receptor (APLNR) in Human Ovary: Expression, Signaling, and Regulation of Steroidogenesis in Primary Human Luteinized Granulosa Cells. Biol Reprod 2016; 95: 104-104.

[38] O'Dowd BF, Heiber M, Chan A, et al. A human gene that shows identity with the gene encoding the angiotensin receptor is located on chromosome 11. Gene 1993; 136: 35560 .

[39] Devic E, Rizzoti K, Bodin S, et al. Amino acid sequence and embryonic expression of msr/apj, the mouse homolog of Xenopus X-msr and human APJ. Mech Dev 1999; 84: 199-203.

[40] Pauli A, Norris ML, Valen E, et al. Toddler: an embryonic signal that promotes cell movement via Apelin receptors. Science 2014; 343: 1248636.

[41] Pope GR, Roberts EM, Lolait SJ, et al. Central and peripheral apelin receptor distribution in the mouse: Species differences with rat. Peptides 2012; 33: 139-148.

[42] Medhurst AD, Jennings CA, Robbins MJ, et al. Pharmacological and immunohistochemical characterization of the APJ receptor and its endogenous ligand apelin. J Neurochem 2003; 84: 1162-1172. 
[43] Matsumoto M, Hidaka K, Akiho H, et al. Low stringency hybridization study of the dopamine D4 receptor revealed D4-like mRNA distribution of the orphan seventransmembrane receptor, APJ, in human brain. Neurosci Lett 1996; 219: 119-122.

[44] Edinger AL, Hoffman TL, Sharron M, et al. An orphan seven-transmembrane domain receptor expressed widely in the brain functions as a coreceptor for human immunodeficiency virus type 1 and simian immunodeficiency virus. J Virol 1998; 72: 7934-40.

[45] O'Carroll AM, Selby TL, Palkovits M, et al. Distribution of mRNA encoding B78/apj, the rat homologue of the human APJ receptor, and its endogenous ligand apelin in brain and peripheral tissues. Biochim Biophys Acta 2000; 1492: 72-80.

[46] Zeng XJ, Yu SP, Zhang L, et al. Neuroprotective effect of the endogenous neural peptide apelin in cultured mouse cortical neurons. Exp Cell Res 2010; 316: 1773-83.

[47] Hazell GGJ, Hindmarch CC, Pope GR, et al. G protein-coupled receptors in the hypothalamic paraventricular and supraoptic nuclei - serpentine gateways to neuroendocrine homeostasis. Front Neuroendocrinol 2012; 33: 45-66.

[48] O’Donnell L a, Agrawal A, Sabnekar P, et al. Apelin, an endogenous neuronal peptide, protects hippocampal neurons against excitotoxic injury. J Neurochem 2007; 102: 1905-17.

[49] Lee DK, Lanca AJ, Cheng R, et al. Agonist-independent Nuclear Localization of the Apelin, Angiotensin AT 1, and Bradykinin B2 Receptors. J Biol Chem 2004; 279: 7901-7908.

[50] Puffer B, Sharron M, Coughlan CM, et al. Expression and coreceptor function of APJ for primate immunodeficiency viruses. Virology 2000; 276: 435-444.

[51] Choe W, Albright A, Sulcove J, et al. Functional expression of the seventransmembrane HIV-1 co-receptor APJ in neural cells. J Neurovirol 2000; 6 Suppl 1: S61-9.

[52] Bai B, Cai X, Jiang Y, et al. Heterodimerization of apelin receptor and neurotensin receptor 1 induces phosphorylation of ERK $1 / 2$ and cell proliferation via Gaqmediated mechanism. J Cell Mol Med 2014; 18: 2071-2081.

[53] Loot AE, Fleming I. A Novel APJ Signaling Cascade That Regulates Cardiovascular Development. Circ Res 2013; 113: 4-6.

[54] Hosoya M, Kawamata Y, Fukusumi S, et al. Molecular and functional characteristics of APJ: Tissue distribution of mRNA and interaction with the endogenous ligand apelin. J Biol Chem 2000; 275: 21061-21067.

[55] Masri B, Lahlou H, Mazarguil H, et al. Apelin (65-77) activates extracellular signalregulated kinases via a PTX-sensitive G protein. Biochem Biophys Res Commun 2002; 
290: 539-45.

[56] Szokodi I, Tavi P, Földes G, et al. Apelin, the novel endogenous ligand of the orphan receptor APJ, regulates cardiac contractility. Circ Res 2002; 91: 434-440.

[57] Kang Y, Kim J-D, Anderson JP, et al. Apelin-APJ Signaling Is a Critical Regulator of Endothelial MEF2 Activation in Cardiovascular Development. Circ Res 2013; 113 : 22-31.

[58] Chaves-Almagro C, Castan-Laurell I, Dray C, et al. Apelin receptors: From signaling to antidiabetic strategy. Eur J Pharmacol 2015; 763: 1-11.

[59] Lee DK, Ferguson SSG, George SR, et al. The fate of the internalized apelin receptor is determined by different isoforms of apelin mediating differential interaction with beta-arrestin. Biochem Biophys Res Commun 2010; 395: 185-189.

[60] Bai B, Tang J, Liu H, et al. Apelin-13 induces ERK1-2 but not p38 MAPK activation through coupling of the human apelin receptor to the Gi2 pathway. Acta Biochim Biophys Sin (Shanghai) 2008; 40: 311-318.

[61] Sun X, Iida S, Yoshikawa A, et al. Non-activated APJ suppresses the angiotensin II type 1 receptor, whereas apelin-activated APJ acts conversely. Hypertens Res 2011; 34: 701-6.

[62] Siddiquee K, Hampton J, McAnally D, et al. The apelin receptor inhibits the angiotensin II type 1 receptor via allosteric trans-inhibition. Br J Pharmacol 2013; 168: 1104-17.

[63] Ishida J, Hashimoto T, Hashimoto Y, et al. Regulatory roles for APJ, a seventransmembrane receptor related to angiotensin-type 1 receptor in blood pressure in vivo. J Biol Chem 2004; 279: 26274-26279.

[64] Li Y, Chen J, Bai B, et al. Heterodimerization of human apelin and kappa opioid receptors: roles in signal transduction. Cell Signal 2012; 24: 991-1001.

[65] Sato T, Suzuki T, Watanabe H, et al. Apelin is a positive regulator of ace2 in failing hearts. J Clin Invest 2013; 123: 5203-5211.

[66] Kidoya H, Ueno M, Yamada Y, et al. Spatial and temporal role of the apelin/APJ system in the caliber size regulation of blood vessels during angiogenesis. EMBO J 2008; 27: 522-534.

[67] Pitkin SL, Maguire JJ, Bonner TI, et al. International Union of Basic and Clinical Pharmacology. LXXVIII. Lysophospholipid receptor nomenclature. Pharmacol Rev 2010; 62: 331-342.

[68] Shin K, Pandey A, Liu XQ, et al. Preferential apelin-13 production by the proprotein convertase PCSK3 is implicated in obesity. FEBS Open Bio 2013; 3: 328-333. 
[69] Masri B, Knibiehler B, Audigier Y. Apelin signalling: A promising pathway from cloning to pharmacology. Cell Signal 2005; 17: 415-426.

[70] Habata Y, Fujii R, Hosoya M, et al. Apelin, the natural ligand of the orphan receptor APJ, is abundantly secreted in the colostrum. Biochim Biophys Acta - Mol Cell Res 1999; 1452: 25-35.

[71] Lee DL, Cheng R, Nguyen T, et al. Characterization of apelin, the ligand for the APJ receptor. $J$ Neurochem 2000; 74: 34-41.

[72] Yang P, Maguire JJ, Davenport AP. Apelin, Elabela/Toddler, and biased agonists as novel therapeutic agents in the cardiovascular system. Trends Pharmacol Sci 2015; 36: $560-567$.

[73] Yang P, Kuc RE, Brame AL, et al. [Pyr1] apelin-13(1-12) is a biologically active ACE2 metabolite of the endogenous cardiovascular peptide [Pyr1] apelin-13. Front Neurosci 2017; 11: 1-14.

[74] Wang W, McKinnie SMK, Farhan M, et al. Angiotensin-Converting Enzyme 2 Metabolizes and Partially Inactivates Pyr-Apelin-13 and Apelin-17 Novelty and Significance. Hypertension 2016; 68: 365-377.

[75] Kleinz MJ, Davenport AP. Emerging roles of apelin in biology and medicine. Pharmacol Ther 2005; 107: 198-211.

[76] Shin K, Chapman NA, Sarker M, et al. Bioactivity of the putative apelin proprotein expands the repertoire of apelin receptor ligands. Biochim Biophys Acta - Gen Subj 2017; 1861: 1901-1912.

[77] Pisarenko O, Shulzhenko V, Studneva I, et al. Structural apelin analogues: mitochondrial ROS inhibition and cardiometabolic protection in myocardial ischaemia reperfusion injury. Br J Pharmacol 2015; 172: 2933-2945.

[78] Maguire JJ, Kleinz MJ, Pitkin SL, et al. [Pyr1]apelin-13 identified as the predominant apelin isoform in the human heart: Vasoactive mechanisms and inotropic action in disease. Hypertension 2009; 54: 598-604.

[79] Boucher J, Masri B, Daviaud D, et al. Apelin, a newly identified adipokine upregulated by insulin and obesity. Endocrinology 2005; 146: 1764-71.

[80] Jiang H, Ye XP, Yang ZY, et al. Aldosterone directly affects apelin expression and secretion in adipocytes. $J$ Mol Endocrinol 2013; 51: 37-48.

[81] Ronkainen V-P, Ronkainen JJ, Hänninen SL, et al. Hypoxia inducible factor regulates the cardiac expression and secretion of apelin. FASEB J 2007; 21: 1821-1830.

[82] Glassford AJ, Yue P, Sheikh AY, et al. HIF-1 regulates hypoxia- and insulin-induced expression of apelin in adipocytes. Am J Physiol Endocrinol Metab 2007; 293: E1590- 
E1596.

[83] Geiger K, Muendlein A, Stark N, et al. Hypoxia Induces Apelin Expression in Human Adipocytes. Horm Metab Res 2011; 43: 380-385.

[84] Yang P, Read C, Kuc RE, et al. Elabela/Toddler Is an Endogenous Agonist of the Apelin APJ Receptor in the Adult Cardiovascular System, and Exogenous

Administration of the Peptide Compensates for the Downregulation of Its Expression in Pulmonary Arterial HypertensionClinical Perspective. Circulation 2017; 135: 11601173.

[85] Ho L, Tan SYX, Wee S, et al. ELABELA Is an Endogenous Growth Factor that Sustains hESC Self-Renewal via the PI3K/AKT Pathway. Cell Stem Cell 2015; 17 : 435-447.

[86] Wang Z, Yu D, Wang M, et al. Elabela-Apelin Receptor Signaling Pathway is Functional in Mammalian Systems. Sci Rep 2015; 5: 8170.

[87] Picault FX, Chaves-Almagro C, Projetti F, et al. Tumour co-expression of apelin and its receptor is the basis of an autocrine loop involved in the growth of colon adenocarcinomas. Eur J Cancer 2014; 50: 663-674.

[88] Wan Y, Zeng Z, Xi M, et al. Dysregulated microRNA-224/apelin axis associated with aggressive progression and poor prognosis in patients with prostate cancer. Hum Pathol 2015; 46: 295-303.

[89] Hao Y, Li M, Ning F, et al. APJ Is Associated with Treatment Response in Gastric Cancer Patients Receiving Concurrent Chemoradiotherapy and Endostar Therapy. Cancer Biother Radiopharm 2017; 32: 133-138.

[90] Yang L, Su T, Lv D, et al. ERK1/2 mediates lung adenocarcinoma cell proliferation and autophagy induced by apelin-13. Acta Biochim Biophys Sin (Shanghai) 2014; 46: $100-111$.

[91] Ni Y, Liu D, Ge G, et al. Apelin is a novel circulating biomarker for the diagnosis of lung cancer. Int J Clin Exp Pathololgy 2017; 10: 5559-5565.

[92] Diakowska D, Markocka-Mączka K, Szelachowski P, et al. Serum Levels of Resistin, Adiponectin, and Apelin in Gastroesophageal Cancer Patients. Dis Markers 2014; 2014: $1-8$.

[93] Zuurbier L, Rahman A, Cordes M, et al. Apelin: A putative novel predictive biomarker for bevacizumab response in colorectal cancer. Oncotarget 2017; 8: 42949-42961.

[94] Chen T, Liu N, Xu G, et al. Apelin13/APJ promotes proliferation of colon carcinoma by activating Notch3 signaling pathway. Oncotarget 2017; 8: 101697-101706.

[95] Muto J, Shirabe K, Yoshizumi T, et al. The apelin-APJ system induces tumor 
arteriogenesis in hepatocellular carcinoma. Anticancer Res 2014; 34: 5313-20.

[96] Altinkaya SO, Nergiz S, Kucuk M, et al. Apelin levels are higher in obese patients with endometrial cancer. J Obstet Gynaecol Res 2015; 41: 294-300.

[97] Peng X, Li F, Wang P, et al. Apelin-13 induces MCF-7 cell proliferation and invasion via phosphorylation of ERK1/2. Int J Mol Med 2015; 36: 733-8.

[98] Wang Z, Greeley GH, Qiu S. Immunohistochemical localization of apelin in human normal breast and breast carcinoma. J Mol Histol 2008; 39: 121-124.

[99] Zhang HP, Zou J, Xu ZQ, et al. Association of leptin, visfatin, apelin, resistin and adiponectin with clear cell renal cell carcinoma. Oncol Lett 2017; 13: 463-468.

[100] Maden M, Pamuk ON, Pamuk GE. High apelin levels could be used as a diagnostic marker in multiple myeloma: A comparative study. Cancer Biomarkers 2017; 17: 391396.

[101] Hall C, Ehrlich L, Venter J, et al. Inhibition of the apelin/apelin receptor axis decreases cholangiocarcinoma growth. Cancer Lett 2017; 386: 179-188.

[102] Patel SJ, Sanjana NE, Kishton RJ, et al. Identification of essential genes for cancer immunotherapy. Nature 2017; 548: 537-542.

[103] Bailey P, Cushing H. A classification of the tumours of the glioma group on a histogenetic basis, with a correlated study of prognosis. Br J Surg 1927; 14: 554-555.

[104] Louis DN, Holland EC, Cairncross JG. Glioma classification: A molecular reappraisal. Am J Pathol 2001; 159: 779-786.

[105] Thakkar JP, Dolecek TA, Horbinski C, et al. Epidemiologic and molecular prognostic review of glioblastoma. Cancer Epidemiol Biomarkers Prev 2014; 23: 1985-1996.

[106] Reifenberger G, Wirsching H-G, Knobbe-Thomsen CB, et al. Advances in the molecular genetics of gliomas - implications for classification and therapy. Nat Rev Clin Oncol 2016; 14: 434-452.

[107] International Agency for Research on Cancer. WHO Classification of Tumours of the Central Nervous System. 4th editio. World Health Organization, http://publications.iarc.fr/Book-And-Report-Series/Who-Iarc-Classification-OfTumours/Who-Classification-Of-Tumours-Of-The-Central-Nervous-System-2016 (2016).

[108] Stupp R. Promising Survival for Patients With Newly Diagnosed Glioblastoma Multiforme Treated With Concomitant Radiation Plus Temozolomide Followed by Adjuvant Temozolomide. J Clin Oncol 2002; 20: 1375-1382.

[109] Stupp R, Mason WP, van den Bent MJ, et al. Radiotherapy plus Concomitant and 
Adjuvant Temozolomide for Glioblastoma. N Engl J Med 2005; 352: 987-996.

[110] Jiang Y, Uhrbom L. On the origin of glioma. Ups J Med Sci 2012; 117: 113-121.

[111] Zong H, Verhaak RG, Canoll P. The cellular origin for malignant glioma and prospects for clinical advancements. Expert Rev Mol Diagn 2012; 12: 383-394.

[112] Alcantara Llaguno SR, Parada LF. Cell of origin of glioma: biological and clinical implications. Br J Cancer 2016; 115: 1445-1450.

[113] Modrek AS. Brain stem cells as the cell of origin in glioma. World J Stem Cells 2014; 6: $43-52$.

[114] Liu C, Sage J, Miller M. Mosaic Analysis with Double Markers (MADM) Reveals Tumor Cell-of-Origin in Glioma. Cell 2011; 146: 209-221.

[115] Schwartzbaum J a, Fisher JL, Aldape KD, et al. Epidemiology and molecular pathology of glioma. Nat Clin Pract Neurol 2006; 2: 494-503.

[116] Winters JL, Wilson D, Davis DG. Congenital glioblastoma multiforme: A report of three cases and a review of the literature. J Neurol Sci 2001; 188: 13-19.

[117] Yuile P, Dent O, Cook R, et al. Survival of glioblastoma patients related to presenting symptoms, brain site and treatment variables. J Clin Neurosci 2006; 13: 747-51.

[118] Braganza MZ, Kitahara CM, Berrington de $\mathrm{G}$ a, et al. Ionizing radiation and the risk of brain and central nervous system tumors: a systematic review. NeuroOncol 2012; 14 : $1316-1324$.

[119] Weller M. Novel diagnostic and therapeutic approaches to malignant glioma. Swiss Med Wkly 2011; 141: 1-7.

[120] Weller M, van den Bent M, Hopkins K, et al. EANO guideline for the diagnosis and treatment of anaplastic gliomas and glioblastoma. Lancet Oncol 2014; 15: 395-403.

[121] Young RM, Jamshidi A, Davis G, et al. Current trends in the surgical management and treatment of adult glioblastoma. Ann Transl Med 2015; 3: 121.

[122] Ohgaki H, Kleihues P. The definition of primary and secondary glioblastoma. Clin Cancer Res 2013; 19: 764-772.

[123] Bianco J, Bastiancich C, Jankovski A, et al. On glioblastoma and the search for a cure: where do we stand? Cell Mol Life Sci 2017; 74: 2451-2466.

[124] Malzkorn B, Reifenberger G. Practical implications of integrated glioma classification according to the World Health Organization classification of tumors of the central nervous system 2016. Curr Opin Oncol 2016; 28: 494-501.

[125] Adamson C, Kanu OO, Mehta AI, et al. Glioblastoma multiforme: a review of where 
we have been and where we are going. Expert Opin Investig Drugs 2009; 18: 1061-83.

[126] Urbanska K, Sokolowska J, Szmidt M, et al. Glioblastoma multiforme - An overview. Wspolczesna Onkol 2014; 18: 307-312.

[127] Kanu OO, Mehta A, Di C, et al. Glioblastoma multiforme: a review of therapeutic targets. Expert Opin Ther Targets 2009; 13: 701-718.

[128] Baba H, Nakahira K, Morita N, et al. GFAP Gene Expression during Development of Astrocyte. Dev Neurosci 1997; 19: 49-57.

[129] Wilhelmsson U, Eliasson C, Bjerkvig R, et al. Loss of GFAP expression in high-grade astrocytomas does not contribute to tumor development or progression. Oncogene 2003; 22: 3407-3411.

[130] Bhagavan NV, Ha C-E. Carbohydrate Metabolism I. In: Essentials of Medical Biochemistry. Elsevier, pp. 115-133.

[131] Chen J-R, Yao Y, Xu H-Z, et al. Isocitrate Dehydrogenase (IDH)1/2 Mutations as Prognostic Markers in Patients With Glioblastomas. Medicine (Baltimore) 2016; 95: $1-13$.

[132] Parsons DW, Jones S, Zhang X, et al. An integrated genomic analysis of human glioblastoma multiforme. Science (80- ) 2008; 321: 1807-12.

[133] Cancer Genome Atlas Research Network. Comprehensive genomic characterization defines human glioblastoma genes and core pathways. Nature 2008; 455: 1061-8.

[134] Verhaak RG., Hoadley K., Purdom E, et al. An integrated genomic analysis identifies clinically relevant subtypes of glioblastoma characterized by abnormalities in PDGFRA, IDH1, EGFR and NF1. Cancer 2010; 19: 38-46.

[135] Cancer Genome Atlas Research Network, Brat DJ, Verhaak RGW, et al. Comprehensive, Integrative Genomic Analysis of Diffuse Lower-Grade Gliomas. $N$ Engl J Med 2015; 372: 2481-98.

[136] McGirt MJ, Chaichana KL, Gathinji M, et al. Independent association of extent of resection with survival in patients with malignant brain astrocytoma. J Neurosurg 2009; 110: 156-162.

[137] Park JK, Hodges T, Arko L, et al. Scale to predict survival after surgery for recurrent glioblastoma multiforme. J Clin Oncol 2010; 28: 3838-3843.

[138] Johnson BE, Mazor T, Hong C, et al. Mutational Analysis Reveals the Origin and Therapy-Driven Evolution of Recurrent Glioma. Science (80- ) 2014; 343: 189-193.

[139] Holland EC. Glioblastoma multiforme: The terminator. Proc Natl Acad Sci 2000; 97: $6242-6244$. 
[140] Sulman EP, Ismaila N, Armstrong TS, et al. Radiation therapy for glioblastoma: American Society of Clinical Oncology clinical practice guideline endorsement of the American Society for Radiation Oncology guideline. J Clin Oncol 2017; 35: 361-369.

[141] Barani IJ, Larson DA. Radiation Therapy of Glioblastoma. In: Raizer J, Parsa A (eds) Current Understanding and Treatment of Gliomas. Cham: Springer International Publishing, pp. 49-73.

[142] Frosina G. DNA repair and resistance of gliomas to chemotherapy and radiotherapy. Mol Cancer Res 2009; 7: 989-99.

[143] Christmann M, Kaina B. Transcriptional regulation of human DNA repair genes following genotoxic stress: Trigger mechanisms, inducible responses and genotoxic adaptation. Nucleic Acids Res 2013; 41: 8403-8420.

[144] Olson JJ, Nayak L, Ormond DR, et al. The role of cytotoxic chemotherapy in the management of progressive glioblastoma: A systematic review and evidence-based clinical practice guideline. J Neurooncol 2014; 118: 501-555.

[145] Villano JL, Seery TE, Bressler LR. Temozolomide in malignant gliomas: Current use and future targets. Cancer Chemother Pharmacol 2009; 64: 647-655.

[146] Zhang J, Stevens MFG, Bradshaw TD. Temozolomide: Mechanisms of Action, Repair and Resistance. Curr Mol Pharmacol 2011; 5: 102-114.

[147] Sathornsumetee S, Reardon D a., Desjardins A, et al. Molecularly targeted therapy for malignant glioma. Cancer 2007; 110: 13-24.

[148] Vredenburgh JJ, Desjardins A, Reardon D a, et al. Experience with irinotecan for the treatment of malignant glioma. Neuro Oncol 2009; 11: 80-91.

[149] Vredenburgh JJ, Desjardins A, Herndon JE, et al. Bevacizumab plus irinotecan in recurrent glioblastoma multiforme. J Clin Oncol 2007; 25: 4722-4729.

[150] Jiang P, Mukthavavam R, Chao Y, et al. Novel anti-glioblastoma agents and therapeutic combinations identified from a collection of FDA approved drugs. $J$ Transl Med 2014; 12: 13.

[151] Mesti T, Ebert Moltara M, Boc M, et al. Bevacizumab and irinotecan in recurrent malignant glioma, a single institution experience. Radiol Oncol 2015; 49: 4-9.

[152] Schaub C, Kebir S, Junold N, et al. Tumor growth patterns of MGMT-non-methylated glioblastoma in the randomized GLARIUS trial. J Cancer Res Clin Oncol 2018; 0: 0.

[153] Koukourakis MI, Koukouraki S, Fezoulidis I, et al. High intratumoural accumulation of stealth liposomal doxorubicin (Caelyx) in glioblastomas and in metastatic brain tumours. Br J Cancer 2000; 83: 1281-6. 
[154] Lin YL, Wu MT, Yang FY. Pharmacokinetics of doxorubicin in glioblastoma multiforme following ultrasound-Induced blood-brain barrier disruption as determined by microdialysis. J Pharm Biomed Anal 2018; 149: 482-487.

[155] Almubarak M, Newton M, Altaha R. Reinduction of Bevacizumab in Combination with Pegylated Liposomal Doxorubicin in a Patient with Recurrent Glioblastoma Multiforme Who Progressed on Bevacizumab/Irinotecan. J Oncol 2008; 2008: 1-4.

[156] Belhadj Z, Zhan C, Ying M, et al. Multifunctional targeted liposomal drug delivery for efficient glioblastoma treatment. Oncotarget 2017; 8: 66889-66900.

[157] Johnson DR, Fogh SE, Giannini C, et al. Case-Based Review : newly diagnosed glioblastoma. Neuro-Oncology Pract 2015; 2: 106-121.

[158] Wick W, Gorlia T, Bendszus M, et al. Lomustine and Bevacizumab in Progressive Glioblastoma. N Engl J Med 2017; 377: 1954-1963.

[159] Gilbert MR, Dignam JJ, Armstrong TS, et al. A randomized trial of bevacizumab for newly diagnosed glioblastoma. $N$ Engl J Med 2014; 370: 699-708.

[160] Diaz RJ, Ali S, Qadir MG, et al. The role of bevacizumab in the treatment of glioblastoma. J Neurooncol 2017; 133: 455-467.

[161] Ozel O, Kurt M, Ozdemir O, et al. Complete response to bevacizumab plus irinotecan in patients with rapidly progressive GBM: Cases report and literature review. J Oncol Sci 2016; 2: 87-94.

[162] Friedman HS, Prados MD, Wen PY, et al. Bevacizumab alone and in combination with irinotecan in recurrent glioblastoma. J Clin Oncol 2009; 27: 4733-4740.

[163] Stewart LA. Chemotherapy in adult high-grade glioma: a systematic review and metaanalysis of individual patient data from 12 randomised trials. Lancet 2002; 359: 10111018.

[164] Brandes AA, Bartolotti M, Tosoni A, et al. Nitrosoureas in the Management of Malignant Gliomas. Curr Neurol Neurosci Rep 2016; 16: 13.

[165] Ashby LS, Smith KA, Stea B. Gliadel wafer implantation combined with standard radiotherapy and concurrent followed by adjuvant temozolomide for treatment of newly diagnosed high-grade glioma: A systematic literature review. World J Surg Oncol 2016; 14: 1-15.

[166] Taal W, Oosterkamp HM, Walenkamp AME, et al. Single-agent bevacizumab or lomustine versus a combination of bevacizumab plus lomustine in patients with recurrent glioblastoma (BELOB trial): A randomised controlled phase 2 trial. Lancet Oncol 2014; 15: 943-953.

[167] Happold C, Roth P, Wick W, et al. ACNU-based chemotherapy for recurrent glioma in 
the temozolomide era. J Neurooncol 2009; 92: 45-48.

[168] Jakobsen JN, Urup T, Grunnet K, et al. Toxicity and efficacy of lomustine and bevacizumab in recurrent glioblastoma patients. J Neurooncol 2018; 0: 1-8.

[169] Kanner AA, Wong ET, Villano JL, et al. Post hoc analyses of intention-to-treat population in phase III comparison of NovoTTF-100A ${ }^{\mathrm{TM}}$ system versus best physician's choice chemotherapy. Semin Oncol 2014; 41: S25-S34.

[170] Stupp R, Wong ET, Kanner AA, et al. NovoTTF-100A versus physician's choice chemotherapy in recurrent glioblastoma: A randomised phase III trial of a novel treatment modality. Eur J Cancer 2012; 48: 2192-2202.

[171] Kirson ED, Dbalý V, Tovaryš F, et al. Alternating electric fields arrest cell proliferation in animal tumor models and human brain tumors. Proc Natl Acad Sci 2007; 104: 10152-10157.

[172] Zhu P, Zhu J-J. Tumor treating fields: a novel and effective therapy for glioblastoma: mechanism, efficacy, safety and future perspectives. Chinese Clin Oncol 2017; 6: 4141 .

[173] Mehta M, Wen P, Nishikawa R, et al. Critical review of the addition of tumor treating fields (TTFields) to the existing standard of care for newly diagnosed glioblastoma patients. Crit Rev Oncol Hematol 2017; 111: 60-65.

[174] Stupp R, Taillibert S, Kanner A, et al. Effect of tumor-treating fields plus maintenance temozolomide vs maintenance temozolomide alone on survival in patients with glioblastoma a randomized clinical trial. JAMA - J Am Med Assoc 2017; 318: 23062316.

[175] Rong Y, Durden DL, Van Meir EG, et al. 'Pseudopalisading' necrosis in glioblastoma: A familiar morphologic feature that links vascular pathology, hypoxia, and angiogenesis. J Neuropathol Exp Neurol 2006; 65: 529-539.

[176] Talasila KM, Røsland G V., Hagland HR, et al. The angiogenic switch leads to a metabolic shift in human glioblastoma. Neuro Oncol 2017; 19: 383-393.

[177] Zhou YY, Zhou YY, Shingu T, et al. Metabolic alterations in highly tumorigenic glioblastoma cells: Preference for hypoxia and high dependency on glycolysis. J Biol Chem 2011; 286: 32843-32853.

[178] Cairns RA, Harris IS, Mak TW. Regulation of cancer cell metabolism. Nat Rev Cancer 2011; 11: 85-95.

[179] Fulda S. Tumor resistance to apoptosis. Int J Cancer 2009; 124: 511-515.

[180] Fulda S. Evasion of Apoptosis as a Cellular Stress Response in Cancer. Int J Cell Biol 2010; 2010: 1-6. 
[181] Huber-Keener KJ, Yang J-M. Impact of Metabolic and Therapeutic Stresses on Glioma Progression and Therapy. In: Chen C (ed) Advances in the Biology, Imaging and Therapies for Glioblastoma. InTech, pp. 23-52.

[182] Vaupel P, Kallinowski F, Okunieff P. Blood flow, oxygen and nutrient supply, and metabolic microenvironment of human tumors: a review. Cancer Res 1989; 49: 644965 .

[183] Mucaj V, Lee SS, Skuli N, et al. MicroRNA-124 expression counteracts pro-survival stress responses in glioblastoma. Oncogene 2015; 34: 2204-2214.

[184] Huber-keener KJ, Huber-keener KJ. Alterations in gene expression as a response of tumor cells to stresses. Pennsylvania State University, 2013.

[185] Liu J, Liu M, Chen L. Novel pathogenesis: Regulation of apoptosis by Apelin/APJ system. Acta Biochim Biophys Sin (Shanghai) 2017; 49: 471-478.

[186] Mahajan K, Mahajan NP. Cross talk of tyrosine kinases with the DNA damage signaling pathways. Nucleic Acids Res 2015; 43: 10588-10601.

[187] Kao GD, Jiang Z, Fernandes AM, et al. Inhibition of phosphatidylinositol-3-OH kinase/Akt signaling impairs DNA repair in glioblastoma cells following ionizing radiation. J Biol Chem 2007; 282: 21206-12.

[188] Gil Del Alcazar CR, Hardebeck MC, Mukherjee B, et al. Inhibition of DNA doublestrand break repair by the dual PI3K/mTOR inhibitor NVP-BEZ235 as a strategy for radiosensitization of glioblastoma. Clin Cancer Res 2014; 20: 1235-1248.

[189] Westhoff M-A, Karpel-Massler G, Brühl O, et al. A critical evaluation of PI3K inhibition in Glioblastoma and Neuroblastoma therapy. Mol Cell Ther 2014; 2: 32.

[190] Tao J, Zhu W, Li Y, et al. Apelin-13 protects the heart against ischemia-reperfusion injury through inhibition of ER-dependent apoptotic pathways in a time-dependent fashion. AJP Hear Circ Physiol 2011; 301: H1471-H1486.

[191] Li L, Zeng H, Chen J-X. Apelin-13 increases myocardial progenitor cells and improves repair postmyocardial infarction. AJP Hear Circ Physiol 2012; 303: H605H618.

[192] Li L, Zeng H, Hou X, et al. Myocardial Injection of Apelin-Overexpressing Bone Marrow Cells Improves Cardiac Repair via Upregulation of Sirt3 after Myocardial Infarction. PLoS One 2013; 8: 1-11.

[193] Ustunel I, Acar N, Gemici B, et al. The effects of water immersion and restraint stress on the expressions of apelin, apelin receptor (APJR) and apoptosis rate in the rat heart. Acta Histochem 2014; 116: 675-681.

[194] Zhang Z, Yu B, Tao GZ. Apelin protects against cardiomyocyte apoptosis induced by 
glucose deprivation. Chin Med J (Engl) 2009; 122: 2360-2365.

[195] Alastalo TP, Li M, De Jesus Perez V, et al. Disruption of PPAR $\gamma / \beta$-catenin-mediated regulation of apelin impairs BMP-induced mouse and human pulmonary arterial EC survival. J Clin Invest 2011; 121: 3735-3746.

[196] Mottaghi S, Larijani B, Sharifi AM. Apelin 13: A novel approach to enhance efficacy of hypoxic preconditioned mesenchymal stem cells for cell therapy of diabetes. Med Hypotheses 2012; 79: 717-718.

[197] Zou Y, Wang B, Fu W, et al. Apelin-13 Protects PC12 Cells from CorticosteroneInduced Apoptosis Through PI3K and ERKs Activation. Neurochem Res 2016; 41: $1635-1644$.

[198] Gu Q, Zhai L, Feng X, et al. Apelin-36, a potent peptide, protects against ischemic brain injury by activating the PI3K/Akt pathway. Neurochem Int 2013; 63: 535-540.

[199] Lu Q, Jiang YR, Qian J, et al. Apelin-13 regulates proliferation, migration and survival of retinal Müller cells under hypoxia. Diabetes Res Clin Pract 2013; 99: 158-167.

[200] Chen L, Tao Y, Feng J, et al. Apelin Protects Primary Rat Retinal Pericytes from Chemical Hypoxia-Induced Apoptosis. J Ophthalmol 2015; 2015: 1-14.

[201] Tang SY, Xie H, Yuan LQ, et al. Apelin stimulates proliferation and suppresses apoptosis of mouse osteoblastic cell line MC3T3-E1 via JNK and PI3-K/Akt signaling pathways. Peptides 2007; 28: 708-718.

[202] Xie H, Yuan LQ, Luo XH, et al. Apelin suppresses apoptosis of human osteoblasts. Apoptosis 2007; 12: 247-254.

[203] Antushevich H, Krawczynska A, Kapica M, et al. Effect of apelin on mitosis, apoptosis and DNA repair enzyme OGG $1 / 2$ expression in intestinal cell lines IEC-6 and Caco-2. Folia Histochem Cytobiol 2014; 52: 51-59.

[204] Zagzag D, Zhong H, Scalzitti JM, et al. Expression of hypoxia-inducible factor-1 $\alpha$ in brain tumors. Cancer 2000; 88: 2606-2618.

[205] Brat DJ, Castellano-Sanchez AA, Hunter SB, et al. Pseudopalisades in Glioblastoma Are Hypoxic, Express Extracellular Matrix Proteases, and Are Formed by an Actively Migrating Cell Population. Cancer Res 2004; 64: 920-927.

[206] Kaur B, Khwaja FW, Severson E a, et al. Hypoxia and the hypoxia-inducible-factor pathway in glioma growth and angiogenesis. Neuro Oncol 2005; 7: 134-53.

[207] Province P, Griguer C, Han X. Hypoxia, Angiogenesis and Mechanisms for Invasion of Malignant Gliomas. Evol Mol Biol Brain Tumours Ther Implic 2013; 105-123.

[208] Combs S, Schmid T, Vaupel P, et al. Stress Response Leading to Resistance in 
Glioblastoma-The Need for Innovative Radiotherapy (iRT) Concepts. Cancers (Basel) 2016; 8: 15.

[209] Jawhari S, Ratinaud M-H, Verdier M. Glioblastoma, hypoxia and autophagy: a survival-prone 'ménage-à-trois'. Cell Death Dis 2016; 7: e2434.

[210] Jensen RL. Brain tumor hypoxia: Tumorigenesis, angiogenesis, imaging, pseudoprogression, and as a therapeutic target. J Neurooncol 2009; 92: 317-335.

[211] Joseph J V., Conroy S, Pavlov K, et al. Hypoxia enhances migration and invasion in glioblastoma by promoting a mesenchymal shift mediated by the HIF $1 \alpha-Z E B 1$ axis. Cancer Lett 2015; 359: 107-116.

[212] Zagzag D, Lukyanov Y, Lan L, et al. Hypoxia-inducible factor 1 and VEGF upregulate CXCR4 in glioblastoma: implications for angiogenesis and glioma cell invasion. Lab Invest 2006; 86: 1221-1232.

[213] Monteiro A, Hill R, Pilkington G, et al. The Role of Hypoxia in Glioblastoma Invasion. Cells 2017; 6: 45.

[214] Huang W, Chen W, Zhang X. Glioblastoma multiforme: Effect of hypoxia and hypoxia inducible factors on therapeutic approaches (Review). Oncol Lett 2016; 12 : $2283-2288$.

[215] Shweiki D, Itin A, Soffer D, et al. Vascular endothelial growth factor induced by hypoxia may mediate hypoxia-initiated angiogenesis. Nature 1992; 359: 843-845.

[216] Du R, Lu K V., Petritsch C, et al. HIF1?? Induces the Recruitment of Bone MarrowDerived Vascular Modulatory Cells to Regulate Tumor Angiogenesis and Invasion. Cancer Cell 2008; 13: 206-220.

[217] Rajendran JG, Mankoff DA, O'Sullivan F, et al. Hypoxia and glucose metabolism in malignant tumors: evaluation by $[18 \mathrm{~F}]$ fluoromisonidazole and [18F]fluorodeoxyglucose positron emission tomography imaging. Clin Cancer Res 2004; 10: 2245-52.

[218] Colwell N, Larion M, Giles AJ, et al. Hypoxia in the glioblastoma microenvironment: Shaping the phenotype of cancer stem-like cells. Neuro Oncol 2017; 19: 887-896.

[219] Manini I, Caponnetto F, Bartolini A, et al. Role of Microenvironment in Glioma Invasion: What We Learned from In Vitro Models. Int J Mol Sci 2018; 19: 147.

[220] Persano L, Pistollato F, Rampazzo E, et al. BMP2 sensitizes glioblastoma stem-like cells to Temozolomide by affecting HIF-1 $\alpha$ stability and MGMT expression. Cell Death Dis 2012; 3: e412.

[221] Yang L, Lin C, Wang L, et al. Hypoxia and hypoxia-inducible factors in glioblastoma multiforme progression and therapeutic implications. Exp Cell Res 2012; 318: 2417- 
2426.

[222] Sheikh AY, Chun HJ, Glassford AJ, et al. In vivo genetic profiling and cellular localization of apelin reveals a hypoxia-sensitive, endothelial-centered pathway activated in ischemic heart failure. Am J Physiol Heart Circ Physiol 2008; 294: H8898 .

[223] Kapitsinou PP, Rajendran G, Astleford L, et al. The Endothelial Prolyl-4-Hydroxylase Domain 2/Hypoxia-Inducible Factor 2 Axis Regulates Pulmonary Artery Pressure in Mice. Mol Cell Biol 2016; 36: 1584-1594.

[224] Lu He, Jing Xu, Linxi Chen LLP, He L, Xu J, et al. Apelin/APJ signaling in hypoxiarelated diseases. Clin Chim Acta 2015; 451: 191-8.

[225] Kidoya H, Kunii N, Naito H, et al. The apelin/APJ system induces maturation of the tumor vasculature and improves the efficiency of immune therapy. Oncogene 2012; 31: 3254-64.

[226] Chen L, Lin ZX, Lin GS, et al. Classification of microvascular patterns via cluster analysis reveals their prognostic significance in glioblastoma. Hum Pathol 2015; 46: $120-128$.

[227] Gi T, Sato Y, Tokumitsu T, et al. Microvascular proliferation of brain metastases mimics glioblastomas in squash cytology. Cytopathology 2017; 28: 228-234.

[228] Korkolopoulou P, Patsouris E, Kavantzas N, et al. Prognostic implications of microvessel morphometry in diffuse astrocytic neoplasms. Neuropathol Appl Neurobiol 2002; 28: 57-66.

[229] Casals G, Fernández-Varo G, Melgar-Lesmes P, et al. Factors involved in extracellular matrix turnover in human derived cardiomyocytes. Cell Physiol Biochem 2013; 32: $1125-1136$.

[230] Kong $\mathrm{H}$ liang, Li Z quan, Zhao S mei, et al. Apelin-APJ effects of ginsenoside-Rb1 depending on hypoxia-induced factor $1 \alpha$ in hypoxia neonatal cardiomyocytes. Chin $J$ Integr Med 2014; 21: 139-146.

[231] Cox CM, D'Agostino SL, Miller MK, et al. Apelin, the ligand for the endothelial Gprotein-coupled receptor, APJ, is a potent angiogenic factor required for normal vascular development of the frog embryo. Dev Biol 2006; 296: 177-189.

[232] Zhang J, Liu Q, Hu X, et al. Apelin/APJ signaling promotes hypoxia-induced proliferation of endothelial progenitor cells via phosphoinositide-3 kinase/Akt signaling. Mol Med Rep 2015; 12: 3829-3834.

[233] Kunduzova O, Alet N, Delesque-Touchard N, et al. Apelin/APJ signaling system: a potential link between adipose tissue and endothelial angiogenic processes. FASEB J 2008; 22: 4146-4153. 
[234] Yang F, Bai Y, Jiang Y. Effects of Apelin on RAW264.7 cells under both normal and hypoxic conditions. Peptides 2015; 69: 133-43.

[235] Melgar-Lesmes P, Pauta M, Reichenbach V, et al. Hypoxia and proinflammatory factors upregulate apelin receptor expression in human stellate cells and hepatocytes. Gut 2011; 60: 1404-1411.

[236] Li L, Li L, Zhang Z, et al. Hypoxia promotes bone marrow-derived mesenchymal stem cell proliferation through apelin/APJ/autophagy pathway. Acta Biochim Biophys Sin (Shanghai) 2015; 47: 362-367.

[237] Hou X, Liu Y, Liu H, et al. PERK silence inhibits glioma cell growth under low glucose stress by blockage of p-AKT and subsequent HK2's mitochondria translocation. Sci Rep 2015; 5: 9065.

[238] Sun H, Zhang M, Cheng K, et al. Resistance of glioma cells to nutrient-deprived microenvironment can be enhanced by CD133-mediated autophagy. Oncotarget 2016; 7: 76238-76249.

[239] Lathia JD, Mack SC, Mulkearns-Hubert EE, et al. Cancer stem cells in glioblastoma. Genes Dev 2015; 29: 1203-17.

[240] Graham NA, Tahmasian M, Kohli B, et al. Glucose deprivation activates a metabolic and signaling amplification loop leading to cell death. Mol Syst Biol 2012; 8: 1-16.

[241] Jones RG, Thompson CB. Tumor suppressors and cell metabolism: a recipe for cancer growth. Genes Dev 2009; 23: 537-548.

[242] Zhang Z, Rahme GJ, Chatterjee PD, et al. ID2 promotes survival of glioblastoma cells during metabolic stress by regulating mitochondrial function. Cell Death Dis 2017; 8: e2615.

[243] Kathagen A, Schulte A, Balcke G, et al. Hypoxia and oxygenation induce a metabolic switch between pentose phosphate pathway and glycolysis in glioma stem-like cells. Acta Neuropathol 2013; 126: 763-780.

[244] Ewa Obuchowicz AMB. Chronic Physiological Hypoxia and High Glucose Concentration Promote Resistance of T98G Glioblastoma Cell Line to Temozolomide. Drug Des Open Access; 03. Epub ahead of print 2014. DOI: 10.4172/21690138.1000117.

[245] Lu J, Tan M, Cai Q. The Warburg effect in tumor progression: Mitochondrial oxidative metabolism as an anti-metastasis mechanism. Cancer Lett 2015; 356: 156164.

[246] Godlewski J, Nowicki MO, Bronisz A, et al. MicroRNA-451 Regulates LKB1/AMPK Signaling and Allows Adaptation to Metabolic Stress in Glioma Cells. Mol Cell 2010; 37: $620-632$. 
[247] Yang C, Sudderth J, Dang T, et al. Glioblastoma cells require glutamate dehydrogenase to survive impairments of glucose metabolism or Akt signaling. Cancer Res 2009; 69: 7986-7993.

[248] Flavahan WA, Wu Q, Hitomi M, et al. Brain tumor initiating cells adapt to restricted nutrition through preferential glucose uptake. Nat Neurosci 2013; 16: 1373-1382.

[249] Ameri K, Jahangiri A, Rajah AM, et al. HIGD1A Regulates Oxygen Consumption, ROS Production, and AMPK Activity during Glucose Deprivation to Modulate Cell Survival and Tumor Growth. Cell Rep 2015; 10: 891-899.

[250] Wang XL, Tao Y, Lu Q, et al. Apelin supports primary rat retinal Müller cells under chemical hypoxia and glucose deprivation. Peptides 2012; 33: 298-306.

[251] Jiao H, Zhang Z, Ma Q, et al. Mechanism underlying the inhibitory effect of Apelin-13 on glucose deprivation-induced autophagy in rat cardiomyocytes. Exp Ther Med 2013; 5: 797-802.

[252] Zeng X, Yu SP, Taylor T, et al. Protective effect of apelin on cultured rat bone marrow mesenchymal stem cells against apoptosis. Stem Cell Res 2012; 8: 357-367.

[253] Lai J-H, Jan H-J, Liu L-W, et al. Nodal regulates energy metabolism in glioma cells by inducing expression of hypoxia-inducible factor 1 $\alpha$. Neuro Oncol 2013; 15: 1330-41.

[254] R. Stupp, W. P. Mason MJ van den B et al. "Radiotherapy plus concomitant and adjuvant temozolomide for glioblastoma,". New Engl J Med vol 352, no 10 2005; 987996.

[255] Wang Y, Chen X, Zhang Z, et al. Comparison of the clinical efficacy of temozolomide (TMZ) versus nimustine (ACNU)-based chemotherapy in newly diagnosed glioblastoma. Neurosurg Rev 2014; 37: 73-78.

[256] Weatherbee JL, Kraus J, Ross AH. ER stress in temozolomide-treated glioblastomas interferes with DNA repair and induces apoptosis. Oncotarget 2016; 7: 43820-43834.

[257] Lin CJ, Lee CC, Shih YL, et al. Resveratrol enhances the therapeutic effect of temozolomide against malignant glioma in vitro and in vivo by inhibiting autophagy. Free Radic Biol Med 2012; 52: 377-391.

[258] Jeong K, Oh Y, Kim S-J, et al. Apelin is transcriptionally regulated by ER stressinduced ATF4 expression via a p38 MAPK-dependent pathway. Apoptosis 2014; 19 : 1399-1410.

[259] Than A, Zhang X, Leow MK-S, et al. Apelin attenuates oxidative stress in human adipocytes. J Biol Chem 2014; 289: 3763-3774.

[260] Zhang P, Yi LH, Meng GY, et al. Apelin-13 attenuates cisplatin-induced cardiotoxicity through inhibition of ROS-mediated DNA damage and regulation of MAPKs and AKT 
pathways. Free Radic Res 2017; 51: 449-459.

[261] Sanai N, Berger MS. Glioma Extent of Resection and its Impact on Patient Outcome. Neurosurgery 2008; 62: 753-766.

[262] Ho Kim E, Sook Song H, Hoon Yoo S, et al. Tumor treating fields inhibit glioblastoma cell migration, invasion and angiogenesis. Oncotarget 2016; 7: 65125-65136.

[263] Claes A, Idema AJ, Wesseling P. Diffuse glioma growth: A guerilla war. Acta Neuropathol 2007; 114: 443-458.

[264] Smith CL, Kilic O, Schiapparelli P, et al. Migration Phenotype of Brain-Cancer Cells Predicts Patient Outcomes. Cell Rep 2016; 15: 2616-2624.

[265] Zhang H, Gong Y, Wang Z, et al. Apelin inhibits the proliferation and migration of rat PASMCs via the activation of PI3K/Akt/mTOR signal and the inhibition of autophagy under hypoxia. J Cell Mol Med 2014; 18: 542-553.

[266] Wang C, Wen J, Zhou Y, et al. Apelin induces vascular smooth muscle cells migration via a PI3K/Akt/FoxO3a/MMP-2 pathway. Int J Biochem Cell Biol 2015; 69: 173-182.

[267] Qin D, Zheng X, Jiang Y. Apelin-13 induces proliferation, migration, and collagen I mRNA expression in human RPE cells via PI3K/Akt and MEK/Erk signaling pathways. Mol Vis 2013; 19: 2227-36.

[268] Koh I, Cha J, Park J, et al. The mode and dynamics of glioblastoma cell invasion into a decellularized tissue-derived extracellular matrix-based three-dimensional tumor model. Sci Rep 2018; 8: 1-12.

[269] Kislin KL, McDonough WS, Eschbacher JM, et al. NHERF-1: modulator of glioblastoma cell migration and invasion. Neoplasia 2009; 11:377-387.

[270] Harford-Wright E, Andre-Gregoire G, Jacobs KA, et al. Pharmacological targeting of apelin impairs glioblastoma growth. Brain 2017; 1-16.

[271] Calabrese C, Poppleton H, Kocak M, et al. A perivascular niche for brain tumor stem cells. Cancer Cell 2007; 11: 69-82.

[272] Liu H-T, Chen M, Yu J, et al. Serum Apelin Level Predicts the Major Adverse Cardiac Events in Patients With ST Elevation Myocardial Infarction Receiving Percutaneous Coronary Intervention. Medicine (Baltimore) 2015; 94: e449.

[273] Du J-H, Li X, Li R, et al. Elevation of serum apelin-13 associated with proliferative diabetic retinopathy in type 2 diabetic patients. Int J Ophthalmol 2014; 7: 968-73.

[274] El-Mesallamy HO, Hamdy NM, Rizk HH, et al. Apelin Serum Level in Egyptian Patients with Chronic Hepatitis C. Mediators Inflamm 2011; 2011: 1-7.

[275] $\mathrm{Ba} \mathrm{HJ}$, Chen HS, Su Z, et al. Associations between serum apelin-12 levels and obesity- 
related markers in Chinese children. PLoS One 2014; 9: 1-8.

[276] Soriguer F, Garrido-sanchez L, Garcia-serrano S, et al. Apelin Levels Are Increased in Morbidly Obese Subjects with Type 2 Diabetes Mellitus. 2009; 1574-1580.

[277] Dray C, Debard C, Jager J, et al. Apelin and APJ regulation in adipose tissue and skeletal muscle of type 2 diabetic mice and humans. Am J Physiol Endocrinol Metab 2010; 298: E1161-9.

[278] Ayada C, Toru Ü, Genç O, et al. Increased serum level of apelin in chronic obstructive pulmonary disease. Eur Respir J 2015; 46: PA396.

[279] Kadoglou NPE, Lampropoulos S, Kapelouzou A, et al. Serum levels of apelin and ghrelin in patients with acute coronary syndromes and established coronary artery disease--KOZANI STUDY. Transl Res 2010; 155: 238-46.

[280] Lacquaniti A, Altavilla G, Picone A, et al. Apelin beyond kidney failure and hyponatremia: a useful biomarker for cancer disease progression evaluation. Clin Exp Med 2015; 15: 97-105.

[281] Zhen EY, Higgs RE, Gutierrez J a. Pyroglutamyl apelin-13 identified as the major apelin isoform in human plasma. Anal Biochem 2013; 442: 1-9.

[282] Mesmin C, Fenaille F, Becher F, et al. Identification and characterization of apelin peptides in bovine colostrum and milk by liquid chromatography-mass spectrometry. $J$ Proteome Res 2011; 10: 5222-31.

[283] Mesmin C, Dubois M, Becher F, et al. Liquid chromatography/tandem mass spectrometry assay for the absolute quantification of the expected circulating apelin peptides in human plasma. Rapid Commun Mass Spectometry 2010; 24: 2875-2884.

[284] Phoenix Pharmaceuticals I. Phoenix Pharmaceuticals website: Apelin-12 (Human, Rat, Mouse, Bovine) - EIA kit, http://www.phoenixpeptide.com/catalog/product_info.php?products_id=4322 (2013, accessed 18 August 2016).

[285] Dalzell JR, Jackson CE, Chong KS, et al. Do plasma concentrations of apelin predict prognosis in patients with advanced heart failure? Biomark Med 2014; 8: 807-813.

[286] Falcone C, Buzzi MP, D’Angelo A, et al. Apelin Plasma Levels Predict Arrhythmia Recurrence in Patients with Persistent Atrial Fibrillation. Int J Immunopathol Pharmacol 2010; 23: 917-925.

[287] Lim YL, Choi E, Jang YO, et al. Clinical implications of the serum apelin level on portal hypertension and prognosis of liver cirrhosis. Gut Liver 2016; 10: 109-116.

[288] Chong KS, Gardner RS, Morton JJ, et al. Plasma concentrations of the novel peptide apelin are decreased in patients with chronic heart failure. Eur J Heart Fail 2006; 8: 
$355-360$.

[289] Yunn N-O, Koh A, Han S, et al. Agonistic aptamer to the insulin receptor leads to biased signaling and functional selectivity through allosteric modulation. Nucleic Acids Res 2015; 43: 7688-7701.

[290] Thiviyanathan V, Gorenstein DG. Aptamers and the next generation of diagnostic reagents. Proteomics - Clin Appl 2012; 6: 563-573.

[291] Ma H, Liu J, Ali MM, et al. Nucleic acid aptamers in cancer research, diagnosis and therapy. Chem Soc Rev 2015; 44: 1240-1256.

[292] Kratschmer C, Levy M. Effect of Chemical Modifications on Aptamer Stability in Serum. Nucleic Acid Ther 2017; 00: nat.2017.0680.

[293] Wang RE, Wu H, Niu Y, et al. Improving the stability of aptamers by chemical modification. Curr Med Chem 2011; 18: 4126-38.

[294] Shigdar S, Macdonald J, O'Connor M, et al. Aptamers as theranostic agents: Modifications, serum stability and functionalisation. Sensors (Switzerland) 2013; 13: $13624-13637$.

[295] Song S, Wang L, Li J, et al. Aptamer-based biosensors. TrAC Trends Anal Chem 2008; 27: $108-117$.

[296] Lenting K, Verhaak R, ter Laan M, et al. Glioma: experimental models and reality. Acta Neuropathol 2017; 133: 263-282.

[297] Huszthy PC, Daphu I, Niclou SP, et al. In vivo models of primary brain tumors: pitfalls and perspectives. Neuro Oncol 2012; 14: 979-993.

[298] Simeonova I, Huillard E. In vivo models of brain tumors: roles of genetically engineered mouse models in understanding tumor biology and use in preclinical studies. Cell Mol Life Sci 2014; 71: 4007-4026.

[299] Khaitan D, Chandna S, Arya MB, et al. Establishment and characterization of multicellular spheroids from a human glioma cell line; implications for tumor therapy. J Transl Med 2006; 4: 1-13.

[300] Pontén J, Macintyre EH. Long term culture of normal and neoplastic human glia. Acta Pathol Microbiol Scand 1968; 74: 465-486.

[301] Diserens AC, de Tribolet N, Martin-Achard A, et al. Characterization of an established human malignant glioma cell line: LN-18. Acta Neuropathol 1981; 53: 21-8.

[302] Xie Y, Bergström T, Jiang Y, et al. The Human Glioblastoma Cell Culture Resource: Validated Cell Models Representing All Molecular Subtypes. EBioMedicine 2015; 2: 1351-1363. 
[303] Candolfi M, Curtin JF, Nichols WS, et al. Intracranial glioblastoma models in preclinical neuro-oncology: Neuropathological characterization and tumor progression. J Neurooncol 2007; 85: 133-148.

[304] Szatmári T, Lumniczky K, Désaknai S, et al. Detailed characterization of the mouse glioma GL261 tumor model for experimental glioblastoma therapy. Cancer Sci 2006; 97: 546-553.

[305] Newcomb EW, Zagzag D. The Murine GL261 Glioma Experimental Model to Assess Novel Brain Tumor Treatments. In: Van Meir EG (ed) CNS Cancer. Totowa, NJ: Humana Press, pp. 227-241.

[306] Wang J, Miletic H, Sakariassen PØ, et al. A reproducible brain tumour model established from human glioblastoma biopsies. BMC Cancer 2009; 9: 465.

[307] Mahesparan R, Read T-A, Lund-Johansen M, et al. Expression of extracellular matrix components in a highly infiltrative in vivo glioma model. Acta Neuropathol 2003; 105: $49-57$.

[308] Ross DT, Scherf U, Eisen MB, et al. Systematic variation in gene expression patterns in human cancer cell lines. Nat Genet 2000; 24: 227-35.

[309] Jacobs VL, Valdes PA, Hickey WF, et al. Current review of in vivo GBM rodent models: emphasis on the CNS-1 tumour model. ASN Neuro 2011; 3: e00063.

[310] Clark MJ, Homer N, O’Connor BD, et al. U87MG decoded: the genomic sequence of a cytogenetically aberrant human cancer cell line. PLoS Genet 2010; 6: e1000832.

[311] Allen M, Bjerke M, Edlund H, et al. Origin of the U87MG glioma cell line: Good news and bad news. Sci Transl Med 2016; 8: 354re3.

[312] Yu S-C, Ping Y-F, Yi L, et al. Isolation and characterization of cancer stem cells from a human glioblastoma cell line U87. Cancer Lett 2008; 265: 124-134.

[313] Lee J, Kotliarova S, Kotliarov Y, et al. Tumor stem cells derived from glioblastomas cultured in bFGF and EGF more closely mirror the phenotype and genotype of primary tumors than do serum-cultured cell lines. Cancer Cell 2006; 9: 391-403.

[314] Oh T, Fakurnejad S, Sayegh ET, et al. Immunocompetent murine models for the study of glioblastoma immunotherapy. J Transl Med 2014; 12: 107.

[315] Yi L, Zhou C, Wang B, et al. Implantation of GL261 neurospheres into C57/BL6 mice: A more reliable syngeneic graft model for research on glioma-initiating cells. Int $J$ Oncol 2013; 43: 477-484.

[316] Wu A, Oh S, Wiesner SM, et al. Persistence of CD133+ cells in human and mouse glioma cell lines: detailed characterization of GL261 glioma cells with cancer stem cell-like properties. Stem Cells Dev 2008; 17: 173-84. 
[317] Maes W, Van Gool SW. Experimental immunotherapy for malignant glioma: Lessons from two decades of research in the GL261 model. Cancer Immunol Immunother 2011; 60: 153-160.

[318] Villa GR, Mischel PS. Shared Intelligence: A Patient-Derived, Deeply Characterized Glioblastoma Cell Line Resource. EBioMedicine 2015; 2: 1274-1275.

[319] Gillet J-P, Varma S, Gottesman MM. The Clinical Relevance of Cancer Cell Lines. JNCI J Natl Cancer Inst 2013; 105: 452-458.

[320] Patrizii M, Bartucci M, Pine SR, et al. Utility of Glioblastoma Patient-Derived Orthotopic Xenografts in Drug Discovery and Personalized Therapy. Front Oncol 2018; 8: 23.

[321] Goodspeed A, Heiser LM, Gray JW, et al. Tumor-Derived Cell Lines as Molecular Models of Cancer Pharmacogenomics. Mol Cancer Res 2016; 14: 3-13.

[322] Hunn MK, Farrand KJ, Broadley KWR, et al. Vaccination with irradiated tumor cells pulsed with an adjuvant that stimulates NKT cells is an effective treatment for glioma. Clin Cancer Res 2012; 18: 6446-6459.

[323] Authier A, Farrand KJ, Broadley KWR, et al. Enhanced immunosuppression by therapy-exposed glioblastoma multiforme tumor cells. Int J Cancer 2015; 136: 25662578 .

[324] Ryan DJ, Watts C. In Vitro Models of Brain Cancer. In: Emerging Concepts in NeuroOncology. London: Springer London, pp. 75-86.

[325] Bigner SH, Mark J, Bigner DD. Chromosomal progression of malignant human gliomas from biopsy to establishment as permanent lines in vitro. Cancer Genet Cytogenet 1987; 24: 163-176.

[326] Rey JA, Bello MJ, de Campos JM, et al. Cytogenetic follow-up from direct preparation to advanced in vitro passages of a human malignant glioma. Cancer Genet Cytogenet 1989; 41: 175-83.

[327] Pandita A, Aldape KD, Zadeh G, et al. Contrasting in Vivo and in Vitro Fates of Glioblastoma Cell Subpopulations with Amplified EGFR. Genes Chromosom Cancer 2004; 39: 29-36.

[328] Monterey MD, Szerlip NJ, Mathupala SP. Low-cost media formulation for culture of brain tumor spheroids (neurospheres). Biotechniques 2013; 55: 83-88.

[329] Fael Al-Mayhani TM, Ball SLR, Zhao JW, et al. An efficient method for derivation and propagation of glioblastoma cell lines that conserves the molecular profile of their original tumours. J Neurosci Methods 2009; 176: 192-199.

[330] Anton D, Burckel H, Josset E, et al. Three-dimensional cell culture: A breakthrough in 
vivo. Int J Mol Sci 2015; 16: 5517-5527.

[331] Gomez-Roman N, Stevenson K, Gilmour L, et al. A novel 3D human glioblastoma cell culture system for modeling drug and radiation responses. Neuro Oncol 2017; 19: 229-241.

[332] Hoarau-Véchot J, Rafii A, Touboul C, et al. Halfway between 2D and Animal Models: Are 3D Cultures the Ideal Tool to Study Cancer-Microenvironment Interactions? Int $J$ Mol Sci 2018; 19: 181.

[333] Osmond TL, Broadley KWR, McConnell MJ. Glioblastoma cells negative for the antiCD133 antibody AC133 express a truncated variant of the CD133 protein. Int J Mol Med 2010; 25: 883-888.

[334] Castro ML, McConnell MJ, Herst PM. Radiosensitisation by pharmacological ascorbate in glioblastoma multiforme cells, human glial cells, and HUVECs depends on their antioxidant and DNA repair capabilities and is not cancer specific. Free Radic Biol Med 2014; 74: 200-209.

[335] Nehoff H, Parayath NN, Mcconnell MJ, et al. A combination of tyrosine kinase inhibitors, crizotinib and dasatinib for the treatment of glioblastoma multiforme. Oncotarget 2015; 6: 37948 .

[336] Untergasser A, Cutcutache I, Koressaar T, et al. Primer3-new capabilities and interfaces. Nucleic Acids Res 2012; 40: 1-12.

[337] Attané C, Daviaud D, Dray C, et al. Apelin stimulates glucose uptake but not lipolysis in human adipose tissue ex vivo. J Mol Endocrinol 2011; 46: 21-8.

[338] Niu X, Zhang T, Liao L, et al. The von Hippel-Lindau tumor suppressor protein regulates gene expression and tumor growth through histone demethylase JARID1C. Oncogene 2012; 31: 776-786.

[339] King C a., Li X, Barbachano-Guerrero A, et al. STAT3 Regulates Lytic Activation of Kaposi's Sarcoma-associated Herpesvirus. J Virol 2015; 89: JVI.02008-15.

[340] Kenig S, Faoro V, Bourkoula E, et al. Topoisomerase II $\beta$ mediates the resistance of glioblastoma stem cells to replication stress-inducing drugs. Cancer Cell Int 2016; 16: 58 .

[341] Wang G, Anini Y, Wei W, et al. Apelin, a New Enteric Peptide: Localization in the Gastrointestinal Tract, Ontogeny, and Stimulation of Gastric Cell Proliferation and of Cholecystokinin Secretion. Endocrinology 2004; 145: 1342-1348.

[342] Nishide K, Nakatani Y, Kiyonari H, et al. Glioblastoma formation from cell population depleted of prominin1-expressing cells. PLoS One; 4. Epub ahead of print 2009. DOI: 10.1371/journal.pone.0006869. 
[343] Seagroves TN, Peacock DL, Liao D, et al. VHL deletion impairs mammary alveologenesis but is not sufficient for mammary tumorigenesis. Am J Pathol 2010; 176: 2269-2282.

[344] Livak KJ, Schmittgen TD. Analysis of relative gene expression data using real-time quantitative PCR and the $2^{\wedge}(-\Delta \Delta \mathrm{CT})$ method. Methods 2001; 25: 402-408.

[345] Schmittgen TD, Livak KJ. Analyzing real-time PCR data by the comparative CT method. Nat Protoc 2008; 3: 1101-1108.

[346] Valente V, Teixeira SA, Neder L, et al. Selection of suitable housekeeping genes for expression analysis in glioblastoma using quantitative RT-PCR. BMC Mol Biol 2009; 10: 17 .

[347] Aithal MGS, Rajeswari N. Validation of housekeeping genes for gene expression analysis in glioblastoma using quantitative real-time polymerase chain reaction. Brain tumor Res Treat 2015; 3: 24-9.

[348] Murza A, Belleville K, Longpré J-M, et al. Stability and degradation patterns of chemically modified analogs of apelin-13 in plasma and cerebrospinal fluid. Biopolymers 2014; 102: 297-303.

[349] Maloney PR, Khan P, Hedrick M, et al. Discovery of 4-oxo-6-((pyrimidin-2ylthio)methyl)-4H-pyran-3-yl 4-nitrobenzoate (ML221) as a functional antagonist of the apelin (APJ) receptor. Bioorganic Med Chem Lett 2012; 22: 6656-6660.

[350] Francescone III R a., Faibish M, Shao R. A Matrigel-Based Tube Formation Assay to Assess the Vasculogenic Activity of Tumor Cells. J Vis Exp 2011; 2-5.

[351] Chan A, Singh AJ, Northcote PT, et al. Inhibition of human vascular endothelial cell migration and capillary-like tube formation by the microtubule-stabilizing agent peloruside A. Invest New Drugs 2015; 33: 564-574.

[352] Chan A. Cellular Targets and Tumour Suppressive Mechanisms of Peloruside A By. Victoria University of Wellington, http://hdl.handle.net/10063/2533 (2012).

[353] Gebäck T, Schulz MMP, Koumoutsakos P, et al. TScratch: A novel and simple software tool for automated analysis of monolayer wound healing assays. Biotechniques 2009; 46: 265-274.

[354] JoVE, Cambridge M. The Transwell Migration Assay. JoVE Science Education Database 2016; 2-3.

[355] Field CS. Attenuation of Immune Suppression to Complement Glioma Immunotherapy. Victoria University of Wellington, http://hdl.handle.net/10063/4920 (2016).

[356] Chung CT, Niemela SL, Miller RH. One-step preparation of competent Escherichia coli: transformation and storage of bacterial cells in the same solution. Proc Natl Acad 
Sci 1989; 86: 2172-2175.

[357] Liu Q, Hu T, He L, et al. Genetic targeting of sprouting angiogenesis using AplnCreER. Nat Commun 2015; 6: 6020.

[358] Kidoya H, Takakura N. Biology of the apelin-APJ axis in vascular formation. $J$ Biochem 2012; 152: 125-131.

[359] Carvalho KC, Cunha IW, Rocha RM, et al. GLUT1 expression in malignant tumors and its use as an immunodiagnostic marker. Clinics (Sao Paulo) 2011; 66: 965-972.

[360] Nagamatsu S, Sawa H, Wakizaka A, et al. Expression of Facilitative Glucose Transporter Isoforms in Human Brain Tumors. J Neurochem 1993; 61: 2048-2053.

[361] Winkler EA, Nishida Y, Sagare AP, et al. GLUT1 reductions exacerbate Alzheimer's disease vasculo-neuronal dysfunction and degeneration. Nat Neurosci 2015; 18: 521530 .

[362] Labak CM, Wang PY, Arora R, et al. Glucose transport: Meeting the metabolic demands of cancer, and applications in glioblastoma treatment. Am J Cancer Res 2016; 6: $1599-1608$.

[363] Lee WL, Klip A. Shuttling glucose across brain microvessels, with a little help from GLUT1 and AMP kinase. Focus on 'AMP kinase regulation of sugar transport in brain capillary endothelial cells during acute metabolic stress'. AJP Cell Physiol 2012; 303: C803-C805.

[364] Pardrige WM, Boado RJ, Farrell CR. Brain-type Glucose Transporter the Blood-Brain Barrier (GLUT-1) Is Selectively Localized to the Blood-Brain Barrier. J Biol Chem 1990; 265: 18035-18040.

[365] Yeh W, Lin C, Fu W. Enhancement of Glucose Transporter Expression of Brain Endothelial Cells by Vascular Endothelial Growth Factor Derived from Glioma Exposed to Hypoxia. 2008; 73: 170-177.

[366] Jensen RL, Chkheidze R. The Role of Glucose Transporter-1 (GLUT-1) in Malignant Gliomas. In: Hayat MA (ed) Tumors of the Central Nervous System. Dordrecht: Springer Netherlands, pp. 99-108.

[367] Brennan CW, Verhaak RGW, McKenna A, et al. The somatic genomic landscape of glioblastoma. Cell 2013; 155: 462-477.

[368] van Staveren WCG, Solís DYW, Hébrant A, et al. Human cancer cell lines: Experimental models for cancer cells in situ? For cancer stem cells? Biochim Biophys Acta 2009; 1795: 92-103.

[369] Vincent KM, Findlay SD, Postovit LM. Assessing breast cancer cell lines as tumour models by comparison of mRNA expression profiles. Breast Cancer Res 2015; 17: 1- 
12.

[370] Samura M, Morikage N, Suehiro K, et al. Combinatorial Treatment with Apelin-13 Enhances the Therapeutic Efficacy of a Preconditioned Cell-Based Therapy for Peripheral Ischemia. Sci Rep 2016; 6: 19379.

[371] Bülbül M, Sinen O, Gök M, et al. Apelin-13 inhibits gastric motility through vagal cholinergic pathway in rats. Am J Physiol Liver Physiol 2018; 314: G201-G210.

[372] Takakura N, Kidoya H. Maturation of blood vessels by haematopoietic stem cells and progenitor cells: Involvement of apelin/APJ and angiopoietin/Tie2 interactions in vessel caliber size regulation. Thromb Haemost 2009; 101: 999-1005.

[373] Kleinz MJ, Skepper JN, Davenport AP. Immunocytochemical localisation of the apelin receptor, APJ, to human cardiomyocytes, vascular smooth muscle and endothelial cells. Regul Pept 2005; 126: 233-240.

[374] McLean DL, Kim J, Kang Y, et al. Apelin/APJ signaling is a critical regulator of statin effects in vascular endothelial cells-brief report. Arterioscler Thromb Vasc Biol 2012; 32: $2640-2643$.

[375] Busch R, Strohbach A, Pennewitz M, et al. Regulation of the endothelial apelin/APJ system by hemodynamic fluid flow. Cell Signal 2015; 27: 1286-1296.

[376] Itinteang T, Tan ST, Brasch HD, et al. Primitive erythropoiesis in infantile haemangioma. Br J Dermatol 2011; 164: 1097-1100.

[377] Sidney LE, Branch MJ, Dunphy SE, et al. Concise review: Evidence for CD34 as a common marker for diverse progenitors. Stem Cells 2014; 32: 1380-1389.

[378] Fina L, Molgaard H V, Robertson D, et al. Expression of the CD34 gene in vascular endothelial cells. Blood 1990; 75: 2417-26.

[379] Owens GK. Regulation of differentiation of vascular smooth muscle cells. Physiol Rev 1995; 75: 487-517.

[380] Steel R, Day D. Increased apoptosis and secretion of tryptase by mast cells in infantile haemangioma treated with propranolol. Pathology 2014; 46: 496-500.

[381] Itinteang T, Brasch HD, Tan ST, et al. Expression of components of the reninangiotensin system in proliferating infantile haemangioma may account for the propranolol-induced accelerated involution. J Plast Reconstr Aesthetic Surg 2011; 64: 759-765.

[382] Bowman RL, Wang Q, Carro A, et al. GlioVis data portal for visualization and analysis of brain tumor expression datasets. Neuro-Oncology 2017; 19: 139-141.

[383] Ceccarelli M, Barthel FP, Malta TM, et al. Molecular Profiling Reveals Biologically 
Discrete Subsets and Pathways of Progression in Diffuse Glioma. Cell 2016; 164 : 550-563.

[384] Guo Y, Sheng Q, Li J, et al. Large Scale Comparison of Gene Expression Levels by Microarrays and RNAseq Using TCGA Data. PLoS One 2013; 8: e71462.

[385] Shah N, Feng X, Lankerovich M, et al. Data from Ivy GAP. Cancer Imaging Arch. Epub ahead of print 2016. DOI: 10.7937/k9/tcia.2016.xlwan6nl.

[386] Darmanis S, Sloan SA, Croote D, et al. Single-Cell RNA-Seq Analysis of Infiltrating Neoplastic Cells at the Migrating Front of Human Glioblastoma. Cell Rep 2017; 21: 1399-1410.

[387] Darmanis S, Sloan SA, Zhang Y, et al. A survey of human brain transcriptome diversity at the single cell level. Proc Natl Acad Sci 2015; 112: 7285-7290.

[388] Hinkle DE, Wiersma W, Jurs SG. Applied Statistics for the Behavioral Sciences. 5th Ed. Belmont, CA, United States: Houghton Mifflin, 2002.

[389] Boado RJ, Black KL, Pardridge WM. Gene expression of GLUT3 and GLUT1 glucose transporters in human brain tumors. Mol Brain Res 1994; 27: 51-57.

[390] Martínez-González A, Calvo GF, Pérez Romasanta LA, et al. Hypoxic Cell Waves Around Necrotic Cores in Glioblastoma: A Biomathematical Model and Its Therapeutic Implications. Bull Math Biol 2012; 74: 2875-2896.

[391] Bache M, Rot S, Kessler J, et al. mRNA expression levels of hypoxia-induced and stem cell-associated genes in human glioblastoma. Oncol Rep 2015; 33: 3155-3161.

[392] Ausman JI, Shapiro WR, Rall DP. Studies on the chemotherapy of experimental brain tumors: development of an experimental model. Cancer Res 1970; 30: 2394-400.

[393] De Mota N, Lenkei Z, Llorens-Cortès C. Cloning, pharmacological characterization and brain distribution of the rat apelin receptor. Neuroendocrinology 2000; 72: 400407.

[394] Deng C, Chen H, Yang N, et al. Apela regulates fluid homeostasis by binding to the APJ receptor to activate Gi signaling. J Biol Chem 2015; 290: 18261-18268.

[395] Cavallo MG, Sentinelli F, Barchetta I, et al. Altered glucose homeostasis is associated with increased serum apelin levels in type 2 diabetes mellitus. PLoS One 2012; 7: e51236.

[396] Yi Y, Tsai SH, Cheng JC, et al. APELA promotes tumour growth and cell migration in ovarian cancer in a p53-dependent manner. Gynecol Oncol 2017; 147: 663-671.

[397] Shen H, Decollogne S, Dilda PJ, et al. Dual-targeting of aberrant glucose metabolism in glioblastoma. J Exp Clin Cancer Res 2015; 34: 14. 
[398] Rodriguez FJ, Orr B a, Ligon KL, et al. Neoplastic cells are a rare component in human glioblastoma microvasculature. Oncotarget 2012; 3: 98-106.

[399] Ferrarelli LK. Hypoxia micromanages glioma. Sci Signal 2016; 9: ec11-ec11.

[400] Brescia P, Richichi C, Pelicci G. Current Strategies for Identification of Glioma Stem Cells: Adequate or Unsatisfactory? J Oncol 2012; 2012: 1-10.

[401] Novakova V, Sandhu GS, Dragomir-Daescu D, et al. Apelinergic system in endothelial cells and its role in angiogenesis in myocardial ischemia. Vascul Pharmacol 2016; 76: $1-10$.

[402] Cooper R, Sarioğlu S, Sökmen S, et al. Glucose transporter-1 (GLUT-1): a potential marker of prognosis in rectal carcinoma? Br J Cancer 2003; 89: 870-876.

[403] Jin P, Shin S-H, Chun Y-S, et al. Astrocyte-derived CCL20 reinforces HIF-1-mediated hypoxic responses in glioblastoma by stimulating the CCR6-NF- $\mathrm{BB}$ signaling pathway. Oncogene 2018; 37: 3070-3087.

[404] Harris AL. Hypoxia - a Key Regulatory Factor in Tumour Growth. Nat Rev Cancer 2002; $2: 38-47$.

[405] Zhang L, Takara K, Yamakawa D, et al. Apelin as a marker for monitoring the tumor vessel normalization window during antiangiogenic therapy. Cancer Sci 2016; 107 : $36-44$.

[406] Jamal M, Rath BH, Tsang PS, et al. The brain microenvironment preferentially enhances the radioresistance of CD133(+) glioblastoma stem-like cells. Neoplasia 2012; 14: 150-8.

[407] William D, Mokri P, Schneider B, et al. Optimization of Glioblastoma multiforme in vitro culture conditions for preservation of EGFR gene amplification. Klin Pädiatrie 2015; 227: A11.

[408] Talasila KM, Soentgerath A, Euskirchen P, et al. EGFR wild-type amplification and activation promote invasion and development of glioblastoma independent of angiogenesis. Acta Neuropathol 2013; 125: 683-698.

[409] Taylor TE, Furnari FB, Cavenee WK. Targeting EGFR for treatment of glioblastoma: molecular basis to overcome resistance. Curr Cancer Drug Targets 2012; 12: 197209.

[410] Gan HK, Kaye AH, Luwor RB. The EGFRvIII variant in glioblastoma multiforme. $J$ Clin Neurosci 2009; 16: 748-754.

[411] DeCarvalho AC, Nelson K, Lemke N, et al. Gliosarcoma stem cells undergo glial and mesenchymal differentiation in vivo. Stem Cells 2010; 28: 181-190. 
[412] Kidoya H, Naito H, Muramatsu F, et al. APJ Regulates Parallel Alignment of Arteries and Veins in the Skin. Dev Cell 2015; 33: 247-259.

[413] Ando W, Yokomori H, Otori K, et al. The Apelin Receptor APJ in Hematopoietic Stem Cells/Progenitor Cells in the Early Stage of Non-Alcoholic Steatohepatitis. J Clin Med Res 2017; 9: 809-811.

[414] Sofroniew M V., Vinters H V. Astrocytes: Biology and pathology. Acta Neuropathol 2010; 119: 7-35.

[415] Vogel C, De Sousa Abreu R, Ko D, et al. Sequence signatures and mRNA concentration can explain two-thirds of protein abundance variation in a human cell line. Mol Syst Biol 2010; 6: 1-9.

[416] Tian Q, Stepaniants SB, Mao M, et al. Integrated Genomic and Proteomic Analyses of Gene Expression in Mammalian Cells. Mol Cell Proteomics 2004; 3: 960-969.

[417] Lundberg E, Fagerberg L, Klevebring D, et al. Defining the transcriptome and proteome in three functionally different human cell lines. Mol Syst Biol 2010; 6: 1-10.

[418] Schwanhüusser B, Busse D, Li N, et al. Global quantification of mammalian gene expression control. Nature 2011; 473: 337-342.

[419] Maier T, Güell M, Serrano L. Correlation of mRNA and protein in complex biological samples. FEBS Lett 2009; 583: 3966-3973.

[420] Mendes P. Emerging bioinformatics for the metabolome. Brief Bioinform 2002; 3: $134-45$.

[421] Koussounadis A, Langdon SP, Um IH, et al. Relationship between differentially expressed mRNA and mRNA-protein correlations in a xenograft model system. Sci Rep 2015; 5: 10775.

[422] Schiffer D, Mellai M, Annovazzi L, et al. Stem Cell Niches in Glioblastoma: A Neuropathological View. Biomed Res Int 2014; 2014: 1-7.

[423] Čunderlíková B. Issues to be considered when studying cancer in vitro. Crit Rev Oncol Hematol 2013; 85: 95-111.

[424] Hagemann C, Meyer C, Stojic J, et al. High efficiency transfection of glioma cell lines and primary cells for overexpression and RNAi experiments. J Neurosci Methods 2006; 156: 194-202.

[425] Masri B, Morin N, Pedebernade L, et al. The apelin receptor is coupled to Gi1 or Gi2 protein and is differentially desensitized by apelin fragments. J Biol Chem 2006; 281: $18317-18326$.

[426] Wang I-NE, Wang X, Ge X, et al. Apelin enhances directed cardiac differentiation of 
mouse and human embryonic stem cells. PLoS One 2012; 7: e38328.

[427] Justus CR, Leffler N, Ruiz-Echevarria M, et al. In vitro Cell Migration and Invasion Assays. J Vis Exp 2014; 1-8.

[428] Edwards SA, Adamson ED. Isolation of a clone of F9 teratocarcinoma cells 'naturally' resistant to G418. J Cell Physiol 1987; 133: 46-54.

[429] Reeves PJ, Kim J-M, Khorana HG. Structure and function in rhodopsin: a tetracyclineinducible system in stable mammalian cell lines for high-level expression of opsin mutants. Proc Natl Acad Sci U S A 2002; 99: 13413-8.

[430] Zhou N, Zhang X, Fan X, et al. The N-terminal domain of APJ, a CNS-based coreceptor for HIV-1, is essential for its receptor function and coreceptor activity. Virology 2003; 317: 84-94.

[431] Chapman NA, Dupré DJ, Rainey JK. The apelin receptor: physiology, pathology, cell signalling, and ligand modulation of a peptide-activated class A GPCR. Biochem Cell Biol 2014; 92: 431-440.

[432] El Messari S, Iturrioz X, Fassot C, et al. Functional dissociation of apelin receptor signaling and endocytosis: Implications for the effects of apelin on arterial blood pressure. J Neurochem 2004; 90: 1290-1301.

[433] Chen X, Bai B, Tian Y, et al. Identification of Serine 348 on the Apelin Receptor as a Novel Regulatory Phosphorylation Site in Apelin-13-induced G Protein-independent Biased Signaling. J Biol Chem 2014; 289: 31173-31187.

[434] Kawahara H, Naito H, Takara K, et al. Tumor endothelial cell-specific drug delivery system using apelin-conjugated liposomes. PLoS One 2013; 8: e65499.

[435] Witusik-Perkowska M, Rieske P, Hułas-Bigoszewska K, et al. Glioblastoma-derived spheroid cultures as an experimental model for analysis of EGFR anomalies. $J$ Neurooncol 2011; 102: 395-407.

[436] Pope GR, Tilve S, McArdle CA, et al. Agonist-induced internalization and desensitization of the apelin receptor. Mol Cell Endocrinol 2016; 437: 108-119.

[437] Li H, Yang BB. Stress response of glioblastoma cells mediated by miR-17-5p targeting PTEN and the passenger strand miR-17-3p targeting MDM2. Oncotarget 2012; 3: $1653-68$.

[438] Spriggs KA, Bushell M, Willis AE. Translational Regulation of Gene Expression during Conditions of Cell Stress. Mol Cell 2010; 40: 228-237.

[439] Wouters BG, Koritzinsky M, Chiu RK, et al. Modulation of cell death in the tumor microenvironment. Semin Radiat Oncol 2003; 13: 31-41. 
[440] Bar EE. Glioblastoma, Cancer Stem Cells and Hypoxia. Brain Pathol 2011; 21: 119129.

[441] Qiu Y, Li P, Ji C. Cell death conversion under hypoxic condition in tumor development and therapy. Int J Mol Sci 2015; 16: 25536-25551.

[442] Ikemori RY, Machado CML, Furuzawa KM, et al. Galectin-3 up-regulation in hypoxic and nutrient deprived microenvironments promotes cell survival. PLoS One 2014; 9: $1-12$.

[443] Bacher M, Schrader J, Thompson N, et al. Up-Regulation of Macrophage Migration Inhibitory Factor Gene and Protein Expression in Glial Tumor Cells during Hypoxic and Hypoglycemic Stress Indicates a Critical Role for Angiogenesis in Glioblastoma Multiforme. Am J Pathol 2003; 162: 11-17.

[444] Noch E, Bookland M, Khalili K. Astrocyte-elevated gene-1 (AEG-1) induction by hypoxia and glucose deprivation in glioblastoma. Cancer Biol Ther 2011; 11: 32-39.

[445] Griguer CE, Oliva CR, Gobin E, et al. CD133 is a marker of bioenergetic stress in human glioma. PLoS One 2008; 3: 1-11.

[446] Tabu K, Bizen N, Taga T, et al. Gene Regulation of Prominin-1 (CD133) in Normal and Cancerous Tissues. In: Corbeil D (ed) Prominin-1 (CD133): New Insights on Stem \& Cancer Stem Cell Biology. New York, NY: Springer New York, pp. 73-85.

[447] Santra M, Liu XS, Santra S, et al. Ectopic expression of doublecortin protects adult rat progenitor cells and human glioma cells from severe oxygen and glucose deprivation. Neuroscience 2006; 142: 739-752.

[448] Xie K, Huang S. Regulation of cancer metastasis by stress pathways. Clin Exp Metastasis 2003; 20: 31-43.

[449] Seidel S, Garvalov BK, Wirta V, et al. A hypoxic niche regulates glioblastoma stem cells through hypoxia inducible factor $2 \alpha$. Brain 2010; 133: 983-995.

[450] Mayer A, Schneider F, Vaupel P, et al. Differential expression of HIF-1 in glioblastoma multiforme and anaplastic astrocytoma. Int J Oncol 2012; 41: 12601270 .

[451] Foussal C, Lairez O, Calise D, et al. Activation of catalase by apelin prevents oxidative stress-linked cardiac hypertrophy. FEBS Lett 2010; 584: 2363-70.

[452] Xie F, Wu D, Huang S, et al. The endoplasmic reticulum stress-autophagy pathway is involved in apelin-13-induced cardiomyocyte hypertrophy in vitro. Acta Pharmacol Sin 2017; 38: 1589-1600.

[453] Chen C, Pore N, Behrooz A, et al. Regulation of glut1 mRNA by hypoxia-inducible factor-1: Interaction between H-ras and hypoxia. J Biol Chem 2001; 276: 9519-9525. 
[454] Wang E, Zhang C, Polavaram N, et al. The role of Factor Inhibiting HIF (FIH-1) in inhibiting HIF-1 transcriptional activity in glioblastoma multiforme. PLoS One 2014; 9: $1-9$.

[455] Choy W, Nagasawa DT, Trang A, et al. CD133 as a marker for regulation and potential for targeted therapies in glioblastoma Multiforme. Neurosurg Clin N Am 2012; 23: 391-405.

[456] Li Z. CD133: a stem cell biomarker and beyond. Exp Hematol Oncol 2013; 2: 17.

[457] Bar EE, Lin A, Mahairaki V, et al. Hypoxia increases the expression of stem-cell markers and promotes clonogenicity in glioblastoma neurospheres. Am J Pathol 2010; 177: 1491-1502.

[458] Chen H, Luo Z, Dong L, et al. CD133/Prominin-1-Mediated Autophagy and Glucose Uptake Beneficial for Hepatoma Cell Survival. PLoS One 2013; 8: 1-13.

[459] Jang J-W, Song Y, Kim S-H, et al. Potential mechanisms of CD133 in cancer stem cells. Life Sci 2017; 184: 25-29.

[460] Kanzawa T, Germano IM, Kondo Y, et al. Inhibition of telomerase activity in malignant glioma cells correlates with their sensitivity to temozolomide. Br J Cancer 2003; 89: 922-929.

[461] Kanzawa T, Germano IM, Komata T, et al. Role of autophagy in temozolomideinduced cytotoxicity for malignant glioma cells. Cell Death Differ 2004; 11: 448-457.

[462] Qiu ZK, Shen D, Chen YS, et al. Enhanced MGMT expression contributes to temozolomide resistance in glioma stem-like cells. Chin J Cancer 2014; 33: 115-122.

[463] Yuan JS, Reed A, Chen F, et al. Statistical analysis of real-time PCR data. BMC Bioinformatics 2006; 7: 1-12.

[464] Ghasemi A, Zahediasl S. Normality tests for statistical analysis: A guide for nonstatisticians. Int J Endocrinol Metab 2012; 10: 486-489.

[465] Strober W. Trypan Blue Exclusion Test of Cell Viability. Curr Protoc Immunol 2015; 111: 1-3.

[466] Even MS, Sandusky CB, Barnard ND. Serum-free hybridoma culture: Ethical, scientific and safety considerations. Trends Biotechnol 2006; 24: 105-108.

[467] Brunner D, Frank J, Appl H, et al. Serum-free cell culture: the serum-free media interactive online database. ALTEX 2010;27: 53-62.

[468] Jochems CEA, van der Valk JBF, Stafleu FR, et al. The use of fetal bovine serum: ethical or scientific problem? Altern Lab Anim 2002; 30: 219-27.

[469] Angelastro JM, Lamé MW. Overexpression of CD133 promotes drug resistance in C6 
glioma cells. Mol Cancer Res 2010; 8: 1105-15.

[470] Kucharzewska P, Belting M. Emerging roles of extracellular vesicles in the adaptive response of tumour cells to microenvironmental stress. J Extracell vesicles 2013; 2: 20304.

[471] Li Z, Bao S, Wu Q, et al. Hypoxia-Inducible Factors Regulate Tumorigenic Capacity of Glioma Stem Cells. Cancer 2010; 15: 501-513.

[472] Said HM, Hagemann C, Stojic J, et al. GAPDH is not regulated in human glioblastoma under hypoxic conditions. BMC Mol Biol 2007; 8: 55.

[473] Simiantonaki N, Jayasinghe C, Michel-Schmidt R, et al. Hypoxia-induced epithelial VEGF-C/VEGFR-3 upregulation in carcinoma cell lines. Int J Oncol 2008; 32: 58592.

[474] Said HM, Hagemann C, Staab A, et al. Expression patterns of the hypoxia-related genes osteopontin, CA9, erythropoietin, VEGF and HIF-1 $\alpha$ in human glioma in vitro and in vivo. Radiother Oncol 2007; 83: 398-405.

[475] Said HM, Stein S, Hagemann C, et al. Oxygen-dependent regulation of NDRG1 in human glioblastoma cells in vitro and in vivo. Oncol Rep 2009; 21: 237-246.

[476] Mineo M, Ricklefs F, Rooj AK, et al. The Long Non-coding RNA HIF1A-AS2 Facilitates the Maintenance of Mesenchymal Glioblastoma Stem-like Cells in Hypoxic Niches. Cell Rep 2016; 15: 2500-2509.

[477] Guo M, Cai C, Zhao G, et al. Hypoxia promotes migration and induces CXCR4 expression via HIF-1 $\alpha$ activation in human osteosarcoma. PLoS One 2014; 9: 1-9.

[478] Wenger RH, Kvietiko I, Rolfs A, et al. Hypoxia-inducible factor-1 $\alpha$ is regulated at the post-mRNA level. Kidney Int 1997; 51: 560-563.

[479] Masoud GN, Li W. HIF-1 $\alpha$ pathway: Role, regulation and intervention for cancer therapy. Acta Pharm Sin B 2015; 5: 378-389.

[480] Zhang Y, Liu Q, Wang F, et al. Melatonin antagonizes hypoxia-mediated glioblastoma cell migration and invasion via inhibition of HIF-1 $\alpha$. J Pineal Res 2013; 55: 121-30.

[481] Li P, Zhou C, Xu L, et al. Hypoxia enhances stemness of cancer stem cells in Glioblastoma: An in vitro study. Int J Med Sci 2013; 10: 399-407.

[482] Jiang H, White EJ, Conrad C, et al. Chapter 13 Autophagy Pathways in Glioblastoma. In: Klionsky DJ (ed) Autophagy in Disease and Clinical Applications, Part C. Elsevier Inc., pp. 273-286.

[483] Hu YL, DeLay M, Jahangiri A, et al. Hypoxia-induced autophagy promotes tumor cell survival and adaptation to antiangiogenic treatment in glioblastoma. Cancer Res 2012; 
72: $1773-1783$.

[484] Kasai A, Ishimaru Y, Kinjo T, et al. Apelin is a crucial factor for hypoxia-induced retinal angiogenesis. Arterioscler Thromb Vasc Biol 2010; 30: 2182-2187.

[485] Hardee ME, Zagzag D. Mechanisms of Glioma-Associated Neovascularization. Am J Pathol 2012; 181: 1-16.

[486] Chen Y, Jing Z, Luo C, et al. Vasculogenic mimicry-potential target for glioblastoma therapy: An in vitro and in vivo study. Med Oncol 2012; 29: 324-331.

[487] El Hallani S, Boisselier B, Peglion F, et al. A new alternative mechanism in glioblastoma vascularization: tubular vasculogenic mimicry. Brain 2010; 133: $973-$ 982.

[488] Candice A. Shaifer, Jianhua Huang and PCL. Glioblastoma cells incorporate into tumor vasculature and contribute to vascular radioresistance. Int J Cancer 2011; 127: 2063-2075.

[489] Francescone R, Scully S, Bentley B, et al. Glioblastoma-derived tumor cells induce vasculogenic mimicry through Flk-1 protein activation. $J$ Biol Chem 2012; 287: 24821-24831.

[490] McGirt MJ, Chaichana KL, Gathinji M, et al. Persistent outpatient hyperglycemia is independently associated with decreased survival after primary resection of malignant brain astrocytomas. Neurosurgery 2008; 63: 286-291.

[491] Derr RL, Ye X, Islas MU, et al. Association between hyperglycemia and survival in patients with newly diagnosed glioblastoma. J Clin Oncol 2009; 27: 1082-1086.

[492] Tieu MT, Lovblom LE, McNamara MG, et al. Impact of glycemia on survival of glioblastoma patients treated with radiation and temozolomide. J Neurooncol 2015; 124: 119-126.

[493] Abdelwahab MG, Fenton KE, Preul MC, et al. The ketogenic diet is an effective adjuvant to radiation therapy for the treatment of malignant glioma. PLoS One 2012; 7: $1-7$.

[494] Woolf EC, Scheck AC. The ketogenic diet for the treatment of malignant glioma. $J$ Lipid Res 2015; 56: 5-10.

[495] Champ CE, Palmer JD, Volek JS, et al. Targeting metabolism with a ketogenic diet during the treatment of glioblastoma multiforme. J Neurooncol 2014; 117: 125-131.

[496] Schwartz K, Chang HT, Nikolai M, et al. Treatment of glioma patients with ketogenic diets: report of two cases treated with an IRB-approved energy-restricted ketogenic diet protocol and review of the literature. Cancer Metab 2015; 3: 3. 
[497] Winter SF, Loebel F, Dietrich J. Role of ketogenic metabolic therapy in malignant glioma: A systematic review. Crit Rev Oncol Hematol 2017; 112: 41-58.

[498] Bost J, Maroon J, Seyfried T, et al. The role of metabolic therapy in treating glioblastoma multiforme. Surg Neurol Int 2015; 6: 61.

[499] Boado RJ, Pardridge WM. Glucose deprivation and hypoxia increase the expression of the GLUT1 glucose transporter via a specific mRNA cis-acting regulatory element. $J$ Neurochem 2002; 80: 552-554.

[500] Bruckner BA, Ammini C V, Otal MP, et al. Regulation of brain glucose transporters by glucose and oxygen deprivation. Metabolism 1999; 48: 422-31.

[501] Kucharzewska P, Christianson HC, Belting M. Global profiling of metabolic adaptation to hypoxic stress in human glioblastoma cells. PLoS One 2015; 10: e0116740.

[502] Semenza GL. Regulation of cancer cell metabolism by hypoxia-inducible factor 1. Semin Cancer Biol 2009; 19: 12-16.

[503] Hayashi M, Sakata M, Takeda T, et al. Induction of glucose transporter 1 expression through hypoxia-inducible factor $1 \alpha$ under hypoxic conditions in trophoblast-derived cells. J Endocrinol 2004; 183: 145-154.

[504] Jelluma N, Yang X, Stokoe D, et al. Glucose withdrawal induces oxidative stress followed by apoptosis in glioblastoma cells but not in normal human astrocytes. Mol Cancer Res 2006; 4: 319-330.

[505] Goji T, Takahara K, Negishi M, et al. Cystine uptake through the cystine/glutamate antiporter $\mathrm{xCT}$ triggers glioblastoma cell death under glucose deprivation. J Biol Chem 2017; 292: 19721-19732.

[506] Eckert A, Böck BC, Tagscherer KE, et al. The PEA-15/PED protein protects glioblastoma cells from glucose deprivation-induced apoptosis via the ERK/MAP kinase pathway. Oncogene 2008; 27: 1155-1166.

[507] Elstrom RL, Bauer DE, Buzzai M, et al. Akt stimultes aerobic glycolysis in cancer cells. Cancer Res 2004; 64: 3892-3899.

[508] Ansari KI, Ogawa D, Rooj AK, et al. Glucose-Based Regulation of miR-451/AMPK Signaling Depends on the OCT1 Transcription Factor. Cell Rep 2015; 11: 902-909.

[509] Barnes D, Sato G. Serum-free cell culture: a unifying approach. Cell 1980; 22: 649655.

[510] Morfouace M, Lalier L, Bahut M, et al. Comparison of spheroids formed by rat glioma stem cells and neural stem cells reveals differences in glucose metabolism and promising therapeutic applications. J Biol Chem 2012; 287: 33664-33674. 
[511] Lee SY. Temozolomide resistance in glioblastoma multiforme. Genes Dis 2016; 3: 198-210.

[512] Chakravarti A, Erkkinen MG, Nestler U, et al. Temozolomide-mediated radiation enhancement in glioblastoma: A report on underlying mechanisms. Clin Cancer Res 2006; 12: 4738-4746.

[513] Friedman HS, Kerby T, Calvert H. Temozolomide and treatment of malignant glioma. Clin Cancer Res 2000; 6: 2585-97.

[514] Martinho O, Vilaça N, Castro PJG, et al. In vitro and in vivo studies of temozolomide loading in zeolite structures as drug delivery systems for glioblastoma. RSC Adv 2015; 5: 28219-28227.

[515] Agarwala SS. Temozolomide, a Novel Alkylating Agent with Activity in the Central Nervous System, May Improve the Treatment of Advanced Metastatic Melanoma. Oncologist 2000; 5: 144-151.

[516] Zhou Q, Cao J, Chen L. Apelin/APJ system: A novel therapeutic target for oxidative stress-related inflammatory diseases (Review). Int J Mol Med 2016; 37: 1159-1169.

[517] Yin H, Zhou Y, Wen C, et al. Curcumin sensitizes glioblastoma to temozolomide by simultaneously generating ROS and disrupting AKT/mTOR signaling. Oncol Rep 2014; 32: 1610-1616.

[518] Seyfrid M, Marschall V, Fulda S. Reactive oxygen species contribute toward Smac mimetic/temozolomide-induced cell death in glioblastoma cells. Anticancer Drugs 2016; 27: 953-959.

[519] Jayasena SD. Aptamers: An emerging class of molecules that rival antibodies in diagnostics. Clin Chem 1999; 45: 1628-1650.

[520] Brody EN, Gold L. Aptamers as therapeutic and diagnostic agents. J Biotechnol 2000; 74: 5-13.

[521] Nimjee SM, Rusconi CP, Sullenger B a. Aptamers: an emerging class of therapeutics. Annu Rev Med 2005; 56: 555-583.

[522] Keefe AD, Pai S, Ellington A. Aptamers as therapeutics. Nat Rev Drug Discov 2010; 9: $537-550$.

[523] Hong P, Li W, Li J. Applications of aptasensors in clinical diagnostics. Sensors 2012; 12: $1181-1193$.

[524] Dhiman A, Kalra P, Bansal V, et al. Aptamer-based point-of-care diagnostic platforms. Sensors Actuators, B Chem 2017; 246: 535-553.

[525] Zhou W, Jimmy Huang P-J, Ding J, et al. Aptamer-based biosensors for biomedical 
diagnostics. Analyst 2014; 139: 2627.

[526] Zhou J, Rossi J. Aptamers as targeted therapeutics: Current potential and challenges. Nat Rev Drug Discov 2017; 16: 181-202.

[527] Gold L. SELEX: How It Happened and Where It will Go. J Mol Evol 2015; 81: 140143.

[528] Oliphant a R, Struhl K. An efficient method for generating proteins with altered enzymatic properties: application to beta-lactamase. Proc Natl Acad Sci U S A 1989; 86: 9094-8.

[529] Ellington AD, Szostak JW. In vitro selection of RNA molecules that bind specific ligands. Nature 1990; 346: 818-822.

[530] Tuerk C, Gold L. Systematic evolution of ligands by exponential enrichment: RNA ligands to bacteriophage T4 DNA polymerase. Science (80- ) 1990; 249: 505-510.

[531] Mondal B, Ramlal S, Lavu PSR, et al. A combinatorial systematic evolution of ligands by exponential enrichment method for selection of aptamer against protein targets. Appl Microbiol Biotechnol 2015; 99: 9791-9803.

[532] Ohuchi S. Cell-SELEX Technology. Biores Open Access 2012; 1: 265-72.

[533] Sefah K, Shangguan D, Xiong X, et al. Development of DNA aptamers using CellSELEX. Nat Protoc 2010; 5: 1169-85.

[534] Stoltenburg R, Reinemann C, Strehlitz B. SELEX-A (r)evolutionary method to generate high-affinity nucleic acid ligands. Biomol Eng 2007; 24: 381-403.

[535] Oh SS, Ahmad KM, Cho M, et al. Improving Aptamer Selection Efficiency through Volume Dilution, Magnetic Concentration, and Continuous Washing in Microfluidic Channels. Anal Chem 2011; 83: 6883-6889.

[536] Shao K, Ding W, Wang F, et al. Emulsion PCR: a high efficient way of PCR amplification of random DNA libraries in aptamer selection. PLoS One 2011; 6: e24910.

[537] Tolle F, Wilke J, Wengel J, et al. By-product formation in repetitive PCR amplification of DNA libraries during SELEX. PLoS One 2014; 9: 1-12.

[538] Musheev MU, Krylov SN. Selection of aptamers by systematic evolution of ligands by exponential enrichment: addressing the polymerase chain reaction issue. Anal Chim Acta 2006; 564: 91-6.

[539] Williams R, Peisajovich SG, Miller OJ, et al. Amplification of complex gene libraries by emulsion PCR. Nat Protoc 2006; 3: 545-550.

[540] Svobodová M, Pinto A, Nadal P, et al. Comparison of different methods for generation 
of single-stranded DNA for SELEX processes. Anal Bioanal Chem 2012; 404: 1-8.

[541] M.Espelund RAPS and KSJ. A simple method for generating single-stranded DNA probes labeled to high activities. Nucleic Acids Res 1990; 18: 535-8.

[542] Avci-Adali M, Paul A, Wilhelm N, et al. Upgrading SELEX technology by using lambda exonuclease digestion for single-stranded DNA generation. Molecules 2010; 15: $1-11$.

[543] Williams KP, Bartel DP. PCR product with strands of unequal length. Nucleic Acids Res 1995; 23: 4220-4221.

[544] Citartan M, Tang TH, Tan SC, et al. Asymmetric PCR for good quality ssDNA generation towards DNA aptamer production. Songklanakarin J Sci Technol 2012; 34: $125-131$.

[545] Citartan M, Tang T-H, Tan S-C, et al. Conditions optimized for the preparation of single-stranded DNA (ssDNA) employing lambda exonuclease digestion in generating DNA aptamer. World J Microbiol Biotechnol 2011; 27: 1167-1173.

[546] Jing M, Bowser MT. Methods for measuring aptamer-protein equilibria: A review. Anal Chim Acta 2011; 686: 9-18.

[547] Mencin N, Šmuc T, Vraničar M, et al. Optimization of SELEX: Comparison of different methods for monitoring the progress of in vitro selection of aptamers. $J$ Pharm Biomed Anal 2014; 91: 151-159.

[548] Avci-Adali M, Wilhelm N, Perle N, et al. Absolute quantification of cell-bound DNA aptamers during SELEX. Nucleic Acid Ther 2013; 23: 125-30.

[549] Raddatz MSL, Dolf A, Endl E, et al. Enrichment of cell-targeting and populationspecific aptamers by fluorescence-activated cell sorting. Angew Chemie - Int Ed 2008; 47: 5190-5193.

[550] Vivekananda J, Kiel JL. Anti-Francisella tularensis DNA aptamers detect tularemia antigen from different subspecies by Aptamer-Linked Immobilized Sorbent Assay. Lab Invest 2006; 86: 610-8.

[551] Wang R, Zhao J, Jiang T, et al. Selection and characterization of DNA aptamers for use in detection of avian influenza virus H5N1. J Virol Methods 2013; 189: 362-369.

[552] Lee H-J, Kim BC, Kim K-W, et al. A sensitive method to detect Escherichia coli based on immunomagnetic separation and real-time PCR amplification of aptamers. Biosens Bioelectron 2009; 24: 3550-5.

[553] Savory N, Nzakizwanayo J, Abe K, et al. Selection of DNA aptamers against uropathogenic Escherichia coli NSM59 by quantitative PCR controlled Cell-SELEX. $J$ Microbiol Methods 2014; 104: 94-100. 
[554] Mayer G, Ahmed M-SLM, Dolf A, et al. Fluorescence-activated cell sorting for aptamer SELEX with cell mixtures. Nat Protoc 2010; 5: 1993-2004.

[555] Sambrook J, Russell DW. Isolation of DNA Fragments from Polyacrylamide Gels by the Crush and Soak Method. Cold Spring Harb Protoc 2006; 2006: pdb.prot2936pdb.prot2936.

[556] Millipore. Protein Blotting Handbook Tips and Tricks. Merck Millipore Protein blotting Handbook, http://iris.fishersci.ca/LitRepo.nsf/0/029DE90E3A5A41E985257AA1005B29E5/\$file/ 23067759.pdf (2012, accessed 26 September 2016).

[557] Valle A, Hoggard N, Adams AC, et al. Chronic central administration of apelin-13 over 10 days increases food intake, body weight, locomotor activity and body temperature in C57BL/6 mice. J Neuroendocr 2008; 20: 79-84.

[558] Kaltenbach M, Devenish SR a., Hollfelder F. A simple method to evaluate the biochemical compatibility of oil/surfactant mixtures for experiments in microdroplets. Lab Chip 2012; 12: 4185.

[559] Schneider CA, Rasband WS, Eliceiri KW. NIH Image to ImageJ: 25 years of image analysis. Nat Methods 2012; 9: 671-675.

[560] Tawfik DS, Griffiths AD. Man-made cell-like compartments for molecular evolution. Nat Biotechnol 1998; 16: 652-656.

[561] Zhong Q, Bhattacharya S, Kotsopoulos S, et al. Multiplex digital PCR: breaking the one target per color barrier of quantitative PCR. Lab Chip 2011; 11: 2167-74.

[562] Soundy J, Day D. Selection of DNA aptamers specific for live Pseudomonas aeruginosa. PLoS One 2017; 12: 1-11.

[563] Liu J, You M, Pu Y, et al. Recent developments in protein and cell-targeted aptamer selection and applications. Curr Med Chem 2011; 18: 4117-25.

[564] Ku TH, Zhang T, Luo H, et al. Nucleic acid aptamers: An emerging tool for biotechnology and biomedical sensing. Sensors (Switzerland) 2015; 15: 16281-16313.

[565] Vallée-Bélisle A, Plaxco KW. Structure-switching biosensors: Inspired by Nature. Curr Opin Struct Biol 2010; 20: 518-526.

[566] Mendonsa SD, Bowser MT. In vitro selection of high-affinity DNA ligands for human IgE using capillary electrophoresis. Anal Chem 2004; 76: 5387-5392.

[567] Takahashi M, Wu X, Ho M, et al. High throughput sequencing analysis of RNA libraries reveals the influences of initial library and PCR methods on SELEX efficiency. Sci Rep 2016; 6: 33697. 
[568] Kanagawa T. Bias and artifacts in multitemplate polymerase chain reactions (PCR). $J$ Biosci Bioeng 2003; 96: 317-323.

[569] Thiel WH, Bair T, Wyatt Thiel K, et al. Nucleotide bias observed with a short SELEX RNA aptamer library. Nucleic Acid Ther 2011; 21: 253-63.

[570] Bittker JA, Le B V, Liu DR. Nucleic acid evolution and minimization by nonhomologous random recombination. Nat Biotechnol 2002; 20: 1024-9.

[571] Murgha YE, Rouillard JM, Gulari E. Methods for the preparation of large quantities of complex single-stranded oligonucleotide libraries. PLoS One 2014; 9: 1-10.

[572] Arnold S, Pampalakis G, Kantiotou K, et al. One round of SELEX for the generation of DNA aptamers directed against KLK6. Biol Chem 2012; 393: 343-353.

[573] Alexander BM, Cloughesy TF. Adult Glioblastoma. J Clin Oncol 2017; 35: 24022409.

[574] Santosh V, Sravya P, Arivazhagan A. Molecular Pathology of Glioblastoma- An Update. In: Somasundaram K (ed) Advances in Biology and Treatment of Glioblastoma. Cham: Springer International Publishing, pp. 19-55.

[575] Naito H, Takara K, Wakabayashi T, et al. Changes in blood vessel maturation in the fibrous cap of the tumor rim. Cancer Sci 2012; 103: 433-8.

[576] Wu Y, Wang X, Zhou X, et al. Temporal Expression of Apelin/Apelin Receptor in Ischemic Stroke and its Therapeutic Potential. Front Mol Neurosci 2017; 10: 1-8.

[577] Chibon F. Cancer gene expression signatures-The rise and fall? Eur J Cancer 2013; 49: 2000-2009.

[578] Sager R. Expression genetics in cancer: shifting the focus from DNA to RNA. Proc Natl Acad Sci U S A 1997; 94: 952-955.

[579] Liang P, Pardee AB. Analysing differential gene expression in cancer. Nat Rev Cancer 2003; 3: 869-76.

[580] De Witt Hamer PC, Van Tilborg a a G, Eijk PP, et al. The genomic profile of human malignant glioma is altered early in primary cell culture and preserved in spheroids. Oncogene 2008; 27: 2091-2096.

[581] Zhou W, Chen C, Shi Y, et al. Targeting Glioma Stem Cell-Derived Pericytes Disrupts the Blood-Tumor Barrier and Improves Chemotherapeutic Efficacy. Cell Stem Cell 2017; 21: 591-603.e4.

[582] Wang L, Zhu Z-M, Zhang N-K, et al. Apelin: an endogenous peptide essential for cardiomyogenic differentiation of mesenchymal stem cells via activating extracellular signal-regulated kinase 1/2 and 5. Cell Biol Int 2016; 40: 501-514. 
[583] Inda M-M, Bonavia R, Mukasa A, et al. Tumor heterogeneity is an active process maintained by a mutant EGFR-induced cytokine circuit in glioblastoma. Genes Dev 2010; $24: 1731-45$.

[584] Inda M-D-M, Bonavia R, Seoane J. Glioblastoma multiforme: a look inside its heterogeneous nature. Cancers (Basel) 2014; 6: 226-39.

[585] Hubert CG, Rivera M, Spangler LC, et al. A Three-Dimensional Organoid Culture System Derived from Human Glioblastomas Recapitulates the Hypoxic Gradients and Cancer Stem Cell Heterogeneity of Tumors Found In Vivo. Cancer Res 2016; 76: 2465-2477.

[586] Niyazi M, Harter PN, Hattingen E, et al. Bevacizumab and radiotherapy for the treatment of glioblastoma: brothers in arms or unholy alliance? Oncotarget 2016; 7: 2313-28.

[587] Molina JR, Hayashi Y, Stephens C, et al. Invasive Glioblastoma Cells Acquire Stemness and Increased Akt Activation. Neoplasia 2010; 12: 453-IN5.

[588] Xu N, Wang H, Fan L, et al. Supraspinal administration of apelin-13 induces antinociception via the opioid receptor in mice. Peptides 2009; 30: 1153-7.

[589] Stepanenko AA, Heng HH. Transient and stable vector transfection: Pitfalls, off-target effects, artifacts. Mutat Res - Rev Mutat Res 2017; 773: 91-103.

[590] Winnard P, Glackin C, Raman V. Stable integration of an empty vector in MCF-7 cells greatly alters the karyotype. Cancer Genet Cytogenet 2006; 164: 174-176.

[591] Jacobsen LB, Calvin SA, Lobenhofer EK. Transcriptional effects of transfection: The potential for misinterpretation of gene expression data generated from transiently transfected cells. Biotechniques 2009; 47: 617-624.

[592] Yallop CA, Svendsen I. The effects of G418 on the growth and metabolism of recombinant mammalian cell lines. Cytotechnology 2001; 35: 101-114.

[593] Okuyama H, Endo H, Akashika T, et al. Downregulation of c-MYC protein levels contributes to cancer cell survival under dual deficiency of oxygen and glucose. Cancer Res 2010; 70: 10213-10223.

[594] Majmundar AJ, Wong WJ, Simon MC. Hypoxia-Inducible Factors and the Response to Hypoxic Stress. Mol Cell 2010; 40: 294-309.

[595] Méndez O, Zavadil J, Esencay M, et al. Knock down of HIF-1alpha in glioma cells reduces migration in vitro and invasion in vivo and impairs their ability to form tumor spheres. Mol Cancer 2010; 9: 133.

[596] Lao Z, Kelly CJ, Yang XY, et al. Improved methods to generate spheroid cultures from tumor cells, tumor cells \& fibroblasts or tumor-fragments: microenvironment, 
microvesicles and MiRNA. PLoS One 2015; 10: 1-19.

[597] Shoval H, Karsch-Bluman A, Brill-Karniely Y, et al. Tumor cells and their crosstalk with endothelial cells in 3D spheroids. Sci Rep 2017; 7: 1-11.

[598] Cheng V, Esteves F, Chakrabarty A, et al. High-content analysis of tumour cell invasion in three-dimensional spheroid assays. Oncoscience 2015; 2: 596.

[599] Evans SM, Judy KD, Dunphy I, et al. Comparative measurements of hypoxia in human brain tumors using needle electrodes and EF5 binding. Cancer Res 2004; 64: 1886-92.

[600] Richards R, Jenkinson MD, Haylock BJ, et al. Cell cycle progression in glioblastoma cells is unaffected by pathophysiological levels of hypoxia. PeerJ 2016; 4: e1755.

[601] Wolf A, Agnihotri S, Guha A. Targeting metabolic remodeling in glioblastoma multiforme. Oncotarget 2010; 1: 552-62.

[602] Heddleston JM, Li Z, McLendon RE, et al. The hypoxic microenvironment maintains glioblastoma stem cells and promotes reprogramming towards a cancer stem cell phenotype. Cell Cycle 2009; 8: 3274-3284.

[603] Corbeil D, Joester A, Fargeas CA, et al. Expression of distinct splice variants of the stem cell marker prominin-1 (CD133) in glial cells. Glia 2009; 57: 860-874.

[604] Yang ZJ, Chee CE, Huang S, et al. The role of autophagy in cancer: therapeutic implications. Mol Cancer Ther 2011; 10: 1533-41.

[605] Liang D, Han D, Fan W, et al. Therapeutic efficacy of apelin on transplanted mesenchymal stem cells in hindlimb ischemic mice via regulation of autophagy. Sci Rep 2016; 6: 21914.

[606] Ledur PF, Onzi GR, Zong H, et al. Culture conditions defining glioblastoma cells behavior: what is the impact for novel discoveries? Oncotarget 2017; 8: 69185-69197.

[607] Sakimoto S, Kidoya H, Naito H, et al. A role for endothelial cells in promoting the maturation of astrocytes through the apelin/APJ system in mice. Development 2012; 139: 1327-1335.

[608] Fidoamore A, Cristiano L, Antonosante A, et al. Glioblastoma Stem Cells Microenvironment: The Paracrine Roles of the Niche in Drug and Radioresistance. Stem Cells Int 2016; 2016: 6809105.

[609] Codrici E, Enciu A, Popescu I, et al. Glioma Stem Cells and Their Microenvironments: Providers of Challenging Therapeutic Targets. Stem Cells Int 2016; 2016: 1-20.

[610] Gilbertson R, Rich J. Making a tumour's bed: glioblastoma stem cells and the vascular niche. Nat Rev Cancer 2007; 7: 733-736.

[611] Roesch S, Rapp C, Dettling S, et al. When Immune Cells Turn Bad-Tumor- 
Associated Microglia/Macrophages in Glioma. Int J Mol Sci 2018; 19: 436.

[612] Obara S, Akifusa S, Ariyoshi W, et al. Pyroglutamated Apelin-13 Inhibits Lipopolysaccharide-Induced Production of Pro-Inflammatory Cytokines in Murine. Sci Res 2014; 3: 59-66.

[613] Shen X, Burguillos MA, Osman AM, et al. Glioma-induced inhibition of caspase-3 in microglia promotes a tumor-supportive phenotype. Nat Immunol 2016; 17: 1282-1290.

[614] Hambardzumyan D, Gutmann DH, Kettenmann H. The role of microglia and macrophages in glioma maintenance and progression. Nat Neurosci 2015; 19: 20-27.

[615] Skov Jensen S, Aaberg-Jessen C, Pind I, et al. Three-Dimensional In Vitro Models in Glioma Research - Focus on Spheroids. In: Ghosh A (ed) Glioma - Exploring Its Biology and Practical Relevance. InTech, p. 33.

[616] Riffle S, Hegde RS. Modeling tumor cell adaptations to hypoxia in multicellular tumor spheroids. J Exp Clin Cancer Res 2017; 36: 1-10.

[617] Langan LM, Dodd NJF, Owen SF, et al. Correction: Direct measurements of oxygen gradients in spheroid culture system using electron paramagnetic resonance oximetry. PLoS One 2016; 11: 1-13.

[618] Browne SM, Al-Rubeai M. Selection methods for high-producing mammalian cell lines. Trends Biotechnol 2007; 25: 425-432.

[619] Mathupala SP, Sloan AE. An agarose-based cloning-ring anchoring method for isolation of viable cell clones. Biotechniques 2009; 46: 305-307.

[620] Strukov YG, Belmont AS. Development of mammalian cells lines with lac operatortagged chromosomes. Cold Spring Harb Protoc 2008; 3: 1-9.

[621] Natarajan S, Chow TT, Shay JW, et al. Replica plating of mammalian cells using low melt agarose. Cytotechnology 2007; 54: 145-147.

[622] Davis JM, Pennington JE, Kubler AM, et al. A simple, single-step technique for selecting and cloning hybridomas for the production of monoclonal antibodies. $J$ Immunol Methods 1982; 50: 161-171.

[623] Puck TT, Marcus PI. a Rapid Method for Viable Cell Titration and Clone Production With Hela Cells in Tissue Culture: the Use of X-Irradiated Cells To Supply Conditioning Factors*. Proc Natl Acad Sci U S A 1955; 41: 432-437.

[624] Staszewski R. Cloning by limiting dilution: an improved estimate that an interesting culture is monoclonal. Yale J Biol Med 1984; 57: 865-8.

[625] Underwood PA, Bean PA. Hazards of the limiting-dilution method of cloning hybridomas. J Immunol Methods 1988; 107: 119-128. 
[626] Freimark D, Jérôme V, Freitag R. A GFP-based method facilitates clonal selection of transfected CHO cells. Biotechnol J 2010; 5: 24-31.

[627] Sun B, Zhang S, Zhang D, et al. Vasculogenic mimicry is associated with high tumor grade, invasion and metastasis, and short survival in patients with hepatocellular carcinoma. Oncol Rep 2006; 16: 693-8.

[628] Mao JM, Liu J, Guo G, et al. Glioblastoma vasculogenic mimicry: signaling pathways progression and potential anti-angiogenesis targets. Biomark Res 2015; 3: 8 .

[629] Meenu Jain, Ali S Arbab, BR Achyut. Vascular Mimicry: The Next Big Glioblastoma Target. Biochem Physiol Open Access 2015; 04: 4-9.

[630] Chen YS, Chen ZP. Vasculogenic mimicry: A novel target for glioma therapy. Chin J Cancer 2014; 33: 74-79.

[631] Kibbey MC. Maintenance of the EHS sarcoma and Matrigel preparation. J Tissue Cult Methods 1994; 16: 227-230.

[632] Arnaoutova I, George J, Kleinman HK, et al. The endothelial cell tube formation assay on basement membrane turns 20: State of the science and the art. Angiogenesis 2009; 12: $267-274$. 